\title{
Enantioselective Catalytic Allylation of Acyclic Ketiminoesters: Synthesis of $\alpha$-Fully-Substituted Amino Esters
}

\author{
Urmibhusan Bhakta, ${ }^{\dagger}$ Padmanabha V. Kattamuri, ${ }^{\dagger}$ Juha H. Siitonen, ${ }^{\dagger}$ Lawrence B. Alemany $y^{\#, \dagger}$ \\ and László Kürti๋*
}

${ }^{\dagger}$ Department of Chemistry, Rice University, BioScience Research Collaborative, Houston, TX 77005, USA.

"Shared Equipment Authority, Rice University, 6100 Main Street, Houston, TX 77005, USA.

\section{Correspondence to:}

kurti.laszlo@ rice.edu 


\section{TABLE OF CONTENTS}

1. General Experimental Procedures:

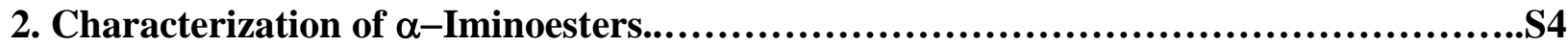

3. Attempted Enantioselective Ag-catalyzed Allylation of $\alpha$-Ketiminoesters by Following Yamamoto Procedure........................................................................S10

4. Optimization Table for Catalytic Asymmetric Allylboration of $\alpha$-Ketiminoesters......S10

5. General Procedure for Catalytic Asymmetric Allylboration of Keiminoesters 1a-u....S12

6. Optimization Table for Catalytic Asymmetric Allylboration of $\alpha$-Trifluoromethyl $\alpha$ -

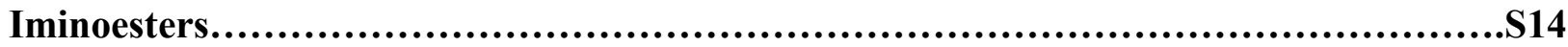

7. General Procedure for Catalytic Asymmetric Allylboration of $\alpha$-Trifluoromethyl $\alpha$ Iminoesters 4a-i.

8. General Procedure for Hydroboration-Oxidation of Homoallylic aminoesters...

9. Transformations of the Homoallylic Amine Products into the Pyrrolidine Structures

10. Characterization of Catalytic Asymmetric Allylation Products in Table 2............S17

11. Characterization of Catalytic Asymmetric Allylation Products in Table 3............S35

12. Transformation of the Chiral Homoallylic Amine Products into the Pyrrolidine Structures.............................................................................S47

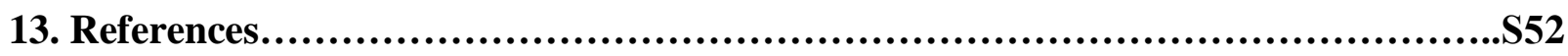

14. NMR Spectra of All the compounds...........................................S53

15. HPLC Chromatograms 


\section{General Experimental Procedures:}

All the reagents were purchased at the highest quality from commercially available sources and used without further purification. All the ligands were purchased from commercially available sources. All the solvents for the reactions were obtained from pure process technology solvent system by passing the previously degassed solvents through an activated alumina column under argon. All reactions were carried out in either flame-dried or oven-dried glass ware under an atmosphere of argon with magnetic stirring. All reactions were monitored by thin layer chromatography (TLC) carried out on $0.25 \mathrm{~mm}$ pre-coated E. Merck silica plates (60F-254), using shortwave UV light as a visualizing agent and $\mathrm{KMnO}_{4}$ or phosphomolybdic acid (PMA) and heat as developing agents. Flash column chromatography was performed using Biotage Isolera One automated chromatograph with pre-packed KP-Sil cartridges. $1 \mathrm{D}{ }^{1} \mathrm{H}$ and ${ }^{13} \mathrm{C}$ NMR spectra were recorded on Bruker Avance III HD 600 spectrometer operating at $600 \mathrm{MHz}$ for ${ }^{1} \mathrm{H}$ and $151 \mathrm{MHz}$ for ${ }^{13} \mathrm{C}$. The spectra were calibrated using either TMS or the solvent as an internal reference (residual $\mathrm{CHCl}_{3}: 7.26 \mathrm{ppm}{ }^{1} \mathrm{H} \mathrm{NMR} ; \mathrm{CDCl}_{3}: 77.00 \mathrm{ppm}{ }^{13} \mathrm{C} \mathrm{NMR}$ ). ${ }^{19} \mathrm{~F}$ NMR spectra at $471 \mathrm{MHz}$ were recorded on the $500 \mathrm{MHz}$ spectrometer, and the chemical shifts were relative to $\mathrm{CF}^{35} \mathrm{Cl}_{3}$ defined as 0 ppm. For reporting NMR peak multiplicities, the following abbreviations were used: $\mathrm{s}=$ singlet, $\mathrm{d}=$ doublet, $\mathrm{t}=$ triplet, $\mathrm{q}=$ quartet, $\mathrm{p}=$ pentet, hept $=$ heptet, $\mathrm{m}=$ multiplet. Highresolution mass spectra (HRMS) were recorded on an Agilent UHPLC TOF mass spectrometer using electrospray ionization time-of-flight (ESI-TOF) or chemical ionization time-of-flight (CITOF) reflectron experiments. IR spectra were recorded with Bruker Alpha Platinum ATR single reflector spectrometer and the compound was applied as a thin film directly on the ATR unit (neat or in dichloromethane). Data are represented as the most characteristic absorption frequencies in $\mathrm{cm}^{-1}$.The melting points and ranges were recorded on Mettler Toledo MP50 melting point system, and are uncorrected. Enantiomeric excesses of the compounds were determined using Agilent chiral stationary phase (CSP) HPLC or SFC on AD, IA or IB columns. $\alpha$-iminoesters were synthesized by following the literature procedure ${ }^{1 \mathrm{a}, 1 \mathrm{~b}}$ and their spectral data $\left[(\mathbf{1 a}, \mathbf{1 b}, \mathbf{1 j}, \mathbf{1 n}, \mathbf{1 r}){ }^{1}\right.$ $(\mathbf{1 c}, \mathbf{1 d}, \mathbf{1 f}, \mathbf{1 i}, \mathbf{1 j}) ;^{2}(\mathbf{1 e}, \mathbf{1 t}) ;^{3}(\mathbf{1 m})^{4}$ and $(\mathbf{4 a}-\mathbf{4 e}, \mathbf{4 j})^{1 \mathrm{~b}}$ ] agreed with those reported previously. 


\section{Synthesis and Characterization of $\alpha$-Iminoesters:}

All new imines were prepared using the literature procedure with no modifications. ${ }^{\text {a,b }}$

1. Methyl (Z)-4-((2-methoxy-2-oxm1-phenylethylidene)amino)benzoate (1g):

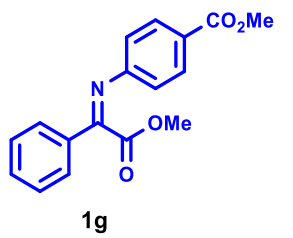

Yield: $66 \%, 1.96 \mathrm{~g} \mathrm{(10} \mathrm{mmol} \mathrm{scale,} \mathrm{16:1} \mathrm{Z:E);}$

Physical State: Yellowish white solid;

M.P: $107-109^{\circ} \mathrm{C}$;

$\boldsymbol{R}_{\mathbf{f}}=0.48(10 \% \mathrm{EA} / \mathrm{Hex})$

${ }^{1} \mathrm{H}$ NMR of major isomer $(600 \mathrm{MHz}$, Chloroform- $d) \delta 8.03(\mathrm{~d}, J=8.5 \mathrm{~Hz}, 2 \mathrm{H}), 7.88(\mathrm{~d}, J=7.3$ $\mathrm{Hz}, 2 \mathrm{H}), 7.55$ (t, $J=7.4 \mathrm{~Hz}, 1 \mathrm{H}), 7.48(\mathrm{t}, J=7.6 \mathrm{~Hz}, 2 \mathrm{H}), 6.98(\mathrm{~d}, J=8.5 \mathrm{~Hz}, 2 \mathrm{H}), 3.91(\mathrm{~s}, 3 \mathrm{H})$, $3.63(\mathrm{~s}, 3 \mathrm{H})$;

${ }^{13}$ C NMR of major isomer (151 MHz, Chloroform- $\left.d\right) \delta$ 166.7, 164.7, 160.4, 154.3, 133.3, 132.2, $130.6,128.8,128.2,126.5,119.2,52.0,51.9$;

FTIR (ATR, $\left.\mathbf{c m}^{-1}\right):$ 1737, 1705, 1598, 1449, 1436, 1429, 1281, 1230, 1199, 1151, 1115, 1101, $1005,868,74,695,685$.

HRMS (ESI-TOF): calc'd for $\mathrm{C}_{17} \mathrm{H}_{16} \mathrm{NO}_{4}[\mathrm{M}+\mathrm{H}]^{+}$298.1074; found 298.1076.

2. Methyl (Z)-2-(naphthalen-2-ylimino)-2-phenylacetate (1h):

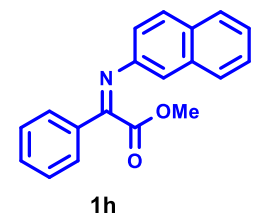

Yield: $55 \%, 1.59 \mathrm{~g}(10 \mathrm{mmol}$ scale, $20: 1 \mathrm{Z:E})$;

Physical State: Yellowish grey solid;

M.P: $93-95{ }^{\circ} \mathrm{C}$;

$\boldsymbol{R}_{\mathbf{f}}=0.55(10 \% \mathrm{EA} / \mathrm{Hex})$ 
${ }^{\mathbf{1}} \mathrm{H}$ NMR of major isomer $(\mathbf{6 0 0} \mathrm{MHz}$, Chloroform- $\boldsymbol{d}) \delta 8.03(\mathrm{~d}, J=7.7 \mathrm{~Hz}, 3 \mathrm{H}), 7.85(\mathrm{~d}, J=8.1$ $\mathrm{Hz}, 1 \mathrm{H}), 7.66(\mathrm{~d}, J=8.3 \mathrm{~Hz}, 1 \mathrm{H}), 7.58(\mathrm{t}, J=7.3 \mathrm{~Hz}, 1 \mathrm{H}), 7.53(\mathrm{t}, J=8.3 \mathrm{~Hz}, 3 \mathrm{H}), 7.49(\mathrm{t}, J=7.0$ $\mathrm{Hz}, 1 \mathrm{H}), 7.40$ (t, $J=7.8 \mathrm{~Hz}, 1 \mathrm{H}), 6.90(\mathrm{~d}, J=7.2 \mathrm{~Hz}, 1 \mathrm{H}), 3.53$ (s, 3H);

${ }^{13} \mathrm{C}$ NMR of major isomer (151 MHz, Chloroform-d) $\delta$ 165.4, 160.0, 146.6, 133.86, 133.85, $131.9,128.8,128.2,127.7,127.2,126.4,125.7,125.5,125.2,124.0,112.8,51.8$;

FTIR (ATR, $\left.\mathbf{c m}^{-1}\right): 1731,1708,1597,1449,1428,1282,1232,1200,1160,1116,1102,1004$, $868,774,692$.

HRMS (ESI-TOF): calc'd for $\mathrm{C}_{19} \mathrm{H}_{16} \mathrm{NO}_{2}[\mathrm{M}+\mathrm{H}]^{+}$290.1176; found 290.1183.

3. Methyl (Z)-2-(4-methoxyphenyl)-2-((4-(trifluoromethyl)phenyl)imino)acetate (1k):

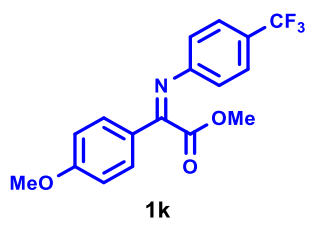

Yield: $53 \%, 1.78 \mathrm{~g}(10 \mathrm{mmol}$ scale, $20: 1 \mathrm{Z:E})$;

Physical State: Grey solid;

M.P: $70-72^{\circ} \mathrm{C}$;

$\boldsymbol{R}_{\mathbf{f}}=0.51(10 \% \mathrm{EA} / \mathrm{Hex})$

${ }^{1} \mathbf{H}$ NMR of major isomer $(600 \mathrm{MHz}$, Chloroform- $d) \delta 7.82(\mathrm{~d}, J=8.8 \mathrm{~Hz}, 2 \mathrm{H}), 7.58(\mathrm{~d}, J=8.3$ $\mathrm{Hz}, 2 \mathrm{H}), 7.01(\mathrm{~d}, J=8.2 \mathrm{~Hz}, 2 \mathrm{H}), 6.98(\mathrm{~d}, J=8.8 \mathrm{~Hz}, 2 \mathrm{H}), 3.88(\mathrm{~s}, 3 \mathrm{H}), 3.63(\mathrm{~s}, 3 \mathrm{H})$;

${ }^{13}$ C NMR of major isomer (151 MHz, Chloroform- $d$ ) $\delta 164.9,163.0,160.1,153.4,130.1,126.5$ $(\mathrm{q}, J=32.6 \mathrm{~Hz}), 126.1-125.8(\mathrm{~m}), 124.3(\mathrm{q}, J=271.6 \mathrm{~Hz}), 119.8,114.2,55.5,51.9$;

FTIR (ATR, $\left.\mathbf{c m}^{-1}\right):$ 1737, 1709, 1593, 1515, 1448, 1319, 1231, 1201, 1160, 1101, 1013, 1004, $851,835,684$.

HRMS (ESI-TOF): calc'd for $\mathrm{C}_{17} \mathrm{H}_{15} \mathrm{~F}_{3} \mathrm{NO}_{3}[\mathrm{M}+\mathrm{H}]^{+}$338.0999; found 338.1008.

4. Methyl (Z)-2-((4-chlorophenyl)imino)-2-(4-methoxyphenyl)acetate (11): 


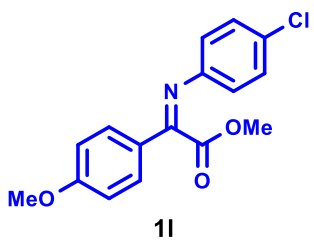

Yield: 67\%, $2.04 \mathrm{~g}$ (10 mmol scale, 14:1 Z:E);

Physical State: Yellow solid;

M.P: $65-67^{\circ} \mathrm{C}$;

$\boldsymbol{R}_{\mathbf{f}}=0.51(10 \% \mathrm{EA} / \mathrm{Hex})$

${ }^{1}$ H NMR of major isomer (600 MHz, Chloroform- $\left.d\right) \delta 7.80(\mathrm{~d}, J=8.8 \mathrm{~Hz}, 2 \mathrm{H}), 7.28(\mathrm{~d}, J=8.5$ $\mathrm{Hz}, 2 \mathrm{H}), 6.97$ (d, $J=8.8 \mathrm{~Hz}, 2 \mathrm{H}), 6.88$ (d, $J=8.5 \mathrm{~Hz}, 2 \mathrm{H}), 3.87$ (s, 3H), 3.66 (s, 3H);

${ }^{13}$ C NMR of major isomer (151 MHz, Chloroform-d) $\delta$ 165.4, 162.8, 159.8, 148.8, 130.1, 129.9, $128.9,126.3,121.1,114.2,55.5,51.9$;

FTIR (ATR, $\left.\mathbf{c m}^{-1}\right):$ 1728, 1608, 1504, 1437, 1317, 1290, 1250, 1231, 1197, 1175, 1163, 1031, $1012,830,671,736$.

HRMS (ESI-TOF): calc'd for $\mathrm{C}_{16} \mathrm{H}_{15} \mathrm{ClNO}_{3}[\mathrm{M}+\mathrm{H}]^{+}$304.0735; found 304.0745.

5. Methyl (Z)-2-((3-bromophenyl)imino)-2-(4-chlorophenyl)acetate (10):

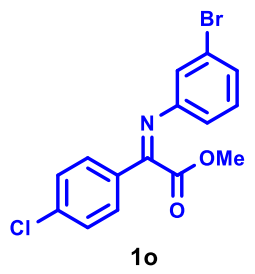

Yield: 79\%, $2.76 \mathrm{~g}$ (10 mmol scale, 19:1 Z:E);

Physical State: Yellow solid;

M.P: $66-69^{\circ} \mathrm{C}$;

$\boldsymbol{R}_{\mathbf{f}}=0.58(10 \% \mathrm{EA} / \mathrm{Hex})$

${ }^{\mathbf{1}} \mathrm{H}$ NMR of major isomer $(\mathbf{6 0 0} \mathrm{MHz}$, Chloroform- $d) \delta 7.80(\mathrm{~d}, J=8.5 \mathrm{~Hz}, 2 \mathrm{H}), 7.45(\mathrm{~d}, J=8.5$ $\mathrm{Hz}, 2 \mathrm{H}), 7.28(\mathrm{~d}, J=8.0 \mathrm{~Hz}, 1 \mathrm{H}), 7.20(\mathrm{t}, J=7.9 \mathrm{~Hz}, 1 \mathrm{H}), 7.13(\mathrm{~s}, 1 \mathrm{H}), 6.87(\mathrm{~d}, J=8.5 \mathrm{~Hz}, 1 \mathrm{H})$, $3.68(\mathrm{~s}, 3 \mathrm{H})$; 
${ }^{13}$ C NMR of major isomer (151 MHz, Chloroform- $\left.\boldsymbol{d}\right) \boldsymbol{\delta} 164.6,159.5,151.0,138.5,131.9,130.2$, $129.4,129.1,128.0,122.6,122.5,118.1,52.1$;

FTIR (ATR, $\mathbf{c m}^{-\mathbf{1}}$ ): 1723, 1617, 1579, 1563, 1492, 1463, 1435, 1403, 121, 1234, 1195, 1171, 1088, 1019, 1009, 9238, 966, 826, 770, 712, 693, 669.

HRMS (ESI-TOF): calc'd for $\mathrm{C}_{15} \mathrm{H}_{12} \mathrm{BrClNO}_{2}[\mathrm{M}+\mathrm{H}]^{+} 351.9734$; found 351.9728 .

6. Methyl (Z)-2-(4-chlorophenyl)-2-((3-fluorophenyl)imino)acetate (1p):

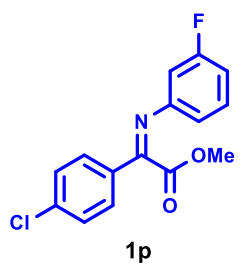

Yield: 59\%, $1.71 \mathrm{~g} \mathrm{(10} \mathrm{mmol} \mathrm{scale,} \mathrm{19:1} \mathrm{Z:E);}$

Physical State: Yellow solid;

M.P: $56-58^{\circ} \mathrm{C}$;

$\boldsymbol{R}_{\mathbf{f}}=0.60(10 \% \mathrm{EA} / \mathrm{Hex})$

${ }^{1} \mathrm{H}$ NMR of major isomer $(600 \mathrm{MHz}$, Chloroform- $d) \delta 7.81(\mathrm{~d}, J=8.4 \mathrm{~Hz}, 2 \mathrm{H}), 7.45(\mathrm{~d}, J=8.4$ $\mathrm{Hz}, 2 \mathrm{H}), 7.32-7.22(\mathrm{~m}, 1 \mathrm{H}), 6.86(\mathrm{t}, J=7.6 \mathrm{~Hz}, 1 \mathrm{H}), 6.70(\mathrm{t}, J=8.1 \mathrm{~Hz}, 2 \mathrm{H}), 3.67(\mathrm{~s}, 3 \mathrm{H})$;

${ }^{13} \mathrm{C}$ NMR of major isomer (151 MHz, Chloroform-d) $\delta 164.6,163.0(\mathrm{~d}, J=246.7 \mathrm{~Hz}), 159.4$, $151.4(\mathrm{~d}, J=9.3 \mathrm{~Hz}), 138.4,131.9,130.2(\mathrm{~d}, J=9.3 \mathrm{~Hz}), 129.4,129.1,115.0(\mathrm{~d}, J=2.9 \mathrm{~Hz}), 111.8$ $(\mathrm{d}, J=21.3 \mathrm{~Hz}), 107.1(\mathrm{~d}, J=23.6 \mathrm{~Hz}), 52.1$;

FTIR (ATR, $\left.\mathbf{c m}^{-1}\right):$ 1723, 1617, 1579, 1564, 1492, 1563, 1435, 1403, 1322, 1234, 1196, 1171, 1089, 1019, 1008, 928, 896, 866, 926, 771, 693, 670 .

HRMS (ESI-TOF): calc'd for $\mathrm{C}_{15} \mathrm{H}_{12} \mathrm{ClFNO}_{2}[\mathrm{M}+\mathrm{H}]^{+} 292.0535$; found 292.0542 .

7. Methyl (Z)-2-(4-fluorophenyl)-2-(phenylimino)acetate (1q):

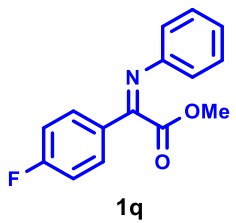

Yield: $75 \%, 1.93 \mathrm{~g}$ (10 mmol scale, 16:1 Z:E); 
Physical State: Yellow solid;

M.P: $57-59^{\circ} \mathrm{C}$;

$\boldsymbol{R}_{\mathbf{f}}=0.61(10 \% \mathrm{EA} / \mathrm{Hex})$

${ }^{1} \mathrm{H}$ NMR of major isomer $(600 \mathrm{MHz}$, Chloroform- $\boldsymbol{d}) \delta 7.93-7.87(\mathrm{~m}, 2 \mathrm{H}), 7.34(\mathrm{t}, J=7.9 \mathrm{~Hz}$, $2 \mathrm{H}), 7.18-7.12(\mathrm{~m}, 3 \mathrm{H}), 6.98-6.92(\mathrm{~m}, 2 \mathrm{H}), 3.63(\mathrm{~s}, 3 \mathrm{H})$;

${ }^{13} \mathrm{C}$ NMR of major isomer (151 MHz, Chloroform- $\left.d\right) \delta 165.3,165.0(\mathrm{~d}, J=253.3 \mathrm{~Hz}), 158.5$, $149.9,130.2(\mathrm{~d}, J=8.9 \mathrm{~Hz}), 130.1(\mathrm{~d}, J=3.1 \mathrm{~Hz}), 128.9,125.0,119.5,115.9(\mathrm{~d}, J=21.9 \mathrm{~Hz})$, 51.9

FTIR (ATR, cm$\left.^{-1}\right):$ 1729, 1589, 1508, 1482, 1411, 1316, 1225, 1193, 1167, 1154, 1007, 850, 809, 766, 698.

HRMS (ESI-TOF): calc'd for $\mathrm{C}_{15} \mathrm{H}_{13} \mathrm{FNO}_{2}[\mathrm{M}+\mathrm{H}]^{+}$258.0925; found 258.0938 .

8. Methyl (Z)-2-(phenylimino)-2-(p-tolyl)acetate (1s):

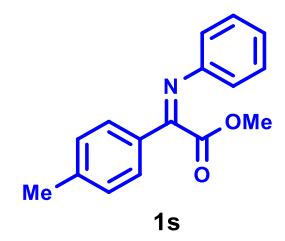

Yield: $73 \%, 1.84 \mathrm{~g} \mathrm{(10} \mathrm{mmol} \mathrm{scale,} \mathrm{16:1} \mathrm{Z:E);}$

Physical State: Pale yellow solid;

M.P: $56-58^{\circ} \mathrm{C}$;

$\boldsymbol{R}_{\mathbf{f}}=0.52(10 \% \mathrm{EA} / \mathrm{Hex})$

${ }^{1} \mathbf{H}$ NMR of major isomer $(600 \mathrm{MHz}$, Chloroform- $d) \delta 7.76(\mathrm{~d}, J=8.2 \mathrm{~Hz}, 2 \mathrm{H}), 7.33(\mathrm{t}, J=7.9$ $\mathrm{Hz}, 2 \mathrm{H}), 7.27(\mathrm{~d}, J=8.0 \mathrm{~Hz}, 2 \mathrm{H}), 7.13(\mathrm{t}, J=7.4 \mathrm{~Hz}, 1 \mathrm{H}), 6.96(\mathrm{~d}, J=7.4 \mathrm{~Hz}, 2 \mathrm{H}), 3.63(\mathrm{~s}, 3 \mathrm{H})$, $2.42(\mathrm{~s}, 3 \mathrm{H})$;

${ }^{13} \mathbf{C}$ NMR of major isomer (151 MHz, Chloroform- $d$ ) $\delta$ 165.5, 159.9, 150.2, 142.4, 131.2, 129.4, $128.8,128.0,124.8,119.6,51.8,21.6$;

FTIR (ATR, cm$\left.^{-1}\right)$ : 1728, 1608, 1504, 1437, 1289, 1250, 1232, 1196, 1175, 1162, 630, 760.

HRMS (ESI-TOF): calc'd for $\mathrm{C}_{16} \mathrm{H}_{16} \mathrm{NO}_{2}[\mathrm{M}+\mathrm{H}]^{+} 254.1176$; found 254.1193.

9. Ethyl (E)-3,3,3-trifluoro-2-((4-(methylthio)phenyl)imino)propanoate (4f): 


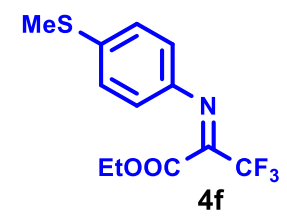

Yield: 95\%, $1.73 \mathrm{~g}(6.25 \mathrm{mmol}$ scale $)$;

Physical State: Brownish yellow colored wax;

$\mathbf{R}_{f}=0.45$ (10\% EtOAc/hexanes);

${ }^{1}$ H NMR (600 MHz, Chloroform-d): $\delta 7.25-7.22(\mathrm{~m}, 2 \mathrm{H}), 6.95-6.91(\mathrm{~m}, 2 \mathrm{H}), 4.22$ (q, $J=$ $7.1 \mathrm{~Hz}, 2 \mathrm{H}), 2.49$ (s, 3H), $1.15(\mathrm{t}, J=7.1 \mathrm{~Hz}, 3 \mathrm{H})$;

${ }^{13}$ C NMR (151 MHz, Chloroform-d): $\delta$ 159.6, 148.2 (q, $J=36.9$ Hz), 143.2, 138.3, 126.8, $120.5,118.2(\mathrm{q}, J=278.4 \mathrm{~Hz}), 62.8,15.7,13.6$;

${ }^{19}$ F NMR (471 MHz, Chloroform-d): $-69.6(\mathrm{~s})$;

FTIR (ATR, $\left.\mathbf{c m}^{-1}\right)$ : 1739, 1488, 1321, 1247, 1229, 1179, 1153, 1134, 1094, 1033, 856, 837, 710.

HRMS (ESI-TOF): calc'd for $\mathrm{C}_{12} \mathrm{H}_{13} \mathrm{~F}_{3} \mathrm{NO}_{2} \mathrm{~S}[\mathrm{M}+\mathrm{H}]^{+} 292.0614$; Found 292.0613.

10. Ethyl (E)-2-((3-chlorophenyl)imino)-3,3,3-trifluoropropanoate (4g):

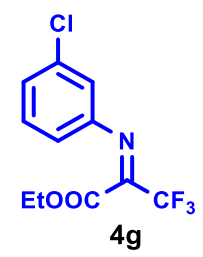

Yield: $71 \%, 0.77 \mathrm{~g}(3.86 \mathrm{mmol}$ scale $)$;

Physical State: Yellow colored oily liquid;

$\boldsymbol{R}_{\mathbf{f}}=0.57(10 \% \mathrm{EtOAc/hexanes)}$

${ }^{1}$ H NMR (600 MHz, Chloroform- $\left.d\right)$ : $\delta 7.30(\mathrm{t}, J=8.0 \mathrm{~Hz}, 1 \mathrm{H}), 7.24-7.21(\mathrm{~m}, 1 \mathrm{H}), 6.96(\mathrm{t}, J=$ $1.8 \mathrm{~Hz}, 1 \mathrm{H}), 6.85-6.80(\mathrm{~m}, 1 \mathrm{H}), 4.22(\mathrm{q}, J=7.1 \mathrm{~Hz}, 2 \mathrm{H}), 1.12(\mathrm{t}, J=7.1 \mathrm{~Hz}, 3 \mathrm{H})$;

${ }^{13}$ C NMR (151 MHz, Chloroform-d): $\delta$ 158.7, 150.0 (q, $J=37.3 \mathrm{~Hz}$ ), 147.5, 135.0, 130.2, $126.8,119.2,118.0(\mathrm{q}, J=278.7 \mathrm{~Hz}), 117.3,63.0,13.6$;

${ }^{19}$ F NMR (471 MHz, Chloroform-d): -69.9 (s); 
FTIR (ATR, cm$^{-1}$ ): 2963, 746, 1325, 1257, 1188, 1156, 1030, 1011, 789, 685.

HRMS (ESI-TOF): calc'd for $\mathrm{C}_{11} \mathrm{H}_{10} \mathrm{ClF}_{3} \mathrm{NO}_{2}[\mathrm{M}+\mathrm{H}]^{+} 280.0347$; Found 280.0333 .

11. Ethyl (E)-2-((2-bromophenyl)imino)-3,3,3-trifluoropropanoate: (4h)

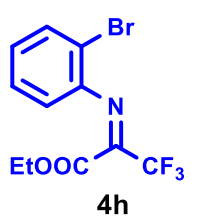

Yield: $99 \%, 0.37 \mathrm{~g}(1.15 \mathrm{mmol}$ scale $)$;

Physical State: Yellow colored oily liquid;

$\boldsymbol{R}_{\mathbf{f}}=0.43(10 \% \mathrm{EtOAc} / \mathrm{hexanes}) ;$

${ }^{1}$ H NMR (600 MHz, Chloroform-d): $\delta 7.62-7.58(\mathrm{~m}, 1 \mathrm{H}), 7.31-7.26(\mathrm{~m}, 1 \mathrm{H}), 7.08$ (td, $J=$ 8.0, $1.4 \mathrm{~Hz}, 1 \mathrm{H}), 6.77(\mathrm{dd}, J=7.9,1.3 \mathrm{~Hz}, 1 \mathrm{H}), 4.16(\mathrm{q}, J=7.1 \mathrm{~Hz}, 2 \mathrm{H}), 1.05(\mathrm{t}, J=7.1 \mathrm{~Hz}, 3 \mathrm{H})$;

${ }^{13}$ C NMR (151 MHz, Chloroform-d): $\delta 157.7,150.1$ (q, $\left.J=37.0 \mathrm{~Hz}\right), 145.7,133.0,127.8$, $127.4,118.2,118.0(\mathrm{q}, J=278.7 \mathrm{~Hz}), 113.0,62.9,13.4$;

${ }^{19}$ F NMR (471 MHz, Chloroform- $d$ ): -69.7 (s);

FTIR (ATR, cm$^{-1}$ ): 2963, 1747, 1467, 1257, 1010, 789, 755.

HRMS (ESI-TOF): calc'd for $\mathrm{C}_{11} \mathrm{H}_{10} \mathrm{BrF}_{3} \mathrm{NO}_{2}[\mathrm{M}+\mathrm{H}]^{+} 323.9842$; Found 323.9838 .

12. Ethyl (E)-3,3,3-trifluoro-2-((2-iodophenyl)imino)propanoate: (4h)

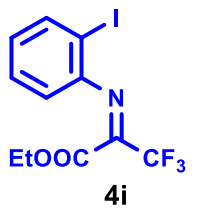

Yield: $87 \%, 0.99 \mathrm{~g}(3.10 \mathrm{mmol}$ scale);

Physical State: Yellow colored oily liquid;

$\boldsymbol{R}_{\mathbf{f}}=0.51(10 \%$ EtOAc/hexanes);

${ }^{1}$ H NMR (600 MHz, Chloroform- $\left.\boldsymbol{d}\right): \delta 7.87(\mathrm{~d}, J=7.9 \mathrm{~Hz}, 1 \mathrm{H}), 7.32(\mathrm{t}, J=7.6 \mathrm{~Hz}, 1 \mathrm{H}), 6.94$ $(\mathrm{td}, J=7.7,1.3 \mathrm{~Hz}, 1 \mathrm{H}), 6.73(\mathrm{dd}, J=7.8,1.2 \mathrm{~Hz}, 1 \mathrm{H}), 4.17(\mathrm{q}, J=7.1 \mathrm{~Hz}, 2 \mathrm{H}), 1.05(\mathrm{t}, J=7.1$ $\mathrm{Hz}, 3 \mathrm{H})$; 
${ }^{13}$ C NMR (151 MHz, Chloroform-d): $\delta$ 158.0, 149.7 (q, $J=37.1 \mathrm{~Hz}$ ), 148.6, 139.2, 128.7, $127.7,118.0(\mathrm{q}, J=278.9 \mathrm{~Hz}), 117.3,88.0,62.9,13.5$;

FTIR (ATR, $\left.\mathbf{c m}^{-1}\right):$ 2965, 1742, 1641, 1325, 1250, 1189, 1150, 1047, 1033, 1018, 756.

${ }^{19}$ F NMR (471 MHz, Chloroform-d): -69.7(s);

HRMS (ESI-TOF): calc'd for $\mathrm{C}_{11} \mathrm{H}_{10} \mathrm{IF}_{3} \mathrm{NO}_{2}[\mathrm{M}+\mathrm{H}]^{+} 371.9703$; Found 371.9704 .

3. Attempted Enantioselective Ag-catalyzed Allylation of $\alpha$-Ketiminoesters by Following Yamamoto Procedure: 5
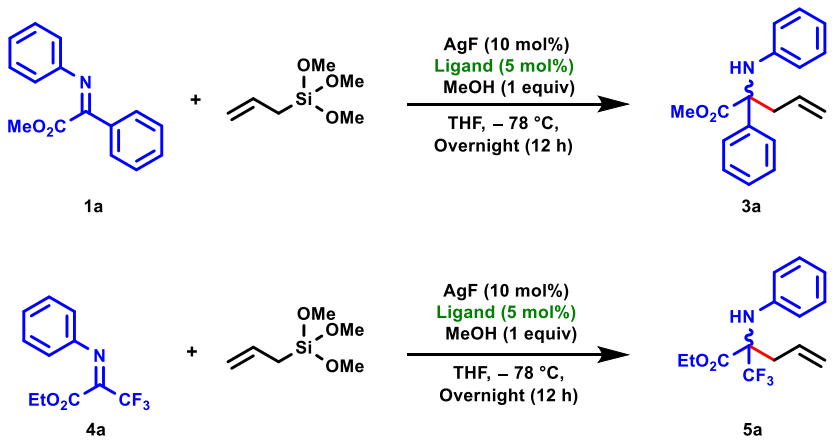

\begin{tabular}{ccccc}
\hline Ligand & $\begin{array}{c}\text { Yield }(\%) \\
(\mathbf{3 a})\end{array}$ & $\begin{array}{c}e e(\%) \\
(\mathbf{3 a})\end{array}$ & $\begin{array}{c}\text { Yield }(\%) \\
(\mathbf{5 a})\end{array}$ & $\begin{array}{c}e e(\%) \\
(\mathbf{5 a})\end{array}$ \\
\hline$R$-BINAP & 85 & - & 82 & - \\
$R$-DIFLUOROPHOS & 88 & - & 90 & - \\
\hline
\end{tabular}

4. Optimization Table for Catalytic Asymmetric Allylboration of $\alpha$-Ketiminoesters:

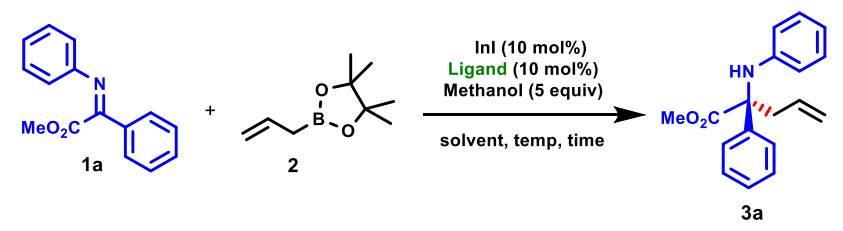

\begin{tabular}{|c|c|c|c|c|c|}
\hline Ligand $^{a}$ & Solvent $^{\mathbf{b}}$ & $\begin{array}{l}\text { Additive } \\
\text { (equiv.) }\end{array}$ & Temp (Time) & Yield (\%) & $e e(\%)$ \\
\hline$(R)$-BINAP & Toluene & None & rt (24 h) & - & - \\
\hline$(R)$-BINAP & Toluene & $\mathrm{MeOH}(5)$ & $\mathrm{rt}(24 \mathrm{~h})$ & - & - \\
\hline$(R)$-BINAP & THF & None & $\mathrm{rt}(24 \mathrm{~h})$ & Incomplete & - \\
\hline$(R)$-BINAP & THF & $\mathrm{MeOH}(5)$ & rt (24 h) & 77 & - \\
\hline$(R)$-SEGPHOS & THF & $\mathrm{MeOH}(5)$ & $\mathrm{rt}(24 \mathrm{~h})$ & 80 & - \\
\hline
\end{tabular}




\begin{tabular}{|c|c|c|c|c|c|}
\hline$(R)$-DIFLUORPHOS & THF & $\mathrm{MeOH}(5)$ & $\mathrm{rt}(24 \mathrm{~h})$ & 75 & - \\
\hline A & Toluene & $\mathrm{MeOH}(5)$ & $\mathrm{rt}(24 \mathrm{~h})$ & - & - \\
\hline $\mathbf{A}$ & $\mathrm{THF}$ & $\mathrm{MeOH}(5)$ & $\mathrm{rt}(24 \mathrm{~h})$ & 85 & 64 \\
\hline $\mathbf{A}$ & DCM & $\mathrm{MeOH}(5)$ & $\mathrm{rt}(26 \mathrm{~h})$ & 86 & 67 \\
\hline $\mathbf{A}$ & $\mathrm{Et}_{2} \mathrm{O}$ & $\mathrm{MeOH}(5)$ & $\mathrm{rt}(36 \mathrm{~h})$ & 86 & 58 \\
\hline $\mathbf{A}$ & THF & $\mathrm{MeOH}(5)$ & $-5^{\circ} \mathrm{C}(80 \mathrm{~h})$ & 82 & 65 \\
\hline $\mathbf{A}$ & THF & $\mathrm{MeOH}(5)$ & $-5^{\circ} \mathrm{C}(82 \mathrm{~h})$ & 82 & 71 \\
\hline $\mathbf{A}$ & 2-Me-THF & $\mathrm{MeOH}(5)$ & $\mathrm{rt}(48 \mathrm{~h})$ & 79 & 53 \\
\hline A & Dioxane & $\mathrm{MeOH}(5)$ & $\mathrm{rt}(50 \mathrm{~h})$ & 88 & 62 \\
\hline B & $\mathrm{THF}$ & $\mathrm{MeOH}(5)$ & $\mathrm{rt}(11 \mathrm{~h})$ & 88 & 95 \\
\hline B & DCM & $\mathrm{MeOH}(5)$ & $\mathrm{rt}(19 \mathrm{~h})$ & 90 & 97 \\
\hline B & $\mathrm{Et}_{2} \mathrm{O}$ & $\mathrm{MeOH}(5)$ & $\mathrm{rt}(24 \mathrm{~h})$ & - & - \\
\hline B & DME & $\mathrm{MeOH}(5)$ & $\mathrm{rt}(24 \mathrm{~h})$ & - & - \\
\hline B & 2-Me-THF & $\mathrm{MeOH}(5)$ & $\mathrm{rt}(12 \mathrm{~h})$ & 84 & 95 \\
\hline B & Dioxane & $\mathrm{MeOH}(5)$ & $\mathrm{rt}(17 \mathrm{~h})$ & 89 & 92 \\
\hline C & THF & $\mathrm{MeOH}(5)$ & rt (12 h) & 90 & 96 \\
\hline C & DCM & $\mathrm{MeOH}(5)$ & rt (12 h) & 93 & 99 \\
\hline $\mathbf{C}$ & $\mathrm{Et}_{2} \mathrm{O}$ & $\mathrm{MeOH}(5)$ & $\mathrm{rt}(24 \mathrm{~h})$ & - & - \\
\hline $\mathbf{C}$ & DME & $\mathrm{MeOH}(5)$ & $\mathrm{rt}(24 \mathrm{~h})$ & - & - \\
\hline $\mathbf{C}$ & 2-Me-THF & $\mathrm{MeOH}(5)$ & $\mathrm{rt}(16 \mathrm{~h})$ & 94 & 97 \\
\hline C & Dioxane & $\mathrm{MeOH}(5)$ & $\mathrm{rt}(14 \mathrm{~h})$ & 94 & 96 \\
\hline $\mathbf{C}$ & $\mathrm{DCM}^{\mathrm{c}}$ & $\mathrm{MeOH}(5)$ & $\mathrm{rt}(12 \mathrm{~h})$ & 90 & 99 \\
\hline $\mathbf{C}$ & $\mathrm{DCM}^{\mathrm{d}}$ & $\mathrm{MeOH}(5)$ & $\mathrm{rt}(12 \mathrm{~h})$ & 88 & 99 \\
\hline $\mathbf{C}^{\mathrm{e}}$ & DCM & МeOH (5) & rt (12 h) & 94 & 99 \\
\hline $\mathbf{C}^{\mathrm{e}}$ & DCM & $\mathrm{EtOH}(5)$ & $\mathrm{rt}(12 \mathrm{~h})$ & 93 & 99 \\
\hline $\mathrm{C}^{\mathrm{e}}$ & DCM & TFE (5) & $\mathrm{rt}(12 \mathrm{~h})$ & - & - \\
\hline $\mathbf{D}^{\mathrm{e}}$ & $\mathrm{THF}$ & $\mathrm{MeOH}(5)$ & $\mathrm{rt}(9 \mathrm{~h})$ & 93 & -99 \\
\hline $\mathbf{D}^{\mathrm{e}}$ & DCM & $\mathrm{MeOH}(5)$ & $\mathrm{rt}(9 \mathrm{~h})$ & 95 & -99 \\
\hline $\mathbf{E}^{\mathrm{e}}$ & DCM & $\mathrm{MeOH}(5)$ & $\mathrm{rt}(26 \mathrm{~h})$ & 82 & $r a c$ \\
\hline $\mathbf{E}^{\mathrm{e}}$ & DCM & $\mathrm{MeOH}(5)$ & $\mathrm{rt}(28 \mathrm{~h})$ & 82 & rac \\
\hline $\mathbf{F}^{\mathbf{e}}$ & DCM & $\mathrm{MeOH}(5)$ & $\mathrm{rt}(29 \mathrm{~h})$ & 79 & $r a c$ \\
\hline $\mathbf{G}^{\mathrm{e}}$ & DCM & $\mathrm{MeOH}(5)$ & $\mathrm{rt}(29 \mathrm{~h})$ & 84 & $r a c$ \\
\hline $\mathbf{H}^{\mathrm{e}}$ & DCM & $\mathrm{MeOH}(5)$ & $\mathrm{rt}(28 \mathrm{~h})$ & 86 & rac \\
\hline $\mathbf{I}^{\mathbf{e}}$ & DCM & $\mathrm{MeOH}(5)$ & $\mathrm{rt}(35 \mathrm{~h})$ & 88 & $r a c$ \\
\hline $\mathbf{J}^{\mathbf{e}}$ & DCM & $\mathrm{MeOH}(5)$ & $\mathrm{rt}(30 \mathrm{~h})$ & 81 & $r a c$ \\
\hline
\end{tabular}

Conditions: $0.5 \mathrm{mmol}$ of 1a, the ligand $(10 \mathrm{~mol} \%)$ and $\mathrm{InI}(10 \mathrm{~mol} \%)$ were added in $1.5 \mathrm{~mL}$ of solvent. Then $0.75 \mathrm{mmol}$ of 2 was added into that mixture. $\mathrm{MeOH}$ (2.5 mmol, 5 equiv) was added at the end and ran at $\mathrm{rt}$. The reaction was monitored by TLC. All the yields are isolated yields. The enantiomeric excesses were determined by chiral HPLC. ${ }^{a} 10 \mathrm{~mol} \%$ of ligand with $10 \mathrm{~mol} \%$ of InI ${ }^{\mathrm{b}} 0.33 \mathrm{M}^{\mathrm{c}} 0.16 \mathrm{M}^{\mathrm{d}} 0.66 \mathrm{M}^{\mathrm{e}} 5 \mathrm{~mol} \%$ of ligand with $5 \mathrm{~mol} \%$ of InI. 


\section{Ligands:}
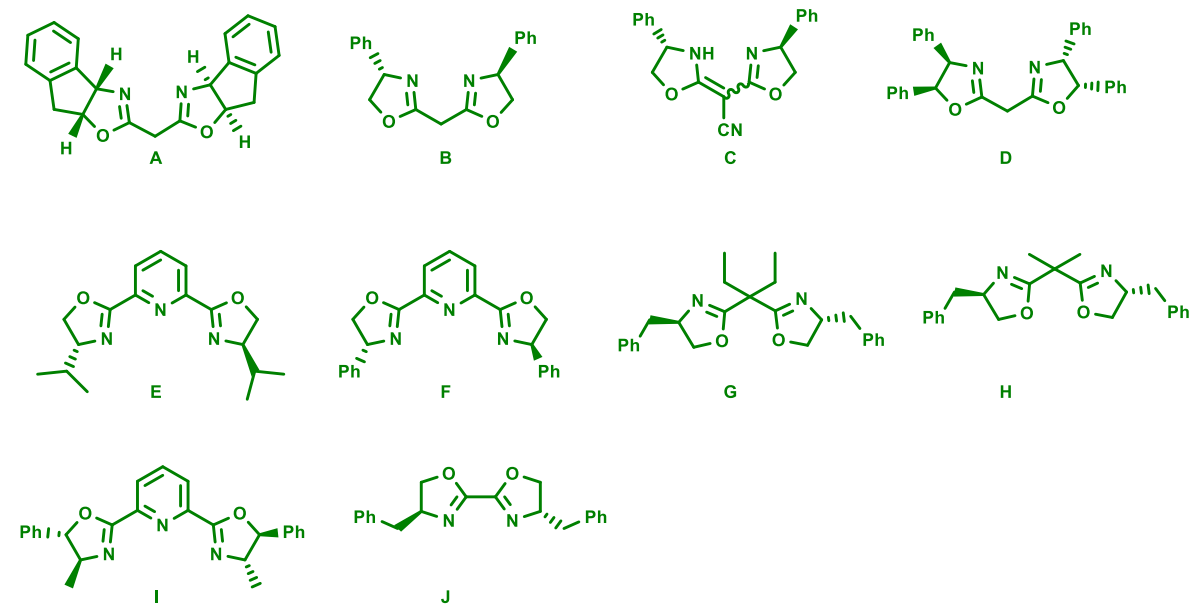

\section{General Procedure for Catalytic Asymmetric Allylboration of Keiminoesters 1a-u:}

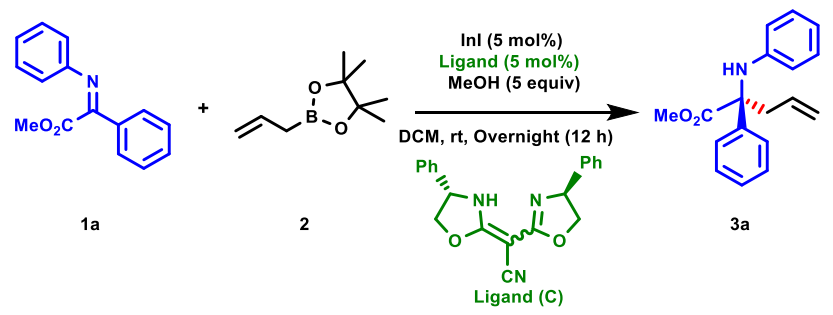

In a Chemglass $8 \mathrm{~mL}$ reaction vial, dried overnight in an oven, the corresponding ketiminoester 1a $(0.5 \mathrm{mmol}, 1$ equiv, $120 \mathrm{mg})$, ligand $\mathbf{C}(0.025 \mathrm{mmol}, 0.05$ equiv, $8.3 \mathrm{mg}), \mathrm{InI}(0.025 \mathrm{mmol}$, 0.05 equiv, $6 \mathrm{mg}$ ) and allylboronic acid pinacol ester $2(0.75 \mathrm{mmol}, 1.5$ equiv, $140 \mu \mathrm{L})$ were dissolved in DCM $(1.5 \mathrm{~mL}, 0.33 \mathrm{M})$ forming a yellow colored solution. $\mathrm{MeOH}$ ( $2.5 \mathrm{mmol}, 5$ equiv, $101 \mu \mathrm{L}$ ) was added dropwise to this solution. The reaction mixture was stirred for 12 hours, during which the solution turned colorless and transparent. After confirming the complete consumption of starting material by TLC, the reaction mixture was passed through a pad of celite and the celite pad was rinsed with DCM $(3 \times 10 \mathrm{~mL})$. All the solvent was removed under reduced pressure. The crude reaction mixture was purified by automated flash chromatography.

Note: For gram scale reaction (for the synthesis of 3a): In a Chemglass $20 \mathrm{~mL}$ reaction vial, dried overnight in an oven, the corresponding ketiminoester 1a (4 mmol, 1 equiv, $960 \mathrm{mg}$ ), ligand $\mathbf{C}$ ( $0.2 \mathrm{mmol}, 0.05$ equiv, $67 \mathrm{mg}), \mathrm{InI}(0.2 \mathrm{mmol}, 0.05$ equiv, $49 \mathrm{mg})$ and allylboronic acid pinacol ester 2 (6 mmol, 1.5 equiv, $1.12 \mathrm{~mL})$ were dissolved in DCM (12 mL, $0.33 \mathrm{M})$. MeOH (20 mmol, 
5 equiv, $0.81 \mathrm{~mL}$ ) was added dropwise to this solution. The rest of the workup procedure is same as mentioned in the procedure described above.

Note: The racemic compounds (1a-1u) were prepared by following exactly the above procedure but without employing the chiral ligand. The observations during the reaction were similar to that of the corresponding asymmetric versions. The workup procedure also is similar as the procedure described above. 
6. Optimization Table for Catalytic Asymmetric Allylboration of $\alpha$-Trifluoromethyl $\alpha$ Iminoesters:

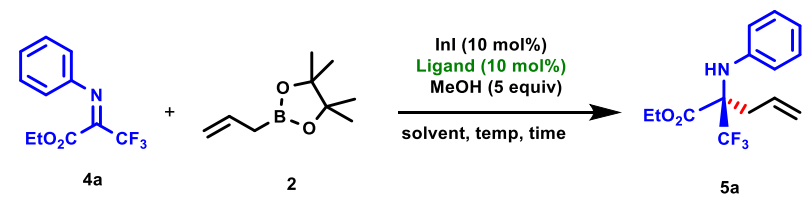

\begin{tabular}{|c|c|c|c|c|}
\hline Ligand $^{a}$ & Solvent $^{\mathrm{b}}$ & $\begin{array}{l}\text { Temperature } \\
\text { (Time) }\end{array}$ & Yield (\%) & $e e(\%)$ \\
\hline A & Toluene & $\mathrm{rt}(24 \mathrm{~h})$ & - & - \\
\hline A & THF & rt $(6$ h) & 86 & 66 \\
\hline $\mathbf{A}$ & DCM & rt $(6 \mathrm{~h})$ & 88 & 56 \\
\hline A & $\mathrm{Et}_{2} \mathrm{O}$ & rt $(14 \mathrm{~h})$ & 87 & 59 \\
\hline A & $\mathrm{THF}$ & $-5^{\circ} \mathrm{C}(17 \mathrm{~h})$ & 83 & 69 \\
\hline A & DCM & $-5^{\circ} \mathrm{C}(24 \mathrm{~h})$ & 82 & 69 \\
\hline A & 2-Me-THF & $\mathrm{rt}(12 \mathrm{~h})$ & 80 & 58 \\
\hline $\mathbf{A}$ & 1,4-Dioxane & $\mathrm{rt}(12 \mathrm{~h})$ & 89 & 76 \\
\hline B & $\mathrm{THF}$ & rt (14 h) & 92 & 97 \\
\hline B & DCM & rt (17 h) & 89 & 95 \\
\hline B & $\mathrm{Et}_{2} \mathrm{O}$ & rt $(24 \mathrm{~h})$ & - & - \\
\hline B & DME & $\mathrm{rt}(24 \mathrm{~h})$ & - & - \\
\hline B & 2-Me-THF & rt $(14 \mathrm{~h})$ & 86 & 97 \\
\hline B & 1,4-Dioxane & rt $(22 \mathrm{~h})$ & 93 & 93 \\
\hline C & $\mathbf{T H F}^{\mathrm{b}, \mathrm{f}}$ & rt $(17 \mathrm{~h})$ & 91 & 97 \\
\hline $\mathbf{C}$ & $\mathrm{DCM}^{\mathrm{b}}$ & rt $(23 \mathrm{~h})$ & 90 & 96 \\
\hline $\mathbf{C}$ & $\mathrm{Et}_{2} \mathrm{O}^{\mathrm{b}}$ & $\mathrm{rt}(24 \mathrm{~h})$ & - & - \\
\hline $\mathbf{C}$ & $\mathrm{DME}^{\mathrm{b}}$ & $\mathrm{rt}(24 \mathrm{~h})$ & - & - \\
\hline C & 2-Me-THF & rt (17 h) & 90 & 96 \\
\hline C & 1,4-Dioxane ${ }^{b}$ & $\mathrm{rt}(17 \mathrm{~h})$ & 75 & 96 \\
\hline C & $\mathrm{THF}^{\mathrm{c}}$ & $\mathrm{rt}(17 \mathrm{~h})$ & 91 & 97 \\
\hline $\mathbf{C}$ & $\mathrm{THF}^{\mathrm{d}}$ & $\mathrm{rt}(17 \mathrm{~h})$ & 86 & 97 \\
\hline $\mathbf{C}^{\mathbf{e}}$ & $\mathrm{THF}^{\mathrm{b}}$ & rt $(24 \mathrm{~h})$ & 89 & 96 \\
\hline $\mathbf{D}^{\mathbf{a}}$ & $\mathrm{THF}^{\mathrm{b}}$ & $\mathrm{rt}(6 \mathrm{~h})$ & 89 & -96 \\
\hline $\mathbf{E}^{\mathbf{a}}$ & $\mathrm{THF}^{\mathrm{b}}$ & rt $(34 \mathrm{~h})$ & 85 & $\mathrm{rac}$ \\
\hline $\mathbf{F}^{\mathbf{a}}$ & $\mathrm{THF}^{\mathrm{b}}$ & rt $(26 \mathrm{~h})$ & 72 & $\mathrm{rac}$ \\
\hline $\mathbf{G}^{\mathbf{a}}$ & $\mathrm{THF}^{\mathrm{b}}$ & rt $(27 \mathrm{~h})$ & 75 & $\mathrm{rac}$ \\
\hline $\mathbf{H}^{\mathbf{a}}$ & $\mathrm{THF}^{\mathrm{b}}$ & rt $(27 \mathrm{~h})$ & 77 & $\mathrm{rac}$ \\
\hline $\mathbf{I}^{\mathbf{a}}$ & $\mathrm{THF}^{\mathrm{b}}$ & rt $(33 \mathrm{~h})$ & 73 & $\mathrm{rac}$ \\
\hline $\mathbf{J}^{\mathbf{a}}$ & $\mathrm{THF}^{\mathrm{b}}$ & rt $(28 \mathrm{~h})$ & 79 & $\mathrm{rac}$ \\
\hline
\end{tabular}

Conditions: $0.5 \mathrm{mmol}$ of $\mathbf{4 a}$, the ligand $(10 \mathrm{~mol} \%)$ and $\mathrm{InI}(10 \mathrm{~mol} \%)$ were added in $1.5 \mathrm{~mL}$ of solvent. Then $0.75 \mathrm{mmol}$ of 2 was added into that mixture. $\mathrm{MeOH}$ (2.5 mmol, 5 equiv) was added at the end and ran at rt. The reaction was monitored by TLC. All the yields are isolated yields. The 
enantiomeric excesses were determined by chiral HPLC of the corresponding amino alcohol derived via hydroboration and oxidation of the olefin unit. ${ }^{\text {a }} 10 \mathrm{~mol} \%$ of ligand with $10 \mathrm{~mol} \%$ of InI. ${ }^{\mathrm{b}} 0.33 \mathrm{M}$. ${ }^{\mathrm{c}} 0.16 \mathrm{M}$. ${ }^{\mathrm{d}} 0.66 \mathrm{M}$. ${ }^{\mathrm{e}} 5 \mathrm{~mol} \%$ of ligand with $5 \mathrm{~mol} \%$ of InI. ${ }^{\mathrm{f}}$ only 1 or 2 equiv of $\mathrm{MeOH}$ as additive leads to a much slower and less cleaner reaction.

\section{General Procedure for Catalytic Asymmetric Allylboration of $\alpha$-Trifluoromethyl $\alpha$ - Iminoesters 4a-i:}

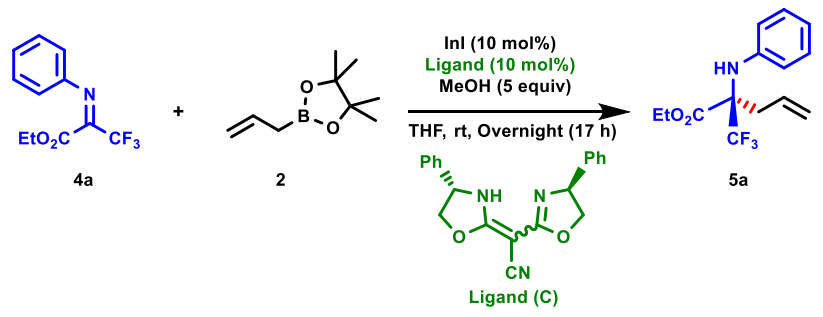

In a Chemglass $8 \mathrm{~mL}$ reaction vial, dried overnight in an oven, the corresponding ketiminoester 4a (0.5 mmol, 1 equiv, $123 \mathrm{mg})$, ligand $\mathbf{C}$ (0.05 mmol, 0.1 equiv, $17 \mathrm{mg})$, InI (0.05 mmol, 0.1 equiv, $12 \mathrm{mg})$ and allylboronic acid pinacol ester $2(0.75 \mathrm{mmol}, 1.5$ equiv, $140 \mu \mathrm{L})$ were dissolved in THF (1.5 mL, $0.33 \mathrm{M})$ forming a yellow colored solution. $\mathrm{MeOH}(2.5 \mathrm{mmol}, 5$ equiv, $101 \mu \mathrm{L})$ was added dropwise to this solution. The reaction mixture was stirred for 17 hours, during which the solution turned colorless and transparent. After confirming the complete consumption of starting material by TLC, the reaction mixture was passed through a pad of celite to remove traces of residual InI and the celite pad was rinsed with DCM $(3 \times 10 \mathrm{~mL})$. All the solvent was removed under reduced pressure. The crude reaction mixture was purified by automated flash chromatography.

Note: For gram scale reaction (for the synthesis of 5a): In a Chemglass $20 \mathrm{~mL}$ reaction vial, dried overnight in an oven, the corresponding ketiminoester 4a (4 mmol, 1 equiv, $984 \mathrm{mg}$ ), ligand $\mathbf{C}$ ( $0.4 \mathrm{mmol}, 0.1$ equiv, $136 \mathrm{mg}), \mathrm{InI}(0.4 \mathrm{mmol}, 0.1$ equiv, $96 \mathrm{mg})$ and allylboronic acid pinacol ester 2 (6 mmol, 1.5 equiv, $1.12 \mathrm{~mL}$ ) were dissolved in THF (12 mL, 0.33 M). MeOH (20 mmol, 5 equiv, $0.81 \mathrm{~mL}$ ) was added dropwise to this solution. The rest of the workup procedure is same as mentioned in the procedure described above. 
Note: The racemic compounds (5a-5i) were prepared by following exactly the above procedure but without employing the chiral ligand. The observations during the reaction were similar to that of the corresponding asymmetric versions. The workup procedure also is similar as the procedure described above.

\section{General Procedure for Hydroboration-Oxidation of Homoallylic aminoesters:}
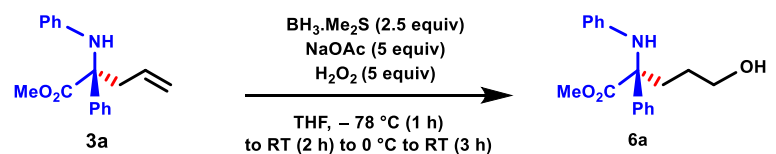

In a Chemglass $20 \mathrm{~mL}$ reaction vial, dried overnight in an oven, the corresponding homoallylic amino ester 3a $(0.8 \mathrm{mmol}, 1$ equiv, $225 \mathrm{mg})$ was dissolved in $6 \mathrm{~mL}$ THF $(0.13 \mathrm{M})$ and cooled at $-78{ }^{\circ} \mathrm{C}$. $\mathrm{BH}_{3} \cdot \mathrm{DMS}(2.0 \mathrm{mmol}, 2.5$ equiv, $190 \mu \mathrm{L})$ was added dropwise to the reaction mixture. The reaction mixture was stirred at this temperature for $1 \mathrm{~h}$, then allowed to warm to room temperature and stirred for additional $2 \mathrm{~h}$. Then the reaction was cooled at $0{ }^{\circ} \mathrm{C}$ and $\mathrm{NaOAc}(4$ mmol, 5 equiv, $329 \mathrm{mg})$ solution in $2 \mathrm{~mL}$ of water mixed with $0.5 \mathrm{~mL}$ of $\mathrm{H}_{2} \mathrm{O}_{2}\left(30 \%\right.$ in $\left.\mathrm{H}_{2} \mathrm{O}\right)$ was added dropwise into the reaction mixture. The reaction was then allowed to warm to room temperature and stirred overnight. The aqueous layer was extracted with EtOAc $(3 \times 10 \mathrm{~mL})$ and dried over anhydrous $\mathrm{Na}_{2} \mathrm{SO}_{4}$. After filtration all the solvent was removed under reduced pressure. The crude reaction mixture was purified by automated flash chromatography.

\section{Transformations of the Homoallylic Amine Products into the Pyrrolidine Structures:}

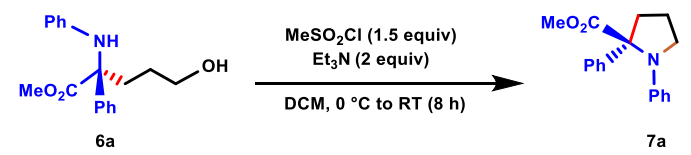

In a Chemglass $20 \mathrm{~mL}$ reaction vial, dried overnight in an oven, the corresponding homoallylic amino alcohol 6a (0.56 mmol, 1 equiv, $168 \mathrm{mg})$ was dissolved in $5 \mathrm{~mL} \mathrm{DCM} \mathrm{(0.11} \mathrm{M)} \mathrm{and} \mathrm{cooled}$ at $0{ }^{\circ} \mathrm{C} . \mathrm{MeSO}_{2} \mathrm{Cl}(0.84 \mathrm{mmol}, 1.5$ equiv, $65 \mu \mathrm{L})$ was added dropwise into it followed by dropwise addition of $\mathrm{Et}_{3} \mathrm{~N}(1.12 \mathrm{mmol}, 2$ equiv, $160 \mu \mathrm{L})$. The reaction mixture was then allowed to warm to room temperature and stirred for $8 \mathrm{~h}$. After confirming the complete consumption of starting material by TLC, the reaction was quenched with saturated aqueous solution of $\mathrm{NaHCO}_{3}(5 \mathrm{~mL})$. 
The aqueous layer was extracted with EtOAc $(2 \times 10 \mathrm{~mL})$ and dried over anhydrous $\mathrm{Na}_{2} \mathrm{SO}_{4}$. After filtration all the solvent was removed under reduced pressure. The crude reaction mixture was purified by automated flash chromatography.

10. Characterization of Catalytic Asymmetric Allylation Products in Table 2:

1. a) Methyl (S)-2-phenyl-2-(phenylamino)pent-4-enoate (3a):

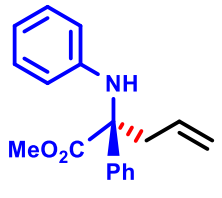

$3 a$

Yield: $95 \%, 1.07 \mathrm{~g} \mathrm{(4} \mathrm{mmol} \mathrm{scale);}$

Physical State: Colorless oily liquid;

$\boldsymbol{R}_{\mathbf{f}}=0.50(10 \% \mathrm{EA} / \mathrm{Hex})$

${ }^{1}$ H NMR (600 MHz, Chloroform- $\left.d\right): \delta 7.64(\mathrm{~d}, J=7.6 \mathrm{~Hz}, 2 \mathrm{H}), 7.39(\mathrm{t}, J=7.5 \mathrm{~Hz}, 2 \mathrm{H}), 7.32(\mathrm{t}$, $J=7.1 \mathrm{~Hz}, 1 \mathrm{H}), 7.04(\mathrm{t}, J=7.6 \mathrm{~Hz}, 2 \mathrm{H}), 6.66(\mathrm{t}, J=7.2 \mathrm{~Hz}, 1 \mathrm{H}), 6.41(\mathrm{~d}, J=7.9 \mathrm{~Hz}, 2 \mathrm{H}), 5.71$ (dq, $J=16.9,7.9 \mathrm{~Hz}, 1 \mathrm{H}), 5.34(\mathrm{~s}, 1 \mathrm{H}), 5.12(\mathrm{~d}, J=10.1 \mathrm{~Hz}, 1 \mathrm{H}), 5.07(\mathrm{~d}, J=17.1 \mathrm{~Hz}, 1 \mathrm{H}), 3.69$ (s, $3 \mathrm{H}), 3.33(\mathrm{dd}, J=13.4,6.3 \mathrm{~Hz}, 1 \mathrm{H}), 3.28(\mathrm{dd}, J=13.4,8.1 \mathrm{~Hz}, 1 \mathrm{H})$;

${ }^{13}$ C NMR (151 MHz, Chloroform- $d$ ): $\delta$ 173.7, 144.3, 140.2, 132.3, 128.8, 128.6, 127.6, 127.0, $119.3,117.5,115.2,66.3,53.0,37.7$;

FTIR (ATR, cm$^{-1}$ ): 3410, 2954, 2844, 1729, 1602, 1446, 1436, 1286, 1233, 1182, 1033, 944, 748691.

HRMS (ESI-TOF): calc'd for $\mathrm{C}_{18} \mathrm{H}_{20} \mathrm{NO}_{2}[\mathrm{M}+\mathrm{H}]^{+} 282.1489$; found 282.1483;

HPLC Analysis: Chiralpak IA, $0.3 \% \mathrm{IP} /$ Hexanes, continuous flow at $0.3 \mathrm{~mL} / \mathrm{min}, 230 \mathrm{~nm} ; t_{\text {major }}$ $=20.3 \mathrm{~min}, t_{\mathrm{minor}}=22.4 \mathrm{~min}$, ee $99 \%$

$[\alpha]_{\mathrm{D}^{20}}=+22.15^{\circ}\left(C=0.8, \mathrm{CHCl}_{3}\right)$.

b) Methyl (R)-2-phenyl-2-(phenylamino)pent-4-enoate (3a'): 


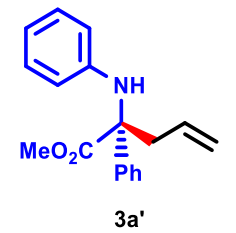

Yield: $95 \%, 133 \mathrm{mg}(0.5 \mathrm{mmol}$ scale $)$;

Physical State: Colorless oily liquid;

HPLC Analysis: Chiralpak IA, $0.3 \%$ IP/Hexanes, continuous flow at $0.3 \mathrm{~mL} / \mathrm{min}, 230 \mathrm{~nm}$; $t_{\text {major }}$ $=22.6 \mathrm{~min}, t_{\mathrm{minor}}=20.6 \mathrm{~min}$, ee $97 \%$;

$[\alpha]_{\mathrm{D}}^{\mathbf{2 0}}=-33.6^{\circ}\left(C=0.63, \mathrm{CHCl}_{3}\right)$.

2. a) Methyl (S)-2-((4-methoxyphenyl)amino)-2-phenylpent-4-enoate (3b):

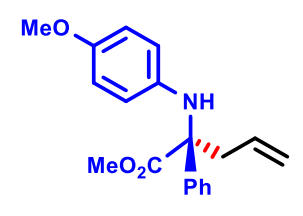

$3 \mathbf{b}$

Yield: 98\%, $1.2 \mathrm{~g}$ (4 mmol scale);

Physical State: Colorless oily liquid;

$\boldsymbol{R}_{\mathbf{f}}=0.38(10 \% \mathrm{EA} / \mathrm{Hex})$

${ }^{1}$ H NMR (600 MHz, Chloroform- $\left.d\right): \delta 7.60(\mathrm{~d}, J=8.1 \mathrm{~Hz}, 2 \mathrm{H}), 7.36(\mathrm{t}, J=7.6 \mathrm{~Hz}, 2 \mathrm{H}), 7.30(\mathrm{t}$, $J=7.0 \mathrm{~Hz}, 1 \mathrm{H}), 6.61(\mathrm{~d}, J=8.6 \mathrm{~Hz}, 2 \mathrm{H}), 6.34(\mathrm{~d}, J=8.6 \mathrm{~Hz}, 2 \mathrm{H}), 5.72-5.60(\mathrm{~m}, 1 \mathrm{H}), 5.08(\mathrm{~d}, J$ $=10.1 \mathrm{~Hz}, 1 \mathrm{H}), 5.05(\mathrm{~s}, 1 \mathrm{H}), 5.03(\mathrm{~d}, J=17.1 \mathrm{~Hz}, 1 \mathrm{H}), 3.68(\mathrm{~s}, 3 \mathrm{H}), 3.66(\mathrm{~s}, 3 \mathrm{H}), 3.20(\mathrm{~d}, J=6.8$ $\mathrm{Hz}, 2 \mathrm{H})$;

${ }^{13}$ C NMR (151 MHz, Chloroform-d): $\delta$ 173.8, 152.2, 140.7, 138.2, 132.4, 128.6, 127.6, 127.0, $119.2,116.8,114.4,66.7,55.5,52.9,38.1$;

FTIR (ATR, $\left.\mathbf{c m}^{-1}\right):$ 2951, 2832, 1731, 1509, 1445, 1233, 117, 1030, 820, 756, 697.

HRMS (ESI-TOF): calc'd for $\mathrm{C}_{19} \mathrm{H}_{22} \mathrm{NO}_{3}[\mathrm{M}+\mathrm{H}]^{+}$312.1594; found 312.1592; 
HPLC Analysis: Chiralpak IA, 5\% IP/Hexanes, continuous flow at $0.4 \mathrm{~mL} / \mathrm{min}, 250 \mathrm{~nm} ; t_{\text {major }}=$ $15.6 \mathrm{~min}, t_{\mathrm{minor}}=18.3 \mathrm{~min}$, ee $98 \%$;

$[\alpha]_{\mathrm{D}^{20}}=+26.11^{\circ}\left(C=0.67, \mathrm{CHCl}_{3}\right)$.

\section{Determination of absolute configuration:}

Methyl (S)-2-((4-methoxyphenyl)amino)-2-phenylpent-4-enoate (3b) was converted to the corresponding homoallylic amine (3b-1) by removing the $p$-methoxyphenyl group on the $N$-atom with ceric ammonium nitrate. ${ }^{1 \text { a }}$ Comparison of the optical rotation to the literature value ${ }^{5,6}$ established the $(S)$ configuration in product $\mathbf{3 b - 1}$. All other absolute configurations of the products from Table 2 were assigned by analogy.

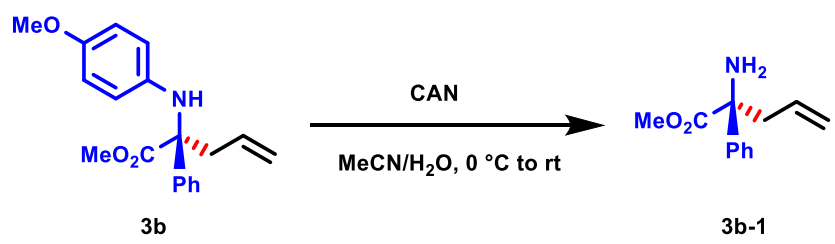

Yield: $66 \%, 36 \mathrm{mg}(0.27 \mathrm{mmol}$ scale $)$;

Physical State: Pale yellow oily liquid;

${ }^{1} \mathbf{H}$ NMR of 3b-1 (600 MHz, Chloroform-d): $\delta 7.52(\mathrm{~d}, J=7.9 \mathrm{~Hz}, 2 \mathrm{H}), 7.35(\mathrm{t}, J=7.6 \mathrm{~Hz}, 2 \mathrm{H})$, $7.28(\mathrm{t}, J=7.3 \mathrm{~Hz}, 1 \mathrm{H}), 5.69(\mathrm{td}, J=17.2,7.3 \mathrm{~Hz}, 1 \mathrm{H}), 5.18(\mathrm{dd}, J=23.2,13.6 \mathrm{~Hz}, 2 \mathrm{H}), 3.71$ (s, 3H), 2.99 (dd, $J=13.7,6.7 \mathrm{~Hz}, 1 \mathrm{H}), 2.69$ (dd, $J=13.6,7.8 \mathrm{~Hz}, 1 \mathrm{H}), 2.49$ (brs, 2H);

${ }^{13}$ C NMR (151 MHz, Chloroform-d): $\delta$ 175.3, 142.3, 132.7, 128.4, 127.6, 125.4, 120.1, 63.3, 52.5, 44.3;

FTIR (ATR, $\left.\mathbf{c m}^{-1}\right):$ 2952, 1845, 1729, 1597, 1505, 1446, 1357, 1216, 1032, 1023, 994, 922, 734.

HRMS (ESI-TOF): calc'd for $\mathrm{C}_{12} \mathrm{H}_{16} \mathrm{NO}_{2}[\mathrm{M}+\mathrm{H}]^{+}$206.1176; found 206.1178;

$[\alpha]_{\mathrm{D}^{\mathbf{2 0}}}=-36.9^{\circ}\left(C=0.8, \mathrm{CHCl}_{3}\right) ;[\alpha]_{\mathrm{D}, \mathrm{lit}}=-13.5^{\circ}\left(C=2, \mathrm{CHCl}_{3}\right) .{ }^{5,6}$

b) Methyl (R)-2-((4-methoxyphenyl)amino)-2-phenylpent-4-enoate (3b'):

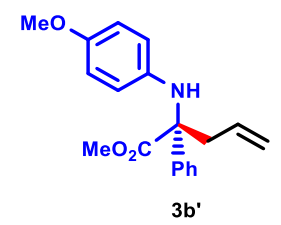

Yield: $98 \%, 151 \mathrm{mg}(0.5 \mathrm{mmol}$ scale $)$; 
Physical State: Colorless oily liquid;

HPLC Analysis: Chiralpak IA, 5\% IP/Hexanes, continuous flow at $0.4 \mathrm{~mL} / \mathrm{min}, 250 \mathrm{~nm} ; t_{\text {major }}=$ $18.2 \mathrm{~min}, t_{\mathrm{minor}}=15.6 \mathrm{~min}$, ee $97 \%$;

$[\alpha]_{\mathbf{D}^{\mathbf{2 0}}}=-29.1^{\circ}\left(C=0.82, \mathrm{CHCl}_{3}\right)$.

3. Methyl (S)-2-phenyl-2-(p-tolylamino)pent-4-enoate (3c):

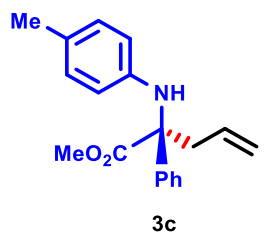

Yield: $93 \%, 147 \mathrm{mg}(0.5 \mathrm{mmol}$ scale $)$;

Physical State: Colorless oily liquid;

$\boldsymbol{R}_{\mathbf{f}}=0.51(10 \% \mathrm{EA} / \mathrm{Hex})$

${ }^{1}$ H NMR (600 MHz, Chloroform- $\left.d\right): \delta 7.62(\mathrm{~d}, J=7.6 \mathrm{~Hz}, 2 \mathrm{H}), 7.37(\mathrm{t}, J=7.6 \mathrm{~Hz}, 2 \mathrm{H}), 7.30(\mathrm{t}$, $J=7.3 \mathrm{~Hz}, 1 \mathrm{H}), 6.84(\mathrm{~d}, J=8.2 \mathrm{~Hz}, 2 \mathrm{H}), 6.31(\mathrm{~d}, J=8.4 \mathrm{~Hz}, 2 \mathrm{H}), 5.81-5.56(\mathrm{~m}, 1 \mathrm{H}), 5.17(\mathrm{~s}$, $1 \mathrm{H}), 5.09(\mathrm{~d}, J=10.2 \mathrm{~Hz}, 1 \mathrm{H}), 5.05(\mathrm{~d}, J=17.1 \mathrm{~Hz}, 1 \mathrm{H}), 3.67(\mathrm{~s}, 3 \mathrm{H}), 3.28(\mathrm{dd}, J=13.5,6.3 \mathrm{~Hz}$, $1 \mathrm{H}), 3.23(\mathrm{dd}, J=13.5,8.1 \mathrm{~Hz}, 1 \mathrm{H}), 2.18(\mathrm{~s}, 3 \mathrm{H})$;

${ }^{13}$ C NMR (151 MHz, Chloroform- $\left.d\right): \delta$ 173.8, 141.9, 140.4, 132.4, 129.3, 128.6, 127.64, 127.0, 126.7, 119.2, 115.3, 66.4, 52.9, 37.9, 20.3;

FTIR (ATR, cm$\left.^{-1}\right)$ : 3386, 2950, 2866, 1728, 1615, 1519, 1435, 1284, 1225, 1181, 1033, 920, 737.

HRMS (ESI-TOF): calc'd for $\mathrm{C}_{19} \mathrm{H}_{22} \mathrm{NO}_{2}[\mathrm{M}+\mathrm{H}]^{+}$296.1645; found 296.1635;

HPLC Analysis: CSP SFC (AD-H), 10\% Methanol, continuous flow at $2 \mathrm{~mL} / \mathrm{min}, 35^{\circ} \mathrm{C}, 160$ bar, $210 \mathrm{~nm} ; t_{\text {major }}=1.9 \mathrm{~min}, t_{\text {minor }}=1.38 \mathrm{~min}$, ee $98 \%$;

$[\alpha] \mathbf{D}^{\mathbf{2 0}}=+25.37^{\circ}\left(C=0.73, \mathrm{CHCl}_{3}\right)$.

4. Methyl (S)-2-((3,5-dimethylphenyl)amino)-2-phenylpent-4-enoate (3d): 


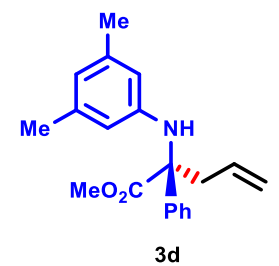

Yield: 93\%, $143 \mathrm{mg}(0.5 \mathrm{mmol}$ scale $)$;

Physical State: Colorless oily liquid;

$\boldsymbol{R}_{\mathbf{f}}=0.52(10 \% \mathrm{EA} / \mathrm{Hex})$

${ }^{1}$ H NMR (600 MHz, Chloroform- $\left.d\right): \delta 7.62(\mathrm{~d}, J=7.4 \mathrm{~Hz}, 2 \mathrm{H}), 7.37(\mathrm{t}, J=7.6 \mathrm{~Hz}, 2 \mathrm{H}), 7.30(\mathrm{t}$, $J=7.3 \mathrm{~Hz}, 1 \mathrm{H}), 6.32(\mathrm{~s}, 1 \mathrm{H}), 6.04(\mathrm{~s}, 2 \mathrm{H}), 5.78-5.62(\mathrm{~m}, 1 \mathrm{H}), 5.22(\mathrm{~s}, 1 \mathrm{H}), 5.14-5.05(\mathrm{~m}, 2 \mathrm{H})$, $3.67(\mathrm{~s}, 3 \mathrm{H}), 3.33(\mathrm{dd}, J=13.5,6.0 \mathrm{~Hz}, 1 \mathrm{H}), 3.23(\mathrm{dd}, J=13.5,8.3 \mathrm{~Hz}, 1 \mathrm{H}), 2.12(\mathrm{~s}, 6 \mathrm{H})$;

${ }^{13}$ C NMR (151 MHz, Chloroform-d): $\delta$ 173.8, 144.3, 140.4, 138.3, 132.6, 128.6, 127.5, 127.0, 119.6, 119.1, 113.1, 66.3, 52.9, 37.8, 21.4;

FTIR (ATR, cm$\left.^{-1}\right):$ 3412, 2957, 2923, 1721, 1601, 1446, 1434, 1338, 1232, 1200, 828, 734.

HRMS (ESI-TOF): calc'd for $\mathrm{C}_{20} \mathrm{H}_{24} \mathrm{NO}_{2}[\mathrm{M}+\mathrm{H}]^{+} 310.1802$; found 310.1822;

HPLC Analysis: CSP SFC (AD-H), 10\% Methanol, continuous flow at $2 \mathrm{~mL} / \mathrm{min}, 35{ }^{\circ} \mathrm{C}, 160$ bar, $250 \mathrm{~nm} ; t_{\text {major }}=1.9 \mathrm{~min}, t_{\mathrm{minor}}=1.0 \mathrm{~min}$, ee $97 \%$;

$[\alpha]_{\mathbf{D}^{20}}=+21.3^{\circ}\left(C=0.78, \mathrm{CHCl}_{3}\right)$.

5. Methyl (S)-2-((4-chlorophenyl)amino)-2-phenylpent-4-enoate (3e):

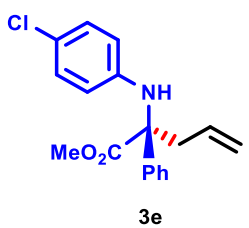

Yield: $88 \%, 138 \mathrm{mg}(0.5 \mathrm{mmol}$ scale $)$;

Physical State: Colorless oily liquid;

$\boldsymbol{R}_{\mathbf{f}}=0.55(10 \% \mathrm{EA} / \mathrm{Hex})$ 
${ }^{1}$ H NMR (600 MHz, Chloroform- $\left.d\right)$ : $\delta 7.58(\mathrm{~d}, J=7.3 \mathrm{~Hz}, 2 \mathrm{H}), 7.37(\mathrm{t}, J=7.6 \mathrm{~Hz}, 2 \mathrm{H}), 7.31(\mathrm{t}$, $J=7.3 \mathrm{~Hz}, 1 \mathrm{H}), 6.96(\mathrm{~d}, J=8.9 \mathrm{~Hz}, 2 \mathrm{H}), 6.30(\mathrm{~d}, J=8.9 \mathrm{~Hz}, 2 \mathrm{H}), 5.65(\mathrm{ddt}, J=14.4,10.2,7.2$ $\mathrm{Hz}, 1 \mathrm{H}), 5.36$ (s, 1H), $5.11(\mathrm{~d}, J=10.2 \mathrm{~Hz}, 1 \mathrm{H}), 5.05$ (d, $J=17.1 \mathrm{~Hz}, 1 \mathrm{H}), 3.68(\mathrm{~s}, 3 \mathrm{H}), 3.25$ (m, $2 \mathrm{H})$;

${ }^{13}$ C NMR (151 MHz, Chloroform-d): $\delta$ 173.4, 142.9, 139.7, 132.0, 128.8, 128.7, 127.8, 126.9, 122.2, 119.6, 116.1, 66.3, 53.1, 37.7;

FTIR (ATR, $\left.\mathbf{c m}^{-1}\right)$ : 3410, 2952, 1728, 1598, 1495, 1224, 1182, 923, 817, 731.

HRMS (ESI-TOF): calc'd for $\mathrm{C}_{18} \mathrm{H}_{19} \mathrm{ClNO}_{2}[\mathrm{M}+\mathrm{H}]^{+}$316.1099; found 316.1085;

HPLC Analysis: CSP SFC (AD-H), 10\% Methanol, continuous flow at $2 \mathrm{~mL} / \mathrm{min}, 35^{\circ} \mathrm{C}, 160$ bar, $210 \mathrm{~nm} ; t_{\text {major }}=1.8 \mathrm{~min}, t_{\mathrm{minor}}=1.4 \mathrm{~min}$, ee $96 \%$;

$[\alpha]_{\mathbf{D}^{\mathbf{2 0}}}=+27.3^{\circ}\left(C=0.81, \mathrm{CHCl}_{3}\right)$.

6. Methyl (S)-2-phenyl-2-((4-(trifluoromethyl)phenyl)amino)pent-4-enoate (3f):

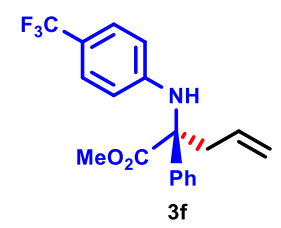

Yield: $90 \%, 156 \mathrm{mg}(0.5 \mathrm{mmol}$ scale $)$;

Physical State: Colorless oily liquid;

$\boldsymbol{R}_{\mathbf{f}}=0.59(10 \% \mathrm{EA} / \mathrm{Hex})$

${ }^{1}$ H NMR (600 MHz, Chloroform- $\left.d\right)$ : $\delta 7.56(\mathrm{~d}, J=7.5 \mathrm{~Hz}, 2 \mathrm{H}), 7.38(\mathrm{t}, J=7.6 \mathrm{~Hz}, 2 \mathrm{H}), 7.32(\mathrm{t}$, $J=7.3 \mathrm{~Hz}, 1 \mathrm{H}), 7.24(\mathrm{~d}, J=8.5 \mathrm{~Hz}, 2 \mathrm{H}), 6.39(\mathrm{~d}, J=8.5 \mathrm{~Hz}, 2 \mathrm{H}), 5.70(\mathrm{~s}, 1 \mathrm{H}), 5.68-5.59(\mathrm{~m}$, $1 \mathrm{H}), 5.11(\mathrm{~d}, J=10.1 \mathrm{~Hz}, 1 \mathrm{H}), 5.05(\mathrm{~d}, J=16.4 \mathrm{~Hz}, 1 \mathrm{H}), 3.69(\mathrm{~s}, 3 \mathrm{H}), 3.32-3.25(\mathrm{~m}, 2 \mathrm{H})$;

${ }^{13}$ C NMR (151 MHz, Chloroform-d): $\delta$ 173.3, 146.9, 139.2, 131.8, 128.9, 128.0, 126.7, 126.1 (q, $J=3.7 \mathrm{~Hz}), 124.8(\mathrm{q}, J=270.3 \mathrm{~Hz}), 119.8,119.0(\mathrm{q}, J=32.6 \mathrm{~Hz}), 114.1,66.2,53.3,37.4$;

${ }^{19}$ F NMR (471 MHz, Chloroform-d): $-60.2(\mathrm{~m})$;

FTIR (ATR, $\left.\mathbf{c m}^{-1}\right)$ : 2921, 2853, 1462, 1377, 1189, 970, 836.

HRMS (ESI-TOF): calc'd for $\mathrm{C}_{19} \mathrm{H}_{19} \mathrm{~F}_{3} \mathrm{NO}_{2}[\mathrm{M}+\mathrm{H}]^{+}$350.1362; found 350.1355. 
$[\alpha] \mathbf{D}^{\mathbf{2 0}}=+29.4^{\circ}\left(C=0.70, \mathrm{CHCl}_{3}\right)$.

Determination of ee via Hydroboration-Oxidation:

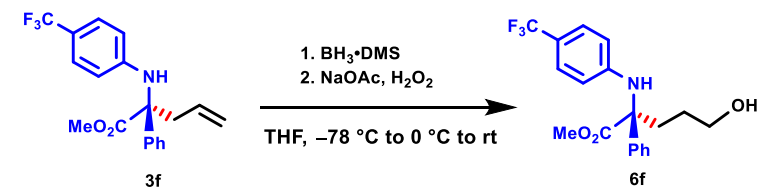

Yield: $71 \%, 81 \mathrm{mg}(0.31 \mathrm{mmol} \mathrm{scale})$;

${ }^{1}$ H NMR (600 MHz, Chloroform- $\left.d\right): \delta 7.55(\mathrm{~d}, J=7.5 \mathrm{~Hz}, 2 \mathrm{H}), 7.35(\mathrm{t}, J=7.6 \mathrm{~Hz}, 2 \mathrm{H}), 7.30$ $(\mathrm{t}, J=7.3 \mathrm{~Hz}, 1 \mathrm{H}), 7.23(\mathrm{~d}, J=8.6 \mathrm{~Hz}, 2 \mathrm{H}), 6.38(\mathrm{~d}, J=8.6 \mathrm{~Hz}, 2 \mathrm{H}), 5.81(\mathrm{~s}, 1 \mathrm{H}), 3.69(\mathrm{~s}, 3 \mathrm{H})$, $3.65-3.57(\mathrm{~m}, 2 \mathrm{H}), 2.72(\mathrm{td}, J=12.6,11.6,4.5 \mathrm{~Hz}, 1 \mathrm{H}), 2.57(\mathrm{td}, J=13.5,12.8,4.7 \mathrm{~Hz}, 1 \mathrm{H})$, 1.59 (ddt, $J=17.5,11.2,6.0 \mathrm{~Hz}, 1 \mathrm{H}), 1.46$ (brs, $1 \mathrm{H}), 1.38$ (ddt, $J=18.1,12.8,6.5 \mathrm{~Hz}, 1 \mathrm{H}$ );

${ }^{13}$ C NMR (151 MHz, Chloroform- $d$ ): $\delta$ 173.9, 146.8, 139.5, 128.9, 128.0, 126.7, $126.2(\mathrm{q}, J=$ $3.7 \mathrm{~Hz}), 124.8$ (q, $J=270.4 \mathrm{~Hz}), 118.9$ (q, $J=32.6 \mathrm{~Hz}), 113.9$, 66.0, 62.4, 53.4, 29.3, 27.4;

${ }^{19}$ F NMR (471 MHz, Chloroform-d): $-60.2(\mathrm{~m})$;

FTIR (ATR, cm$^{-1}$ ): 3401, 2954, 2881, 1728, 1615, 1531, 1448, 1320, 1274, 1188, 1158, 1101, $1064,827,729,700$.

HRMS (ESI-TOF): calc'd for $\mathrm{C}_{19} \mathrm{H}_{21} \mathrm{~F}_{3} \mathrm{NO}_{3}[\mathrm{M}+\mathrm{H}]^{+}$368.1468; found 368.1485;

HPLC Analysis: Chiralpak IA, 5\% IP/Hexanes, continuous flow at $1.0 \mathrm{~mL} / \mathrm{min}, 250 \mathrm{~nm}$; $t_{\text {major }}=$ $19.8 \mathrm{~min}, t_{\mathrm{minor}}=22.2 \mathrm{~min}$, ee $96 \%$;

$[\alpha]_{\mathbf{D}^{\mathbf{2 0}}}=+19.8^{\circ}\left(C=0.70, \mathrm{CHCl}_{3}\right)$.

7. a) Methyl (S)-4-((1-methoxy-1-oxo-2-phenylpent-4-en-2-yl)amino)benzoate (3g):

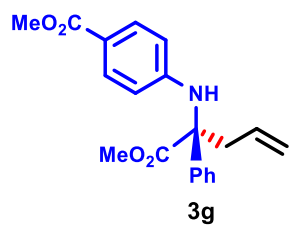

Yield: $90 \%, 1.21 \mathrm{~g}$ (4 mmol scale);

Physical State: Colorless oily liquid;

$\boldsymbol{R}_{\mathbf{f}}=0.47(10 \% \mathrm{EA} / \mathrm{Hex})$ 
${ }^{1}$ H NMR (600 MHz, Chloroform- $\left.d\right): \delta 7.70(\mathrm{~d}, J=8.8 \mathrm{~Hz}, 2 \mathrm{H}), 7.54(\mathrm{~d}, J=7.5 \mathrm{~Hz}, 2 \mathrm{H}), 7.36(\mathrm{t}$, $J=7.5 \mathrm{~Hz}, 2 \mathrm{H}), 7.31(\mathrm{t}, J=7.3 \mathrm{~Hz}, 1 \mathrm{H}), 6.35(\mathrm{~d}, J=8.8 \mathrm{~Hz}, 2 \mathrm{H}), 5.82(\mathrm{~s}, 1 \mathrm{H}), 5.64$ (ddt, $J=14.5$, $10.1,7.2 \mathrm{~Hz}, 1 \mathrm{H}), 5.10(\mathrm{~d}, J=10.1 \mathrm{~Hz}, 1 \mathrm{H}), 5.04(\mathrm{~d}, J=17.0 \mathrm{~Hz}, 1 \mathrm{H}), 3.80(\mathrm{~s}, 3 \mathrm{H}), 3.68(\mathrm{~s}, 3 \mathrm{H})$, $3.41-3.17(\mathrm{~m}, 2 \mathrm{H})$;

${ }^{13}$ C NMR (151 MHz, Chloroform-d): $\delta$ 173.2, 167.1, 148.3, 139.1, 131.8, 131.0, 128.9, 128.0, 126.7, 119.8, 118.7, 113.9, 66.2, 53.3, 51.4, 37.6;

FTIR (ATR, $\left.\mathbf{c m}^{-1}\right):$ 3385, 3949, 2844, 1724, 1705, 1064, 1527, 1435, 1287, 1226, 1178, 1145 , 1114, 1033, 919, 847, 766, 707.

HRMS (ESI-TOF): calc'd for $\mathrm{C}_{20} \mathrm{H}_{22} \mathrm{NO}_{4}[\mathrm{M}+\mathrm{H}]^{+}$340.1543; found 340.1547;

SFC Analysis: CSP SFC (AD-H), 10\% Methanol, continuous flow at $2 \mathrm{~mL} / \mathrm{min}, 35^{\circ} \mathrm{C}, 160 \mathrm{bar}$, $210 \mathrm{~nm} ; t_{\text {major }}=2.1 \mathrm{~min}, t_{\text {minor }}=2.5 \mathrm{~min}$, ee $98 \%$;

$[\alpha]_{\mathbf{D}^{20}}=+23.0^{\circ}\left(C=0.69, \mathrm{CHCl}_{3}\right)$.

b) Methyl (R)-4-((1-methoxy-1-oxo-2-phenylpent-4-en-2-yl)amino)benzoate (3g'):

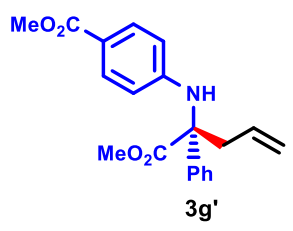

Yield: $88 \%, 148 \mathrm{mg}(0.5 \mathrm{mmol}$ scale $)$;

Physical State: Colorless oily liquid;

SFC Analysis: CSP SFC (AD-H), 10\% Methanol, continuous flow at $2 \mathrm{~mL} / \mathrm{min}, 35^{\circ} \mathrm{C}, 160 \mathrm{bar}$, $210 \mathrm{~nm} ; t_{\text {major }}=2.6 \mathrm{~min}, t_{\text {minor }}=2.1 \mathrm{~min}$, ee $97 \%$;

$[\alpha]_{\mathbf{D}^{20}}=-22.6^{\circ}\left(C=0.75, \mathrm{CHCl}_{3}\right)$.

8. Methyl (S)-2-(naphthalen-1-ylamino)-2-phenylpent-4-enoate (3h):

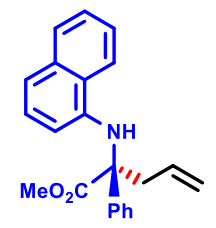

$3 \mathbf{h}$

Yield: $92 \%, 151 \mathrm{mg}(0.5 \mathrm{mmol})$; 
Physical State: Colorless oily liquid;

$\boldsymbol{R}_{\mathbf{f}}=0.53(10 \% \mathrm{EA} / \mathrm{Hex})$

${ }^{1}$ H NMR (600 MHz, Chloroform- $d$ ): $\delta 8.07(\mathrm{~d}, J=8.4 \mathrm{~Hz}, 1 \mathrm{H}), 7.79(\mathrm{~d}, J=7.9 \mathrm{~Hz}, 1 \mathrm{H}), 7.66$ $(\mathrm{d}, J=7.5 \mathrm{~Hz}, 2 \mathrm{H}), 7.52(\mathrm{t}, J=7.0 \mathrm{~Hz}, 1 \mathrm{H}), 7.48(\mathrm{t}, J=7.0 \mathrm{~Hz}, 1 \mathrm{H}), 7.38(\mathrm{t}, J=7.6 \mathrm{~Hz}, 2 \mathrm{H}), 7.32$ $(\mathrm{t}, J=7.3 \mathrm{~Hz}, 1 \mathrm{H}), 7.17(\mathrm{~d}, J=8.1 \mathrm{~Hz}, 1 \mathrm{H}), 7.04(\mathrm{t}, J=7.9 \mathrm{~Hz}, 1 \mathrm{H}), 6.26(\mathrm{~s}, 1 \mathrm{H}), 6.07(\mathrm{~d}, J=7.6$ $\mathrm{Hz}, 1 \mathrm{H}), 5.65(\mathrm{dtd}, J=17.6,8.2,6.3 \mathrm{~Hz}, 1 \mathrm{H}), 5.09-5.00(\mathrm{~m}, 2 \mathrm{H}), 3.73(\mathrm{~s}, 3 \mathrm{H}), 3.51$ (dd, $J=13.6$, $6.2 \mathrm{~Hz}, 1 \mathrm{H}), 3.34(\mathrm{dd}, J=13.6,8.2 \mathrm{~Hz}, 1 \mathrm{H})$;

${ }^{13}$ C NMR (151 MHz, Chloroform-d): $\delta$ 174.1, 139.8, 138.8, 134.5, 132.4, 128.7, 128.7, 127.7, $126.9,125.9,125.6,124.9,124.0,120.0,119.2,117.3,107.8,66.5,53.2,37.3$;

HRMS (ESI-TOF): calc'd for $\mathrm{C}_{22} \mathrm{H}_{22} \mathrm{NO}_{2}[\mathrm{M}+\mathrm{H}]^{+} 332.1645$; found 332.1650;

FTIR (ATR, $\left.\mathbf{c m}^{-1}\right)$ : 3424, 3384, 2949, 1726, 1706, 1604, 1526, 1434, 1287, 1227, 1178, 1146, 1033, 921, 767, 694 .

SFC Analysis: CSP SFC (AD-H), 10\% Methanol, continuous flow at $2 \mathrm{~mL} / \mathrm{min}, 35^{\circ} \mathrm{C}, 160 \mathrm{bar}$, $250 \mathrm{~nm} ; t_{\text {major }}=1.3 \mathrm{~min}, t_{\text {minor }}=1.7 \mathrm{~min}$, ee $99 \%$;

$[\alpha]_{\mathrm{D}^{20}}=+23.2^{\circ}\left(C=0.68, \mathrm{CHCl}_{3}\right)$.

9. a) Methyl (S)-2-(4-methoxyphenyl)-2-(phenylamino)pent-4-enoate (3i):

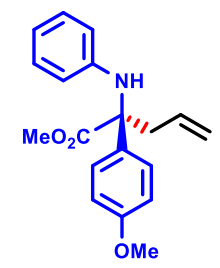

$3 \mathbf{i}$

Yield: $91 \%, 140 \mathrm{mg}$ scale $(0.5 \mathrm{mmol}$ scale);

Physical State: Colorless oily liquid;

$\boldsymbol{R}_{\mathbf{f}}=0.38(10 \% \mathrm{EA} / \mathrm{Hex})$

${ }^{1}$ H NMR (600 MHz, Chloroform- $\left.d\right): \delta 7.52(\mathrm{~d}, J=8.9 \mathrm{~Hz}, 2 \mathrm{H}), 7.03(\mathrm{t}, J=7.9 \mathrm{~Hz}, 2 \mathrm{H}), 6.89(\mathrm{~d}$, $J=8.9 \mathrm{~Hz}, 2 \mathrm{H}), 6.64(\mathrm{t}, J=7.3 \mathrm{~Hz}, 1 \mathrm{H}), 6.40(\mathrm{~d}, J=7.9 \mathrm{~Hz}, 2 \mathrm{H}), 5.72-5.61(\mathrm{~m}, 1 \mathrm{H}), 5.28(\mathrm{~s}$, $1 \mathrm{H}), 5.08(\mathrm{~d}, J=10.0 \mathrm{~Hz}, 1 \mathrm{H}), 5.04(\mathrm{~d}, J=17.1 \mathrm{~Hz}, 1 \mathrm{H}), 3.81(\mathrm{~s}, 3 \mathrm{H}), 3.67(\mathrm{~s}, 3 \mathrm{H}), 3.28(\mathrm{dd}, J=$ $13.6,6.3 \mathrm{~Hz}, 1 \mathrm{H}), 3.21(\mathrm{dd}, J=13.6,8.0 \mathrm{~Hz}, 1 \mathrm{H})$; 
${ }^{13}$ C NMR (151 MHz, Chloroform-d): $\delta$ 173.9, 159.0, 144.4, 132.4, 132.1, 128.8, 128.2, 119.2, $117.5,115.2,114.0,65.9,55.2,52.9,37.8$;

FTIR (ATR, $\left.\mathbf{c m}^{-1}\right):$ 2951, 2843, 1730, 1602, 1504, 1249, 1223, 1177, 1033, 839, 747.

HRMS (ESI-TOF): calc'd for $\mathrm{C}_{19} \mathrm{H}_{22} \mathrm{NO}_{3}[\mathrm{M}+\mathrm{H}]^{+}$312.1594; found 312.1599;

SFC Analysis: CSP SFC (AD-H), 10\% Methanol, continuous flow at $2 \mathrm{~mL} / \mathrm{min}, 35^{\circ} \mathrm{C}, 160$ bar, $250 \mathrm{~nm} ; t_{\text {major }}=2.9 \mathrm{~min}, t_{\text {minor }}=1.6 \mathrm{~min}$, ee $98 \%$;

$[\alpha]_{\mathbf{D}^{20}}=+27.1^{\circ}\left(C=0.73, \mathrm{CHCl}_{3}\right)$.

b) Methyl (R)-2-(4-methoxyphenyl)-2-(phenylamino)pent-4-enoate (3i'):

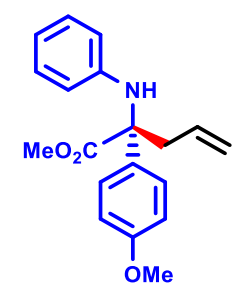

$3 i^{\prime}$

Yield: $96 \%, 148 \mathrm{mg}(0.5 \mathrm{mmol} \mathrm{scale})$;

Physical State: Colorless oily liquid;

SFC Analysis: CSP SFC (AD-H), 10\% Methanol, continuous flow at $2 \mathrm{~mL} / \mathrm{min}, 35^{\circ} \mathrm{C}, 160 \mathrm{bar}$, $250 \mathrm{~nm} ; \mathrm{t}_{\text {major }}=1.6 \mathrm{~min}, \mathrm{t}_{\mathrm{minor}}=3.0 \mathrm{~min}$, ee $98 \%$;

$[\alpha]_{\mathrm{D}^{20}}=-49.6^{\circ}\left(C=0.62, \mathrm{CHCl}_{3}\right)$.

10. a) Methyl (S)-2-(4-methoxyphenyl)-2-((4-methoxyphenyl)amino)pent-4-enoate (3j):

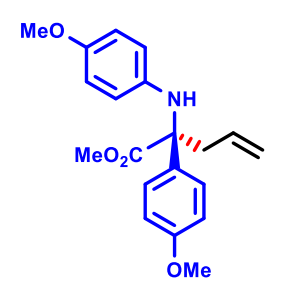

3j

Yield: $95 \%, 171 \mathrm{mg}(0.5 \mathrm{mmol}$ scale $)$;

Physical State: Colorless oily liquid; 
$\boldsymbol{R}_{\mathbf{f}}=0.28(10 \% \mathrm{EA} / \mathrm{Hex})$

${ }^{1}$ H NMR (600 MHz, Chloroform-d): $\delta 7.51(\mathrm{~d}, J=8.8 \mathrm{~Hz}, 2 \mathrm{H}), 6.89(\mathrm{~d}, J=8.9 \mathrm{~Hz}, 2 \mathrm{H}), 6.62$ $(\mathrm{d}, J=8.9 \mathrm{~Hz}, 2 \mathrm{H}), 6.35(\mathrm{~d}, J=8.9 \mathrm{~Hz}, 2 \mathrm{H}), 5.66(\mathrm{ddt}, J=17.1,10.1,7.2 \mathrm{~Hz}, 1 \mathrm{H}), 5.07(\mathrm{~d}, J=$ $10.2 \mathrm{~Hz}, 1 \mathrm{H}), 5.02(\mathrm{~d}, J=17.1 \mathrm{~Hz}, 1 \mathrm{H}), 5.01$ (missing NH) (brs, 1H), 3.81 (s, 3H), $3.68(\mathrm{~s}, 3 \mathrm{H})$, $3.66(\mathrm{~s}, 3 \mathrm{H}), 3.25-3.10(\mathrm{~m}, 2 \mathrm{H})$;

${ }^{13}$ C NMR (151 MHz, Chloroform-d): $\delta$ 174.0, 158.9, 152.1, 138.4, 132.6, 132.5, 128.2, 119.2 , $116.8,114.4,113.9,66.2,55.5,55.2,52.8,38.1$;

FTIR (ATR, $\mathbf{c m}^{-1}$ ): 2951, 2833, 1731, 1608, 1508, 1439, 1234, 1175, 1030, 921, 822, 774.

HRMS (ESI-TOF): calc'd for $\mathrm{C}_{20} \mathrm{H}_{24} \mathrm{NO}_{4}[\mathrm{M}+\mathrm{Na}]^{+}$364.1519; found 364.1495;

SFC Analysis: CSP SFC (AD-H), 10\% Methanol, continuous flow at $2 \mathrm{~mL} / \mathrm{min}, 35^{\circ} \mathrm{C}, 160 \mathrm{bar}$, $250 \mathrm{~nm} ; t_{\text {major }}=9.7 \mathrm{~min}, t_{\text {minor }}=2.4 \mathrm{~min}$, ee $97 \%$;

$[\alpha]_{\mathbf{D}^{20}}=+42.3^{\circ}\left(C=0.85, \mathrm{CHCl}_{3}\right)$

b) Methyl (R)-2-(4-methoxyphenyl)-2-((4-methoxyphenyl)amino)pent-4-enoate (3j’):

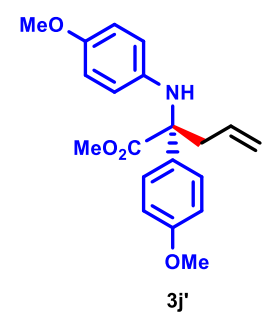

Yield: 95\%, $171 \mathrm{mg}(0.5 \mathrm{mmol}$ scale $)$;

Physical State: Colorless oily liquid;

SFC Analysis: CSP SFC (AD-H), 10\% Methanol, continuous flow at $2 \mathrm{~mL} / \mathrm{min}, 35^{\circ} \mathrm{C}, 160$ bar, $250 \mathrm{~nm} ; t_{\text {major }}=2.4 \mathrm{~min}, t_{\text {minor }}=9.9 \mathrm{~min}$, ee $98 \%$;

$[\alpha]_{\mathrm{D}^{20}}=-52.3^{\circ}\left(C=0.68, \mathrm{CHCl}_{3}\right)$

11. Methyl (S)-2-(4-methoxyphenyl)-2-((4-(trifluoromethyl)phenyl)amino)pent-4-enoate (3k): 


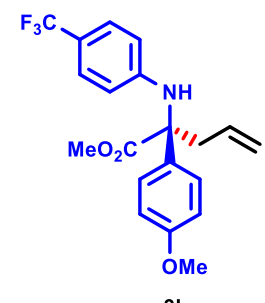

$3 \mathbf{k}$

Yield: $90 \%, 170 \mathrm{mg}(0.5 \mathrm{mmol}$ scale $)$;

Physical State: Colorless oily liquid;

$\boldsymbol{R}_{\mathbf{f}}=0.33(10 \%$ EA/Hex $)$

${ }^{1}$ H NMR (600 MHz, Chloroform- $\left.d\right): \delta 7.48(\mathrm{~d}, J=8.8 \mathrm{~Hz}, 2 \mathrm{H}), 7.27$ (d, $\left.J=8.5 \mathrm{~Hz}, 2 \mathrm{H}\right), 6.91$ $(\mathrm{d}, J=8.8 \mathrm{~Hz}, 2 \mathrm{H}), 6.43(\mathrm{~d}, J=8.5 \mathrm{~Hz}, 2 \mathrm{H}), 5.71(\mathrm{~s}, 1 \mathrm{H}), 5.70-5.62(\mathrm{~m}, 1 \mathrm{H}), 5.12(\mathrm{~d}, J=9.9 \mathrm{~Hz}$, $1 \mathrm{H}), 5.06(\mathrm{~d}, J=17.0 \mathrm{~Hz}, 1 \mathrm{H}), 3.82(\mathrm{~s}, 3 \mathrm{H}), 3.70(\mathrm{~s}, 3 \mathrm{H}), 3.31-3.24(\mathrm{~m}, 2 \mathrm{H})$;

${ }^{13}$ C NMR (151 MHz, Chloroform-d): $\delta$ 173.5, 159.2, 147.0, 131.9, 131.1, 127.9, 126.1 (q, $J=$ $3.6 \mathrm{~Hz}), 124.9$ (q, $J=270.4 \mathrm{~Hz}), 119.6,118.8$ (q, $J=32.6 \mathrm{~Hz}), 114.2,114.1,65.7,55.1,53.1,37.4$;

${ }^{19}$ F NMR (471 MHz, Chloroform-d): -60.2 (m);

FTIR (ATR, cm$\left.^{-1}\right):$ 3403, 2955, 2843, 1732, 1615, 1529, 1509, 1441, 1431, 1251, 1225, 1179, 1159, 1103, 1065, 1033, 924, 827.

HRMS (ESI-TOF): calc'd for $\mathrm{C}_{20} \mathrm{H}_{21} \mathrm{~F}_{3} \mathrm{NO}_{3}[\mathrm{M}+\mathrm{H}]^{+}$380.1468; found 380.1452;

HPLC Analysis: Chiralpak IA, 4\% IP/Hexanes, continuous flow at $0.4 \mathrm{~mL} / \mathrm{min}, 230 \mathrm{~nm}$; $t_{\text {major }}=$ $14.5 \mathrm{~min}, t_{\mathrm{minor}}=15.5 \mathrm{~min}$, ee $98 \%$;

$[\alpha]_{\mathbf{D}^{20}}=+25.7^{\circ}\left(C=0.73, \mathrm{CHCl}_{3}\right)$.

12. Methyl (S)-2-((4-chlorophenyl)amino)-2-(4-methoxyphenyl)pent-4-enoate (3l):

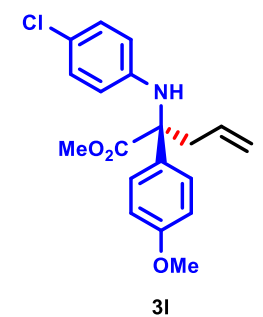

Yield: $92 \%, 158 \mathrm{mg}(0.5 \mathrm{mmol} \mathrm{scale})$; 
Physical State: Colorless oily liquid;

$\boldsymbol{R}_{\mathbf{f}}=0.31(10 \% \mathrm{EA} / \mathrm{Hex})$

${ }^{1}$ H NMR (600 MHz, Chloroform-d): $\delta 7.48(\mathrm{~d}, J=8.8 \mathrm{~Hz}, 2 \mathrm{H}), 6.97(\mathrm{~d}, J=8.8 \mathrm{~Hz}, 2 \mathrm{H}), 6.89$ $(\mathrm{d}, J=8.8 \mathrm{~Hz}, 2 \mathrm{H}), 6.31(\mathrm{~d}, J=8.8 \mathrm{~Hz}, 2 \mathrm{H}), 5.64(\mathrm{ddt}, J=17.3,10.1,7.2 \mathrm{~Hz}, 1 \mathrm{H}), 5.33(\mathrm{~s}, 1 \mathrm{H})$, $5.09(\mathrm{~d}, J=10.0 \mathrm{~Hz}, 1 \mathrm{H}), 5.04(\mathrm{~d}, J=17.1 \mathrm{~Hz}, 1 \mathrm{H}), 3.81(\mathrm{~s}, 3 \mathrm{H}), 3.67$ (s, 3H), 3.21 (dt, $J=10.6$, $5.8 \mathrm{~Hz}, 2 \mathrm{H})$;

${ }^{13}$ C NMR (151 MHz, Chloroform-d): $\delta$ 173.7, 159.1, 143.0, 132.1, 131.6, 128.6, 128.1, 122.1, $119.5,116.2,114.1,65.8,55.2,53.0,37.7$

FTIR (ATR, $\mathbf{c m}^{-1}$ ): 292, 8328, 1731, 1598, 1497, 1439, 1296, 1250, 1223, 1177, 1033, 923, 817, 762.

HRMS (ESI-TOF): calc'd for $\mathrm{C}_{19} \mathrm{H}_{21} \mathrm{ClNO}_{3}[\mathrm{M}+\mathrm{H}]^{+}$346.1204; found 346.1224;

SFC Analysis: CSP SFC (AD-H), 10\% Methanol, continuous flow at $2 \mathrm{~mL} / \mathrm{min}, 35^{\circ} \mathrm{C}, 160$ bar, $250 \mathrm{~nm} ; t_{\text {major }}=5.4 \mathrm{~min}, t_{\text {minor }}=2.0 \mathrm{~min}$, ee $96 \%$;

$[\alpha]_{\mathbf{D}^{20}}=+23.0^{\circ}\left(C=0.73, \mathrm{CHCl}_{3}\right)$.

13. Methyl (S)-2-(4-chlorophenyl)-2-(phenylamino)pent-4-enoate (3m):

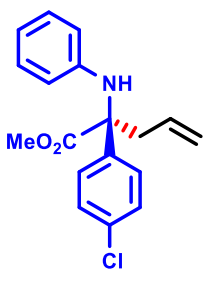

$3 m$

Yield: $93 \%, 145 \mathrm{mg}(0.5 \mathrm{mmol}$ scale $)$;

Physical State: Colorless oily liquid;

$\boldsymbol{R}_{\mathbf{f}}=0.70(10 \% \mathrm{EA} / \mathrm{Hex})$

${ }^{1}$ H NMR (600 MHz, Chloroform- $\left.d\right): \delta 7.56(\mathrm{~d}, J=8.7 \mathrm{~Hz}, 2 \mathrm{H}), 7.34(\mathrm{~d}, J=8.7 \mathrm{~Hz}, 2 \mathrm{H}), 7.06-$ $7.02(\mathrm{~m}, 2 \mathrm{H}), 6.67(\mathrm{t}, J=7.3 \mathrm{~Hz}, 1 \mathrm{H}), 6.38(\mathrm{~d}, J=8.6 \mathrm{~Hz}, 2 \mathrm{H}), 5.77-5.57(\mathrm{~m}, 1 \mathrm{H}), 5.26(\mathrm{~s}, 1 \mathrm{H})$, $5.10(\mathrm{~d}, J=11.1 \mathrm{~Hz}, 1 \mathrm{H}), 5.05(\mathrm{~d}, J=17.1 \mathrm{~Hz}, 1 \mathrm{H}), 3.68(\mathrm{~s}, 3 \mathrm{H}), 3.26(\mathrm{dd}, J=13.5,6.3 \mathrm{~Hz}, 1 \mathrm{H})$, $3.19(\mathrm{dd}, J=13.6,8.0 \mathrm{~Hz}, 1 \mathrm{H})$; 
${ }^{13}$ C NMR (151 MHz, Chloroform-d): $\delta$ 173.2, 144.0, 138.8, 133.6, 131.9, 128.9, 128.8, 128.6, 119.6, 117.8, 115.2, 66.0, 53.1, 38.2;

FTIR (ATR, $\mathbf{c m}^{-1}$ ): 2952, 2844, 1732, 1601, 1502, 1490, 1432, 1281, 1223, 1183, 1093, 1012, $841,747,692$.

HRMS (ESI-TOF): calc'd for $\mathrm{C}_{18} \mathrm{H}_{19} \mathrm{ClNO}_{2}[\mathrm{M}+\mathrm{H}]^{+}$316.1099; found 316.1078;

SFC Analysis: CSP SFC (AD-H), 10\% Methanol, continuous flow at $2 \mathrm{~mL} / \mathrm{min}, 35^{\circ} \mathrm{C}, 160$ bar, $250 \mathrm{~nm} ; t_{\text {major }}=2.3 \mathrm{~min}, t_{\operatorname{minor}}=1.7 \mathrm{~min}$, ee $98 \%$;

$[\alpha]_{\mathbf{D}^{\mathbf{2 0}}}=+27.2^{\circ}\left(C=0.63, \mathrm{CHCl}_{3}\right)$.

14. Methyl (S)-2-(4-chlorophenyl)-2-((4-methoxyphenyl)amino)pent-4-enoate (3n):

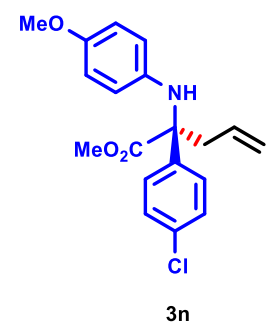

Yield: 90\%, $154 \mathrm{mg}(0.5 \mathrm{mmol}$ scale);

Physical State: Colorless oily liquid;

$\boldsymbol{R}_{\mathbf{f}}=0.28(10 \% \mathrm{EA} / \mathrm{Hex})$

${ }^{1}$ H NMR (600 MHz, Chloroform-d): $\delta 7.56(\mathrm{~d}, J=8.6 \mathrm{~Hz}, 2 \mathrm{H}), 7.33(\mathrm{~d}, J=8.6 \mathrm{~Hz}, 2 \mathrm{H}), 6.64$ $(\mathrm{d}, J=8.9 \mathrm{~Hz}, 2 \mathrm{H}), 6.33(\mathrm{~d}, J=8.9 \mathrm{~Hz}, 2 \mathrm{H}), 5.63(\mathrm{td}, J=17.0,7.2 \mathrm{~Hz}, 1 \mathrm{H}), 5.09(\mathrm{~d}, J=10.1 \mathrm{~Hz}$, $1 \mathrm{H}), 5.03(\mathrm{~d}, J=17.0 \mathrm{~Hz}, 1 \mathrm{H}), 4.94(\mathrm{~s}, 1 \mathrm{H}), 3.69(\mathrm{~s}, 3 \mathrm{H}), 3.67$ (s, 3H), $3.15(\mathrm{dq}, J=13.6,8.0,7.5$ $\mathrm{Hz}, 1 \mathrm{H})$;

${ }^{13}$ C NMR (151 MHz, Chloroform-d): $\delta$ 173.4, 152.3, 139.2, 137.9, 133.5, 132.0, 128.7, 128.7, $119.5,116.8,114.4,66.3,55.5,53.0,38.4$;

FTIR (ATR, cm-1): 2951, 2844, 1731, 1598, 1496, 1439, 1249, 1223, 1177, 1030, 922, 816, 762.

HRMS (ESI-TOF): calc'd for $\mathrm{C}_{19} \mathrm{H}_{21} \mathrm{ClNO}_{3}[\mathrm{M}+\mathrm{H}]^{+}$346.1204; found 346.1197;

HPLC Analysis: Chiralpak IA, 4\% IP/Hexanes, continuous flow at $0.4 \mathrm{~mL} / \mathrm{min}, 230 \mathrm{~nm}$; $t_{\text {major }}=$ $16.0 \mathrm{~min}, t_{\mathrm{minor}}=18.2 \mathrm{~min}$, ee $98 \%$; 
$[\alpha]_{\mathbf{D}^{20}}=+27.7^{\circ}\left(C=0.65, \mathrm{CHCl}_{3}\right)$.

15. a) Methyl (S)-2-((3-bromophenyl)amino)-2-(4-chlorophenyl)pent-4-enoate (3o):<smiles>C=CC(C=C)(Nc1cccc(Br)c1)c1ccc(Cl)cc1</smiles>

Yield: 94\%, $184 \mathrm{mg}(0.5 \mathrm{mmol}$ scale $)$;

Physical State: Colorless oily liquid;

$\boldsymbol{R}_{\mathbf{f}}=0.58(10 \% \mathrm{EA} / \mathrm{Hex})$

${ }^{1}$ H NMR (600 MHz, Chloroform-d): $\delta 7.50(\mathrm{~d}, J=8.6 \mathrm{~Hz}, 2 \mathrm{H}), 7.34(\mathrm{~d}, J=8.6 \mathrm{~Hz}, 2 \mathrm{H}), 6.85(\mathrm{t}$, $J=8.0 \mathrm{~Hz}, 1 \mathrm{H}), 6.77(\mathrm{~d}, J=7.9 \mathrm{~Hz}, 1 \mathrm{H}), 6.58(\mathrm{~s}, 1 \mathrm{H}), 6.21(\mathrm{~d}, J=9.5 \mathrm{~Hz}, 1 \mathrm{H}), 5.62(\mathrm{td}, J=17.1$, $7.2 \mathrm{~Hz}, 1 \mathrm{H}), 5.37(\mathrm{~s}, 1 \mathrm{H}), 5.11(\mathrm{~d}, J=10.1 \mathrm{~Hz}, 1 \mathrm{H}), 5.06(\mathrm{~d}, J=17.0 \mathrm{~Hz}, 1 \mathrm{H}), 3.68$ (s, 3H), $3.24-3.17(\mathrm{~m}, 2 \mathrm{H})$;

${ }^{13}$ C NMR (151 MHz, Chloroform-d): $\delta$ 172.9, 145.3, 138.1, 133.9, 131.5, 130.1, 129.0, 128.4, $122.8,120.7,119.9,117.9,113.4,65.9,53.3,37.8$;

FTIR (ATR, $\left.\mathbf{c m}^{-1}\right):$ 2952, 2844, 1732, 1594, 1890, 1840, 1279, 1224, 1187, 1145, 1092, 1033, 1013, 987, 924, 850764 .

HRMS (ESI-TOF): calc'd for $\mathrm{C}_{18} \mathrm{H}_{18} \mathrm{BrClNO}_{2}[\mathrm{M}+\mathrm{H}]^{+}$394.0204; found 394.0217;

SFC Analysis: CSP SFC (AD-H), 10\% Methanol, continuous flow at $2 \mathrm{~mL} / \mathrm{min}, 35^{\circ} \mathrm{C}, 160 \mathrm{bar}$, $250 \mathrm{~nm} ; t_{\text {major }}=3.0 \mathrm{~min}, t_{\text {minor }}=2.5 \mathrm{~min}$, ee $96 \%$;

$[\alpha]_{\mathrm{D}^{20}}=+27.6^{\circ}\left(C=0.68, \mathrm{CHCl}_{3}\right)$.

b) Methyl (R)-2-((3-bromophenyl)amino)-2-(4-chlorophenyl)pent-4-enoate (3o'):<smiles>C=CCC(C=C)(Nc1cccc(Br)c1)c1ccc(Cl)cc1</smiles> 
Yield: $97 \%, 196 \mathrm{mg}(0.5 \mathrm{mmol}$ scale);

Physical State: Colorless oily liquid;

SFC Analysis: CSP SFC (AD-H), 10\% Methanol, continuous flow at $2 \mathrm{~mL} / \mathrm{min}, 35^{\circ} \mathrm{C}, 160 \mathrm{bar}$, $250 \mathrm{~nm} ; t_{\text {major }}=2.5 \mathrm{~min}, t_{\text {minor }}=3.0 \mathrm{~min}, e e 98 \%$;

$[\alpha] \mathrm{D}^{20}=-25.6^{\circ}\left(\mathrm{C}=0.82, \mathrm{CHCl}_{3}\right)$.

16. Methyl (S)-2-(4-chlorophenyl)-2-((3-fluorophenyl)amino)pent-4-enoate (3p):

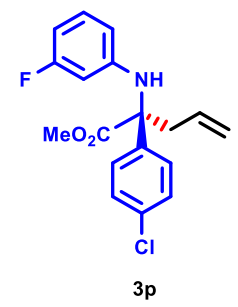

Yield: $90 \%, 167 \mathrm{mg}(0.5 \mathrm{mmol} \mathrm{scale})$;

Physical State: Colorless oily liquid;

$\boldsymbol{R}_{\mathbf{f}}=0.58(10 \% \mathrm{EA} / \mathrm{Hex}) ;$

${ }^{1}$ H NMR (600 MHz, Chloroform- $d$ ): $\delta 7.51(\mathrm{~d}, J=8.6 \mathrm{~Hz}, 2 \mathrm{H}), 7.34(\mathrm{~d}, J=8.6 \mathrm{~Hz}, 2 \mathrm{H}), 6.96$ $(\mathrm{q}, J=8.1 \mathrm{~Hz}, 1 \mathrm{H}), 6.34(\mathrm{td}, J=8.4,2.0 \mathrm{~Hz}, 1 \mathrm{H}), 6.16(\mathrm{dd}, J=8.2,1.7 \mathrm{~Hz}, 1 \mathrm{H}), 6.01(\mathrm{dt}, J=11.7$, $2.0 \mathrm{~Hz}, 1 \mathrm{H}), 5.62$ (ddt, $J=14.4,10.1,7.3 \mathrm{~Hz}, 1 \mathrm{H}), 5.41(\mathrm{~s}, 1 \mathrm{H}), 5.11(\mathrm{~d}, J=10.1 \mathrm{~Hz}, 1 \mathrm{H}), 5.05$ (d, $J=17.0 \mathrm{~Hz}, 1 \mathrm{H}), 3.68(\mathrm{~s}, 2 \mathrm{H}), 3.21(\mathrm{qd}, J=13.6,7.2 \mathrm{~Hz}, 1 \mathrm{H})$;

${ }^{13}$ C NMR (151 MHz, Chloroform- $d$ ): $\delta$ 173.0, $163.5(\mathrm{~d}, J=242.7 \mathrm{~Hz}), 145.8(\mathrm{~d}, J=10.9 \mathrm{~Hz})$, $138.2,133.9,131.6,130.0(\mathrm{~d}, J=10.1 \mathrm{~Hz}), 129.0,128.4,119.9,111.0(\mathrm{~d}, J=2.5 \mathrm{~Hz}), 104.4(\mathrm{~d}, J$ $=21.5 \mathrm{~Hz}), 101.9(\mathrm{~d}, J=25.9 \mathrm{~Hz}), 66.0,53.3,37.9$;

${ }^{19}$ F NMR (471 MHz, Chloroform- $d$ ): -111.7 (dddd);

FTIR (ATR, cm$\left.^{-1}\right)$ : 2952, 2844, 1732, 1615, 1588, 1491, 135, 1286, 1226, 1191, 1151, 1093, 1053, 1013, 924, 832, 761.

HRMS (ESI-TOF): calc'd for $\mathrm{C}_{18} \mathrm{H}_{18} \mathrm{ClFNO}_{2}[\mathrm{M}+\mathrm{H}]^{+}$334.1005; found 334.0984;

SFC Analysis: CSP SFC (AD-H), 10\% Methanol, continuous flow at $2 \mathrm{~mL} / \mathrm{min}, 35^{\circ} \mathrm{C}, 160$ bar, $250 \mathrm{~nm} ; t_{\text {major }}=1.7 \mathrm{~min}, t_{\text {minor }}=1.5 \mathrm{~min}$, ee $95 \%$; 
$[\alpha] \mathrm{D}^{\mathbf{2 0}}=+42.3^{\circ}\left(C=0.56, \mathrm{CHCl}_{3}\right)$

17. Methyl (S)-2-(4-fluorophenyl)-2-(phenylamino)pent-4-enoate (3q):

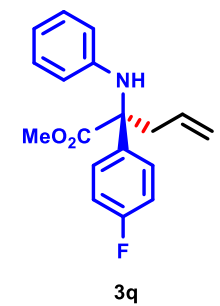

Yield: $93 \%, 138 \mathrm{mg}(0.5 \mathrm{mmol}$ scale $)$;

Physical State: Colorless oily liquid;

$\boldsymbol{R}_{\mathbf{f}}=0.48(10 \% \mathrm{EA} / \mathrm{Hex})$

${ }^{1}$ H NMR (600 MHz, Chloroform-d): $\delta 7.61(\mathrm{dd}, J=8.9,5.3 \mathrm{~Hz}, 2 \mathrm{H}), 7.06(\mathrm{dd}, J=8.0,7.4 \mathrm{~Hz}$, 4H), $6.68(\mathrm{t}, J=7.3 \mathrm{~Hz}, 1 \mathrm{H}), 6.39(\mathrm{~d}, J=7.8 \mathrm{~Hz}, 2 \mathrm{H}), 5.76-5.59(\mathrm{~m}, 1 \mathrm{H}), 5.29(\mathrm{~s}, 1 \mathrm{H}), 5.11(\mathrm{~d}$, $J=10.1 \mathrm{~Hz}, 1 \mathrm{H}), 5.06(\mathrm{~d}, J=17.1 \mathrm{~Hz}, 1 \mathrm{H}), 3.69$ (s, 3H), 3.29 (dd, $J=13.5,6.4 \mathrm{~Hz}, 1 \mathrm{H}), 3.22$ (dd, $J=13.6,8.0 \mathrm{~Hz}, 1 \mathrm{H})$;

${ }^{13}$ C NMR (151 MHz, Chloroform- $d$ ): $\delta$ 173.4, $162.2(\mathrm{~d}, J=247.0 \mathrm{~Hz}), 144.1,135.9(\mathrm{~d}, J=3.2$ $\mathrm{Hz}), 132.0,128.8$ (d, $J=7.5 \mathrm{~Hz}), 128.8,119.5,117.7,115.5(\mathrm{~d}, J=21.3 \mathrm{~Hz}), 115.2,65.9,53.0$, 38.2 ;

${ }^{19}$ F NMR (471 MHz, Chloroform-d): -113.9 (tt);

FTIR (ATR, cm$\left.^{-1}\right)$ : 3390, 2950, 2844, 1730, 1602, 1499, 1284, 1221, 1184, 1160, 1033, 928, 843, 747,688 .

HRMS (ESI-TOF): calc'd for $\mathrm{C}_{18} \mathrm{H}_{19} \mathrm{FNO}_{2}[\mathrm{M}+\mathrm{H}]^{+}$300.1394; found 300.1400;

SFC Analysis: CSP SFC (AD-H), 10\% Methanol, continuous flow at $2 \mathrm{~mL} / \mathrm{min}, 35^{\circ} \mathrm{C}, 160 \mathrm{bar}$, $250 \mathrm{~nm} ; t_{\text {major }}=1.9 \mathrm{~min}, t_{\text {minor }}=1.2 \mathrm{~min}$, ee $98 \%$;

$[\alpha]_{\mathrm{D}^{20}}=+20.5^{\circ}\left(C=0.68, \mathrm{CHCl}_{3}\right)$.

18. Methyl (S)-2-(4-fluorophenyl)-2-((4-methoxyphenyl)amino)pent-4-enoate (3r): 


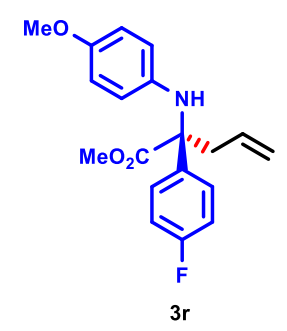

Yield: $93 \%, 1.22 \mathrm{~g}$ (4 mmol scale);

Physical State: Colorless oily liquid;

$\boldsymbol{R}_{\mathbf{f}}=0.41(10 \% \mathrm{EA} / \mathrm{Hex})$;

${ }^{1}$ H NMR (600 MHz, Chloroform- $d$ ): $\delta 7.61-7.56(\mathrm{~m}, 2 \mathrm{H}), 7.07-7.01(\mathrm{~m}, 2 \mathrm{H}), 6.63$ (d, $J=8.9$ $\mathrm{Hz}, 2 \mathrm{H}), 6.33(\mathrm{~d}, J=8.9 \mathrm{~Hz}, 2 \mathrm{H}), 5.64$ (ddt, $J=14.4,10.2,7.4 \mathrm{~Hz}, 1 \mathrm{H}), 5.09$ (d, $J=9.1 \mathrm{~Hz}, 1 \mathrm{H})$, $5.03(\mathrm{~d}, J=17.1 \mathrm{~Hz}, 1 \mathrm{H}), 4.94(\mathrm{~s}, 1 \mathrm{H}), 3.69(\mathrm{~s}, 3 \mathrm{H}), 3.67$ (s, 3H), 3.19-3.13 (m, 2H);

${ }^{13}$ C NMR (151 MHz, Chloroform- $\left.d\right): \delta 173.6,162.2$ (d, $\left.J=246.9 \mathrm{~Hz}\right), 152.3,138.1,136.4(\mathrm{~d}, J$ $=3.0 \mathrm{~Hz}), 132.1,128.9(\mathrm{~d}, J=8.1 \mathrm{~Hz}), 119.4,116.8,115.4(\mathrm{~d}, J=21.3 \mathrm{~Hz}), 114.4,66.3,55.5$, 52.9, 38.6;

${ }^{19}$ F NMR (471 MHz, Chloroform-d): -114.1 (m);

FTIR (ATR, $\left.\mathbf{c m}^{-1}\right):$ 2952, 2833, 1732, 1601, 1506, 1221, 1179, 1033, 922, 820.

HRMS (ESI-TOF): calc'd for $\mathrm{C}_{19} \mathrm{H}_{21} \mathrm{FNO}_{3}[\mathrm{M}+\mathrm{H}]^{+}$330.1500; found 330.1522;

HPLC Analysis: Chiralpak IA, 5\% IP/Hexanes, continuous flow at $0.4 \mathrm{~mL} / \mathrm{min}, 250 \mathrm{~nm}$; $t_{\text {major }}=$ $15.5 \mathrm{~min}, t_{\mathrm{minor}}=18.8 \mathrm{~min}$, ee $98 \%$;

$[\alpha]_{\mathbf{D}^{20}}=+25.6^{\circ}\left(C=0.62, \mathrm{CHCl}_{3}\right)$.

19. Methyl (S)-2-(4-fluorophenyl)-2-((4-methoxyphenyl)amino)pent-4-enoate (3s):

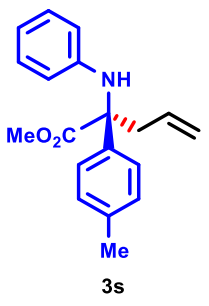

Yield: $94 \%, 138 \mathrm{mg}(0.5 \mathrm{mmol}$ scale);

Physical State: Colorless oily liquid; 
$\boldsymbol{R}_{\mathbf{f}}=0.50(10 \% \mathrm{EA} / \mathrm{Hex})$

${ }^{1}$ H NMR (600 MHz, Chloroform-d): $\delta 7.50(\mathrm{~d}, J=8.2 \mathrm{~Hz}, 2 \mathrm{H}), 7.19(\mathrm{~d}, J=8.1 \mathrm{~Hz}, 2 \mathrm{H}), 7.03(\mathrm{t}$, $J=7.9 \mathrm{~Hz}, 2 \mathrm{H}), 6.65(\mathrm{t}, J=7.3 \mathrm{~Hz}, 1 \mathrm{H}), 6.41(\mathrm{~d}, J=8.0 \mathrm{~Hz}, 2 \mathrm{H}), 5.77-5.61(\mathrm{~m}, 1 \mathrm{H}), 5.31(\mathrm{~s}$, $1 \mathrm{H}), 5.09(\mathrm{~d}, J=10.0 \mathrm{~Hz}, 1 \mathrm{H}), 5.05(\mathrm{~d}, J=17.1 \mathrm{~Hz}, 1 \mathrm{H}), 3.68(\mathrm{~s}, 3 \mathrm{H}), 3.30(\mathrm{dd}, J=13.6,6.3 \mathrm{~Hz}$, $1 \mathrm{H}), 3.24(\mathrm{dd}, J=13.6,8.0 \mathrm{~Hz}, 1 \mathrm{H}), 2.37$ (s, 3H);

${ }^{13}$ C NMR (151 MHz, Chloroform-d): $\delta$ 173.8, 144.4, 137.3, 137.2, 132.4, 129.4, 128.8, 126.8, $119.1,117.4,115.2,66.2,53.0,37.7,21.0$;

FTIR (ATR, cm-1): 3412, 2956, 2844, 1727, 1603, 1505, 1436, 129, 1240, 1185, 1033, 944, 744, 690.

HRMS (ESI-TOF): calc'd for $\mathrm{C}_{19} \mathrm{H}_{22} \mathrm{NO}_{2}[\mathrm{M}+\mathrm{H}]^{+}$296.1645; found 296.1626;

SFC Analysis: CSP SFC (AD-H), 10\% Methanol, continuous flow at $2 \mathrm{~mL} / \mathrm{min}, 35^{\circ} \mathrm{C}, 160$ bar, $250 \mathrm{~nm} ; t_{\text {major }}=1.5 \mathrm{~min}, t_{\mathrm{minor}}=1.3 \mathrm{~min}$, ee $98 \%$;

$[\alpha]_{\mathrm{D}^{20}}=+23.0^{\circ}\left(C=0.73, \mathrm{CHCl}_{3}\right)$

20. Methyl (S)-2-((4-methoxyphenyl)amino)-2-(p-tolyl)pent-4-enoate (3t):

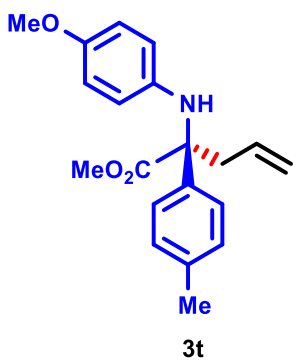

Yield: 91\%, $147 \mathrm{mg}(0.5 \mathrm{mmol}$ scale $)$;

Physical State: Colorless oily liquid;

$\boldsymbol{R}_{\mathbf{f}}=0.37(10 \% \mathrm{EA} / \mathrm{Hex})$

${ }^{1}$ H NMR (600 MHz, Chloroform-d): $\delta 7.49(\mathrm{~d}, J=8.2 \mathrm{~Hz}, 2 \mathrm{H}), 7.18(\mathrm{~d}, J=8.1 \mathrm{~Hz}, 2 \mathrm{H}), 6.63$ $(\mathrm{d}, J=9.0 \mathrm{~Hz}, 2 \mathrm{H}), 6.36(\mathrm{~d}, J=9.0 \mathrm{~Hz}, 2 \mathrm{H}), 5.68(\mathrm{ddt}, J=17.1,10.2,7.2 \mathrm{~Hz}, 1 \mathrm{H}), 5.08(\mathrm{~d}, J=$ $8.7 \mathrm{~Hz}, 1 \mathrm{H}), 5.03(\mathrm{~d}, J=17.1 \mathrm{~Hz}, 1 \mathrm{H}), 4.98(\mathrm{~s}, 1 \mathrm{H}), 3.69(\mathrm{~s}, 3 \mathrm{H}), 3.67(\mathrm{~s}, 3 \mathrm{H}), 3.26-3.13(\mathrm{~m}$, $2 \mathrm{H}), 2.36(\mathrm{~s}, 3 \mathrm{H})$; 
${ }^{13}$ C NMR (151 MHz, Chloroform-d): $\delta$ 174.0, 152.1, 138.4, 137.7, 137.3, 132.5, 129.3, 126.9, $119.1,116.8,114.4,66.5,55.5,52.8,38.0,21.0$;

FTIR (ATR, cm$^{-1}$ ): 2950, 2866, 1731, 1509, 1234, 1177, 1057, 1014, 921, 819, 765.

HRMS (ESI-TOF): calc'd for $\mathrm{C}_{20} \mathrm{H}_{24} \mathrm{NO}_{3}[\mathrm{M}+\mathrm{H}]^{+} 326.1751$; found 326.1740;

HPLC Analysis: Chiralpak IA, 5\% IP/Hexanes, continuous flow at $0.4 \mathrm{~mL} / \mathrm{min}, 250 \mathrm{~nm} ; t_{\text {major }}=$ $16.1 \mathrm{~min}, t_{\mathrm{minor}}=20.2 \mathrm{~min}$, ee $97 \%$;

$[\alpha]_{\mathrm{D}^{20}}=+42.1^{\circ}\left(\mathrm{C}=0.68, \mathrm{CHCl}_{3}\right)$.

21. Methyl (S)-2-([1,1'-biphenyl]-4-ylamino)-2-(p-tolyl)pent-4-enoate (3u):

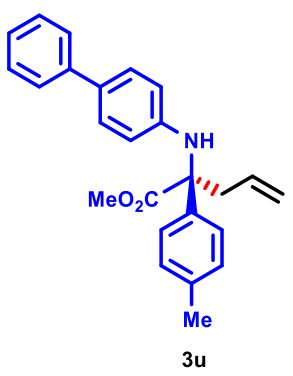

Yield: $85 \%, 157 \mathrm{mg}(0.5 \mathrm{mmol} \mathrm{scale})$;

Physical State: Colorless oily liquid;

$\boldsymbol{R}_{\mathbf{f}}=0.45(10 \% \mathrm{EA} / \mathrm{Hex})$

${ }^{1} \mathrm{H}$ NMR (600 MHz, Chloroform- $\left.\boldsymbol{d}\right): \delta 7.52(\mathrm{~d}, J=8.2 \mathrm{~Hz}, 2 \mathrm{H}), 7.49(\mathrm{~d}, J=7.3 \mathrm{~Hz}, 2 \mathrm{H}), 7.36(\mathrm{t}$, $J=7.7 \mathrm{~Hz}, 2 \mathrm{H}), 7.31(\mathrm{~d}, J=8.6 \mathrm{~Hz}, 2 \mathrm{H}), 7.24(\mathrm{t}, J=7.3 \mathrm{~Hz}, 1 \mathrm{H}), 7.20(\mathrm{~d}, J=8.1 \mathrm{~Hz}, 2 \mathrm{H}), 6.48$ $(\mathrm{d}, J=8.6 \mathrm{~Hz}, 2 \mathrm{H}), 5.80-5.63(\mathrm{~m}, 1 \mathrm{H}), 5.43(\mathrm{~s}, 1 \mathrm{H}), 5.12(\mathrm{~d}, J=10.2 \mathrm{~Hz}, 1 \mathrm{H}), 5.08(\mathrm{~d}, J=17.1$ $\mathrm{Hz}, 1 \mathrm{H}), 3.70(\mathrm{~s}, 3 \mathrm{H}), 3.34(\mathrm{dd}, J=13.6,6.3 \mathrm{~Hz}, 1 \mathrm{H}), 3.27(\mathrm{dd}, J=13.6,8.1 \mathrm{~Hz}, 1 \mathrm{H}), 2.37(\mathrm{~s}, 3 \mathrm{H})$;

${ }^{13}$ C NMR (151 MHz, Chloroform-d): $\delta$ 173.8, 143.8, 141.0, 137.4, 137.1, 132.4, 130.2, 129.5, $128.5,127.4,126.8,126.1,126.0,119.3,115.4,66.2,53.0,37.7,21.0$;

HRMS (ESI-TOF): calc'd for $\mathrm{C}_{25} \mathrm{H}_{26} \mathrm{NO}_{2}[\mathrm{M}+\mathrm{H}]^{+} 372.1958$; found 372.1935;

FTIR (ATR, cm$\left.^{-1}\right)$ : 3410, 2952, 2844, 1731, 1612, 1489, 1298, 1227, 1213, 1182, 1033, 919, 830, $181,726$.

SFC Analysis: CSP SFC (IA), 10\% Methanol, continuous flow at $2 \mathrm{~mL} / \mathrm{min}, 35^{\circ} \mathrm{C}, 160$ bar, 210 $\mathrm{nm} ; t_{\text {major }}=6.0 \mathrm{~min}, t_{\text {minor }}=5.6 \mathrm{~min}, e e 98 \%$; 
$[\alpha] \mathrm{D}^{\mathbf{2 0}}=+45.8^{\circ}\left(C=0.93, \mathrm{CHCl}_{3}\right)$.

11a. Characterization of Catalytic Asymmetric Allylation Products in Table 3:

1a. Ethyl $(R)-2-($ phenylamino)-2-(trifluoromethyl)pent-4-enoate (5a):

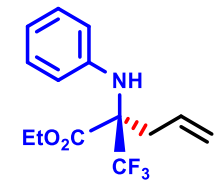

$5 a$

Yield: $91 \%, 1.04 \mathrm{~g}$ (4 mmol scale);

Physical State: Colorless oily liquid;

$\boldsymbol{R}_{\mathbf{f}}=0.55(10 \% \mathrm{EA} / \mathrm{Hex}) ;$

${ }^{1}$ H NMR (600 MHz, Chloroform-d): $\delta 7.22$ - $7.16(\mathrm{~m}, 2 \mathrm{H}), 6.87(\mathrm{tt}, J=7.4,1.0 \mathrm{~Hz}, 1 \mathrm{H}), 6.82$ $(\mathrm{d}, J=7.9 \mathrm{~Hz}, 2 \mathrm{H}), 5.60-5.50(\mathrm{~m}, 1 \mathrm{H}), 5.16-5.07(\mathrm{~m}, 2 \mathrm{H}), 4.69(\mathrm{~s}, 1 \mathrm{H}), 4.37-4.26(\mathrm{~m}, 2 \mathrm{H})$, $3.16(\mathrm{ddt}, J=14.5,6.2,1.2 \mathrm{~Hz}, 1 \mathrm{H}), 2.88(\mathrm{dd}, J=14.5,8.2 \mathrm{~Hz}, 1 \mathrm{H}), 1.29(\mathrm{t}, J=7.1 \mathrm{~Hz}, 3 \mathrm{H})$;

${ }^{13}$ C NMR (151 MHz, Chloroform-d): $\delta$ 168.0, 143.5, 129.8, 129.0, 124.65 (q, $J=288.2 \mathrm{~Hz}$ ), 121.0, 120.3, 117.7, 67.2 (q, $J=27.3 \mathrm{~Hz}), 63.0,34.6,13.8$;

${ }^{19}$ F NMR (471 MHz, Chloroform-d): -71.1(s);

FTIR (ATR, $\left.\mathbf{c m}^{-1}\right):$ 3382, 2984, 1742, 1604, 1499, 1263, 1246, 1218, 1190, 1152, 1033, 926, $750,730,693$.

HRMS (ESI-TOF): calc'd for $\mathrm{C}_{14} \mathrm{H}_{17} \mathrm{~F}_{3} \mathrm{NO}_{2}[\mathrm{M}+\mathrm{H}]^{+}$288.1206; found 288.1206;

$[\boldsymbol{\alpha}] \mathbf{D}^{\mathbf{2 0}}=+8.8^{\circ}\left(C=1.25, \mathrm{CHCl}_{3}\right)$

1b. Ethyl (S)-2-(phenylamino)-2-(trifluoromethyl)pent-4-enoate (5a'):

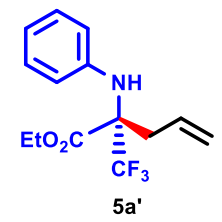

Yield: $89 \%, 127 \mathrm{mg}(0.5 \mathrm{mmol}$ scale);

Physical State: Colorless oily liquid;

$[\boldsymbol{\alpha}]_{\mathbf{D}^{\mathbf{2 0}}}=-11.4^{\circ}\left(C=1.20, \mathrm{CHCl}_{3}\right)$. 
2. Ethyl (R)-2-(naphthalen-2-ylamino)-2-(trifluoromethyl)pent-4-enoate (5b):

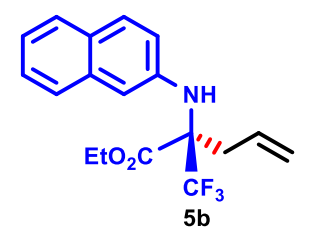

Yield: $94 \%, 158 \mathrm{mg}(0.5 \mathrm{mmol} \mathrm{scale})$;

Physical State: Beige colored viscous oily liquid;

$\boldsymbol{R}_{\mathbf{f}}=0.57(10 \% \mathrm{EA} / \mathrm{Hex})$;

${ }^{1}$ H NMR (600 MHz, Chloroform- $d$ ): $\delta 8.03(\mathrm{~d}, J=8.4 \mathrm{~Hz}, 1 \mathrm{H}), 7.82(\mathrm{~d}, J=7.7 \mathrm{~Hz}, 1 \mathrm{H}), 7.54-$ $7.45(\mathrm{~m}, 2 \mathrm{H}), 7.43(\mathrm{~d}, J=8.2 \mathrm{~Hz}, 1 \mathrm{H}), 7.34(\mathrm{t}, J=7.9 \mathrm{~Hz}, 1 \mathrm{H}), 7.01(\mathrm{~d}, J=7.6 \mathrm{~Hz}, 1 \mathrm{H}), 5.52(\mathrm{~s}$, $1 \mathrm{H}), 5.34$ (tt, $J=17.0,9.0 \mathrm{~Hz}, 1 \mathrm{H}), 5.08-4.95(\mathrm{~m}, 2 \mathrm{H}), 4.37$ (qq, $J=10.7,7.1 \mathrm{~Hz}, 2 \mathrm{H}), 3.33$ (dd, $J=14.6,6.0 \mathrm{~Hz}, 1 \mathrm{H}), 2.95(\mathrm{dd}, J=14.5,8.3 \mathrm{~Hz}, 1 \mathrm{H}), 1.32(\mathrm{t}, J=7.1 \mathrm{~Hz}, 3 \mathrm{H})$;

${ }^{13}$ C NMR (151 MHz, Chloroform- $d$ ): $\delta$ 168.7, 138.5, 134.5, 129.8, 128.7, 126.4, 125.7, 125.6, $124.7\left(\mathrm{q},{ }^{1} J_{\mathrm{CF}}=288.2 \mathrm{~Hz}\right), 120.9,120.7,120.5,112.7(\mathrm{q}, J=3.8 \mathrm{~Hz}), 67.6\left(\mathrm{q},{ }^{2} J_{\mathrm{CF}}=27.5 \mathrm{~Hz}\right)$, 63.2, 33.7, 13.9;

${ }^{19}$ F NMR (471 MHz, Chloroform- $d$ ): -71.3(s);

FTIR (ATR, cm$^{-1}$ ): 3410, 2983, 1734, 1525, 1404, 1251, 1223, 117 1147, 1094, 1032, 993, 936, $783,765,71,673$

HRMS (ESI-TOF): calc'd for $\mathrm{C}_{18} \mathrm{H}_{19} \mathrm{~F}_{3} \mathrm{NO}_{2}[\mathrm{M}+\mathrm{H}]^{+} 338.1362$; found 338.1362;

SFC Analysis: CSP SFC (IA), 2\% Isopropanol, continuous flow at $2 \mathrm{~mL} / \mathrm{min}, 35^{\circ} \mathrm{C}, 160 \mathrm{bar}$, $230 \mathrm{~nm} ; t_{\text {major }}=1.7 \mathrm{~min}, t_{\text {minor }}=2.0 \mathrm{~min}, e e 97 \%$;

$[\boldsymbol{\alpha}] \mathbf{D}^{20}=+50.81^{\circ}\left(C=1.1, \mathrm{CHCl}_{3}\right)$.

3. Ethyl (R)-2-((3,5-dimethoxyphenyl)amino)-2-(trifluoromethyl)pent-4-enoate (5c):

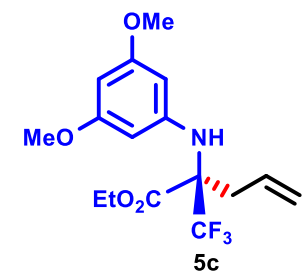

Yield: $92 \%, 159 \mathrm{mg}(0.5 \mathrm{mmol} \mathrm{scale})$; 
Physical State: Beige colored oily liquid;

$\mathbf{R}_{f}=0.28(10 \% \mathrm{EA} / \mathrm{Hex})$;

${ }^{1}$ H NMR (600 MHz, Chloroform- $d$ ): $\delta 6.02(\mathrm{t}, J=2.0 \mathrm{~Hz}, 1 \mathrm{H}), 5.99(\mathrm{~d}, J=2.0 \mathrm{~Hz}, 2 \mathrm{H}), 5.62-$ $5.51(\mathrm{~m}, 1 \mathrm{H}), 5.17-5.09(\mathrm{~m}, 2 \mathrm{H}), 4.71(\mathrm{~s}, 1 \mathrm{H}), 4.36-4.26(\mathrm{~m}, 2 \mathrm{H}), 3.74(\mathrm{~s}, 6 \mathrm{H}), 3.19(\mathrm{dd}, J=$ $14.5,6.1 \mathrm{~Hz}, 1 \mathrm{H}), 2.86(\mathrm{dd}, J=14.5,8.3 \mathrm{~Hz}, 1 \mathrm{H}), 1.30$ (t, $J=7.1 \mathrm{~Hz}, 3 \mathrm{H})$;

${ }^{13}$ C NMR (151 MHz, Chloroform- $\left.\boldsymbol{d}\right): \delta 168.0,161.3,145.3,129.8,124.5\left(\mathrm{q},{ }^{1} J_{\mathrm{CF}}=288.3 \mathrm{~Hz}\right)$, $120.9,96.3,92.4,67.2\left(\mathrm{q},{ }^{2} J_{\mathrm{CF}}=27.5 \mathrm{~Hz}\right), 63.1,55.1,34.5,13.9$;

${ }^{19}$ F NMR (471 MHz, Chloroform- $d$ ): $-71.0(\mathrm{~s})$;

FTIR (ATR, cm$^{-1}$ ): 3392, 2940, 2843, 1742, 1599, 1526, 1482, 1455, 1305, 1203, 1147, 1064, $1032,929,819,755,681$.

HRMS (ESI-TOF): calc'd for $\mathrm{C}_{16} \mathrm{H}_{21} \mathrm{~F}_{3} \mathrm{NO}_{4}[\mathrm{M}+\mathrm{H}]^{+} 348.1417$; found 348.1418;

SFC Analysis: CSP SFC (AD-H), 3\% Isopropanol, continuous flow at $2 \mathrm{~mL} / \mathrm{min}, 35^{\circ} \mathrm{C}, 160$ bar, $250 \mathrm{~nm} ; t_{\text {major }}=1.46 \mathrm{~min}, t_{\text {minor }}=1.28 \mathrm{~min}, e e 93 \%$;

$[\alpha]_{\mathbf{D}^{20}}=+9.27^{\circ}\left(C=1.1, \mathrm{CHCl}_{3}\right)$.

\section{Ethyl (R)-2-(p-tolylamino)-2-(trifluoromethyl)pent-4-enoate (5d):}

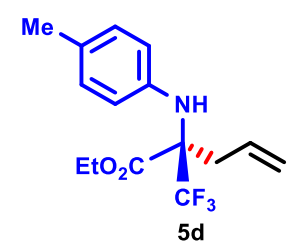

Yield: $99 \%, 149 \mathrm{mg}(0.5 \mathrm{mmol}$ scale $)$;

Physical State: Yellow colored oily liquid;

$\boldsymbol{R}_{\mathbf{f}}=0.55(10 \% \mathrm{EA} / \mathrm{Hex})$;

${ }^{1}$ H NMR (600 MHz, Chloroform-d): $\delta 7.01(\mathrm{~d}, J=8.1 \mathrm{~Hz}, 2 \mathrm{H}), 6.75(\mathrm{~d}, J=8.4 \mathrm{~Hz}, 2 \mathrm{H}), 5.57$ (ddd, $J=17.6,14.9,8.5 \mathrm{~Hz}, 1 \mathrm{H}), 5.15-5.09(\mathrm{~m}, 2 \mathrm{H}), 4.58(\mathrm{~s}, 1 \mathrm{H}), 4.36-4.27(\mathrm{~m}, 2 \mathrm{H}), 3.12$ (dd, $J=14.5,6.1 \mathrm{~Hz}, 1 \mathrm{H}), 2.86(\mathrm{dd}, J=14.5,8.2 \mathrm{~Hz}, 1 \mathrm{H}), 2.26$ (s, 3H), $1.30(\mathrm{t}, J=7.1 \mathrm{~Hz}, 3 \mathrm{H}$ ); ${ }^{13}$ C NMR (151 MHz, Chloroform- $d$ ): $\delta 168.1,141.0,130.03,130.00,129.5,124.6\left(\mathrm{q},{ }^{1} J_{\mathrm{CF}}=\right.$ $288.3 \mathrm{~Hz}), 120.6,118.5,67.5\left(\mathrm{q},{ }^{2} J_{\mathrm{CF}}=26.8 \mathrm{~Hz}\right), 63.0,34.5,20.4,13.9$;

${ }^{19}$ F NMR (471 MHz, Chloroform- $d$ ): - 71.1(s); 
FTIR (ATR, cm$\left.^{-1}\right): 3377,2095.1742,1617,1517,1261,1245,1219,1191,1150,1032,924$, 809.

HRMS (ESI-TOF): calc'd for $\mathrm{C}_{15} \mathrm{H}_{19} \mathrm{~F}_{3} \mathrm{NO}_{2}[\mathrm{M}+\mathrm{H}]^{+}$302.1362; Found 302.1365;

$[\boldsymbol{\alpha}] \mathbf{D}^{\mathbf{2 0}}=-1.8^{\circ}\left(\mathrm{C}=1.0, \mathrm{CHCl}_{3}\right)$.

5. Ethyl $(R)$-2-((4-methoxyphenyl)amino)-2-(trifluoromethyl)pent-4-enoate (5e):

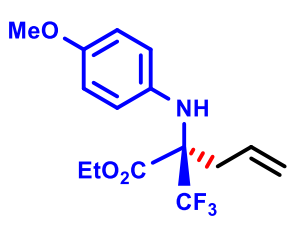

5e

Yield: $98 \%, 620 \mathrm{mg}$ (2 mmol scale);

Physical State: Brownish yellow colored oily liquid;

$\boldsymbol{R}_{\mathbf{f}}=0.46(10 \% \mathrm{EA} / \mathrm{Hex})$

${ }^{1} \mathrm{H}$ NMR (600 MHz, Chloroform-d): $\delta 6.84(\mathrm{~d}, J=8.9 \mathrm{~Hz}, 2 \mathrm{H}), 6.77(\mathrm{~d}, J=8.9 \mathrm{~Hz}, 2 \mathrm{H}), 5.56$ $(\mathrm{dq}, J=16.5,7.7 \mathrm{~Hz}, 1 \mathrm{H}), 5.14-5.07(\mathrm{~m}, 2 \mathrm{H}), 4.45(\mathrm{~s}, 1 \mathrm{H}), 4.35-4.25(\mathrm{~m}, 2 \mathrm{H}), 3.75(\mathrm{~s}, 3 \mathrm{H})$, $3.01(\mathrm{dd}, J=14.6,6.0 \mathrm{~Hz}, 1 \mathrm{H}), 2.82(\mathrm{dd}, J=14.6,8.1 \mathrm{~Hz}, 1 \mathrm{H}), 1.30(\mathrm{t}, J=7.1 \mathrm{~Hz}, 3 \mathrm{H})$;

${ }^{13}$ C NMR (151 MHz, Chloroform- $d$ ): $\delta 168.2,154.7,136.5,130.1,124.6\left(\mathrm{q},{ }^{1} J_{\mathrm{CF}}=288.1 \mathrm{~Hz}\right)$, $121.5,120.4,114.2,68.0\left(\mathrm{q},{ }^{2} J_{\mathrm{CF}}=26.4 \mathrm{~Hz}\right), 63.0,55.4,34.3,13.9$;

${ }^{19}$ F NMR (471 MHz, Chloroform-d): -71.5 (s);

FTIR (ATR, cm$^{-1}$ ): 3376, 2984, 2837, 1741, 1512, 1443, 1239, 1219, 1177, 1149, 1033, 924, 820.

HRMS (ESI-TOF): calc'd for $\mathrm{C}_{15} \mathrm{H}_{19} \mathrm{~F}_{3} \mathrm{NO}_{3}[\mathrm{M}+\mathrm{H}]^{+}$318.1312; Found 318.1313;

SFC Analysis: CSP SFC (AD-H), 6\% Isopropanol, continuous flow at $2 \mathrm{~mL} / \mathrm{min}, 35^{\circ} \mathrm{C}, 160$ bar, $210 \mathrm{~nm} ; t_{\text {major }}=1.08 \mathrm{~min}, t_{\mathrm{minor}}=1.27 \mathrm{~min}, e e 99 \%$;

$[\alpha] \mathbf{D}^{20}=-7.7^{\circ}\left(C=1.0, \mathrm{CHCl}_{3}\right)$.

6a. Ethyl (R)-2-((4-(methylthio)phenyl)amino)-2-(trifluoromethyl)pent-4-enoate (5f): 


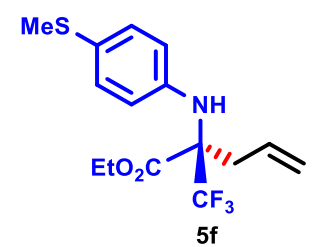

Yield: $92 \%, 153 \mathrm{mg}(0.5 \mathrm{mmol}$ scale $)$;

Physical State: Pale yellow colored oily liquid;

$\boldsymbol{R}_{\mathbf{f}}=0.42(10 \% \mathrm{EA} / \mathrm{Hex})$

${ }^{1}$ H NMR (600 MHz, Chloroform- $\left.d\right): \delta 7.17(\mathrm{~d}, J=8.5 \mathrm{~Hz}, 1 \mathrm{H}), 6.76(\mathrm{~d}, J=8.5 \mathrm{~Hz}, 1 \mathrm{H}), 5.53$ $(\mathrm{dq}, J=16.8,7.7 \mathrm{~Hz}, 1 \mathrm{H}), 5.15-5.07(\mathrm{~m}, 2 \mathrm{H}), 4.68(\mathrm{~s}, 1 \mathrm{H}), 4.37-4.25(\mathrm{~m}, 2 \mathrm{H}), 3.11$ (dd, $J=$ 14.6, 6.1 Hz, 1H), 2.86 (dd, $J=14.5,8.1 \mathrm{~Hz}, 1 \mathrm{H}), 2.42$ (s, 3H), 1.29 (t, $J=7.1 \mathrm{~Hz}, 3 \mathrm{H})$;

${ }^{13}$ C NMR (151 MHz, Chloroform- $\left.d\right): \delta 168.0,141.8,129.67,129.60,128.5,124.6\left(\mathrm{q},{ }^{1} J_{\mathrm{CF}}=\right.$ $288.3 \mathrm{~Hz}), 121.0,118.5,67.3\left(\mathrm{q},{ }^{2} J_{\mathrm{CF}}=27.3 \mathrm{~Hz}\right), 63.1,34.5,17.7,13.9$;

${ }^{19}$ F NMR (471 MHz, Chloroform-d): -71.1 (s);

FTIR (ATR, $\left.\mathbf{c m}^{-1}\right):$ 3377, 2983, 1742, 1599, 1497, 1442, 1259, 1243, 1219, 1152, 1032, 927, 812.

HRMS (ESI-TOF): calc'd for $\mathrm{C}_{15} \mathrm{H}_{19} \mathrm{~F}_{3} \mathrm{NO}_{2} \mathrm{~S}[\mathrm{M}+\mathrm{H}]^{+} 334.1083$; Found 334.1077;

SFC Analysis: CSP SFC (AD-H), 3\% Isopropanol, continuous flow at $2 \mathrm{~mL} / \mathrm{min}, 35^{\circ} \mathrm{C}, 160$ bar, $250 \mathrm{~nm} ; t_{\text {major }}=1.82 \mathrm{~min}, t_{\text {minor }}=2.15 \mathrm{~min}$, ee $95 \%$;

$[\boldsymbol{\alpha}]_{\mathbf{D}^{\mathbf{2 0}}}=-4.7^{\circ}\left(C=1.0, \mathrm{CHCl}_{3}\right)$.

6b. Ethyl (S)-2-((4-(methylthio)phenyl)amino)-2-(trifluoromethyl)pent-4-enoate (5f'):

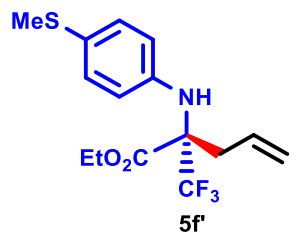

Yield: $92 \%, 159 \mathrm{mg}(0.5 \mathrm{mmol}$ scale $)$;

Physical State: Pale yellow colored oily liquid;

SFC Analysis: CSP SFC (AD-H), 3\% Isopropanol, continuous flow at $2 \mathrm{~mL} / \mathrm{min}, 35^{\circ} \mathrm{C}, 160$ bar, $250 \mathrm{~nm} ; t_{\text {major }}=2.15 \mathrm{~min}, t_{\text {minor }}=1.82 \mathrm{~min}$, ee $97 \%$; 
$[\boldsymbol{\alpha}]_{\mathbf{D}^{\mathbf{2 0}}}=+5.5^{\circ}\left(C=1.0, \mathrm{CHCl}_{3}\right)$.

7. Ethyl (R)-2-((3-chlorophenyl)amino)-2-(trifluoromethyl)pent-4-enoate (5g):<smiles>C=C[C@]([13CH3])(Nc1cccc(Cl)c1)C(=O)OCC</smiles>

Yield: $96 \%, 155 \mathrm{mg}(0.5 \mathrm{mmol}$ scale $)$;

Physical State: Beige colored oily liquid;

$\boldsymbol{R}_{\mathbf{f}}=0.43(10 \% \mathrm{EA} / \mathrm{Hex})$

${ }^{1}$ H NMR (600 MHz, Chloroform-d): $\delta 7.09(\mathrm{t}, J=8.1 \mathrm{~Hz}, 1 \mathrm{H}), 6.86-6.79(\mathrm{~m}, 2 \mathrm{H}), 6.67$ (dd, $J$ $=8.2,2.0 \mathrm{~Hz}, 1 \mathrm{H}), 5.56-5.47(\mathrm{~m}, 1 \mathrm{H}), 5.18-5.08(\mathrm{~m}, 2 \mathrm{H}), 4.78(\mathrm{~s}, 1 \mathrm{H}), 4.37-4.27(\mathrm{~m}, 2 \mathrm{H})$, $3.14(\mathrm{dd}, J=14.6,6.3 \mathrm{~Hz}, 1 \mathrm{H}), 2.89(\mathrm{dd}, J=14.6,8.1 \mathrm{~Hz}, 1 \mathrm{H}), 1.30$ (t, $J=7.1 \mathrm{~Hz}, 3 \mathrm{H})$;

${ }^{13}$ C NMR (151 MHz, Chloroform-d): $\delta$ 167.6, 144.8, 134.6, 129.9, 129.3, 124.5 (q, $J=288.0$ $\mathrm{Hz}), 121.3,120.1,117.3,115.5,67.0$ (q, $J=27.6 \mathrm{~Hz}), 63.3,34.5,13.8$;

${ }^{19}$ F NMR (471 MHz, Chloroform-d): $-71.0(\mathrm{~S})$;

FTIR (ATR, $\left.\mathbf{c m}^{-1}\right)$ : 3385, 2984, 1743, 1598, 1482, 1302, 1218, 118 1155, 1097, 1032, 993, 926, $855,767,682$.

HRMS (ESI-TOF): calc'd for $\mathrm{C}_{14} \mathrm{H}_{16} \mathrm{ClF}_{3} \mathrm{NO}_{2}[\mathrm{M}+\mathrm{H}]^{+}$322.0816; Found 322.0811;

$[\boldsymbol{\alpha}]_{\mathbf{D}^{\mathbf{2 0}}}=+6.86^{\circ}\left(C=1.02, \mathrm{CHCl}_{3}\right)$.

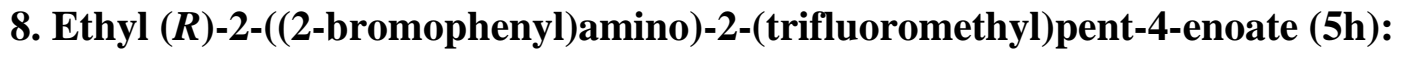

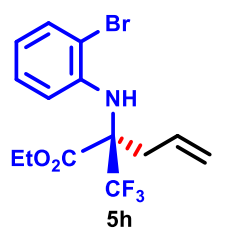

Yield: $93 \%, 832 \mathrm{mg}(2.3 \mathrm{mmol}$ scale);

Physical State: Colorless oily liquid;

$\boldsymbol{R}_{\mathbf{f}}=0.64(10 \% \mathrm{EA} / \mathrm{Hex})$; 
${ }^{1}$ H NMR (600 MHz, Chloroform- $d$ ): $\delta 7.47(\mathrm{dd}, J=7.9,1.5 \mathrm{~Hz}, 1 \mathrm{H}), 7.16-7.11(\mathrm{~m}, 1 \mathrm{H}), 6.87$ $(\mathrm{d}, J=8.2 \mathrm{~Hz}, 1 \mathrm{H}), 6.70(\mathrm{td}, J=7.9,1.4 \mathrm{~Hz}, 1 \mathrm{H}), 5.59-5.50(\mathrm{~m}, 1 \mathrm{H}), 5.48(\mathrm{~s}, 1 \mathrm{H}), 5.23-5.10$ (m, 2H), $4.36-4.26(\mathrm{~m}, 2 \mathrm{H}), 3.14(\mathrm{dd}, J=14.5,6.4 \mathrm{~Hz}, 1 \mathrm{H}), 2.91(\mathrm{dd}, J=14.5,8.0 \mathrm{~Hz}, 1 \mathrm{H})$, $1.28(\mathrm{t}, J=7.1 \mathrm{~Hz}, 3 \mathrm{H})$;

${ }^{13}$ C NMR (151 MHz, Chloroform- $d$ ): $\delta$ 167.5, 141.0, 132.8, 129.1, 127.9, 124.6 (q, $J=288.1$ $\mathrm{Hz}), 121.6,120.5,116.0(\mathrm{q}, J=4.2 \mathrm{~Hz}), 113.0,67.1(\mathrm{q}, J=27.5 \mathrm{~Hz}), 63.1,35.4,13.8$;

${ }^{19}$ F NMR (471 MHz, Chloroform-d): -70.3 (s);

FTIR (ATR, cm$^{-1}$ ): 3348, 2983, 1746, 1591, 1516, 1466, 1444, 1300, 1218, 1187, 1152, 1021 , 929, 743.

HRMS (ESI-TOF): calc'd for $\mathrm{C}_{14} \mathrm{H}_{16} \mathrm{BrF}_{3} \mathrm{NO}_{2}[\mathrm{M}+\mathrm{H}]^{+}$366.0311; Found 366.0310;

$[\alpha]_{\mathbf{D}^{20}}=+13.01^{\circ}\left(C=1.23, \mathrm{CHCl}_{3}\right)$.

9. Ethyl (R)-2-((2-iodophenyl)amino)-2-(trifluoromethyl)pent-4-enoate (5i):

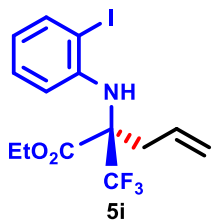

Yield: $94 \%, 193 \mathrm{mg}(0.5 \mathrm{mmol} \mathrm{scale})$;

Physical State: Beige colored oily liquid;

$\boldsymbol{R}_{\mathbf{f}}=0.55(10 \% \mathrm{EA} / \mathrm{Hex})$;

${ }^{1}$ H NMR (600 MHz, Chloroform- $\left.\boldsymbol{d}\right): \delta 7.76-7.69(\mathrm{~m}, 1 \mathrm{H}), 7.19-7.12(\mathrm{~m}, 1 \mathrm{H}), 6.82(\mathrm{~d}, J=$ $8.2 \mathrm{~Hz}, 1 \mathrm{H}), 6.59-6.52(\mathrm{~m}, 1 \mathrm{H}), 5.55(\mathrm{dq}, J=16.9,7.5 \mathrm{~Hz}, 1 \mathrm{H}), 5.26(\mathrm{~s}, 1 \mathrm{H}), 5.23-5.14(\mathrm{~m}$, $2 \mathrm{H}), 4.37-4.25(\mathrm{~m}, 2 \mathrm{H}), 3.14(\mathrm{dd}, J=14.5,6.5 \mathrm{~Hz}, 1 \mathrm{H}), 2.91(\mathrm{dd}, J=14.5,8.0 \mathrm{~Hz}, 1 \mathrm{H}), 1.28(\mathrm{t}$, $J=7.1 \mathrm{~Hz}, 3 \mathrm{H})$;

${ }^{13}$ C NMR (151 MHz, Chloroform- $d$ ): $\delta$ 167.4, 143.5, 139.6, 129.2, 128.8, 124.6 (q, $J=288.3$ $\mathrm{Hz}), 121.8,121.2,115.2(\mathrm{q}, J=4.2 \mathrm{~Hz}), 89.0,67.6(\mathrm{q}, J=27.5 \mathrm{~Hz}), 63.1,35.5,13.8$;

${ }^{19}$ F NMR (471 MHz, Chloroform- $d$ ): -70.1 (s);

FTIR (ATR, cm$^{-1}$ ): 3332, 2983, 1745, 1586, 1514, 1459, 1298, 1261, 1217, 1153, 1008, 929, 745 .

HRMS (ESI-TOF): calc'd for $\mathrm{C}_{14} \mathrm{H}_{16} \mathrm{IF}_{3} \mathrm{NO}_{2}[\mathrm{M}+\mathrm{H}]^{+}$414.0172; Found 414.0168; 
$[\boldsymbol{\alpha}] \mathbf{D}^{\mathbf{2 0}}=+10.55^{\circ}\left(C=1.09, \mathrm{CHCl}_{3}\right)$.

10. Ethyl (R)-2-(trifluoromethyl)-2-((4-(trifluoromethyl)phenyl)amino)pent-4-enoate (5j):<smiles>C=CC(Nc1ccc(C(F)(F)F)cc1)(C(F)(F)F)C(F)(F)F</smiles>

$5 \mathrm{j}$

Yield: $91 \%, 162 \mathrm{mg}(0.5 \mathrm{mmol}$ scale $)$;

Physical State: Beige colored oily liquid;

$\boldsymbol{R}_{\mathbf{f}}=0.37(10 \% \mathrm{EA} / \mathrm{Hex})$

${ }^{1}$ H NMR (500 MHz, Chloroform-d): $\delta 7.42(\mathrm{~d}, J=8.6 \mathrm{~Hz}, 2 \mathrm{H}), 6.81(\mathrm{~d}, J=8.5 \mathrm{~Hz}, 2 \mathrm{H}), 5.57$ $5.48(\mathrm{~m}, 1 \mathrm{H}), 5.21-5.11(\mathrm{~m}, 2 \mathrm{H}), 5.00(\mathrm{~s}, 1 \mathrm{H}), 4.40-4.29(\mathrm{~m}, 2 \mathrm{H}), 3.18(\mathrm{dd}, J=14.6,6.4 \mathrm{~Hz}$, 1H), $2.92(\mathrm{dd}, J=14.6,8.0 \mathrm{~Hz}, 1 \mathrm{H}), 1.30(\mathrm{t}, J=7.1 \mathrm{~Hz}, 3 \mathrm{H})$;

${ }^{13}$ C NMR (126 MHz, Chloroform-d): $\delta$ 167.5, 146.5, 129.1, 126.3 (q, $\left.J=3.8 \mathrm{~Hz}\right), 124.64$ (q, $J$ $=270.7 \mathrm{~Hz}), 124.60(\mathrm{q}, J=288.2 \mathrm{~Hz}), 121.6(\mathrm{q}, J=33.8 \mathrm{~Hz}), 115.8(\mathrm{q}, J=2.1 \mathrm{~Hz}), 66.7(\mathrm{q}, J=$ $27.9 \mathrm{~Hz}), 63.4,34.72(\mathrm{q}, J=1.4 \mathrm{~Hz}), 13.8$;

${ }^{19}$ F NMR (471 MHz, Chloroform-d): $-60.6(\mathrm{p}),-70.7$ (s);

FTIR (ATR, cm$\left.^{-1}\right)$ : 3375, 29991, 1736, 1618, 1537, 1319, 1251, 1154, 118, 1097, 1069, 1007, $923,830,673$.

HRMS (ESI-TOF): calc'd for $\mathrm{C}_{15} \mathrm{H}_{16} \mathrm{~F}_{6} \mathrm{NO}_{2}[\mathrm{M}+\mathrm{H}]^{+}$356.1080; Found 356.1079;

$[\boldsymbol{\alpha}]_{\mathbf{D}^{\mathbf{2 0}}}=+10.30^{\circ}\left(\mathrm{C}=1.0, \mathrm{CHCl}_{3}\right)$.

11b. Characterization of alcohols from Catalytic Asymmetric Allylation Products

1. Ethyl (R)-5-hydroxy-2-(p-tolylamino)-2-(trifluoromethyl)pentanoate (8d):

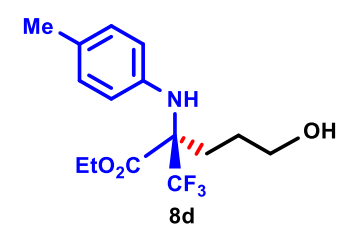

Yield: $40 \%, 58 \mathrm{mg}(0.45 \mathrm{mmol} \mathrm{scale})$;

Physical State: Brown colored viscous oily substance; 
$\boldsymbol{R}_{\mathbf{f}}=0.19(30 \% \mathrm{EA} / \mathrm{Hex})$

${ }^{1}$ H NMR (600 MHz, Chloroform-d): $\delta 6.99(\mathrm{~d}, J=8.1 \mathrm{~Hz}, 2 \mathrm{H}), 6.76(\mathrm{~d}, J=8.3 \mathrm{~Hz}, 2 \mathrm{H}), 4.74$ $(\mathrm{s}, 1 \mathrm{H}), 4.40-4.27(\mathrm{~m}, 2 \mathrm{H}), 3.49$ (tq, $J=7.2,4.4 \mathrm{~Hz}, 2 \mathrm{H}), 2.47-2.39(\mathrm{~m}, 1 \mathrm{H}), 2.31-2.22(\mathrm{~m}$, $4 \mathrm{H}), 1.51-1.40(\mathrm{~m}, 1 \mathrm{H}), 1.40-1.33(\mathrm{~m}, 1 \mathrm{H}), 1.32(\mathrm{t}, J=7.1 \mathrm{~Hz}, 3 \mathrm{H})$;

${ }^{13}$ C NMR (151 MHz, Chloroform- $d$ ): $\delta$ 168.6, 140.8, 130.0, 129.5, 124.8 (q, $\left.J=288.4 \mathrm{~Hz}\right)$, $118.5,67.9$ (q, $J=27.3 \mathrm{~Hz}), 63.2,62.0,26.2,25.6,20.4,13.8$;

${ }^{19}$ F NMR (471 MHz, Chloroform-d): -72.9 (s);

FTIR (ATR, $\left.\mathbf{c m}^{-1}\right):$ 3379, 2982, 2874, 1740, 1617, 1516, 1262, 1191, 1170, 1017, 810, 756.

HRMS (ESI-TOF): calc'd for $\mathrm{C}_{15} \mathrm{H}_{21} \mathrm{~F}_{3} \mathrm{NO}_{3}[\mathrm{M}+\mathrm{H}]^{+}$320.1468; Found 320.1469;

SFC Analysis: CSP SFC (IB), 5\% Isopropanol, continuous flow at $2 \mathrm{~mL} / \mathrm{min}, 35^{\circ} \mathrm{C}, 160 \mathrm{bar}$, $230 \mathrm{~nm} ; t_{\text {major }}=2.81 \mathrm{~min}, t_{\text {minor }}=2.07 \mathrm{~min}$, ee $96 \%$;

$[\boldsymbol{\alpha}] \mathbf{D}^{\mathbf{2 0}}=+29.3^{\circ}\left(C=1.0, \mathrm{CHCl}_{3}\right)$.

2a. Ethyl (R)-2-((3-chlorophenyl)amino)-5-hydroxy-2-(trifluoromethyl)pentanoate (8g):

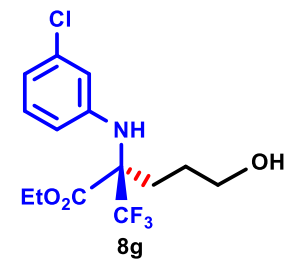

Yield: $23 \%, 36 \mathrm{mg}(0.46 \mathrm{mmol}$ scale $)$;

Physical State: Brown colored viscous oily substance;

$\boldsymbol{R}_{\mathbf{f}}=0.25(30 \%$ EA/Hex $)$

${ }^{1}$ H NMR (600 MHz, Chloroform-d): $\delta 7.09(\mathrm{t}, J=8.3 \mathrm{~Hz}, 1 \mathrm{H}), 6.82(\mathrm{~d}, J=7.0 \mathrm{~Hz}, 2 \mathrm{H}), 6.70$ $(\mathrm{d}, J=7.7 \mathrm{~Hz}, 1 \mathrm{H}), 5.00(\mathrm{~s}, 1 \mathrm{H}), 4.46-4.22(\mathrm{~m}, 2 \mathrm{H}), 3.54(\mathrm{~h}, J=6.5,5.6 \mathrm{~Hz}, 2 \mathrm{H}), 2.52-2.44$ (m, $1 \mathrm{H}), 2.36-2.27(\mathrm{~m}, 1 \mathrm{H}), 1.5^{\prime}-1.4(\mathrm{~m}, 1 \mathrm{H}), 1.39-1.29(\mathrm{~m}, 4 \mathrm{H})$;

${ }^{13}$ C NMR (151 MHz, Chloroform- $d$ ): $\delta$ 168.1, 144.8, 134.7, 130.0, 124.7 (q, $J=288.4 \mathrm{~Hz}$ ), 120.0, 117.2, 115.3, $67.6(\mathrm{q}, J=27.8 \mathrm{~Hz}), 63.5,61.8,26.0,25.8,13.9$;

${ }^{19}$ F NMR (471 MHz, Chloroform-d): -72.7 (s);

FTIR (ATR, cm$\left.^{-1}\right)$ : 3391, 2937, 1741, 1731, 1596, 1526, 1481, 1302, 1289, 1260, 1224, 1180, 1154, 111, 1056, 1016, 855, 834, 771, 756. 
HRMS (ESI-TOF): calc'd for $\mathrm{C}_{14} \mathrm{H}_{18} \mathrm{ClF}_{3} \mathrm{NO}_{3}[\mathrm{M}+\mathrm{H}]^{+}$340.0922; Found 340.0929;

HPLC Analysis: CSP SFC (IB), 5\% Isopropanol, continuous flow at $2 \mathrm{~mL} / \mathrm{min}, 35^{\circ} \mathrm{C}, 160$ bar, $210 \mathrm{~nm} ; t_{\text {major }}=2.79 \mathrm{~min}, t_{\text {minor }}=1.84 \mathrm{~min}$, ee $93 \%$;

$[\boldsymbol{\alpha}] \mathbf{D}^{\mathbf{2 0}}=+32.7^{\circ}\left(C=1.0, \mathrm{CHCl}_{3}\right)$.

2b. Ethyl (S)-2-((3-chlorophenyl)amino)-5-hydroxy-2-(trifluoromethyl)pentanoate (8g'):<smiles>CCOC(=O)C(C)(C)Nc1cccc(Cl)c1</smiles>

Yield: $27 \%, 45 \mathrm{mg}(0.48 \mathrm{mmol} \mathrm{scale})$;

Physical State: Reddish brown colored viscous oily liquid;

SFC Analysis: CSP SFC (IB), 5\% Isopropanol, continuous flow at $2 \mathrm{~mL} / \mathrm{min}, 35^{\circ} \mathrm{C}, 160 \mathrm{bar}$, $210 \mathrm{~nm} ; t_{\text {major }}=1.72 \mathrm{~min}, t_{\text {minor }}=2.30 \mathrm{~min}$, ee $93 \%$;

$[\alpha]_{\mathbf{D}^{20}}=-35.5^{\circ}\left(C=1.0, \mathrm{CHCl}_{3}\right)$.

3. Ethyl (R)-2-((2-bromophenyl)amino)-5-hydroxy-2-(trifluoromethyl)pentanoate (8h):

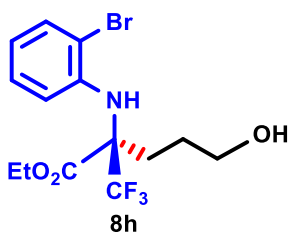

Yield: $35 \%, 63 \mathrm{mg}(0.46 \mathrm{mmol} \mathrm{scale})$;

Physical State: Brown colored viscous oily substance;

$\boldsymbol{R}_{\mathbf{f}}=0.24(30 \% \mathrm{EA} / \mathrm{Hex})$

${ }^{1}$ H NMR (600 MHz, Chloroform-d): $\delta 7.51-7.44(\mathrm{~m}, 1 \mathrm{H}), 7.14(\mathrm{t}, J=7.8 \mathrm{~Hz}, 1 \mathrm{H}), 6.95(\mathrm{~d}, J$ $=8.2 \mathrm{~Hz}, 1 \mathrm{H}), 6.70(\mathrm{t}, J=7.6 \mathrm{~Hz}, 1 \mathrm{H}), 5.67(\mathrm{~s}, 1 \mathrm{H}), 4.42-4.29(\mathrm{~m}, 2 \mathrm{H}), 3.49(\mathrm{t}, J=6.1 \mathrm{~Hz}$, $2 \mathrm{H}), 2.51(\mathrm{ddd}, J=16.1,11.2,5.1 \mathrm{~Hz}, 1 \mathrm{H}), 2.34(\mathrm{ddd}, J=14.8,11.4,5.4 \mathrm{~Hz}, 1 \mathrm{H}), 1.41-1.27$ $(\mathrm{m}, 6 \mathrm{H})$;

${ }^{13}$ C NMR (151 MHz, Chloroform- $\left.d\right): ~ \delta 168.1(\mathrm{q}, J=1.8 \mathrm{~Hz}), 144.8,134.7,130.0,124.6(\mathrm{q}, J=$ $288.6 \mathrm{~Hz}), 120.0,117.2,115.3(\mathrm{q}, J=2.0 \mathrm{~Hz}), 67.6(\mathrm{q}, J=27.8 \mathrm{~Hz}), 63.5,61.8,26.0,25.8,13.9$; 
${ }^{19}$ F NMR (471 MHz, Chloroform-d): -72.6 (s);

FTIR (ATR, $\left.\mathbf{c m}^{-1}\right):$ 3349, 2942, 2875, 1744, 1590, 1518, 1465, 130, 1263, 1223, 1154, 1056, $1020,743$.

HRMS (ESI-TOF): calc'd for $\mathrm{C}_{14} \mathrm{H}_{18} \mathrm{BrF}_{3} \mathrm{NO}_{3}[\mathrm{M}+\mathrm{H}]^{+}$384.0417; Found 384.0436;

SFC Analysis: CSP SFC (IB), 5\% Methanol, continuous flow at $2 \mathrm{~mL} / \mathrm{min}, 35{ }^{\circ} \mathrm{C}, 160 \mathrm{bar}, 210$ $\mathrm{nm} ; t_{\mathrm{major}}=1.56 \mathrm{~min}, t_{\mathrm{minor}}=1.25 \mathrm{~min}$, ee $94 \%$;

$[\boldsymbol{\alpha}] \mathbf{D}^{\mathbf{2 0}}=+49.3^{\circ}\left(C=1.0, \mathrm{CHCl}_{3}\right)$.

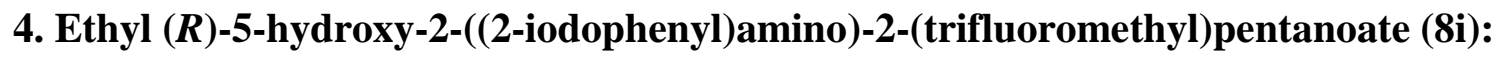

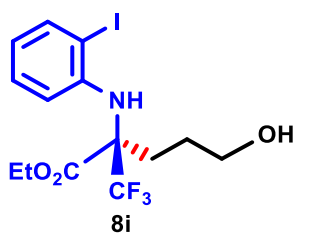

Yield: $26 \%, 22 \mathrm{mg}(0.19 \mathrm{mmol}$ scale $)$;

Physical State: Reddish brown colored oily liquid;

$\boldsymbol{R}_{\mathbf{f}}=0.15(30 \% \mathrm{EA} / \mathrm{Hex})$;

${ }^{1}$ H NMR (600 MHz, Chloroform-d): $\delta 7.74(\mathrm{dd}, J=7.9,1.4 \mathrm{~Hz}, 1 \mathrm{H}), 7.21-7.14(\mathrm{~m}, 1 \mathrm{H}), 6.91$ $(\mathrm{d}, J=8.2 \mathrm{~Hz}, 1 \mathrm{H}), 6.59-6.53(\mathrm{~m}, 1 \mathrm{H}), 5.45(\mathrm{~s}, 1 \mathrm{H}), 4.43-4.30(\mathrm{~m}, 2 \mathrm{H}), 3.51(\mathrm{t}, J=6.1 \mathrm{~Hz}$, 2H), $2.52(\mathrm{ddd}, J=14.6,11.5,4.9 \mathrm{~Hz}, 1 \mathrm{H}), 2.34$ (tt, $J=11.8,5.2 \mathrm{~Hz}, 1 \mathrm{H}), 1.41-1.29$ (m, 5H);

${ }^{13}$ C NMR (126 MHz, Chloroform-d): $\delta 167.8(\mathrm{q}, J=1.8 \mathrm{~Hz}), 143.4,139.7,129.0,124.73$ (q, $J$ $=288.3 \mathrm{~Hz}), 121.4,115.5(\mathrm{q}, J=4.2 \mathrm{~Hz}), 89.4,68.2(\mathrm{q}, J=28.0 \mathrm{~Hz}), 63.5,61.8,26.1,25.6$, 13.9

${ }^{19}$ F NMR (471 MHz, Chloroform-d): -72.4 (s);

FTIR (ATR, $\left.\mathbf{c m}^{-1}\right)$ : 3335, 2924, 1744, 1585, 1516, 1458, 1303, 1264, 1223, 1154, 1055, 1008, 746.

HRMS (ESI-TOF): calc'd for $\mathrm{C}_{14} \mathrm{H}_{18} \mathrm{IF}_{3} \mathrm{NO}_{3}[\mathrm{M}+\mathrm{H}]^{+} 432.0278$; Found 432.0270;

SFC Analysis: CSP SFC (IC), 5\% Isopropanol, continuous flow at $2 \mathrm{~mL} / \mathrm{min}, 35^{\circ} \mathrm{C}, 160 \mathrm{bar}$, $210 \mathrm{~nm} ; t_{\text {major }}=3.07 \mathrm{~min}, t_{\text {minor }}=2.72 \mathrm{~min}$, ee $96 \%$;

$[\alpha]_{\mathbf{D}^{20}}=+40.8^{\circ}\left(C=1.0, \mathrm{CHCl}_{3}\right)$. 
5. Ethyl (R)-5-hydroxy-2-(trifluoromethyl)-2-((4-(trifluoromethyl)phenyl)amino)pentanoate $(\mathbf{8 j})$ :

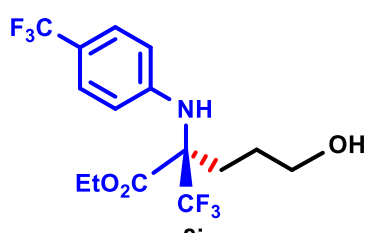

$8 \mathbf{j}$

Yield: $34 \%, 58 \mathrm{mg}(0.45 \mathrm{mmol}$ scale $)$;

Physical State: White colored wax;

$\boldsymbol{R}_{\mathbf{f}}=0.22(40 \%$ EA/Hex $) ;$

${ }^{1}$ H NMR (600 MHz, Chloroform- $\left.d\right): \delta 7.41(\mathrm{~d}, J=8.6 \mathrm{~Hz}, 2 \mathrm{H}), 6.83(\mathrm{~d}, J=8.5 \mathrm{~Hz}, 2 \mathrm{H}), 5.28$ (s, 1H), $4.42-4.30(\mathrm{~m}, 2 \mathrm{H}), 3.56(\mathrm{tt}, J=7.3,3.6 \mathrm{~Hz}, 2 \mathrm{H}), 2.53(\mathrm{ddd}, J=15.8,11.4,4.8 \mathrm{~Hz}, 1 \mathrm{H})$, 2.36 (ddd, $J=15.1,11.4,5.1 \mathrm{~Hz}, 1 \mathrm{H}), 1.53(\mathrm{~s}, 1 \mathrm{H}), 1.49-1.40(\mathrm{~m}, 1 \mathrm{H}), 1.40-1.33(\mathrm{~m}, 1 \mathrm{H})$, $1.32(\mathrm{t}, J=7.1 \mathrm{~Hz}, 3 \mathrm{H})$;

${ }^{13}$ C NMR (151 MHz, Chloroform-d): $\delta$ 168.0, 146.6, 126.4 (q, $\left.J=3.8 \mathrm{~Hz}\right), 124.7$ (q, $J=$ 270.6), 124.6 (q, $J=288.5), 121.4(\mathrm{q}, J=32.8 \mathrm{~Hz}), 115.7,67.4(\mathrm{q}, J=28.1 \mathrm{~Hz}), 63.6,61.7,26.2$, 26.0, 13.8;

${ }^{19}$ F NMR (471 MHz, Chloroform-d): $-61.5(\mathrm{~T}),-72.4(\mathrm{~S})$;

FTIR (ATR, $\left.\mathbf{c m}^{-\mathbf{1}}\right)$ : 3391, 2943, 1742, 1618, 1530, 1324, 1274, 1196, 1158, 11101067, 1010, $830,757$.

HRMS (ESI-TOF): calc'd for $\mathrm{C}_{15} \mathrm{H}_{18} \mathrm{~F}_{6} \mathrm{NO}_{3}[\mathrm{M}+\mathrm{H}]^{+}$374.1185; Found 374.1185;

HPLC Analysis: Chiralpak IA, 5\% IP/hexanes, continuous flow at $1 \mathrm{~mL} / \mathrm{min}, 250 \mathrm{~nm}$; $t_{\text {major }}=$ $10.41 \mathrm{~min}, t_{\operatorname{minor}}=12.24 \mathrm{~min}$, ee $90 \%$;

$[\boldsymbol{\alpha}] \mathbf{D}^{\mathbf{2 0}}=+31.6^{\circ}\left(C=1.0, \mathrm{CHCl}_{3}\right)$.

\section{Determination of absolute configuration:}

Ethyl (R)-2-((4-methoxyphenyl)amino)-2-(trifluoromethyl)pent-4-enoate (5e) was converted to the corresponding homoallylic amine (5e-1) by removing the $p$-methoxyphenyl group on the $N$ atom with ceric ammonium nitrate. ${ }^{1 \text { a }}$ Then it was transformed into the corresponding carboxylic acid (5e-2). ${ }^{7}$ Comparison of the optical rotation to the literature value ${ }^{7,8}$ established the $(R)$ 
configuration in product 5e-2. All other absolute configurations of the products from Table 3 were assigned by analogy.

Ethyl $(R)$-2-amino-2-(trifluoromethyl)pent-4-enoate (5e-1):

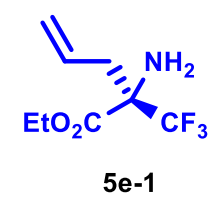

Yield: $56 \%, 118 \mathrm{mg}$ (1 mmol scale);

Physical State: Pale reddish brown colored liquid;

$\boldsymbol{R}_{\mathbf{f}}=0.22(10 \% \mathrm{EA} / \mathrm{Hex})$;

${ }^{1}$ H NMR (600 MHz, Chloroform- ) : $\delta 5.71-5.61(\mathrm{~m}, 1 \mathrm{H}), 5.26-5.19(\mathrm{~m}, 2 \mathrm{H}), 4.27(\mathrm{q}, J=$

$7.1 \mathrm{~Hz}, 2 \mathrm{H}), 2.82(\mathrm{dd}, J=13.8,6.8 \mathrm{~Hz}, 1 \mathrm{H}), 2.46(\mathrm{dd}, J=13.8,7.9 \mathrm{~Hz}, 1 \mathrm{H}), 1.87$ (s, 2H), 1.30 (t, $J=7.1 \mathrm{~Hz}, 3 \mathrm{H})$;

${ }^{13}$ C NMR (151 MHz, Chloroform-d): $\delta 168.8,130.0,124.8(\mathrm{q}, J=285.2 \mathrm{~Hz}), 121.3,64.0(\mathrm{q}, J$ $=26.9 \mathrm{~Hz}), 62.6,37.3(\mathrm{q}, J=1.7 \mathrm{~Hz}), 13.9$;

${ }^{19}$ F NMR (471 MHz, Chloroform-d): -76.4 (s);

FTIR (ATR, cm$\left.^{-1}\right):$ 3378, 2986, 1473, 1517, 1191, 1032, 924, 809.

HRMS (ESI-TOF): calc'd for $\mathrm{C}_{8} \mathrm{H}_{13} \mathrm{~F}_{3} \mathrm{NO}_{2}[\mathrm{M}+\mathrm{H}]^{+}$212.0893; Found 212.0894;

$[\boldsymbol{\alpha}] \mathbf{D}^{\mathbf{2 0}}=+34.0^{\circ}\left(C=1.0, \mathrm{CHCl}_{3}\right)$.

Note: This compound is very volatile.

(R)-2-amino-2-(trifluoromethyl)pent-4-enoic acid hydrochloride (5e-2):

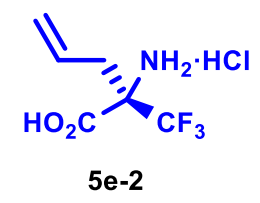

Yield: $87 \%, 9 \mathrm{mg}$ (0.04 mmol scale);

Physical State: Beige colored solid; 
${ }^{1}$ H NMR (600 MHz, Chloroform- $d$ ): $\delta 5.77-5.65(\mathrm{~m}, 1 \mathrm{H}), 5.46-5.34(\mathrm{~m}, 2 \mathrm{H}), 3.10-2.96$ (m, 1H), $2.83-2.67(\mathrm{~m}, 1 \mathrm{H})$;

${ }^{13}$ C NMR (151 MHz, Chloroform- $d$ ): $\delta$ 169.5, 130.5, 126.4, 126.2 (q, $\left.J=283.5 \mathrm{~Hz}\right), 68.21(\mathrm{q}, J$ $=27.2 \mathrm{~Hz}), 37.5$;

${ }^{19}$ F NMR (471 MHz, Chloroform-d): -73.1 (s);

FTIR (ATR, cm-1): 2921 (br), 1719, 1560, 1509, 1438, 1277, 1216, 1159, 935, 696.

HRMS (ESI-TOF): calc'd for $\mathrm{C}_{6} \mathrm{H}_{8} \mathrm{~F}_{3} \mathrm{NO}_{2}[\mathrm{M}+\mathrm{H}]^{+}$184.0580; Found 184.0578;

$[\alpha]_{\mathbf{D}^{20}}=+22.0^{\circ}\left(C=1.0, \mathrm{H}_{2} \mathrm{O}\right) ;[\alpha]_{\mathrm{D}}{ }^{20}{ }_{\text {,it }}=+27.0^{\circ}\left(C=0.69, \mathrm{H}_{2} \mathrm{O}\right){ }^{7,8}$

12. Transformation of the Chiral Homoallylic Amine Products into Proline derivatives:

1. Methyl (S)-5-hydroxy-2-phenyl-2-(phenylamino)pentanoate (6a):

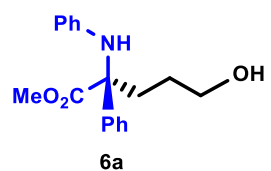

Yield: $67 \%, 160 \mathrm{mg}(0.8 \mathrm{mmol} \mathrm{scale})$;

Physical State: Grey greasy liquid;

$\boldsymbol{R}_{\mathbf{f}}=0.35(35 \%$ EA/Hex $)$

${ }^{1}$ H NMR (600 MHz, Chloroform- $d$ ) $\delta 7.60(\mathrm{~d}, J=7.6 \mathrm{~Hz}, 2 \mathrm{H}), 7.35(\mathrm{t}, J=7.6 \mathrm{~Hz}, 2 \mathrm{H}), 7.28(\mathrm{t}$, $J=7.3 \mathrm{~Hz}, 1 \mathrm{H}), 7.01(\mathrm{t}, J=7.9 \mathrm{~Hz}, 2 \mathrm{H}), 6.62(\mathrm{t}, J=7.3 \mathrm{~Hz}, 1 \mathrm{H}), 6.38(\mathrm{~d}, J=7.9 \mathrm{~Hz}, 2 \mathrm{H}), 5.39$ (s, 1H), $3.68(\mathrm{~s}, 3 \mathrm{H}), 3.62-3.54(\mathrm{~m}, J=6.6 \mathrm{~Hz}, 2 \mathrm{H}), 2.67(\mathrm{td}, J=12.5,11.4,4.6 \mathrm{~Hz}, 1 \mathrm{H}), 2.58$ $(\operatorname{td}, J=13.5,12.7,4.7 \mathrm{~Hz}, 1 \mathrm{H}), 1.60(\mathrm{dp}, J=17.5,6.0 \mathrm{~Hz}, 1 \mathrm{H}), 1.39$ (tq, $J=11.5,6.4 \mathrm{~Hz}, 1 \mathrm{H})$, $1.24(\mathrm{~s}, 1 \mathrm{H})$;

${ }^{13}$ C NMR (151 MHz, Chloroform-d) $\delta$ 174.3, 144.1, 140.5, 128.9, 128.6, 127.6, 126.9, 117.5, $114.9,66.1,62.5,53.2,29.5,27.4$;

FTIR (ATR, cm$^{-1}$ ): 3218, 3060, 1728, 1701, 1607, 1504, 1483, 1250, 1232, 1177, 1100, 832, 761 , 749, 700, 686.

HRMS (ESI-TOF): calc'd for $\mathrm{C}_{18} \mathrm{H}_{22} \mathrm{NO}_{3}[\mathrm{M}+\mathrm{H}]^{+} 300.1594$; found 300.1599 .

$[\alpha]_{\mathbf{D}^{20}}=+17.17^{\circ}\left(C=0.8, \mathrm{CHCl}_{3}\right)$. 
2. Methyl (S)-5-hydroxy-2-((4-methoxyphenyl)amino)-2-phenylpentanoate (6b):

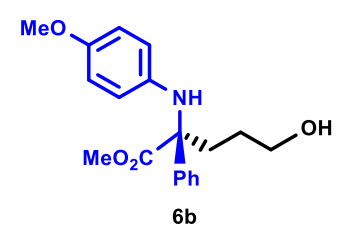

Yield: $65 \%, 172 \mathrm{mg}(0.8 \mathrm{mmol}$ scale $)$;

Physical State: Grey greasy liquid;

$\boldsymbol{R}_{\mathbf{f}}=0.30(35 \% \mathrm{EA} / \mathrm{Hex})$

${ }^{1}$ H NMR (600 MHz, Chloroform-d) $\delta 7.59(\mathrm{~d}, J=7.5 \mathrm{~Hz}, 2 \mathrm{H}), 7.34(\mathrm{t}, J=7.6 \mathrm{~Hz}, 2 \mathrm{H}), 7.28(\mathrm{t}$, $J=7.3 \mathrm{~Hz}, 1 \mathrm{H}), 6.61(\mathrm{~d}, J=9.0 \mathrm{~Hz}, 2 \mathrm{H}), 6.35(\mathrm{~d}, J=8.9 \mathrm{~Hz}, 2 \mathrm{H}), 3.66(\mathrm{~s}, 3 \mathrm{H}), 3.65(\mathrm{~s}, 3 \mathrm{H})$, $3.58-3.52(\mathrm{~m}, 2 \mathrm{H}), 2.61(\mathrm{ddd}, J=13.7,11.5,4.6 \mathrm{~Hz}, 1 \mathrm{H}), 2.54-2.35(\mathrm{~m}, 1 \mathrm{H}), 1.56$ (ddt, $J=17.5$, $11.1,6.1 \mathrm{~Hz}, 1 \mathrm{H}), 1.43-1.35(\mathrm{~m}, 1 \mathrm{H})$;

${ }^{13}$ C NMR (151 MHz, Chloroform-d) $\delta$ 174.4, 152.1, 140.9, 138.0, 128.5, 127.5, 126.9, 116.6, $114.4,66.4,62.4,55.5,53.0,30.0,27.2$;

FTIR (ATR, $\left.\mathbf{c m}^{-1}\right):$ 3402, 2951, 1727, 15010, 1446, 1235, 1175, 1033, 1003, 822, 699.

HRMS (ESI-TOF): calc'd for $\mathrm{C}_{19} \mathrm{H}_{23} \mathrm{NO}_{4}[\mathrm{M}+\mathrm{H}]^{+} 330.1700$; found 330.1714.

$[\alpha]_{\mathbf{D}^{\mathbf{2 0}}}=+20.31^{\circ}\left(C=0.8, \mathrm{CHCl}_{3}\right)$.

3. Methyl (S)-1,2-diphenylpyrrolidine-2-carboxylate (7a):

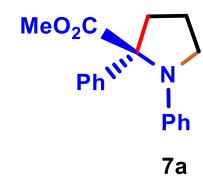

Yield: $87 \%, 122 \mathrm{mg}(0.5 \mathrm{mmol}$ scale $)$;

Physical State: Grey greasy liquid;

$\boldsymbol{R}_{\mathbf{f}}=0.40(5 \% \mathrm{EA} / \mathrm{Hex})$

${ }^{1}$ H NMR (600 MHz, Chloroform- $\left.d\right) \delta 7.47(\mathrm{~d}, J=7.4 \mathrm{~Hz}, 2 \mathrm{H}), 7.33(\mathrm{t}, J=7.5 \mathrm{~Hz}, 2 \mathrm{H}), 7.28(\mathrm{t}$, $J=7.2 \mathrm{~Hz}, 1 \mathrm{H}), 7.15-7.13(\mathrm{~m}, 2 \mathrm{H}), 6.70(\mathrm{t}, J=7.3 \mathrm{~Hz}, 1 \mathrm{H}), 6.48(\mathrm{~d}, J=8.1 \mathrm{~Hz}, 2 \mathrm{H}), 3.77(\mathrm{td}, J$ 
$=8.2,2.7 \mathrm{~Hz}, 1 \mathrm{H}), 3.70(\mathrm{td}, J=8.9,6.8 \mathrm{~Hz}, 1 \mathrm{H}), 3.62(\mathrm{~s}, 3 \mathrm{H}), 2.80(\mathrm{td}, J=11.3,6.7 \mathrm{~Hz}, 1 \mathrm{H}), 2.34$

(ddd, $J=11.7,6.7,3.5 \mathrm{~Hz}, 1 \mathrm{H}), 2.06$ (dtt, $J=13.0,6.5,3.2 \mathrm{~Hz}, 1 \mathrm{H}), 1.98-1.84(\mathrm{~m}, 1 \mathrm{H})$;

${ }^{13}$ C NMR (151 MHz, Chloroform-d) $\delta$ 174.9, 146.1, 140.4, 128.6, 127.9, 127.6, 127.0, 116.6, 113.3, 73.6, 52.3, 50.1, 44.8, 23.0;

FTIR (ATR, cm$\left.^{-1}\right):$ 1728, 1597, 1505, 1367, 1346, 1230, 1216, 1157, 1095, 1033, 1024, 993, 752.

HRMS (ESI-TOF): calc'd for $\mathrm{C}_{18} \mathrm{H}_{20} \mathrm{NO}_{2}[\mathrm{M}+\mathrm{H}]^{+}$282.1489; found 282.1500;

SFC Analysis: CSP SFC (AD-H), 10\% IPA, continuous flow at $2 \mathrm{~mL} / \mathrm{min}, 35{ }^{\circ} \mathrm{C}, 160$ bar, 250 $\mathrm{nm} ; t_{\mathrm{major}}=2.1 \mathrm{~min}, t_{\mathrm{minor}}=1.5 \mathrm{~min}, e e 99 \%$;

$[\alpha] \mathbf{D}^{20}=-3.6^{\circ}\left(C=0.56, \mathrm{CHCl}_{3}\right)$.

4. Methyl (S)-1-(4-methoxyphenyl)-2-phenylpyrrolidine-2-carboxylate (7b):

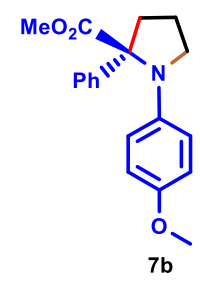

Yield: $76 \%, 118 \mathrm{mg}(0.5 \mathrm{mmol} \mathrm{scale})$;

Physical State: Grey greasy liquid;

$\boldsymbol{R}_{\mathbf{f}}=0.32(10 \% \mathrm{EA} / \mathrm{Hex})$

${ }^{1}$ H NMR (600 MHz, Chloroform-d) $\delta 7.47(\mathrm{~d}, J=7.8 \mathrm{~Hz}, 2 \mathrm{H}), 7.33(\mathrm{t}, J=7.6 \mathrm{~Hz}, 2 \mathrm{H}), 7.29-$ $7.26(\mathrm{~m}, 1 \mathrm{H}), 6.74(\mathrm{~d}, J=9.1 \mathrm{~Hz}, 2 \mathrm{H}), 6.43(\mathrm{~d}, J=8.9 \mathrm{~Hz}, 2 \mathrm{H}), 3.73(\mathrm{~s}, 3 \mathrm{H}), 3.72$ (brs, $1 \mathrm{H}), 3.65$ $(\mathrm{q}, J=8.4 \mathrm{~Hz}, 1 \mathrm{H}), 3.61(\mathrm{~s}, 3 \mathrm{H}), 2.81(\mathrm{td}, J=11.2,10.8,6.8 \mathrm{~Hz}, 1 \mathrm{H}), 2.30(\mathrm{ddd}, J=11.4,6.8,3.7$ $\mathrm{Hz}, 1 \mathrm{H}), 2.05(\mathrm{dtt}, J=13.1,6.5,3.0 \mathrm{~Hz}, 1 \mathrm{H}), 1.95-1.85(\mathrm{~m}, 1 \mathrm{H})$;

${ }^{13}$ C NMR (151 MHz, Chloroform-d) $\delta$ 175.1, 151.2, 141.0, 140.6, 127.9, 127.6, 126.9, 114.3, 114.1, 73.8, 55.6, 52.2, 50.5, 44.8, 23.0;

FTIR (ATR, cm$\left.^{-1}\right)$ : 2949, 2832, 1732, 1445, 1509, 1445, 1288, 1238, 1214, 1097, 1034, 993, 816, 754,700 .

HRMS (ESI-TOF): calc'd for $\mathrm{C}_{19} \mathrm{H}_{22} \mathrm{NO}_{3}[\mathrm{M}+\mathrm{H}]^{+}$312.1594; found 312.1571; 
SFC Analysis: CSP SFC (AD-H), 10\% IPA, continuous flow at $2 \mathrm{~mL} / \mathrm{min}, 35^{\circ} \mathrm{C}, 160$ bar, 250 $\mathrm{nm} ; t_{\text {major }}=2.7 \mathrm{~min}, t_{\operatorname{minor}}=2.3 \mathrm{~min}, e e 99 \%$;

$[\alpha] \mathrm{D}^{20}=-1.4^{\circ}\left(C=0.56, \mathrm{CHCl}_{3}\right)$.

5a. Ethyl (R)-5-hydroxy-2-(phenylamino)-2-(trifluoromethyl)pentanoate (8a):

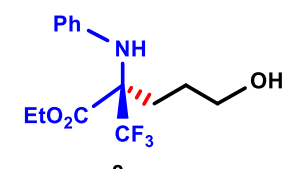

$8 a$

Yield: $61 \%, 148 \mathrm{mg}(0.8 \mathrm{mmol} \mathrm{scale})$;

Physical State: Colorless oily liquid;

$\mathbf{R}_{\mathbf{f}}=0.33(35 \% \mathrm{EA} / \mathrm{Hex})$

${ }^{1}$ H NMR (600 MHz, Chloroform- $\left.d\right) \delta 7.19(\mathrm{t}, J=7.9 \mathrm{~Hz}, 2 \mathrm{H}), 6.92-6.77(\mathrm{~m}, 3 \mathrm{H}), 4.88(\mathrm{~s}, 1 \mathrm{H})$, 4.34 (dddt, $J=17.8,14.3,10.7,7.1 \mathrm{~Hz}, 2 \mathrm{H}$ ), 3.50 (tq, $J=7.4,4.5 \mathrm{~Hz}, 2 \mathrm{H}$ ), 2.50 (ddd, $J=15.9$, $11.8,4.4 \mathrm{~Hz}, 1 \mathrm{H}), 2.29$ (ddt, $J=20.3,13.6,6.6 \mathrm{~Hz}, 1 \mathrm{H}), 1.44(\mathrm{ddt}, J=17.3,11.1,6.0 \mathrm{~Hz}, 1 \mathrm{H})$, $1.36(\mathrm{dt}, J=12.1,6.0 \mathrm{~Hz}, 1 \mathrm{H}), 1.32(\mathrm{t}, J=7.1 \mathrm{~Hz}, 3 \mathrm{H})$;

${ }^{13}$ C NMR (151 MHz, Chloroform-d) $\delta$ 168.5, 143.5, 129.1, 124.8 (q, $\left.J=288.4 \mathrm{~Hz}\right), 120.3$, $117.8,67.8(\mathrm{q}, J=27.6 \mathrm{~Hz}), 63.3,61.9,26.2,25.7,13.9$;

${ }^{19}$ F NMR (471 MHz, Chloroform-d): -71.9 (s);

FTIR (ATR, cm$^{-1}$ ): 3377, 3982, 2875, 1740, 1603, 1499, 1265, 1179, 1055, 1033, 1016, 851, 748.

HRMS (ESI-TOF): calc'd for $\mathrm{C}_{14} \mathrm{H}_{19} \mathrm{~F}_{3} \mathrm{NO}_{3}[\mathrm{M}+\mathrm{H}]^{+}$306.1312; found 306.1319;

HPLC Analysis: Chiralpak IA, 5\% IP/Hexanes, continuous flow at $1.0 \mathrm{~mL} / \mathrm{min}, 250 \mathrm{~nm} ; t_{\text {major }}=$ 9.9 min, $t_{\text {minor }}=11.4$ min, $e e$ 97\%;

$[\alpha] \mathrm{D}^{20}=+52.15^{\circ}\left(C=0.69, \mathrm{CHCl}_{3}\right)$.

5b. Ethyl (S)-5-hydroxy-2-(phenylamino)-2-(trifluoromethyl)pentanoate (8a'): 


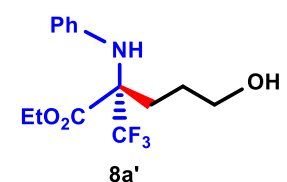

Yield: $58 \%, 87 \mathrm{mg}(0.5 \mathrm{mmol}$ scale $)$;

Physical State: Colorless oily liquid;

HPLC Analysis: Chiralpak IA, 5\% IP/Hexanes, continuous flow at $1.0 \mathrm{~mL} / \mathrm{min}, 250 \mathrm{~nm} ; t_{\text {major }}=$ $11.4 \mathrm{~min}, t_{\mathrm{minor}}=9.9 \mathrm{~min}$, ee $96 \%$;

$[\alpha]_{\mathrm{D}^{20}}=+52.15^{\circ}\left(C=0.69, \mathrm{CHCl}_{3}\right)$.

6. Ethyl (R)-1-phenyl-2-(trifluoromethyl)pyrrolidine-2-carboxylate (9a):

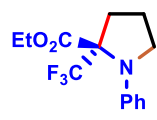

Yield: $77 \%, 110 \mathrm{mg}(0.5 \mathrm{mmol}$ scale $)$;

Physical State: Grey greasy liquid;

$\boldsymbol{R}_{\mathbf{f}}=0.47(5 \% \mathrm{EA} / \mathrm{Hex})$

${ }^{1}$ H NMR (600 MHz, Chloroform-d) $\delta 7.24-7.16(\mathrm{~m}, 2 \mathrm{H}), 6.82(\mathrm{t}, J=7.3 \mathrm{~Hz}, 1 \mathrm{H}), 6.77(\mathrm{~d}, J=$ $8.4 \mathrm{~Hz}, 2 \mathrm{H}), 4.26-4.00(\mathrm{~m}, 2 \mathrm{H}), 3.69(\mathrm{td}, J=8.1,3.7 \mathrm{~Hz}, 1 \mathrm{H}), 3.59(\mathrm{q}, J=8.2 \mathrm{~Hz}, 1 \mathrm{H}), 2.64$ $(\mathrm{ddd}, J=13.1,7.3,3.9 \mathrm{~Hz}, 1 \mathrm{H}), 2.49-2.40(\mathrm{~m}, 1 \mathrm{H}), 2.20(\mathrm{dt}, J=17.3,8.5 \mathrm{~Hz}, 1 \mathrm{H}), 2.16-2.10$ $(\mathrm{m}, 1 \mathrm{H}), 1.09(\mathrm{t}, J=7.1 \mathrm{~Hz}, 3 \mathrm{H})$;

${ }^{13}$ C NMR (151 MHz, Chloroform-d) $\delta 169.6,145.2,128.7,125.6$ (q, $\left.J=287.6 \mathrm{~Hz}\right), 118.7$, 114.2, $71.9(\mathrm{q}, J=27.6 \mathrm{~Hz}), 62.0,51.6,36.4,23.0,13.7$;

${ }^{19}$ F NMR (471 MHz, Chloroform-d): -69.1 (s);

FTIR (ATR, $\mathbf{c m}^{-1}$ ): 2967, 1844, 1731, 1601, 1505, 1316, 12879, 1239, 118,2, 1149, 1083, 945, 744.

HRMS (ESI-TOF): calc'd for $\mathrm{C}_{14} \mathrm{H}_{17} \mathrm{~F}_{3} \mathrm{NO}_{2}[\mathrm{M}+\mathrm{H}]^{+}$288.1206; found 288.1211;

SFC Analysis: CSP SFC (OD-H), 1\% IPA, continuous flow at $0.7 \mathrm{~mL} / \mathrm{min}, 35{ }^{\circ} \mathrm{C}, 160$ bar, 250 $\mathrm{nm} ; t_{\text {major }}=8.2 \mathrm{~min}, t_{\text {minor }}=7.6 \mathrm{~min}, e e 97 \%$; 
$[\alpha] \mathbf{D}^{20}=+22.7^{\circ}\left(C=0.75, \mathrm{CHCl}_{3}\right)$. 


\section{References:}

1. a) Curto, J. M.; Dickstein, J. S.; Berritt, S.; Kozlowski, M. C. Asymmetric Synthesis of $\alpha$-Allyl$\alpha$-Aryl $\alpha$-Amino Acids by Tandem Alkylation/ $\pi$-Allylation of $\alpha$-Iminoesters. Org. Lett. 2014, 16, 1948-1951; b) Fustero, S.; Bello, P.; Javier, M.; Sánchez-Roselló, M.; Maestro, M. A.; González, J.; Pozo, C. Gold catalyzed Stereoselective Tandem Hydroamination-Formal Aza-Diels-Alder Reaction of Propargylic Amino Esters. Chem. Commun., 2013, 49, 1336-1338.

2. Tindall, D. J.; Werle, C.; Goddard, R.; Philipps, P.; Fares, C.; Fürstner A. Structure and Reactivity of Half-Sandwich $\operatorname{Rh}(+3)$ and $\operatorname{Ir}(+3)$ Carbene Complexes. Catalytic Metathesis of Azobenzene Derivatives. J. Am. Chem. Soc. 2018, 140, 1884-1893.

3. Chen, Z.; Akiyama, T. Enantioselective Organocatalytic Transfer Hydrogenation of $\alpha$-Imino Esters by Utilization of Benzothiazoline as Highly Efficient Reducing Agent. Adv. Synth. Catal. 2010, 352, 1846-1850.

4. Huang, H.; Wang, Y.; Chen, Z.; Hu, W. H. A Novel Synthesis of Aryl $\alpha$-Imino Esters from Aryl Diazoacetate. Synlett 2005, 16, 2498-2500.

5. Berger, R.; Duff, K., Leighton, J. L. Enantioselective Allylation of Ketone-Derived Benzoylhydrazones: Practical Synthesis of Tertiary Carbinamines J. Am. Chem. Soc. 2004, 126, 5686-5687.

6. Reyes-Rangel, G.; Bandala, Y.; García-Flores, F.; Juaristi, E. Asymmetric Allylation of $\alpha$ Ketoester-Derived N-Benzoylhydrazones Promoted by Chiral Sulfoxides/N-Oxides Lewis Bases: Highly Enantioselective Synthesis of Quaternary $\alpha$-Substituted $\alpha$-Allyl- $\alpha$-Amino Acids Chirality 2013, 25, 529-540.

7. Asensio, A.; Bravo, P.; Crucianelli, M.; Farina, A.; Fustero, S.; Soler, Juan G.; Meille, Stefano V.; Panzeri, W.; Viani, F.; Volonterio, A.; Zanda, M., Synthesis of Nonracemic $\alpha$ Trifluoromethyl $\alpha$-Amino Acids from Sulfinimines of Trifluoropyruvate. Eur. J. Org. Chem. 2001, 2001, 1449-1458.

8. Chaume, G.; Severen, M-C. V.; Marinkovic, S.; Brigaud, T. Org. Lett. 2006, 8, 6123-6126. 
NMR Spectra of All the Compounds 


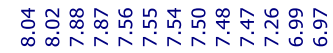

vinntur

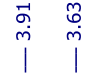

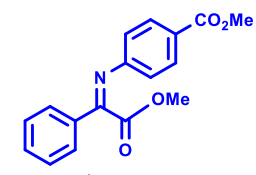

${ }^{1} \mathrm{H} \mathrm{NMR,} \mathrm{CDCl}_{3}, 600 \mathrm{MHz}$

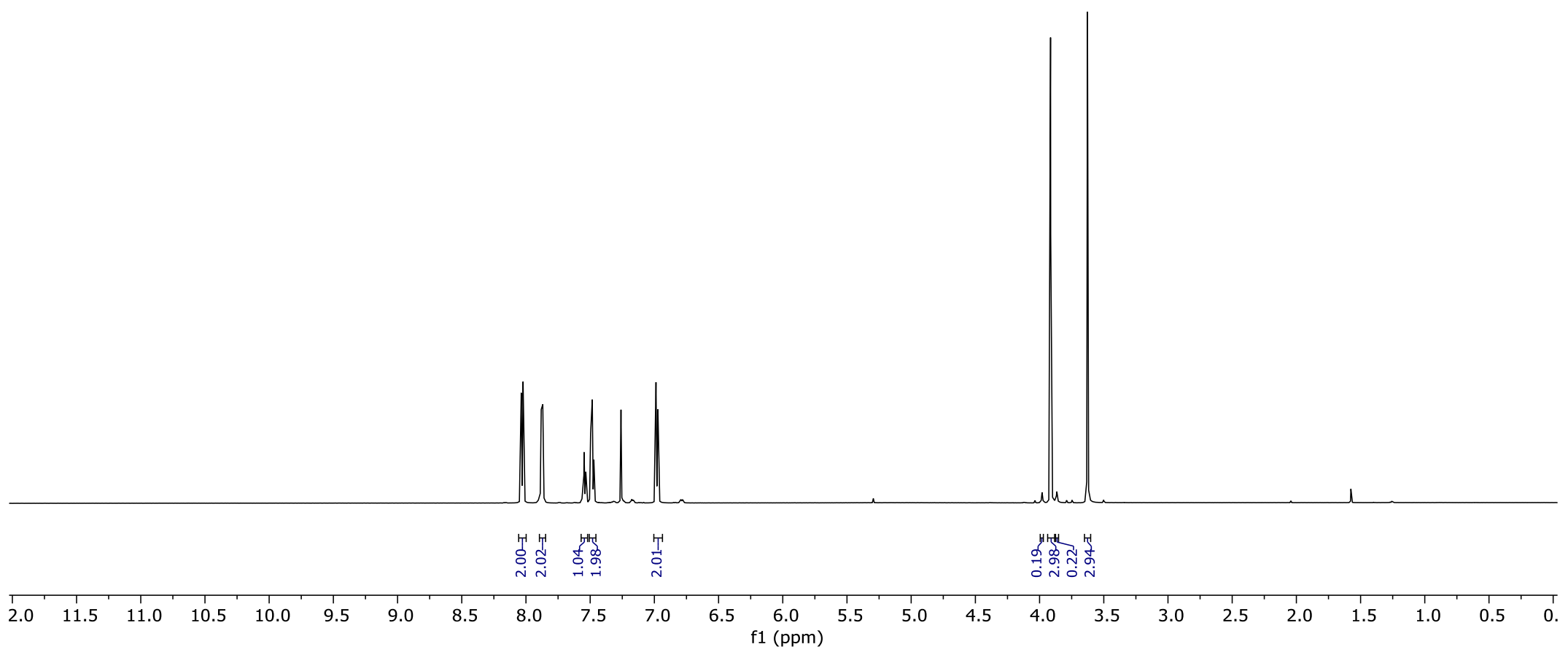



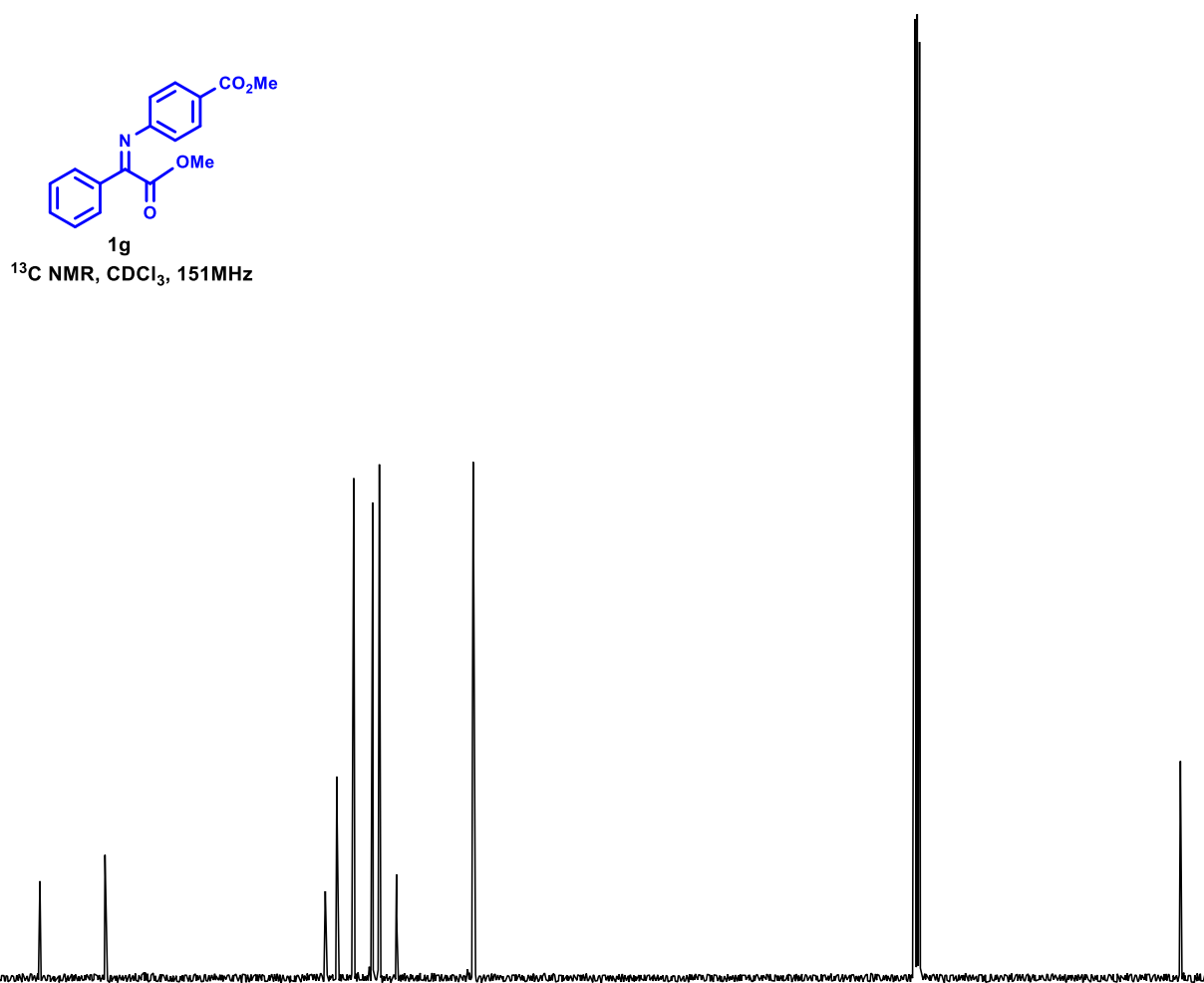

$: 00$ 


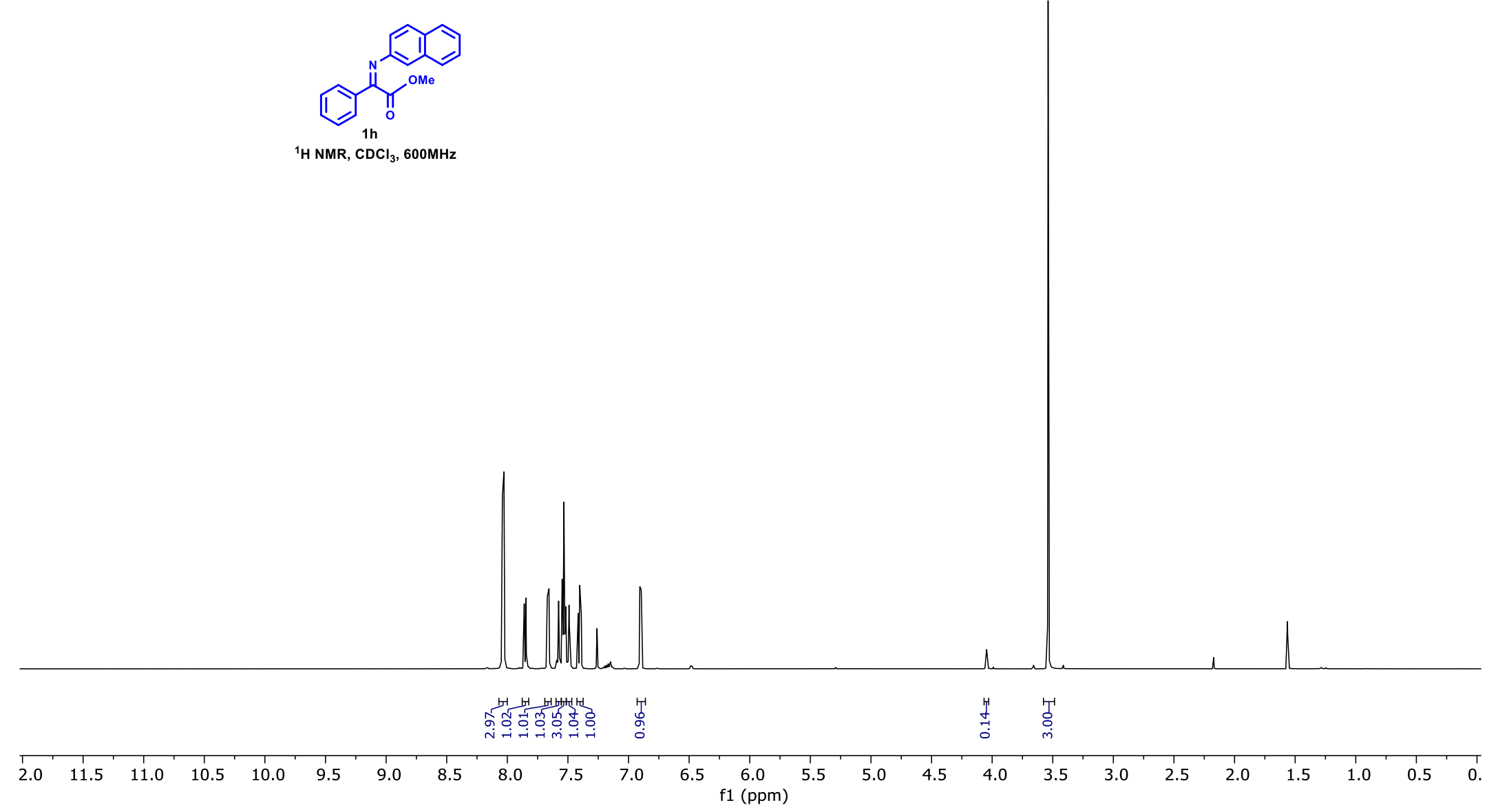

${ }^{1} \mathrm{H} \mathrm{NMR}, \mathrm{CDCl}_{3}, 600 \mathrm{MHz}$ 


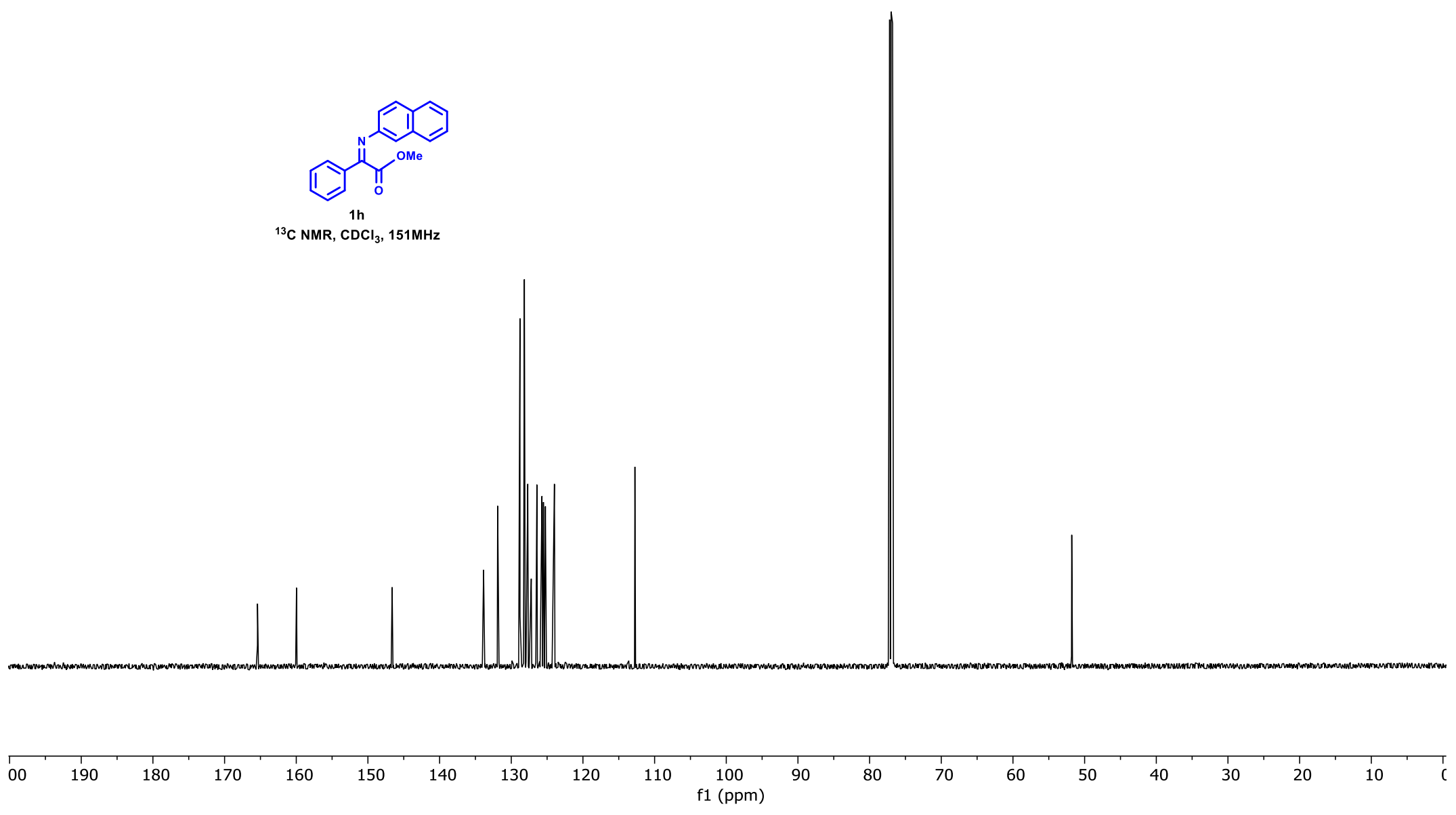




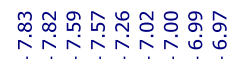

Yर

$\stackrel{\substack{\infty \\ \infty}}{\substack{\infty \\ \dot{m}}}$
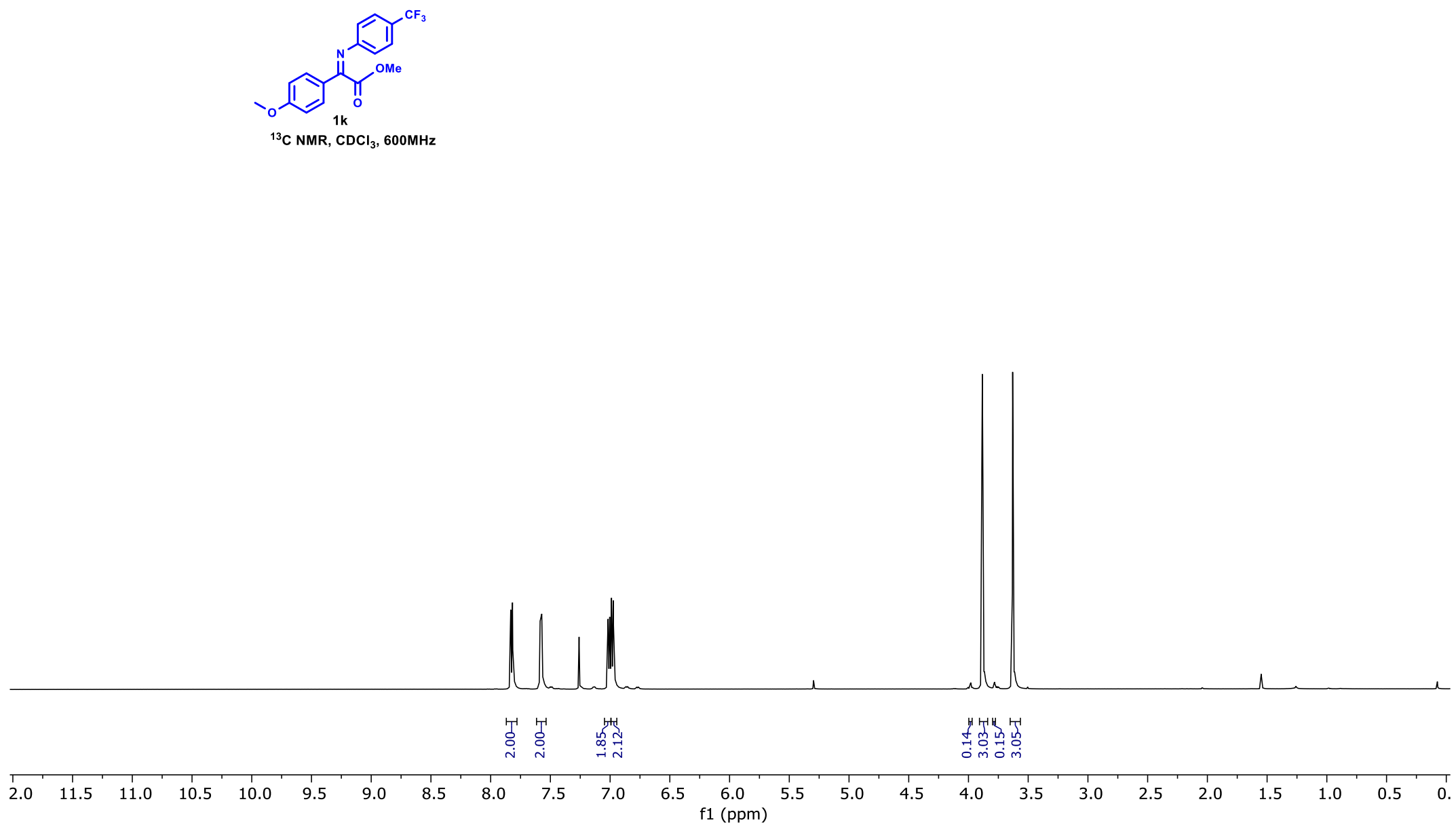


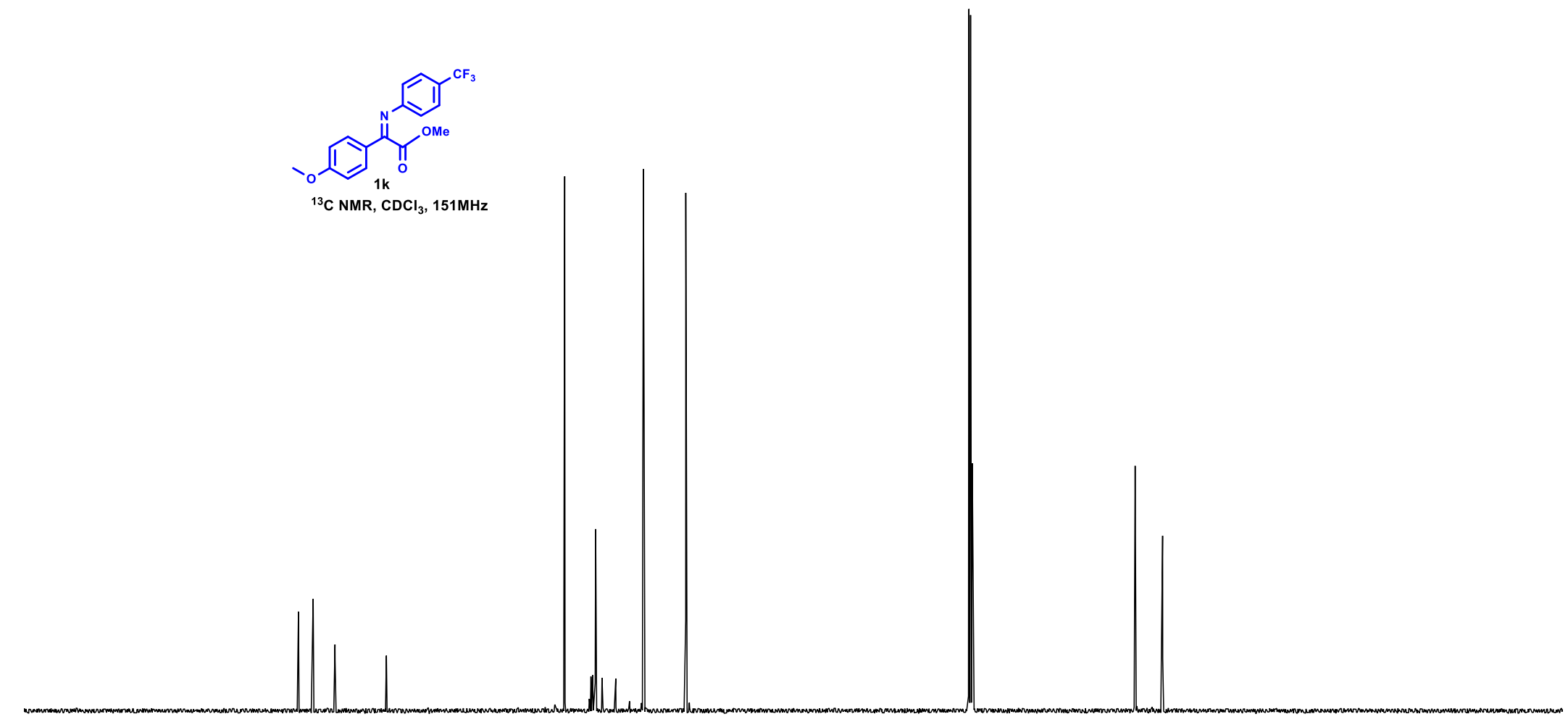

$100 \quad 190-180$

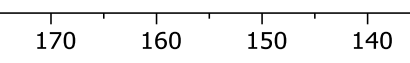

$130 \quad 120$

$\begin{array}{cc}110 & 100 \\ & \mathrm{f} 1(\mathrm{ppm})\end{array}$

$\begin{array}{llll}90 & 80 & 70 & 60\end{array}$

50 40 


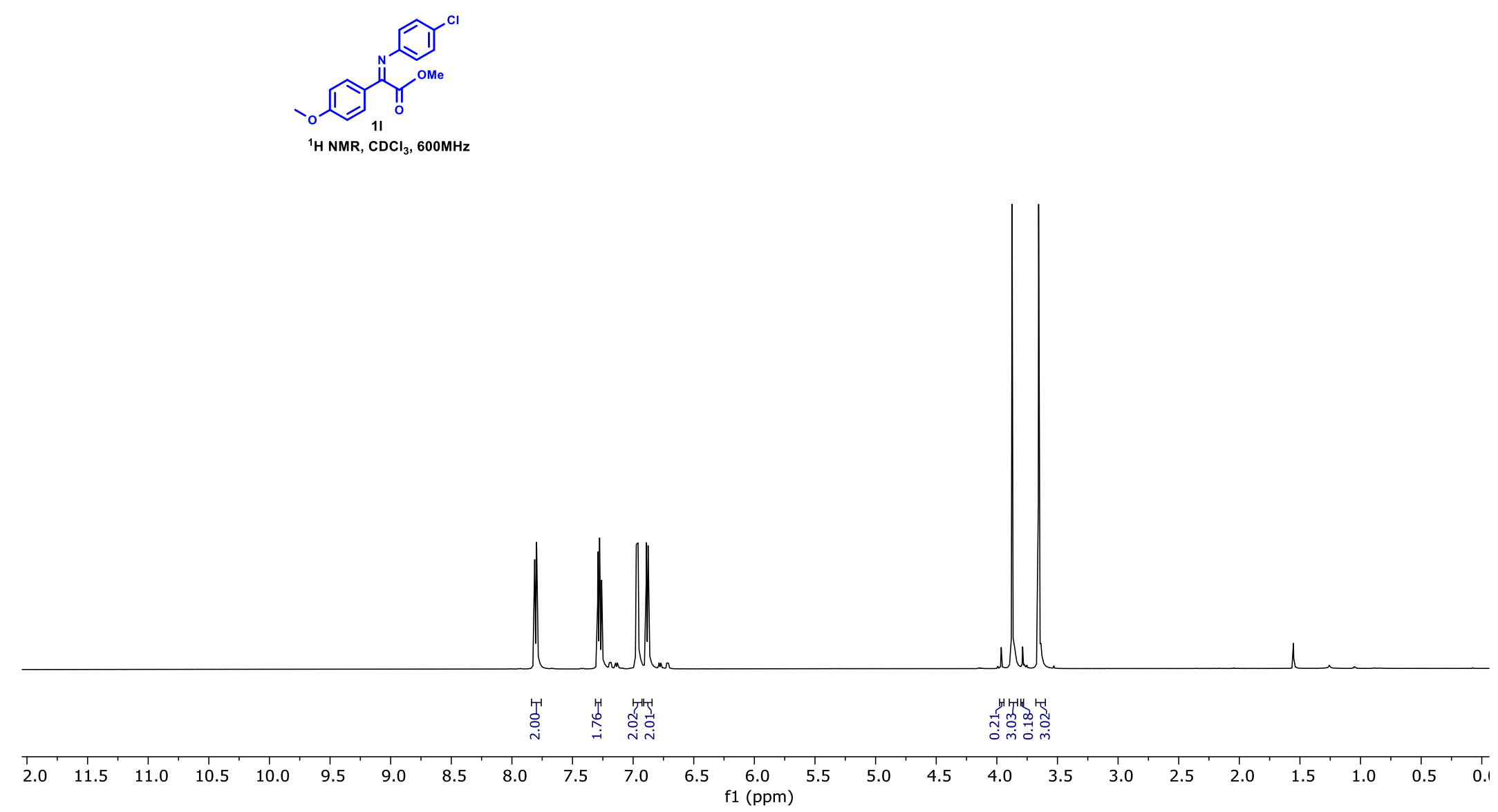




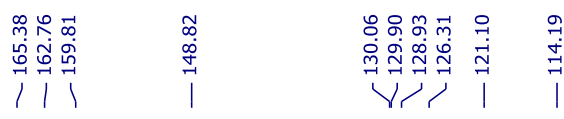
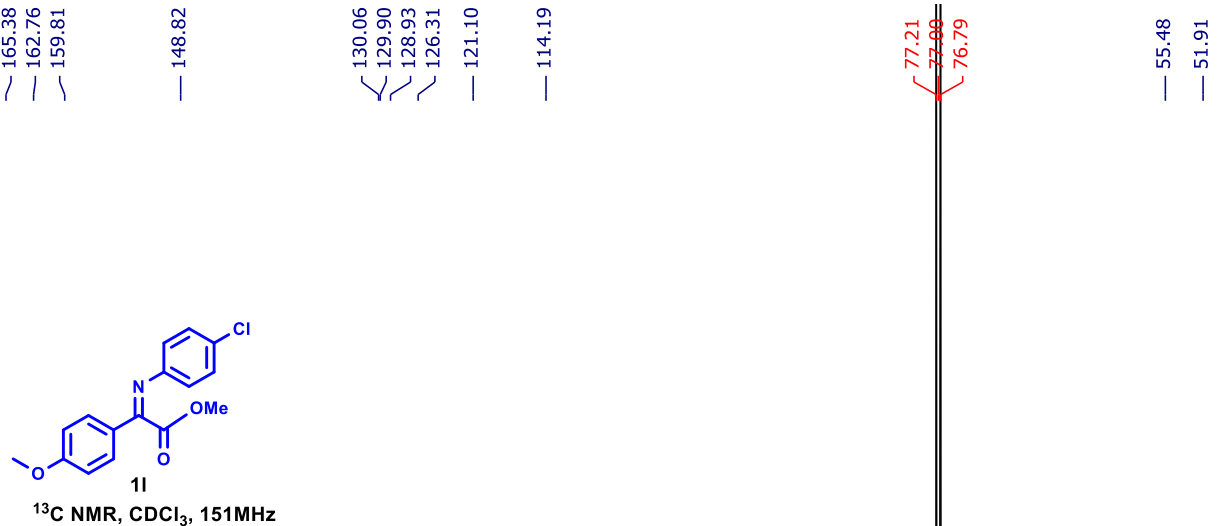

${ }^{13} \mathrm{C} \mathrm{NMR,} \mathrm{CDCl}_{3}, 151 \mathrm{MHz}$

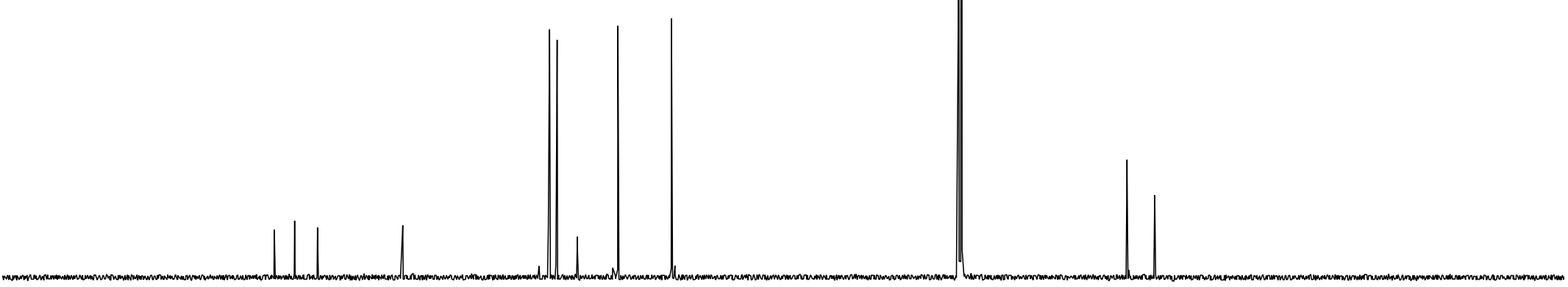

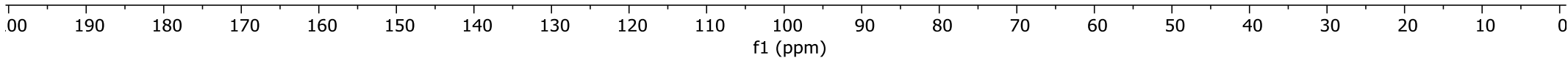




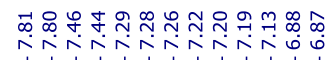

YYV

$\stackrel{\infty}{\sim}$

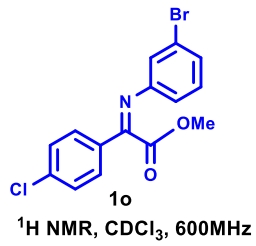

${ }^{1} \mathrm{H} \mathrm{NMR,} \mathrm{CDCl}_{3}, 600 \mathrm{MHz}$

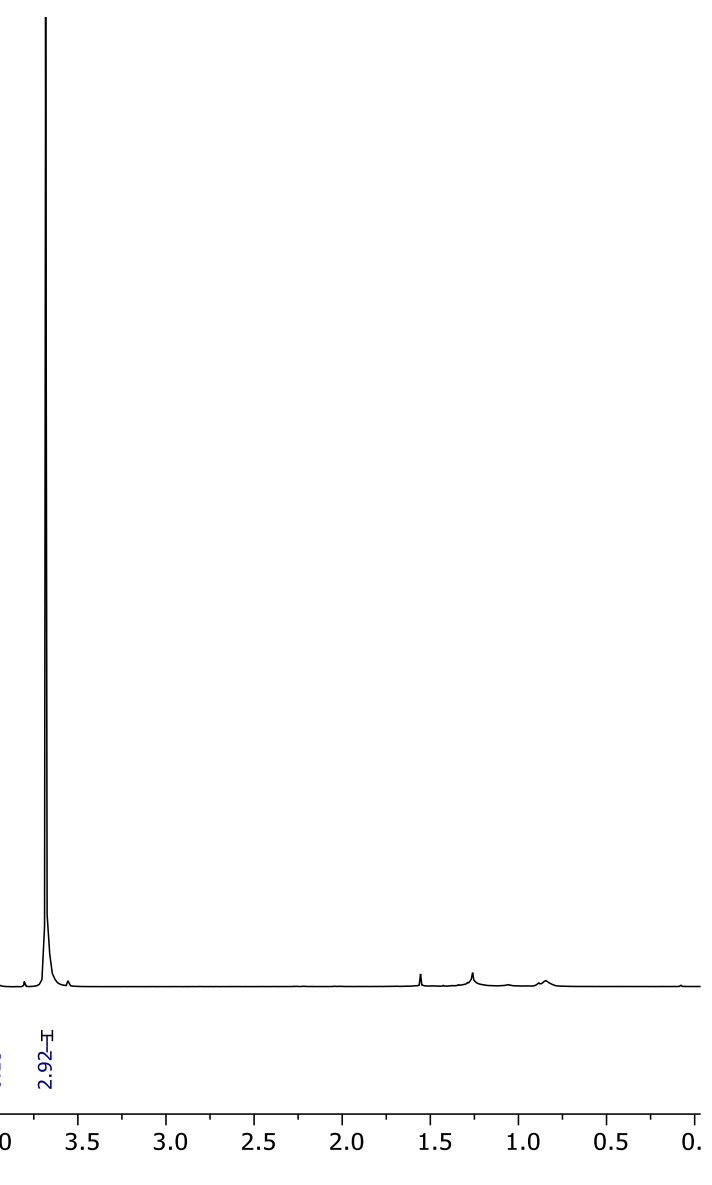



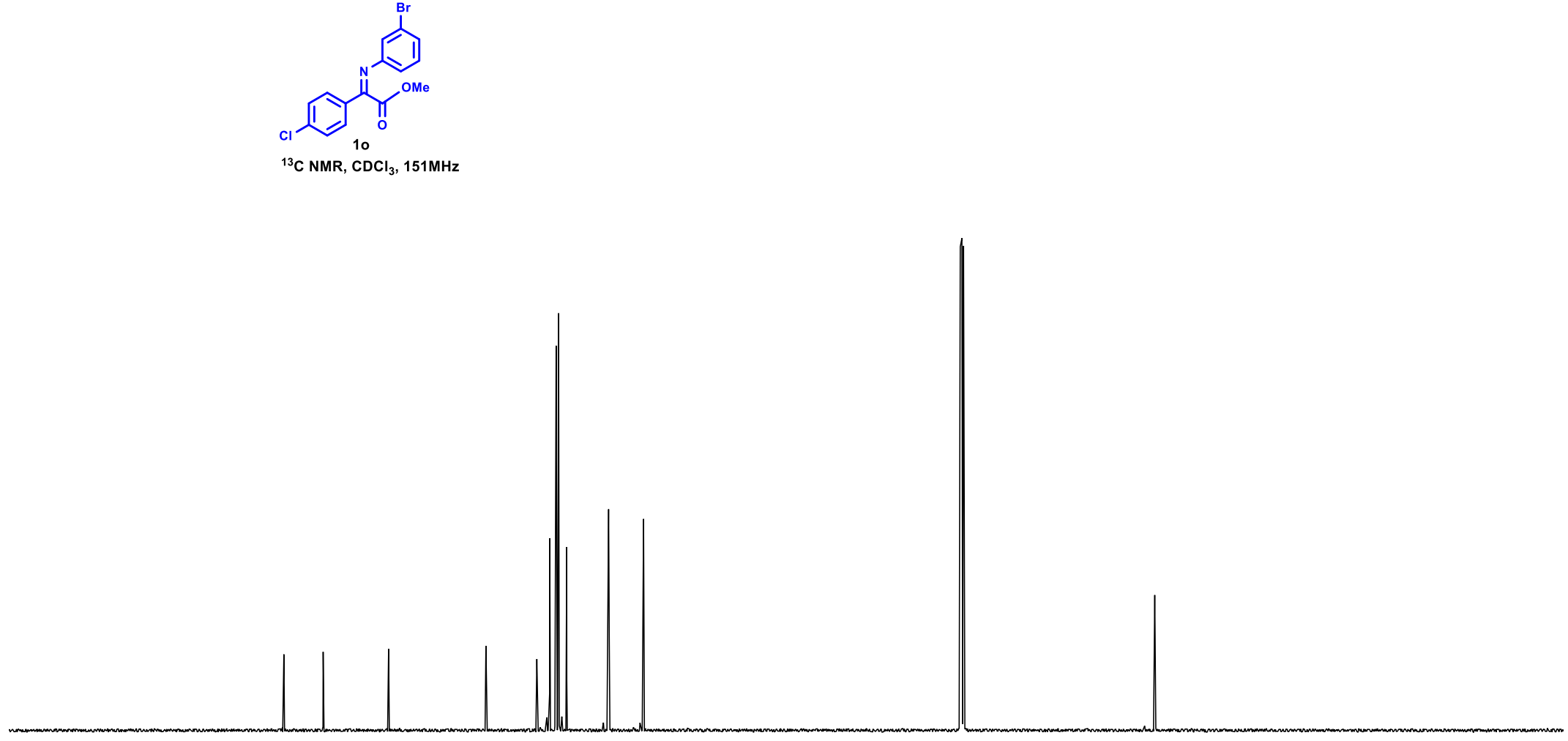

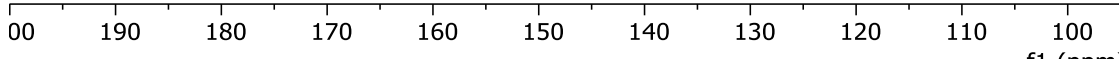
f1 (ppm) 


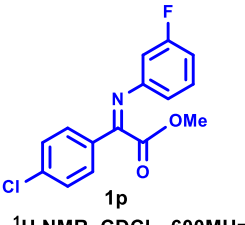

${ }^{1} \mathrm{H}$ NMR, $\mathrm{CDCl}_{3}, 600 \mathrm{MHz}$

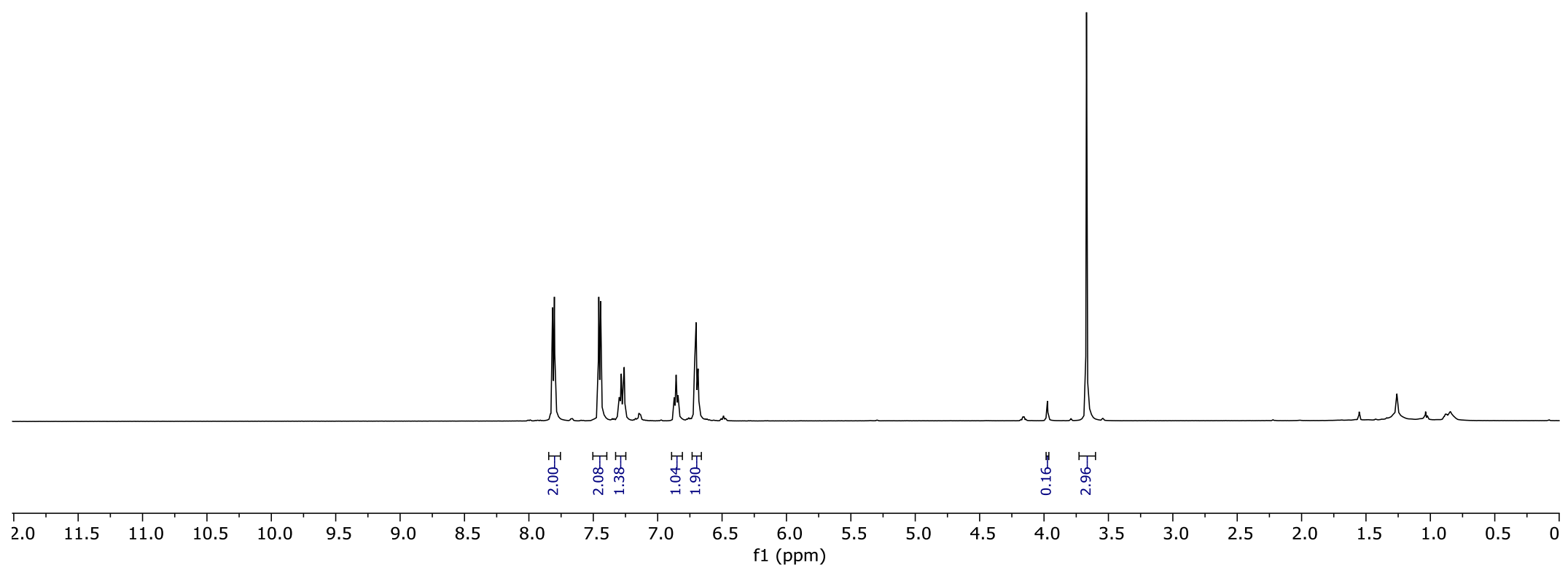




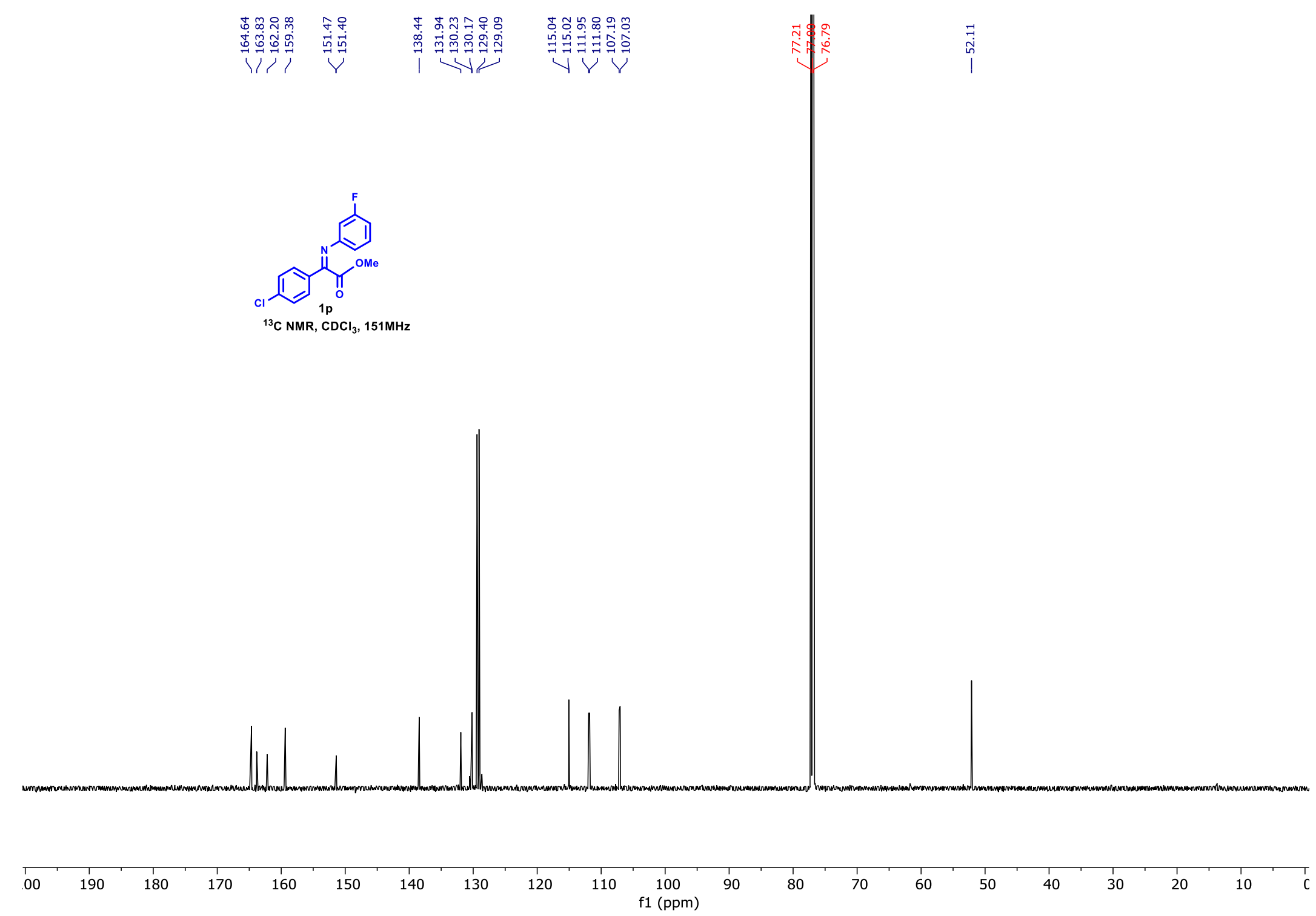




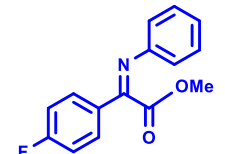

${ }^{1} \mathrm{H} \mathrm{NMR,} \mathrm{CDCl}_{3}, 600 \mathrm{MHz}$

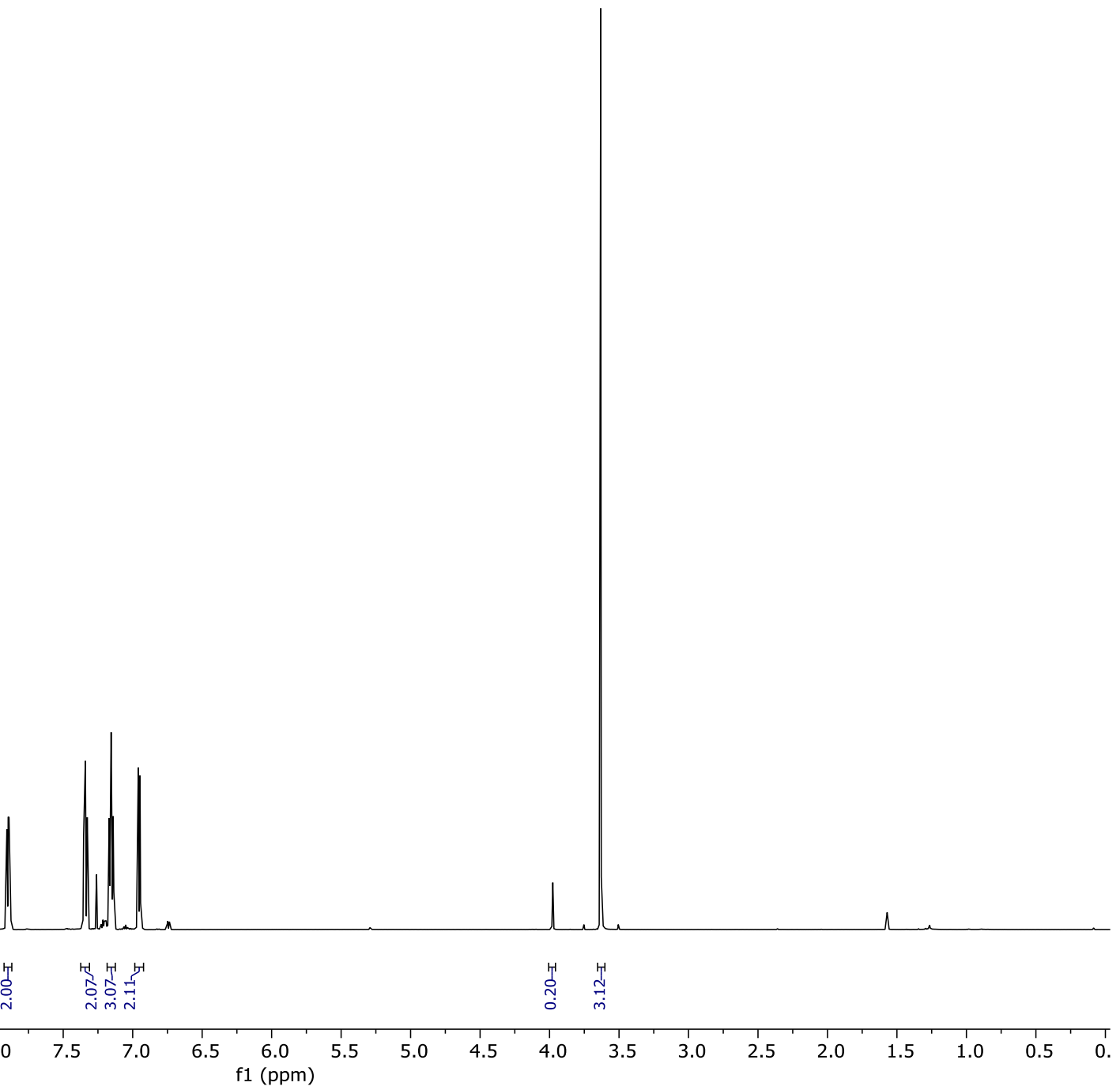




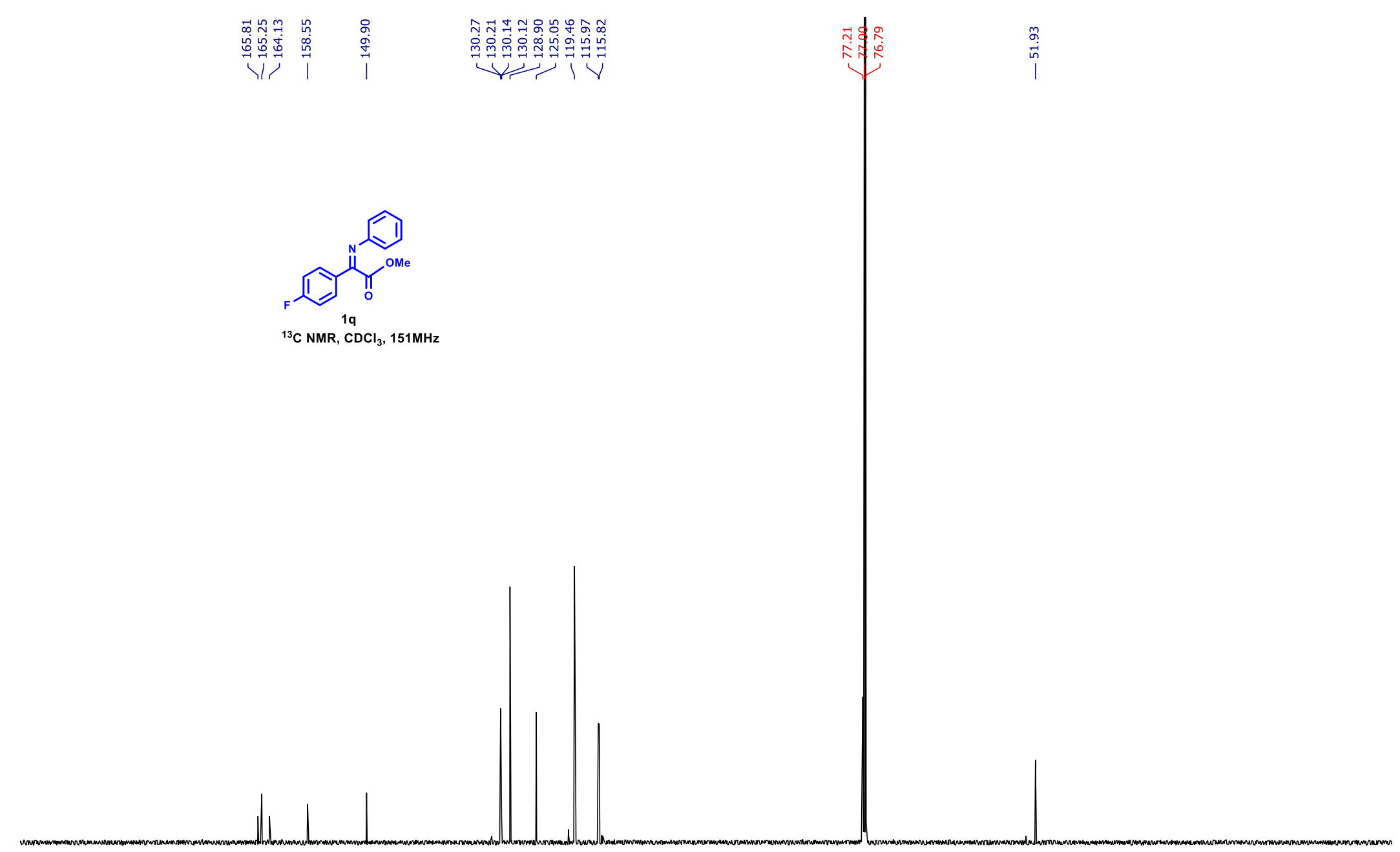

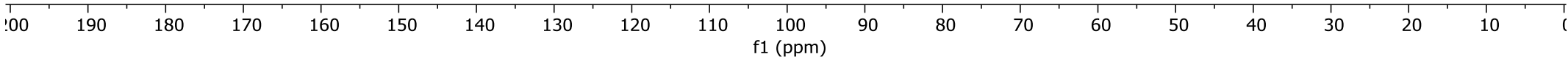




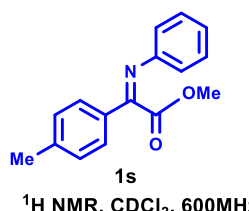

${ }^{1} \mathrm{H} \mathrm{NMR}, \mathrm{CDCl}_{3}, 600 \mathrm{MHz}$

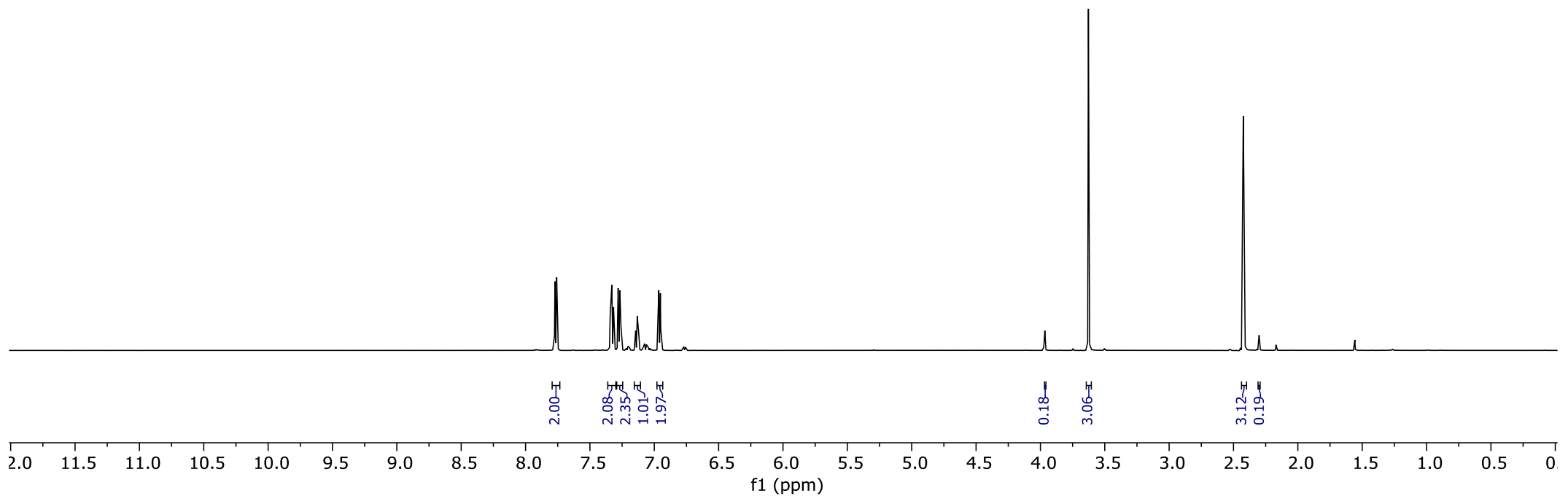



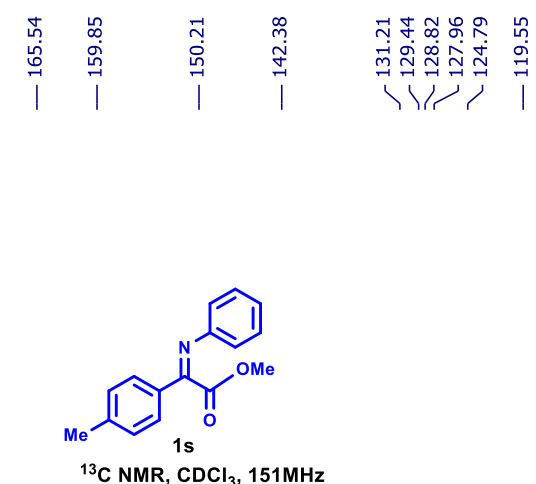

${ }^{13} \mathrm{C} \mathrm{NMR,} \mathrm{CDCl}_{3}, 151 \mathrm{MHz}$

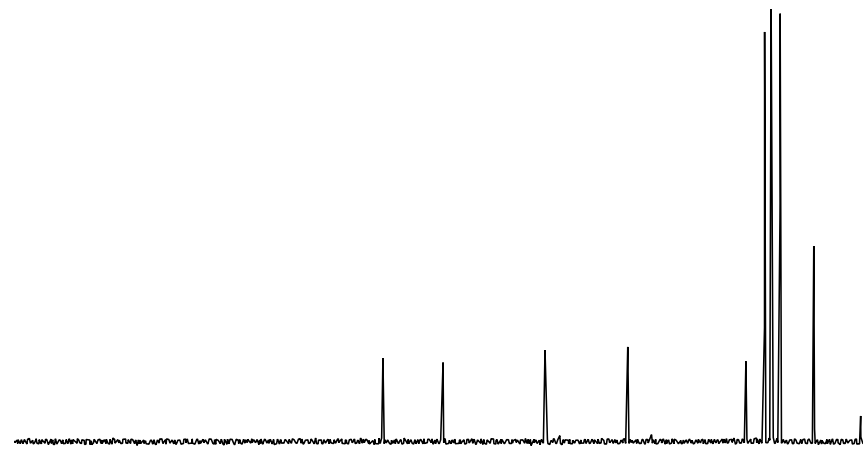

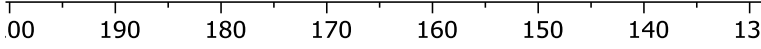

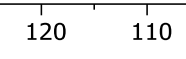

100
$\mathrm{f} 1(\mathrm{ppm})$

$80 \quad 70 \quad 60$

50

40 


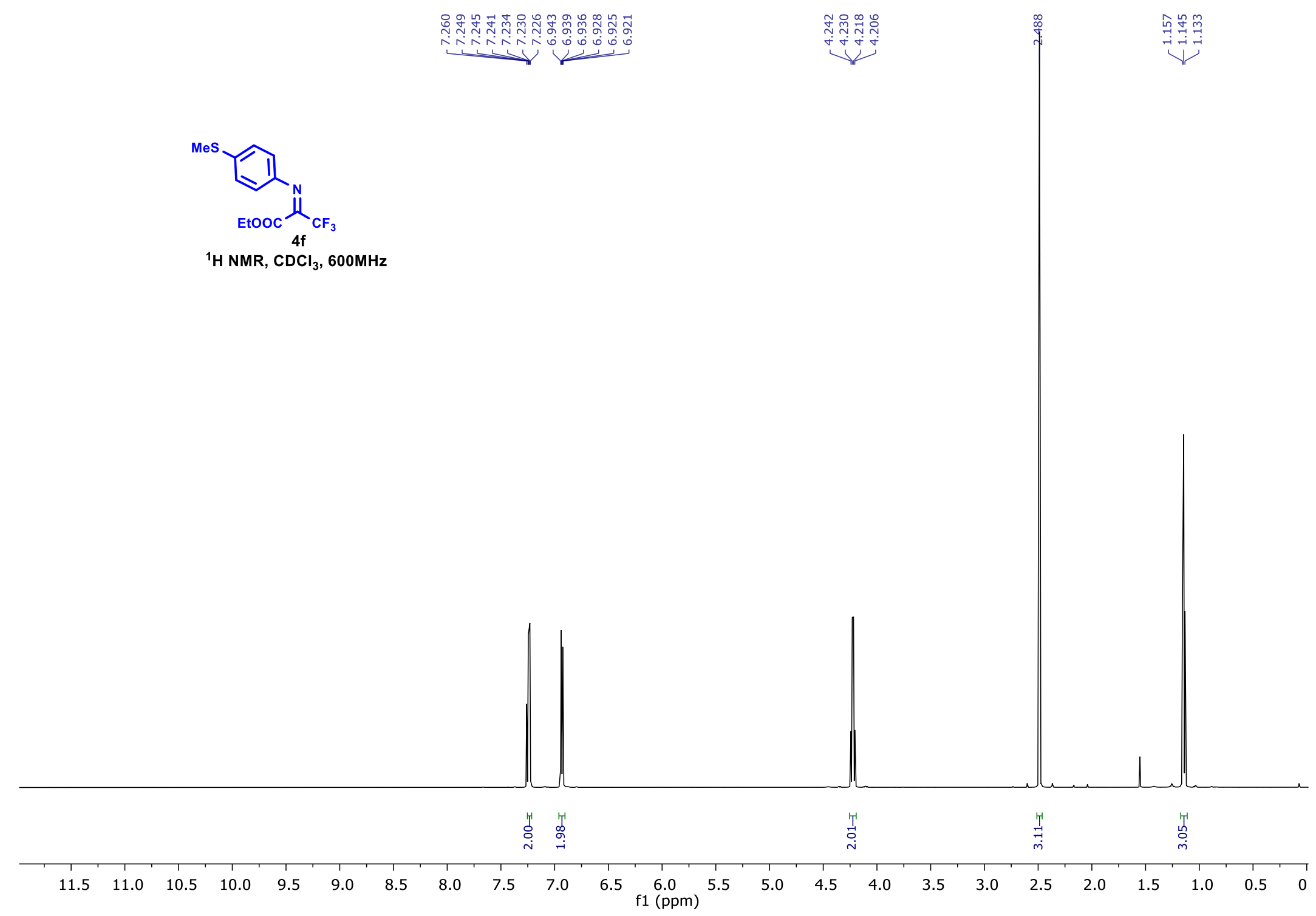




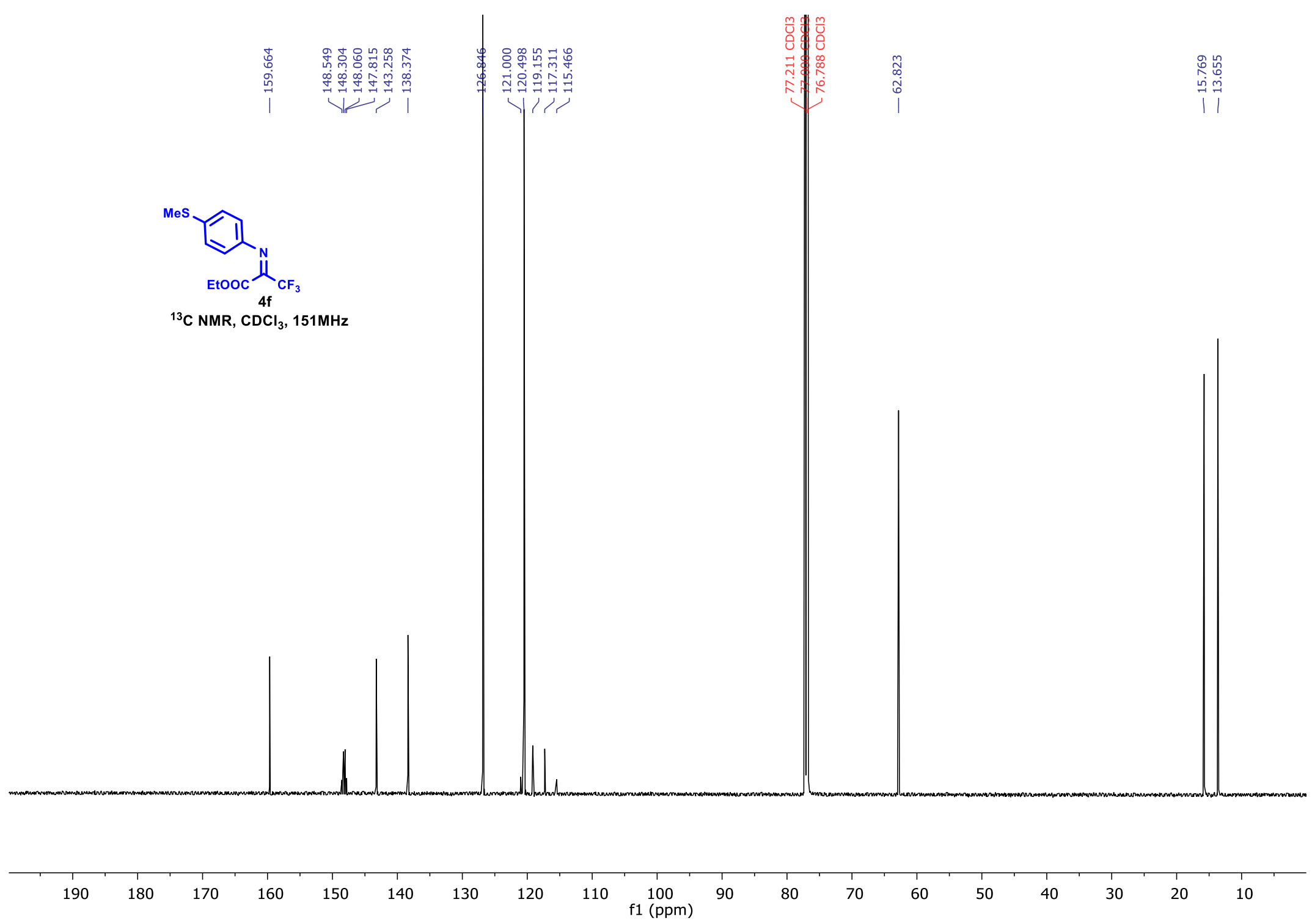


m
$\vdots$
0
0
1
1

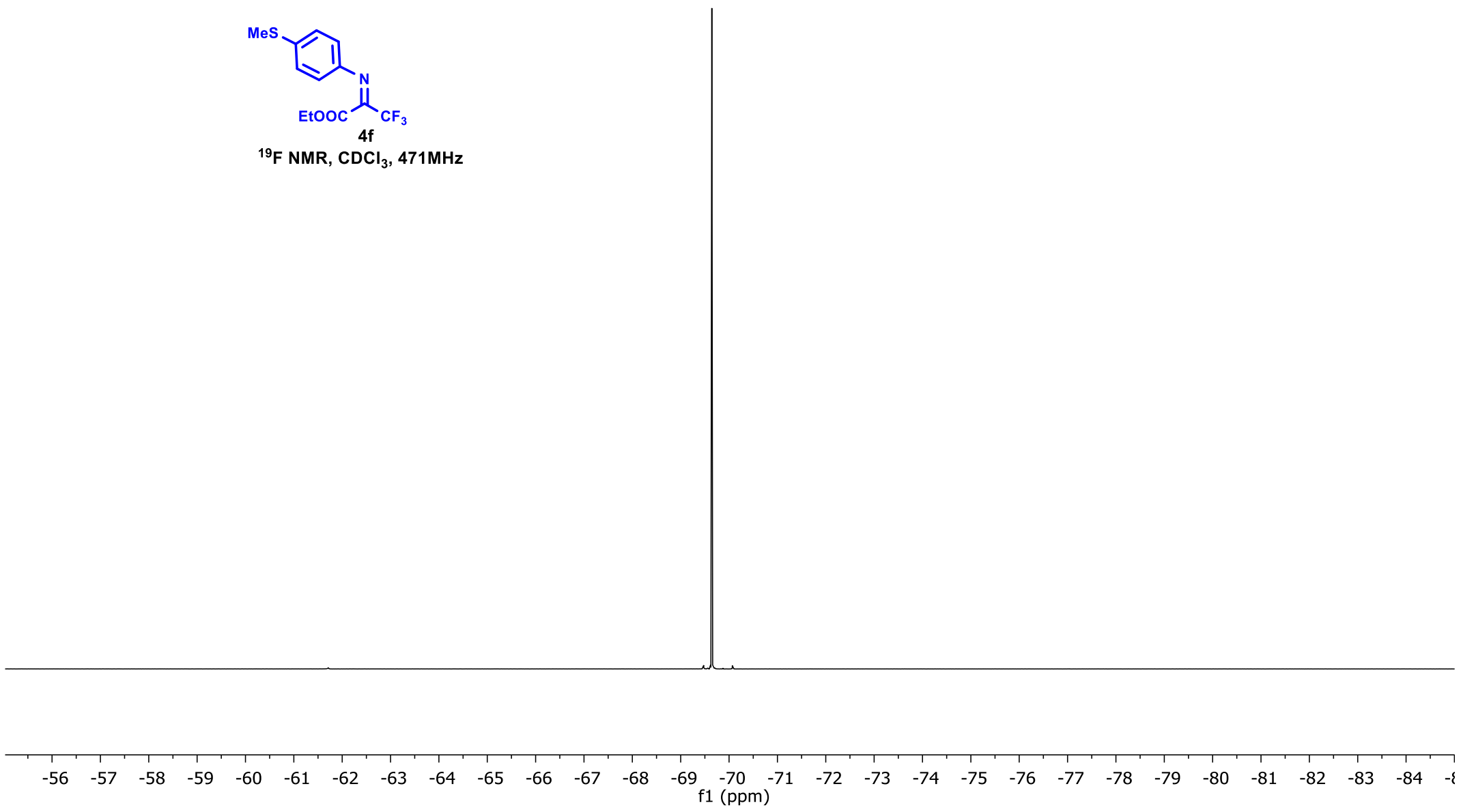

S77 


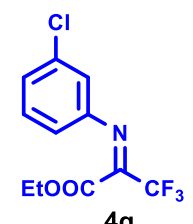

${ }^{1} \mathrm{H} \mathrm{NMR}, \mathrm{CDCl}_{3}, 600 \mathrm{MHz}$

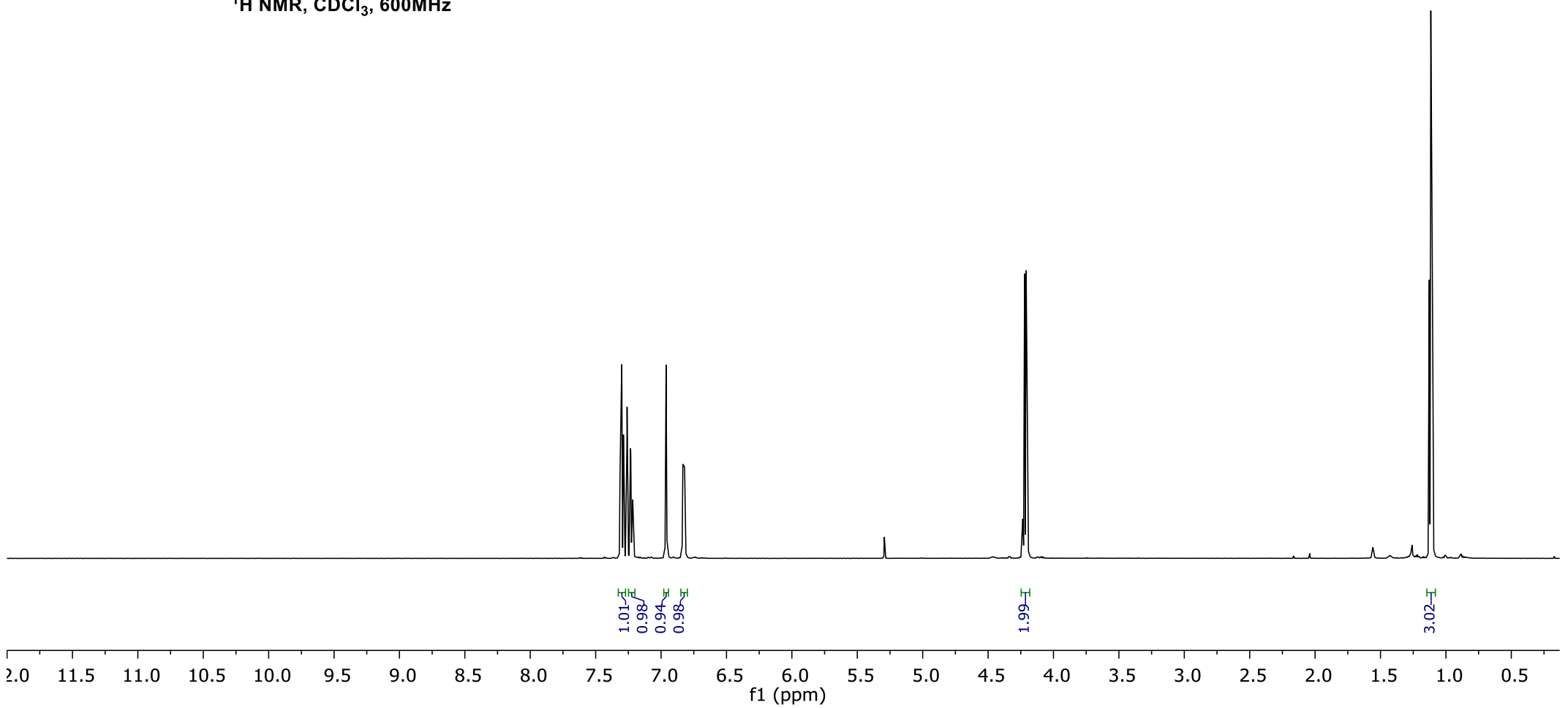




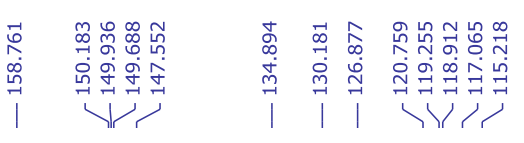

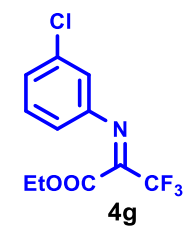

${ }^{13} \mathrm{C} \mathrm{NMR,} \mathrm{CDCl}_{3}, 151 \mathrm{MHz}$

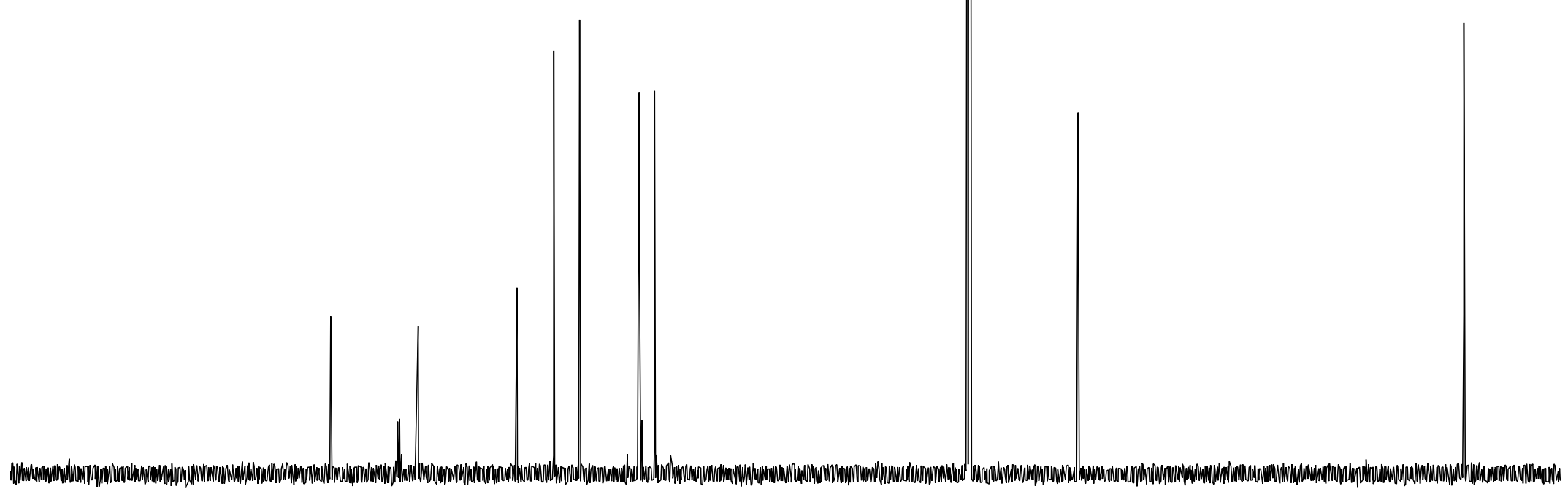




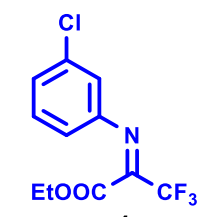

${ }^{19} \mathrm{~F} \mathrm{NMR,} \mathrm{CDCl}_{3}, 471 \mathrm{MHz}$

\begin{tabular}{llllllllllllllllllllllllllllllllllllllllll}
\hline 55 & -56 & -57 & -58 & -59 & -60 & -61 & -62 & -63 & -64 & -65 & -66 & -67 & -68 & -69 & -70 & -71 & -72 & -73 & -74 & -75 & -76 & -77 & -78 & -79 & -80 & -81 & -82 & -83 & -84 & -8
\end{tabular} 


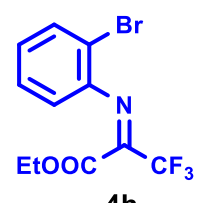

${ }^{1} \mathrm{H} \mathrm{NMR,} \mathrm{CDCl}_{3}, 600 \mathrm{MHz}$

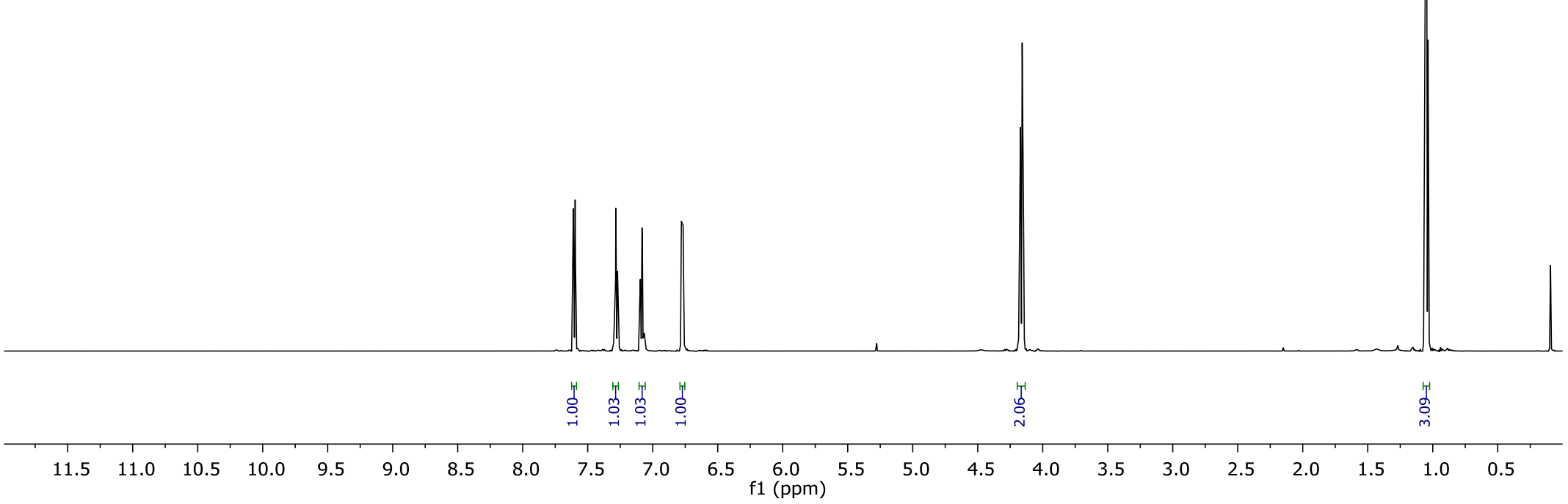




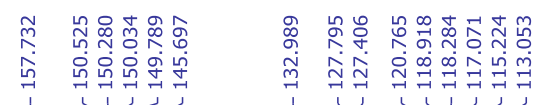

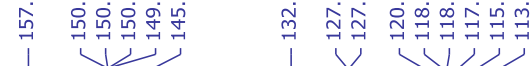

تี

$\stackrel{\substack{\infty \\ m}}{m}$

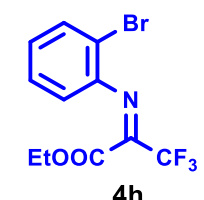

${ }^{13} \mathrm{C} \mathrm{NMR} \mathrm{CDCl}_{3}, 151 \mathrm{MHz}$

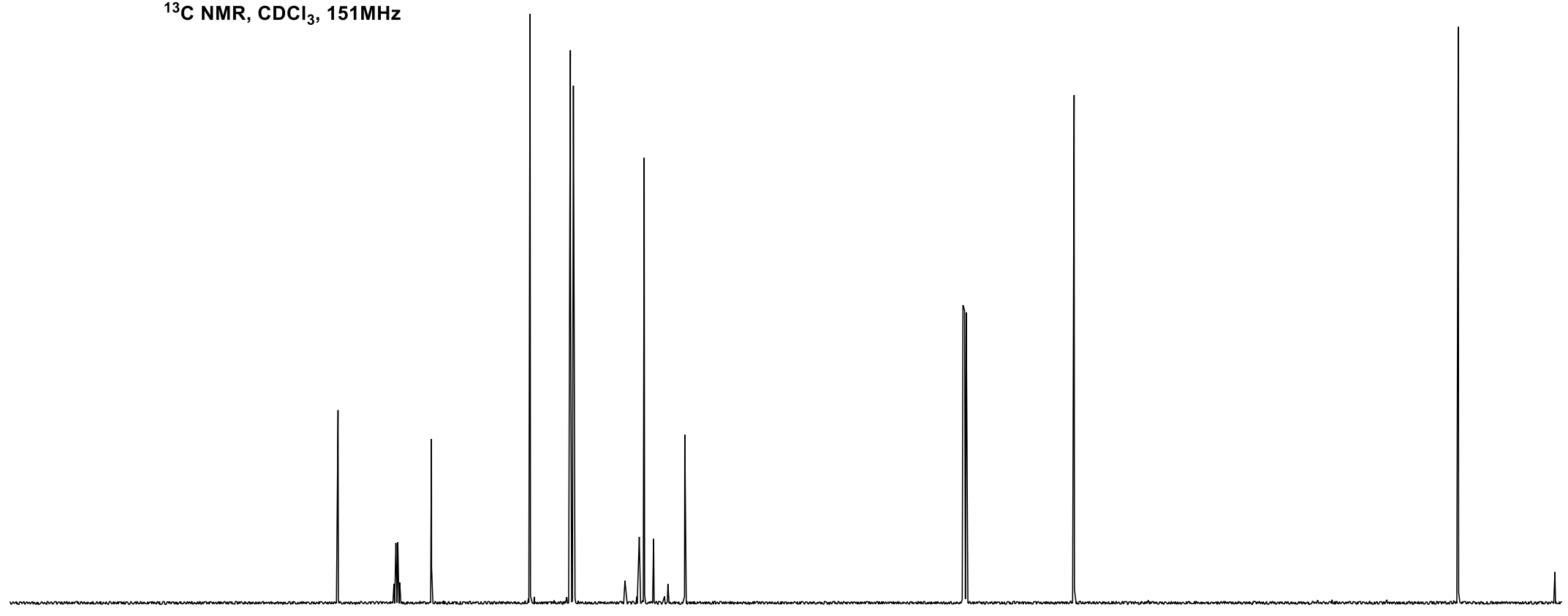

$00 \quad 190 \quad 180 \quad 170 \quad 160$

$\begin{array}{lcc}110 & 100 & 90\end{array}$

$80 \quad 70$

60

50

40

30

20

10 


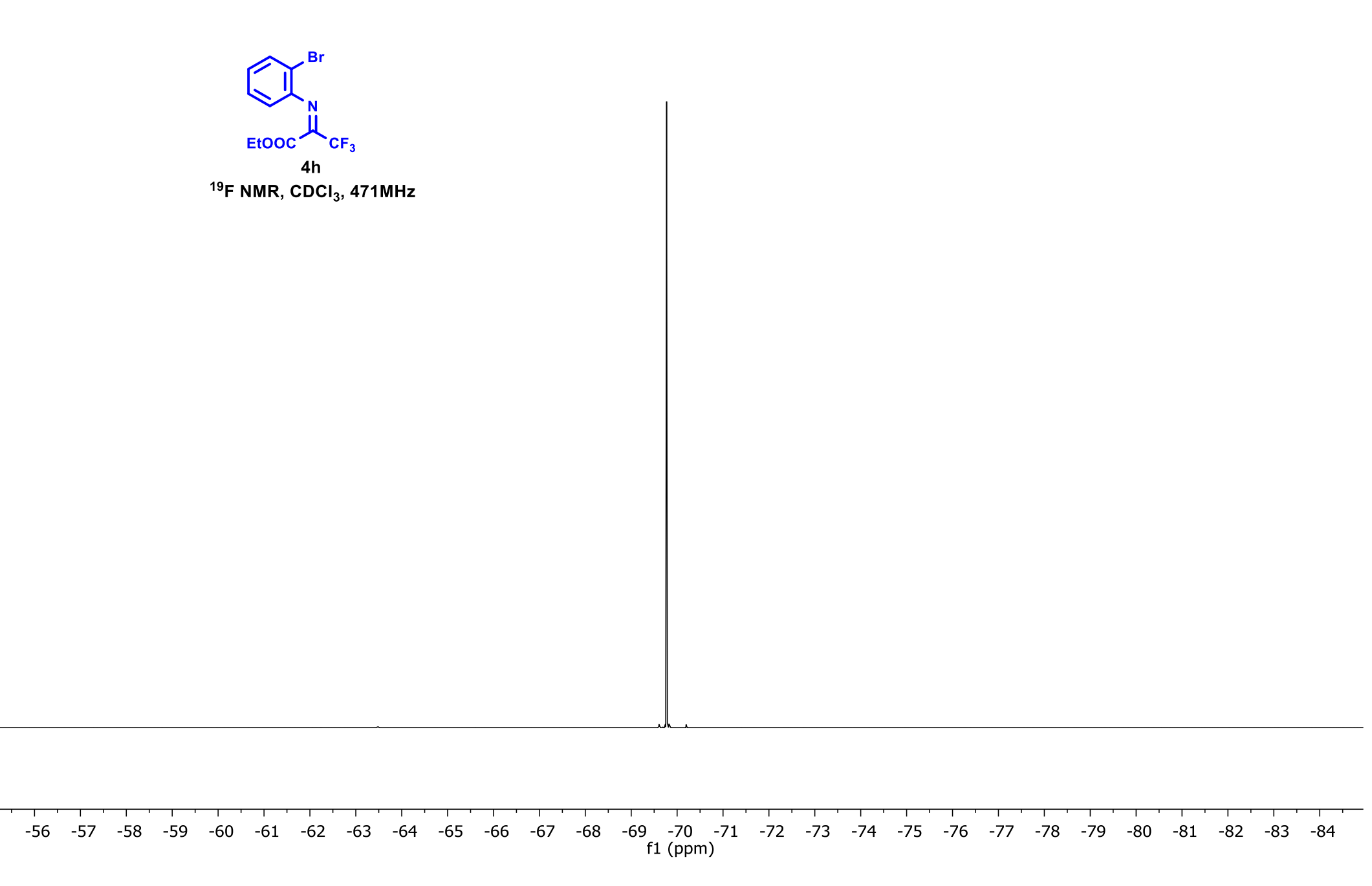




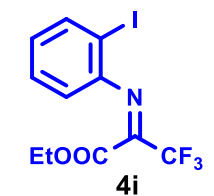

${ }^{1} \mathrm{H} \mathrm{NMR,} \mathrm{CDCl}_{3}, 600 \mathrm{MHz}$

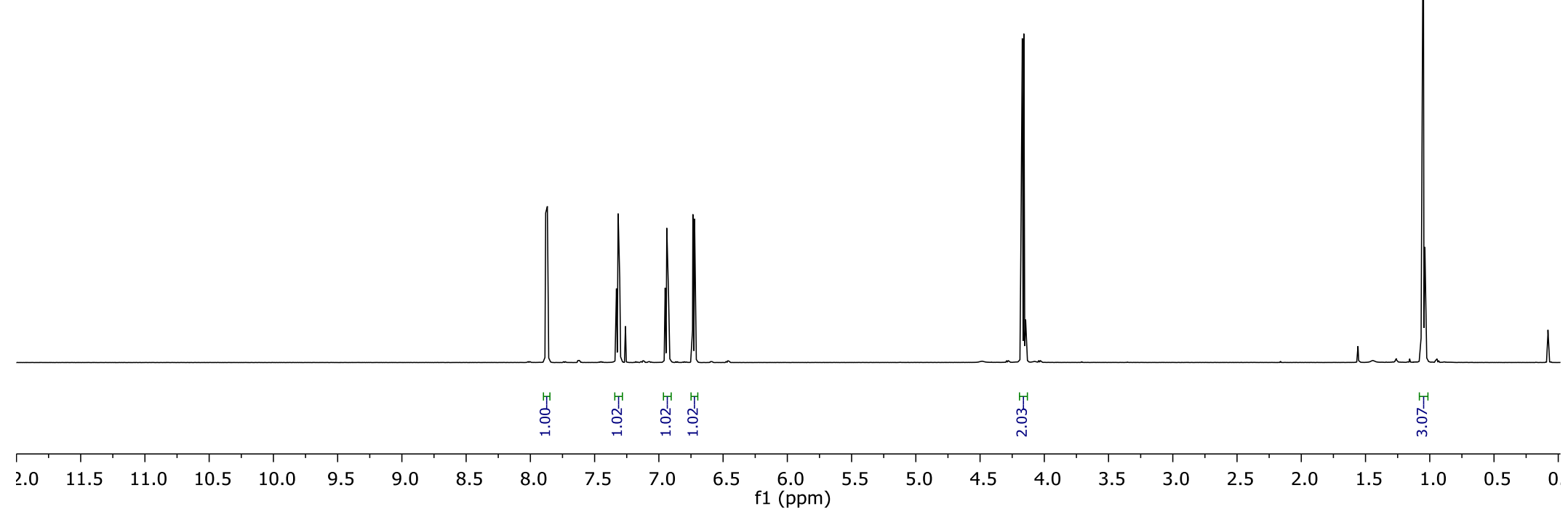




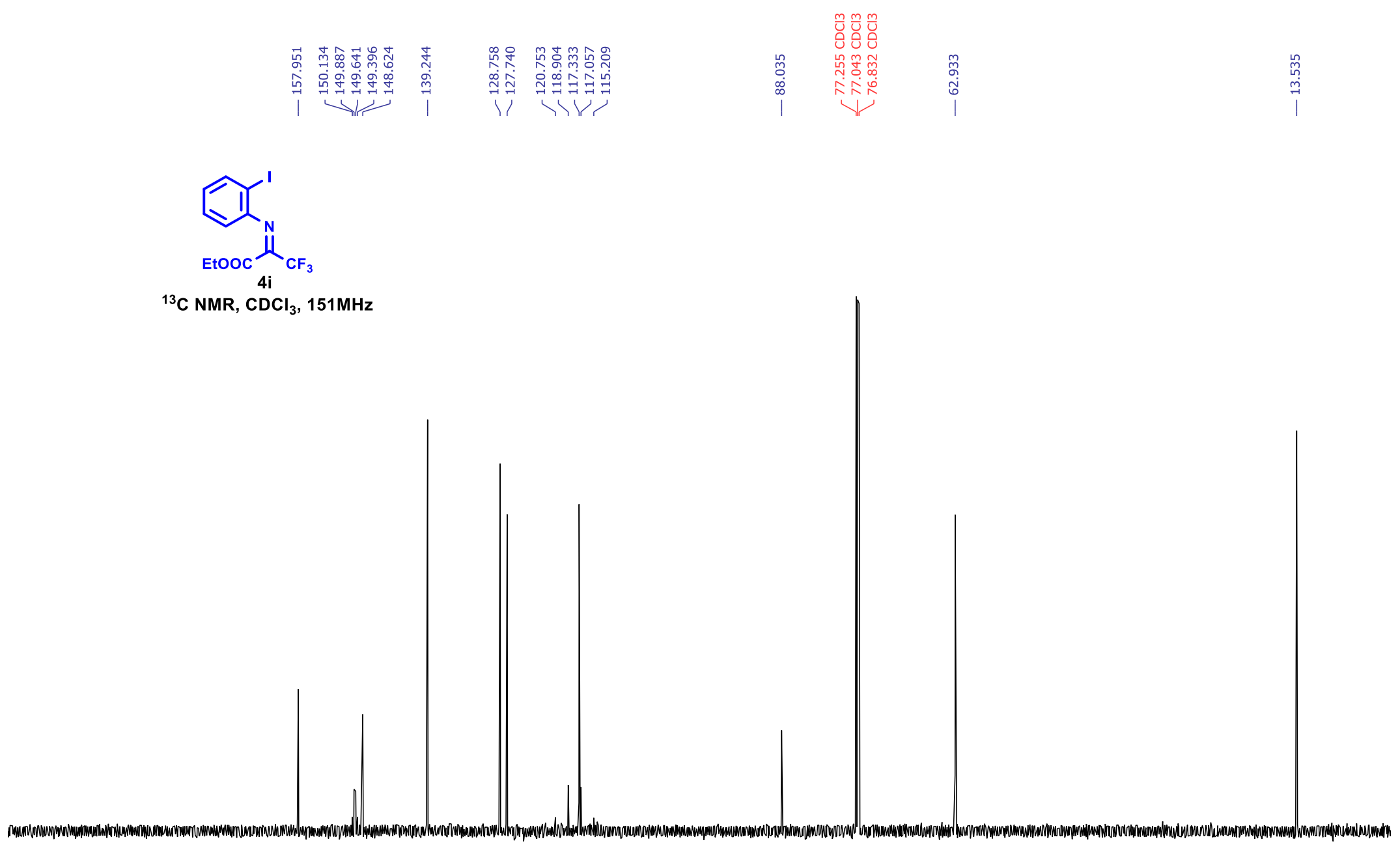

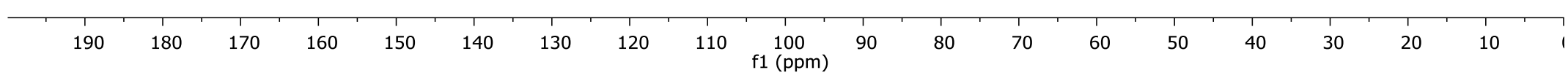




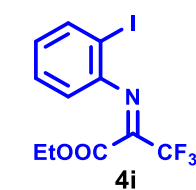

${ }^{19} \mathrm{~F} \mathrm{NMR,} \mathrm{CDCl}_{3}, 471 \mathrm{MHz}$

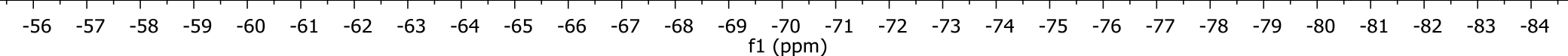


E

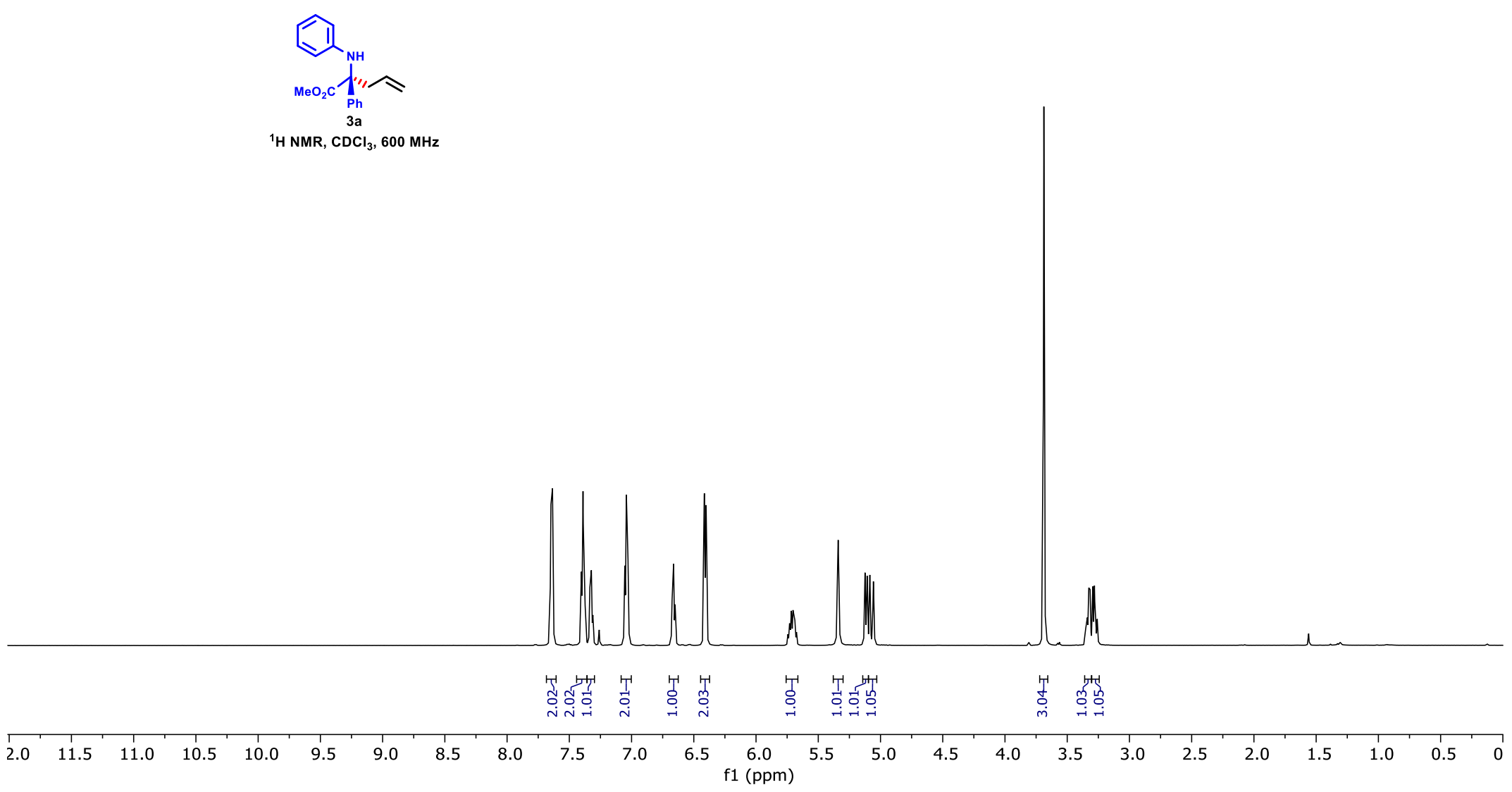

${ }^{1} \mathrm{H} \mathrm{NMR}, \mathrm{CDCl}_{3}, 600 \mathrm{MHz}$ 


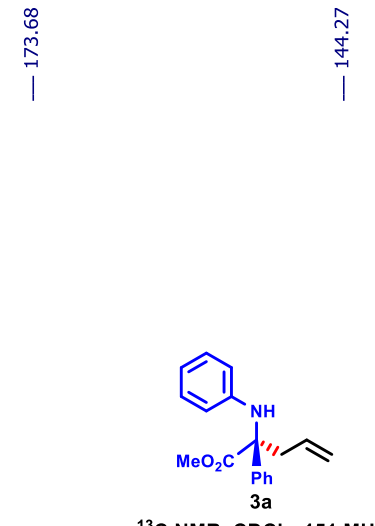

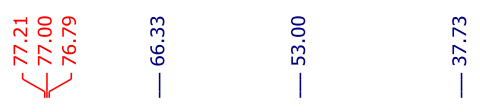

${ }^{13} \mathrm{C} \mathrm{NMR,} \mathrm{CDCl}_{3}, 151 \mathrm{MHz}$

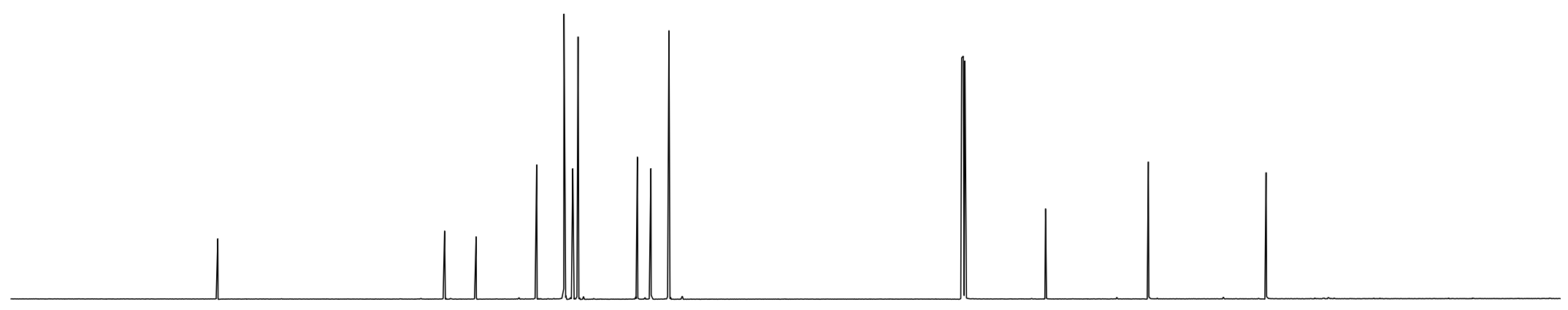

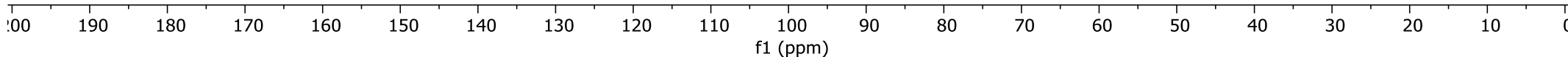




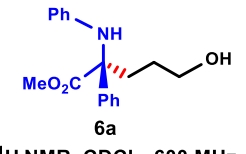

${ }^{1} \mathrm{HNMR}, \mathrm{CDCl}_{3}, 600 \mathrm{MHz}$

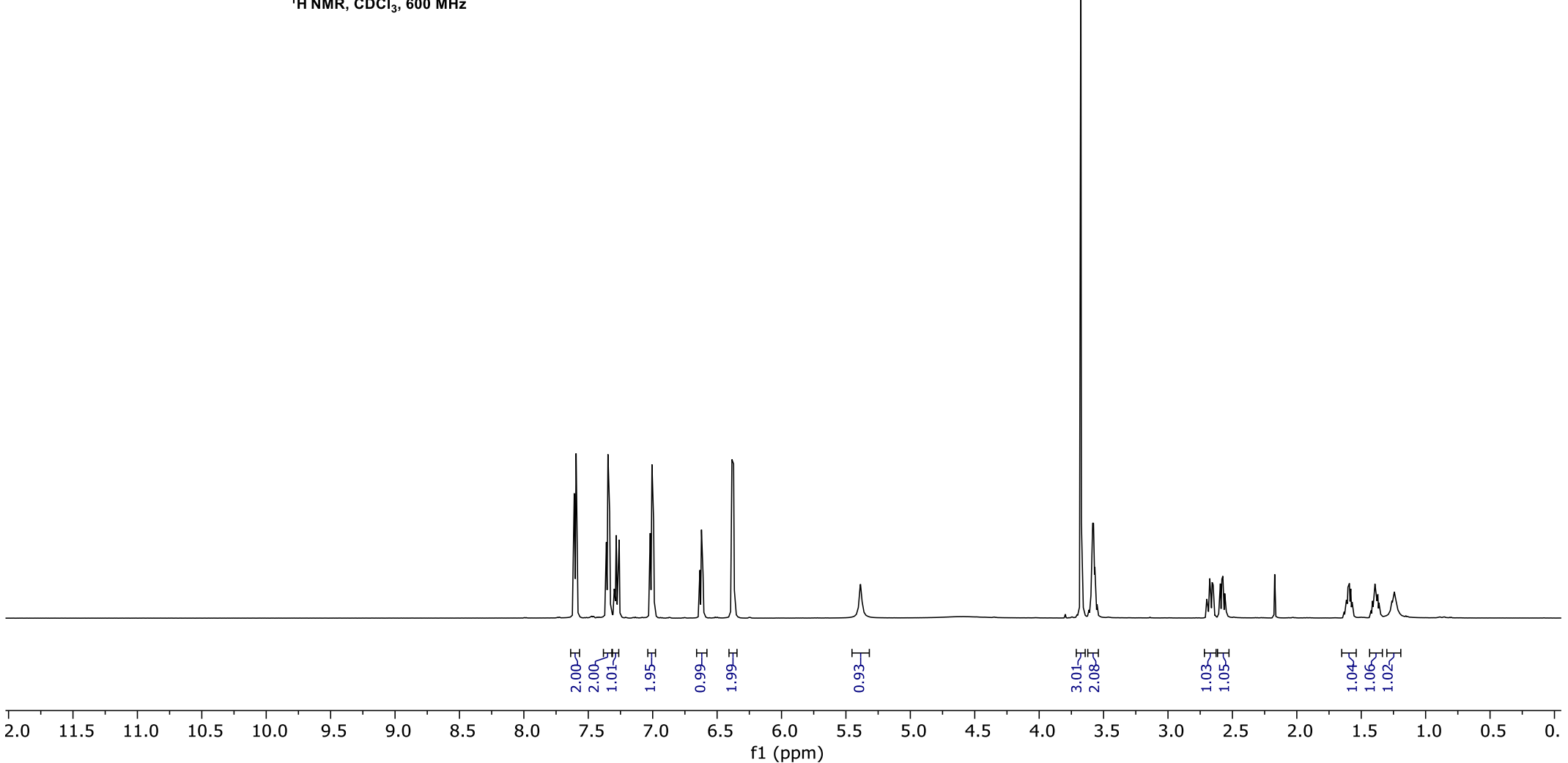


${ }^{13} \mathrm{C} \mathrm{NMR,} \mathrm{CDCl}_{3}, 151 \mathrm{MHz}$

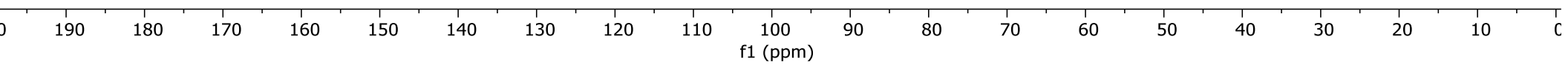




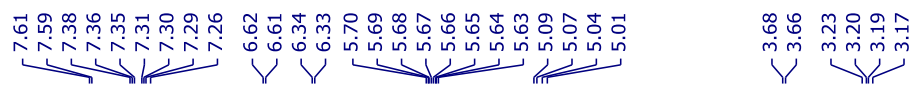

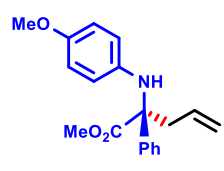

${ }^{1} \mathrm{H} \mathrm{NMR}, \mathrm{CDCl}_{3}, 600 \mathrm{MHz}$

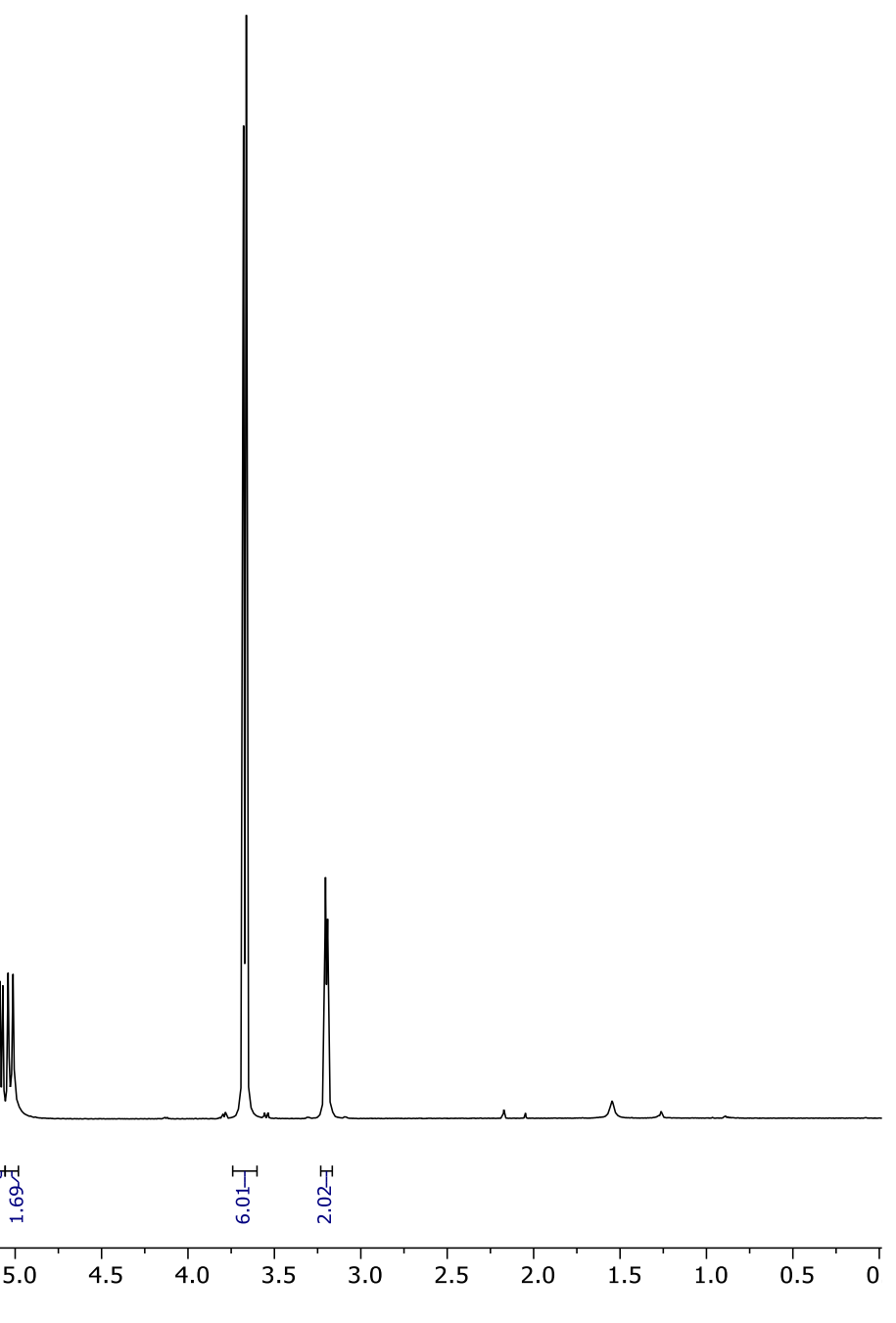




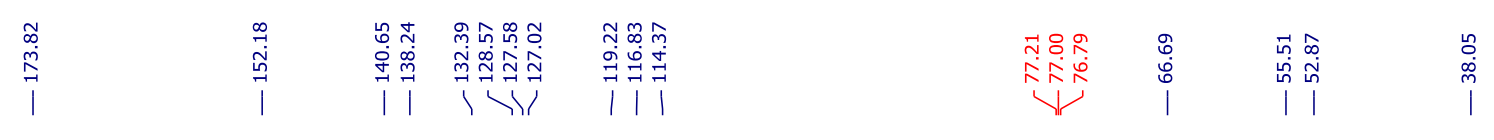

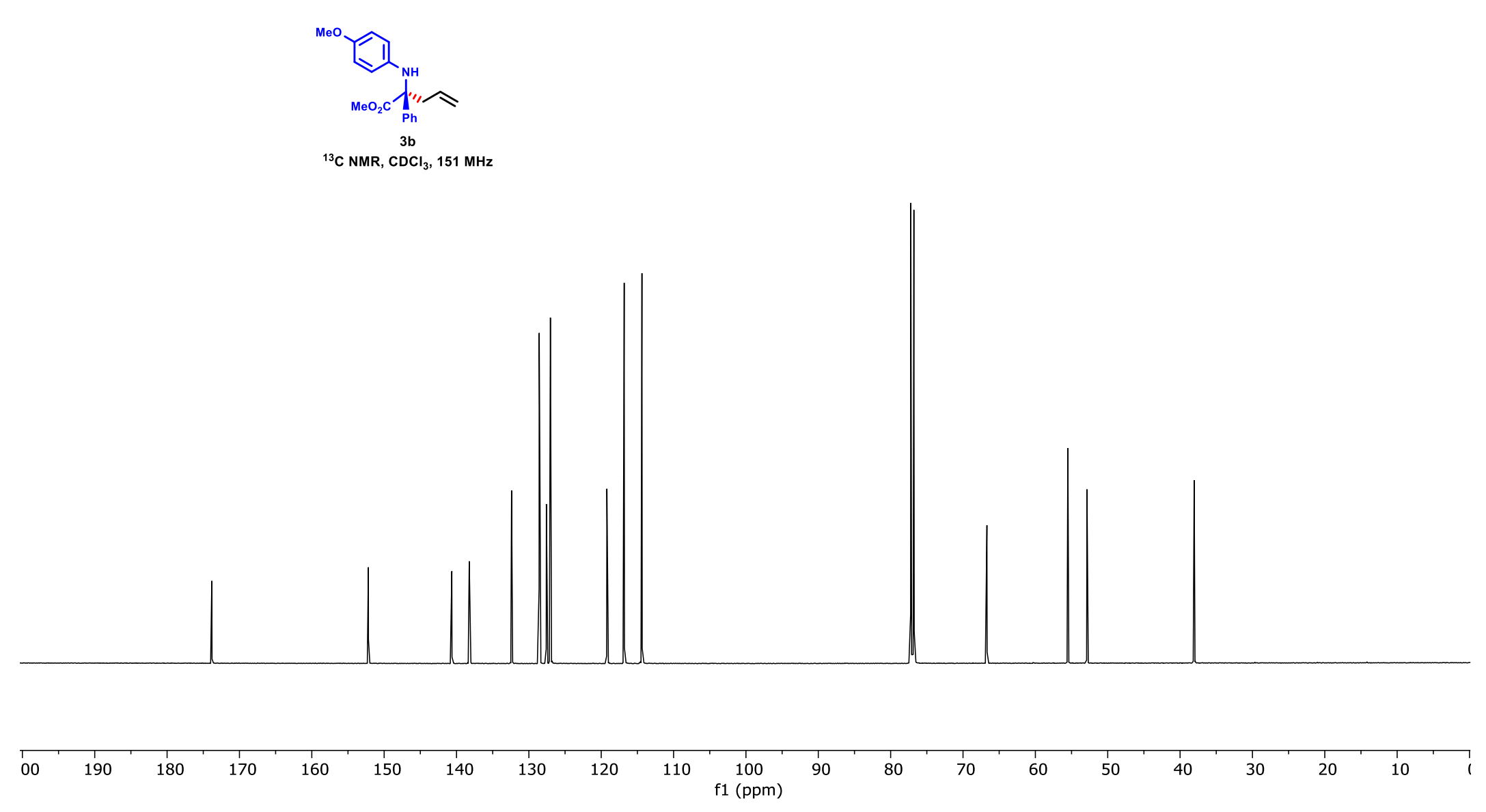




\begin{tabular}{|c|c|}
\hline 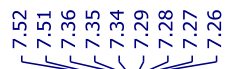 & 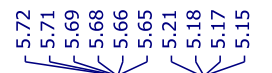 \\
\hline
\end{tabular}

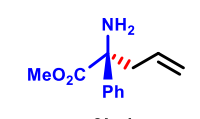

${ }^{1} \mathrm{H} \mathrm{NMR}, \mathrm{CDCl}_{3}, 600 \mathrm{MHz}$

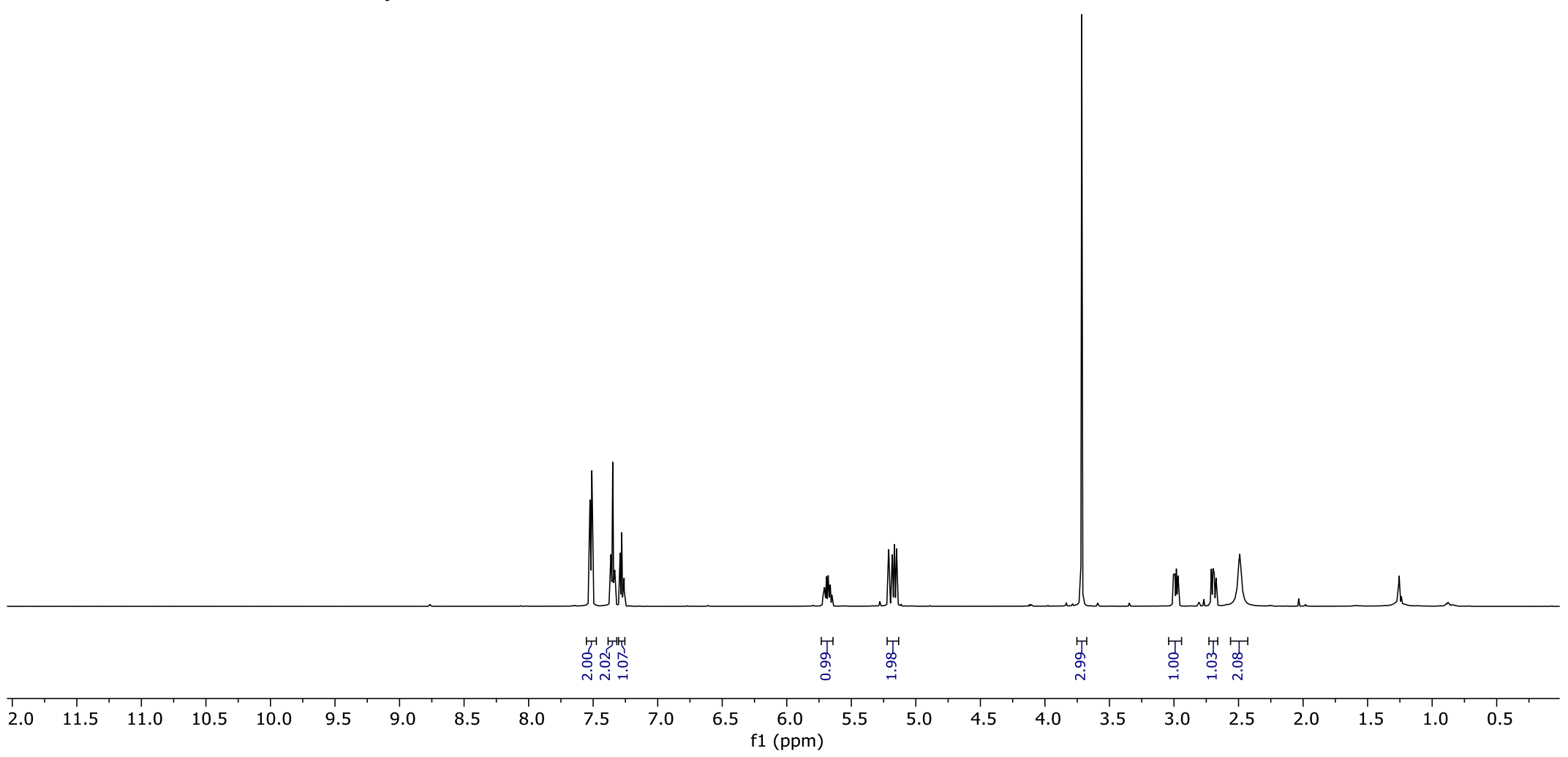




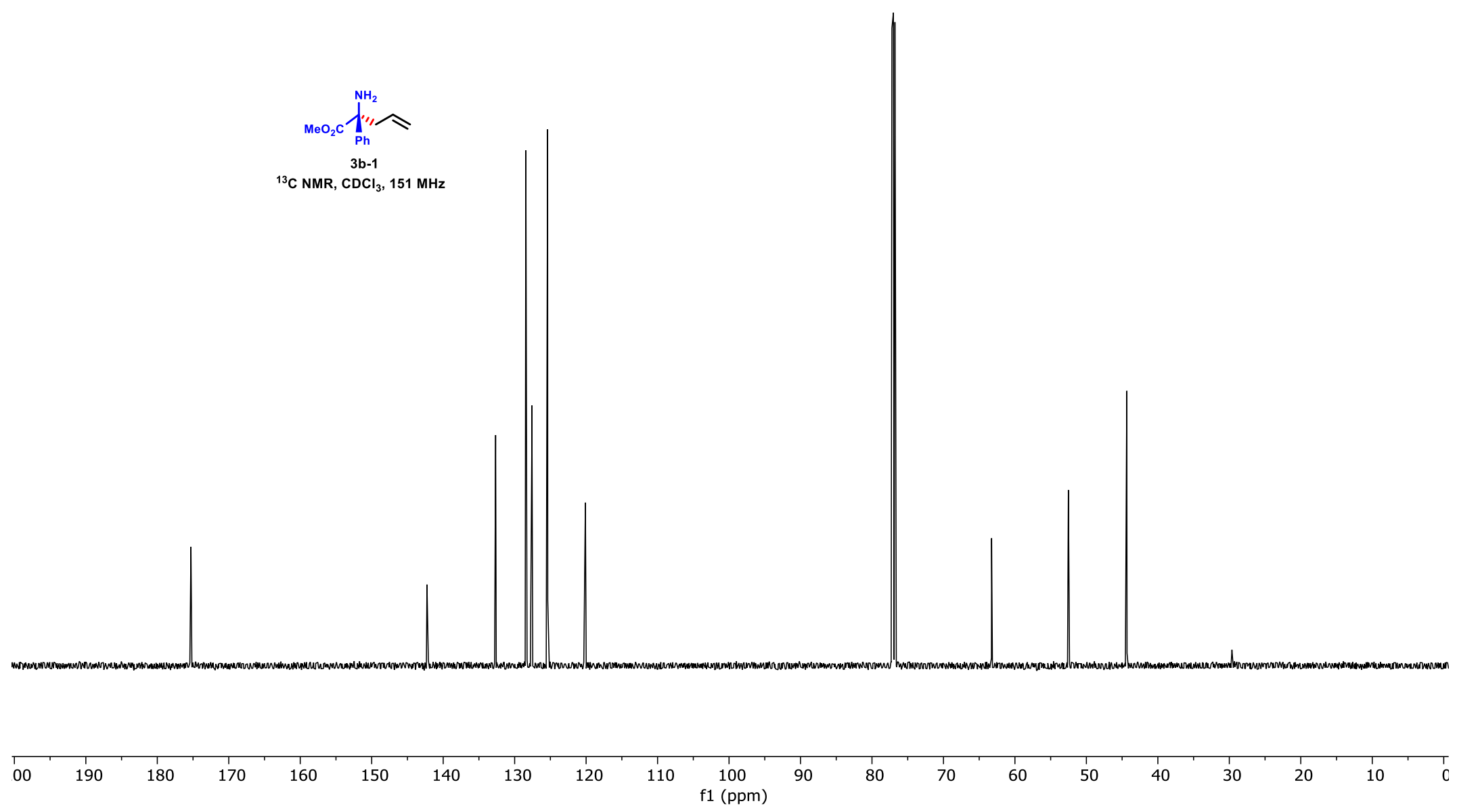




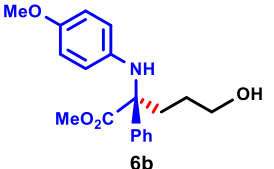

${ }^{1} \mathrm{H} \mathrm{NMR}, \mathrm{CDCl}_{3}, 600 \mathrm{MHz}$

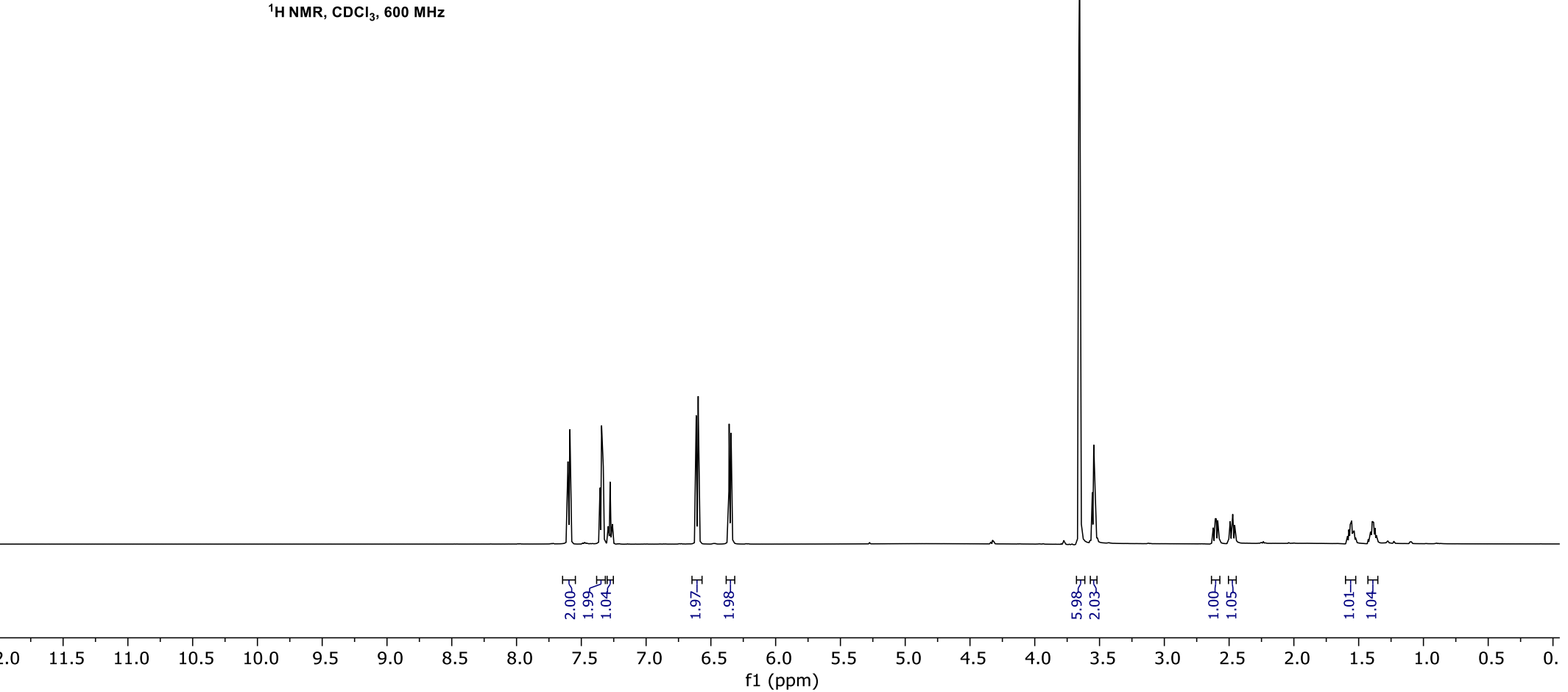



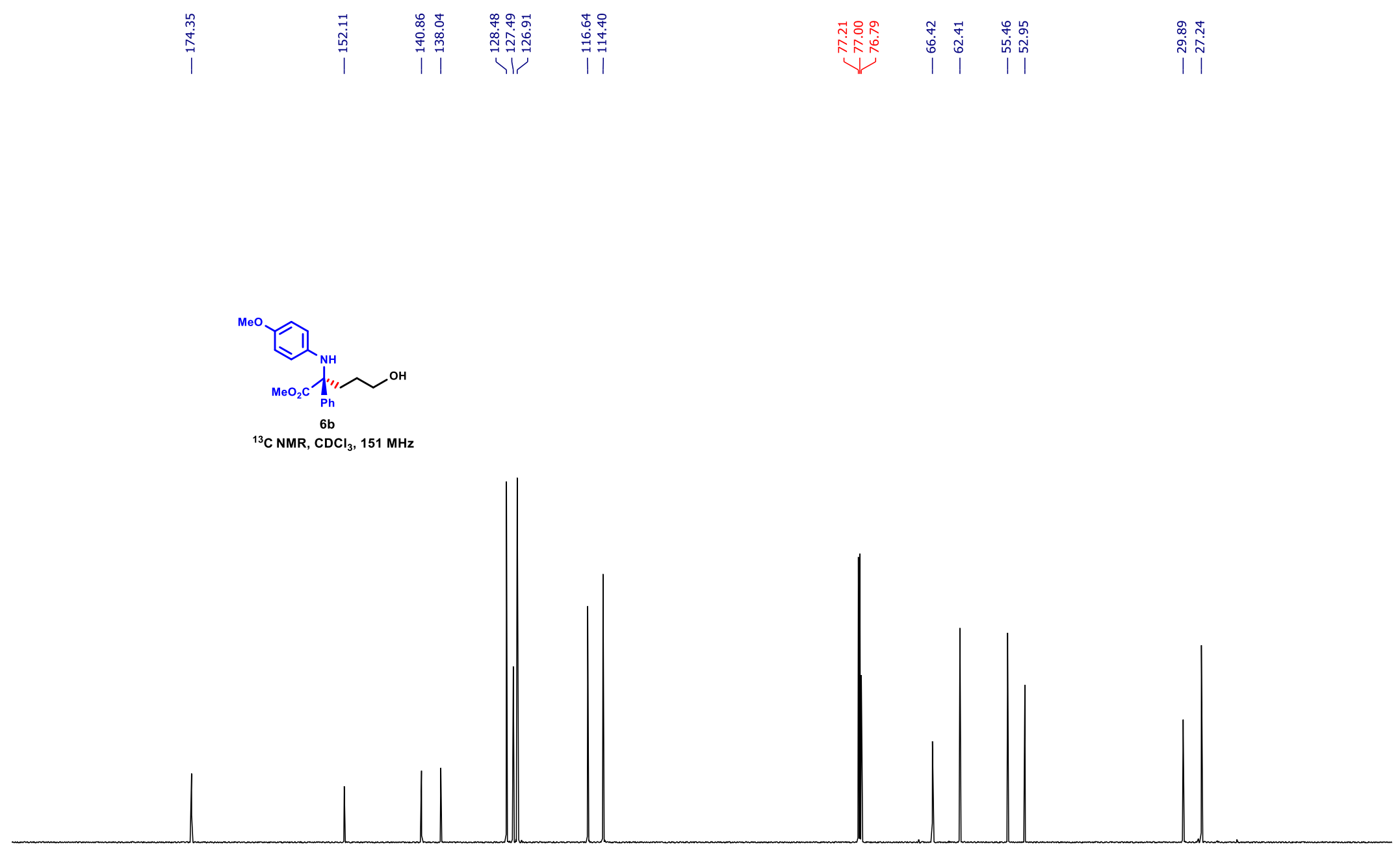

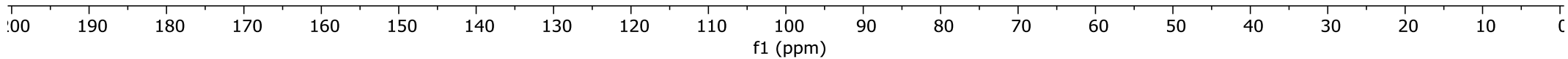




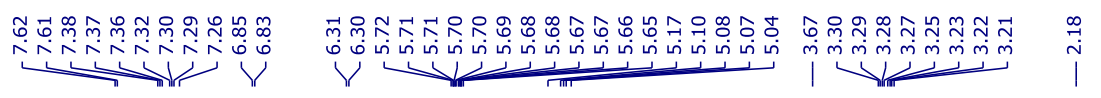

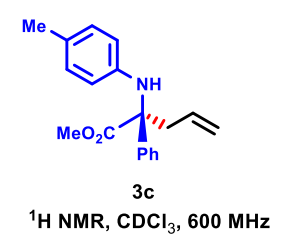

${ }^{1} \mathrm{H} \mathrm{NMR}, \mathrm{CDCl}_{3}, 600 \mathrm{MHz}$

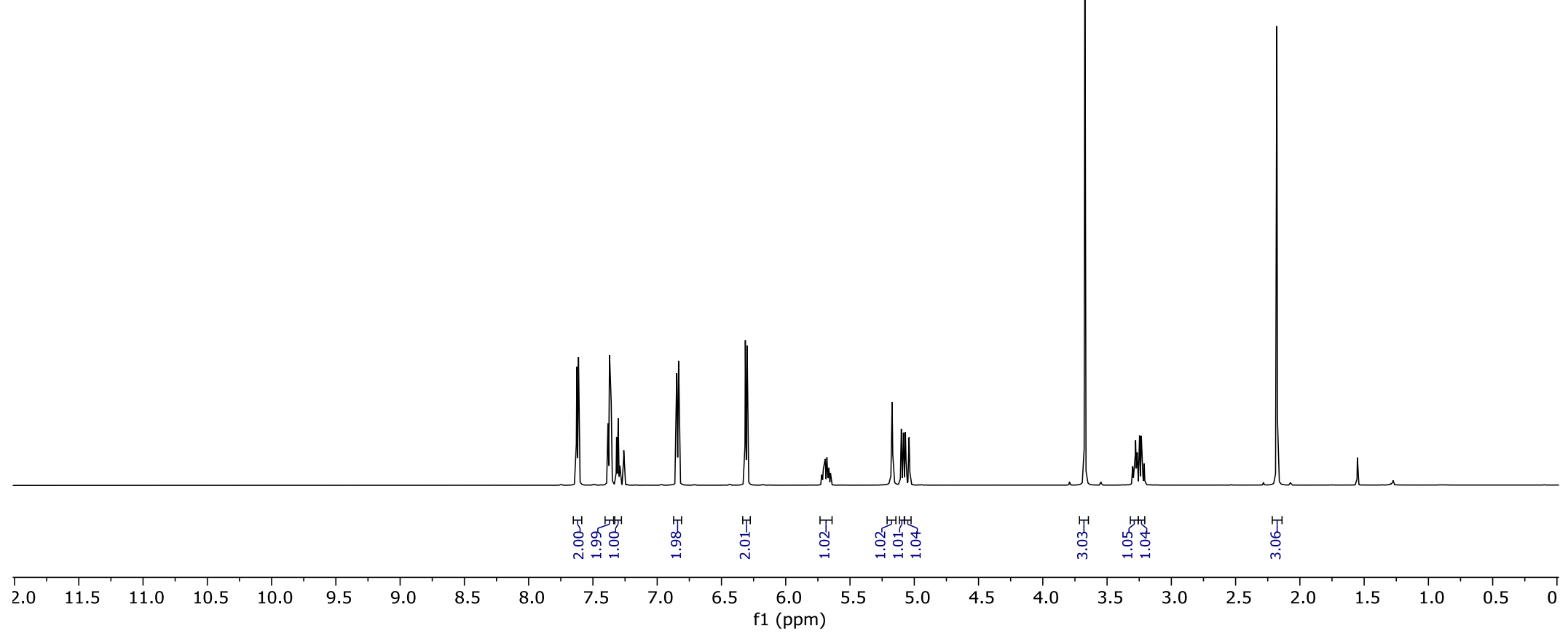




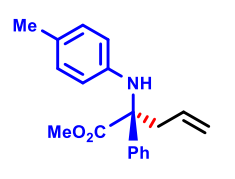

${ }^{13} \mathrm{C} \mathrm{NMR,},{ }^{3 c}$

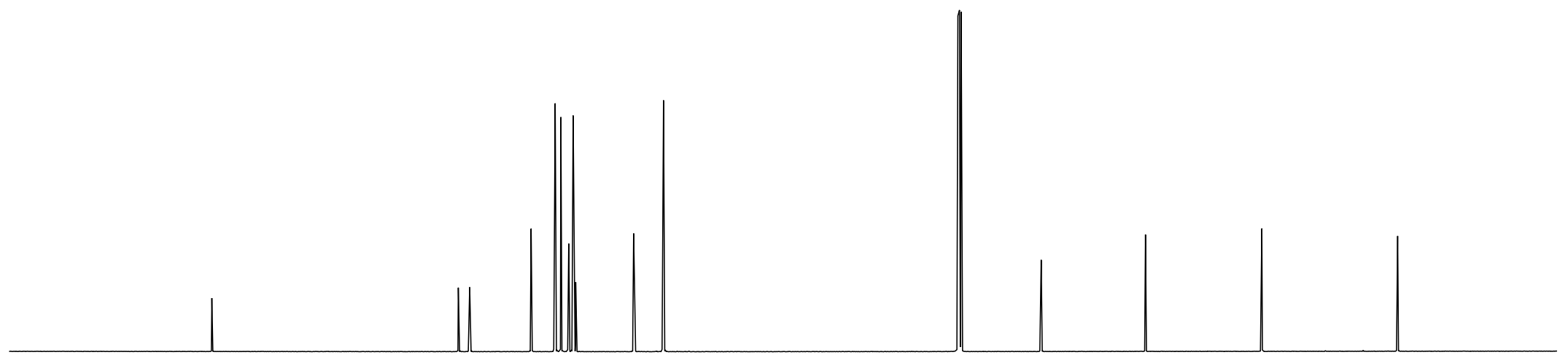

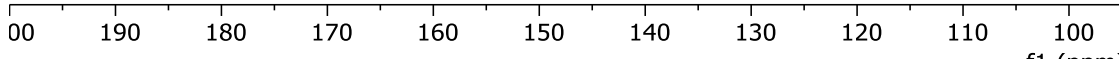
f1 (ppm)

80

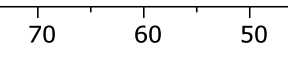

40

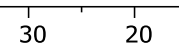

$10 \quad($ 


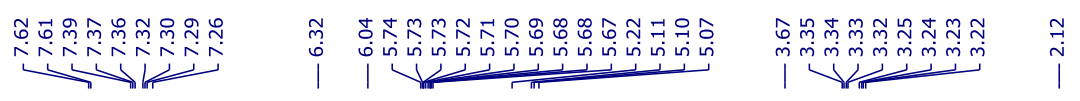

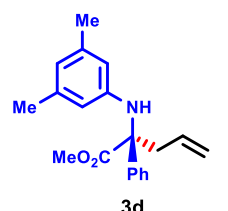

${ }^{1} \mathrm{H} \mathrm{NMR,} \mathrm{CDCl}_{3}, 600 \mathrm{MHz}$

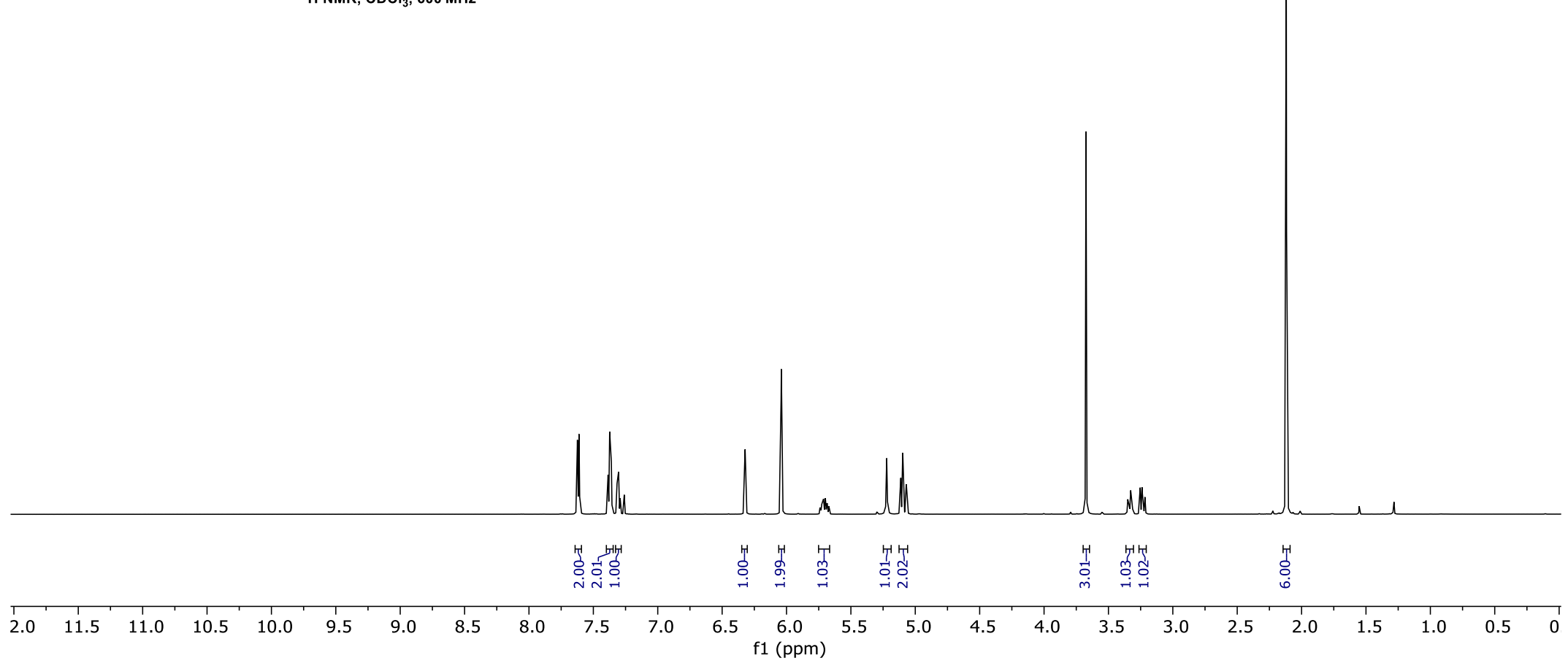



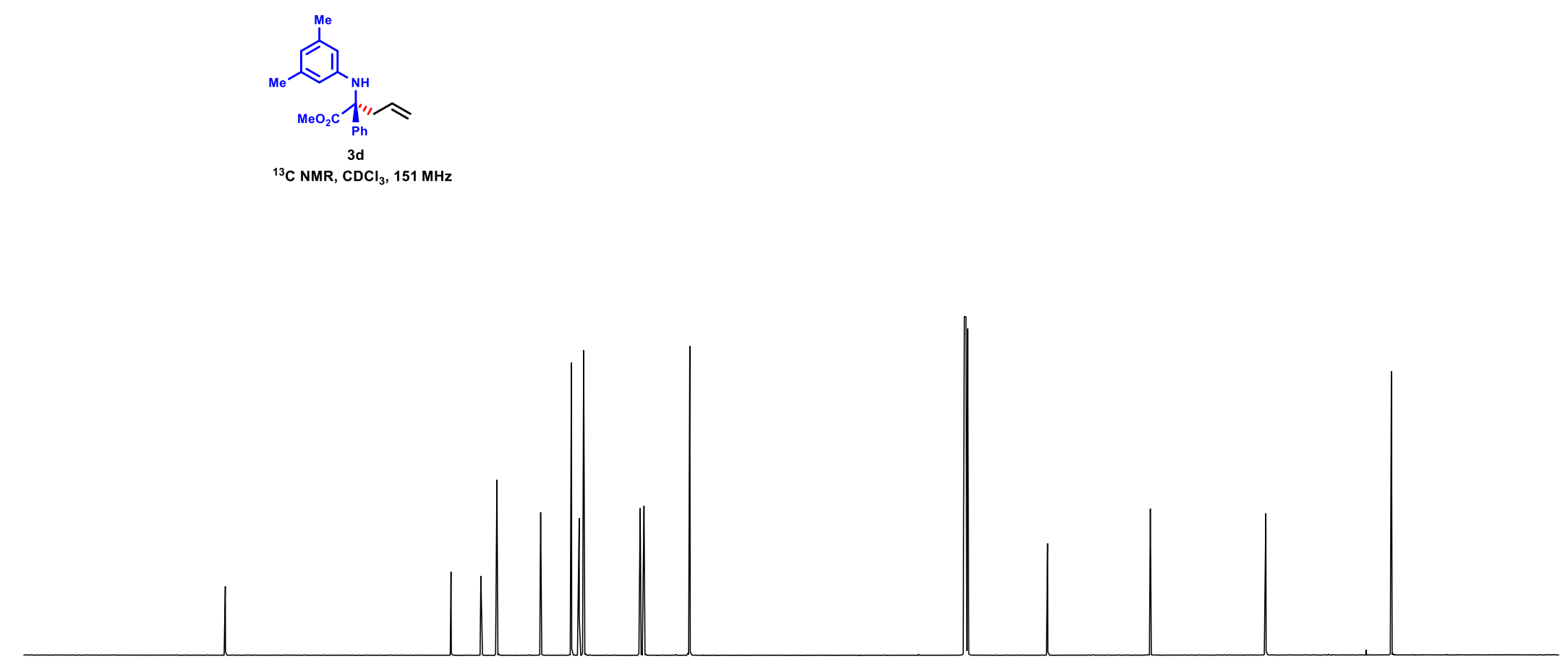

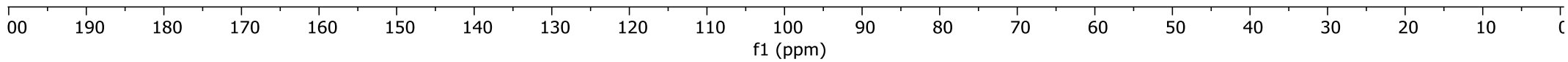




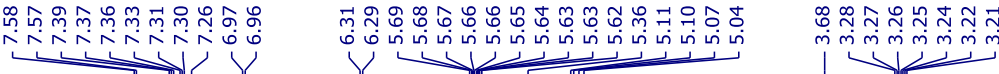

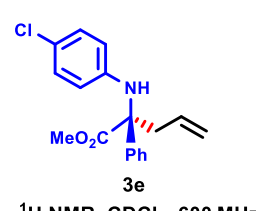

${ }^{1} \mathrm{H} \mathrm{NMR}, \mathrm{CDCl}_{3}, 600 \mathrm{MHz}$

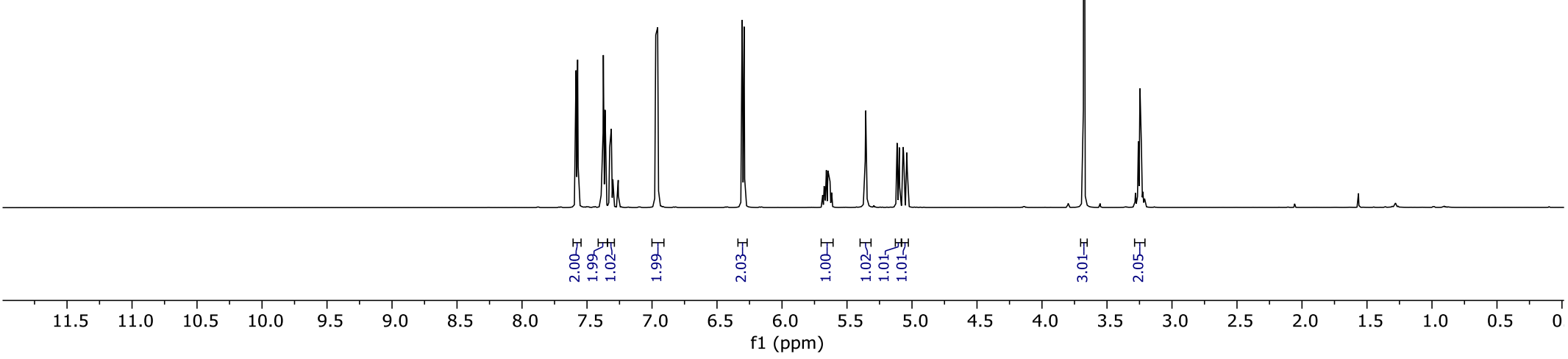


党

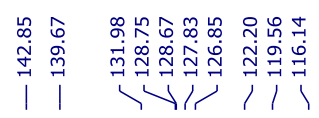

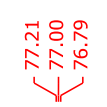

$\underset{\substack{0 \\ 0}}{1}$

ํㅗำ

$\stackrel{\substack{0 \\ \text { i }}}{n}$

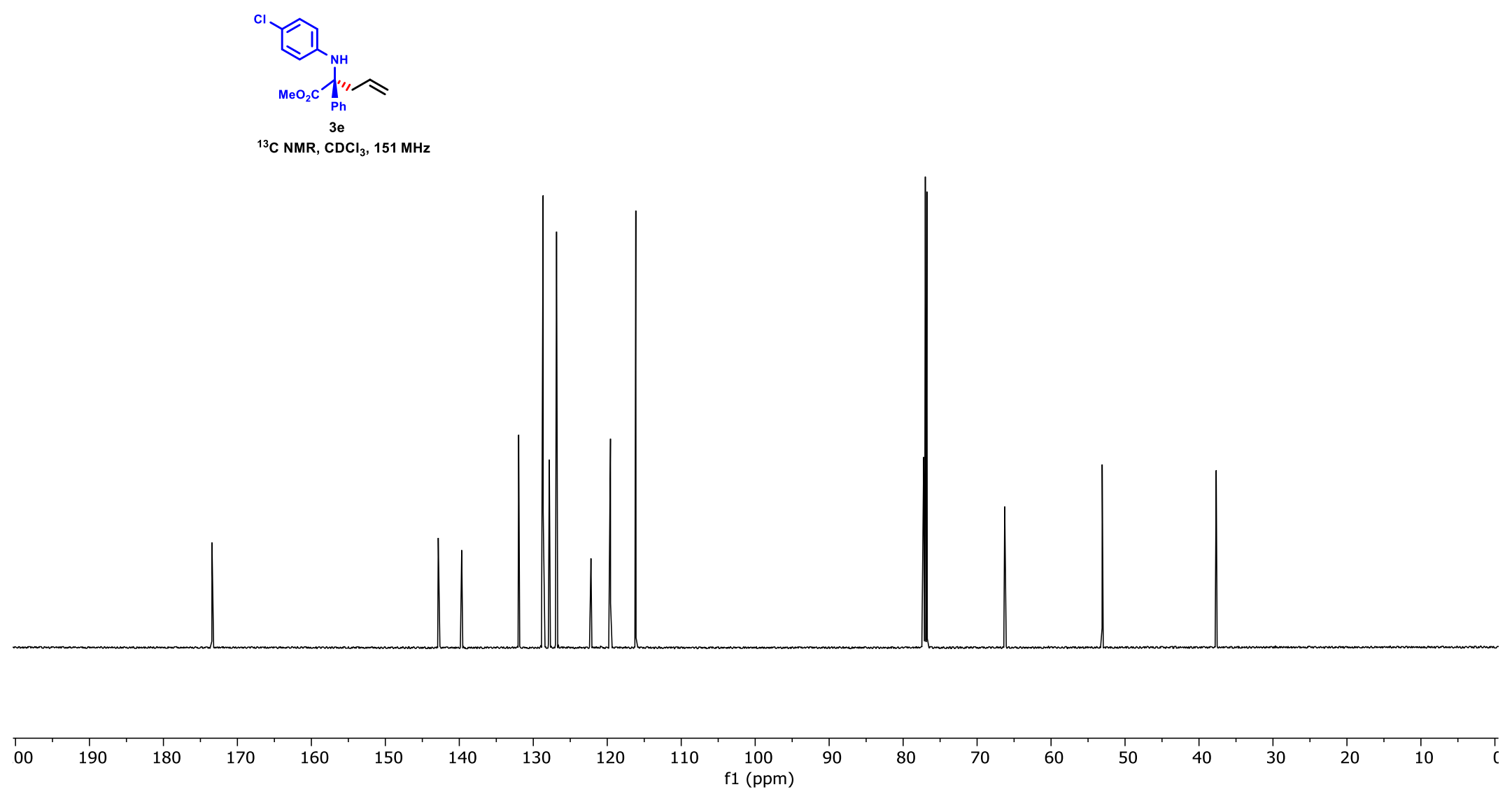

S102 


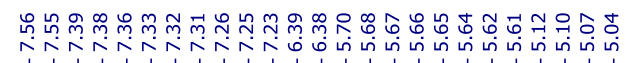

资

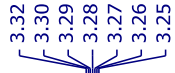

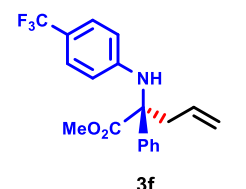

${ }^{1} \mathrm{H} \mathrm{NMR,} \mathrm{CDCl}_{3}, 600 \mathrm{MHz}$

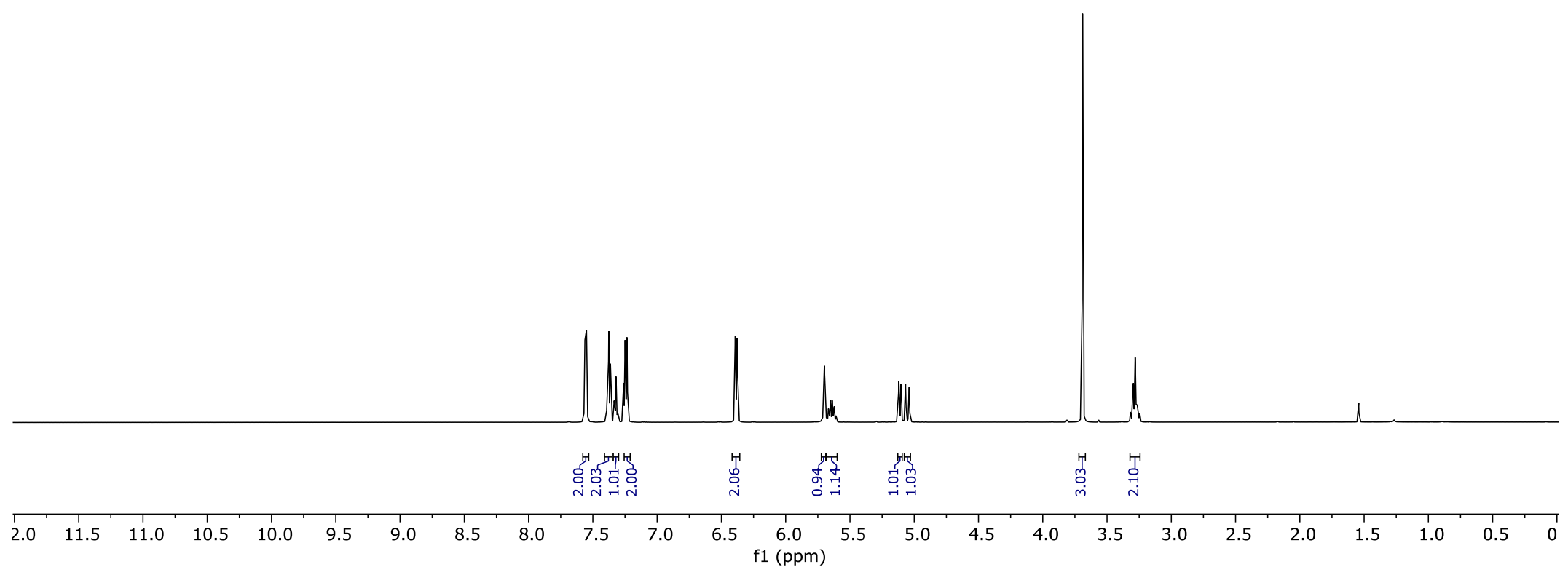




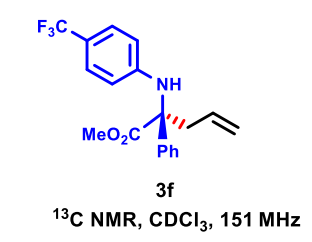

${ }^{13} \mathrm{C} \mathrm{NMR,} \mathrm{CDCl}_{3}, 151 \mathrm{MHz}$

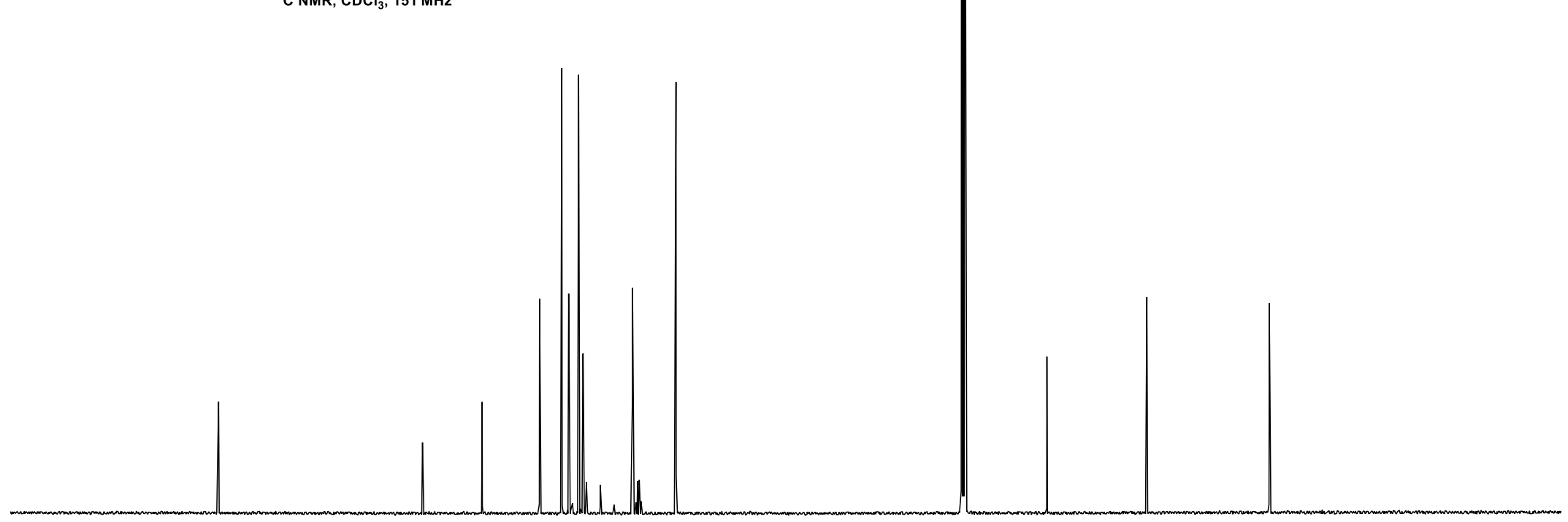

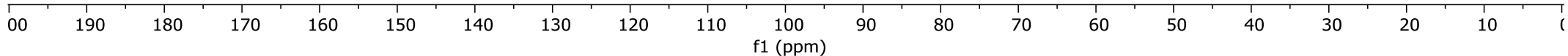


울웅

पें

ن

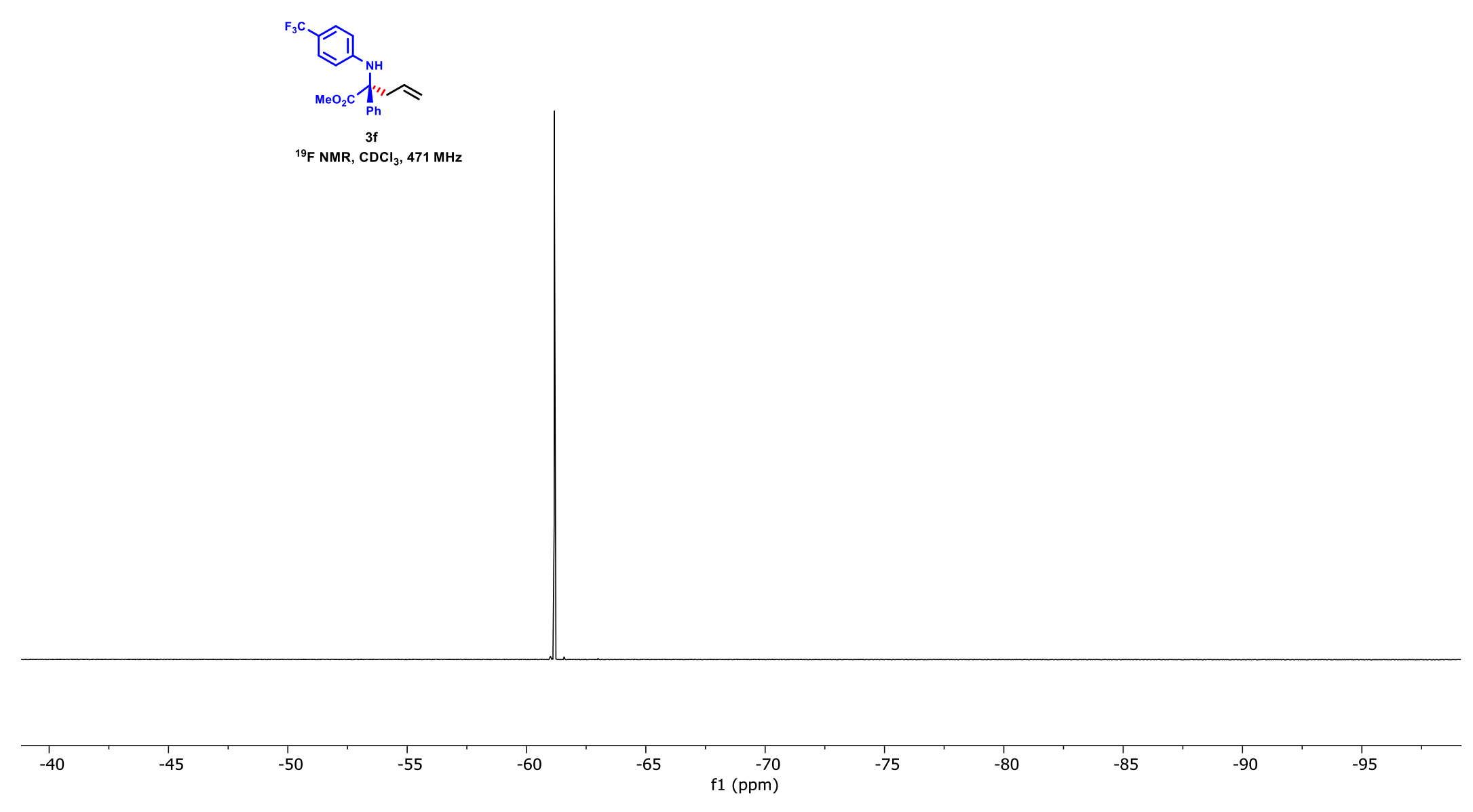




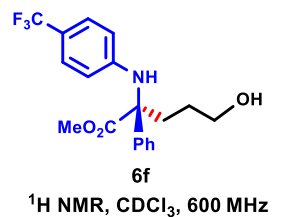

${ }^{1} \mathrm{H} \mathrm{NMR}, \mathrm{CDCl}_{3}, 600 \mathrm{MHz}$

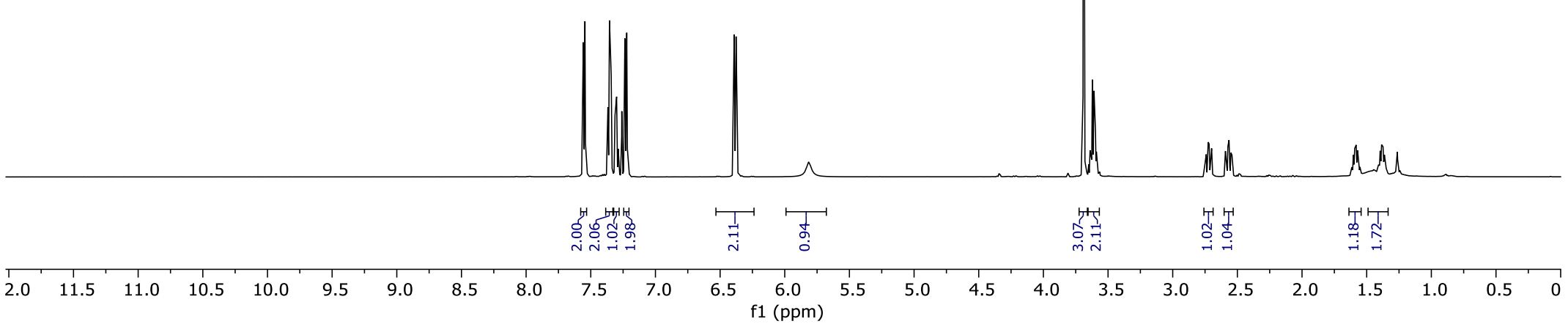



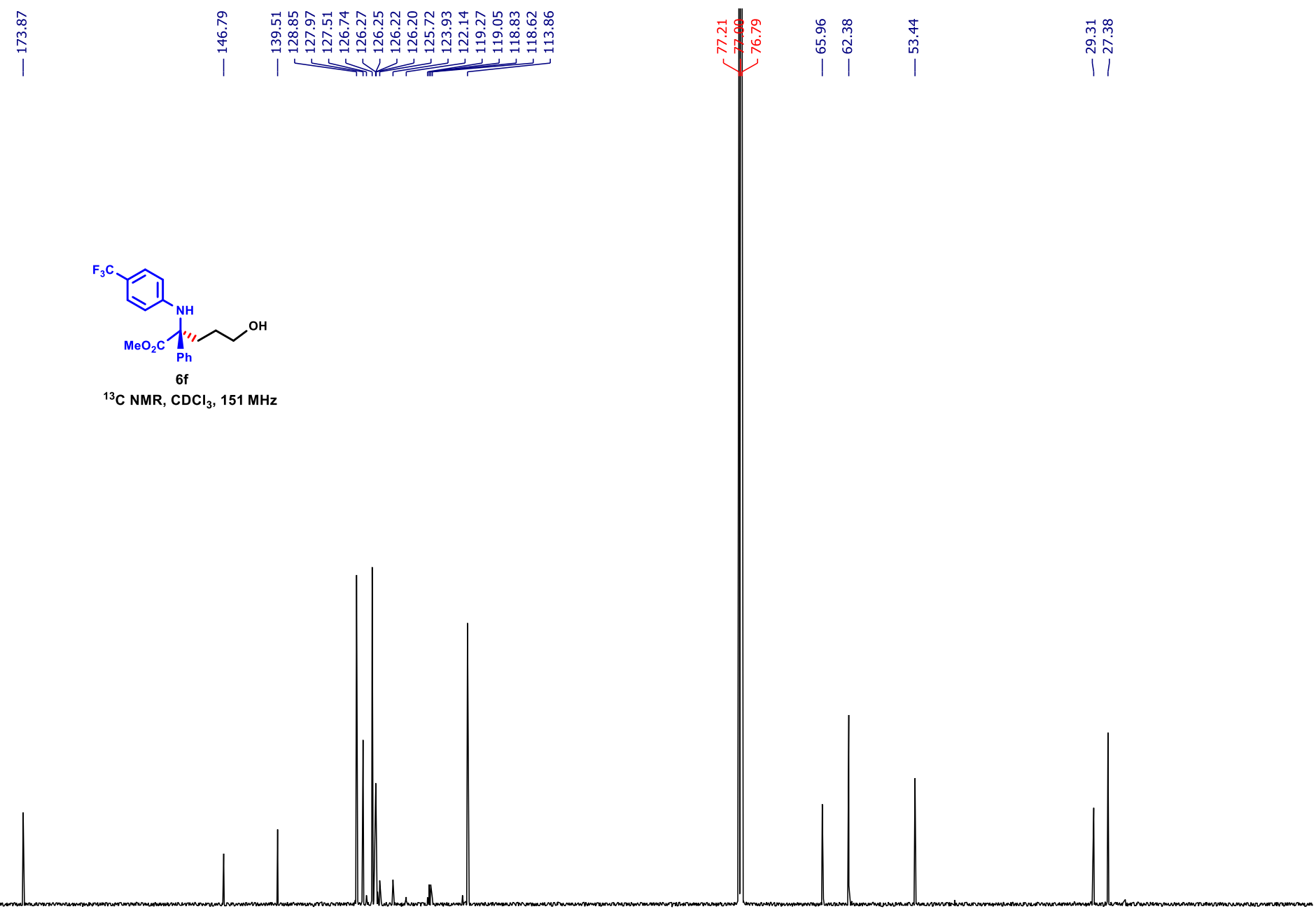

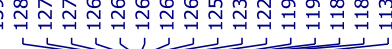

${ }^{13} \mathrm{C} \mathrm{NMR}, \mathrm{CDCl}_{3}, 151 \mathrm{MHz}$

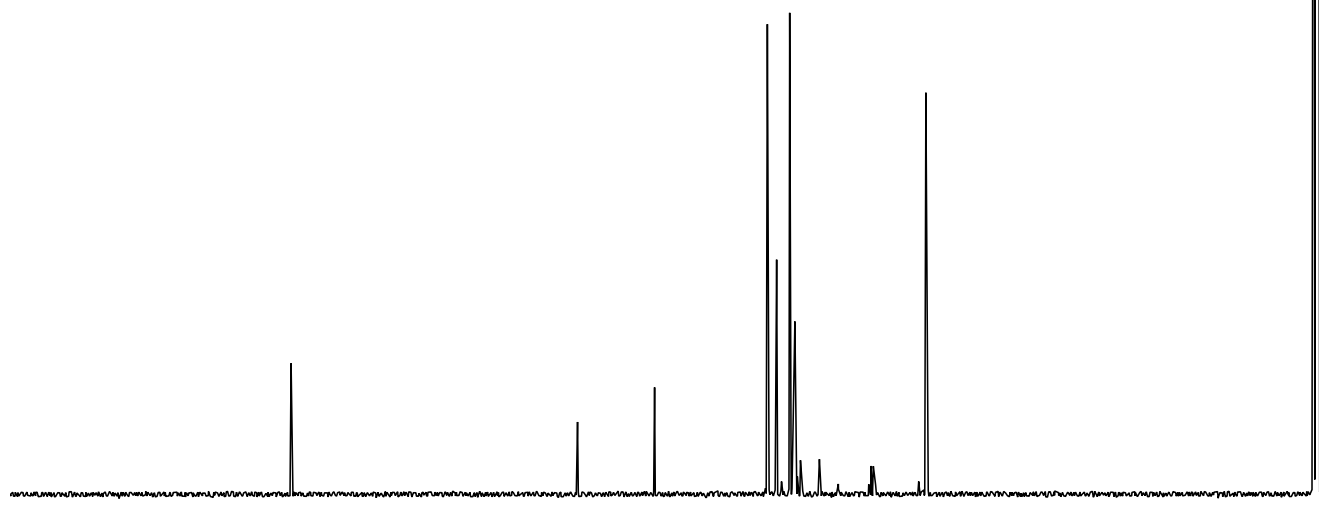

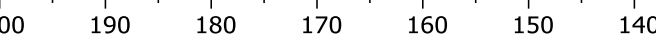

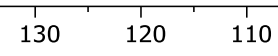

100
f1 $(p p m)$

$\begin{array}{llll}90 & 1 & 1 & 1 \\ & 80 & 70 & 60\end{array}$

50

40

$30 \quad 20 \quad 10$ 
$m m$

نे

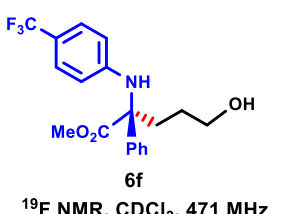

${ }^{19} \mathrm{~F} \mathrm{NMR,} \mathrm{CDCl}_{3}, 471 \mathrm{MHz}$ 


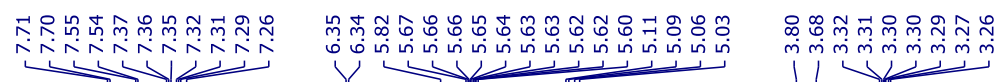

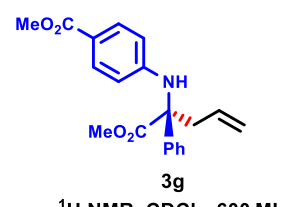

${ }^{1} \mathrm{H} \mathrm{NMR}, \mathrm{CDCl}_{3}, 600 \mathrm{MHz}$

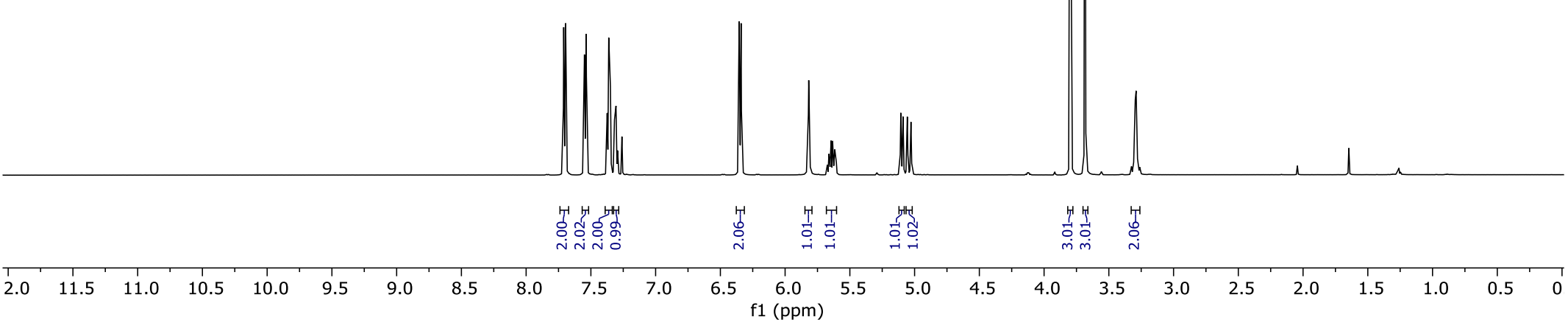




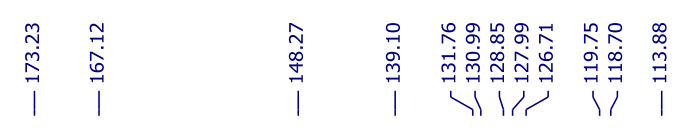

$\underbrace{5}_{\substack{n \\ 8}}$

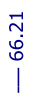

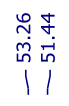

$\stackrel{\substack{n \\ m}}{1}$
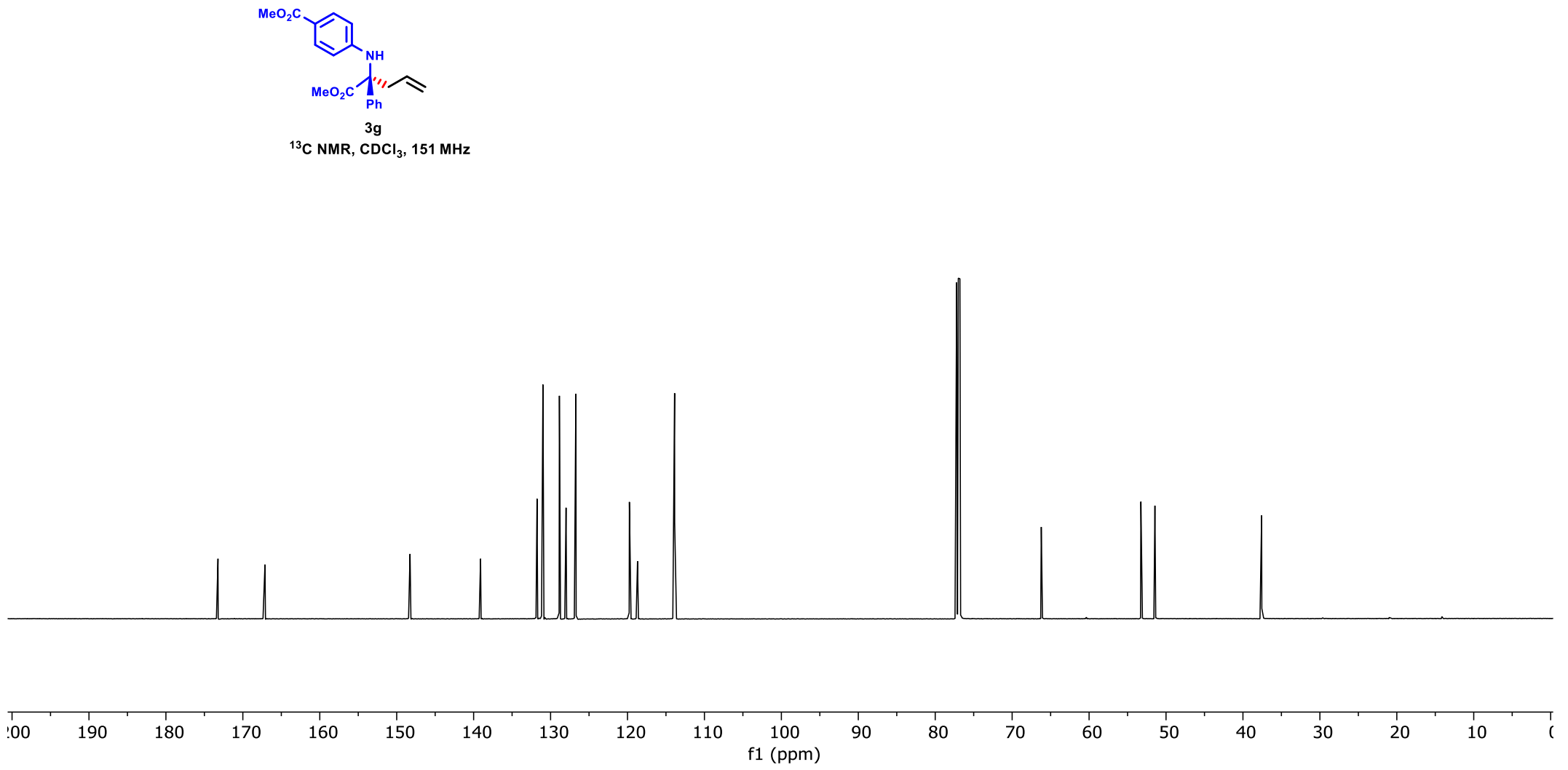

S110 


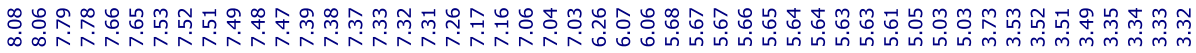

$1 Y \underbrace{2}$

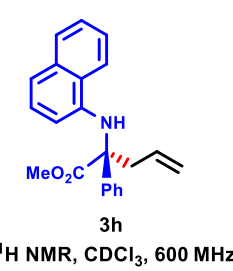

${ }^{1} \mathrm{H} \mathrm{NMR,}, \mathrm{CDCl}_{3}, 600 \mathrm{MHz}$

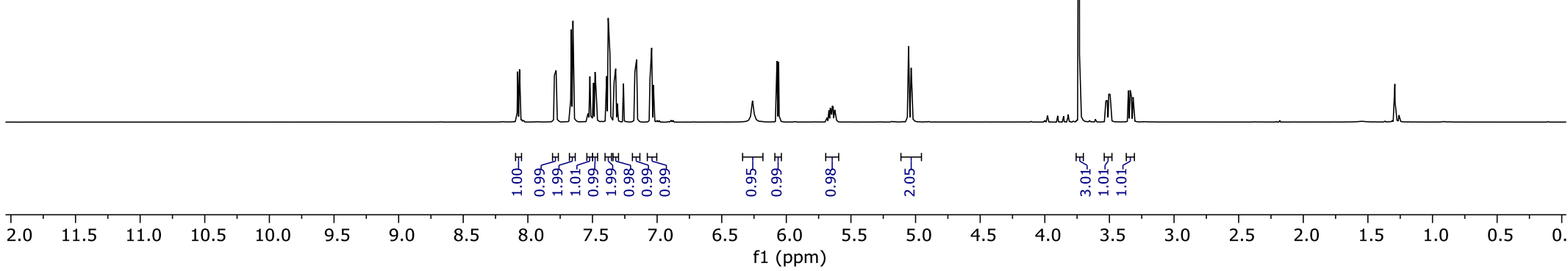


$\stackrel{1}{\stackrel{7}{7}}$

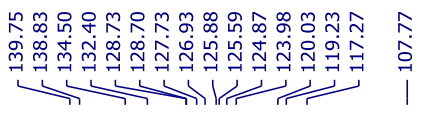

$\underbrace{n}$

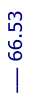

คิ

$\stackrel{\substack{m \\ m}}{n}$
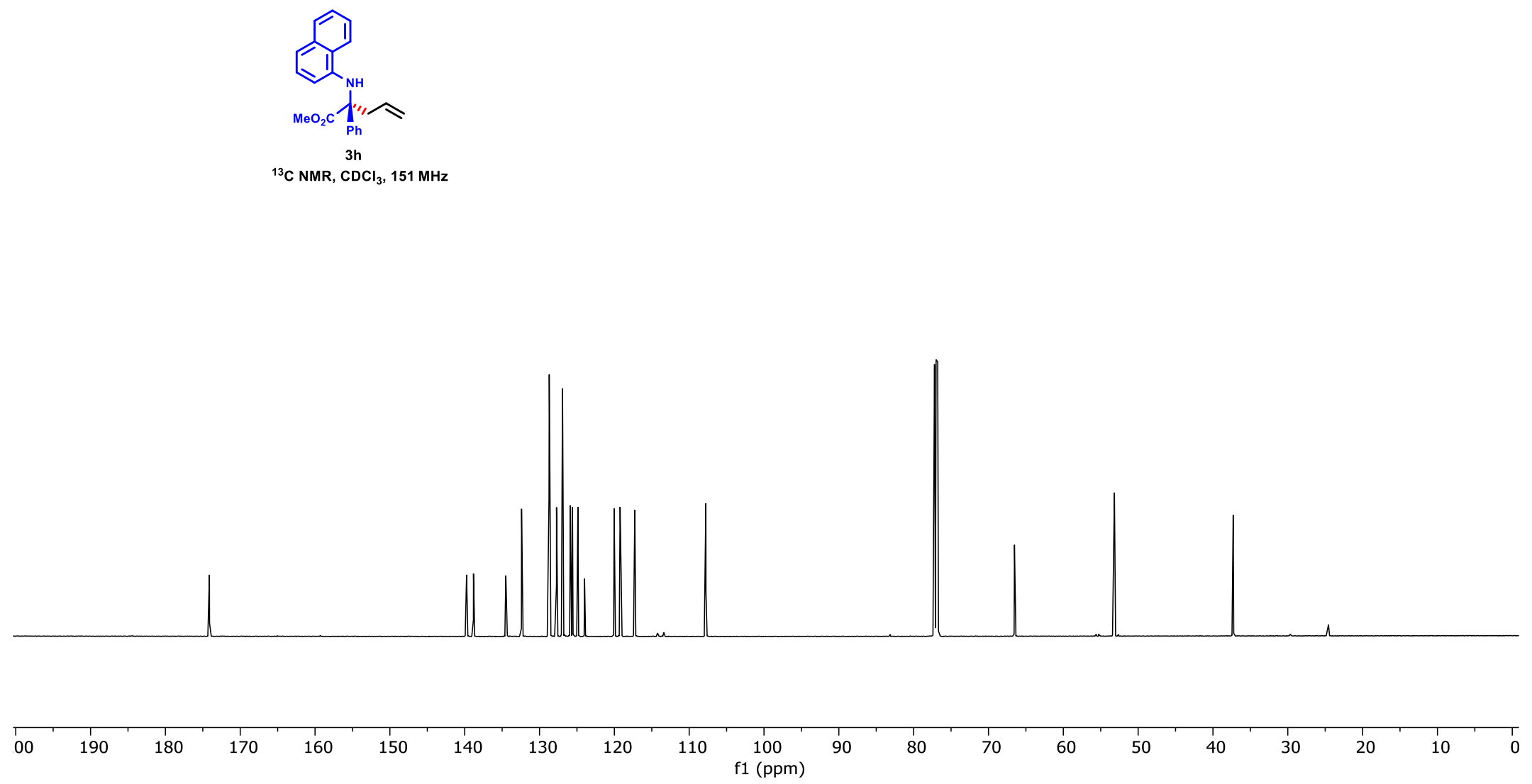

S112 

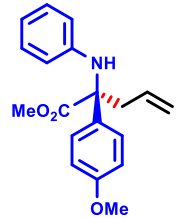

'H NMR, $\mathrm{CDCl}_{3}, 600 \mathrm{MHz}$

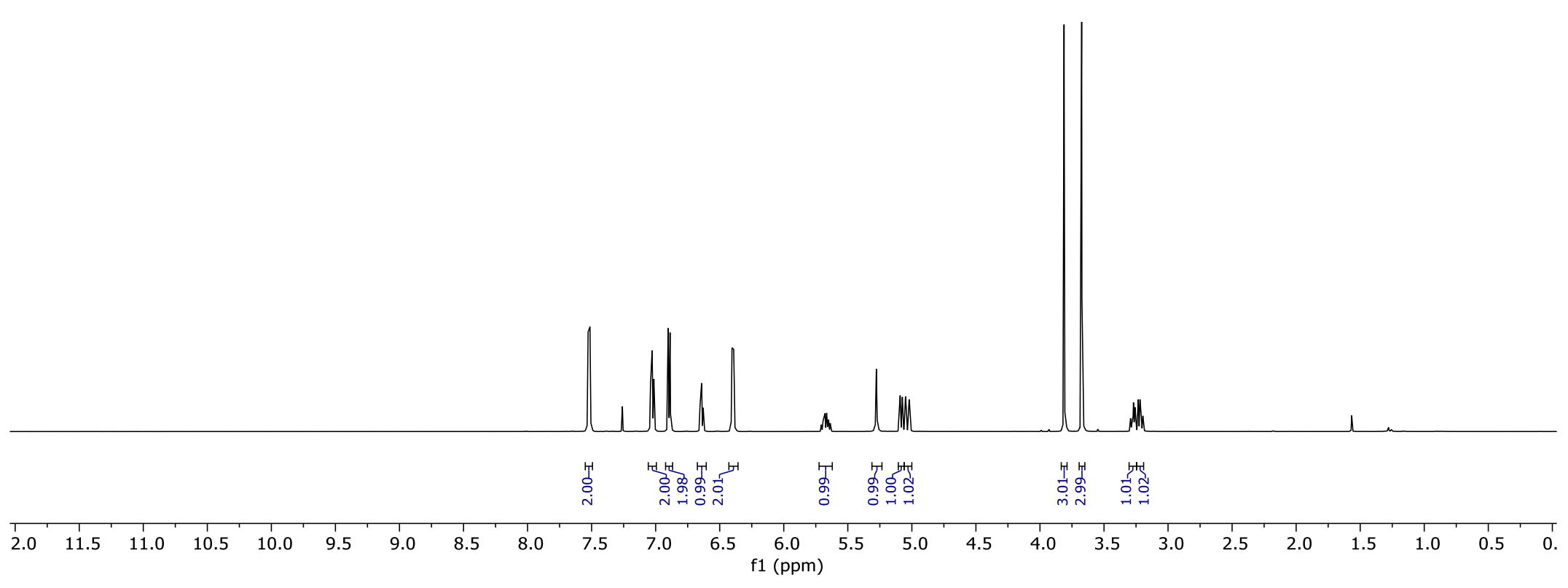




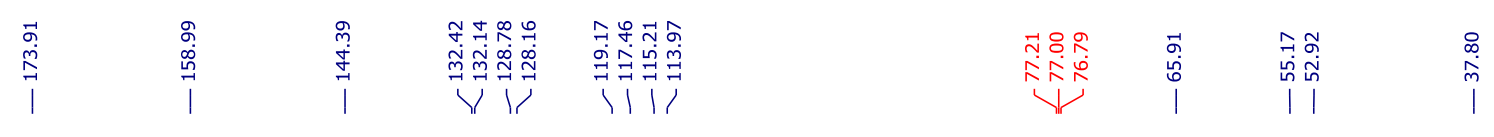

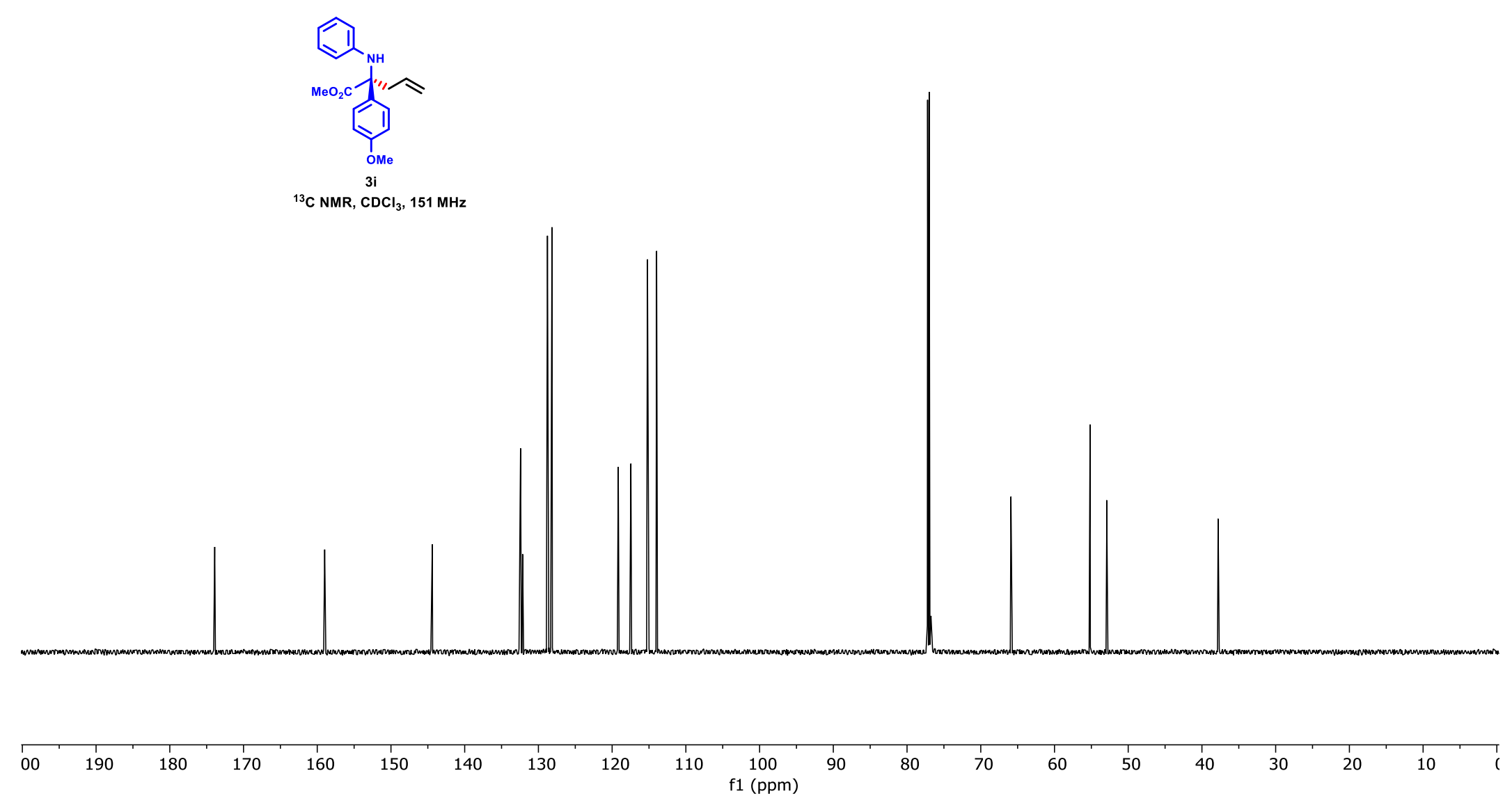



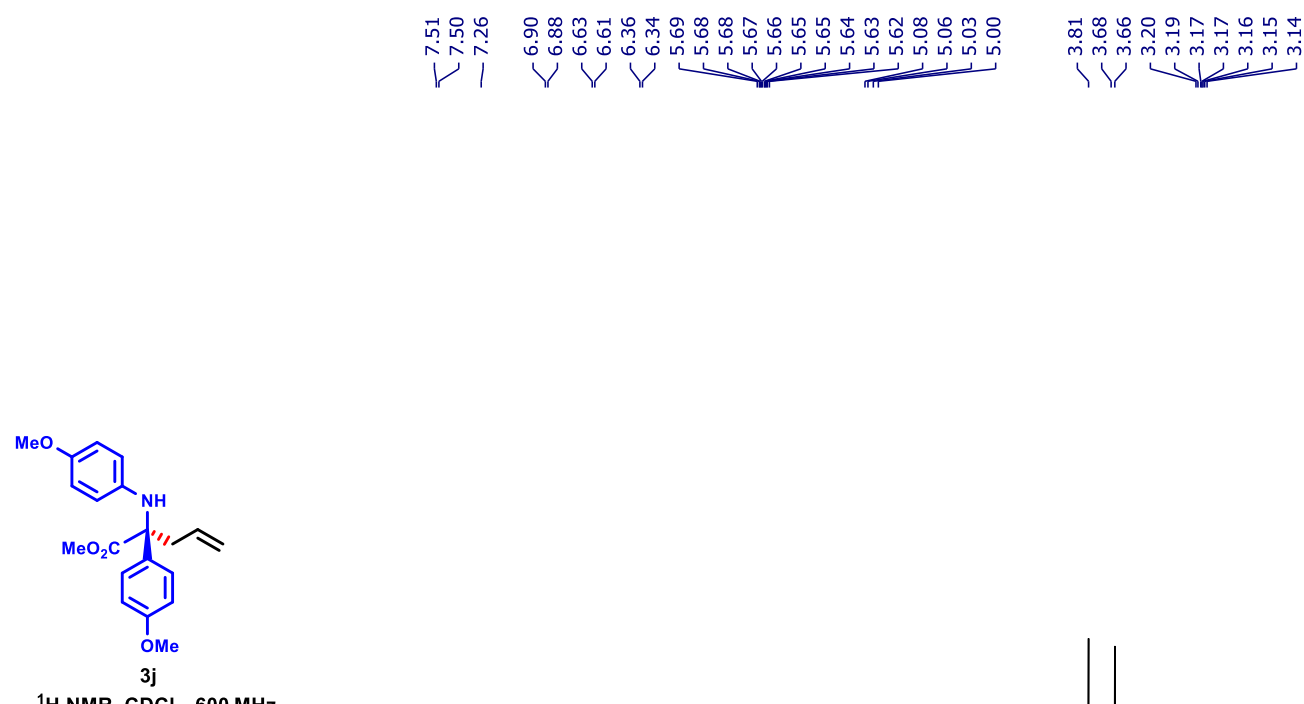

${ }^{1} \mathrm{H} \mathrm{NMR}, \mathrm{CDCl}_{3}, 600 \mathrm{MHz}$

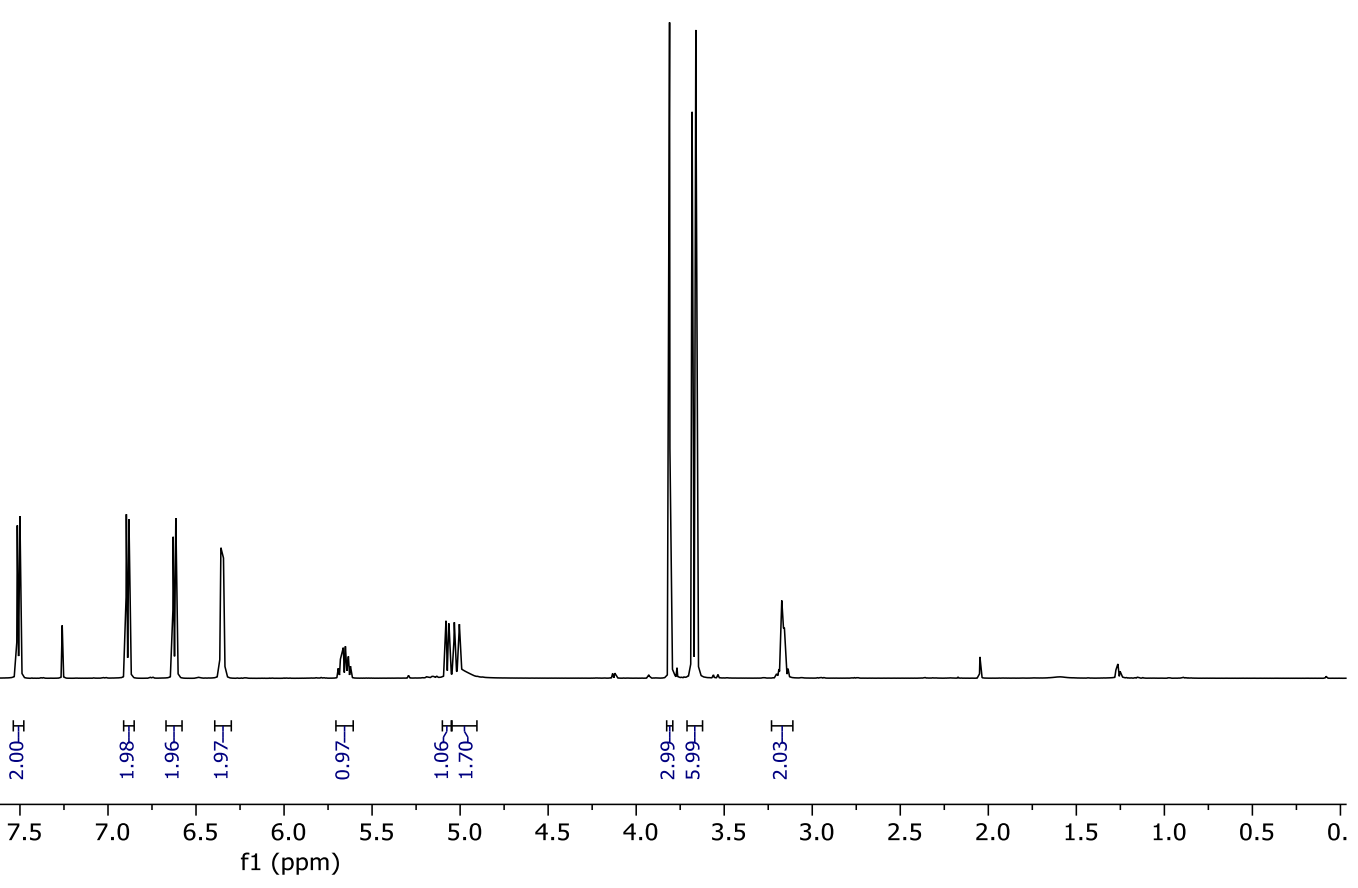

S115 


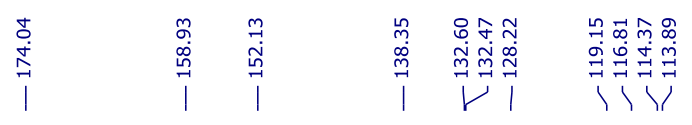

诲

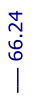

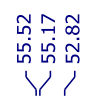

$\underset{\infty}{\infty}$
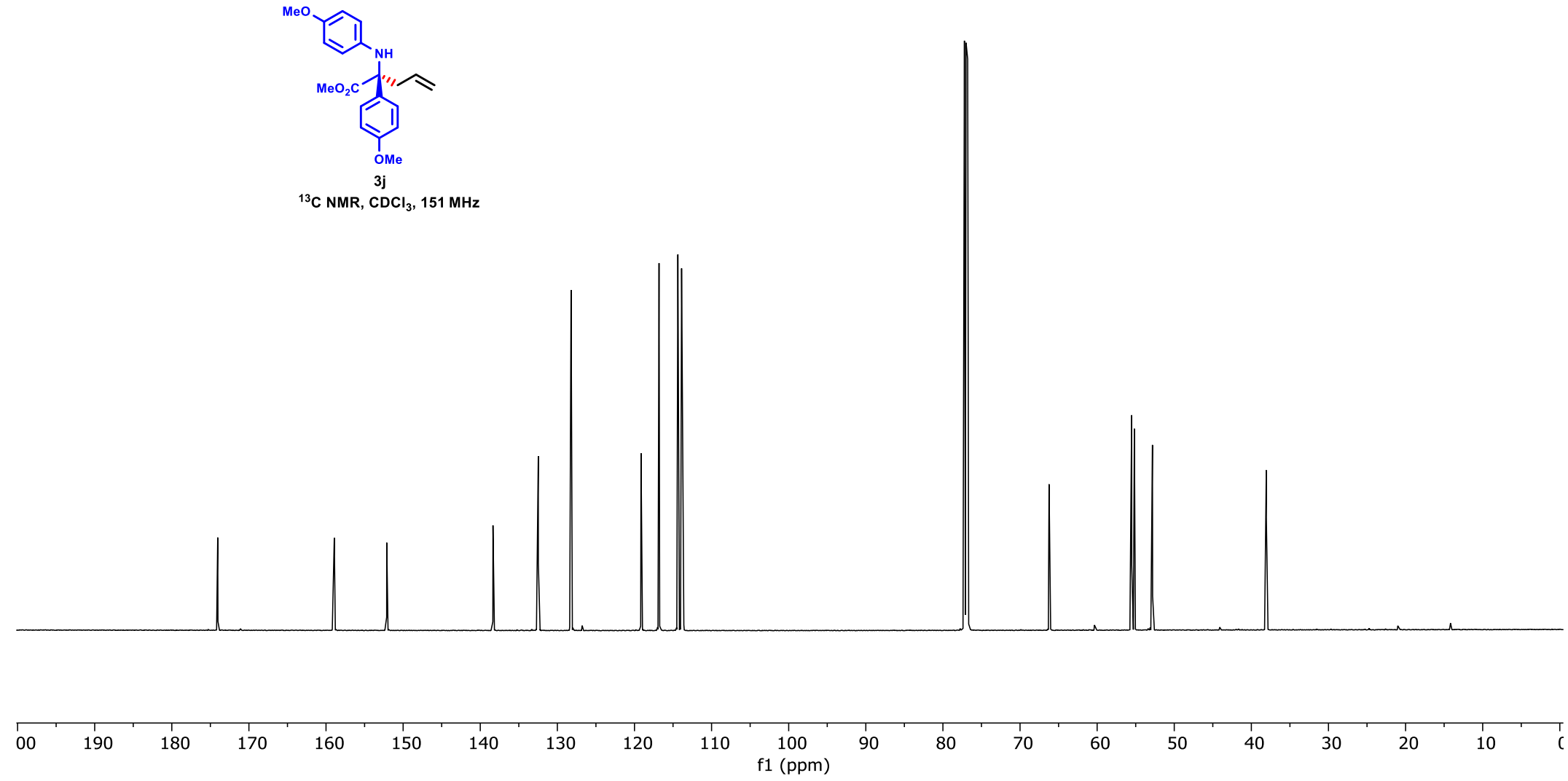

S116 


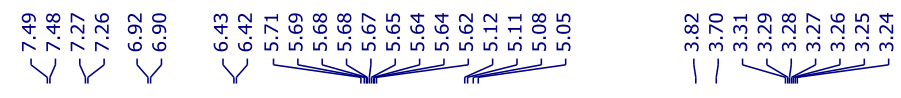

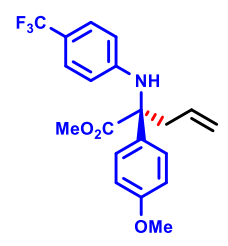

${ }^{1} \mathrm{H} \mathrm{NMR,} \mathrm{CDCl}_{3}, 600 \mathrm{MHz}$

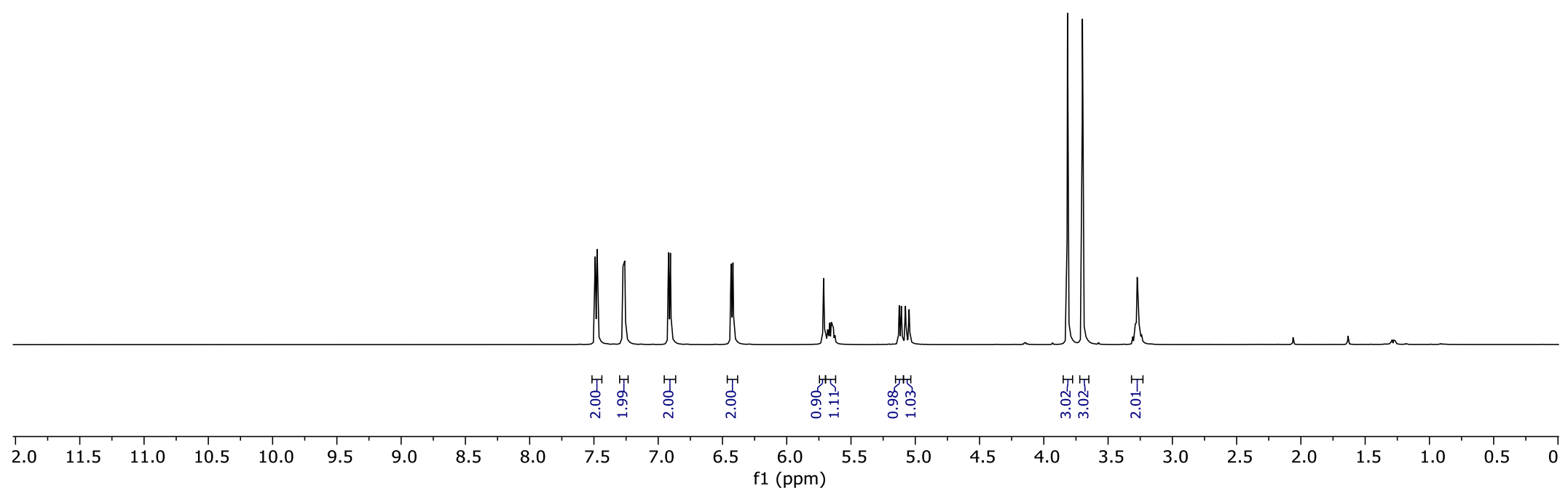



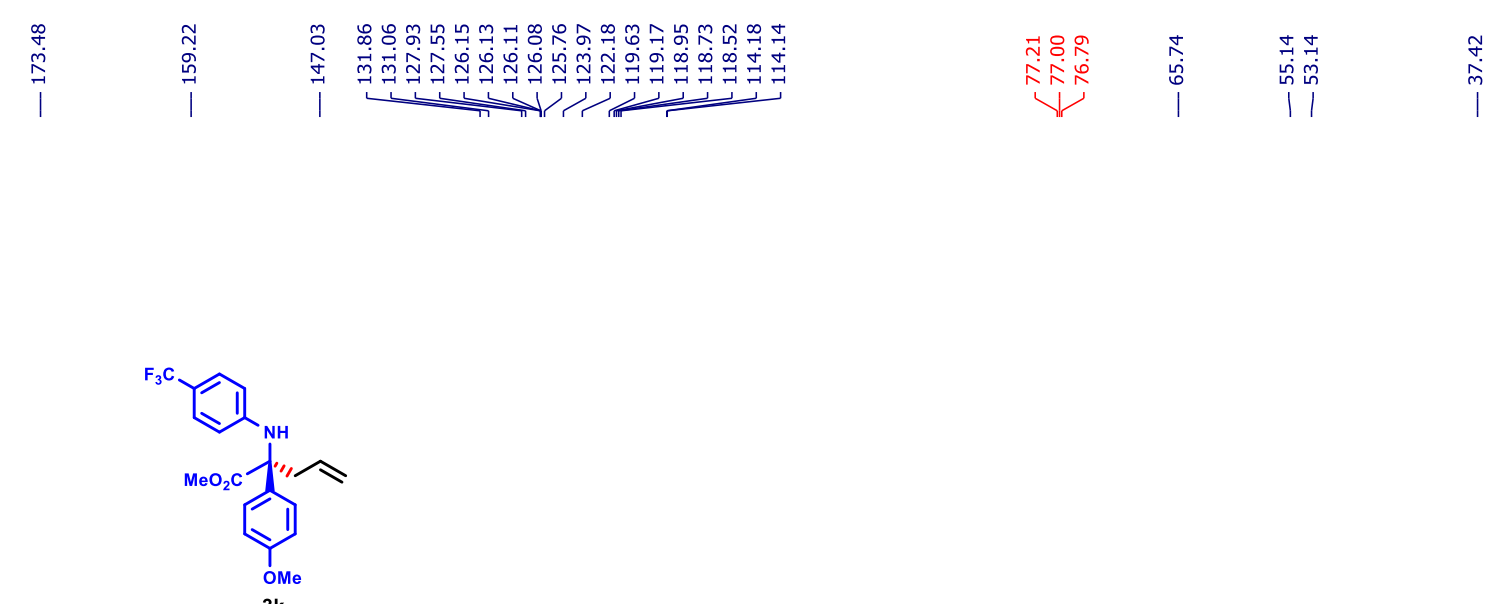

${ }^{13} \mathrm{C} \mathrm{NMR,} \mathrm{CDCl}_{3}, 151 \mathrm{MHz}$

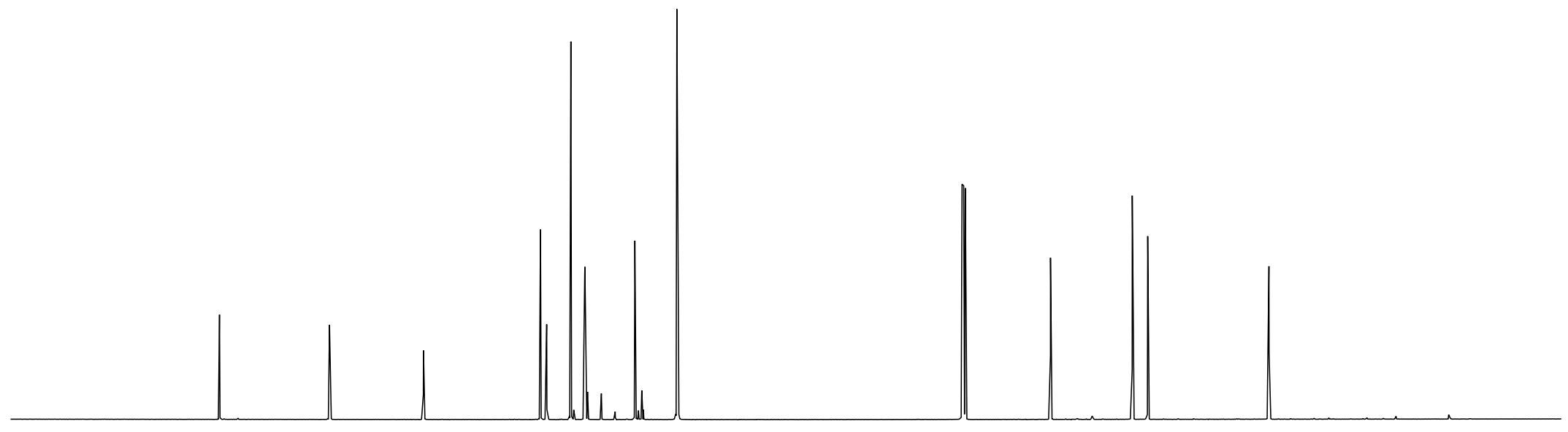

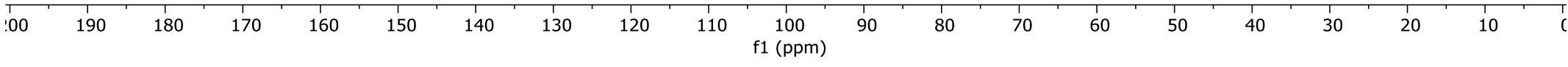




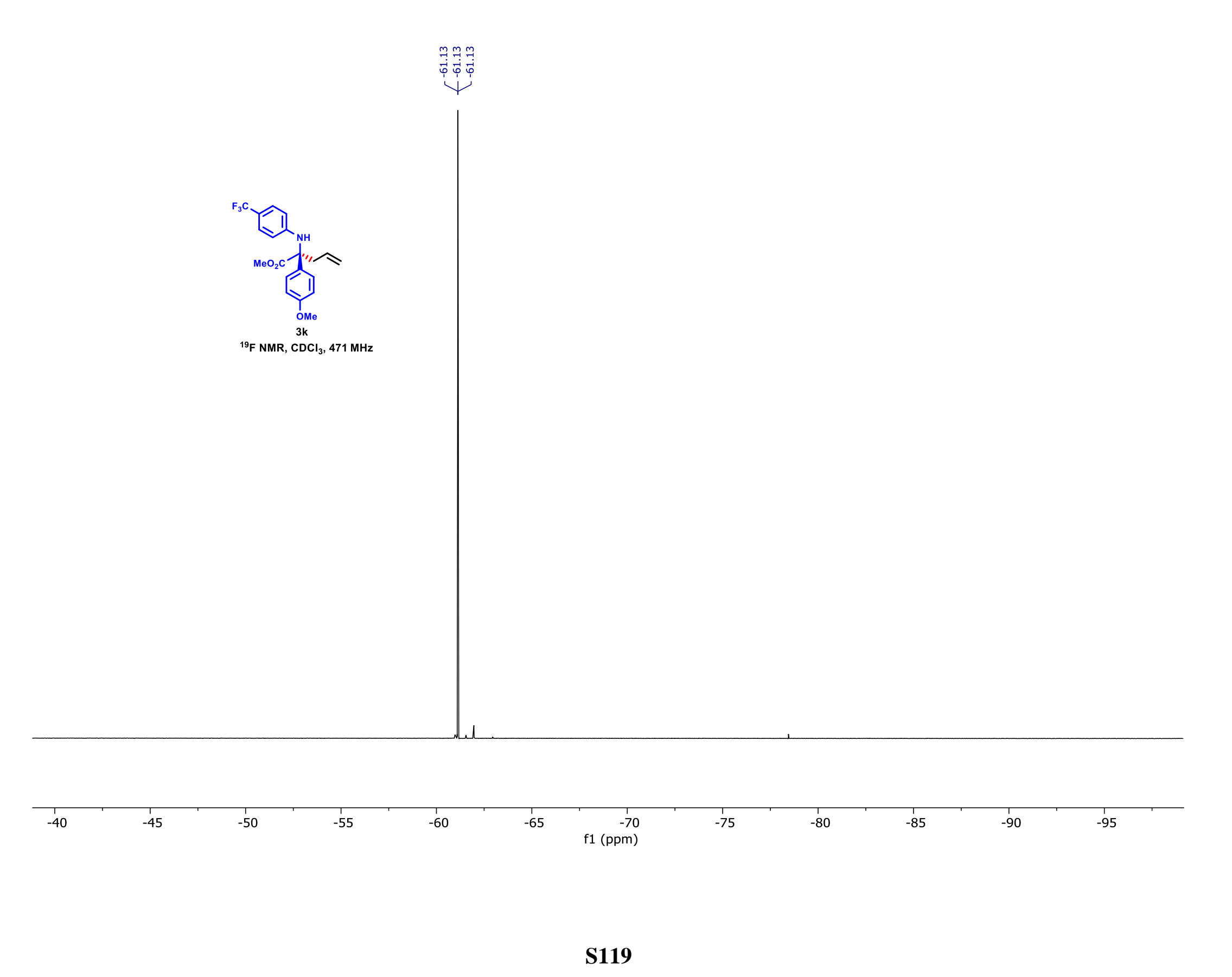




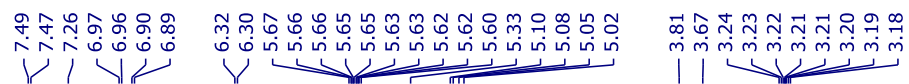

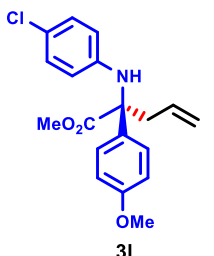

${ }^{1} \mathrm{H} \mathrm{NMR,} \mathrm{CDCl}_{3}, 600 \mathrm{MHz}$

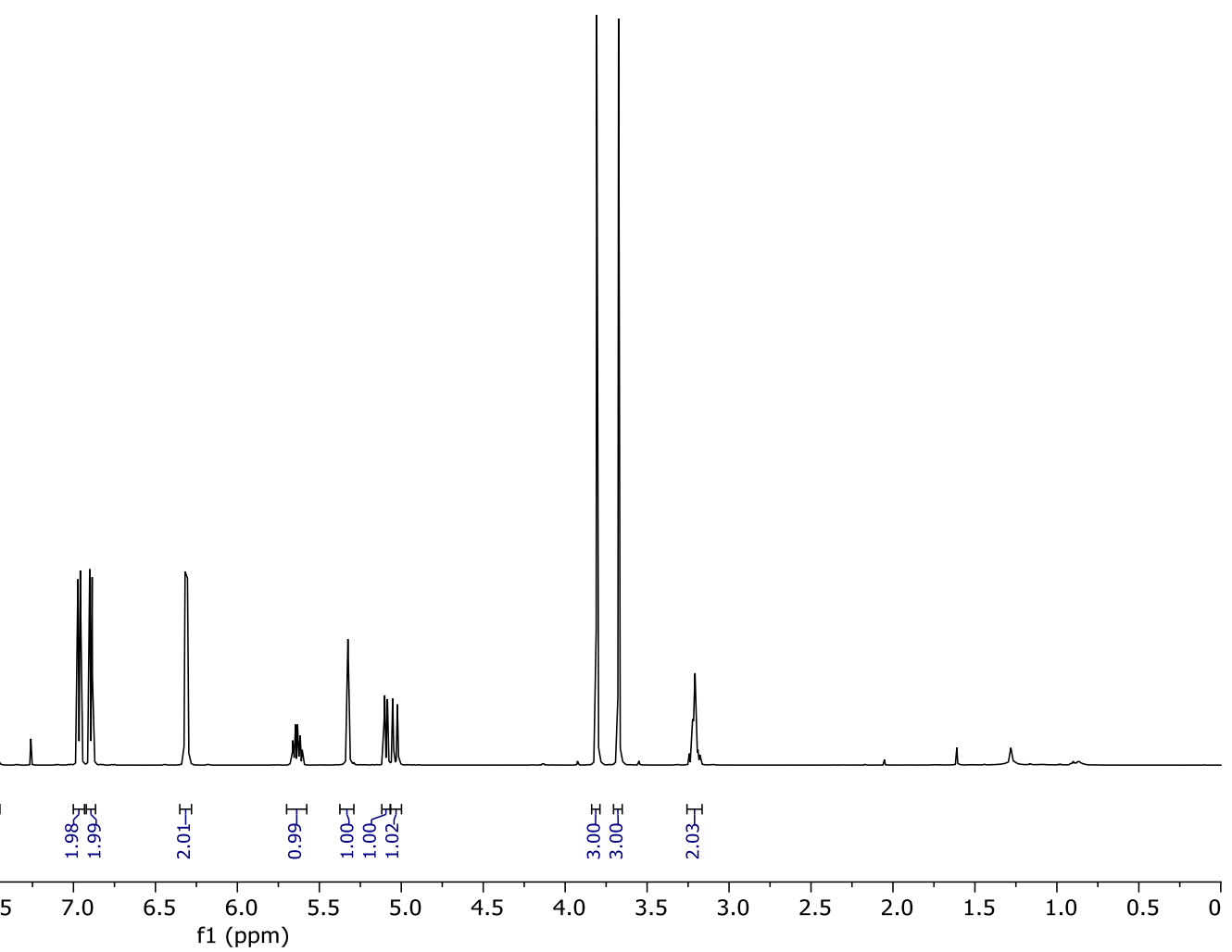




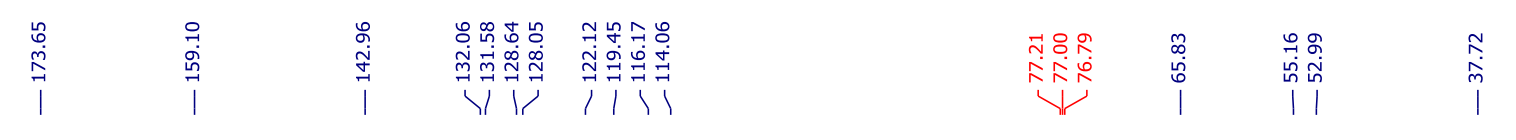

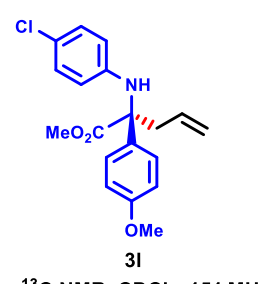

${ }^{13} \mathrm{C} \mathrm{NMR}, \mathrm{CDCl}_{3}, 151 \mathrm{MHz}$

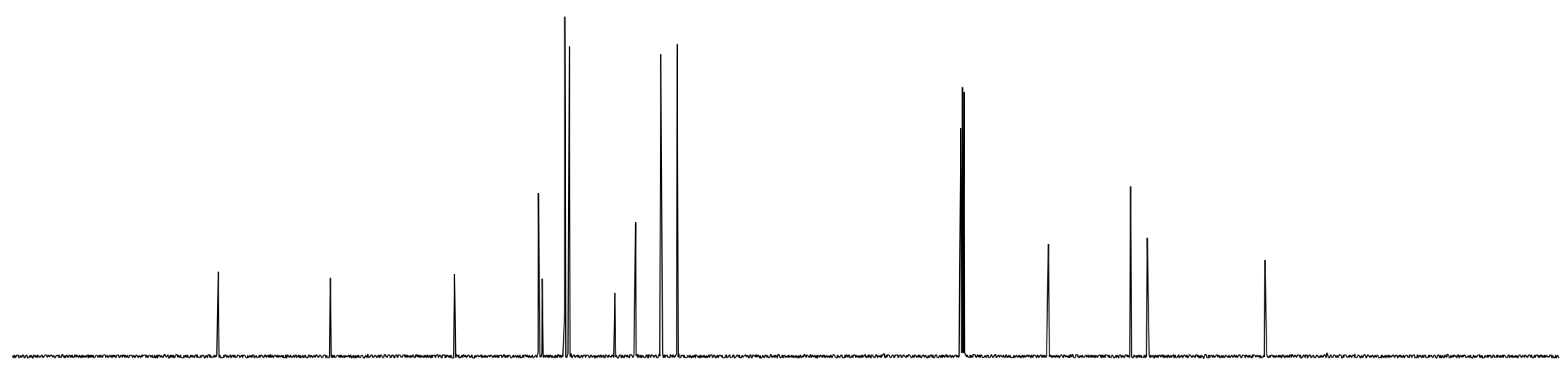

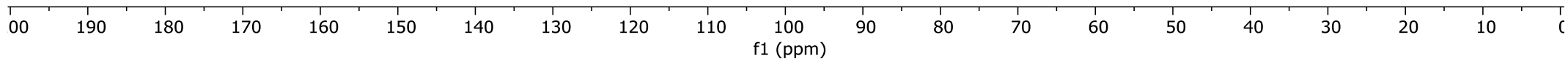

\section{S121}




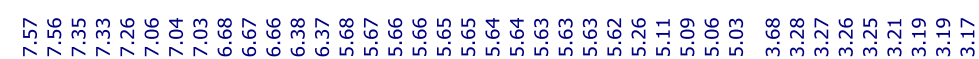

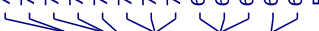

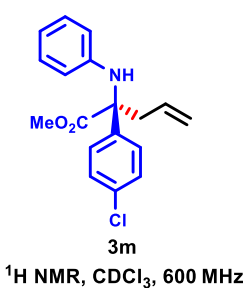

${ }^{1} \mathrm{H}$ NMR, $\mathrm{CDCl}_{3}, 600 \mathrm{MHz}$

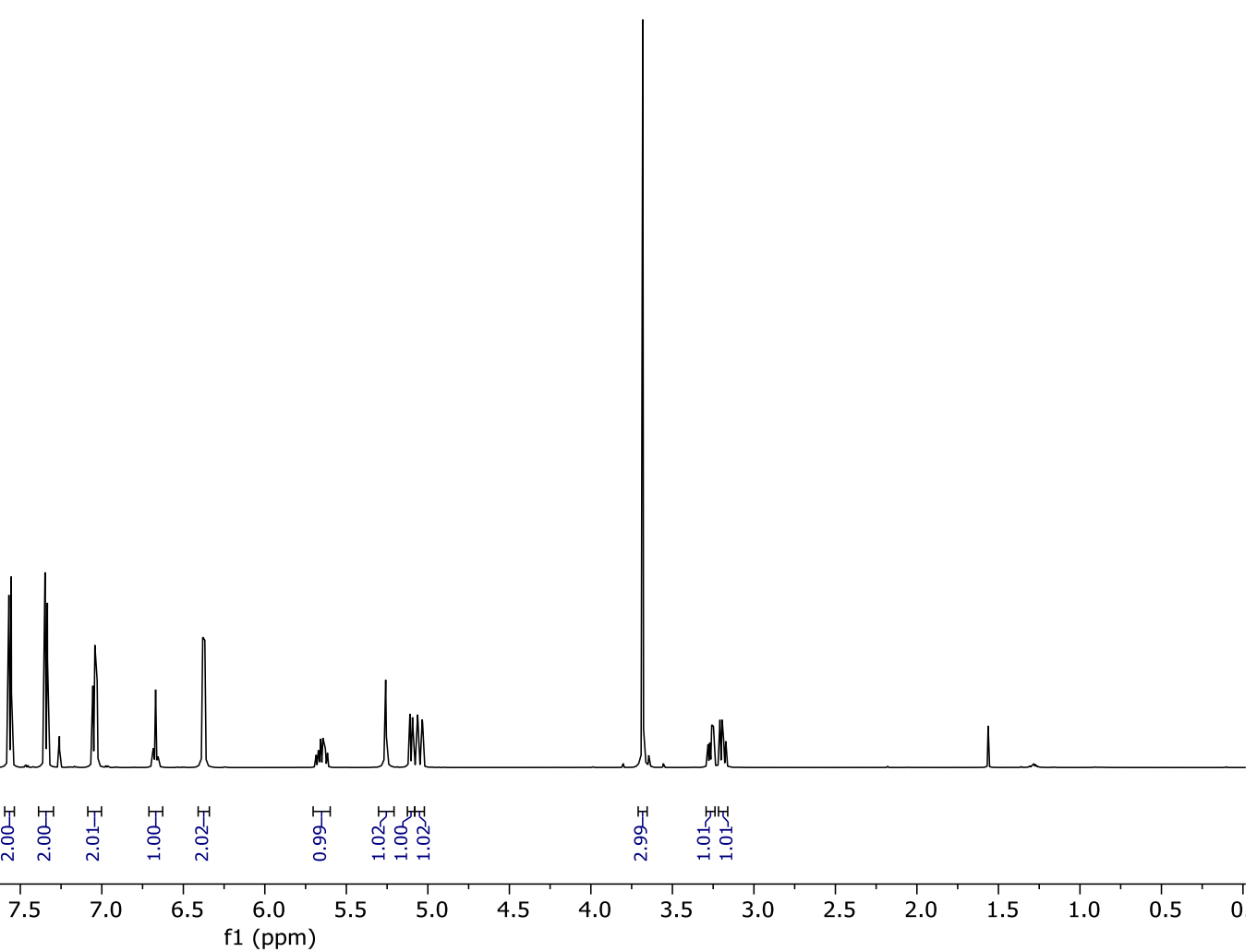




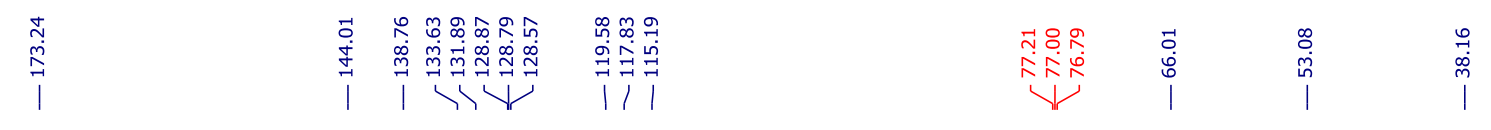
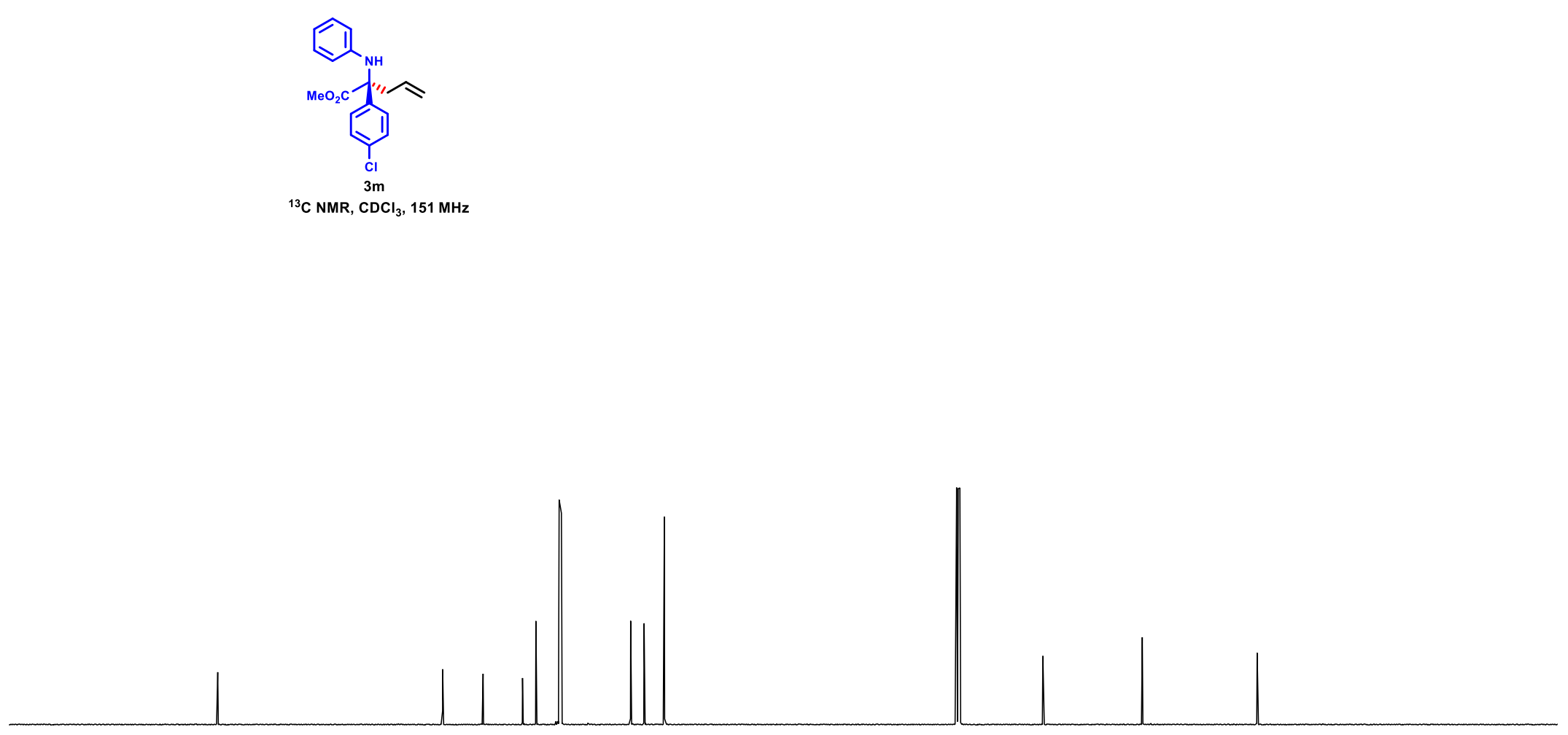

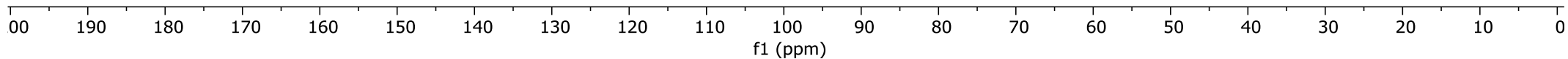




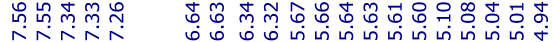

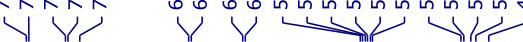

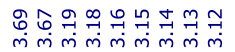

पैum

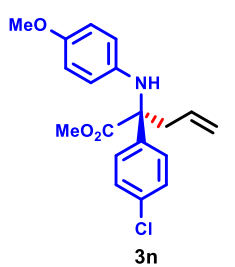

${ }^{1} \mathrm{H} \mathrm{NMR}, \mathrm{CDCl}_{3}, 600 \mathrm{MHz}$

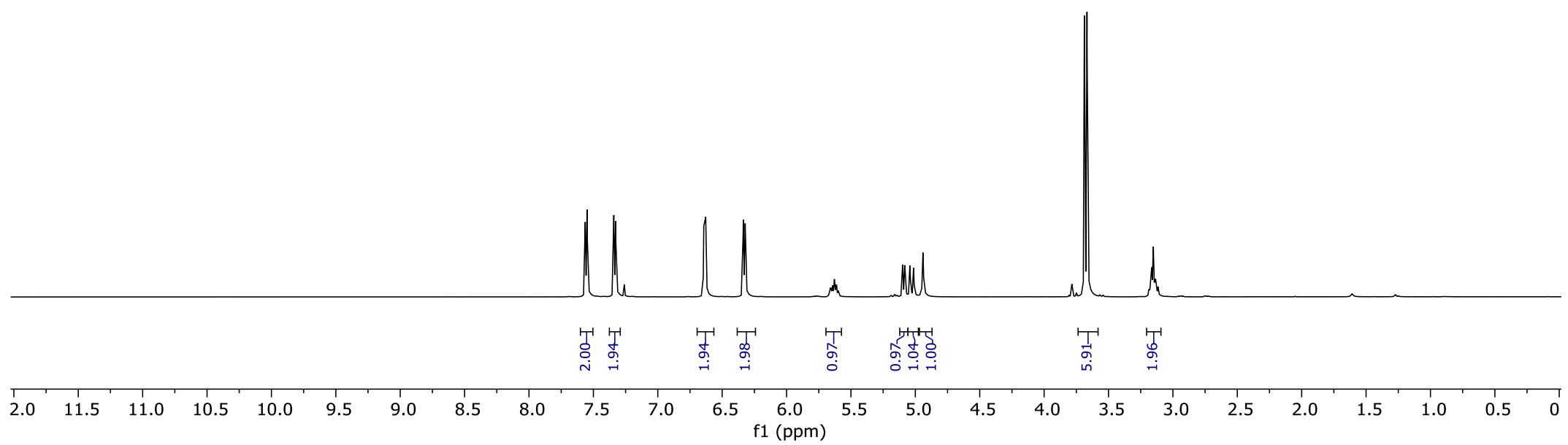

S124 

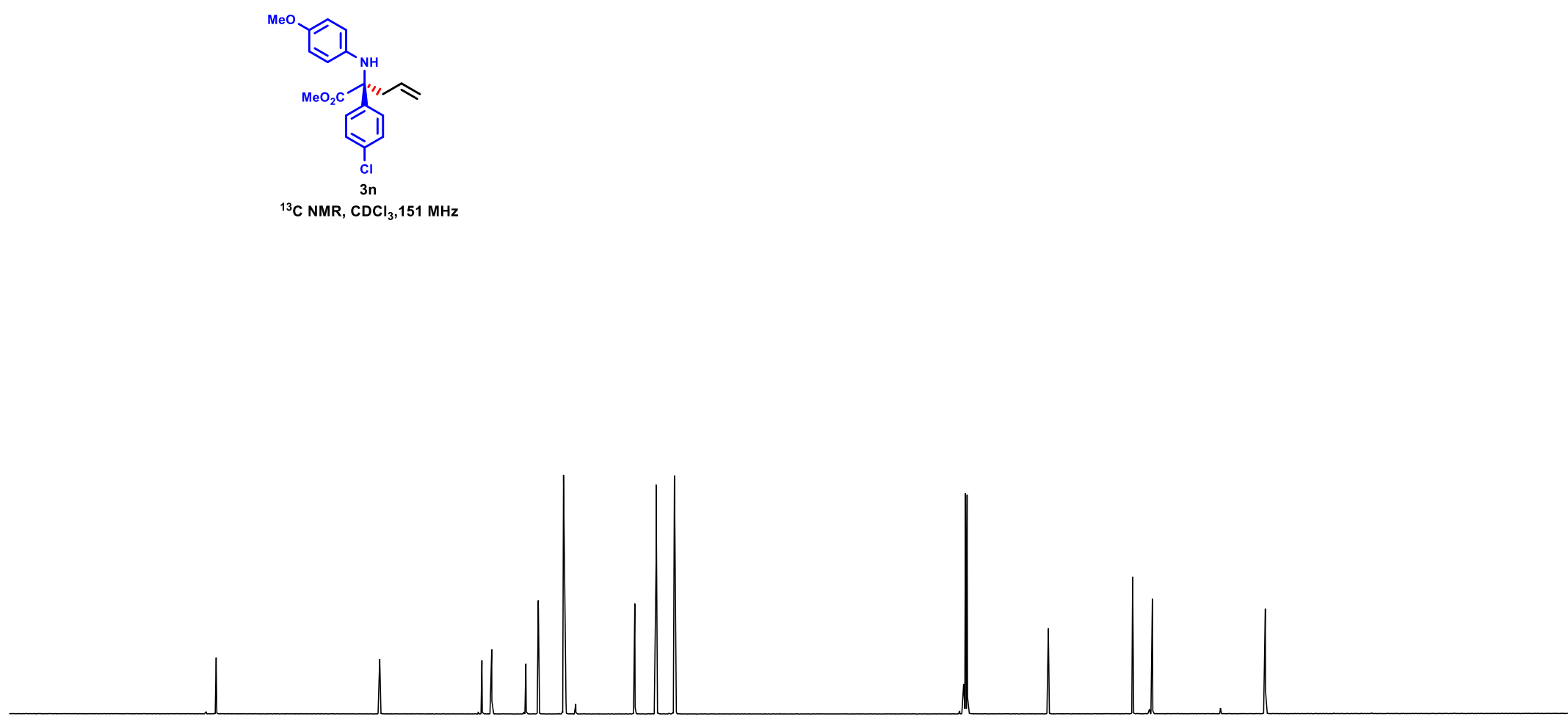


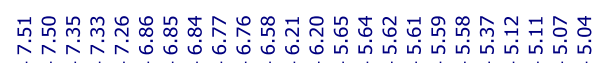

资i

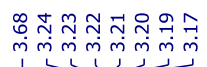

(n)

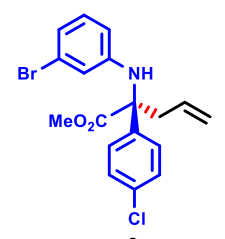

${ }^{1} \mathrm{H} \mathrm{NMR}, \mathrm{CDCl}_{3}, 600 \mathrm{MHz}$

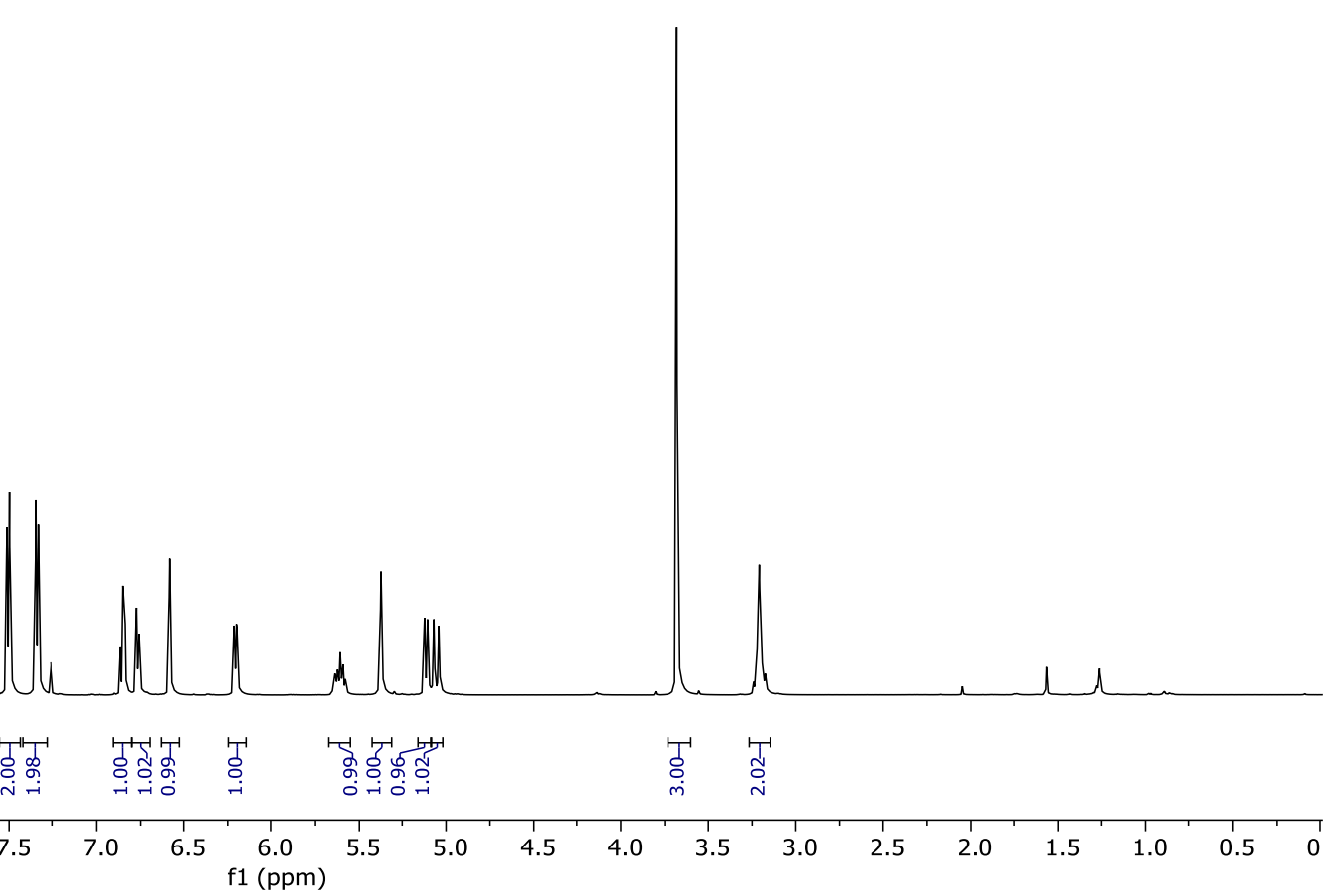

S126 


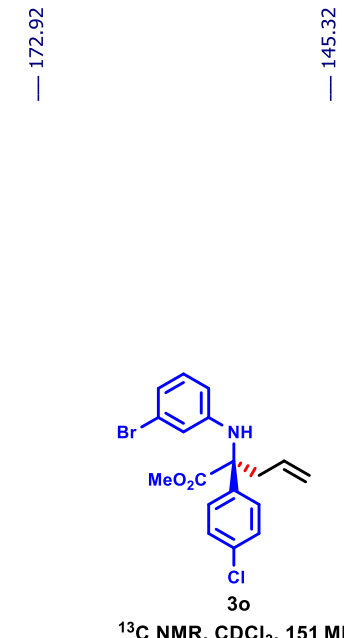

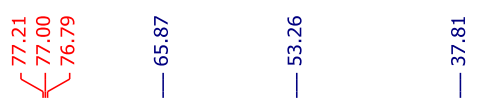

${ }^{13} \mathrm{CNMR}, \mathrm{CDCl}_{3}, 151 \mathrm{MHz}$

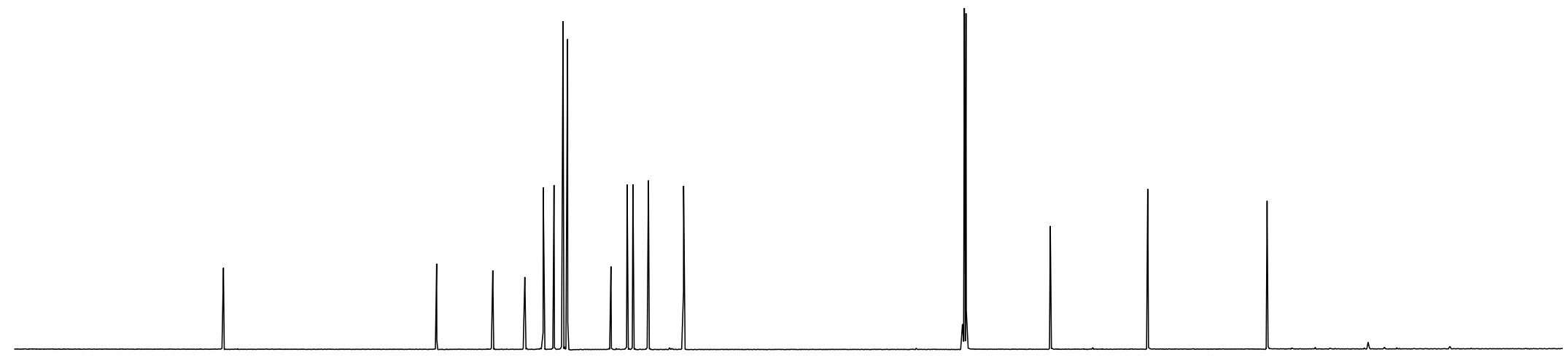

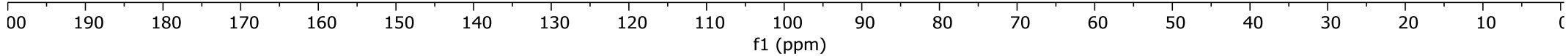




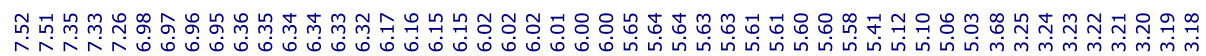

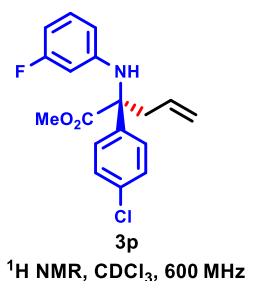

${ }_{1}^{1} \mathrm{H} \mathrm{NMR}, \mathrm{CDCl}_{3}, 600 \mathrm{MHz}$

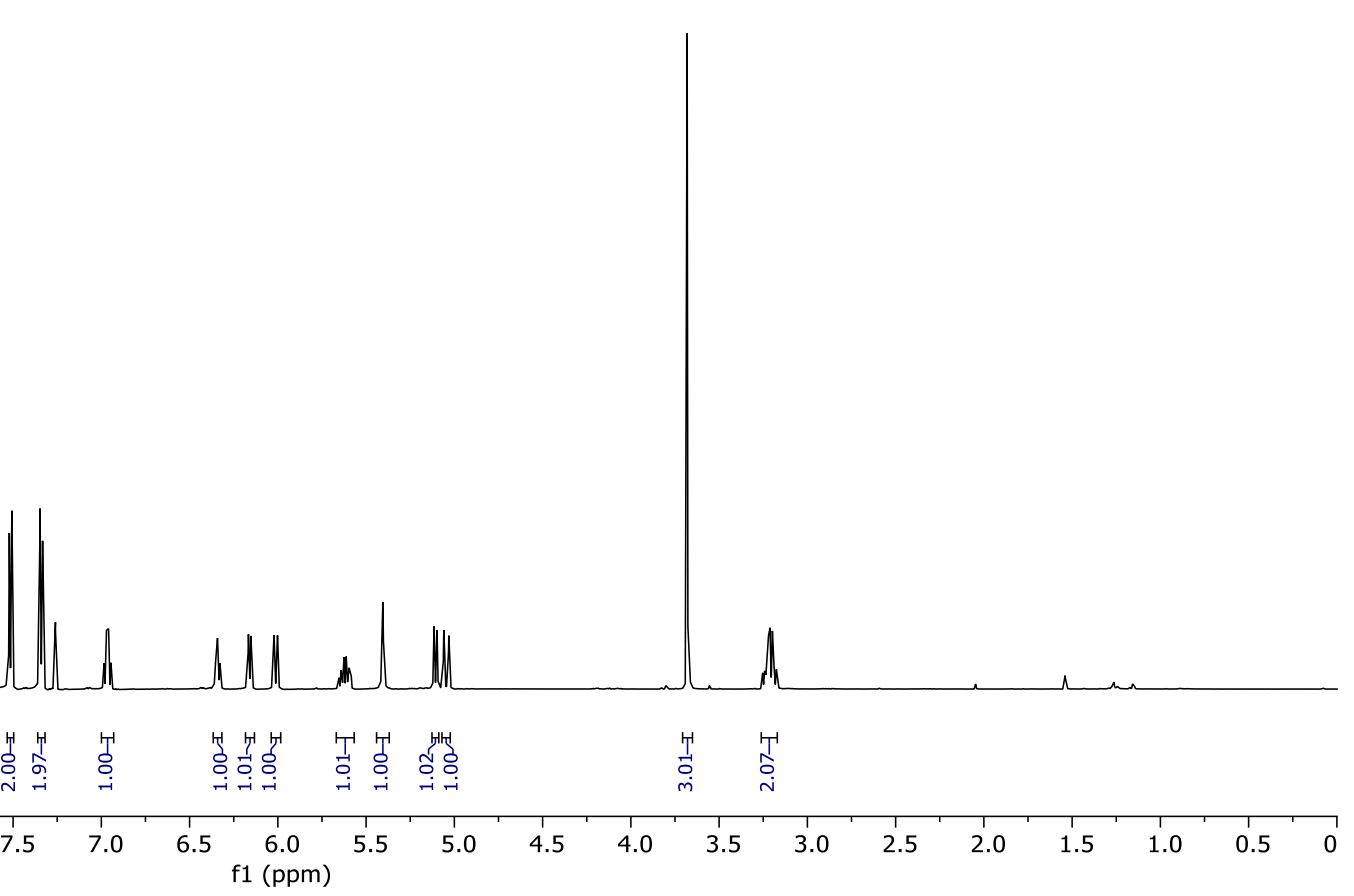

S128 


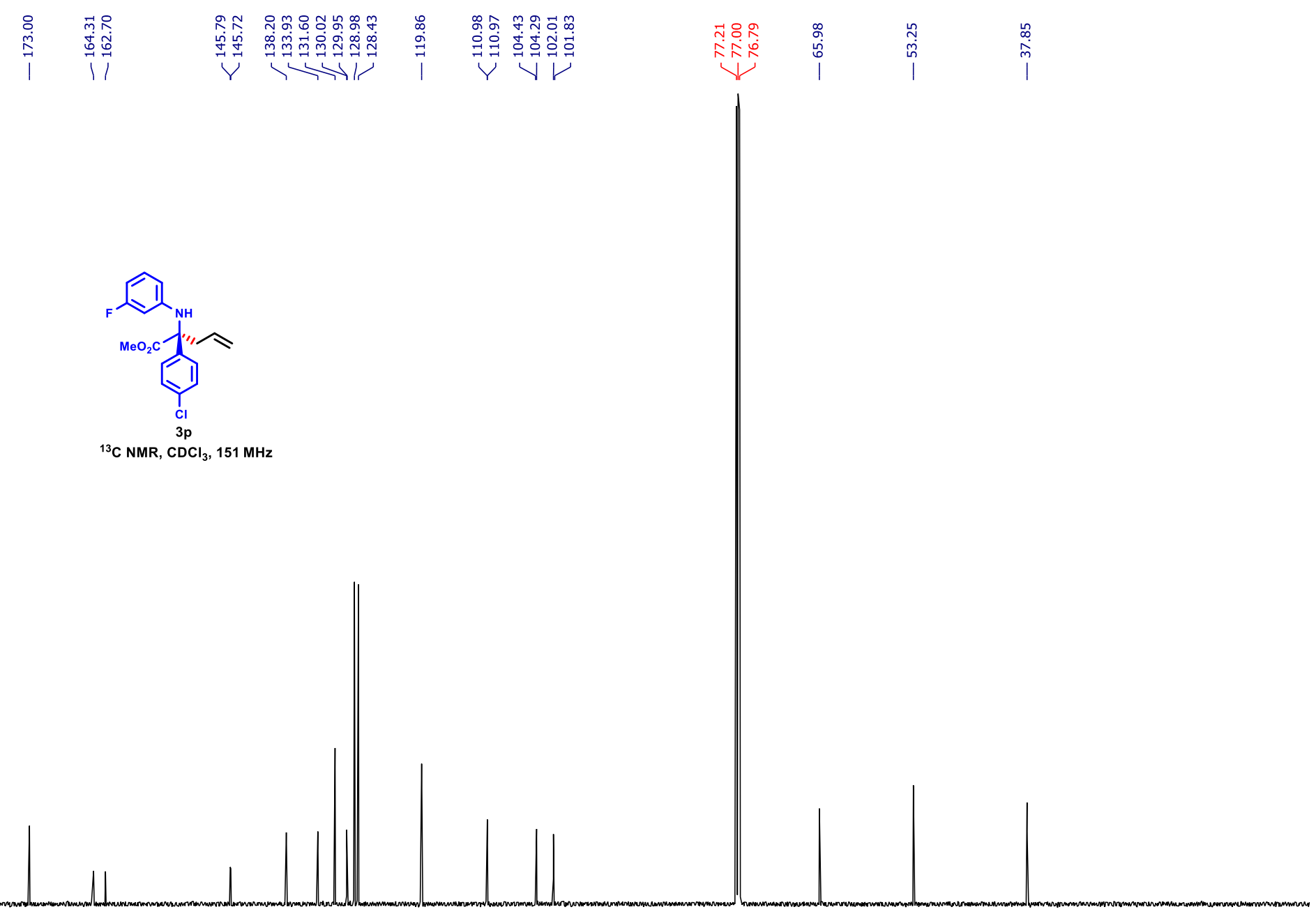

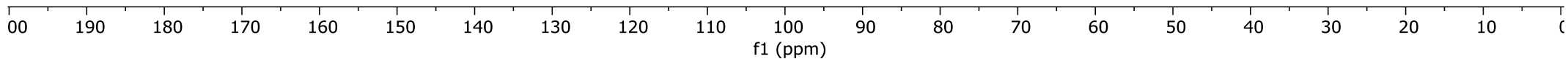




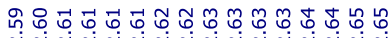

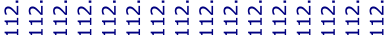

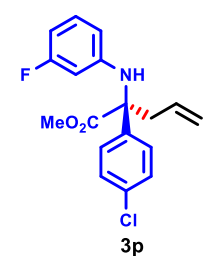

${ }^{19} \mathrm{~F} \mathrm{NMR}, \mathrm{CDCl}_{3}, 471 \mathrm{MHz}$

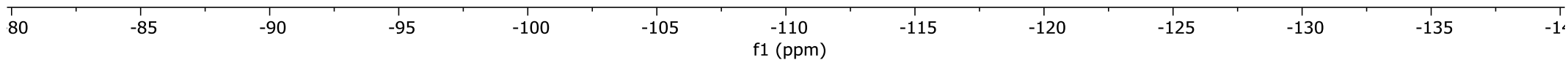




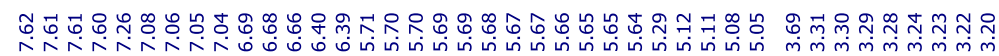

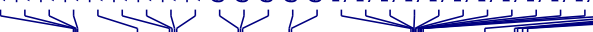

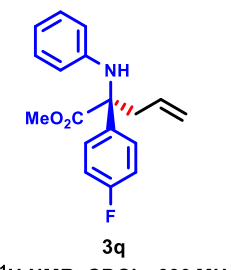

${ }^{1} \mathrm{H} \mathrm{NMR,}, \mathrm{CDCl}_{3}, 600 \mathrm{MHz}$

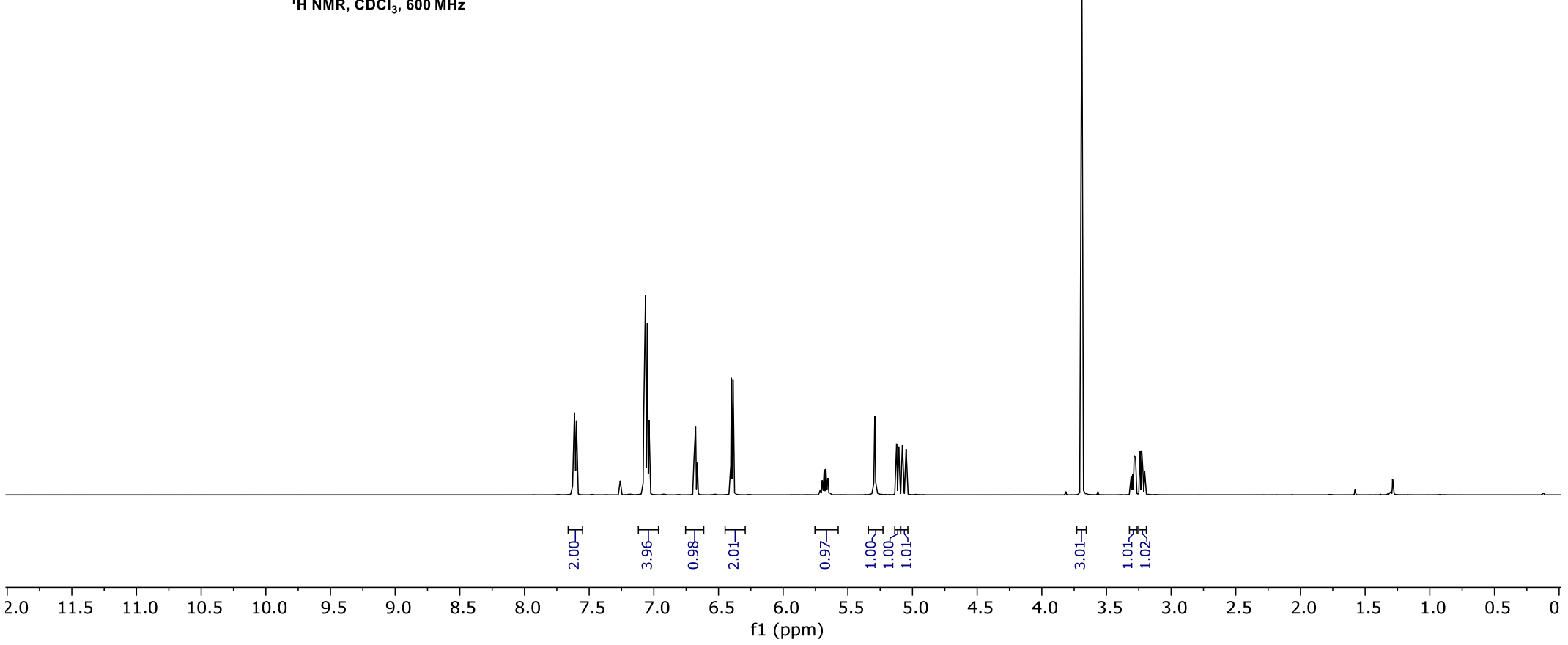

\section{S131}



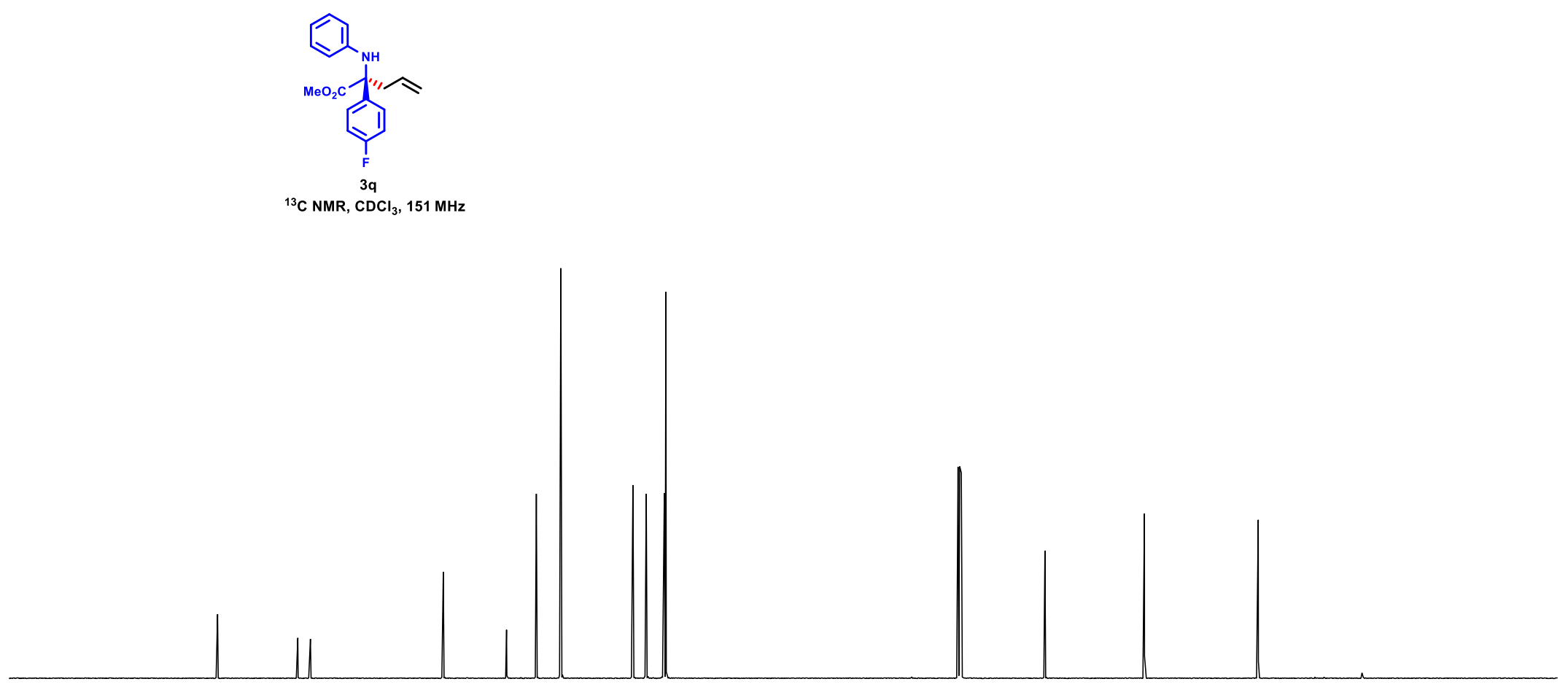

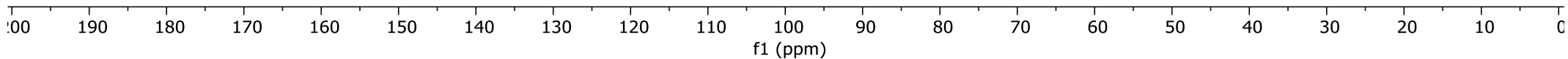




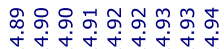

$\Rightarrow=7$

它运正

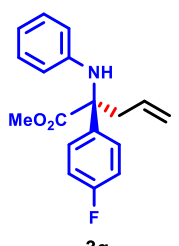

${ }^{19} \mathrm{~F} \mathrm{NMR}, \mathrm{CDCl}_{3}, 471 \mathrm{MHz}$ 


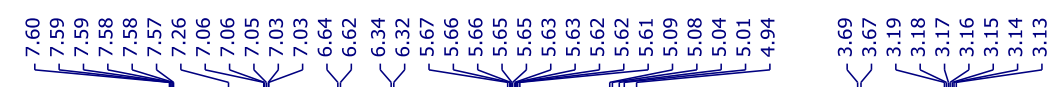

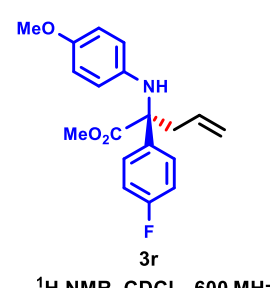

${ }^{1} \mathrm{H}$ NMR, $\mathrm{CDCl}_{3}, 600 \mathrm{MHz}$

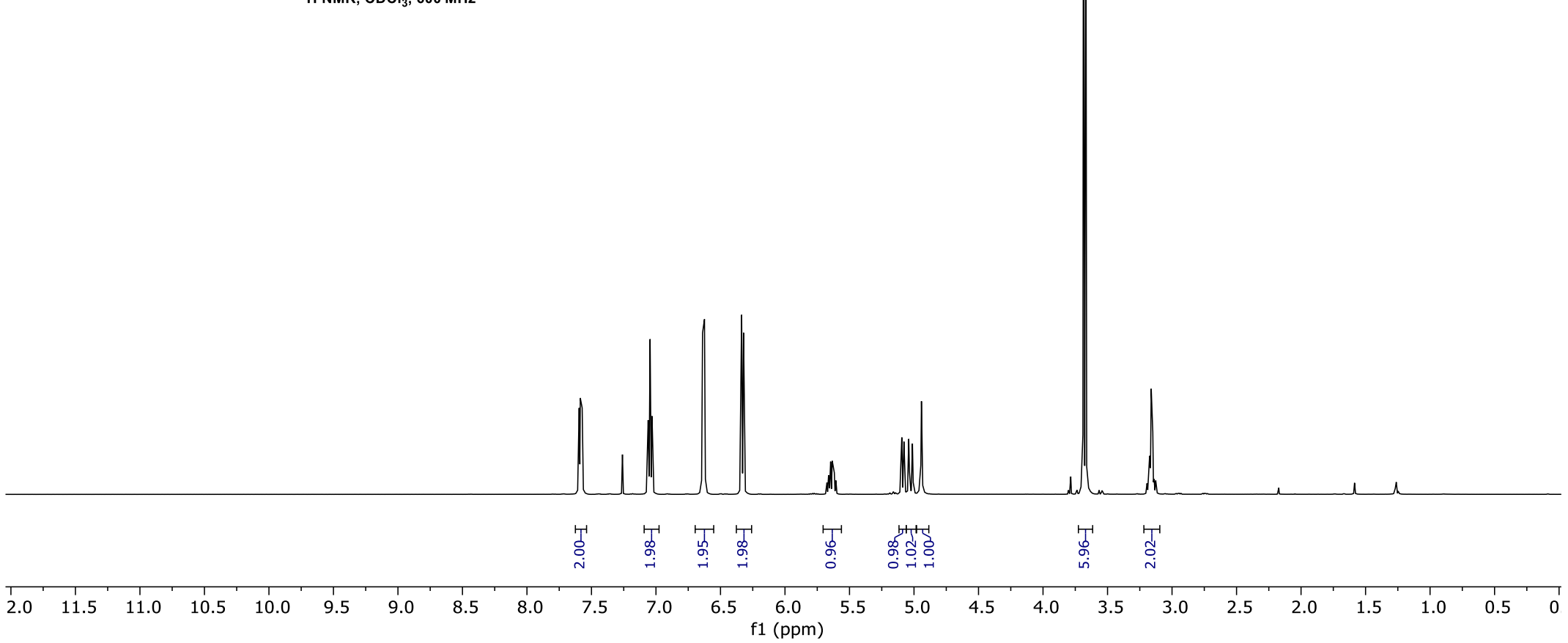

\section{S134}



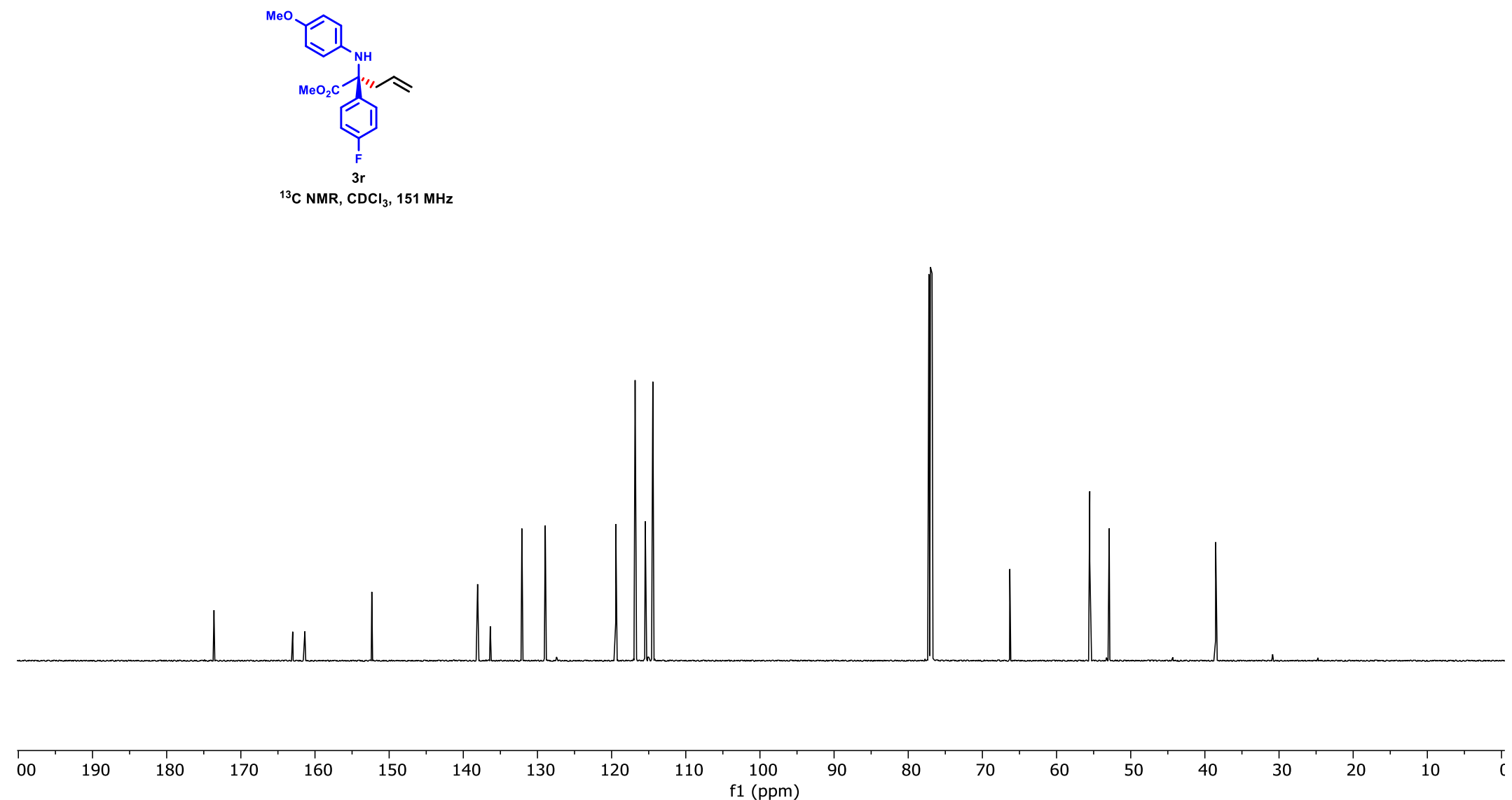


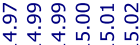

iv

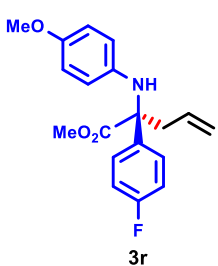

${ }^{19} \mathrm{~F} \mathrm{NMR}, \mathrm{CDCl}_{3}, 471 \mathrm{MHz}$ 


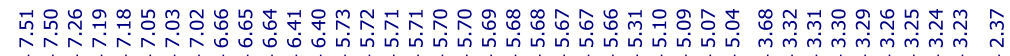

计

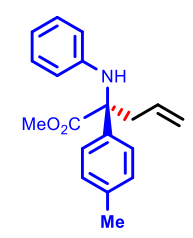

${ }^{1} \mathrm{H} \mathrm{NMR}, \mathrm{CDCl}_{3}, 600 \mathrm{MHz}$

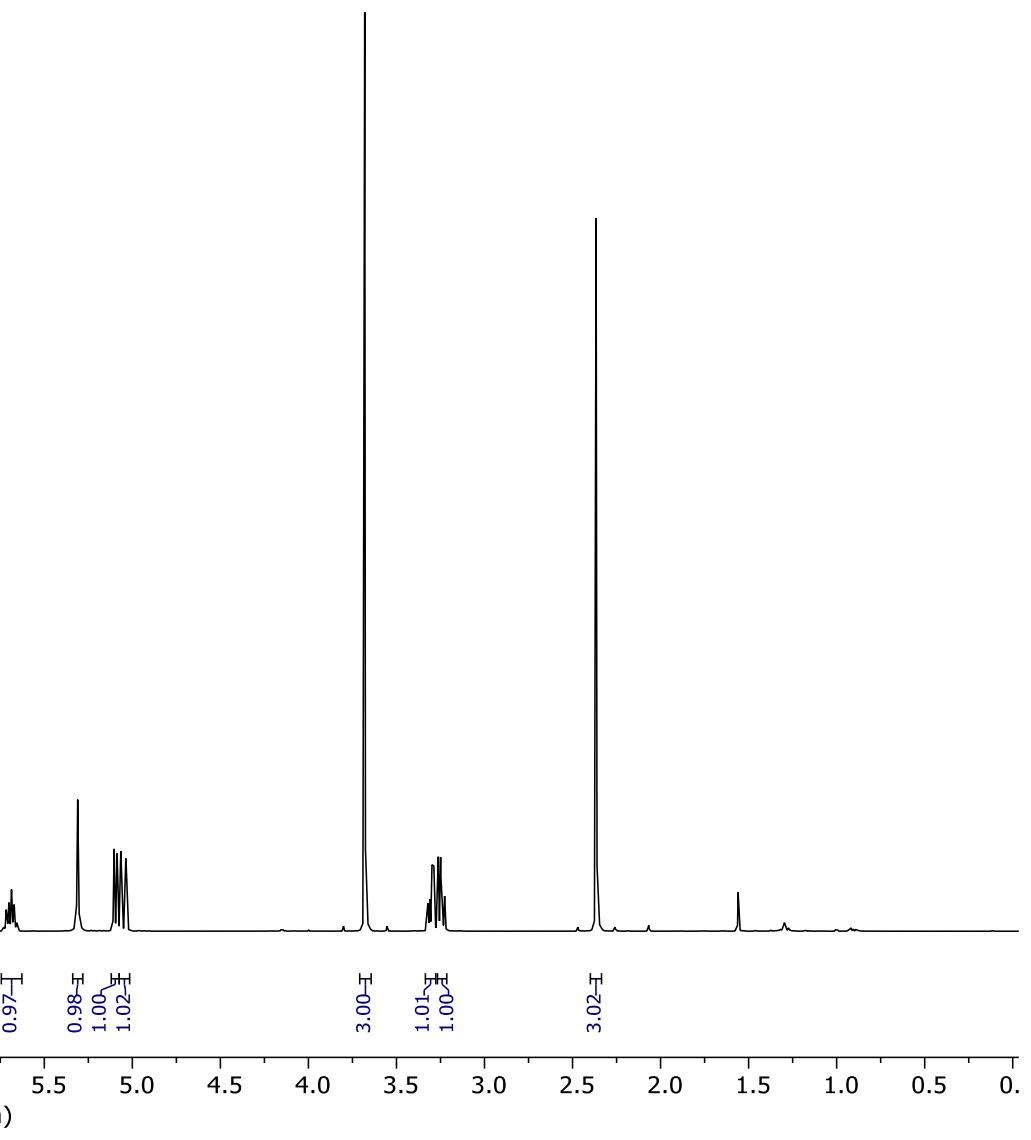




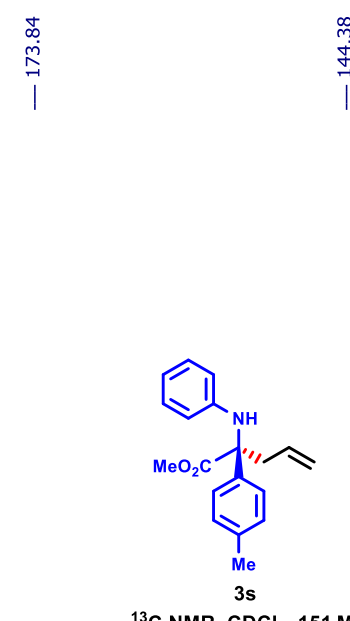

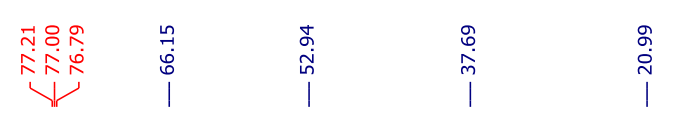

${ }^{13} \mathrm{C} \mathrm{NMR}, \mathrm{CDCl}_{3}, 151 \mathrm{MHz}$

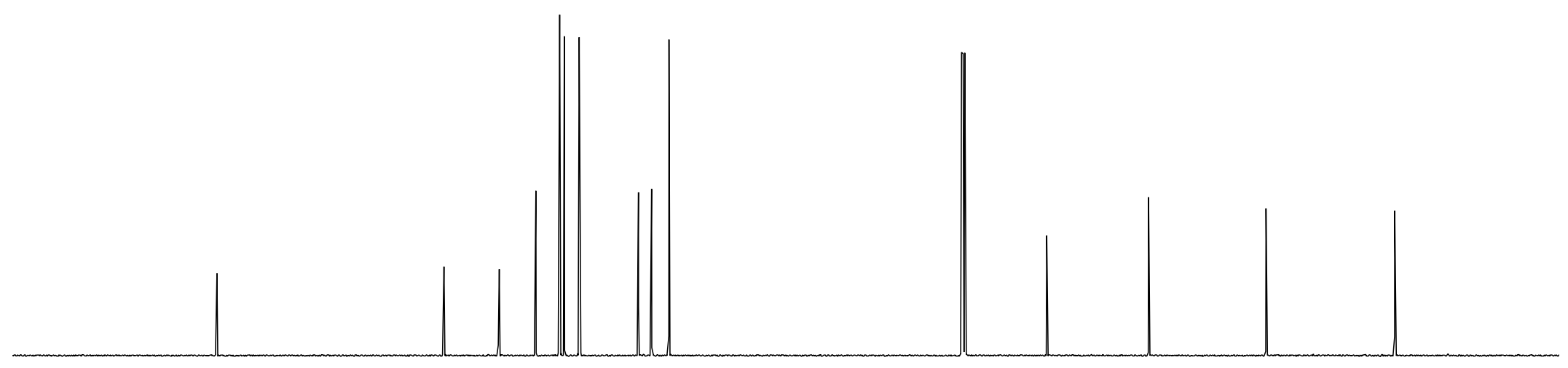

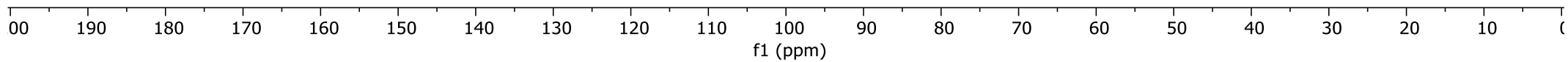

\section{S138}




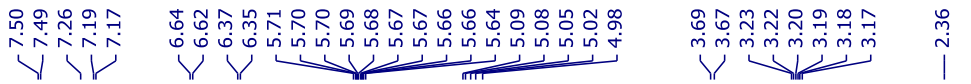

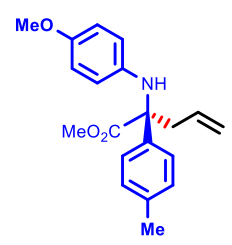

${ }^{1} \mathrm{H} \mathrm{NMR}, \mathrm{CDCl}_{3}, 600 \mathrm{MHz}$

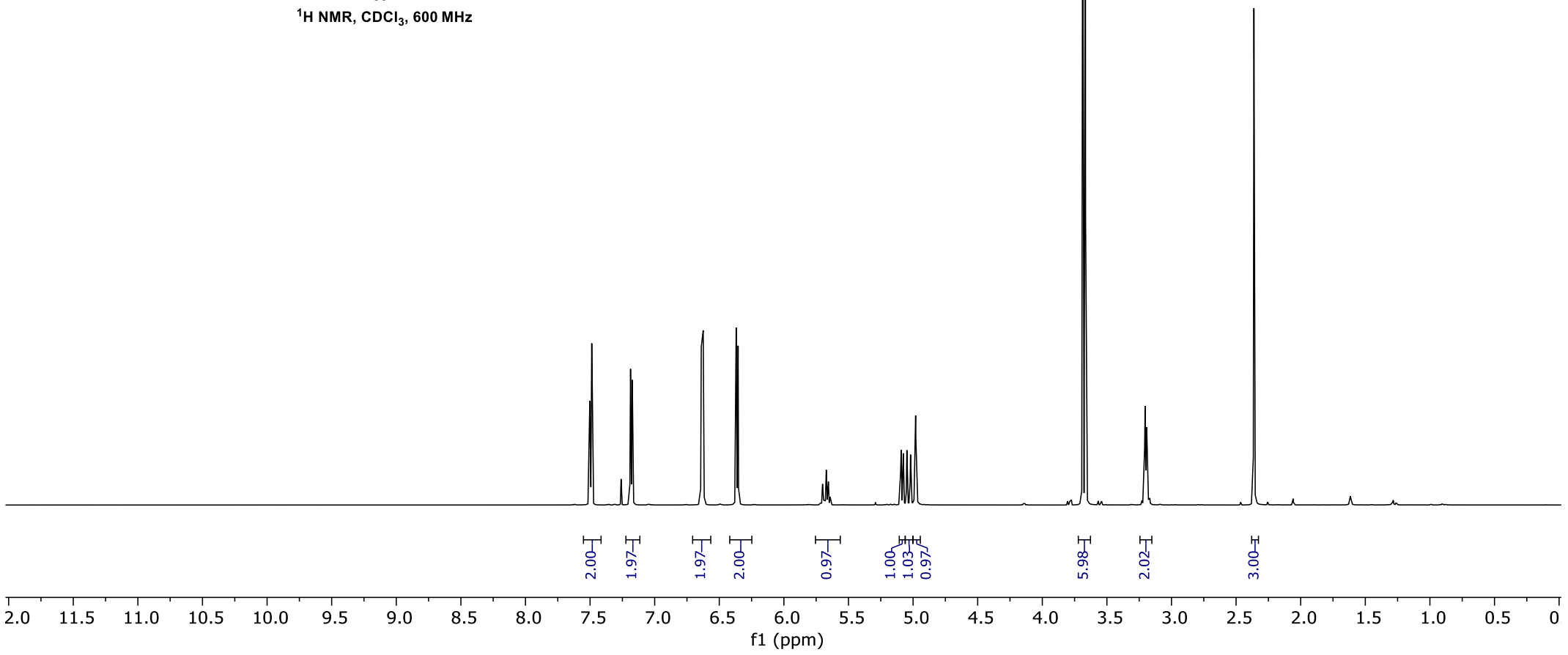




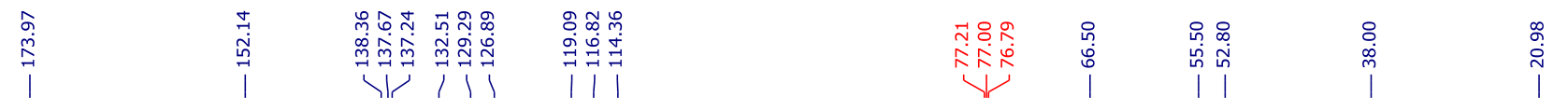
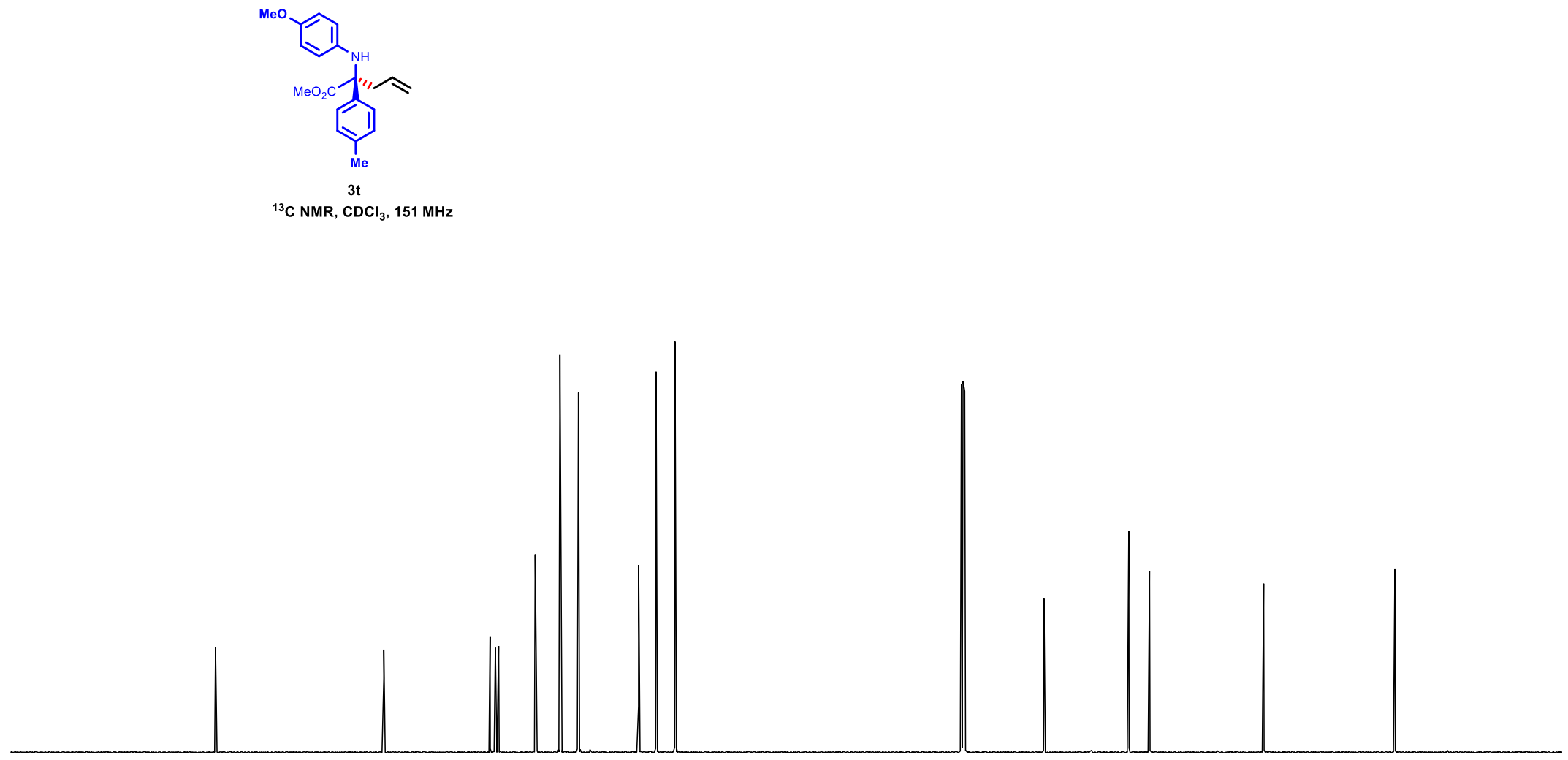

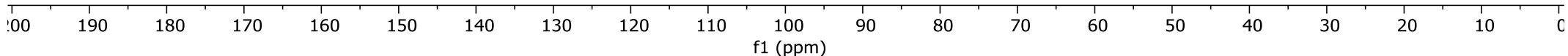




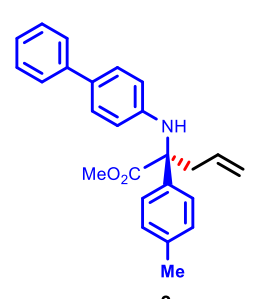

${ }^{1} \mathrm{H} \mathrm{NMR}, \mathrm{CDCl}_{3}, 600 \mathrm{MHz}$

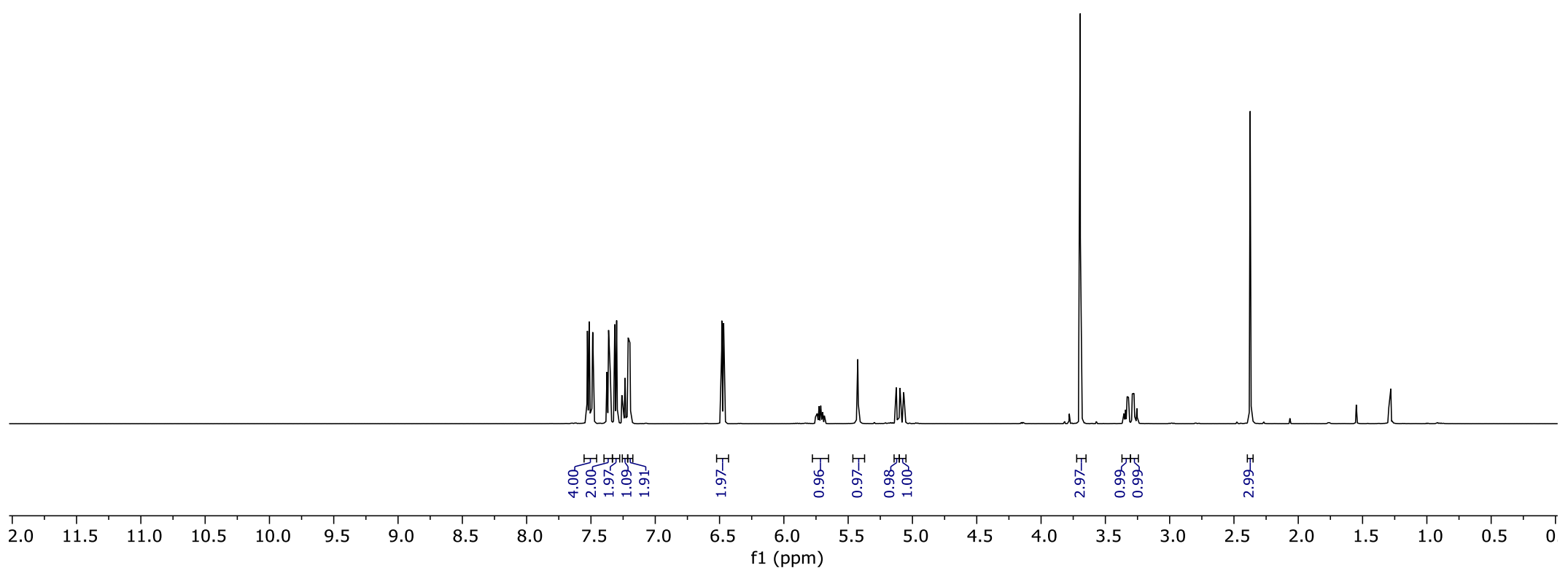




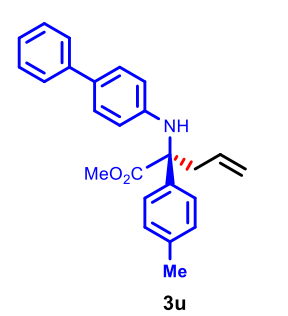

${ }^{13} \mathrm{C} \mathrm{NMR}, \mathrm{CDCl}_{3}, 151 \mathrm{MHz}$

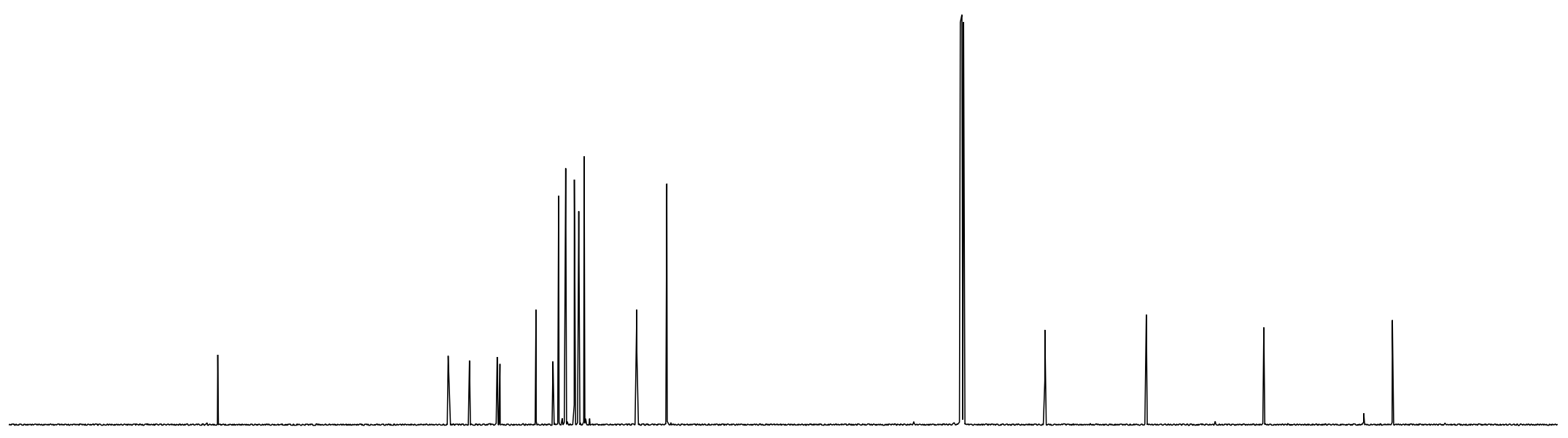

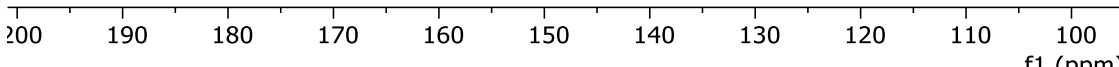
f1 (ppm)

$90 \quad 80$

1
$70 \quad 60$ 50 40 


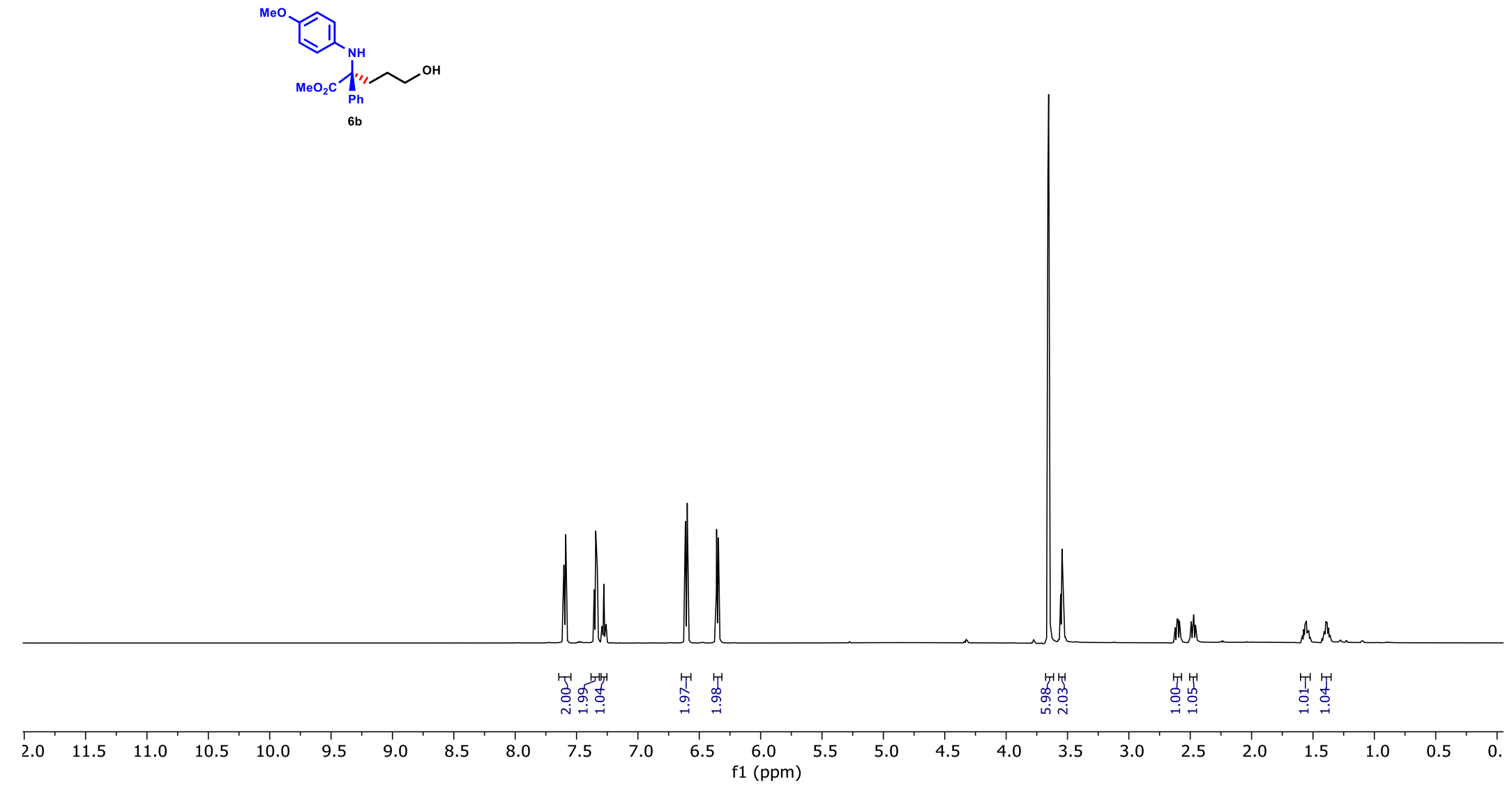



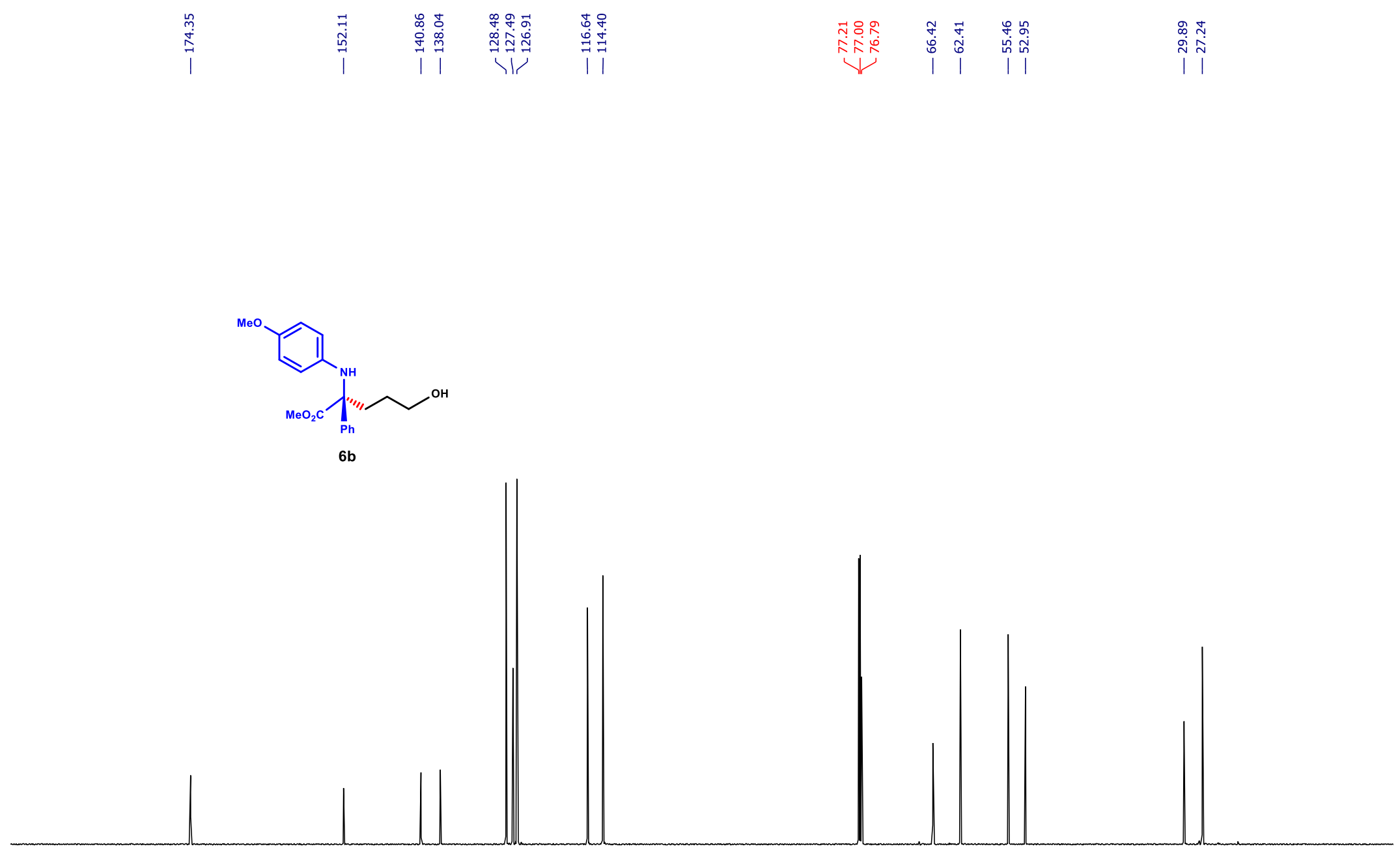

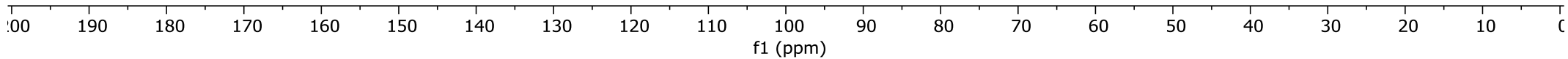

\section{S144}




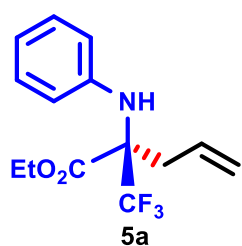

${ }^{1} \mathrm{H} \mathrm{NMR,} \mathrm{CDCl}_{3}, 600 \mathrm{MHz}$

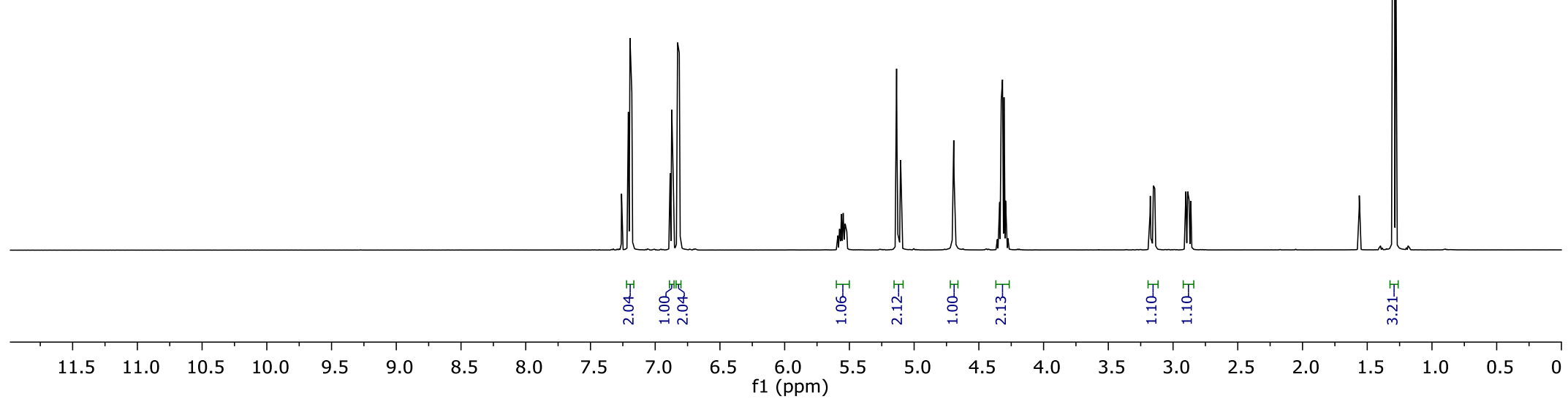



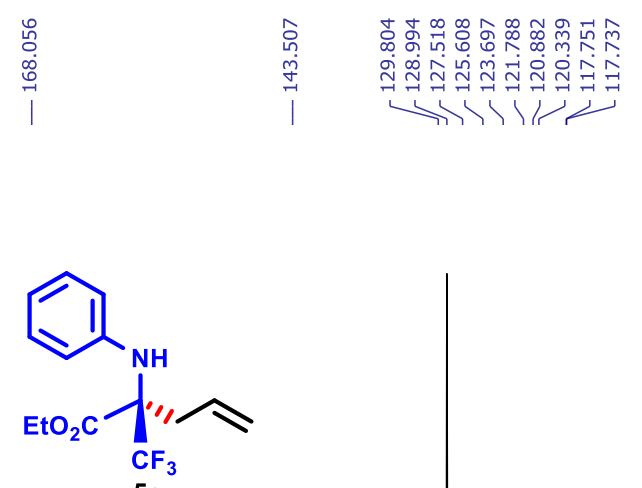

${ }^{13} \mathrm{C} \mathrm{NMR}, \mathrm{CDCl}_{3}, 151 \mathrm{MHz}$ 
81

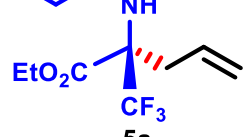

${ }^{19} \mathrm{~F} \mathrm{NMR,} \mathrm{CDCl}_{3}, 471 \mathrm{MHz}$

$\begin{array}{llllllllllllllllllllllllllllll}-69.1 & -69.3 & -69.5 & -69.7 & -69.9 & -70.1 & -70.3 & -70.5 & -70.7 & -70.9 & -71.1 & -71.3 & -71.5 & -71.7 & -71.9 & -72.1 & -72.3 & -72.5 & -72.7 & -72.9\end{array}$ 


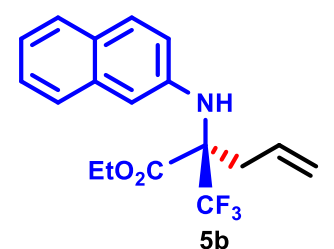

${ }^{1} \mathrm{H} \mathrm{NMR,} \mathrm{CDCl}_{3}, 600 \mathrm{MHz}$

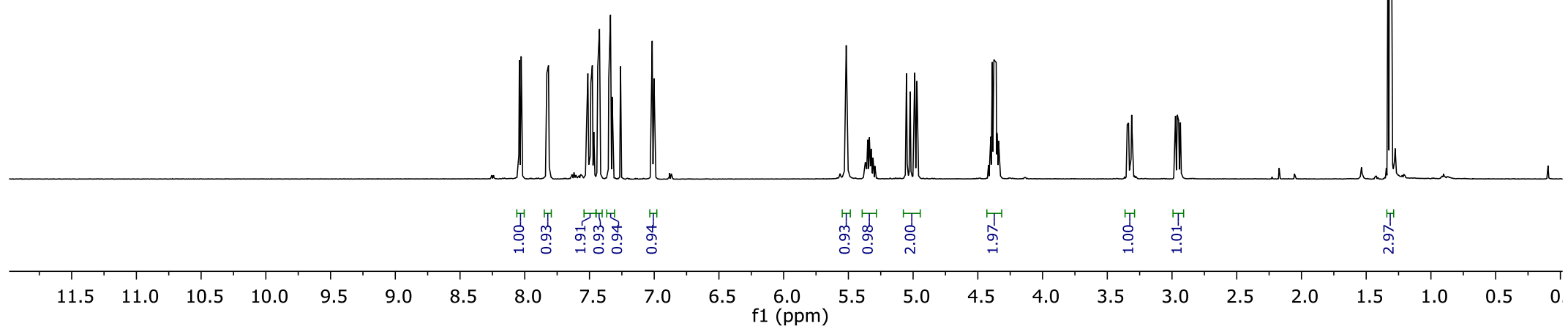



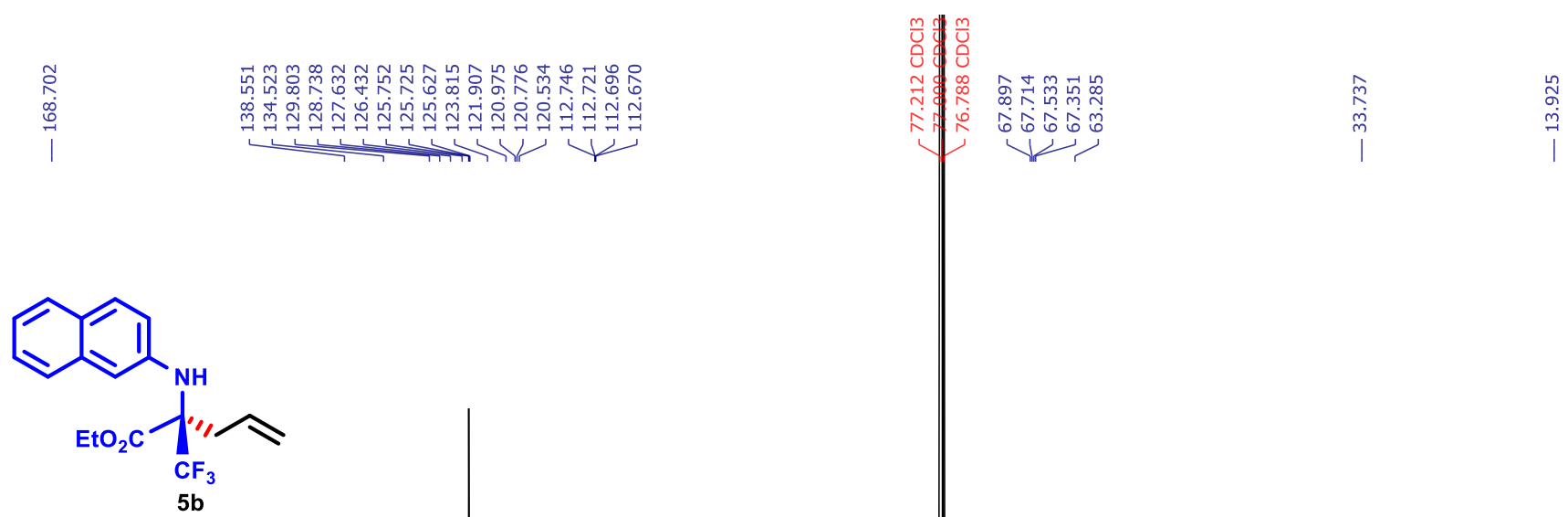

${ }^{13} \mathrm{C} \mathrm{NMR,} \mathrm{CDCl}_{3}, 151 \mathrm{MHz}$

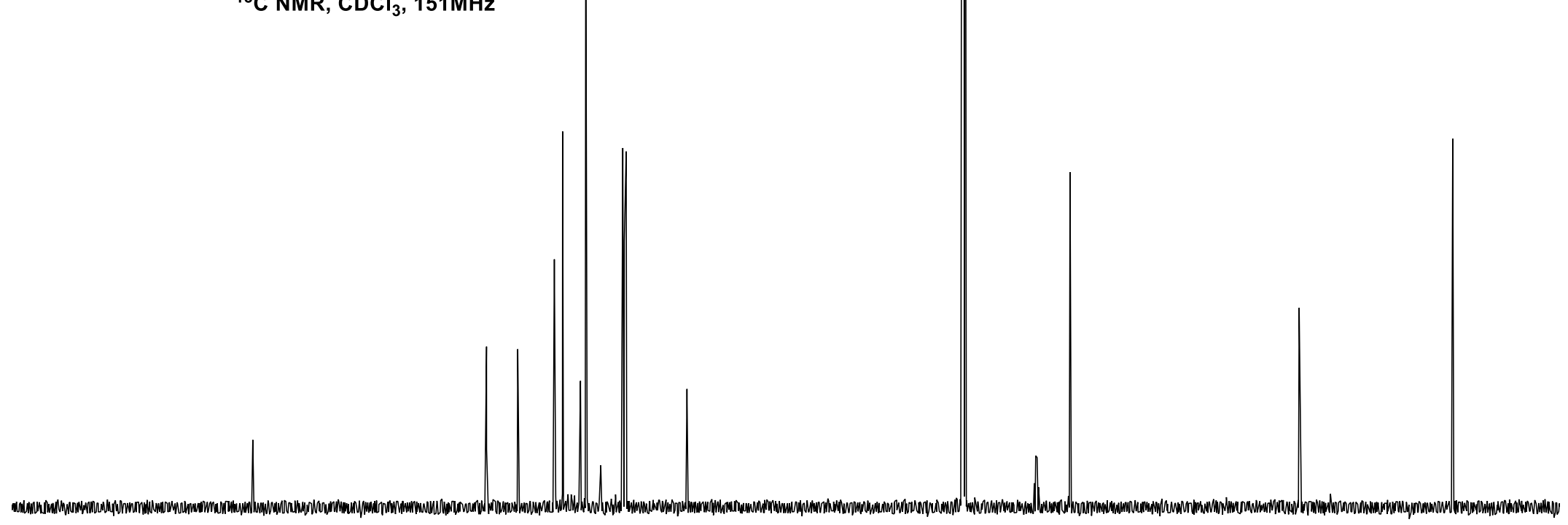

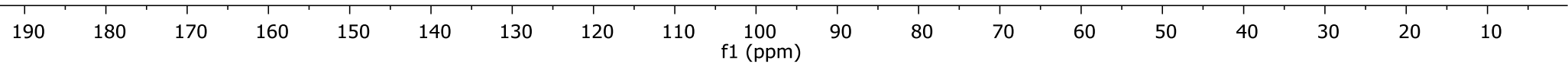




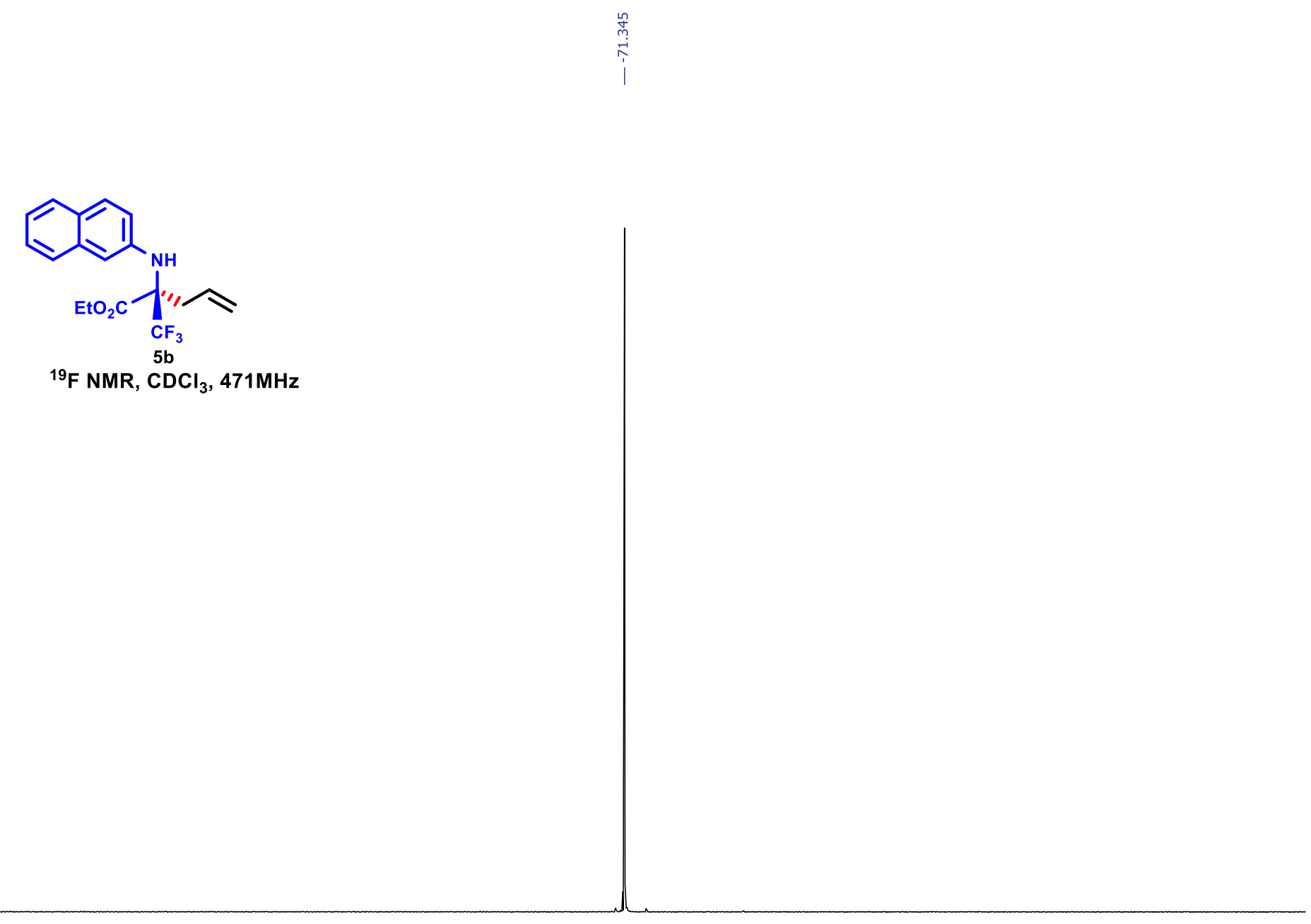

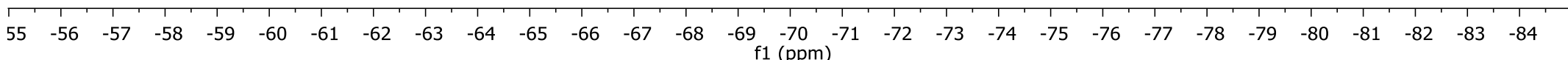



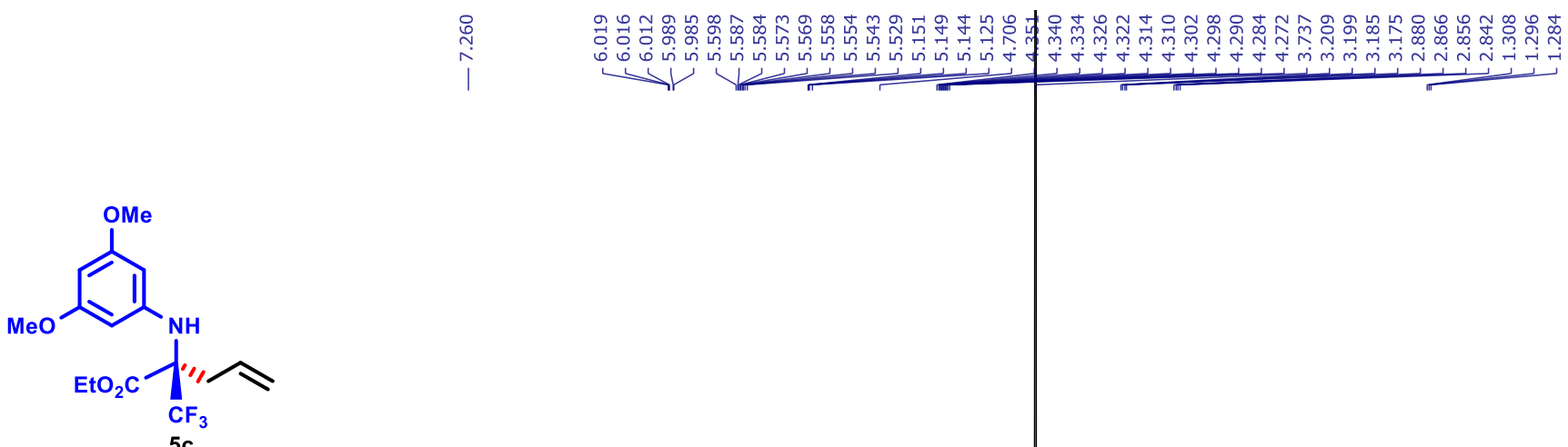

${ }^{1} \mathrm{H} \mathrm{NMR}, \mathrm{CDCl}_{3}, 600 \mathrm{MHz}$

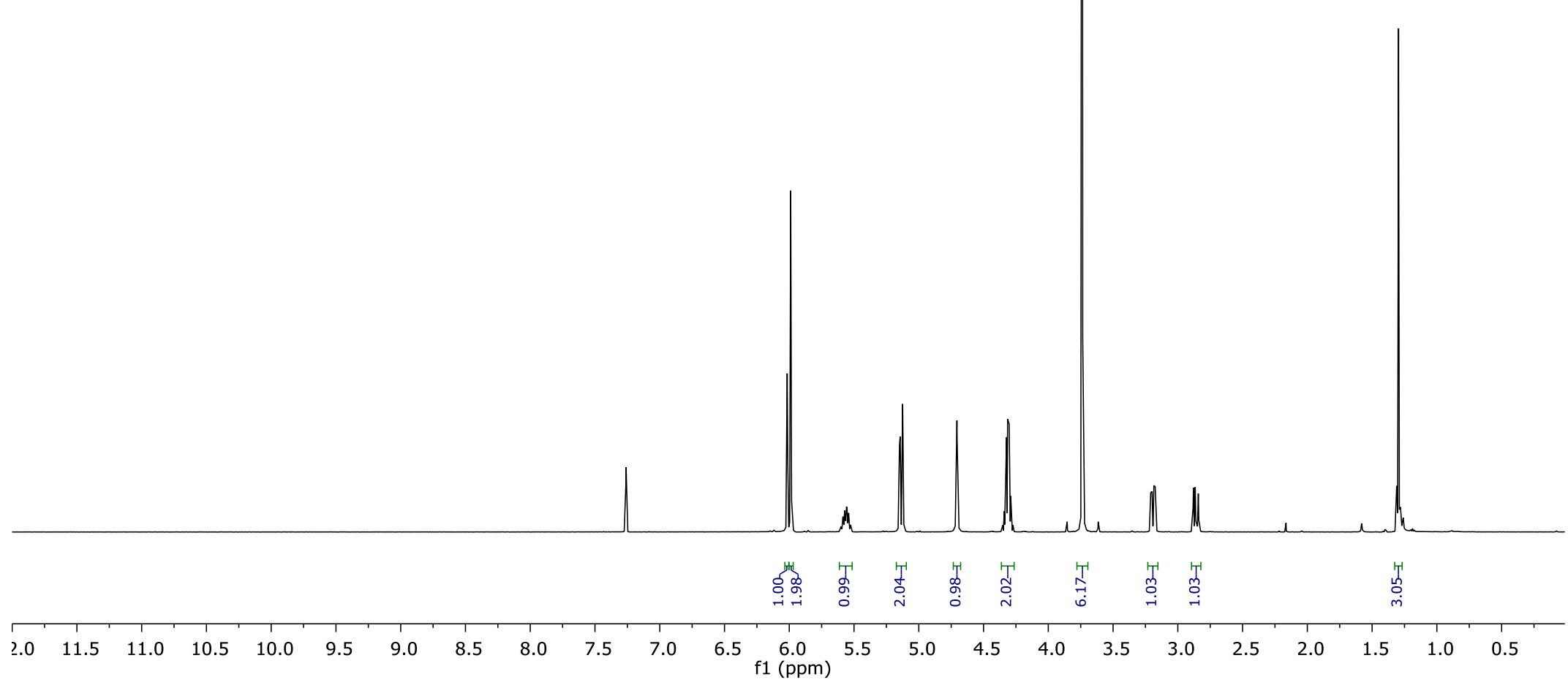




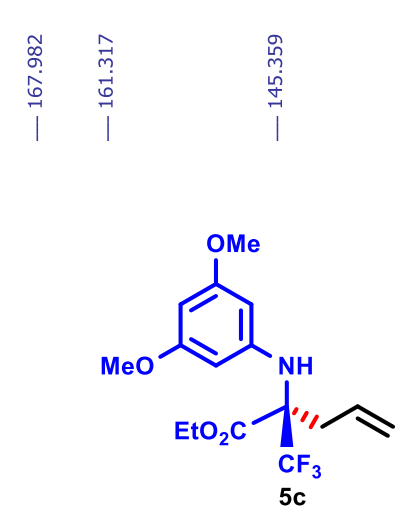

${ }^{13} \mathrm{C} \mathrm{NMR}, \mathrm{CDCl}_{3}, 151 \mathrm{MHz}$
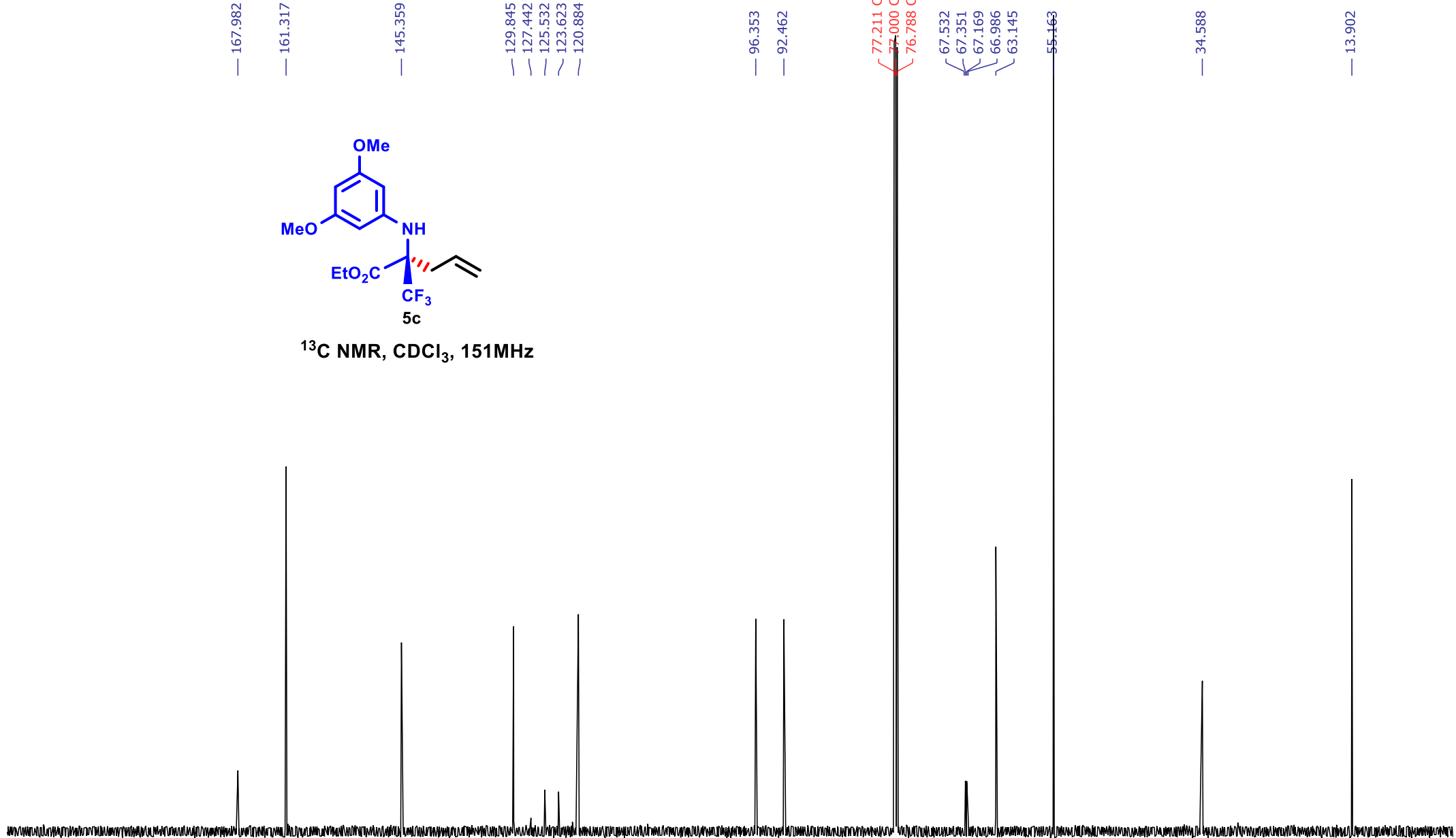


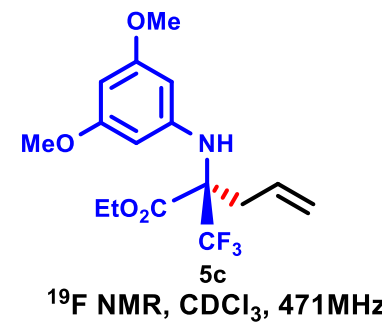

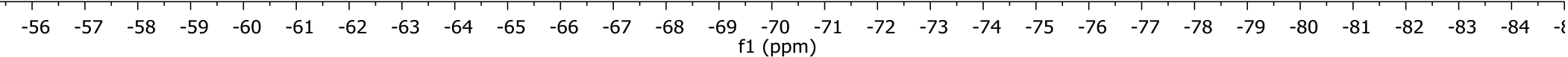




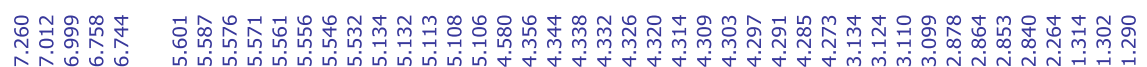

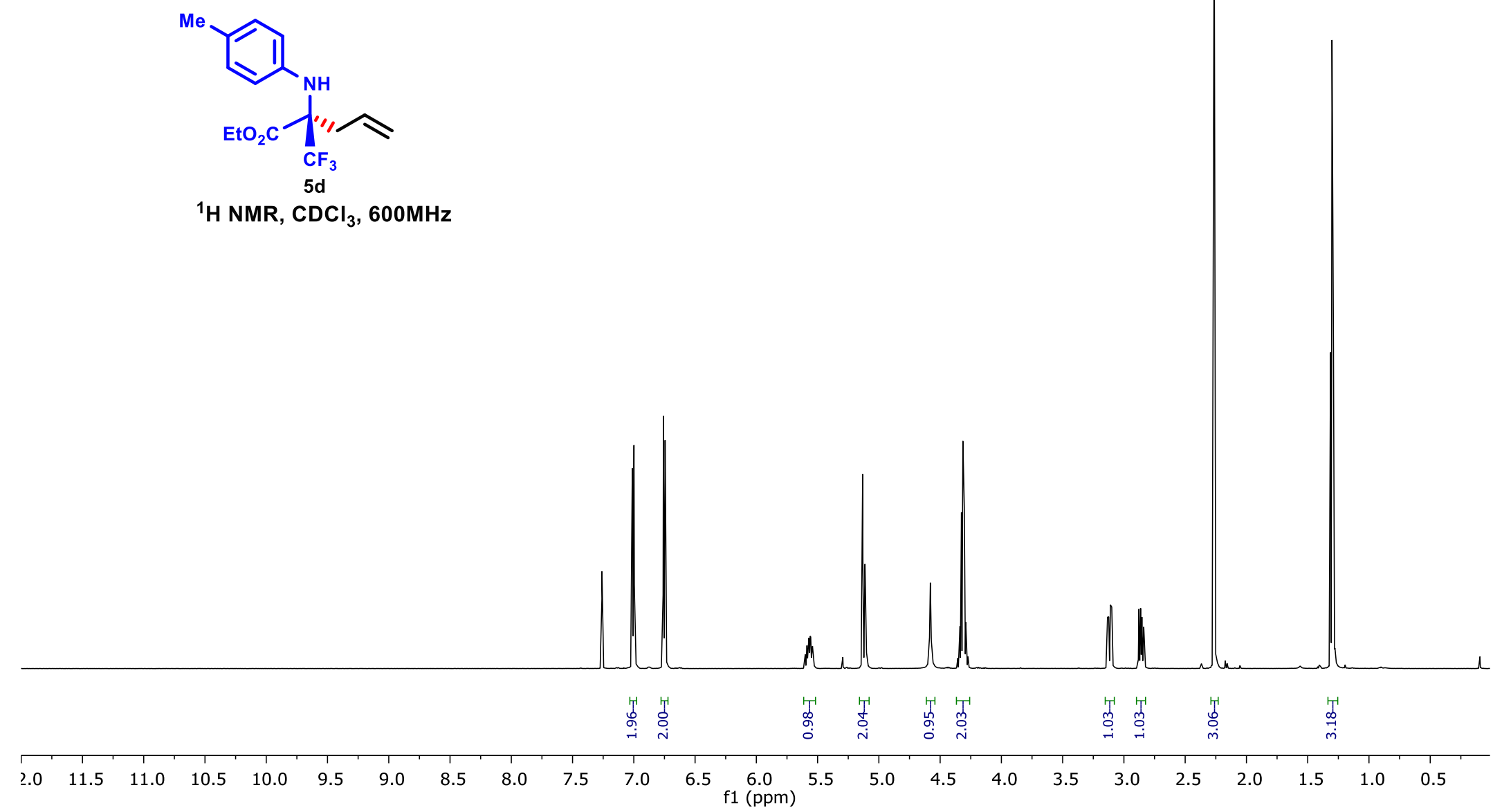

${ }^{1} \mathrm{H} \mathrm{NMR,} \mathrm{CDCl}_{3}, 600 \mathrm{MHz}$

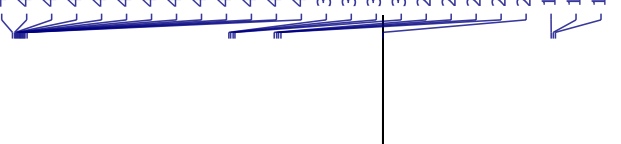




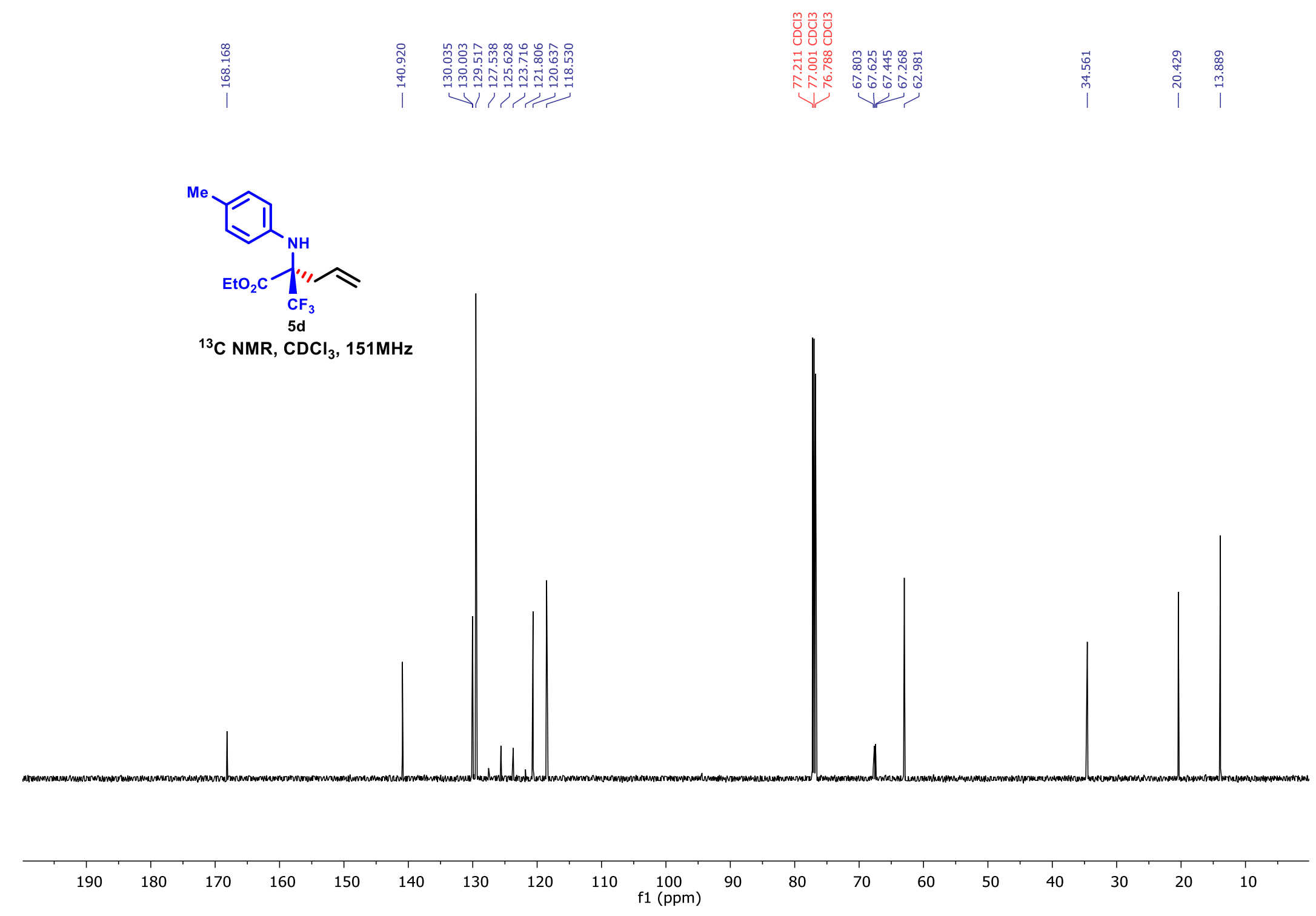




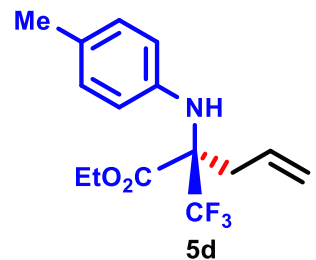

${ }^{19} \mathrm{~F} \mathrm{NMR}, \mathrm{CDCl}_{3}, 471 \mathrm{MHz}$

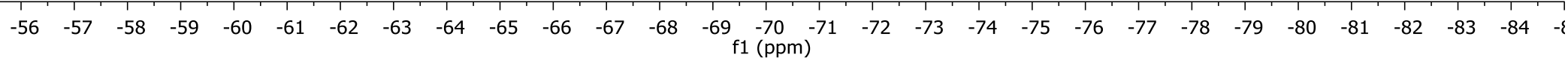




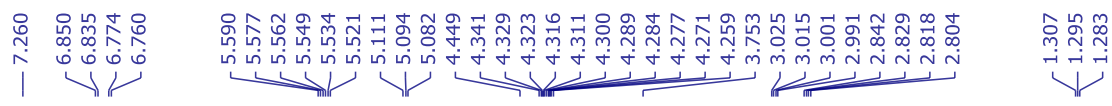

$\mathrm{MeO}$

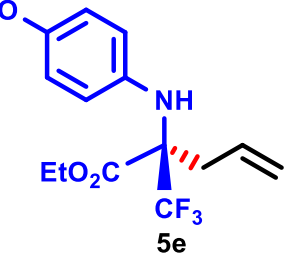

${ }^{1} \mathrm{H}$ NMR, $\mathrm{CDCl}_{3}, 600 \mathrm{MHz}$

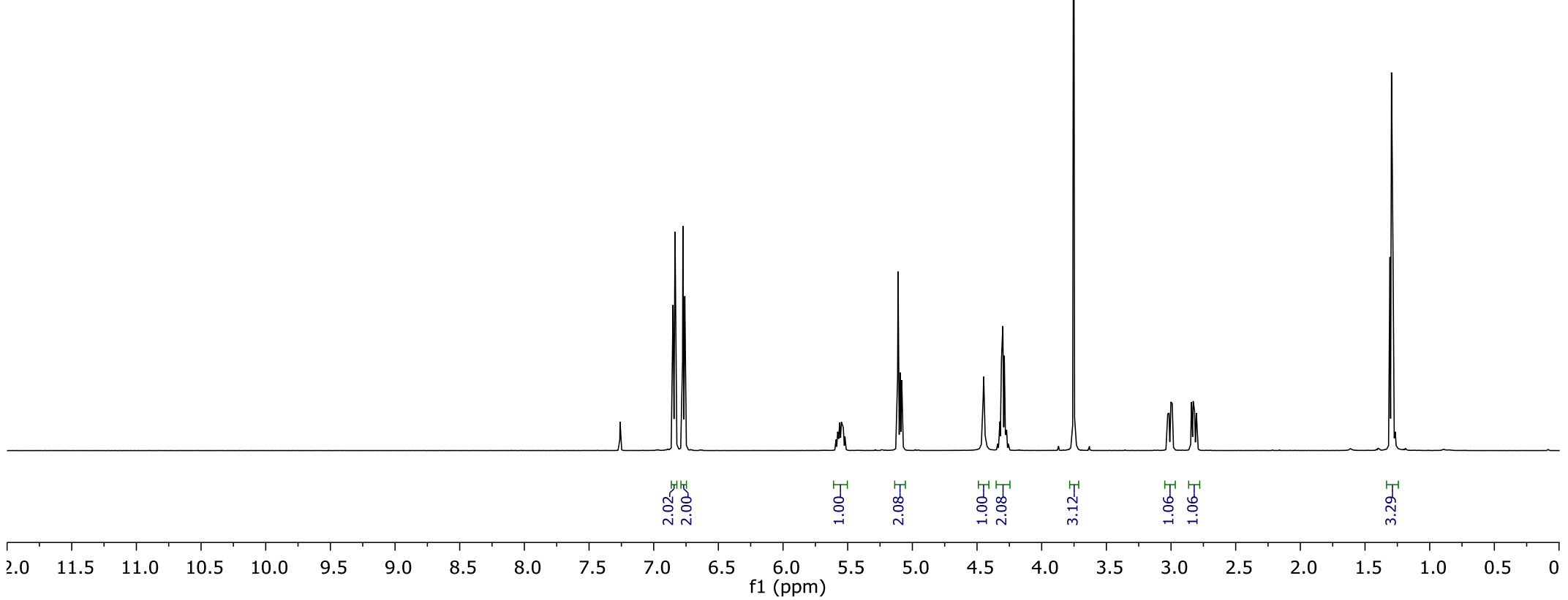




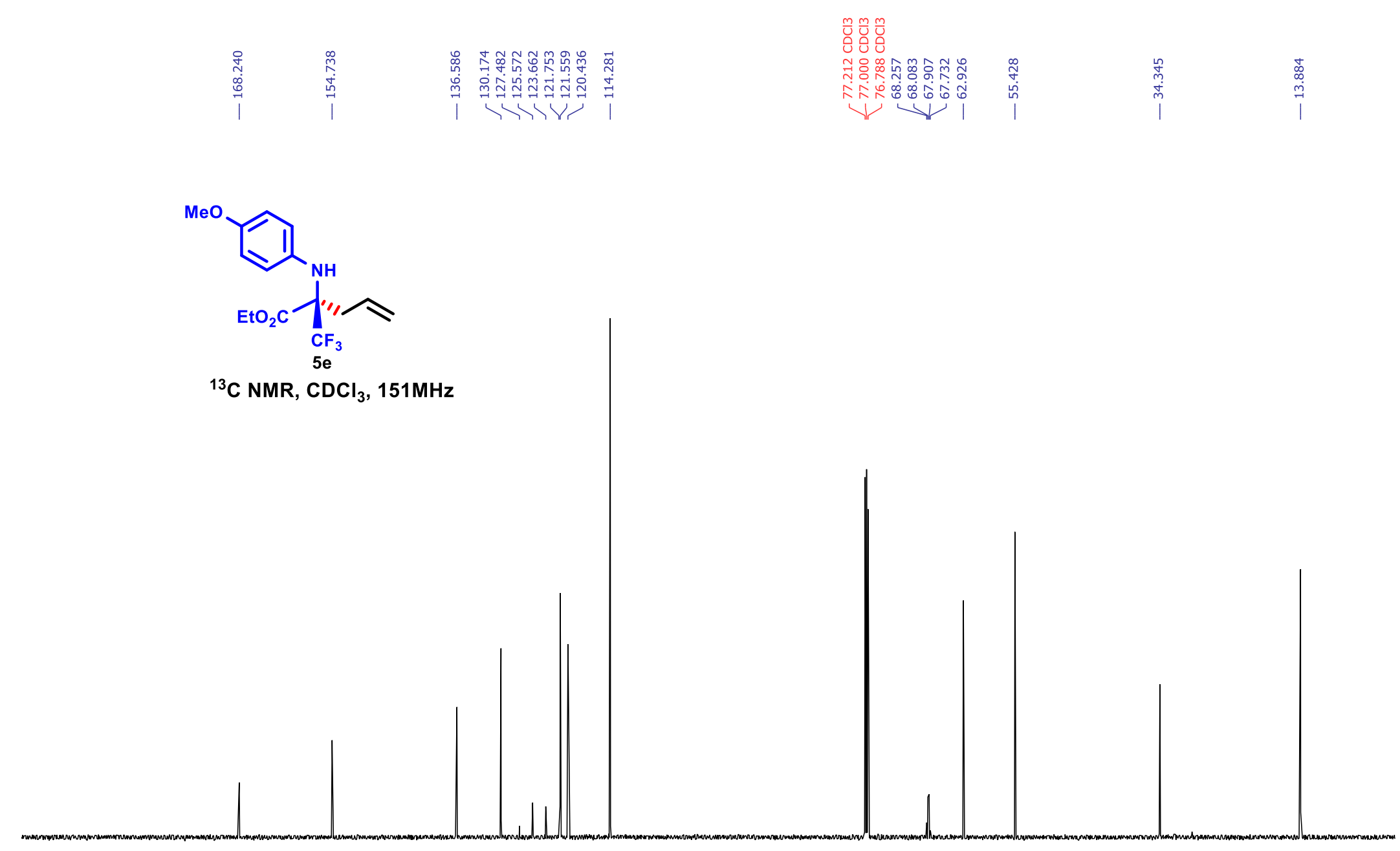


$\mathrm{MeO}$

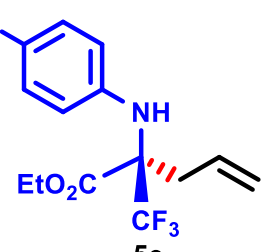

${ }^{19} \mathrm{~F} \mathrm{NMR}, \stackrel{5 \mathrm{e}}{\mathrm{CDCl}}{ }_{3}, 471 \mathrm{MHz}$

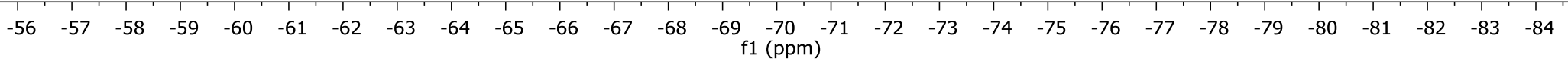



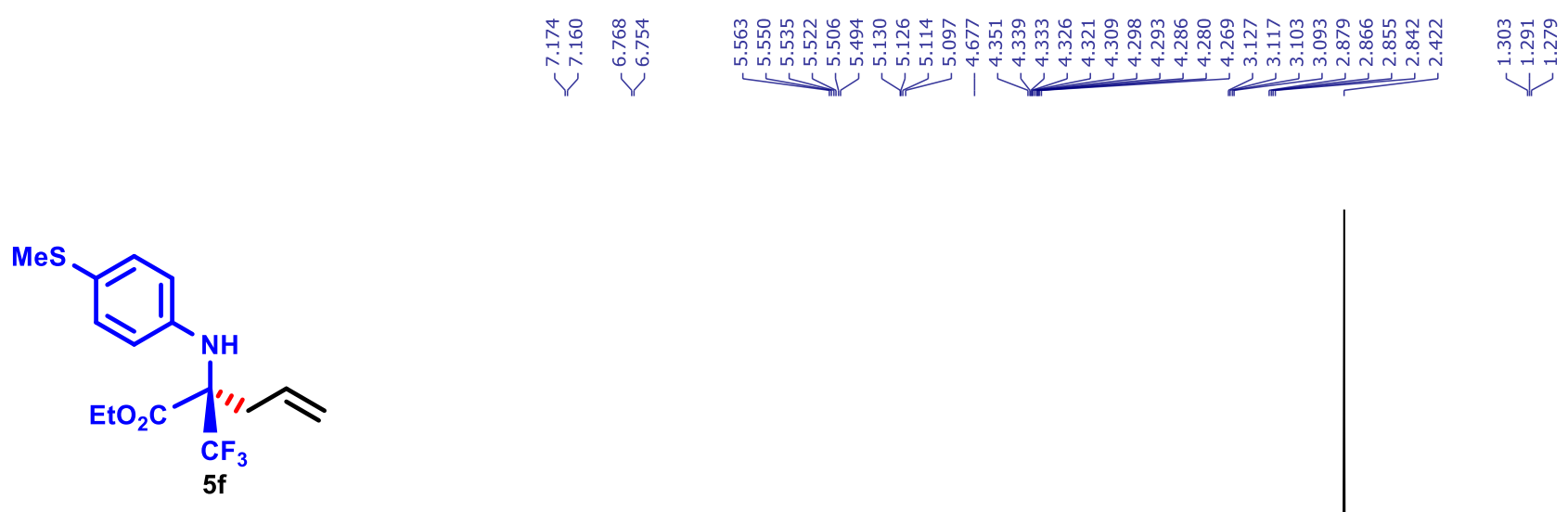

${ }^{1} \mathrm{H} \mathrm{NMR}, \mathrm{CDCl}_{3}, 600 \mathrm{MHz}$

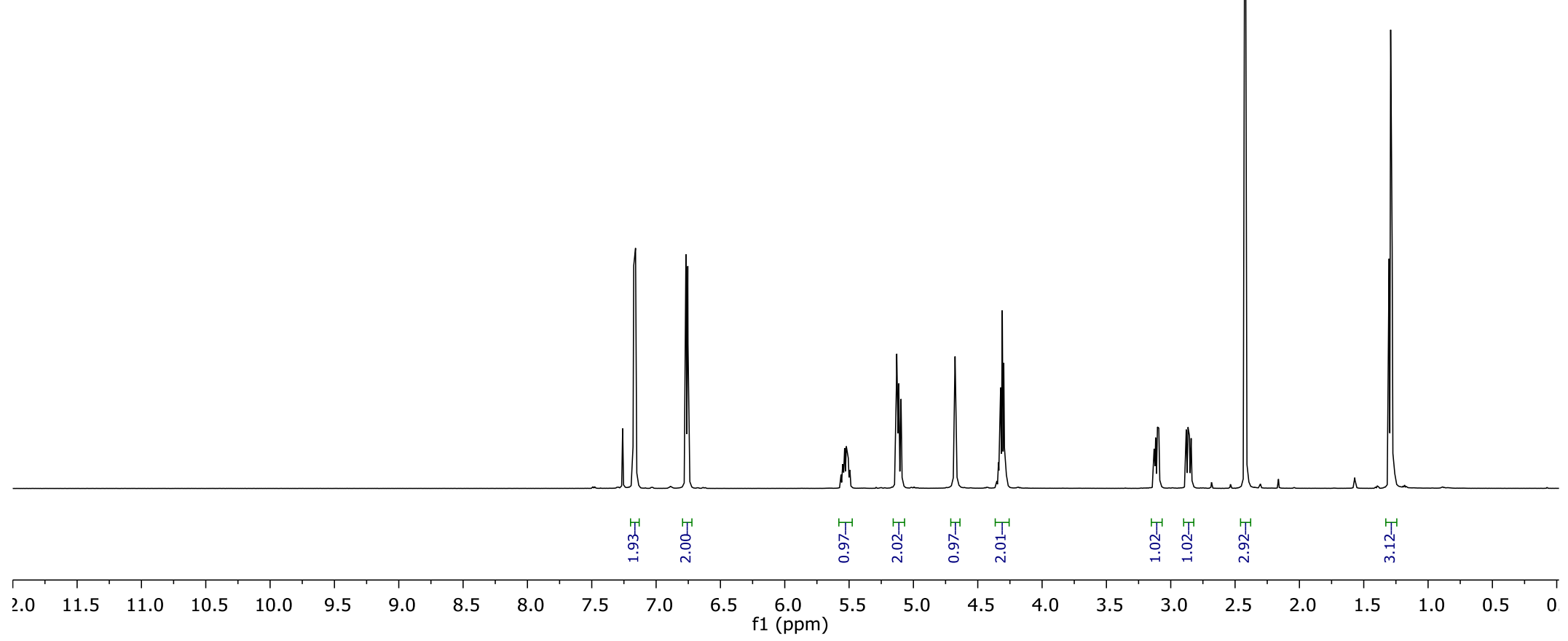




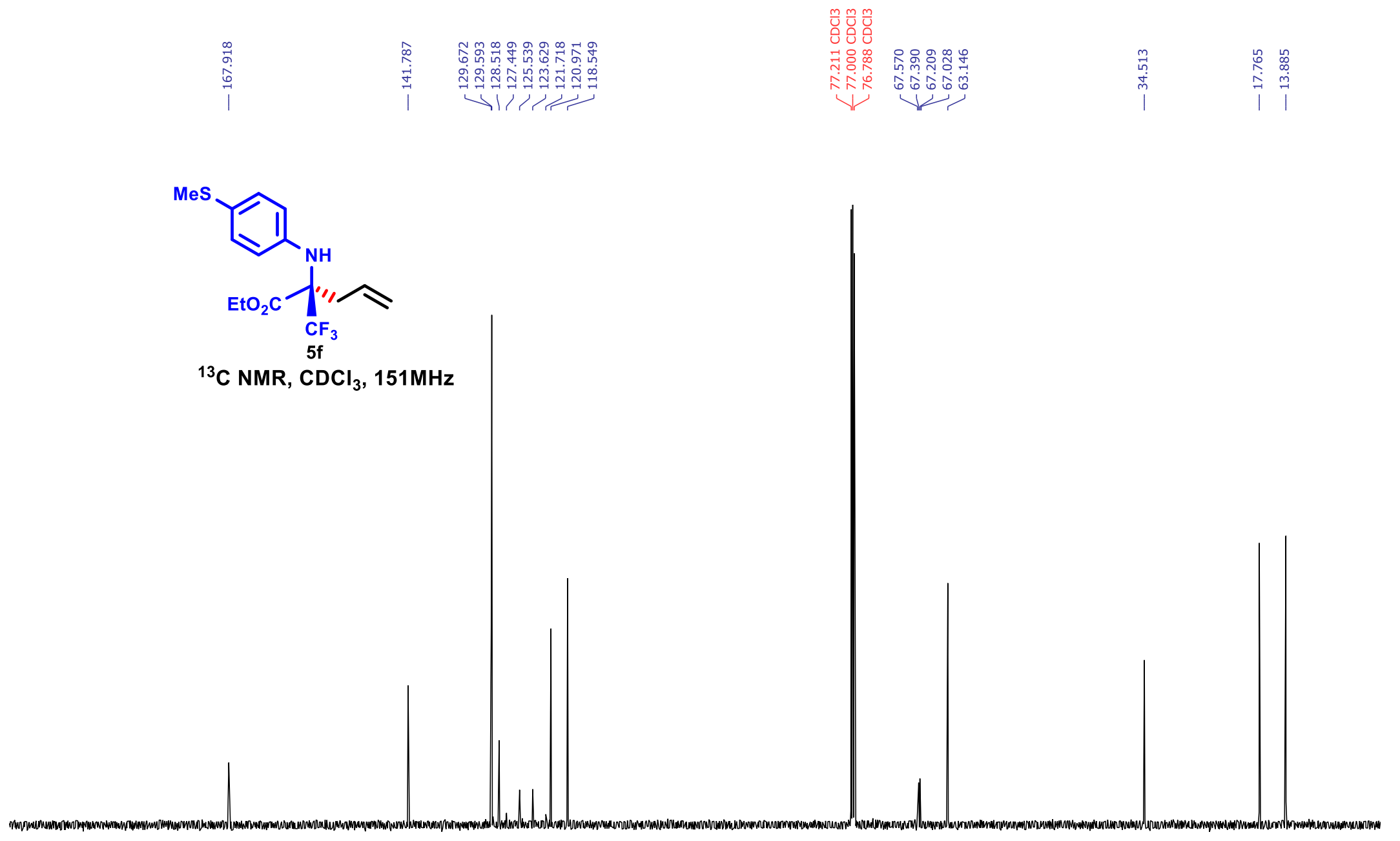


MeS

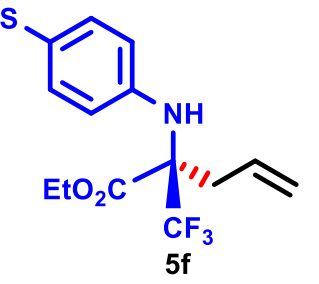

${ }^{19} \mathrm{~F} \mathrm{NMR}, \mathrm{CDCl}_{3}, 471 \mathrm{MHz}$

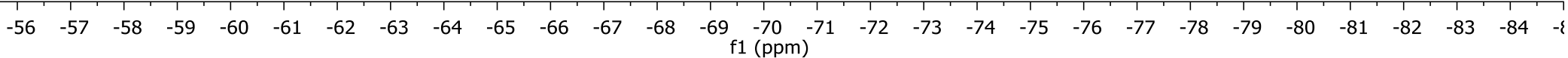




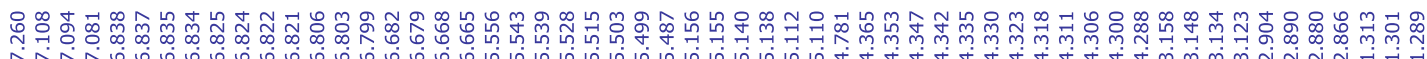

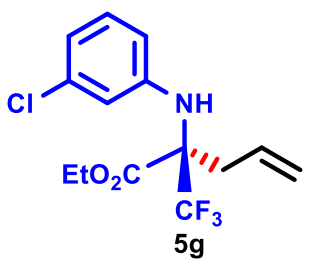

${ }^{1} \mathrm{H} \mathrm{NMR}, \mathrm{CDCl}_{3}, 600 \mathrm{MHz}$

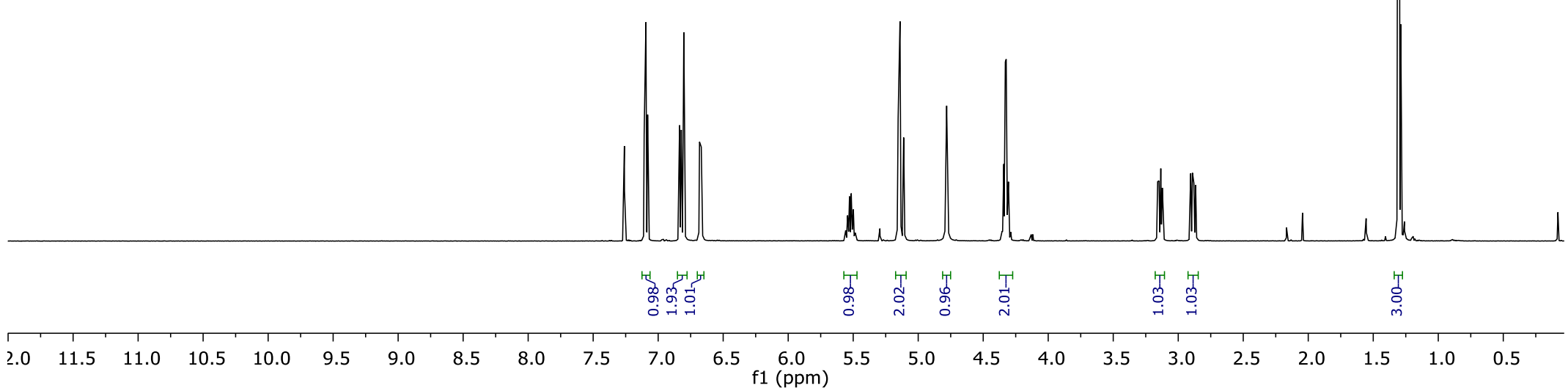




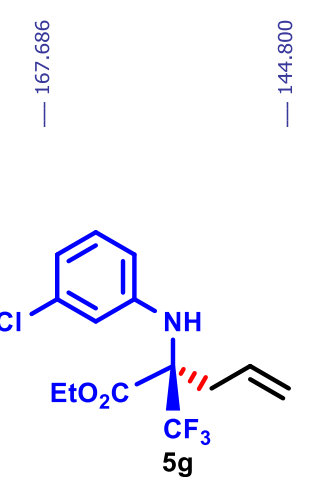

${ }^{13} \mathrm{C} \mathrm{NMR}, \mathrm{CDCl}_{3}, 151 \mathrm{MHz}$

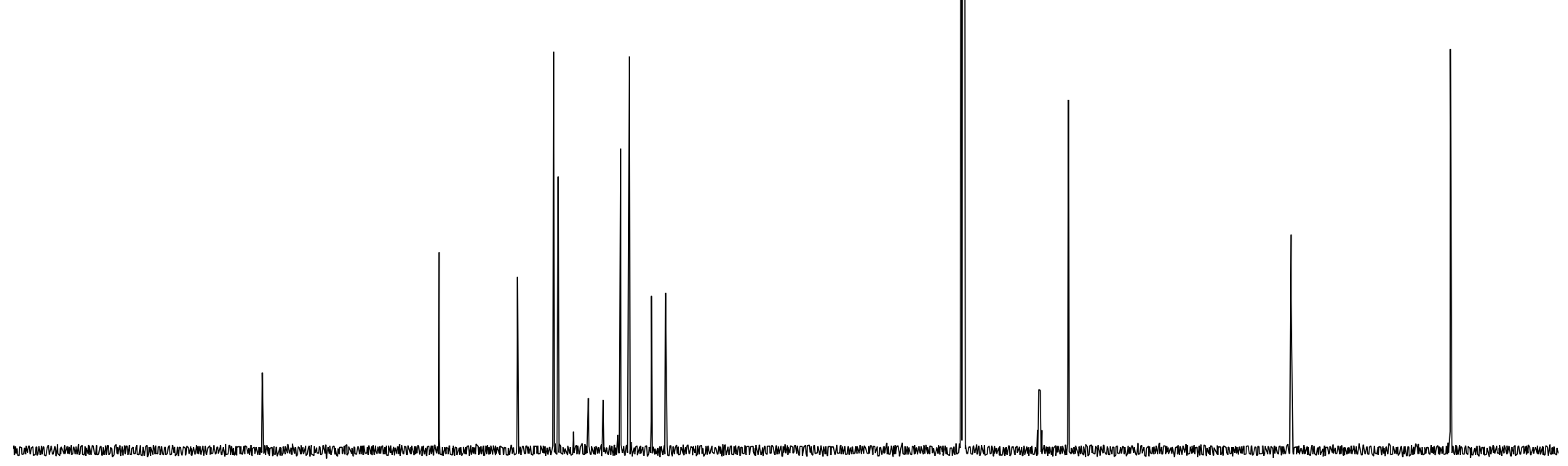




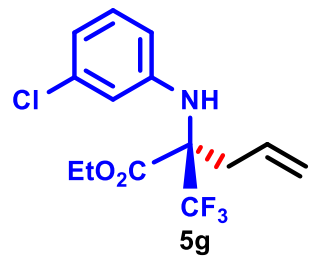

${ }^{19} \mathrm{~F} \mathrm{NMR,} \mathrm{CDCl}_{3}, 471 \mathrm{MHz}$

$\begin{array}{llllllllllllllllllllllllllllll}-56 & -57 & -58 & -59 & -60 & -61 & -62 & -63 & -64 & -65 & -66 & -67 & -68 & -69 & -70 & -71 & -72 & -73 & -74 & -75 & -76 & -77 & -78 & -79 & -80 & -81 & -82 & -83 & -84\end{array}$

\section{S165}




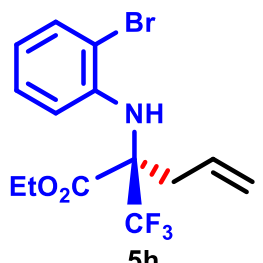

${ }^{1} \mathrm{H} \mathrm{NMR}, \mathrm{CDCl}_{3}, 600 \mathrm{MHz}$

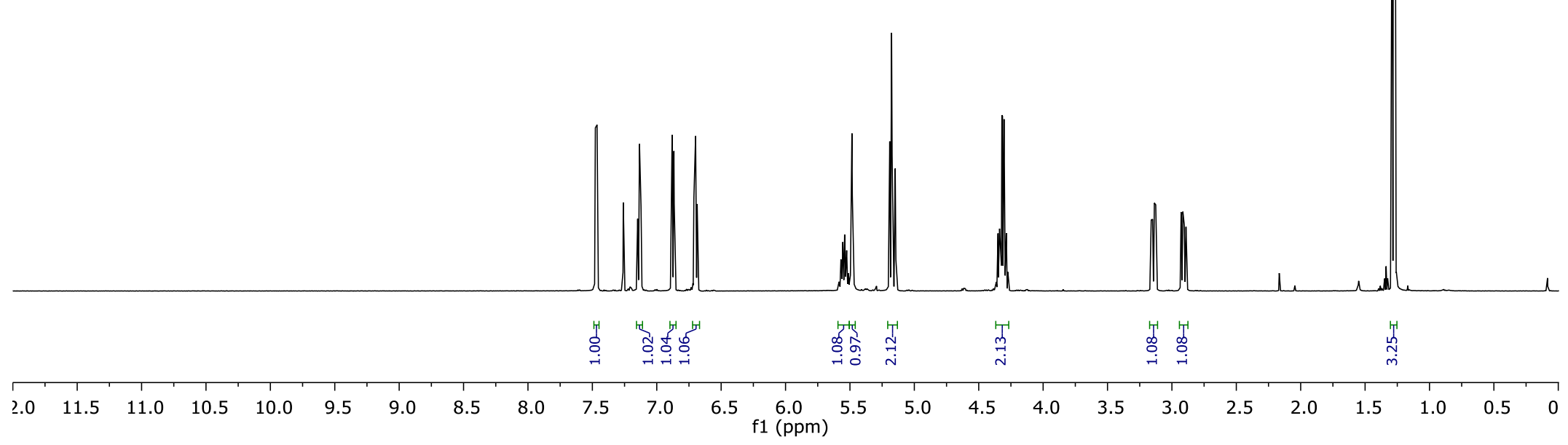



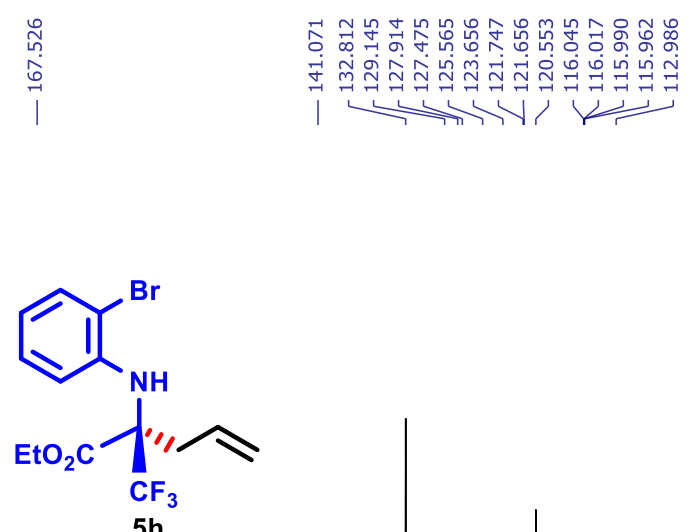

${ }^{13} \mathrm{C}$ NMR, $\stackrel{5 \mathrm{~h}}{\mathrm{CDCl}}{ }_{3}, 151 \mathrm{MHz}$

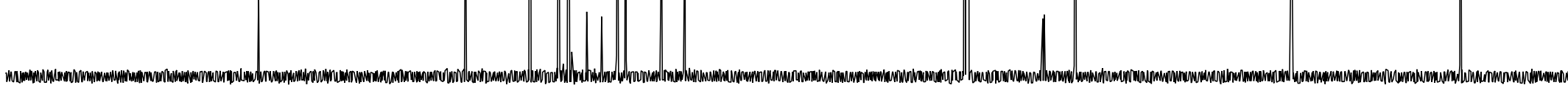




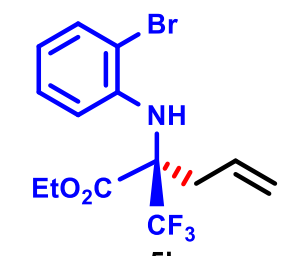

${ }^{19} \mathrm{~F} \mathrm{NMR}, \mathrm{CDCl}_{3}, 471 \mathrm{MHz}$

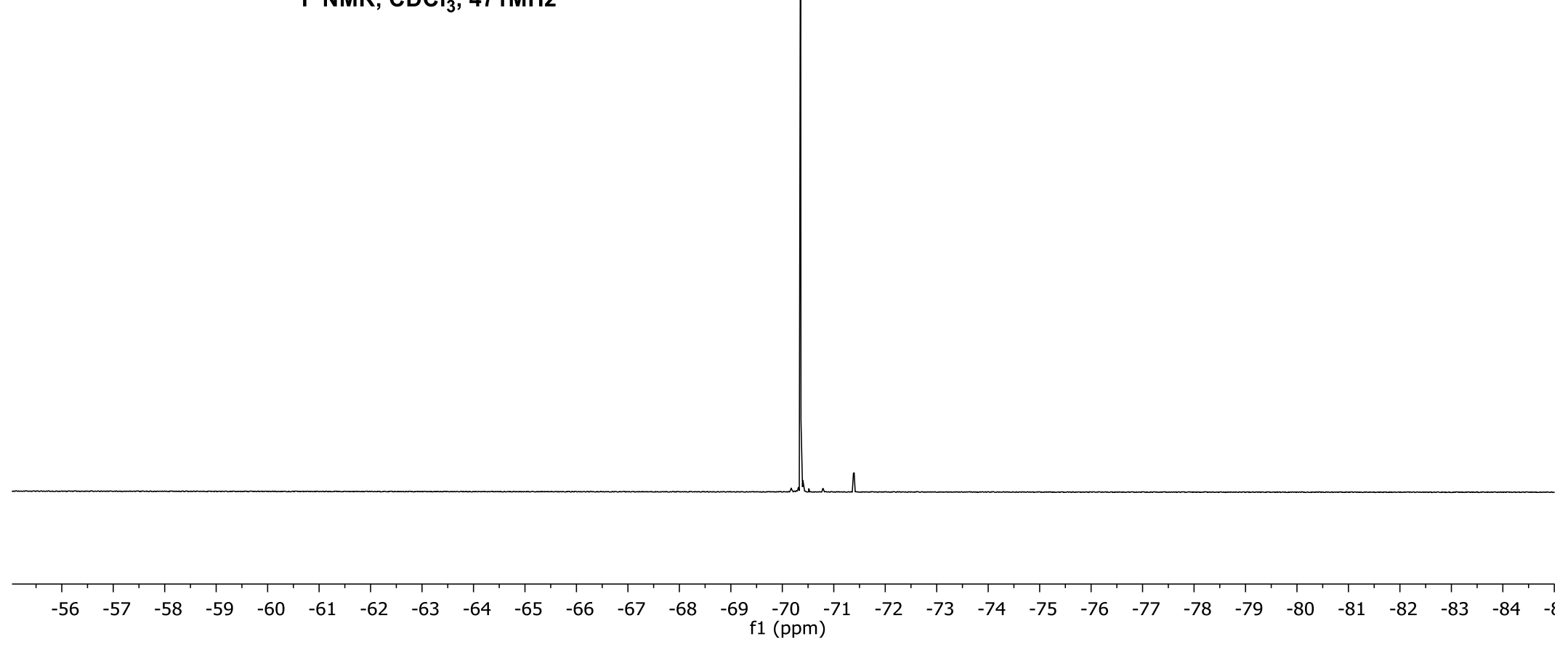




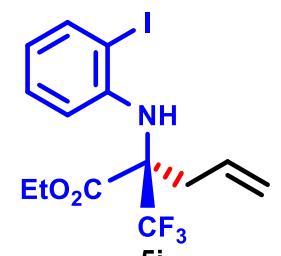

${ }^{1} \mathrm{H}$ NMR, $\mathrm{CDCl}_{3}, 600 \mathrm{MHz}$

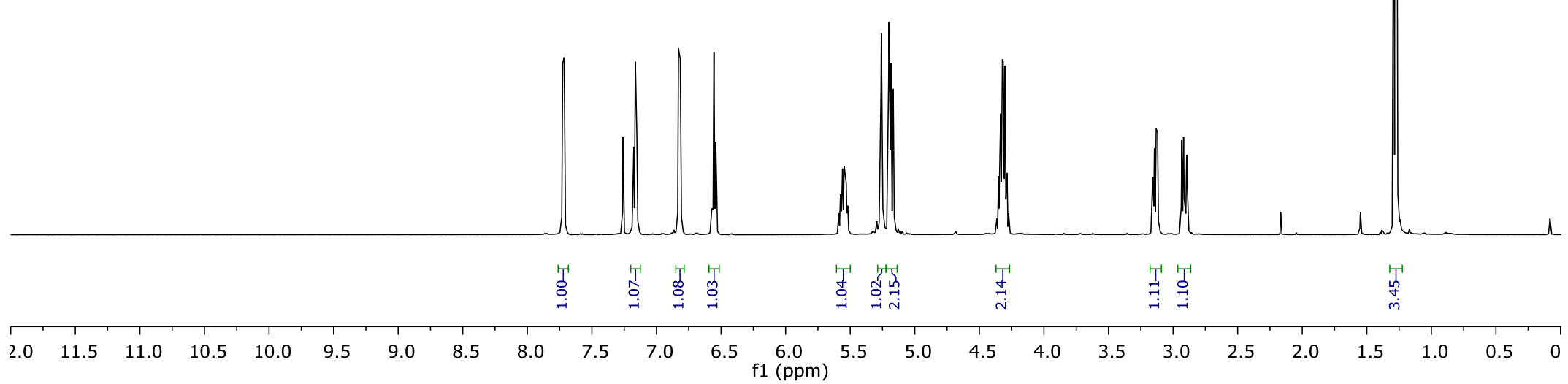




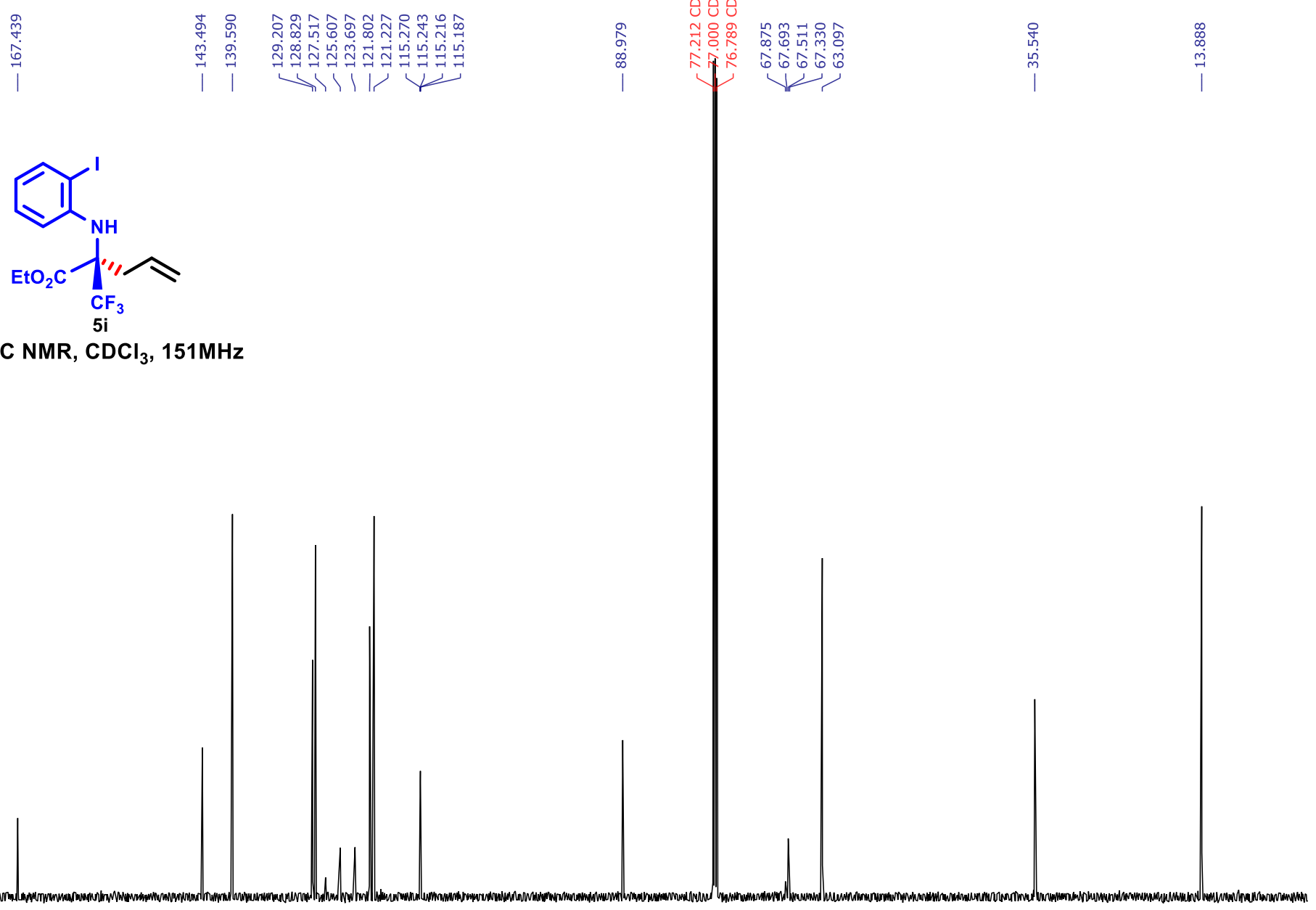

190

18 


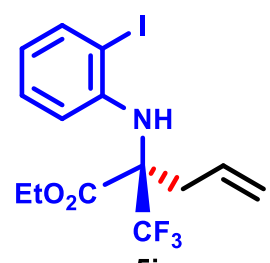

${ }^{19} \mathrm{~F} \mathrm{NMR,} \mathrm{CDCl}_{3}, 471 \mathrm{MHz}$

\begin{tabular}{llllllllllllllllllllllllllllllllllllllllllllllll}
\hline 55 & -56 & -57 & -58 & -59 & -60 & -61 & -62 & -63 & -64 & -65 & -66 & -67 & -68 & -69 & -70 & -71 & -72 & -73 & -74 & -75 & -76 & -77 & -78 & -79 & -80 & -81 & -82 & -83 & -84 & -8
\end{tabular} 

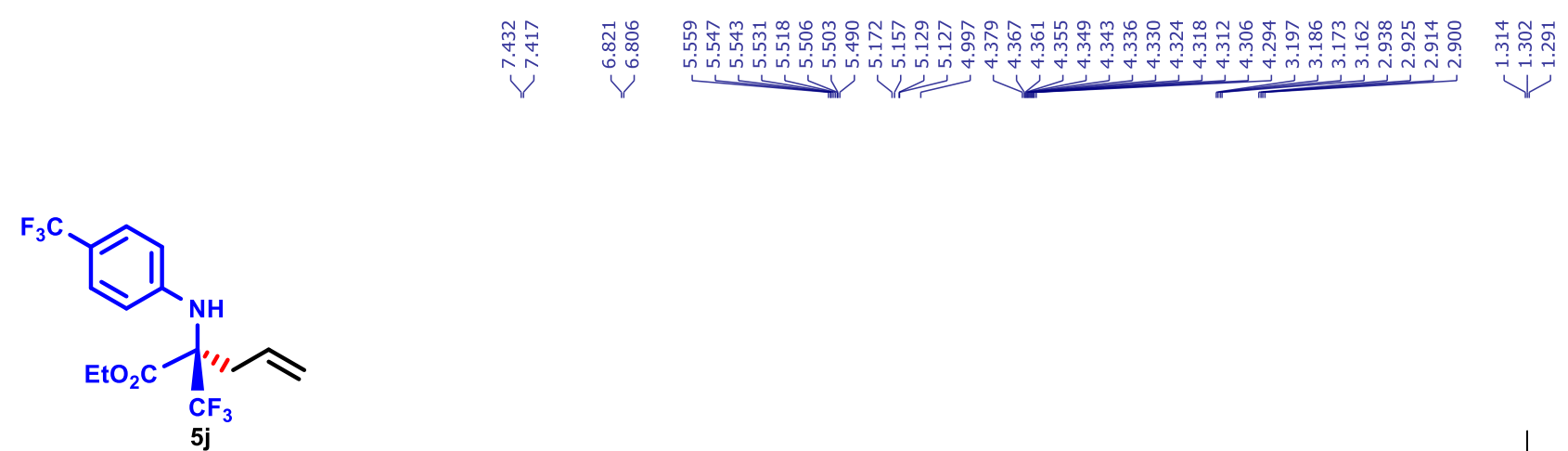

${ }^{1} \mathrm{H} \mathrm{NMR}, \mathrm{CDCl}_{3}, 600 \mathrm{MHz}$

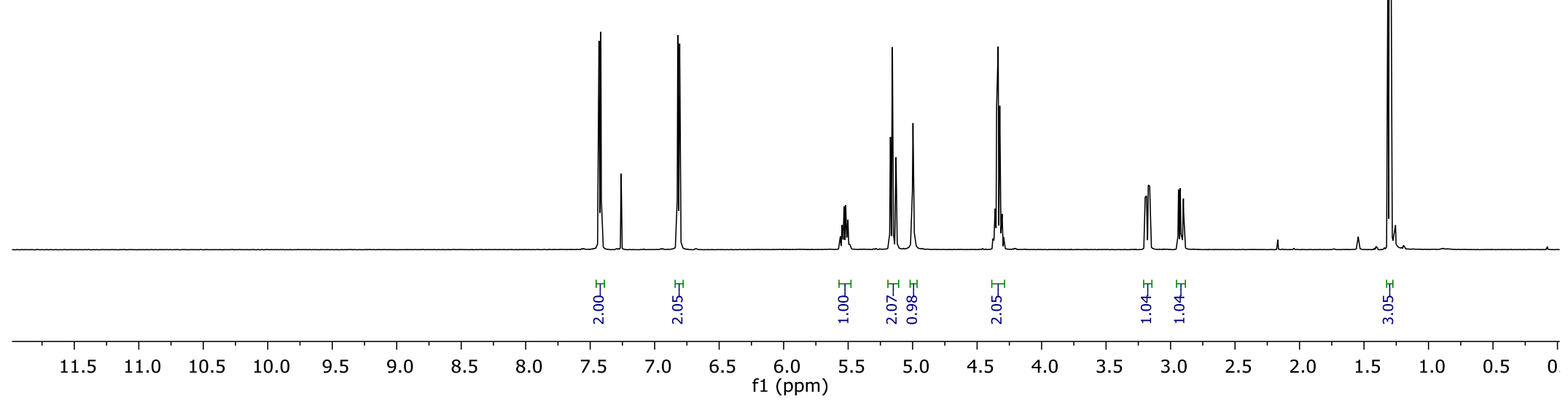



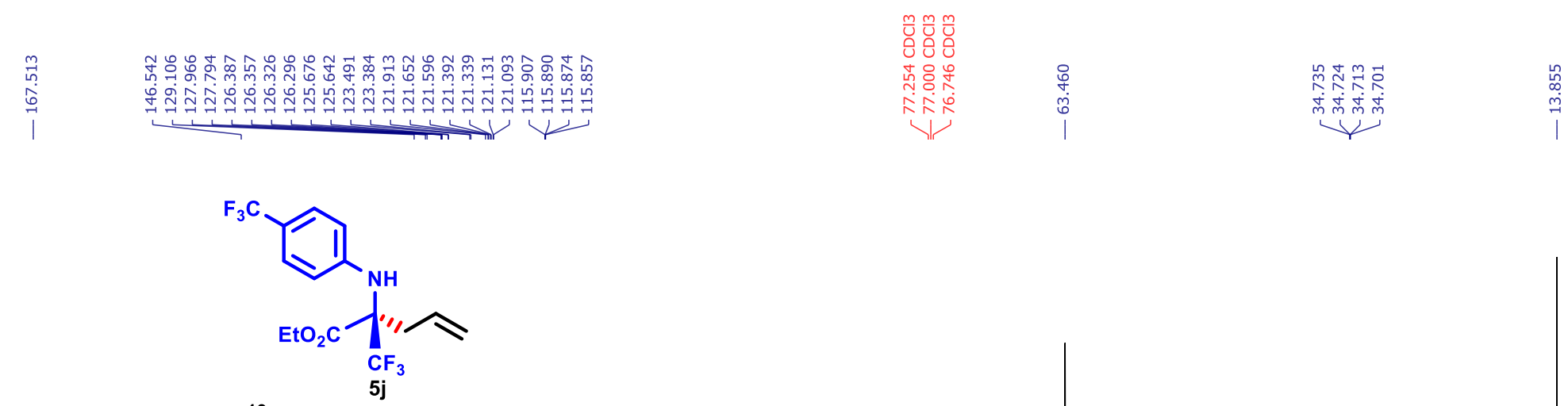

${ }^{13} \mathrm{C} \mathrm{NMR}, \mathrm{CDCl}_{3}, 151 \mathrm{MHz}$

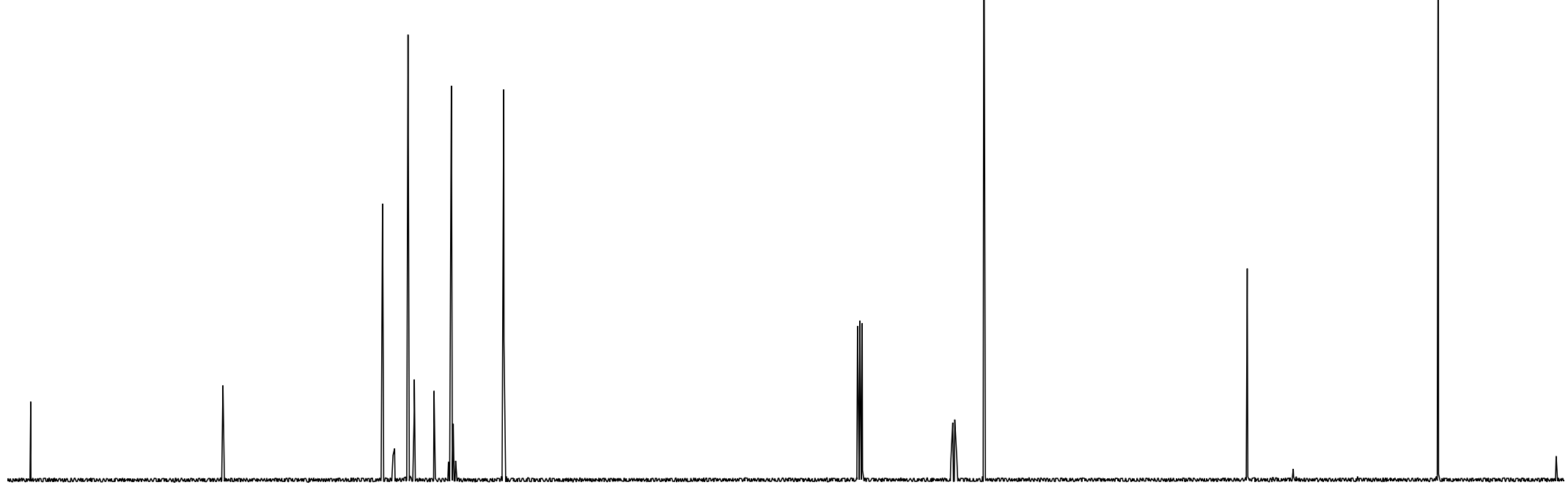

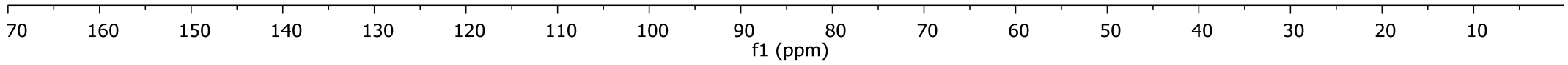




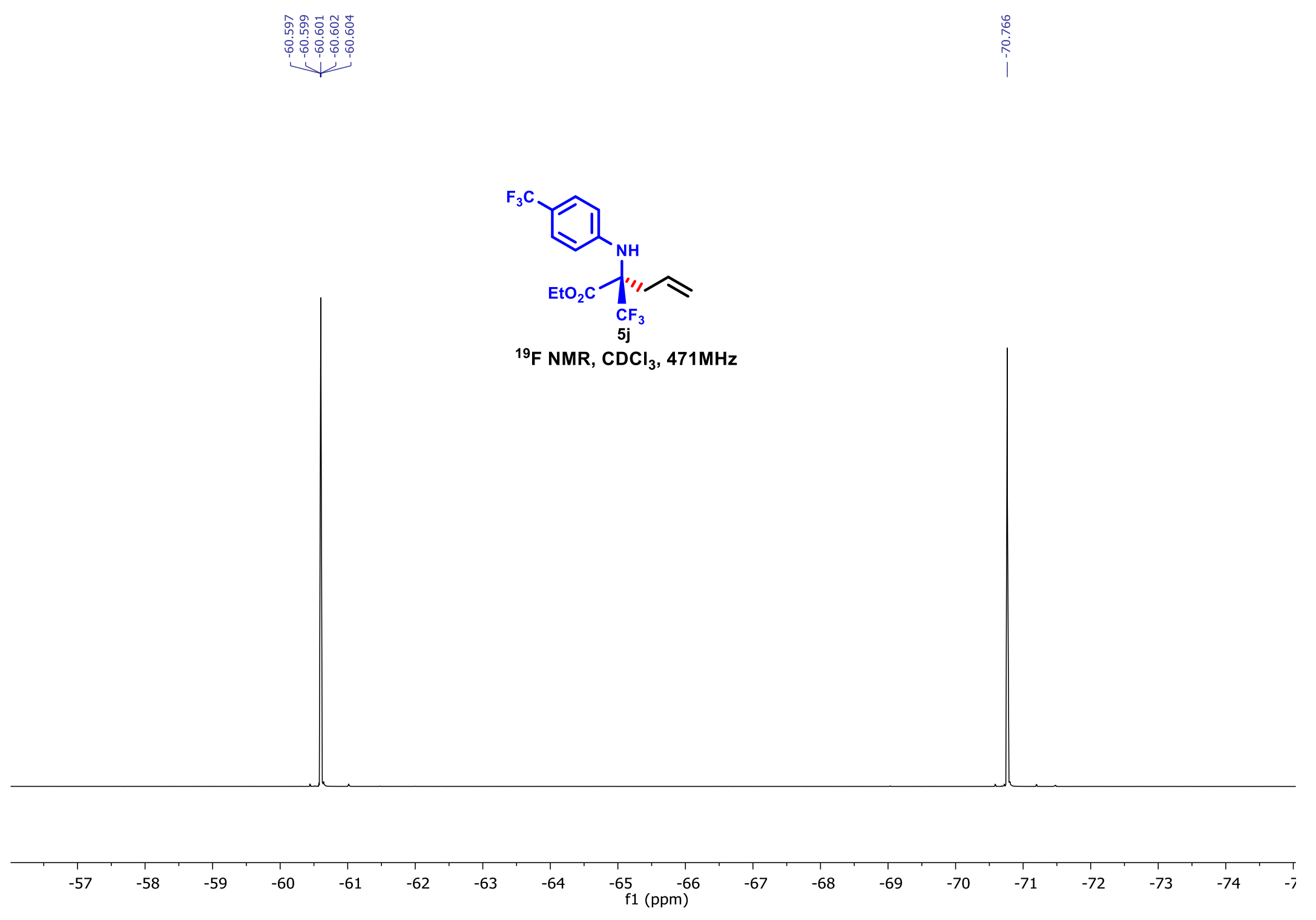




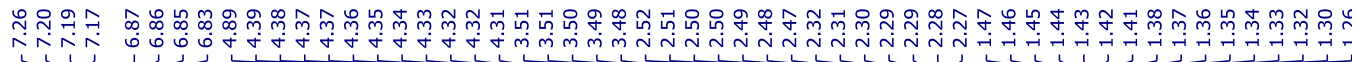

证

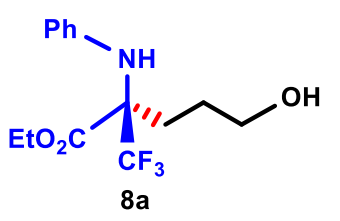

${ }^{1} \mathrm{H}$ NMR, $\mathrm{CDCl}_{3}, 600 \mathrm{MHz}$

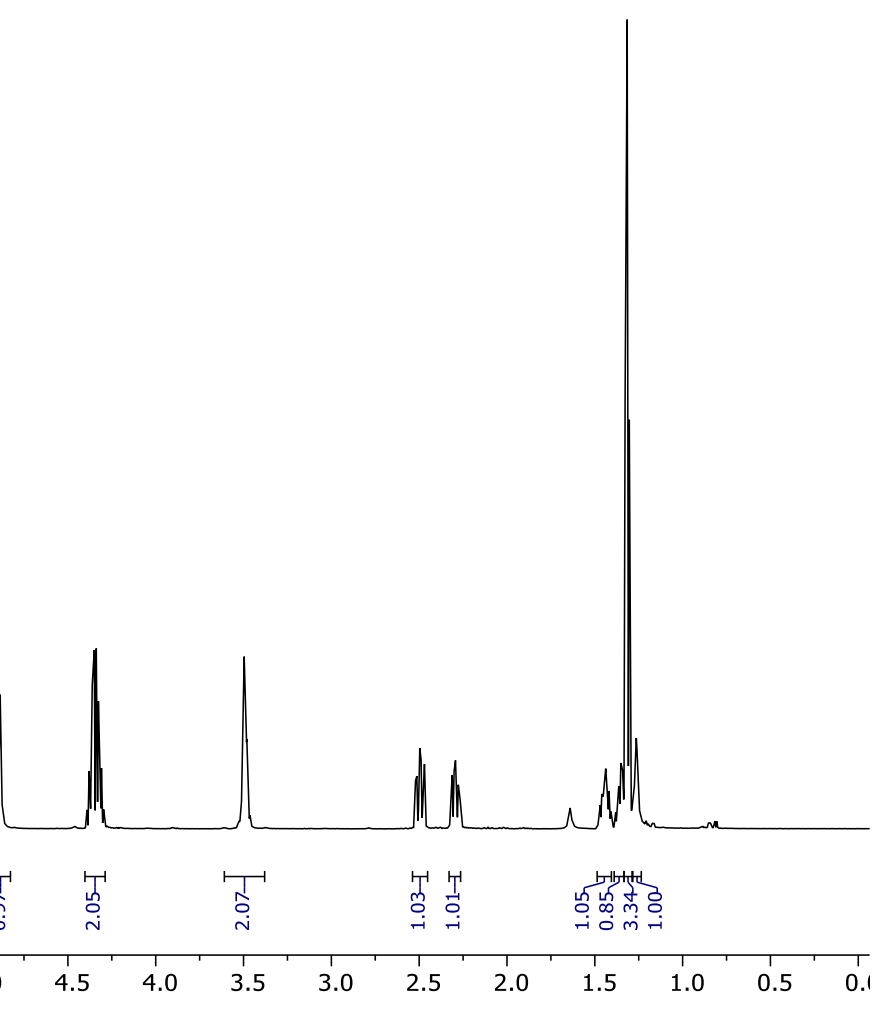




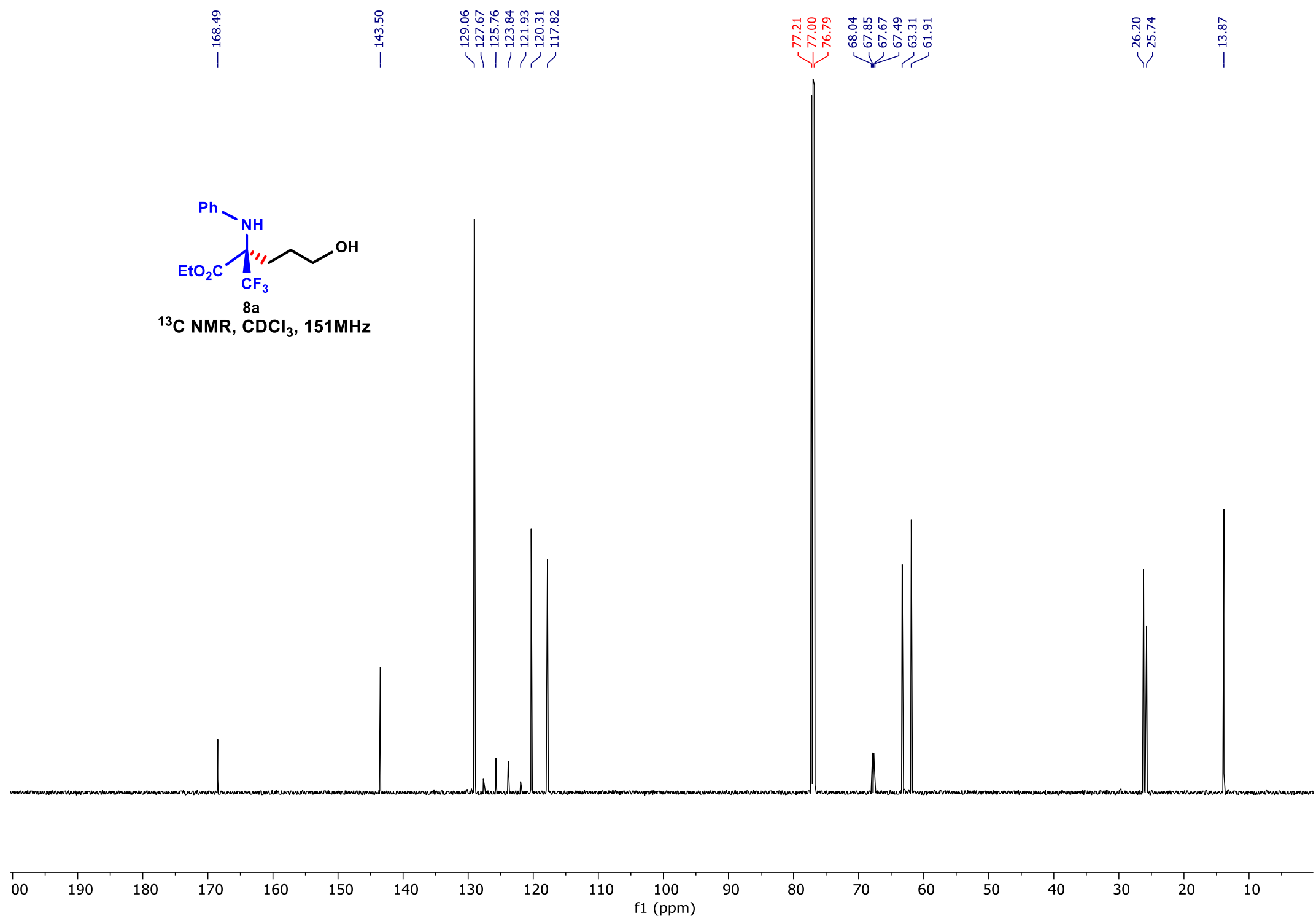




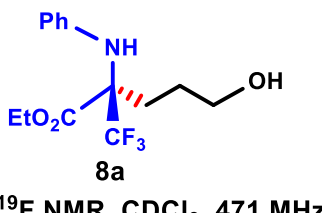

${ }^{19} \mathrm{~F}$ NMR, $\mathrm{CDCl}_{3}, 471 \mathrm{MHz}$ 
Me

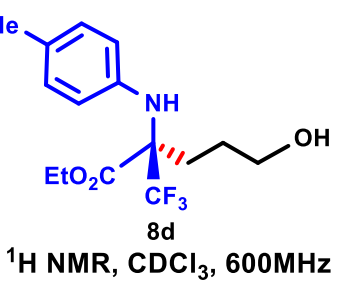

${ }^{1} \mathrm{H} \mathrm{NMR}, \mathrm{CDCl}_{3}, 600 \mathrm{MHz}$

\begin{tabular}{|c|c|c|c|c|c|c|c|c|c|c|c|c|c|c|c|c|c|c|c|c|c|c|}
\hline 11.5 & 11.0 & 10.5 & 10.0 & 9.5 & 9.0 & 8.5 & 8.0 & 7.5 & 7.0 & 6.5 & $\begin{array}{c}6.0 \\
\mathrm{f} 1(\mathrm{ppm})\end{array}$ & 5.5 & 5.0 & 4.5 & 4.0 & 3.5 & 3.0 & 2.5 & 2.0 & 1.5 & 1.0 & 0.5 \\
\hline
\end{tabular}




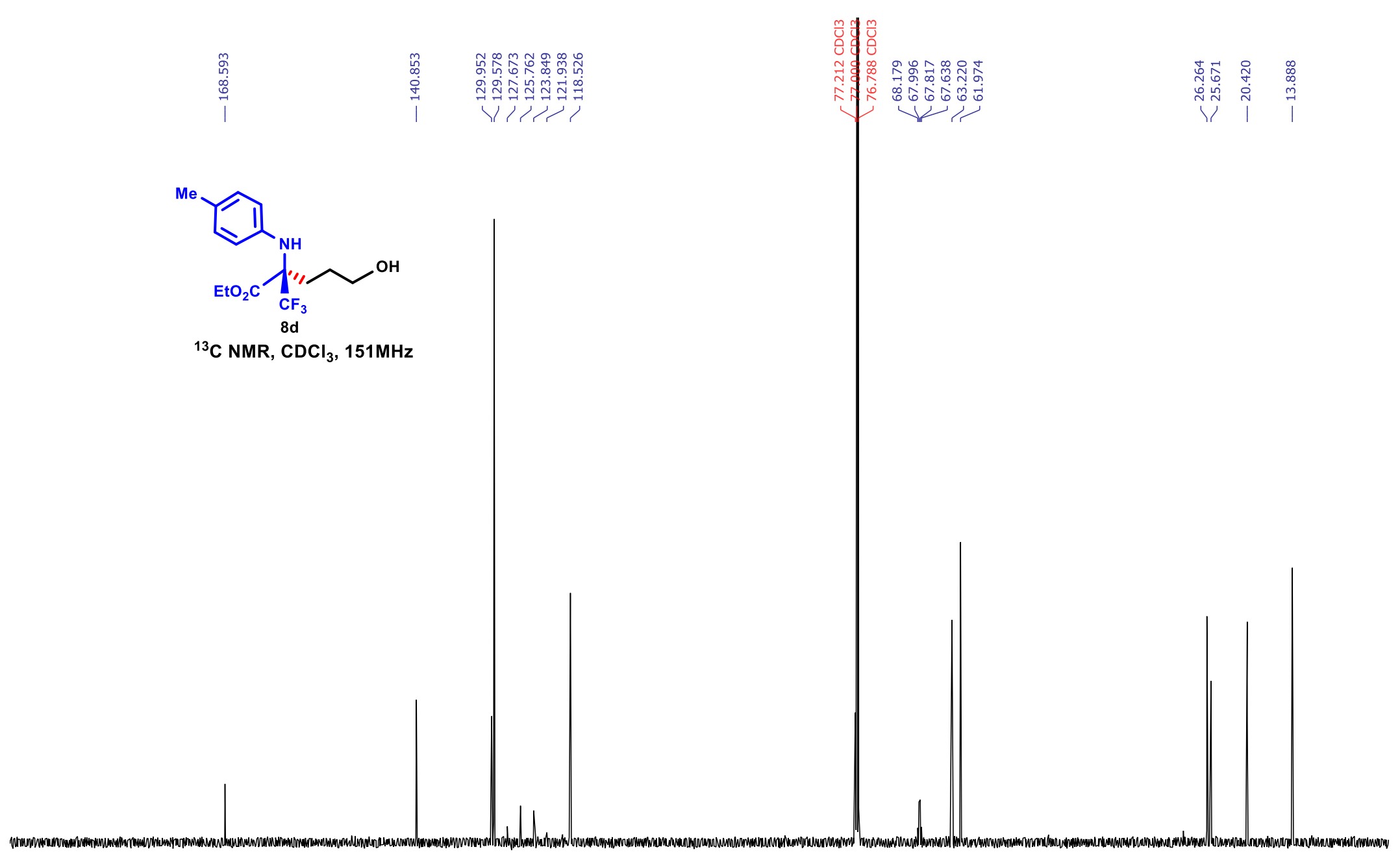

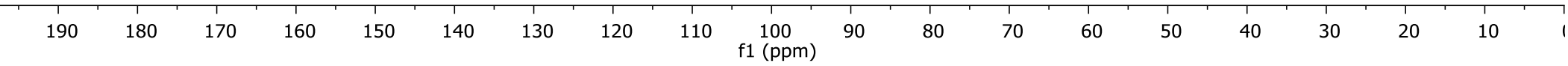




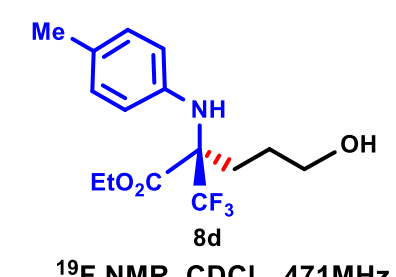

${ }^{19} \mathrm{~F} \mathrm{NMR}, \mathrm{CDCl}_{3}, 471 \mathrm{MHz}$

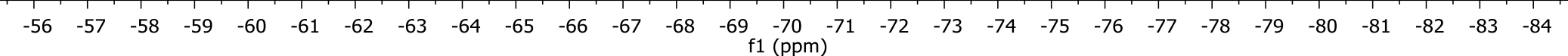




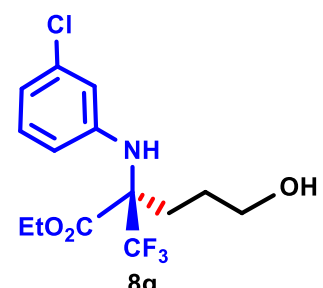

${ }^{1} \mathrm{H}$ NMR, $\mathrm{CDCl}_{3}, 600 \mathrm{MHz}$

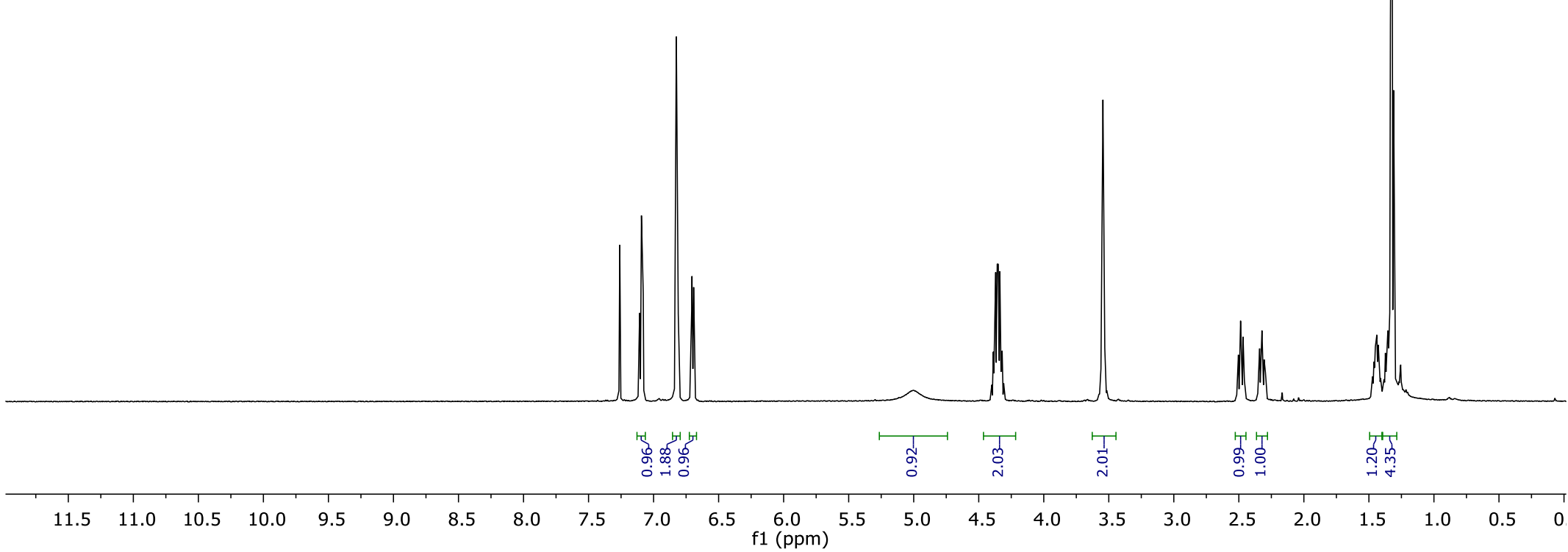



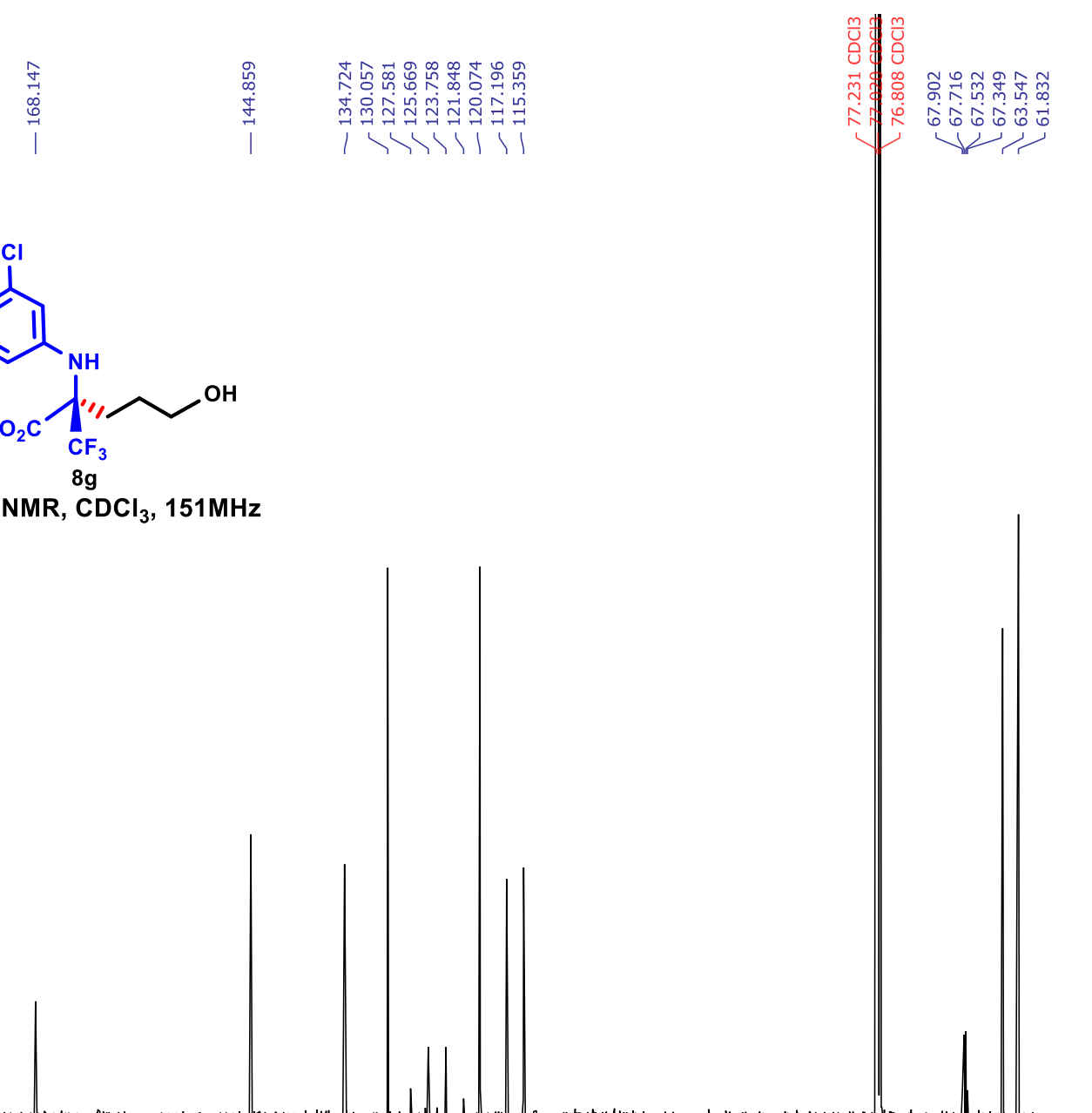

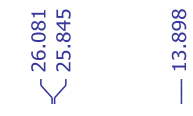

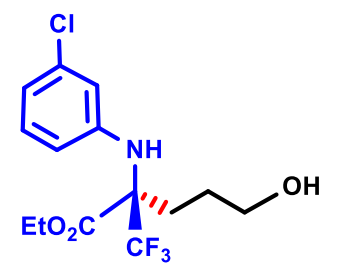

${ }^{13} \mathrm{C} \mathrm{NMR}^{8 \mathrm{~g}} \mathrm{CDCl}_{3}, 151 \mathrm{MHz}$

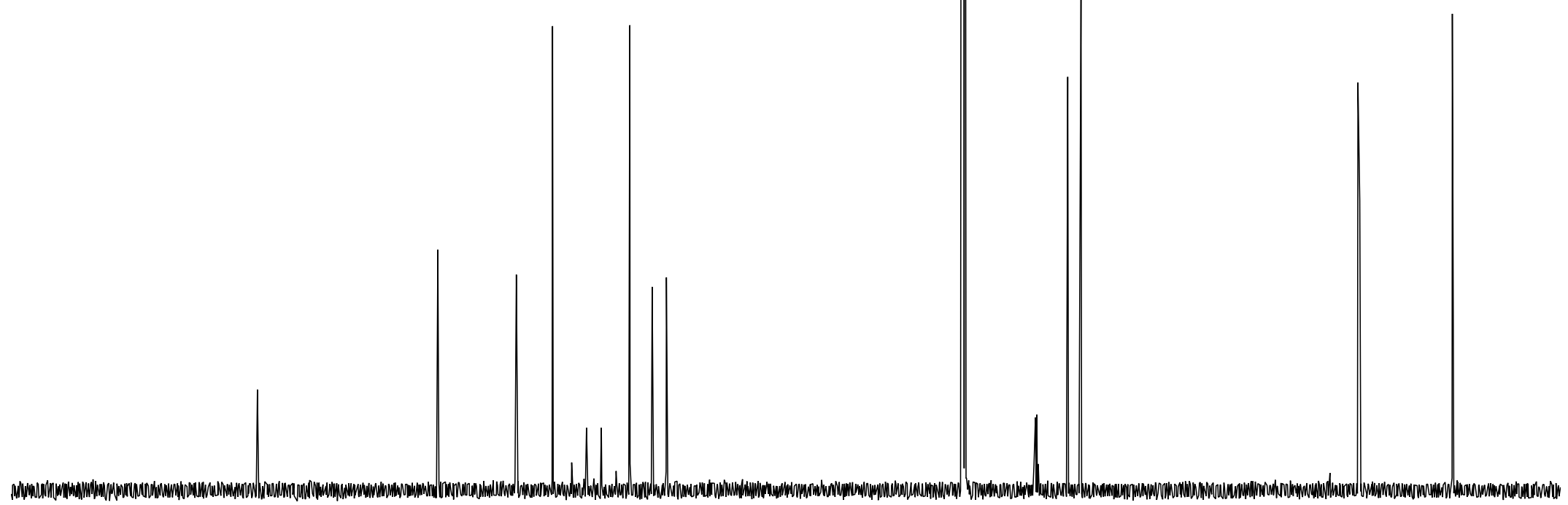

160

150

140

130

120

110

100
$\mathrm{f} 1(\mathrm{ppm})$

90

80

70

60

50

40

30

20

10 


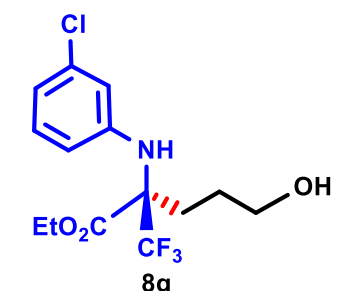

${ }^{19} \mathrm{~F} \mathrm{NMR}, \mathrm{CDCl}_{3}, 471 \mathrm{MHz}$

$\begin{array}{llllllllllllllllllllllllllllllllllllll}-56 & -57 & -58 & -59 & -60 & -61 & -62 & -63 & -64 & -65 & -66 & -67 & -68 & -69 & -70 & -71 & -72 & -73 & -74 & -75 & -76 & -77 & -78 & -79 & -80 & -81 & -82 & -83 & -84\end{array}$ 


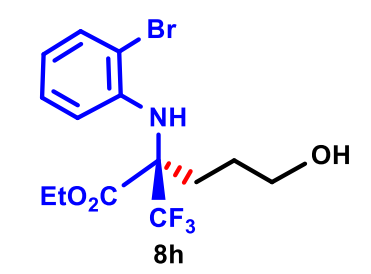

${ }^{1} \mathrm{H} \mathrm{NMR}, \mathrm{CDCl}_{3}, 600 \mathrm{MHz}$

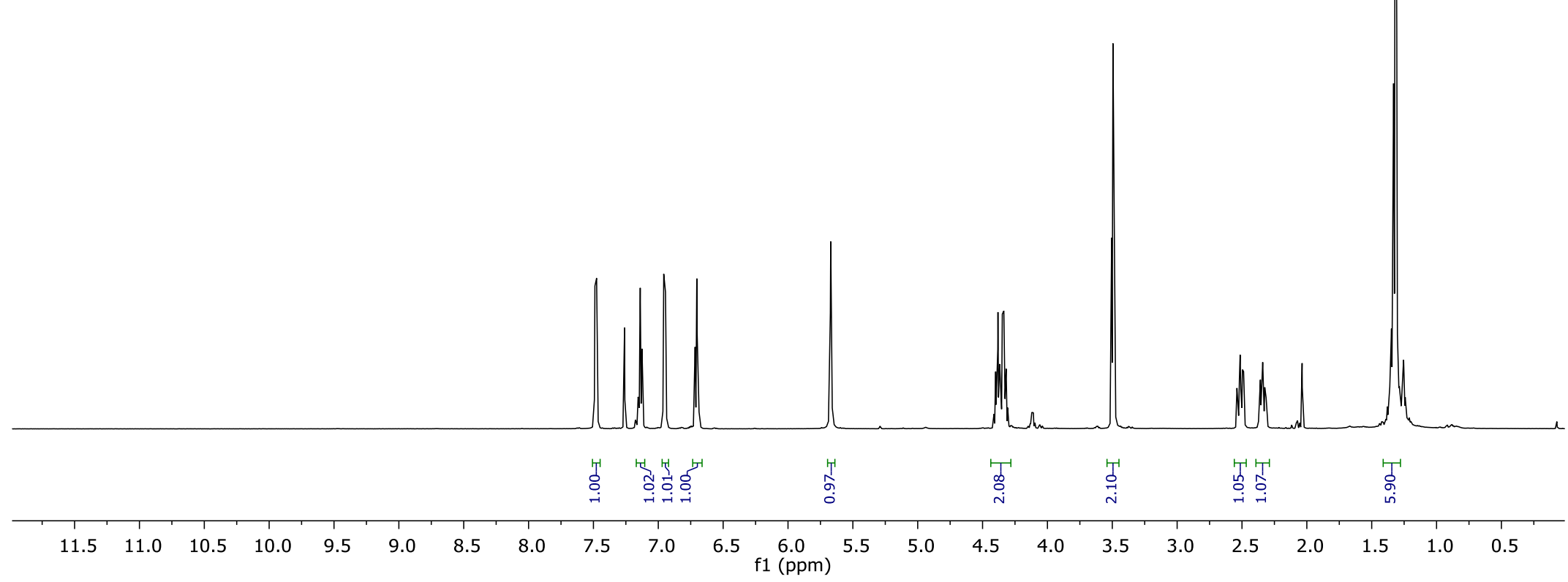



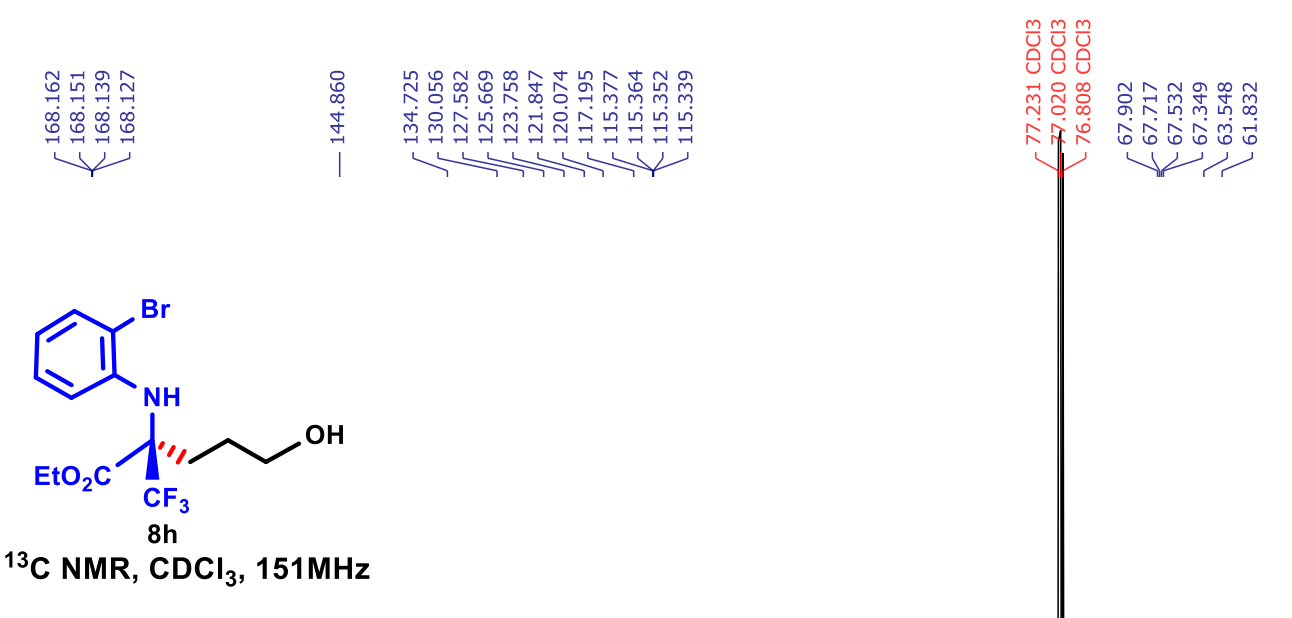

${ }^{13} \mathrm{C} \mathrm{NMR,} \mathrm{CDCl}_{3}, 151 \mathrm{MHz}$

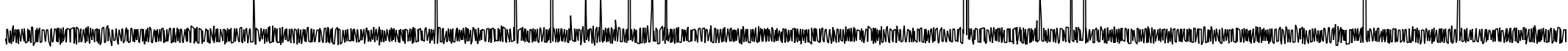

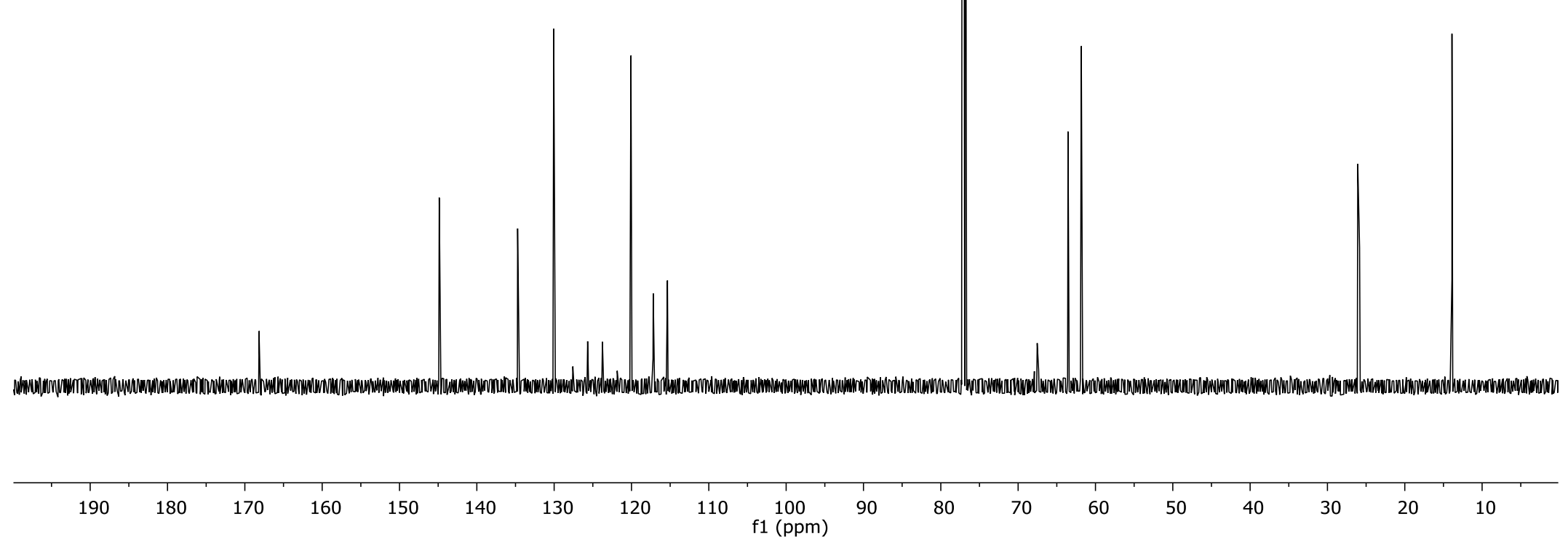




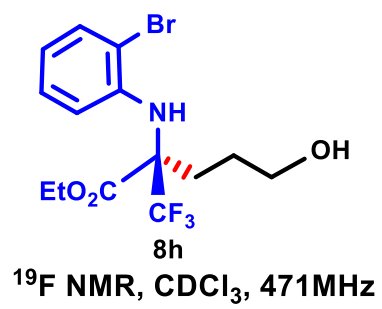

${ }^{19} \mathrm{~F} \mathrm{NMR,} \mathrm{CDCl}_{3}, 471 \mathrm{MHz}$

$\begin{array}{lllllllllllllllllllllllllllllllllll}-56 & -57 & -58 & -59 & -60 & -61 & -62 & -63 & -64 & -65 & -66 & -67 & -68 & -69 & -70 & -71 & -72 & -73 & -74 & -75 & -76 & -77 & -78 & -79 & -80 & -81 & -82 & -83 & -84 & -8\end{array}$ 


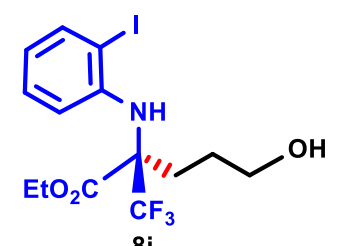

${ }^{1} \mathrm{H} \mathrm{NMR}, \mathrm{CDCl}_{3}, 600 \mathrm{MHz}$

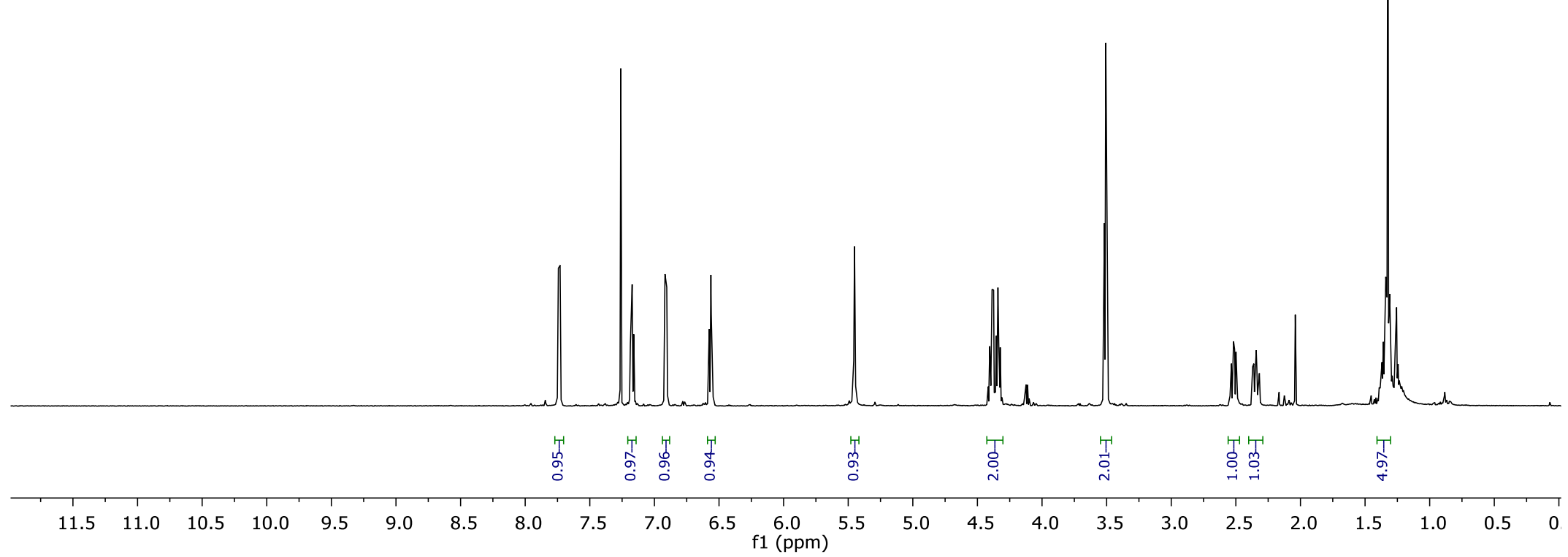




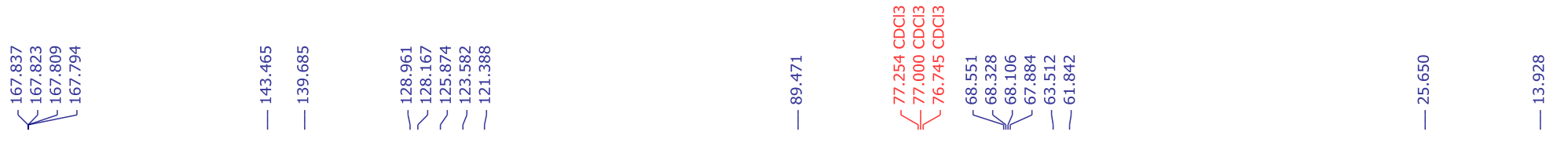

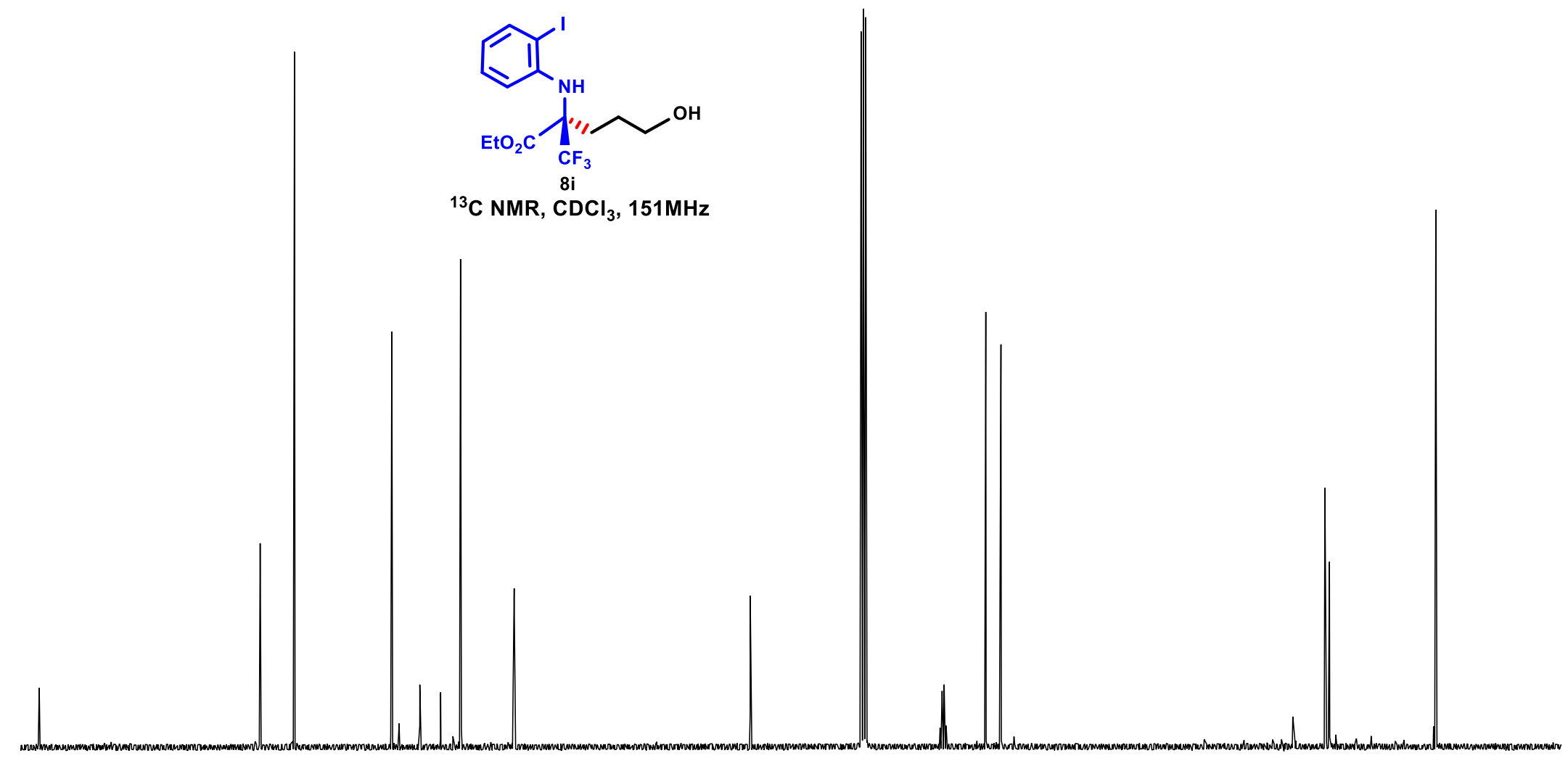

120

110

100

90 f1 (ppm)

70

60

50

40

30

20

10 


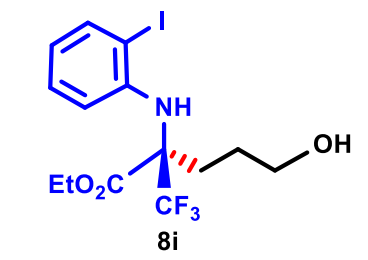

${ }^{19} \mathrm{~F} \mathrm{NMR,} \mathrm{CDCl}_{3}, 471 \mathrm{MHz}$

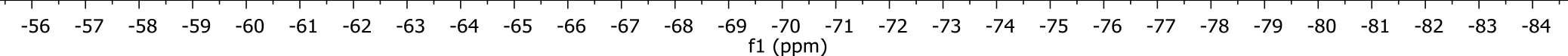




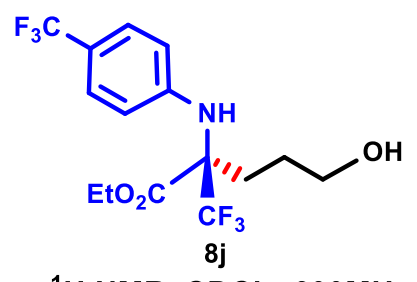

${ }^{1} \mathrm{H}$ NMR, $\mathrm{CDCl}_{3}, 600 \mathrm{MHz}$

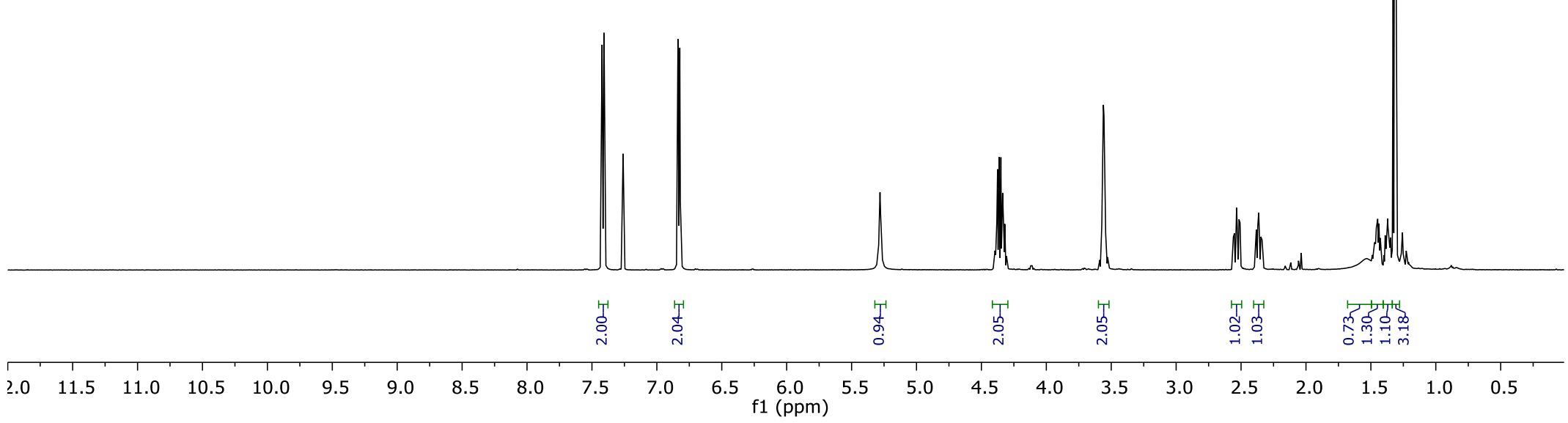




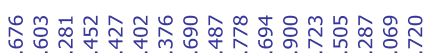

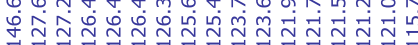

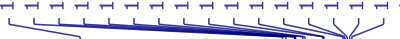

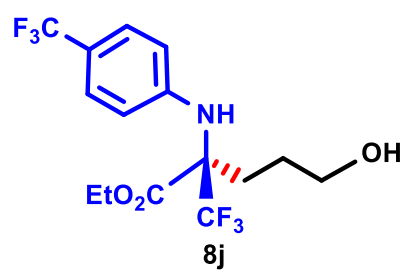

${ }^{13} \mathrm{C} \mathrm{NMR}, \mathrm{CDCl}_{3}, 151 \mathrm{MHz}$

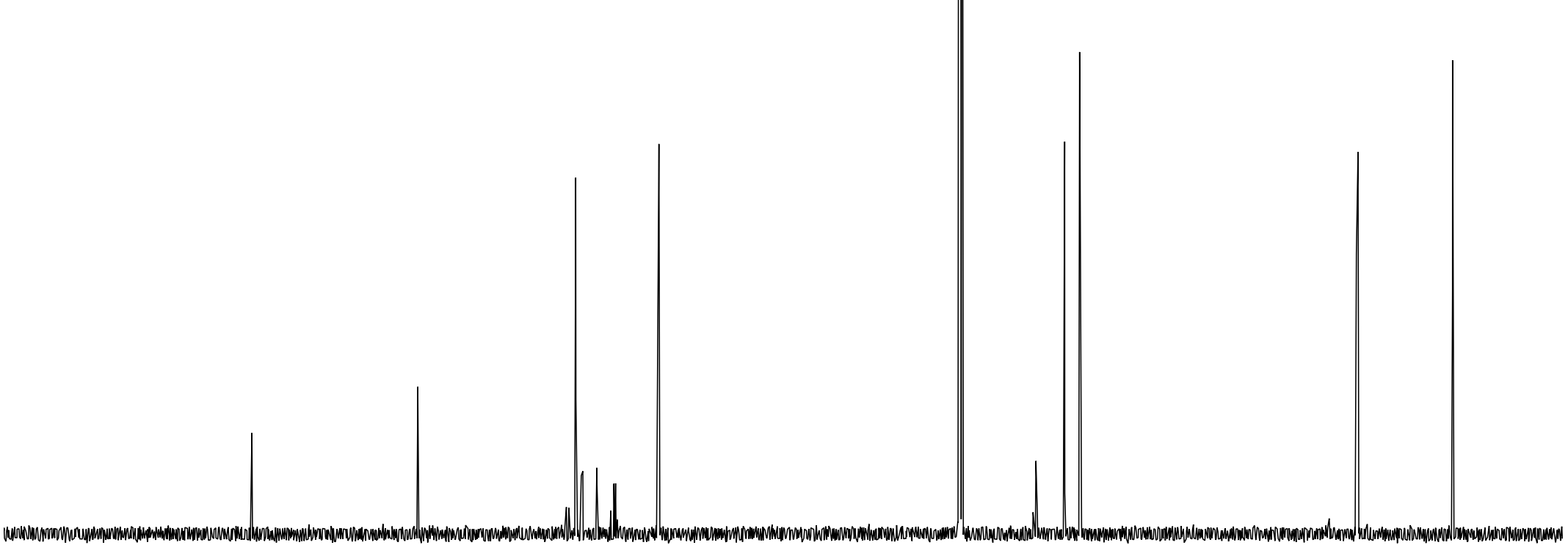




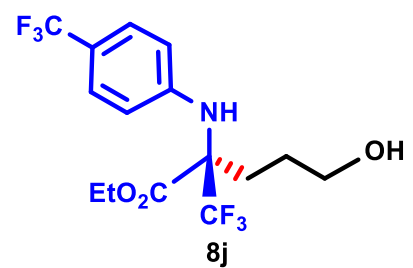

${ }^{1} \mathrm{H}$ NMR, $\mathrm{CDCl}_{3}, 600 \mathrm{MHz}$

$\begin{array}{lllllllllllllllllllllllllllllllllllllllll}-51 & -52 & -53 & -54 & -55 & -56 & -57 & -58 & -59 & -60 & -61 & -62 & -63 & -64 & -65 & -66 & -67 & -68 & -69 & -70 & -71 & -72 & -73 & -74 & -75 & -76 & -77 & -78 & -79 & -80 & -81 & -82\end{array}$ 

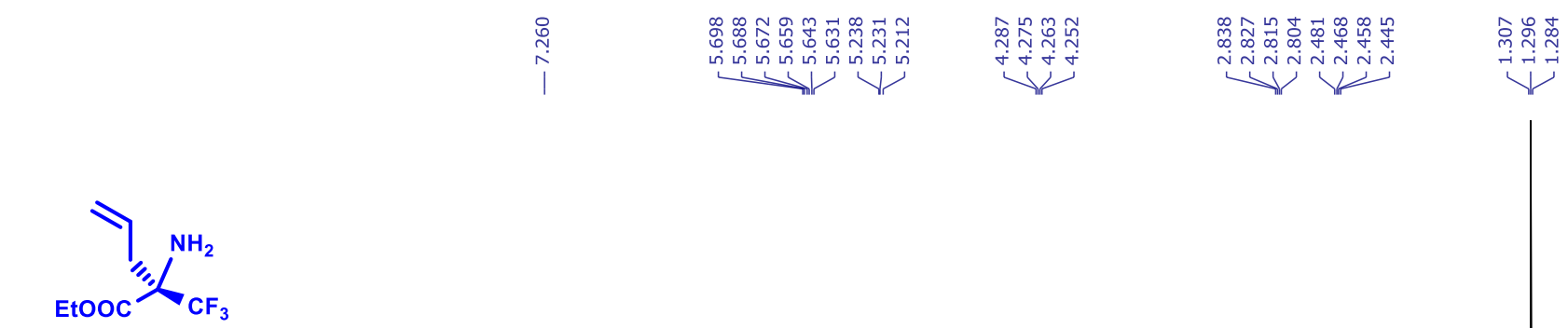

${ }^{1} \mathrm{H}$ NMR, $\mathrm{CDCl}_{3}, 600 \mathrm{MHz}$

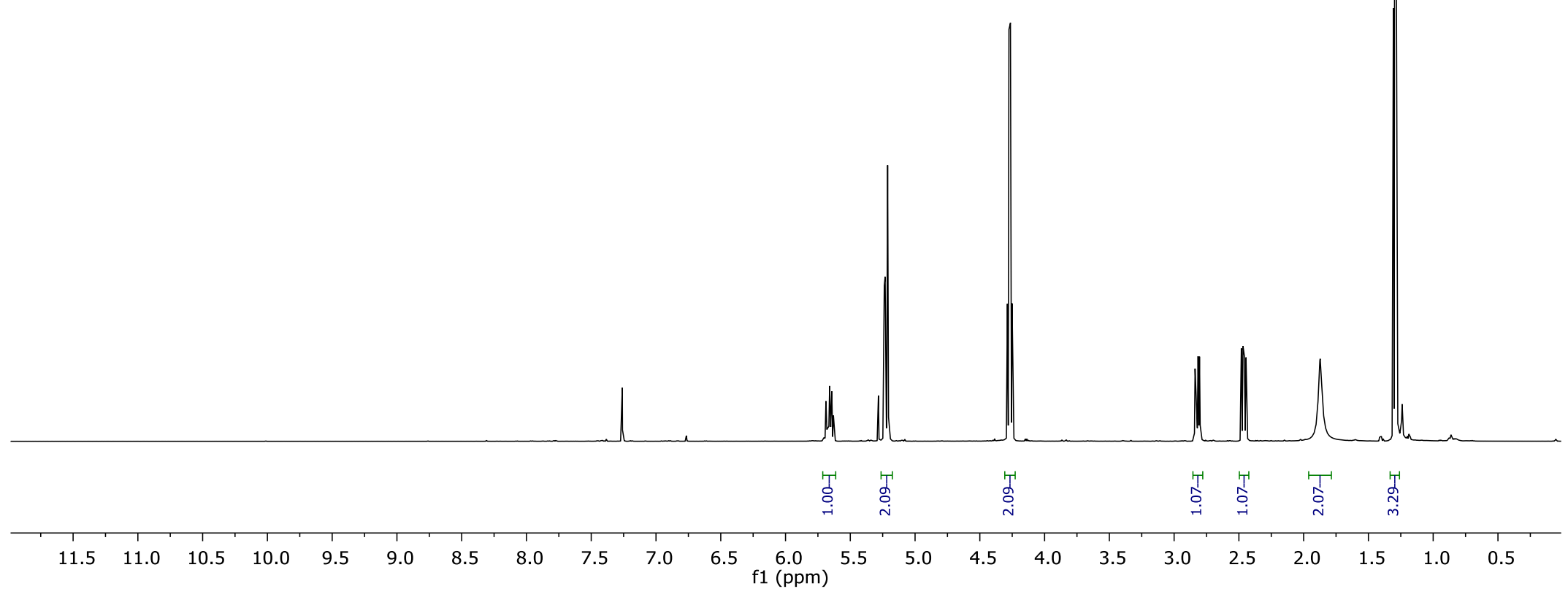




\begin{tabular}{|c|c|c|c|c|}
\hline & 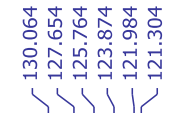 & 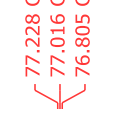 & 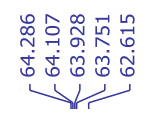 & 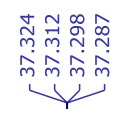 \\
\hline
\end{tabular}

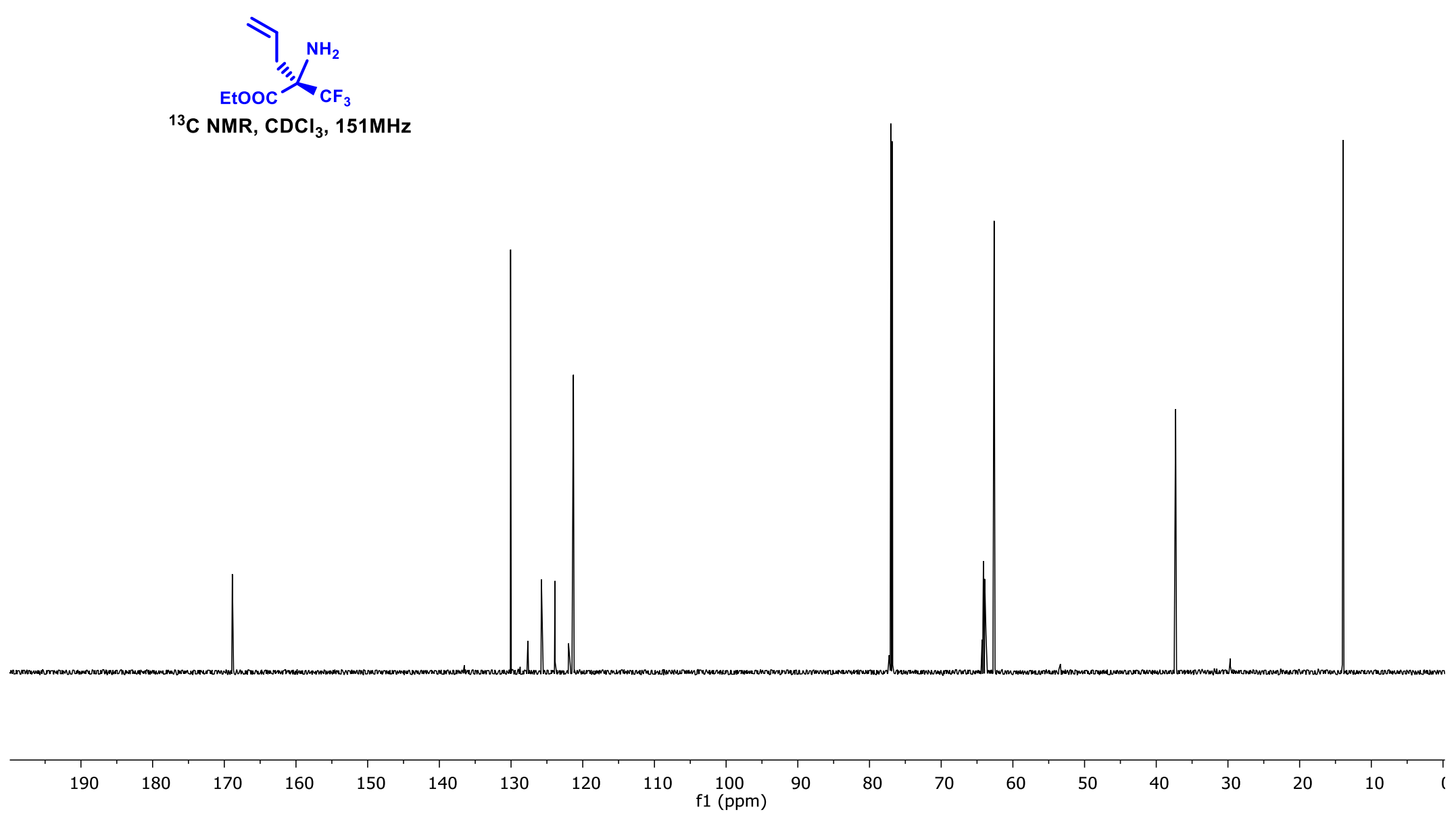


Etooc "र $_{\mathrm{CF}_{3}}^{\mathrm{NH}_{2}}$

${ }^{19} \mathrm{~F} \mathrm{NMR}, \mathrm{CDCl}_{3}, 471 \mathrm{MHz}$ 


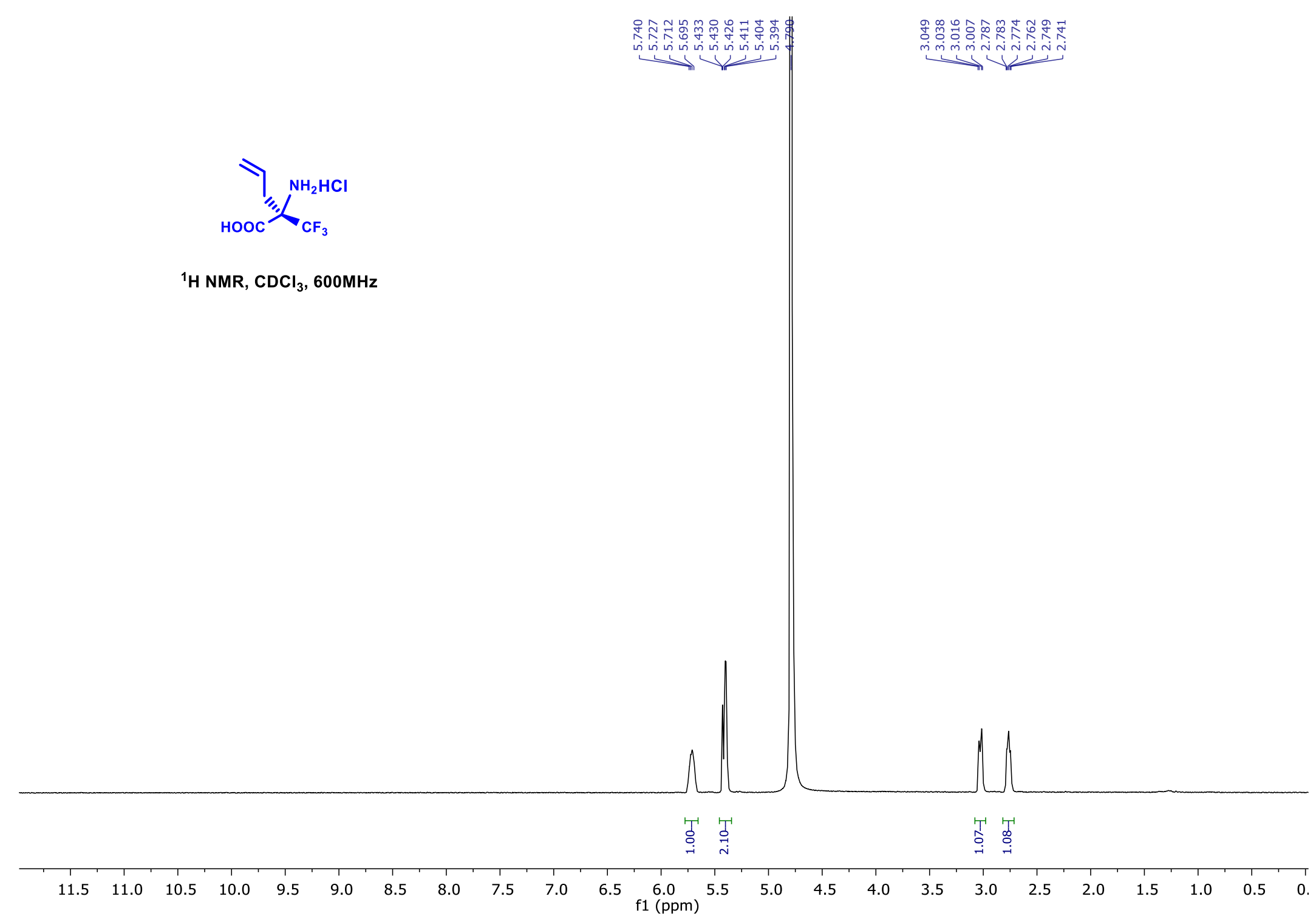




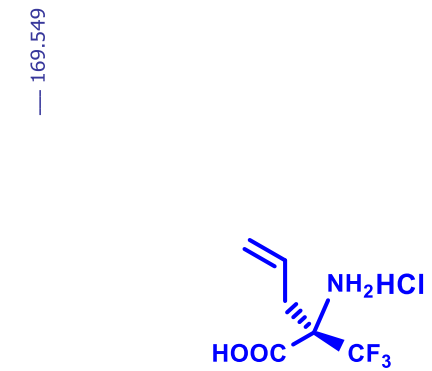

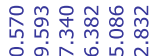

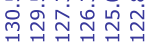

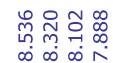

$11 / 1$ ?

ن

${ }^{13} \mathrm{C} \mathrm{NMR,} \mathrm{CDCl}_{3}, 126 \mathrm{MHz}$

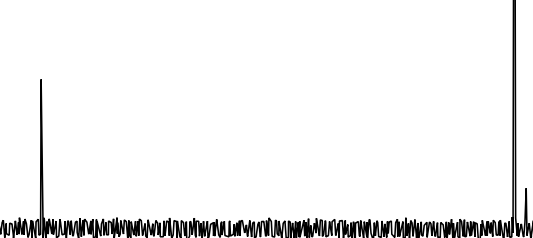

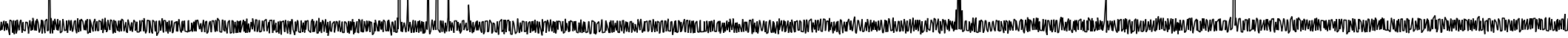

$170 \quad 160 \quad 150 \quad 140$

$120 \quad 110 \quad 100$

$90 \quad 80$

$\stackrel{90}{\mathrm{f} 1(\mathrm{ppm})}$

70

60 


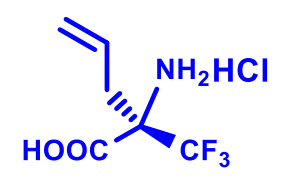

${ }^{19} \mathrm{~F} \mathrm{NMR,} \mathrm{CDCl}_{3}, 471 \mathrm{MHz}$

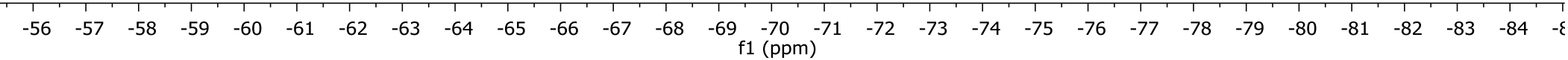




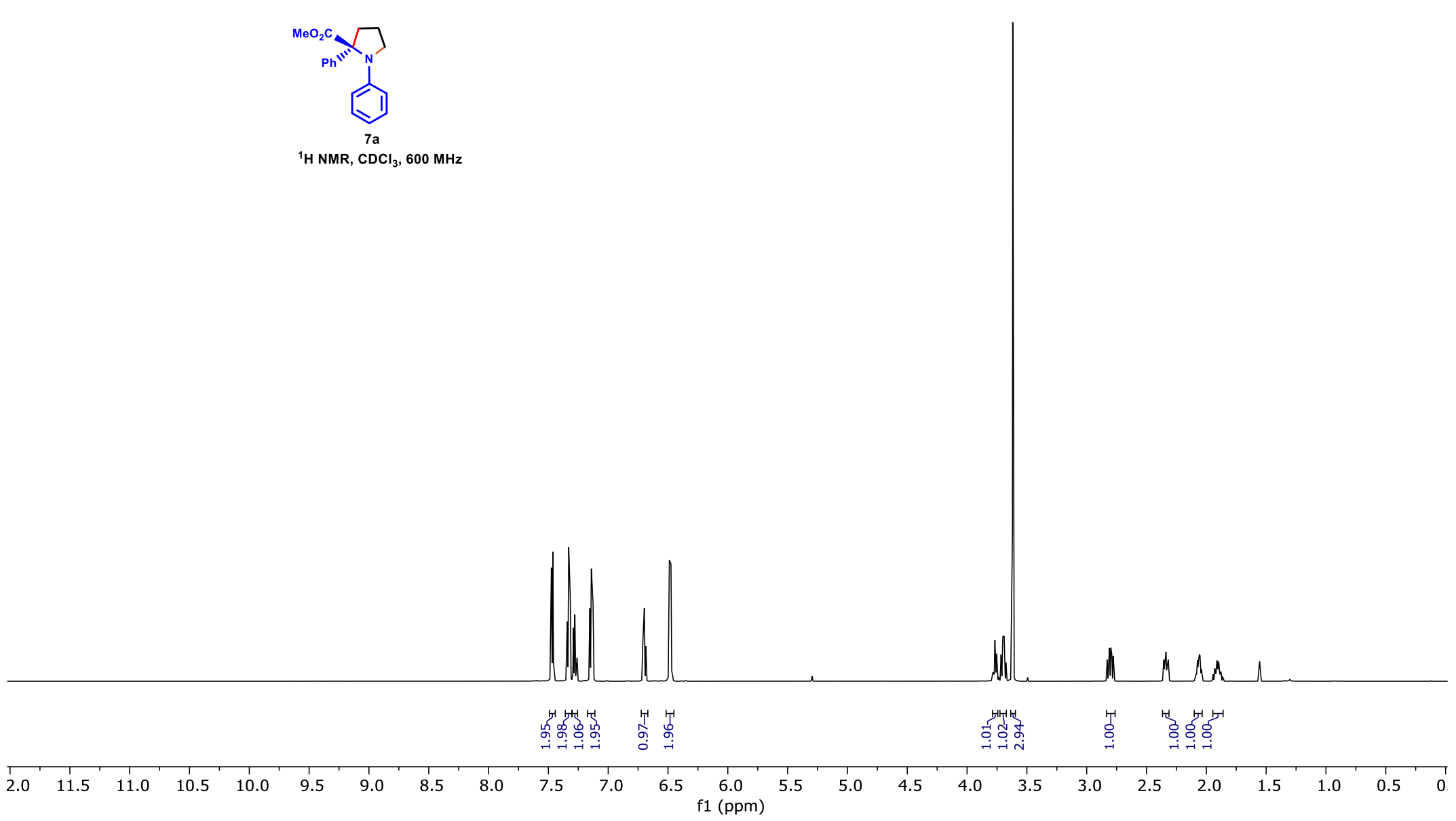

${ }^{1} \mathrm{H} \mathrm{NMR,} \mathrm{CDCl}_{3}, 600 \mathrm{MHz}$ 


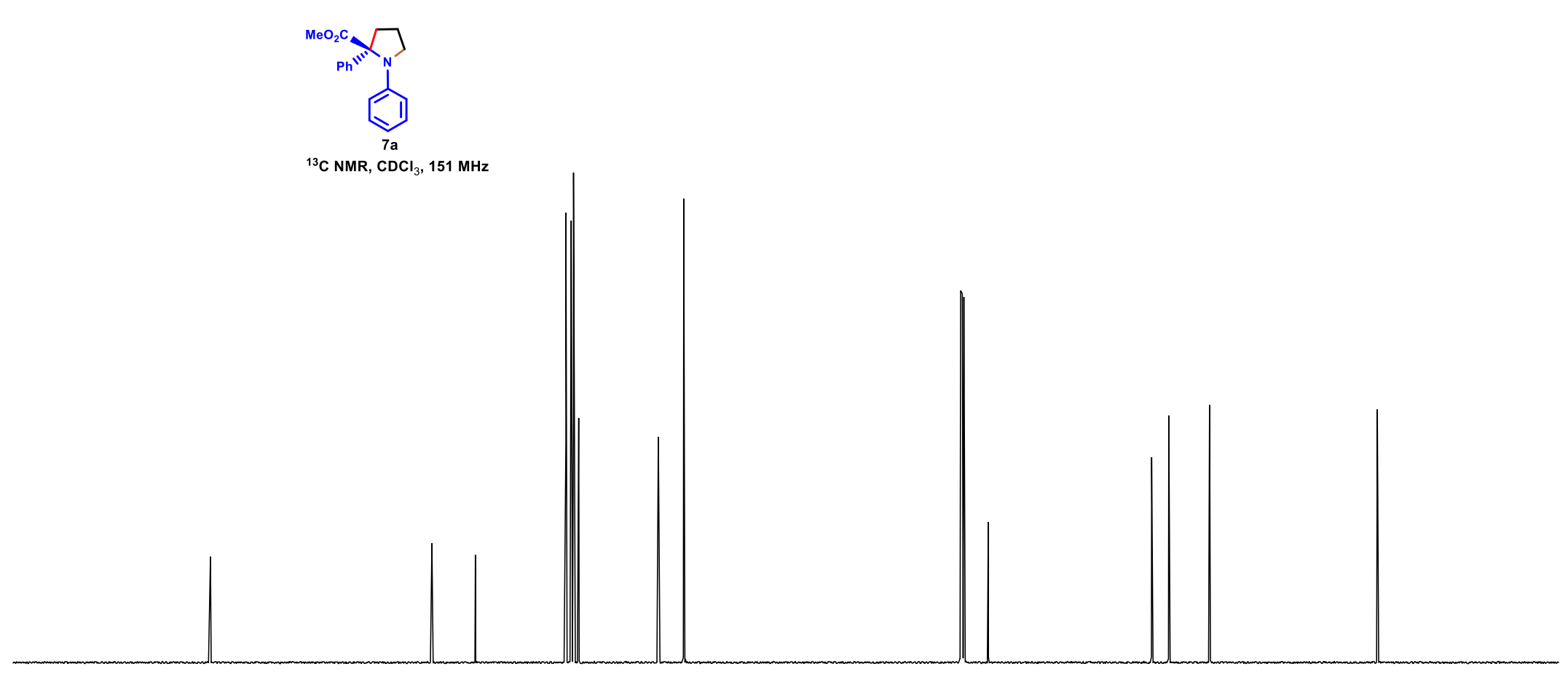

$: 00$ 


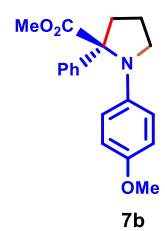

$\mathrm{H}$ NMR, $\mathrm{CDCl}_{3}, 600 \mathrm{MHz}$

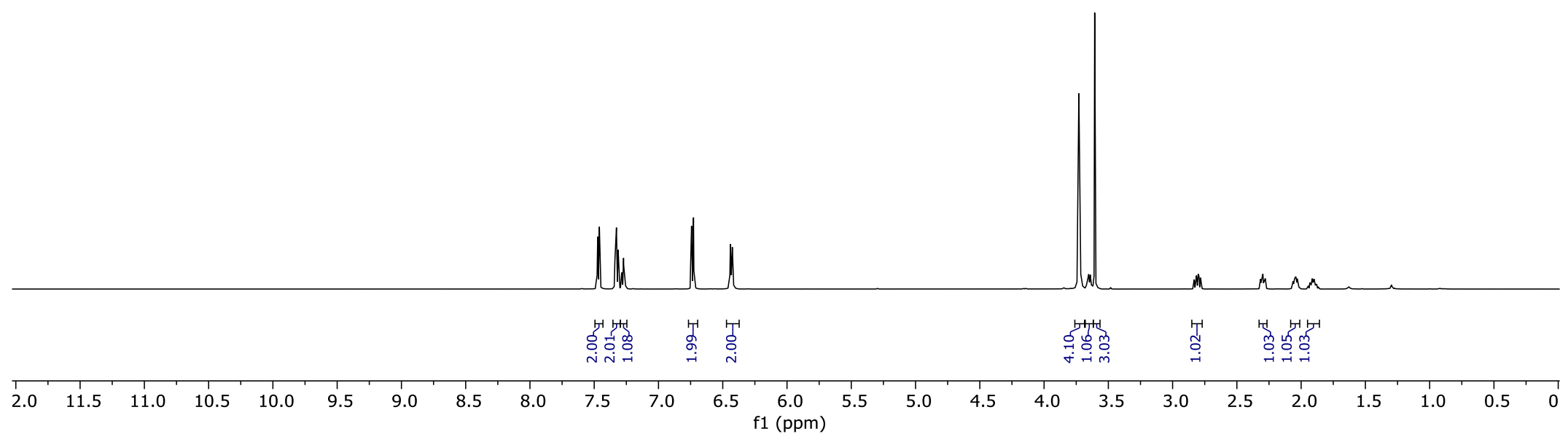



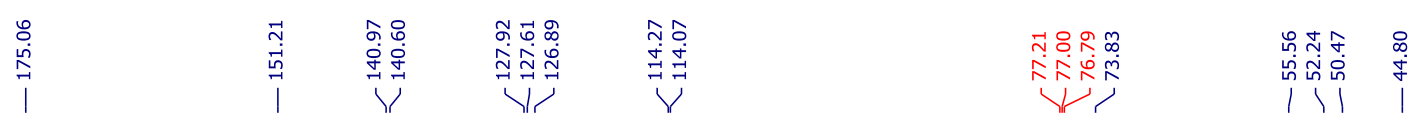

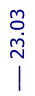

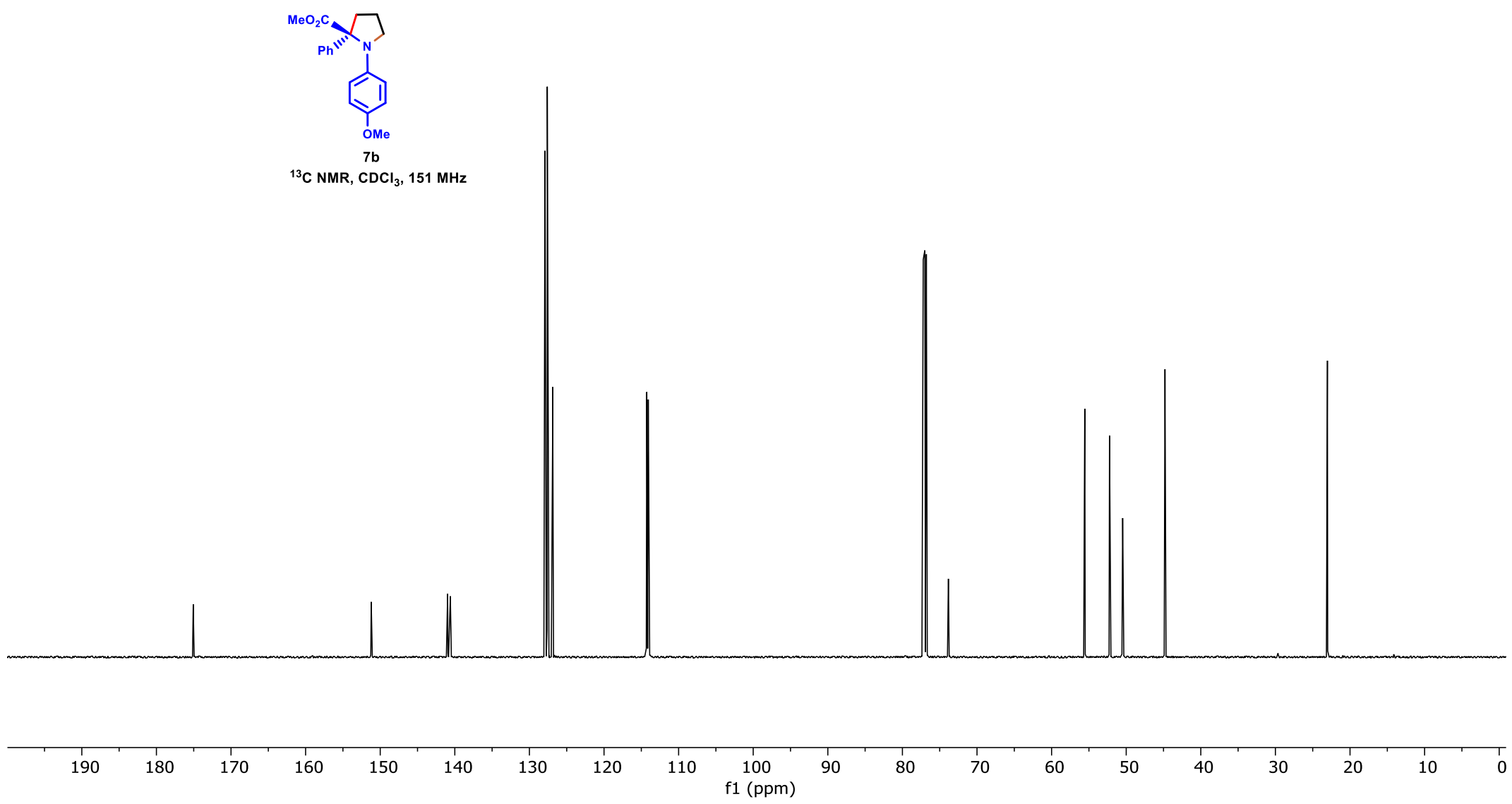

S202 


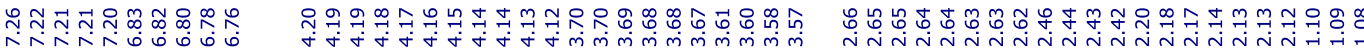 我}

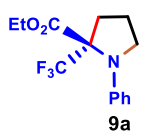

${ }^{1} \mathrm{H} \mathrm{NMR}, \mathrm{CDCl}_{3} 3,600 \mathrm{MHz}$

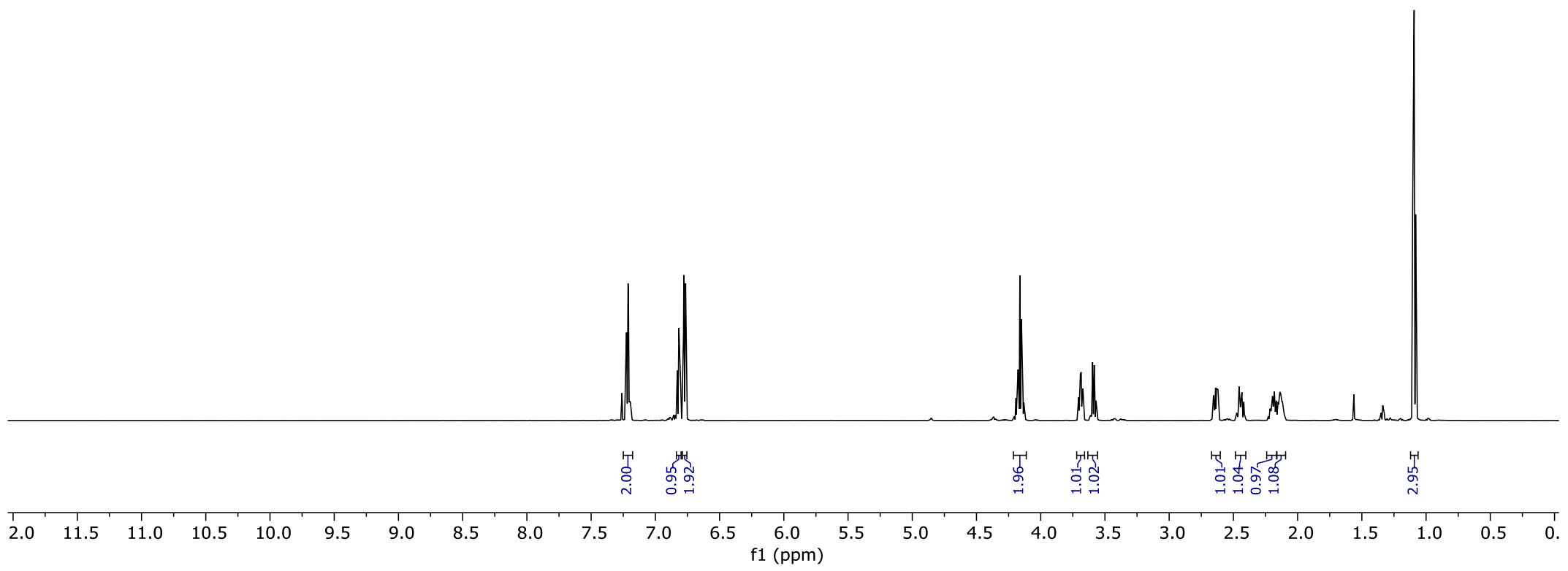




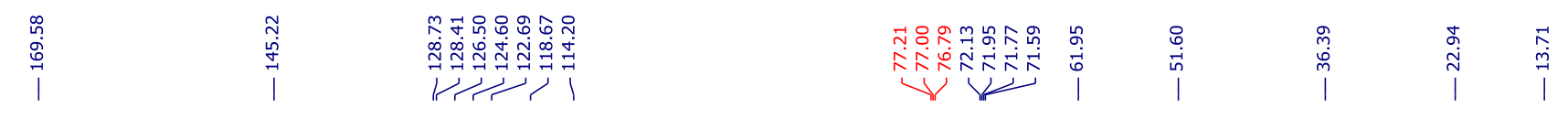
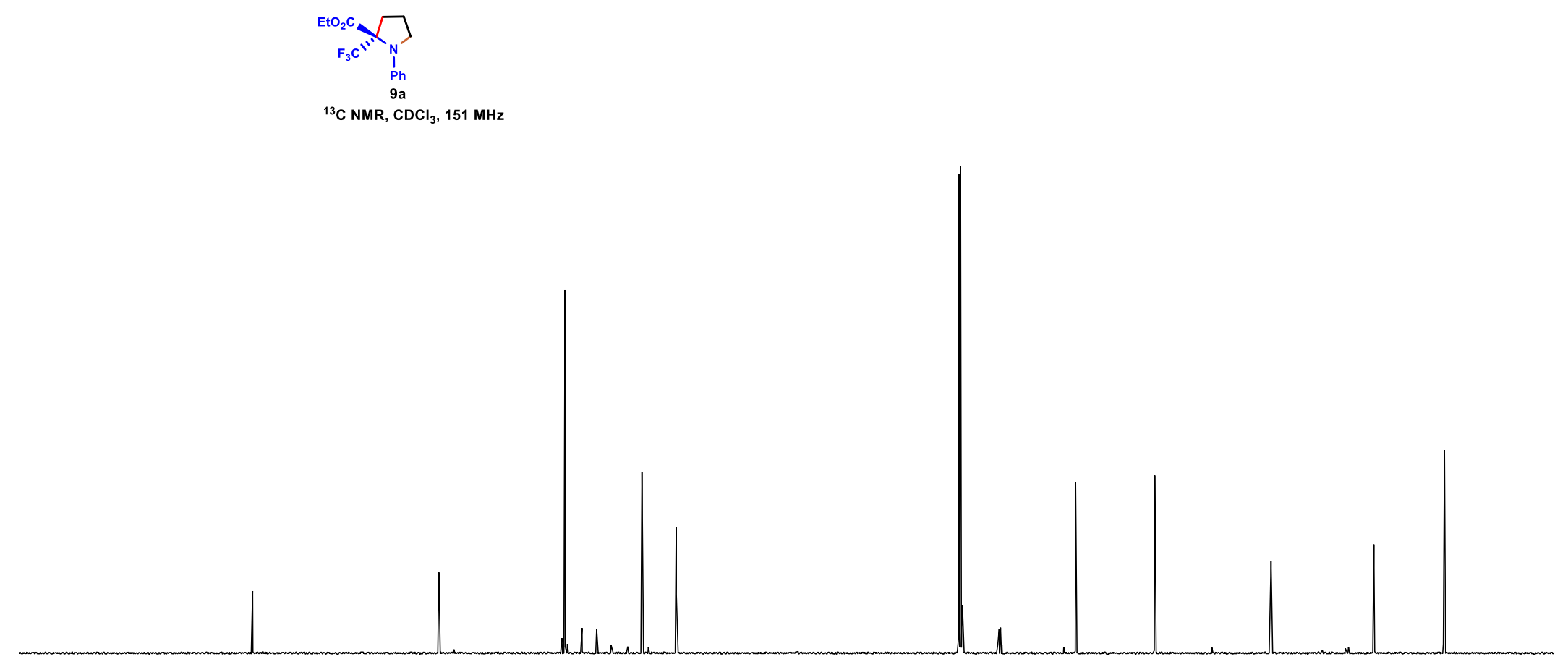

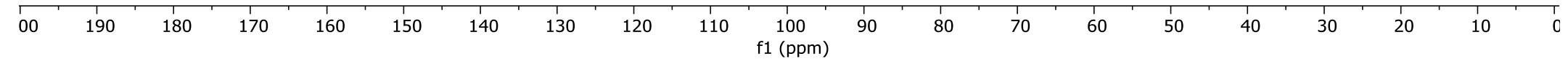




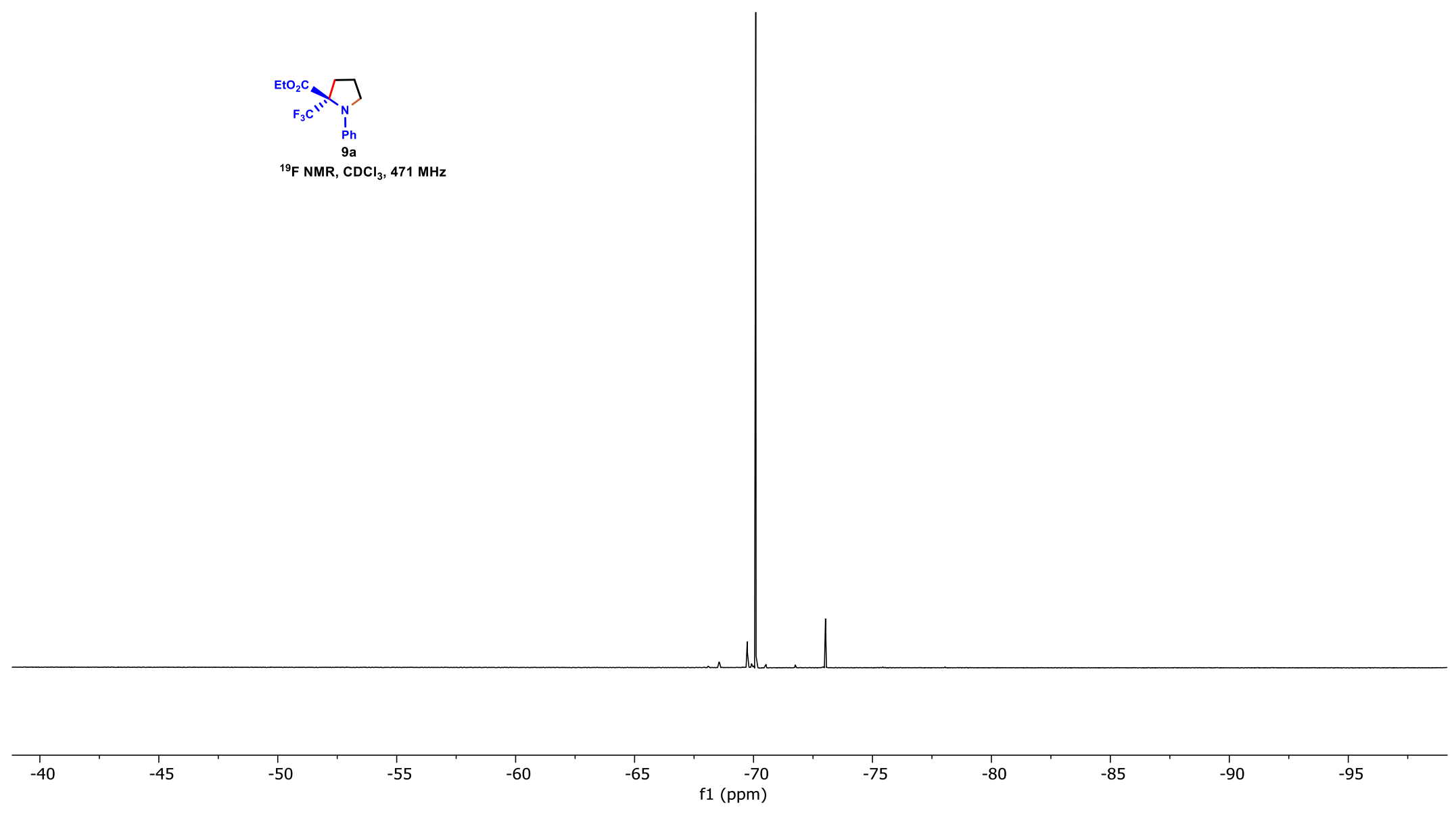




\section{HPLC and SFC Chromatograms}



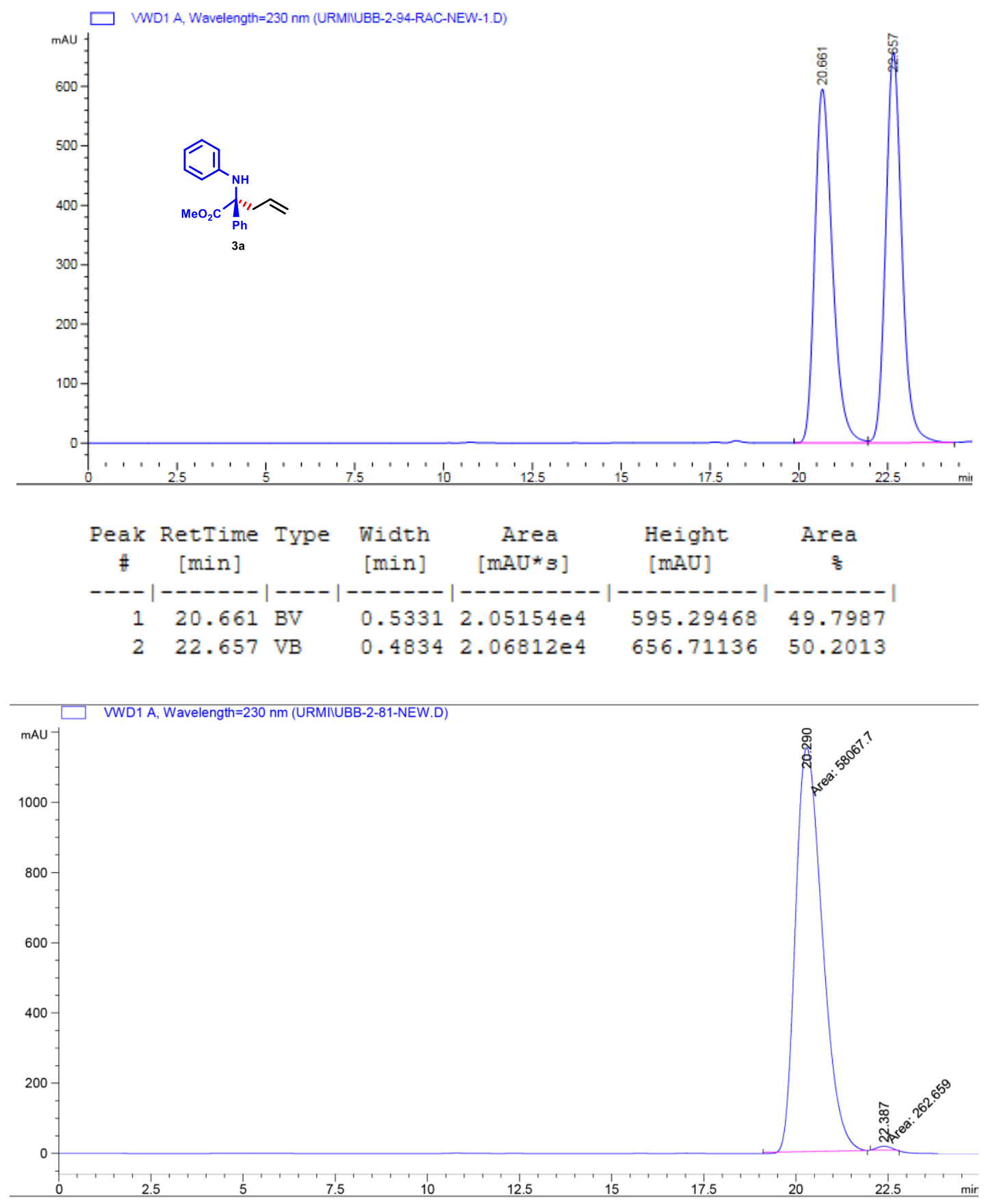

\begin{tabular}{|c|c|c|c|c|c|c|}
\hline \# & $\begin{array}{c}\text { RetTime } \\
\text { [min] }\end{array}$ & ype & $\begin{array}{l}\text { Width } \\
\text { [min] }\end{array}$ & $\begin{array}{c}\text { Area } \\
{\left[\mathrm{mAU}^{*} \mathrm{~s}\right]}\end{array}$ & $\begin{array}{l}\text { Height } \\
{[\mathrm{mAU}]}\end{array}$ & $\begin{array}{c}\text { Area } \\
\frac{\circ}{0}\end{array}$ \\
\hline & & & & & & \\
\hline & 22 . & & 7 & 262.65854 & 10.71130 & 4503 \\
\hline
\end{tabular}




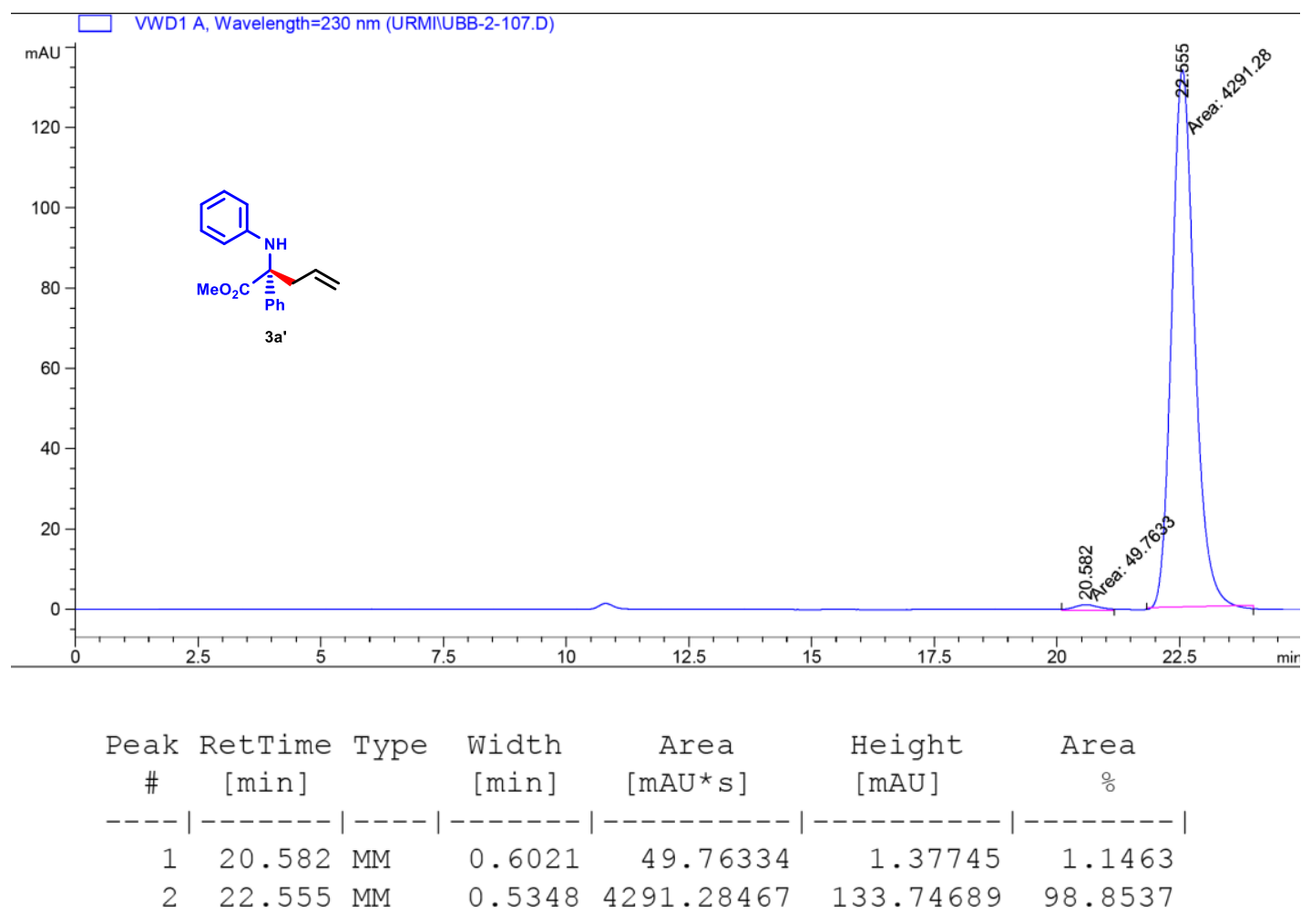



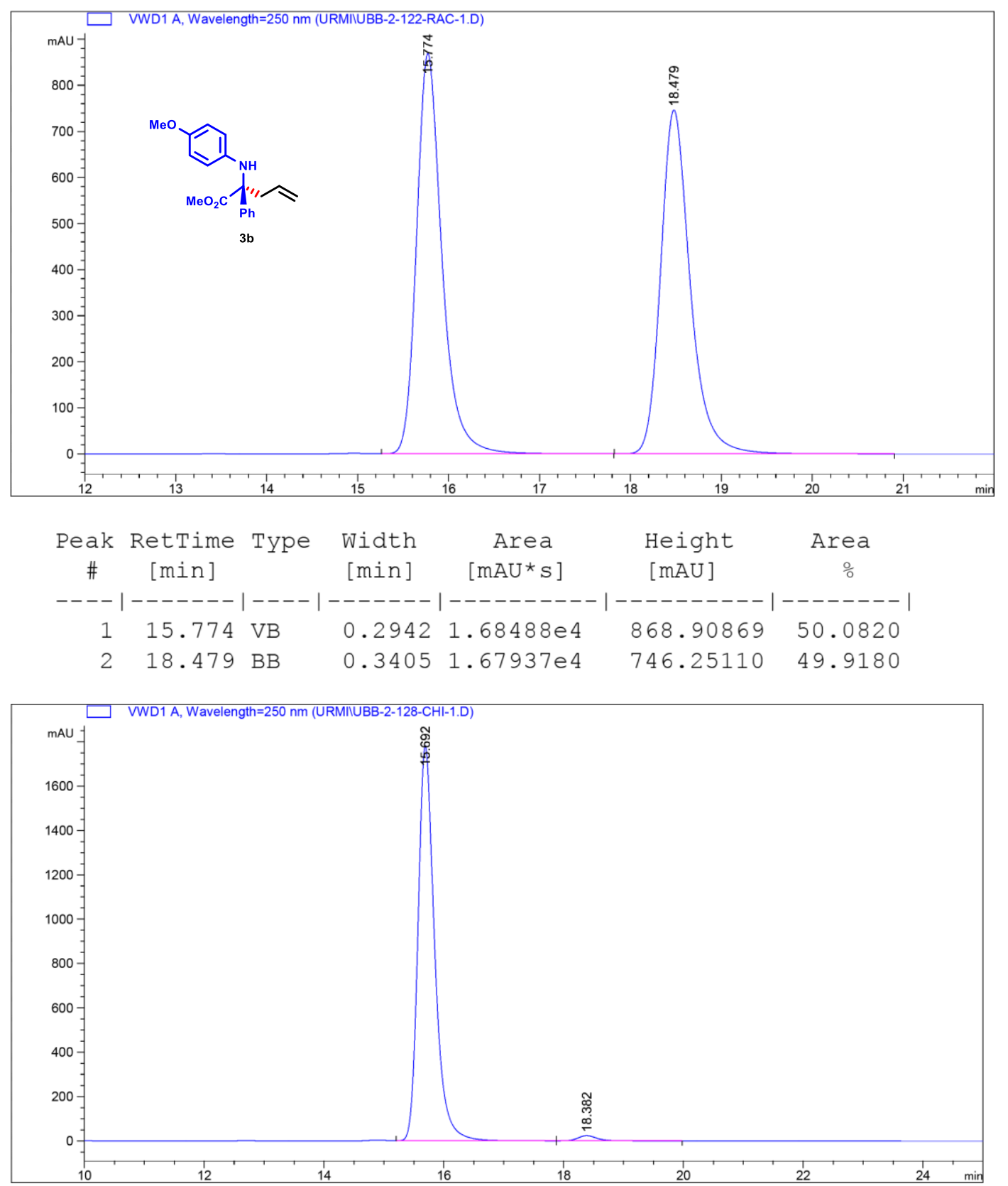

\begin{tabular}{|c|c|c|c|c|c|c|}
\hline $\begin{array}{c}\text { Peak } \\
\text { \# }\end{array}$ & $\begin{array}{c}\text { RetTime } \\
\text { [min] }\end{array}$ & Type & $\begin{array}{l}\text { Width } \\
\text { [min] }\end{array}$ & $\begin{array}{c}\text { Area } \\
{\left[\mathrm{mAU}^{*} \mathrm{~s}\right]}\end{array}$ & $\begin{array}{l}\text { Height } \\
\text { [mAU] }\end{array}$ & $\begin{array}{c}\text { Area } \\
\quad \%\end{array}$ \\
\hline & & & & | ---------- & | ---------- & --- \\
\hline & 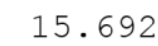 & VB & & $3.42254 \mathrm{e} 4$ & 1782.19617 & 080 \\
\hline & 8.382 & $\mathrm{BB}$ & & 518.36530 & 23.52317 & 4920 \\
\hline
\end{tabular}




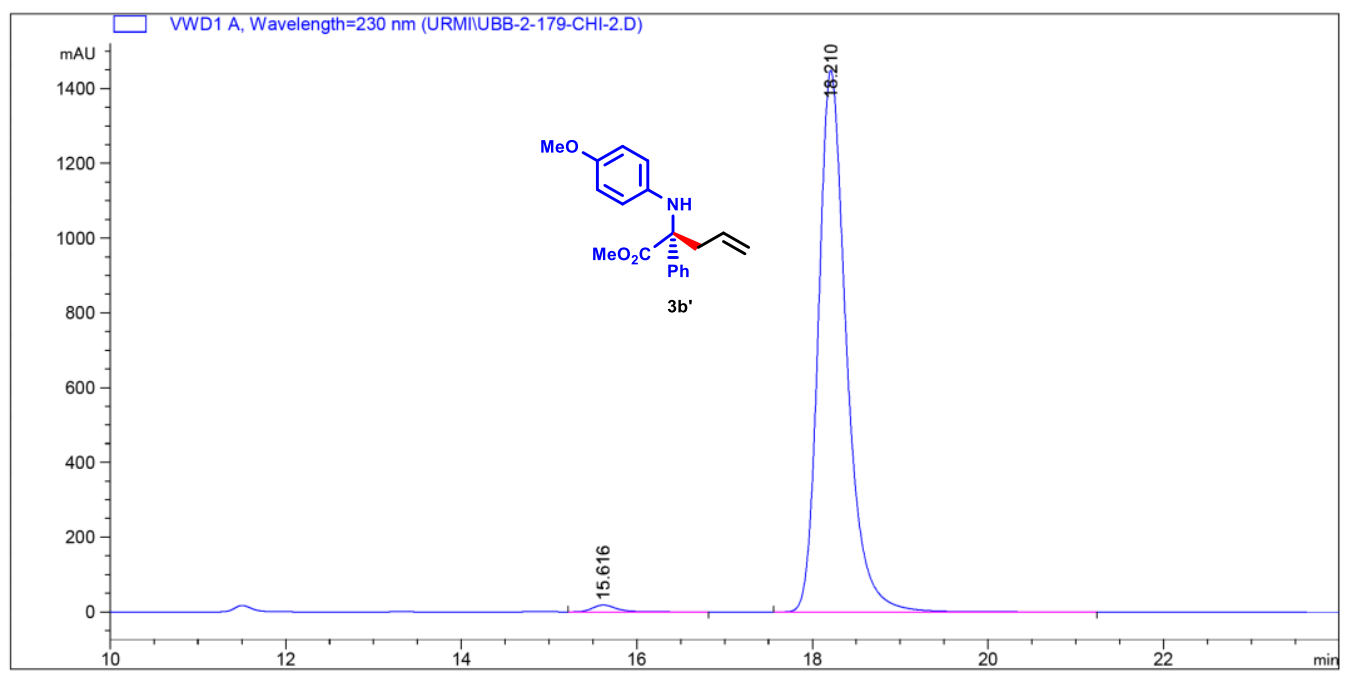

\begin{tabular}{|c|c|c|c|c|c|c|}
\hline $\begin{array}{c}\text { Peak } \\
\text { \# }\end{array}$ & $\begin{array}{c}\text { RetTime } \\
\text { [min] }\end{array}$ & Type & $\begin{array}{l}\text { Width } \\
{[\mathrm{min}]}\end{array}$ & $\begin{array}{c}\text { Area } \\
{\left[\mathrm{mAU}^{*} \mathrm{~S}\right]}\end{array}$ & $\begin{array}{l}\text { Height } \\
{[\mathrm{mAU}]}\end{array}$ & $\begin{array}{c}\text { Area } \\
\frac{\circ}{0}\end{array}$ \\
\hline 1 & 15.61 & & 0.2898 & 349.93997 & 18.24091 & 816 \\
\hline 2 & 18.210 & & 0.3339 & $3.20032 e 4$ & 447.92346 & 98.9184 \\
\hline
\end{tabular}




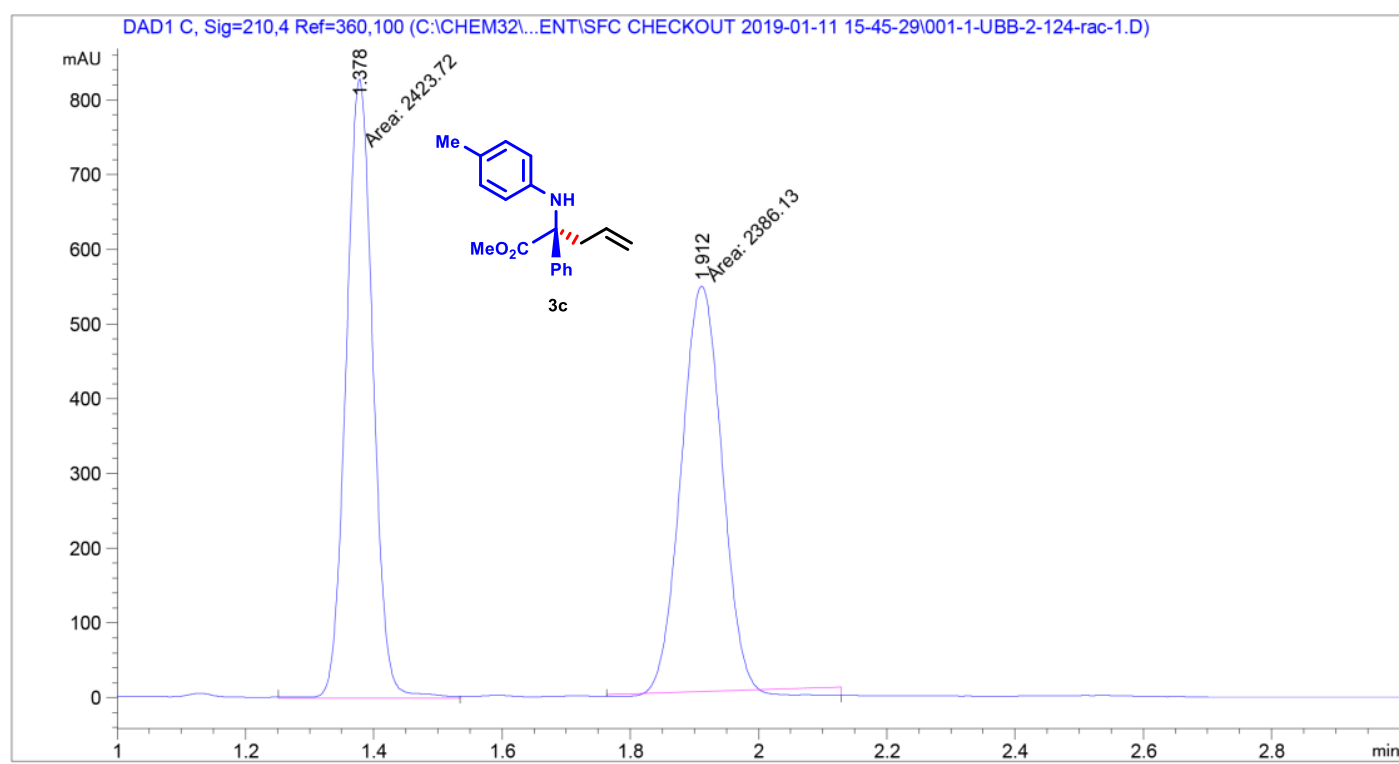

\begin{tabular}{|c|c|c|c|c|c|c|}
\hline $\begin{array}{c}\text { Peak } \\
\text { \# }\end{array}$ & $\begin{array}{c}\text { RetTime } \\
\text { [min] }\end{array}$ & Type & $\begin{array}{l}\text { Width } \\
\text { [min] }\end{array}$ & $\begin{array}{c}\text { Area } \\
{\left[\mathrm{mAU}^{*} \mathrm{~s}\right]}\end{array}$ & $\begin{array}{l}\text { Height } \\
\text { [mAU] }\end{array}$ & $\begin{array}{c}\text { Area } \\
\%\end{array}$ \\
\hline & & & & & & \\
\hline 1 & & MM & .0487 & 2423.71875 & 829.26 & 50.3 \\
\hline 2 & 1.912 & MM & .0732 & 2386.13257 & 543.07568 & 49.6093 \\
\hline
\end{tabular}

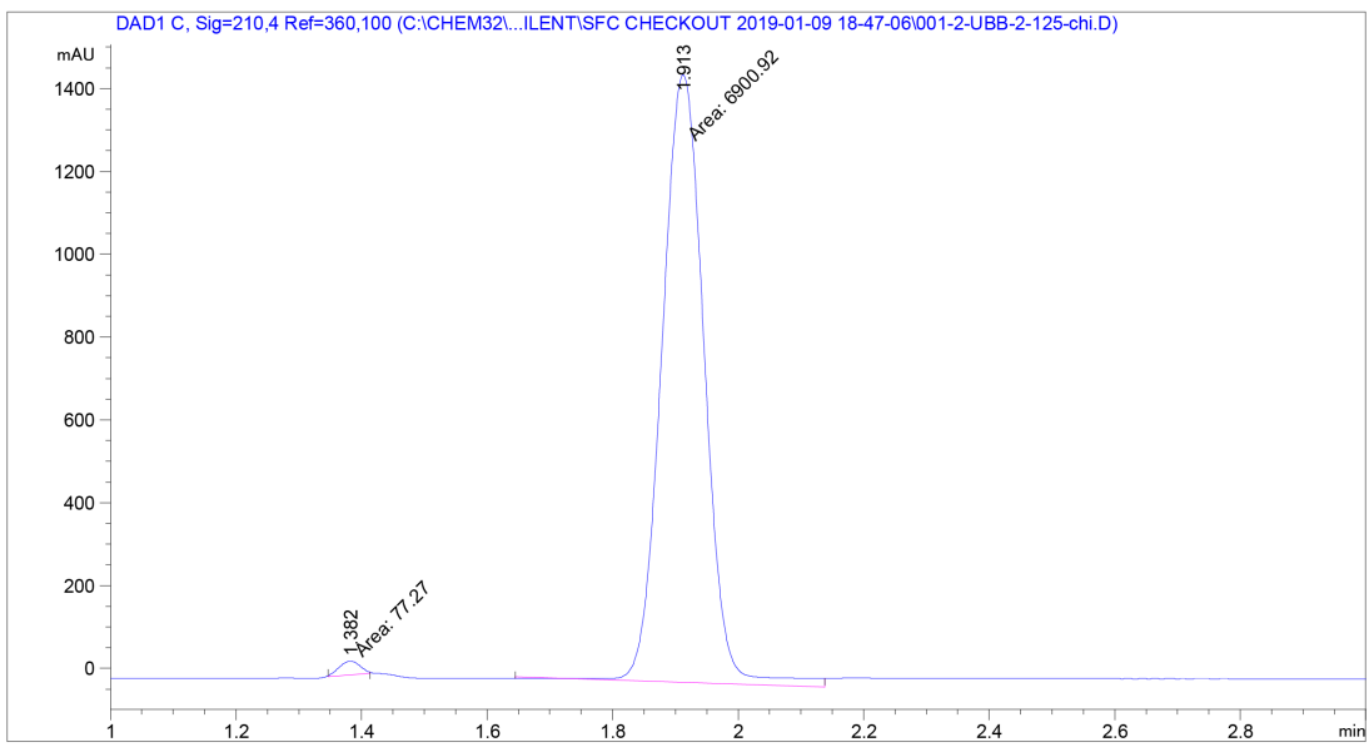

\begin{tabular}{|c|c|c|c|c|c|c|}
\hline $\begin{array}{c}\text { Peak } \\
\#\end{array}$ & $\begin{array}{c}\text { RetTime } \\
\text { [min] }\end{array}$ & Type & $\begin{array}{l}\text { Width } \\
\text { [min] }\end{array}$ & $\begin{array}{c}\text { Area } \\
{\left[\mathrm{mAU}^{*} \mathrm{~s}\right]}\end{array}$ & $\begin{array}{l}\text { Height } \\
\text { [mAU] }\end{array}$ & $\begin{array}{c}\text { Area } \\
\%\end{array}$ \\
\hline & & & & & & \\
\hline 1 & 1.382 & MM & 0.0383 & 77.27002 & 33.60178 & 1.1073 \\
\hline 2 & 1.913 & MM & 0.0783 & 6900.92432 & 1468.30640 & 98.8927 \\
\hline
\end{tabular}



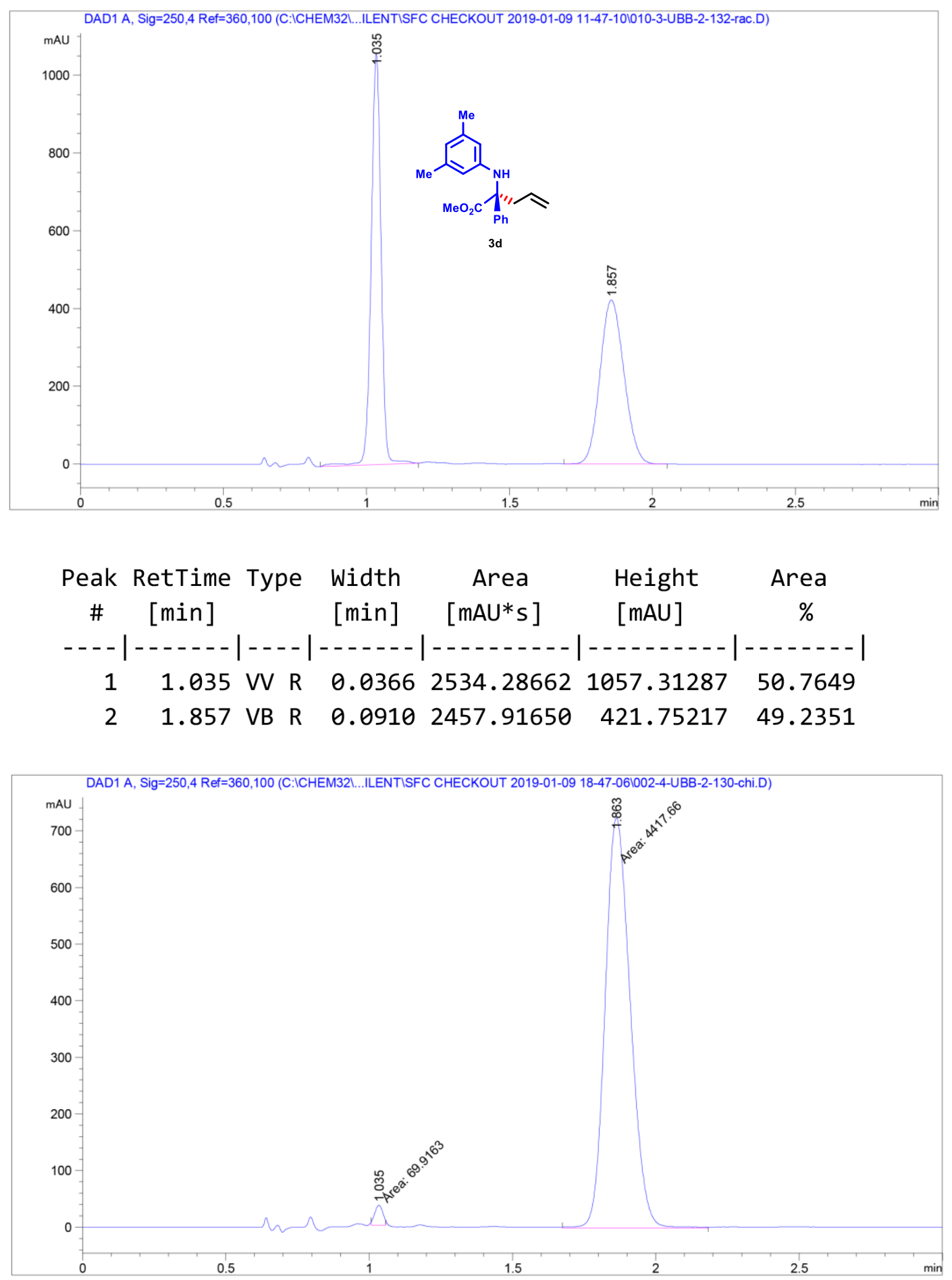

\begin{tabular}{cccccc}
$\begin{array}{c}\text { Peak RetTime Type } \\
\text { \# } \\
\text { [min] }\end{array}$ & $\begin{array}{c}\text { Width } \\
\text { [min] }\end{array}$ & $\begin{array}{c}\text { Area } \\
{[\mathrm{mAU} \text { * }]}\end{array}$ & \multicolumn{1}{c}{$\begin{array}{c}\text { Height } \\
{[\mathrm{mAU}]}\end{array}$} & \multicolumn{1}{c}{$\begin{array}{c}\text { Area } \\
\%\end{array}$} \\
\hline 1 & 1.035 MM & 0.0329 & 69.91634 & 35.40695 & 1.5580 \\
2 & 1.863 MM & 0.1017 & 4417.65625 & 724.15143 & 98.4420
\end{tabular}



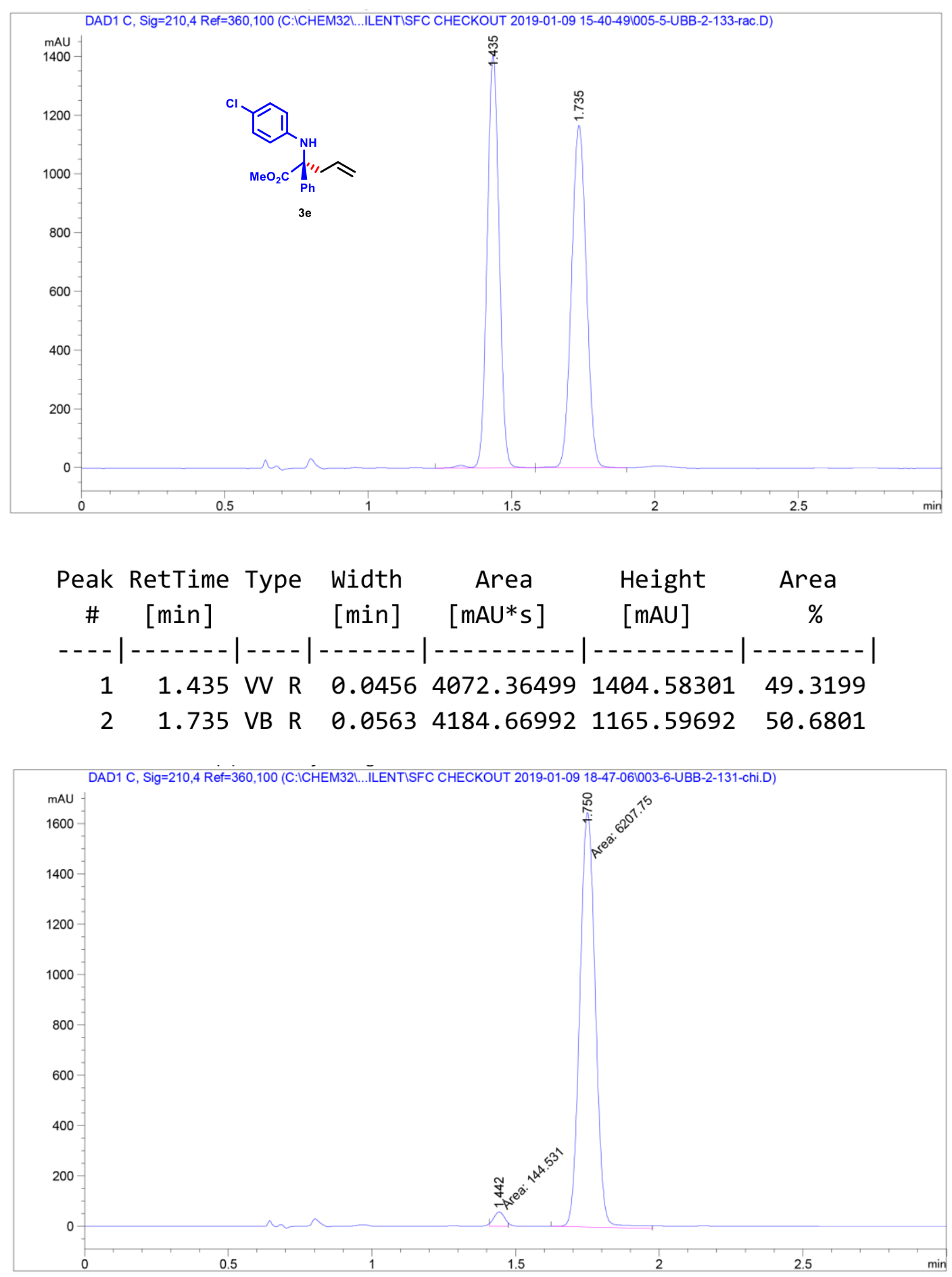

\begin{tabular}{cccccc}
$\begin{array}{c}\text { Peak RetTime Type } \\
\text { Width }\end{array}$ & $\begin{array}{c}\text { Area } \\
{[\mathrm{min}]}\end{array}$ & $\begin{array}{c}\text { Height } \\
{[\mathrm{mAU} \text { *s }]}\end{array}$ & \multicolumn{1}{c}{$\begin{array}{c}\text { Area } \\
{[\mathrm{mAU}]}\end{array}$} & \multicolumn{1}{c}{$\%$} \\
\hline 1 & 1.442 MM & 0.0427 & 144.53101 & 56.38975 & 2.2753 \\
2 & 1.750 MM & 0.0628 & 6207.75391 & 1647.23889 & 97.7247
\end{tabular}




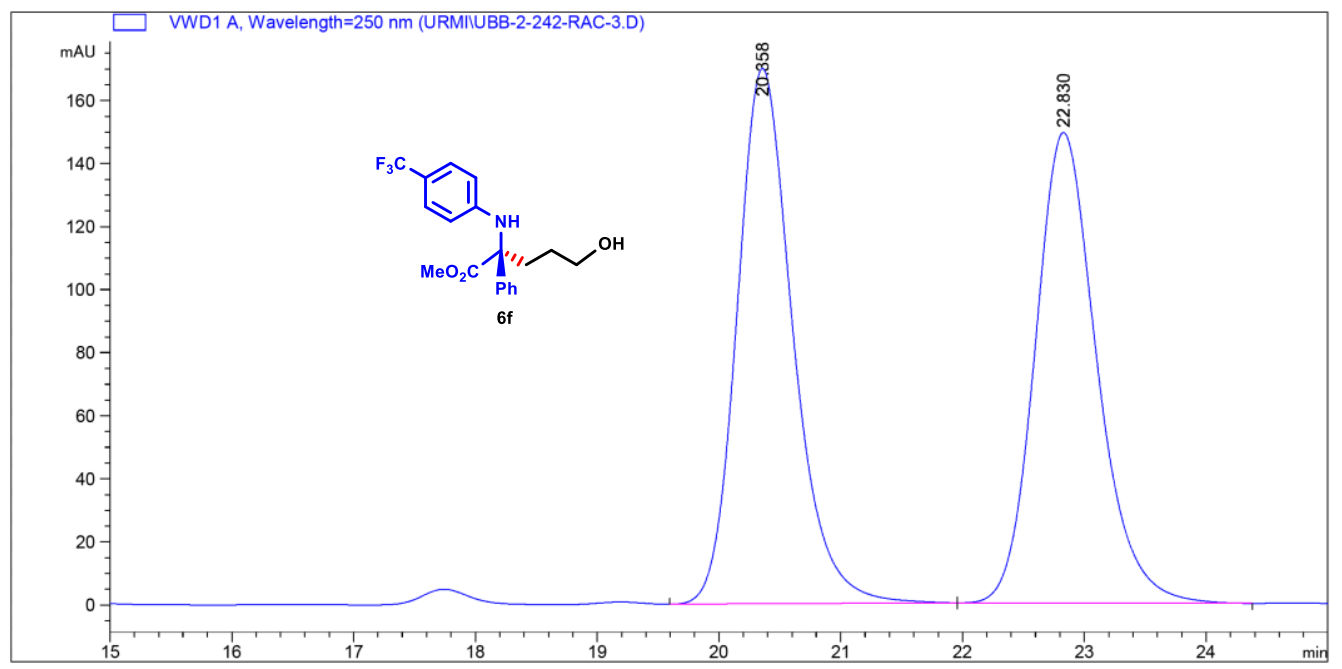

\begin{tabular}{|c|c|c|c|c|c|c|}
\hline $\begin{array}{c}\text { Peak } \\
\#\end{array}$ & $\begin{array}{c}\text { RetTime } \\
\text { [min] }\end{array}$ & Type & $\begin{array}{l}\text { Width } \\
\text { [min] }\end{array}$ & $\begin{array}{c}\text { Area } \\
{\left[m A U^{\star} \mathrm{s}\right]}\end{array}$ & $\begin{array}{l}\text { Height } \\
{[\mathrm{mAU}]}\end{array}$ & $\begin{array}{c}\text { Area } \\
\frac{\circ}{0}\end{array}$ \\
\hline & & & & ---------- & $----------\mid$ & $--------\mid$ \\
\hline 1 & 20.358 & $\mathrm{BB}$ & 0.4852 & 5401.94336 & 169.78180 & 50.8018 \\
\hline 2 & 22.830 & $\mathrm{BB}$ & 0.5361 & 5231.42676 & 149.19852 & 49.1982 \\
\hline
\end{tabular}

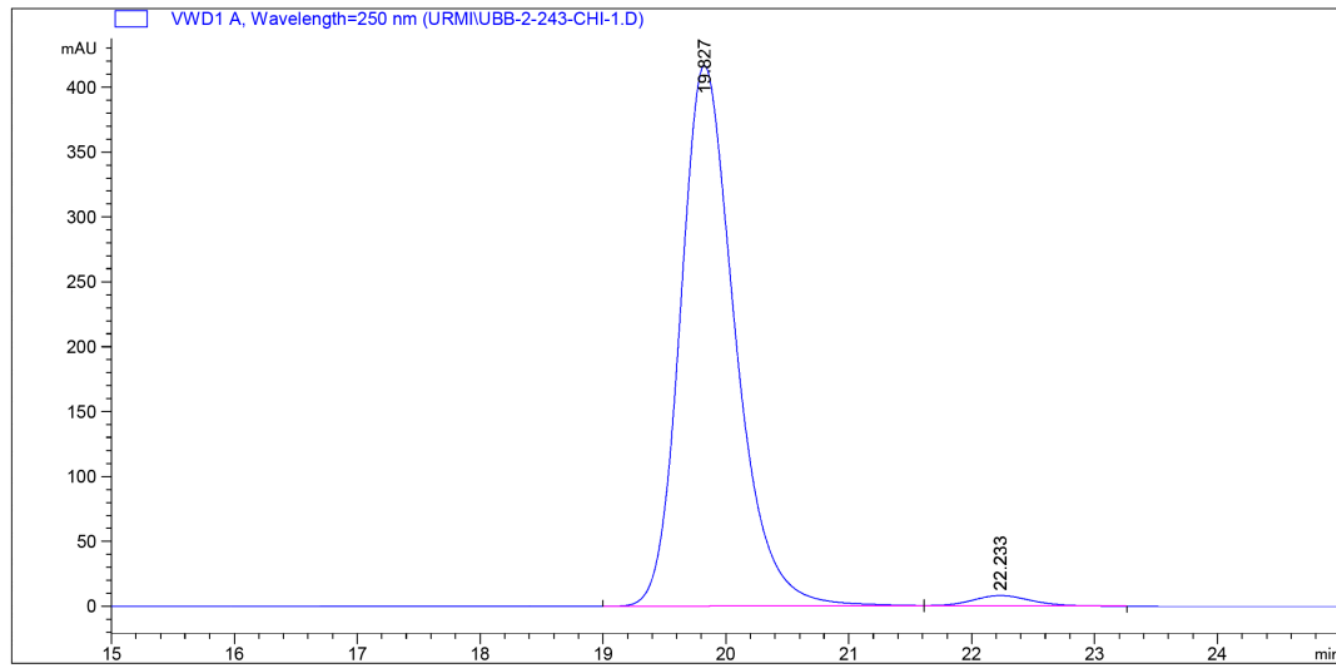

\begin{tabular}{|c|c|c|c|c|c|c|}
\hline $\begin{array}{c}\text { Peak } \\
\#\end{array}$ & $\begin{array}{c}\text { RetTime } \\
\text { [min] }\end{array}$ & Type & $\begin{array}{l}\text { Width } \\
\text { [min] }\end{array}$ & $\begin{array}{c}\text { Area } \\
{\left[m A U^{*} \mathrm{~s}\right]}\end{array}$ & $\begin{array}{l}\text { Height } \\
\text { [mAU] }\end{array}$ & $\begin{array}{c}\text { Area } \\
\frac{\circ}{0}\end{array}$ \\
\hline & & & & ---------- & $----------\mid$ & ---1 \\
\hline 1 & 19 & $R B$ & .4704 & 1.28638 e 4 & 416.47488 & 576 \\
\hline 2 & 22.233 & $\mathrm{BB}$ & 0.4982 & 254.82257 & 7.86141 & 1.9424 \\
\hline
\end{tabular}



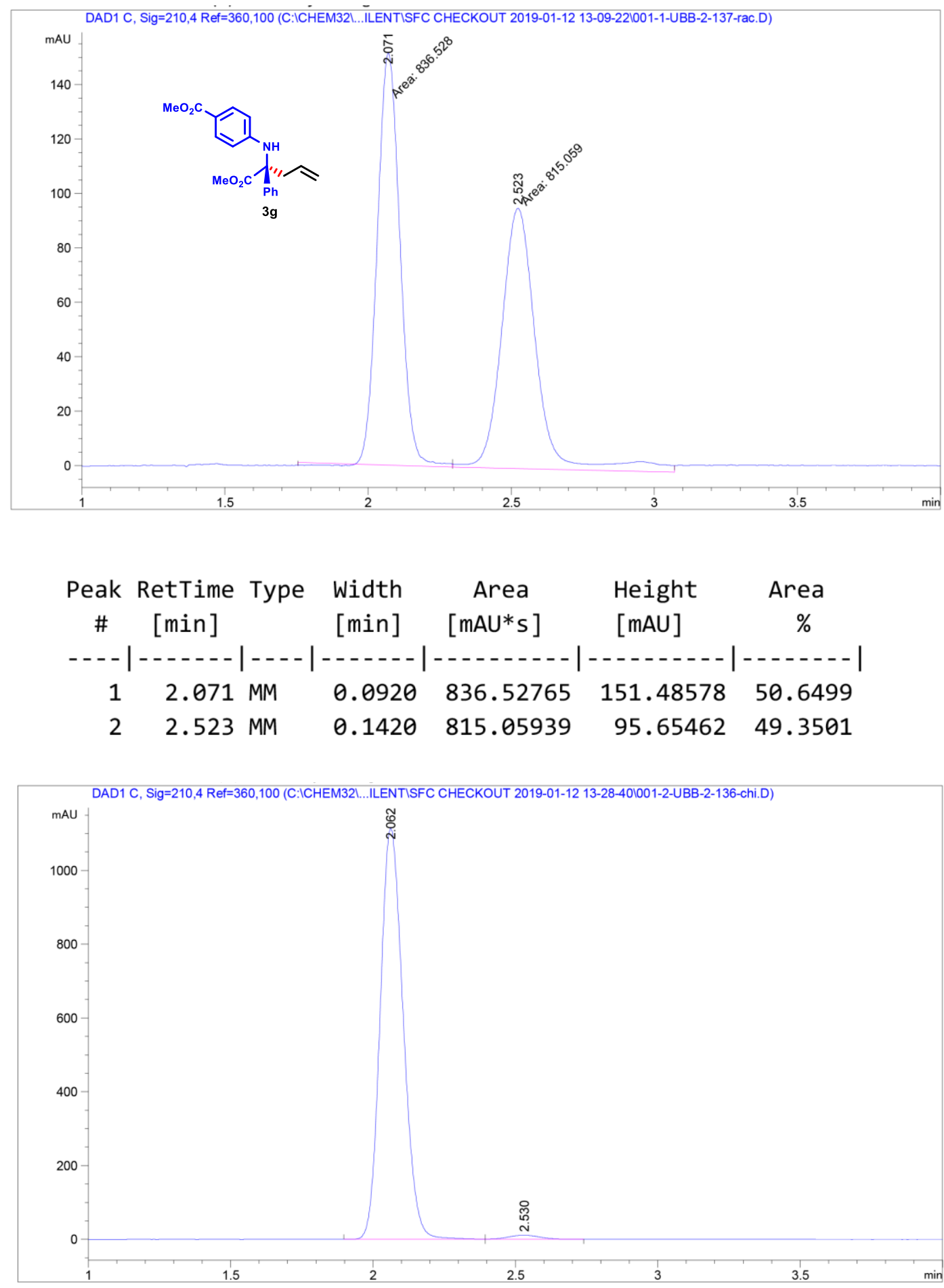

\begin{tabular}{|c|c|c|c|c|c|c|}
\hline $\begin{array}{c}\text { Peak } \\
\quad \#\end{array}$ & $\begin{array}{c}\text { RetTime } \\
\text { [min] }\end{array}$ & Type & $\begin{array}{l}\text { Width } \\
\text { [min] }\end{array}$ & $\begin{array}{c}\text { Area } \\
{\left[\mathrm{mAU}^{*} \mathrm{~s}\right]}\end{array}$ & $\begin{array}{l}\text { Height } \\
\text { [mAU] }\end{array}$ & $\begin{array}{c}\text { Area } \\
\%\end{array}$ \\
\hline & & & & thes & & \\
\hline 1 & 2.062 & $2 \mathrm{~V}$ & 0.0872 & 6231.91211 & 1114.35901 & 98.6585 \\
\hline 2 & 2.530 & VV R & 0.1114 & 84.74036 & 11.05860 & 1.3415 \\
\hline
\end{tabular}




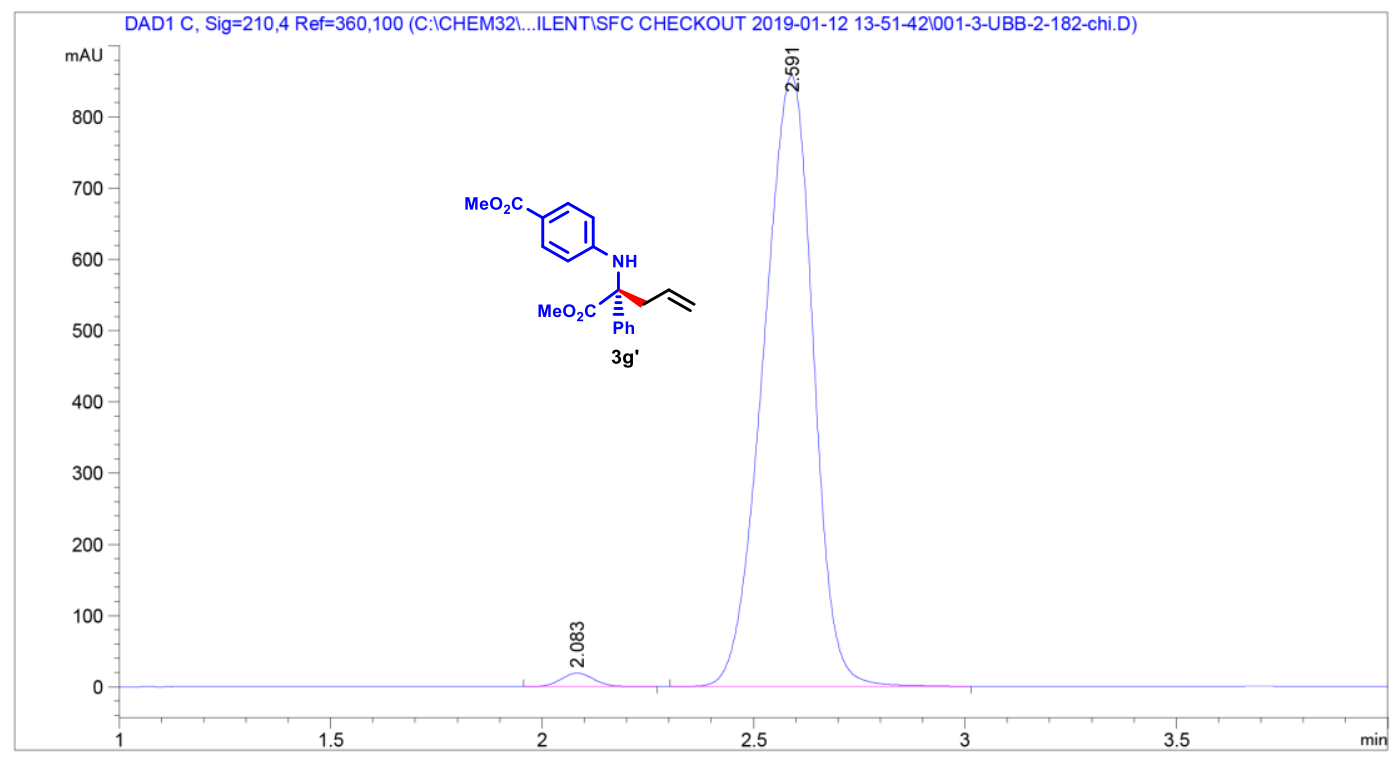

\begin{tabular}{|c|c|c|c|c|c|c|}
\hline $\begin{array}{c}\text { Peak } \\
\text { \# }\end{array}$ & $\begin{array}{c}\text { RetTime } \\
\text { [min] }\end{array}$ & Type & $\begin{array}{l}\text { Width } \\
\text { [min] }\end{array}$ & $\begin{array}{c}\text { Area } \\
{\left[\mathrm{mAU}^{*} \mathrm{~s}\right]}\end{array}$ & $\begin{array}{l}\text { Height } \\
\text { [mAU] }\end{array}$ & $\begin{array}{c}\text { Area } \\
\%\end{array}$ \\
\hline & & & & & & \\
\hline 2 & 2.591 & N R & .1283 & $\begin{array}{r}102.08995 \\
7007.68457\end{array}$ & $\begin{array}{r}18 . / 5689 \\
857.81726\end{array}$ & $\begin{array}{r}1.4359 \\
98.5641\end{array}$ \\
\hline
\end{tabular}




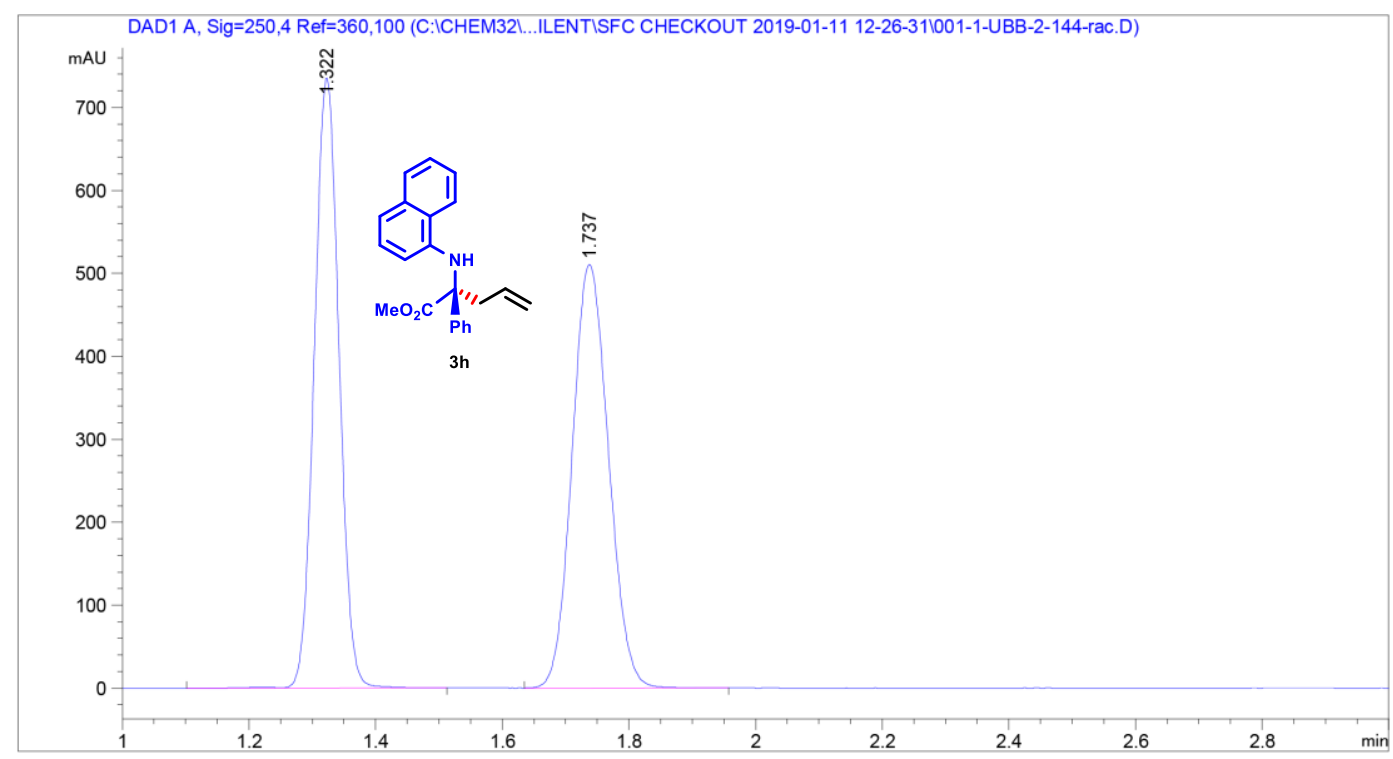

\begin{tabular}{|c|c|c|c|c|c|c|}
\hline $\begin{array}{c}\text { Peak } \\
\quad \#\end{array}$ & $\begin{array}{c}\text { RetTime } \\
\text { [min] }\end{array}$ & Type & $\begin{array}{l}\text { Width } \\
\text { [min] }\end{array}$ & $\begin{array}{c}\text { Area } \\
{[\mathrm{mAU} * \mathrm{~s}]}\end{array}$ & $\begin{array}{l}\text { Height } \\
{[\mathrm{mAU}]}\end{array}$ & $\begin{array}{c}\text { Area } \\
\%\end{array}$ \\
\hline & & & & 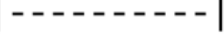 & & 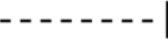 \\
\hline 1 & 1.322 & VB $R$ & 0.0426 & 1992.26819 & 735.55304 & 49.4428 \\
\hline 2 & 1.737 & BB & 0.0620 & 2037.17126 & 510.88382 & 50.5572 \\
\hline
\end{tabular}
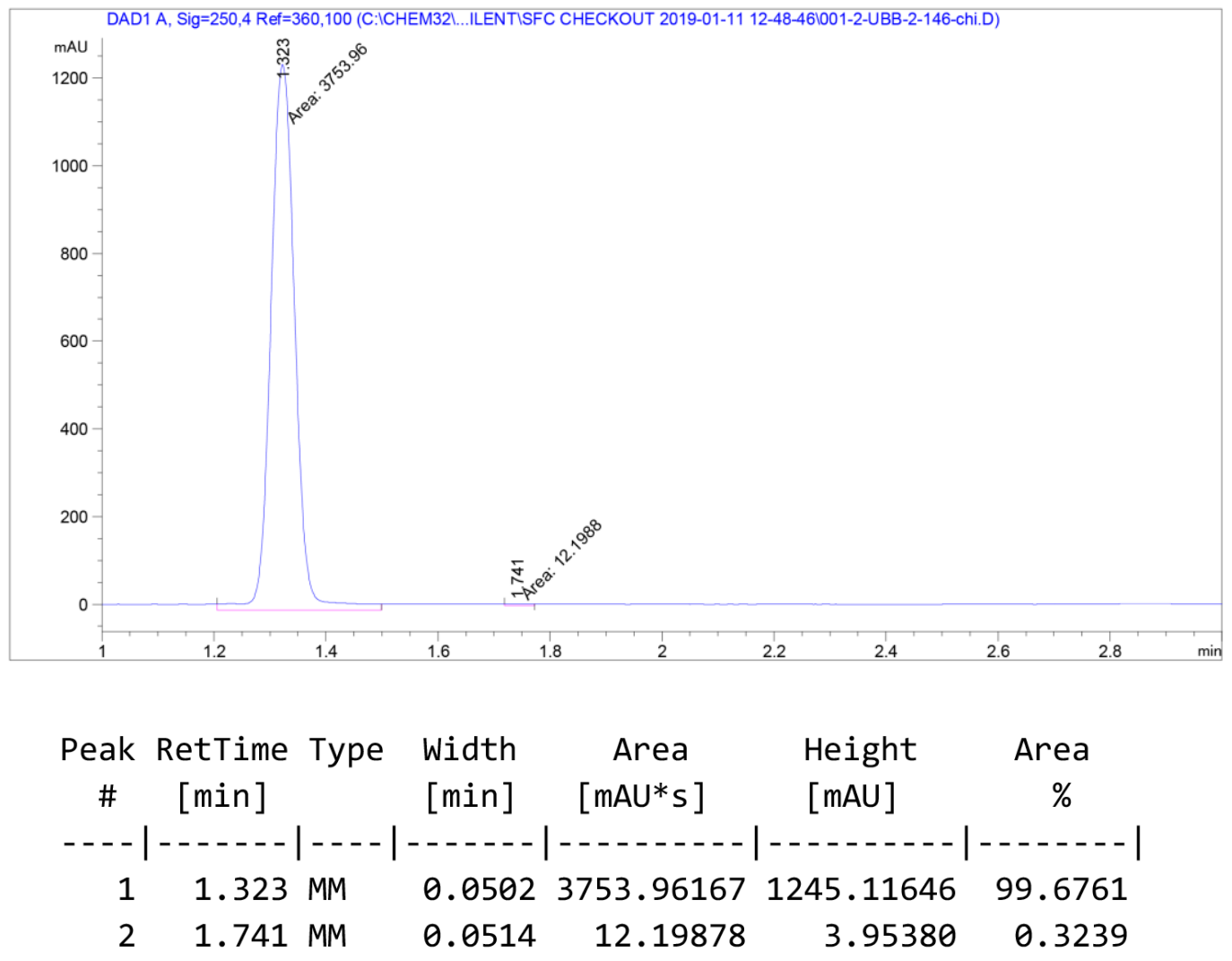

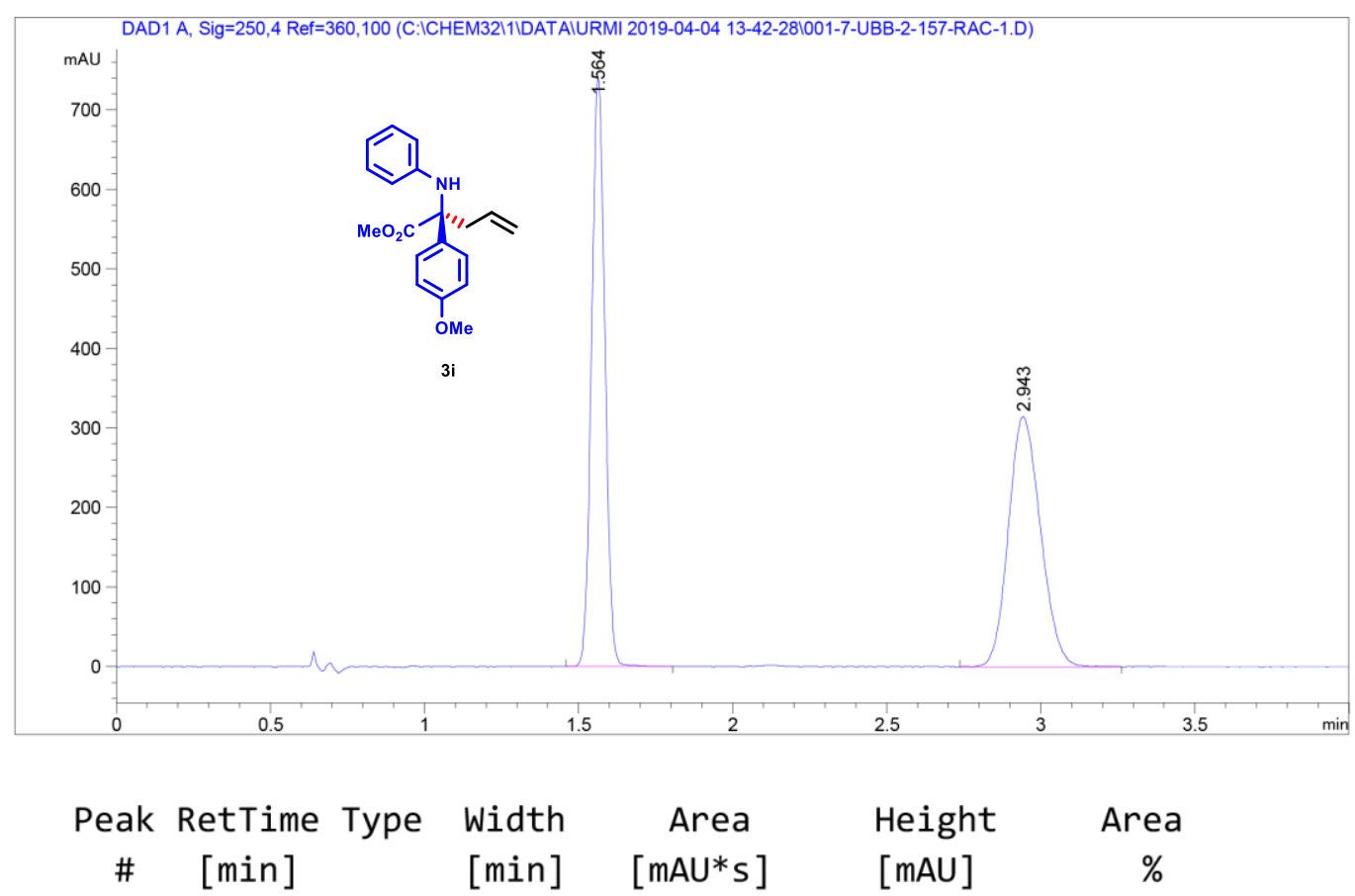

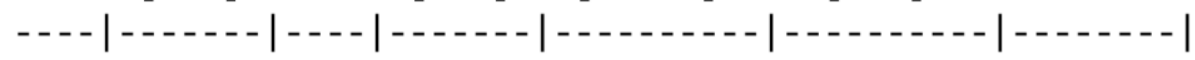

$\begin{array}{lllllll}1 & 1.564 & \text { BV R } & 0.0474 & 2239.71094 & 739.15942 & 49.5721\end{array}$

$\begin{array}{lllllll}2 & 2.943 \\ \text { VV R } & 0.1121 & 2278.37842 & 314.25540 & 50.4279\end{array}$

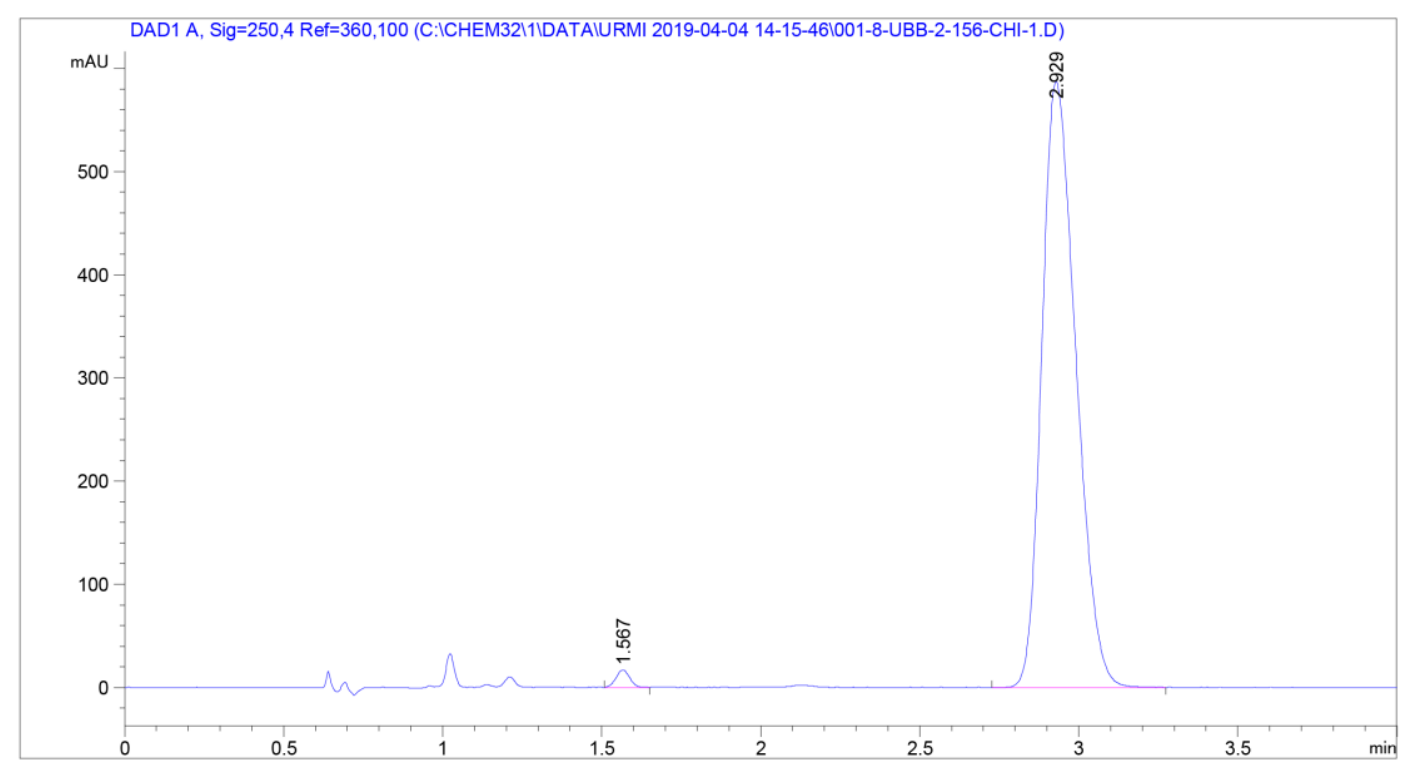

\begin{tabular}{|c|c|c|c|c|c|c|}
\hline $\begin{array}{c}\text { Peak } \\
\quad \#\end{array}$ & $\begin{array}{c}\text { RetTime } \\
\text { [min] }\end{array}$ & Type & $\begin{array}{l}\text { Width } \\
\text { [min] }\end{array}$ & $\begin{array}{c}\text { Area } \\
{\left[\mathrm{mAU}^{*} \mathrm{~s}\right]}\end{array}$ & $\begin{array}{l}\text { Height } \\
\text { [mAU] }\end{array}$ & $\begin{array}{c}\text { Area } \\
\%\end{array}$ \\
\hline & & & & & & \\
\hline 1 & 1.56 & BB & 0.045 & 50.02213 & 16.75908 & 326 \\
\hline 2 & 2.929 & BV $R$ & 0.1163 & 4366.36768 & 586.87823 & 98.8674 \\
\hline
\end{tabular}




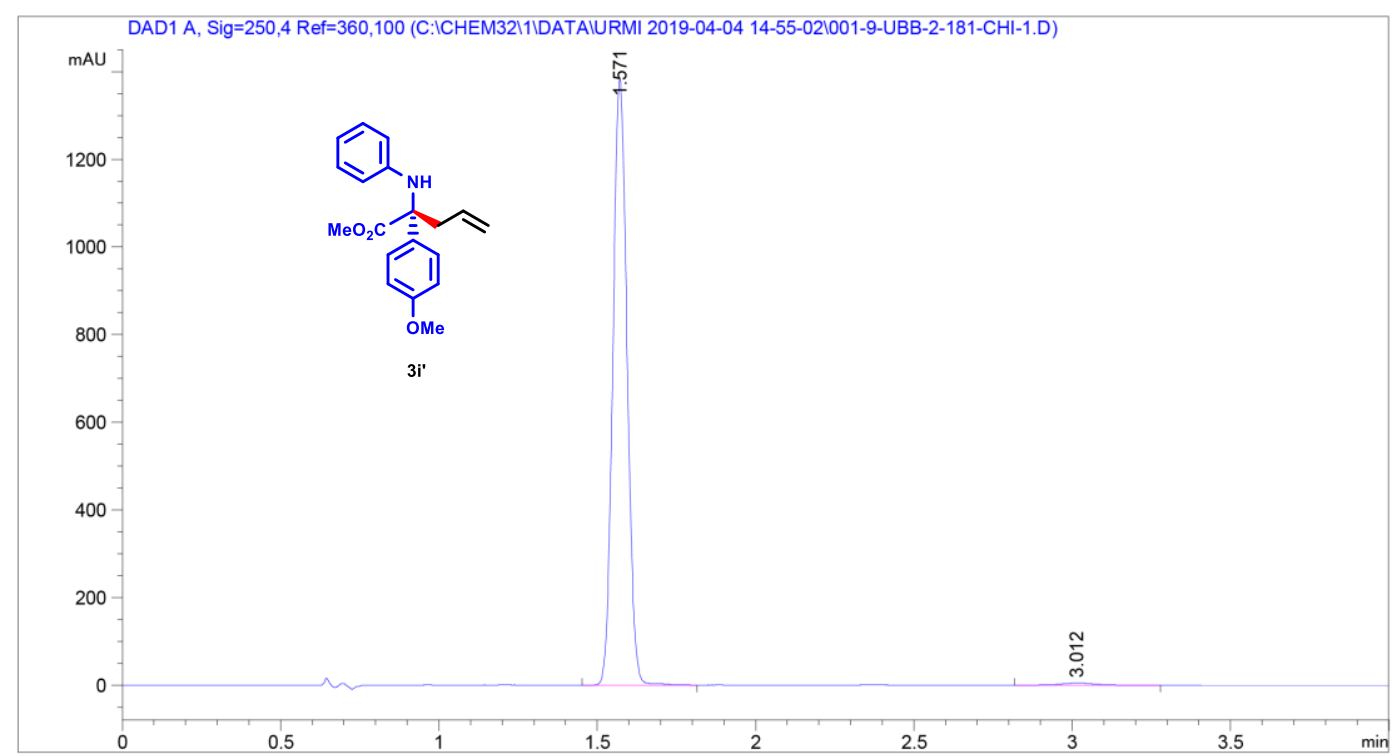

\begin{tabular}{|c|c|c|c|c|c|c|}
\hline $\begin{array}{c}\text { Peak } \\
\#\end{array}$ & $\begin{array}{c}\text { RetTime } \\
\text { [min] }\end{array}$ & Type & $\begin{array}{l}\text { Width } \\
\text { [min] }\end{array}$ & $\begin{array}{c}\text { Area } \\
{[\mathrm{mAU} * \mathrm{~s}]}\end{array}$ & $\begin{array}{l}\text { Height } \\
\text { [mAU] }\end{array}$ & $\begin{array}{c}\text { Area } \\
\%\end{array}$ \\
\hline & & & & 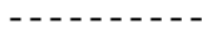 & & \\
\hline 1 & 1.57 & $3 V R$ & & 4308.76855 & 1382.40625 & 1585 \\
\hline 2 & 3.012 & VV R & 0.0931 & 36.56690 & 4.73466 & 0.8415 \\
\hline
\end{tabular}



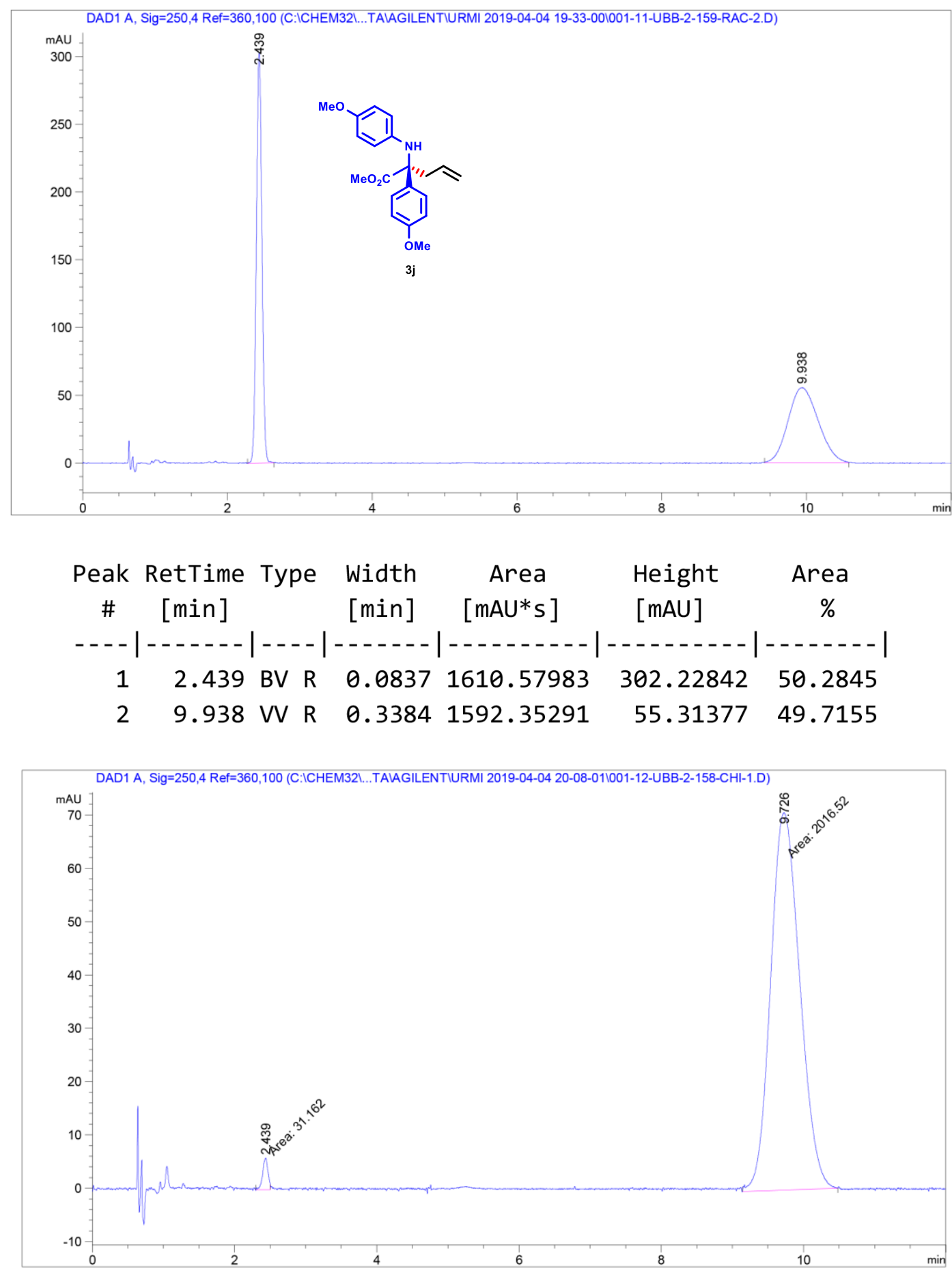

\begin{tabular}{cccccc}
$\begin{array}{c}\text { Peak RetTime Type } \\
\text { Width } \\
{[\mathrm{min}]}\end{array}$ & $\begin{array}{c}\text { Area } \\
{[\mathrm{min}]}\end{array}$ & $\begin{array}{c}\text { Height } \\
{[\mathrm{mAU} \text { s }]}\end{array}$ & $\begin{array}{c}\text { Area } \\
{[\mathrm{mAU}]}\end{array}$ & $\begin{array}{c}\% \\
\%\end{array}$ \\
\hline 1 & 2.439 PM & 0.0871 & 31.16204 & 5.96544 & 1.5218 \\
2 & 9.726 PP & 0.4750 & 2016.51636 & 70.76195 & 98.4782
\end{tabular}




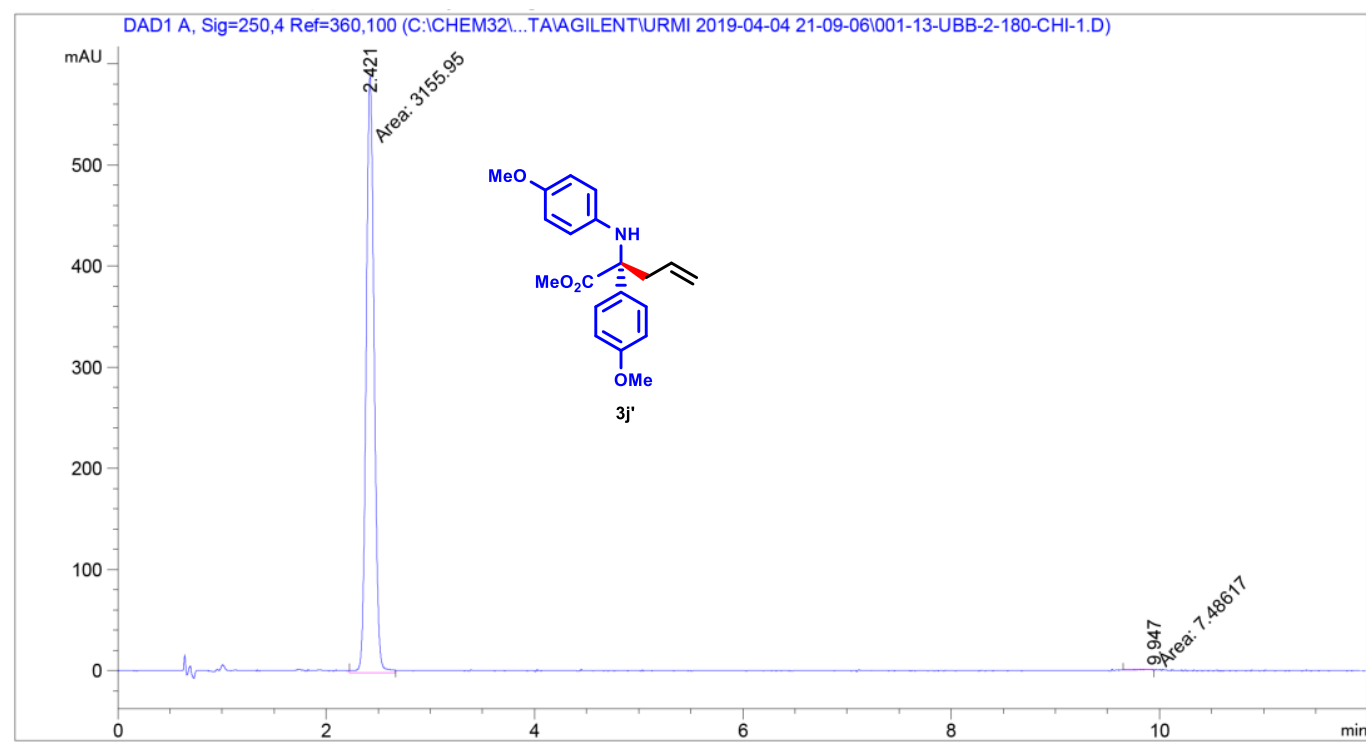

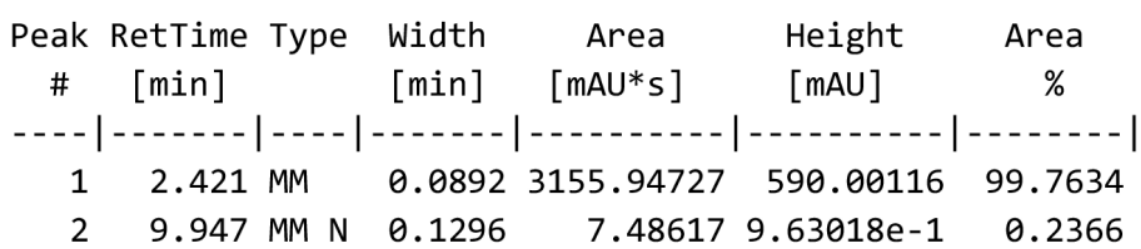




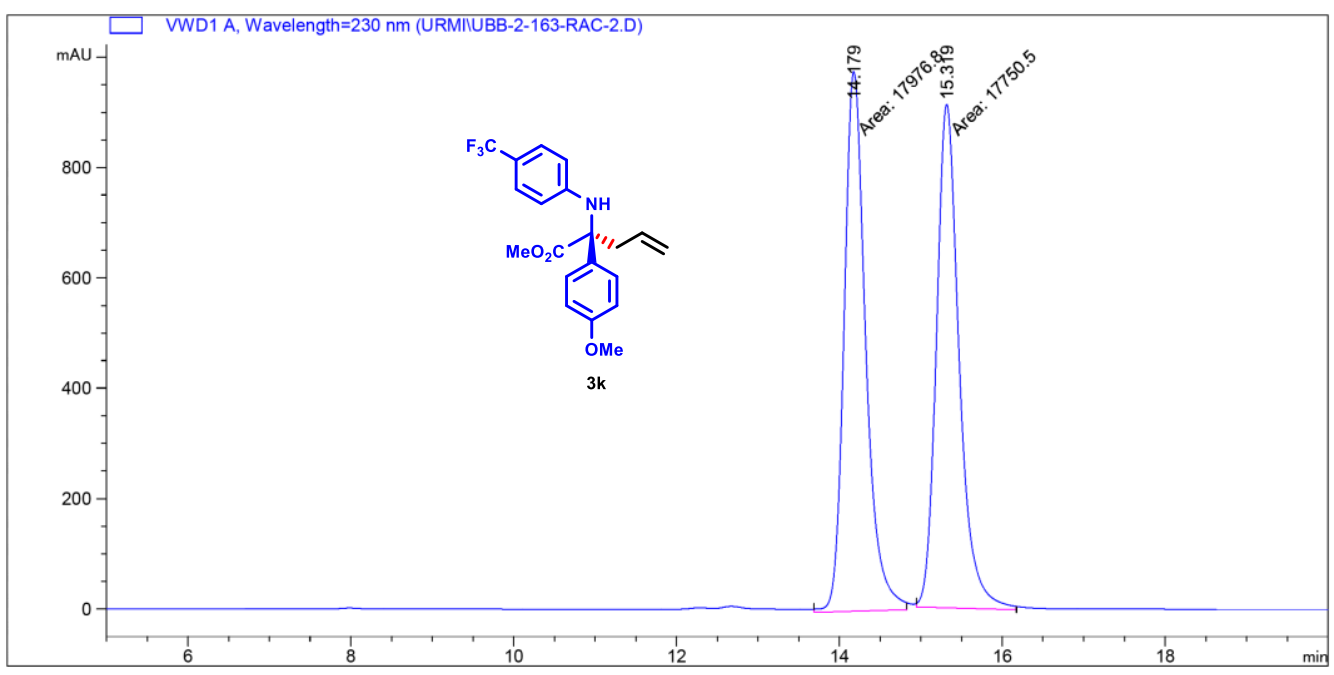

\begin{tabular}{|c|c|c|c|c|c|c|}
\hline $\begin{array}{c}\text { Peak } \\
\quad \#\end{array}$ & $\begin{array}{c}\text { RetTime } \\
\text { [min] }\end{array}$ & Type & $\begin{array}{l}\text { Width } \\
\text { [min] }\end{array}$ & $\begin{array}{c}\text { Area } \\
{\left[\mathrm{mAU}{ }^{*} \mathrm{~s}\right]}\end{array}$ & $\begin{array}{l}\text { Height } \\
{[\mathrm{mAU}]}\end{array}$ & $\begin{array}{c}\text { Area } \\
\quad \%\end{array}$ \\
\hline & & & & --------- & $----------\mid$ & $--------\mid$ \\
\hline 1 & 14.179 & MM & 0.3062 & $1.79768 e 4$ & 978.34601 & 50.3167 \\
\hline 2 & 15.319 & MM & 0.3241 & $1.77505 \mathrm{e} 4$ & 912.86145 & 49.6833 \\
\hline
\end{tabular}

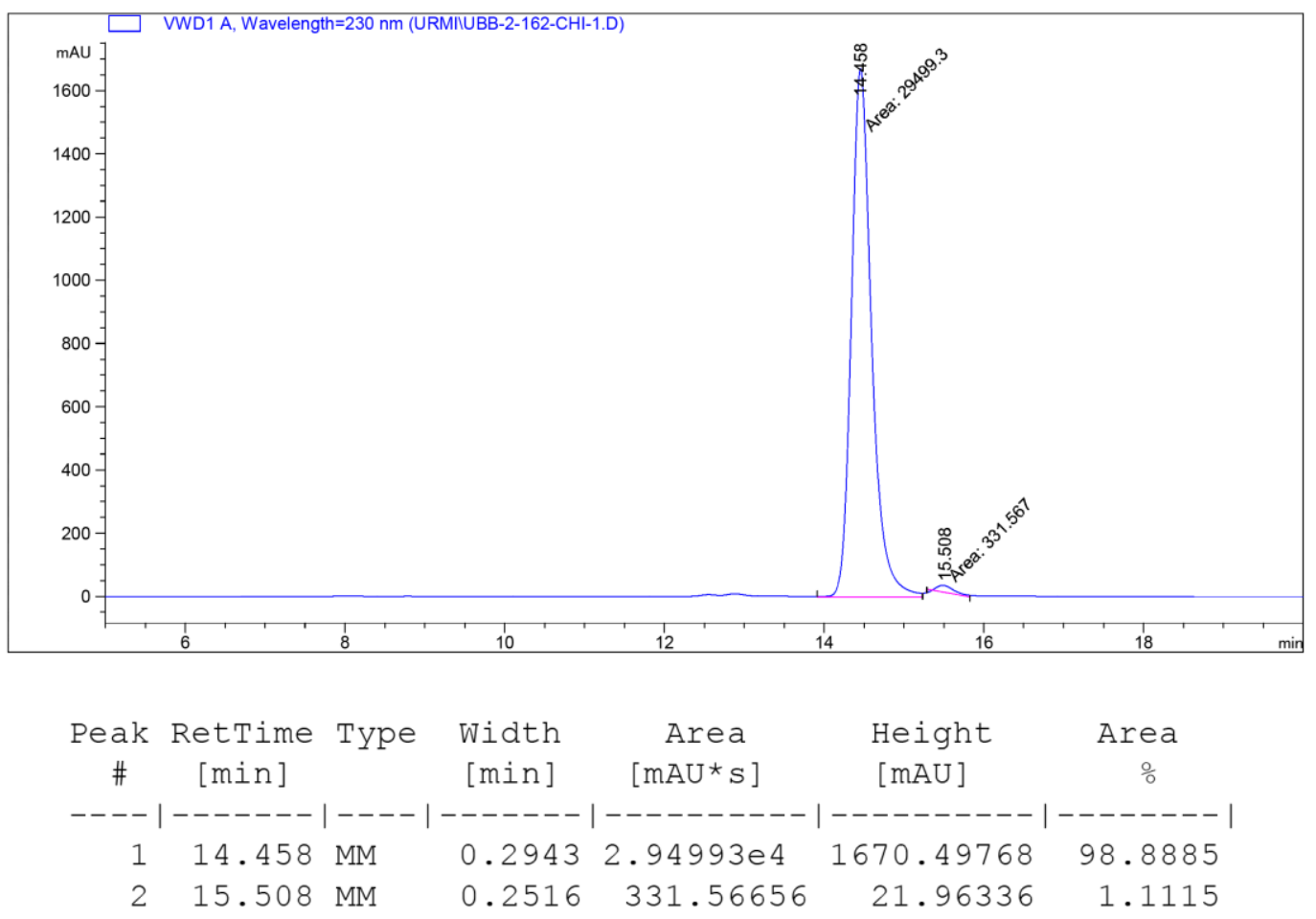



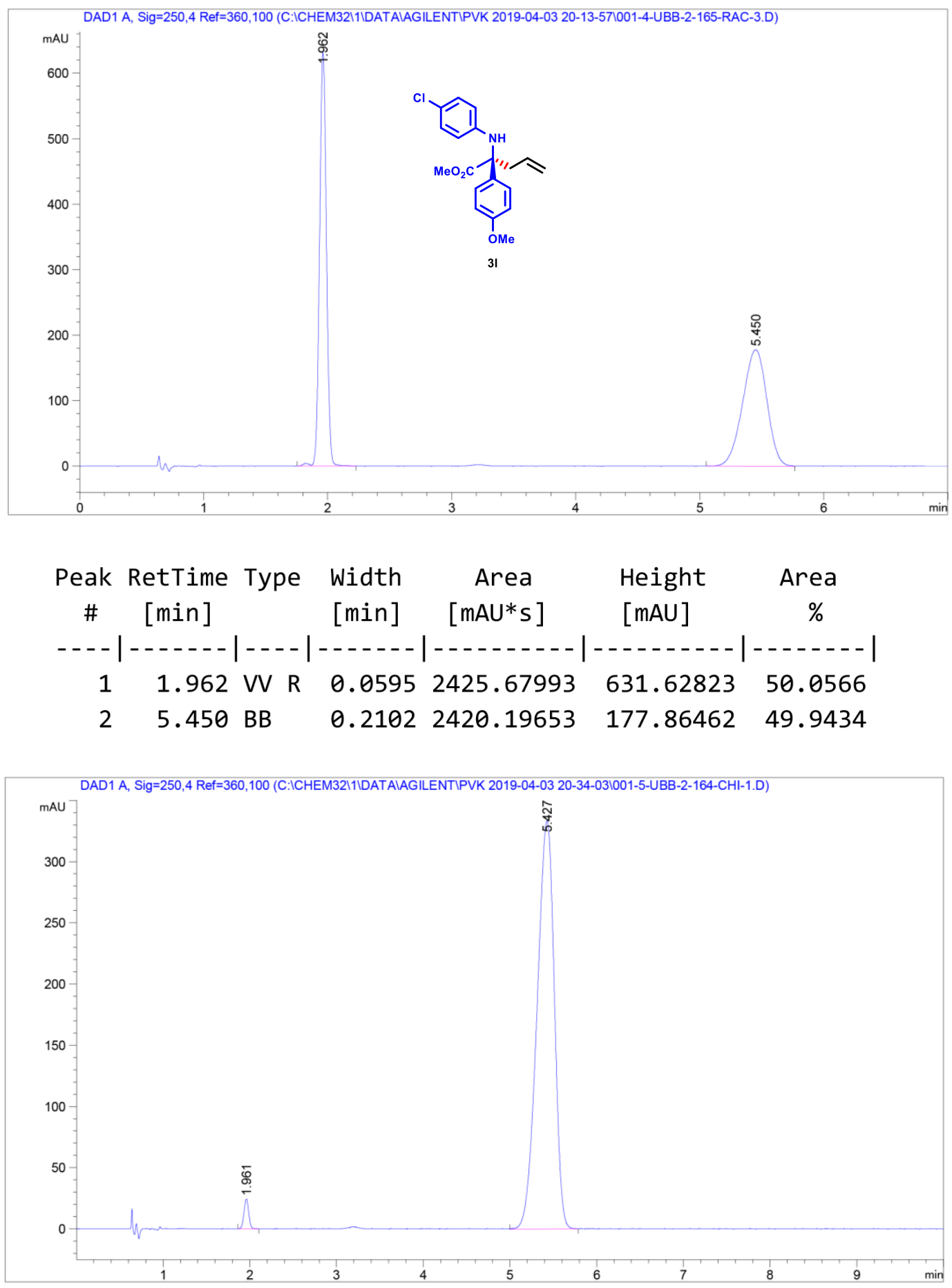

\begin{tabular}{|c|c|c|c|c|c|c|}
\hline $\begin{array}{c}\text { Peak } \\
\text { \# }\end{array}$ & $\begin{array}{c}\text { RetTime } \\
\text { [min] }\end{array}$ & Type & $\begin{array}{l}\text { Width } \\
\text { [min] }\end{array}$ & $\begin{array}{c}\text { Area } \\
{\left[\mathrm{mAU}^{*} \mathrm{~s}\right]}\end{array}$ & $\begin{array}{l}\text { Height } \\
\text { [mAU] }\end{array}$ & $\begin{array}{c}\text { Area } \\
\%\end{array}$ \\
\hline & & & & & & \\
\hline 1 & 1.961 & $3 \mathrm{~V} R$ & 0.0575 & 91.77501 & 24.32663 & 1.9523 \\
\hline 2 & 5.427 & $V V R$ & 0.2188 & 4609.06592 & 333.38510 & 98.0477 \\
\hline
\end{tabular}



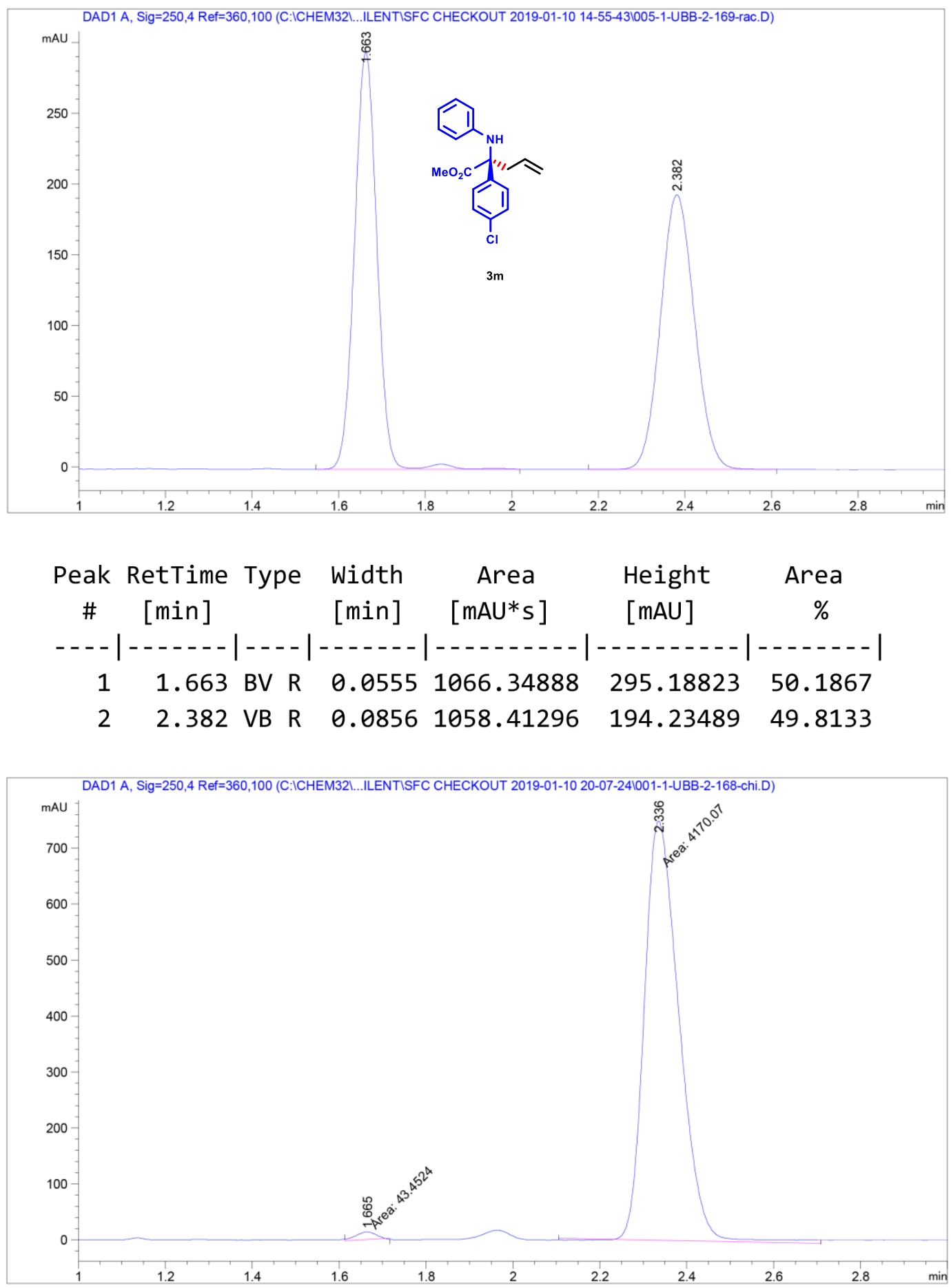

\begin{tabular}{cccccc}
$\begin{array}{c}\text { Peak RetTime Type } \\
\text { Width }\end{array}$ & $\begin{array}{c}\text { Area } \\
{[\mathrm{min}]}\end{array}$ & $\begin{array}{c}\text { Height } \\
{[\mathrm{min}]}\end{array}$ & $\begin{array}{c}\text { Area } \\
{[\mathrm{mAU} \text { s }]}\end{array}$ & \multicolumn{1}{c}{$[\mathrm{mAU}]$} & $\%$ \\
\hline 1 & $1.665 \mathrm{MM}$ & 0.0534 & 43.45244 & 13.56465 & 1.0313 \\
2 & $2.336 \mathrm{MM}$ & 0.0928 & 4170.06982 & 748.67224 & 98.9687
\end{tabular}



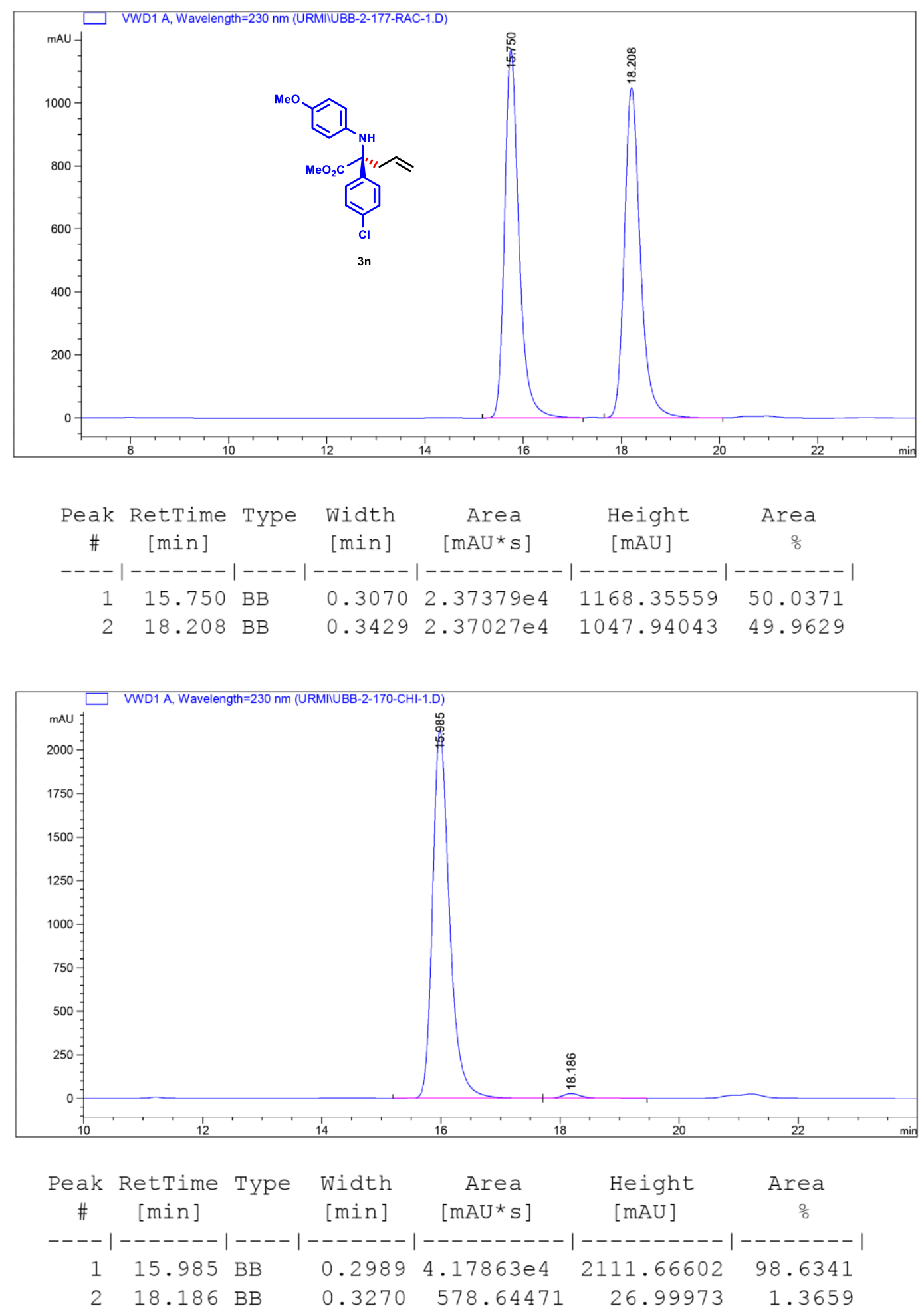

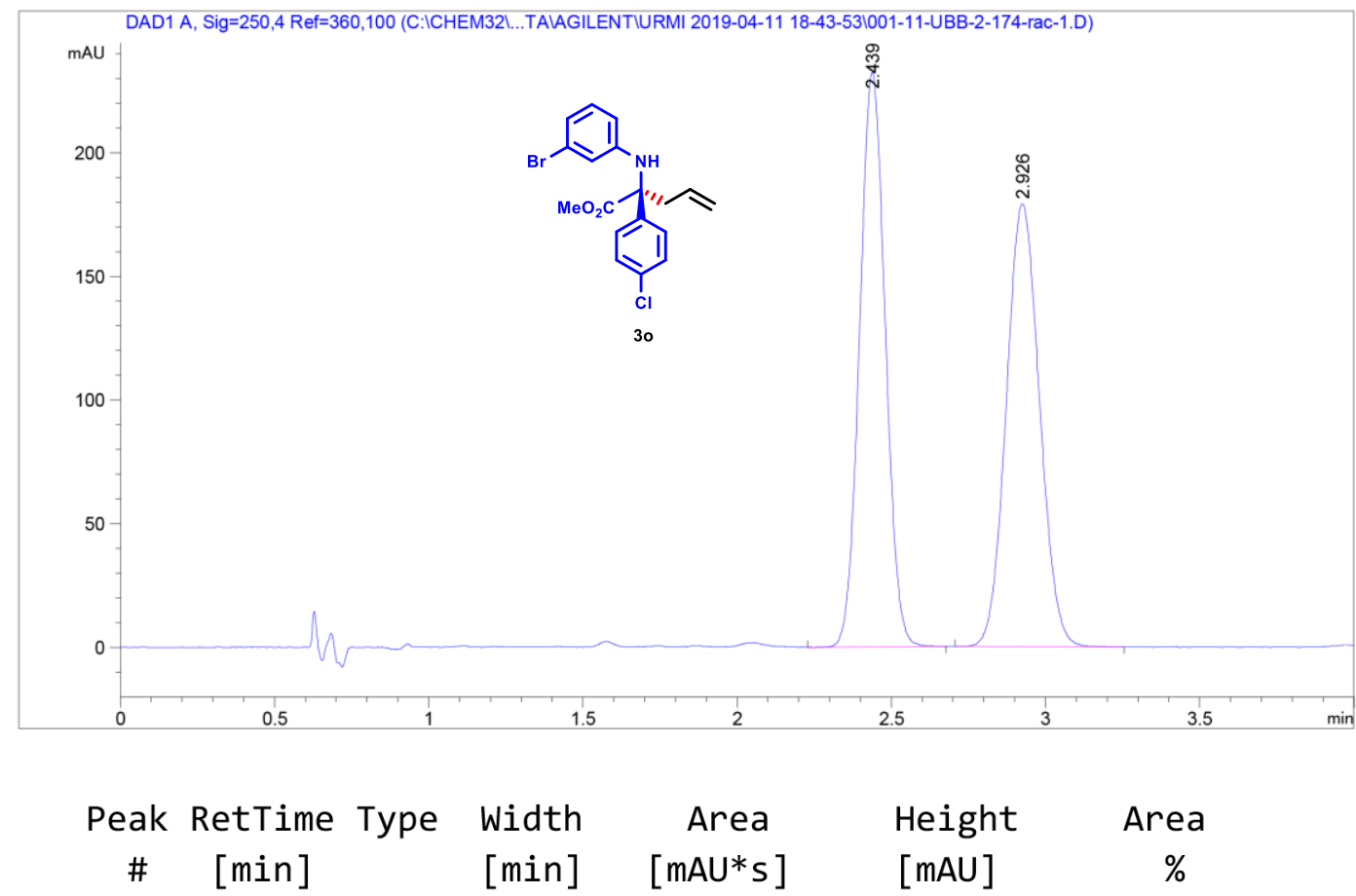

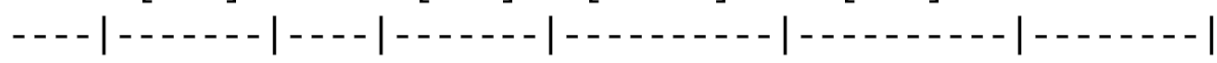

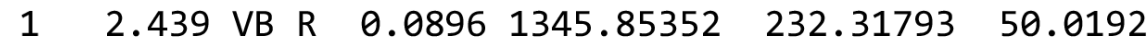

$\begin{array}{llllllll}2 & 2.926 & \text { BV R } & 0.1166 & 1344.81848 & 179.13271 & 49.9808\end{array}$

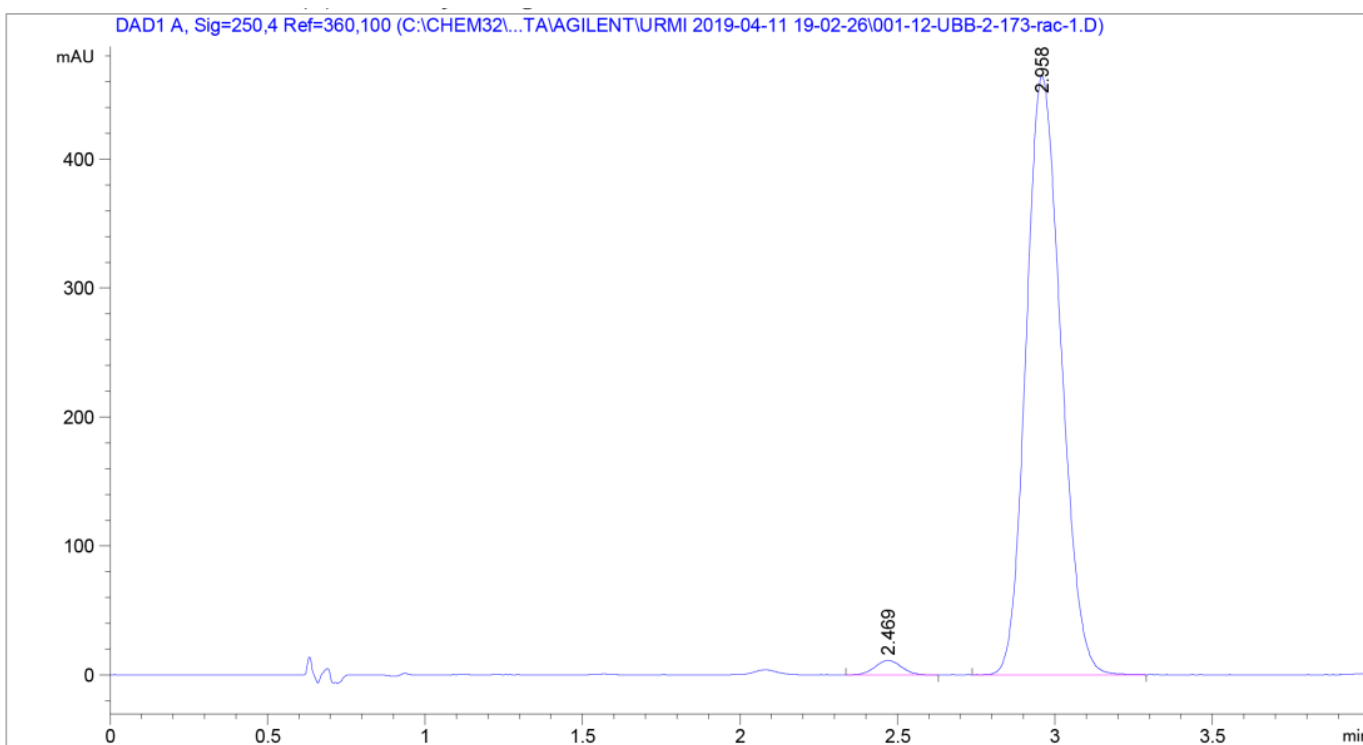

\begin{tabular}{|c|c|c|c|c|c|c|}
\hline $\begin{array}{c}\text { Peak } \\
\text { \# }\end{array}$ & $\begin{array}{c}\text { RetTime } \\
\text { [min] }\end{array}$ & Type & $\begin{array}{l}\text { Width } \\
\text { [min] }\end{array}$ & $\begin{array}{c}\text { Area } \\
{\left[\mathrm{mAU}^{*} \mathrm{~s}\right]}\end{array}$ & $\begin{array}{l}\text { Height } \\
{[\mathrm{mAU}]}\end{array}$ & $\begin{array}{c}\text { Area } \\
\%\end{array}$ \\
\hline & & & & & & \\
\hline 1 & & & $0 . e$ & 64.61723 & 194 & 744 \\
\hline 2 & 2.958 & VV R & 0.1210 & 3576.96484 & 463.91217 & 98.2256 \\
\hline
\end{tabular}




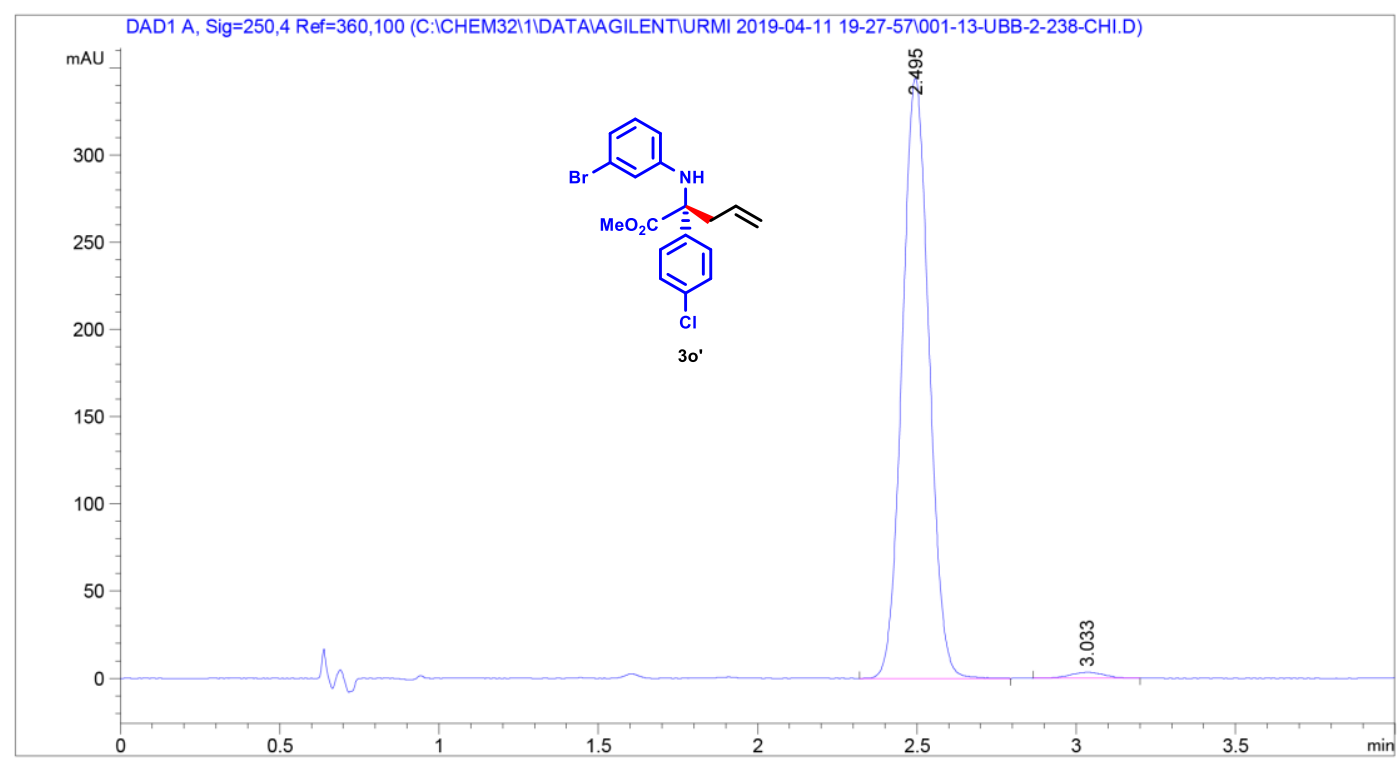

$\begin{array}{ccccc}\text { Peak RetTime Type Width } & \text { Area } & \text { Height } & \text { Area } \\ \#[\text { [min] } & {[\text { min] }} & {[\text { mAU*s }]} & {[\text { mAU] }} & \%\end{array}$

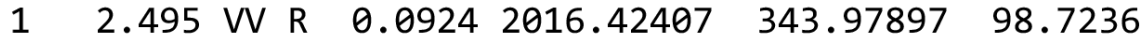

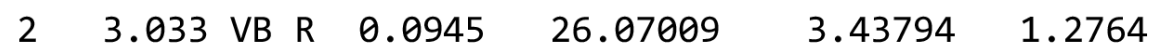



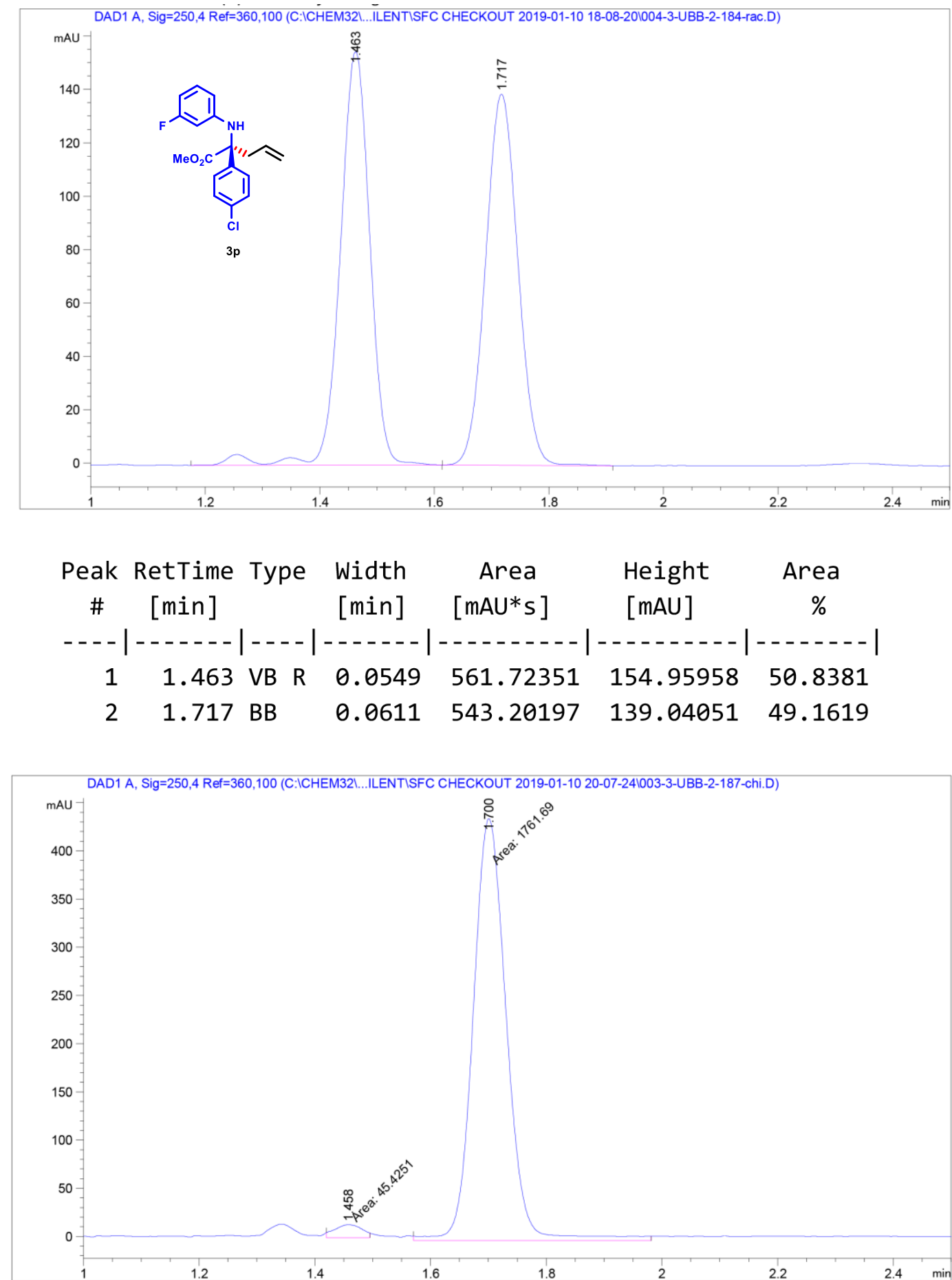

\begin{tabular}{cccccc}
$\begin{array}{c}\text { Peak RetTime Type } \\
\text { \# }\end{array}$ [min] & $\begin{array}{c}\text { Width } \\
{[\mathrm{min}]}\end{array}$ & $\begin{array}{c}\text { Area } \\
{[\text { mAU*s }]}\end{array}$ & $\begin{array}{c}\text { Height } \\
{[\mathrm{mAU}]}\end{array}$ & $\begin{array}{c}\text { Area } \\
\%\end{array}$ \\
\hline 1 & 1.458 MM & 0.0552 & 45.42506 & 13.71776 & 2.5137 \\
2 & 1.700 MM & 0.0671 & 1761.69177 & 437.63898 & 97.4863
\end{tabular}




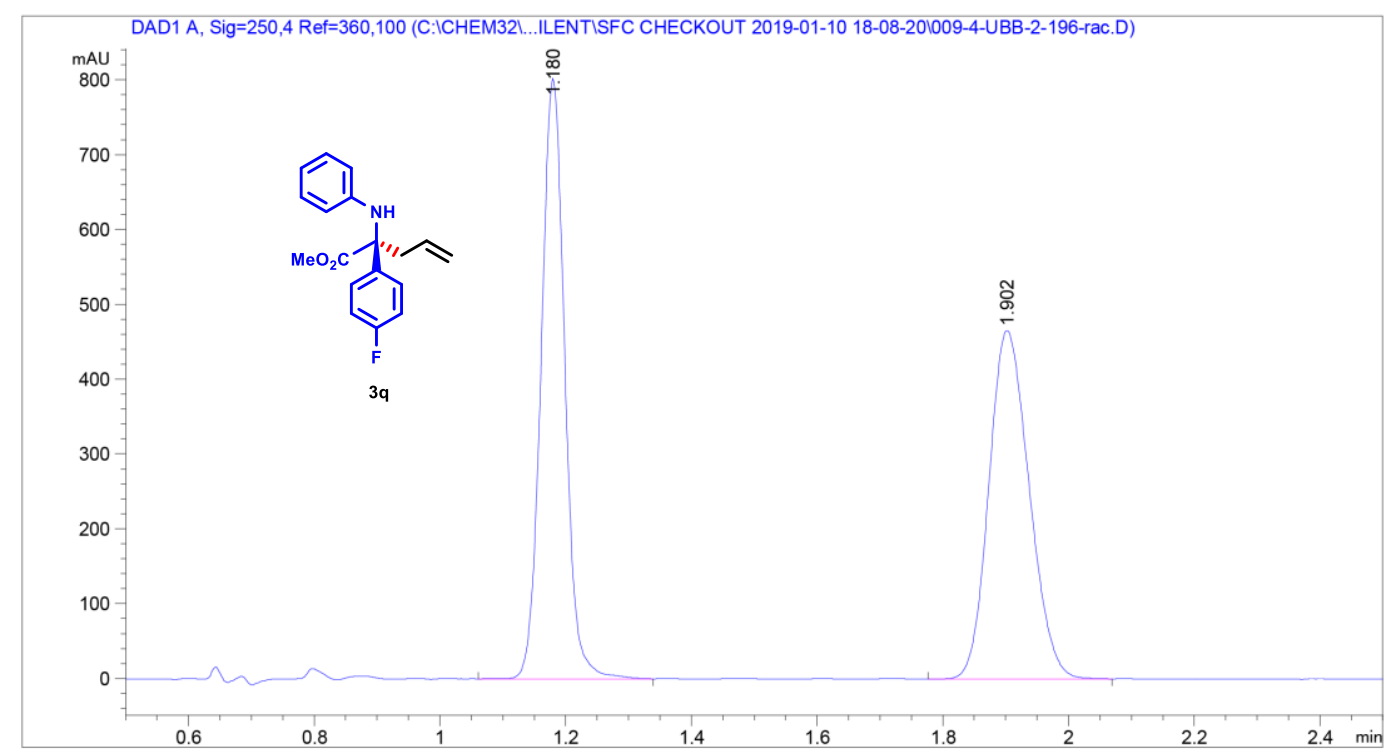

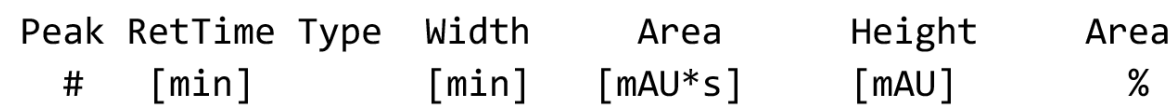

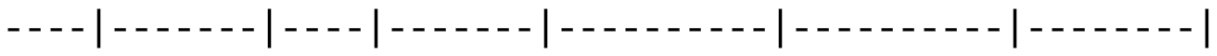

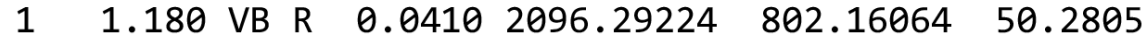

$\begin{array}{llllllll}2 & 1.902 & V B & R & 0.0695 & 2072.89990 & 465.51599 & 49.7195\end{array}$

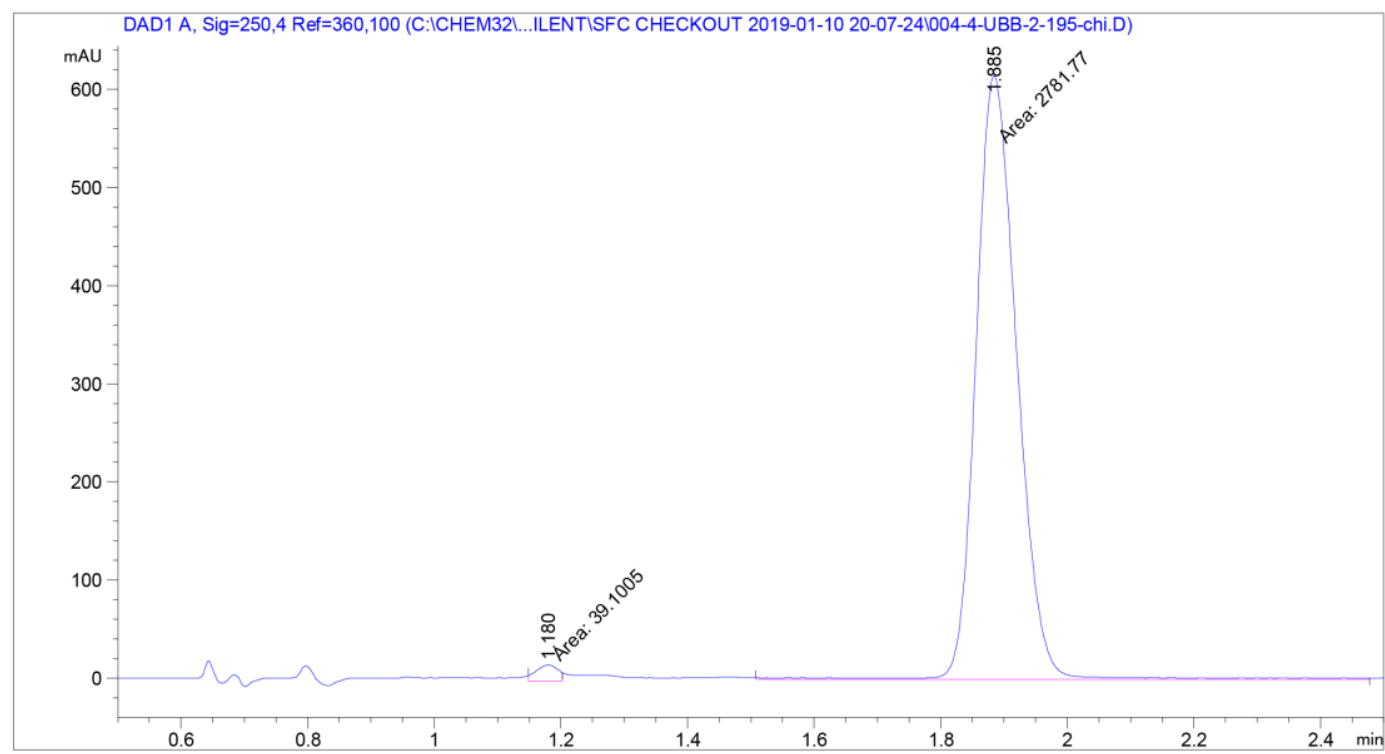

Peak RetTime Type width Area Height Area

\begin{tabular}{rrrrrr}
$\#$ & {$[\mathrm{~min}]$} & {$[\mathrm{min}]$} & {$[\mathrm{mAU} * \mathrm{~s}]$} & \multicolumn{1}{c}{$[\mathrm{mAU}]$} & \multicolumn{1}{c}{$\%$} \\
1 & $1.180 \mathrm{MM}$ & 0.0401 & 39.10055 & 16.24254 & 1.3861 \\
2 & $1.885 \mathrm{MM}$ & 0.0754 & 2781.77246 & 615.16626 & 98.6139
\end{tabular}




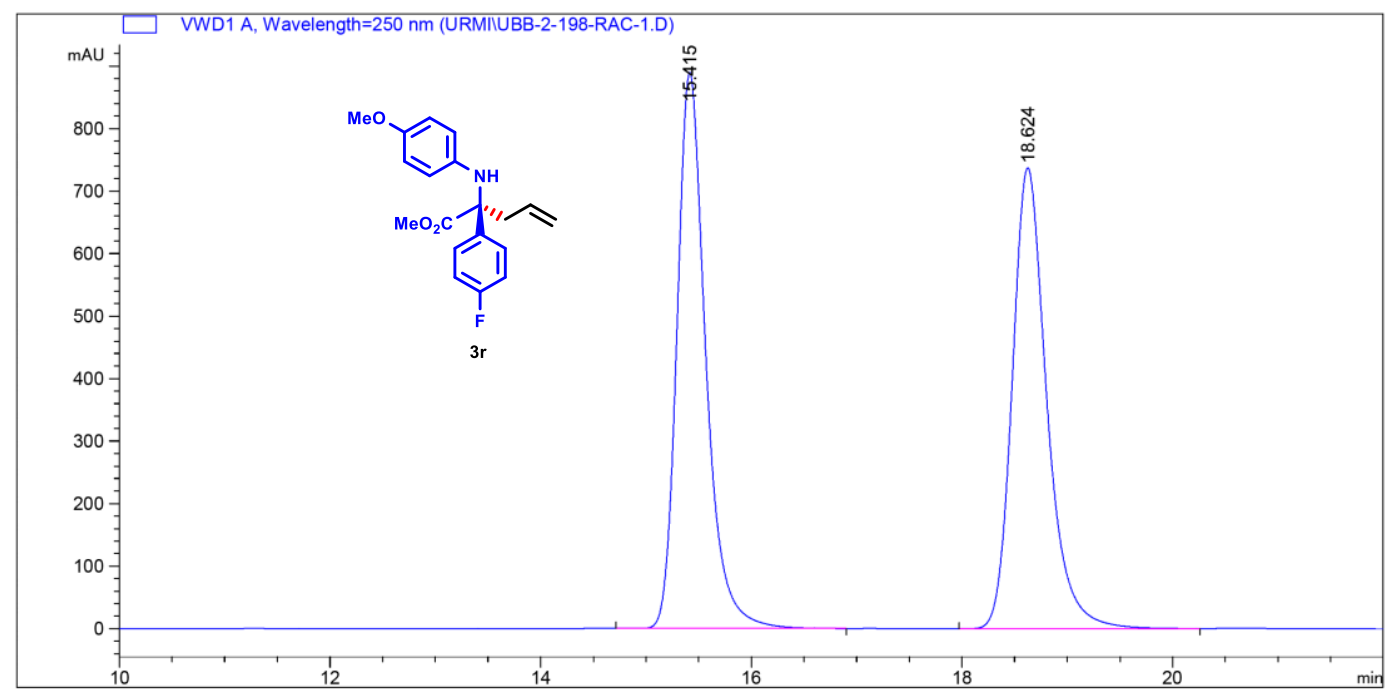

\begin{tabular}{|c|c|c|c|c|c|c|}
\hline $\begin{array}{c}\text { Peak } \\
\text { \# }\end{array}$ & $\begin{array}{c}\text { RetTime } \\
\text { [min] }\end{array}$ & Type & $\begin{array}{l}\text { Width } \\
\text { [min] }\end{array}$ & $\begin{array}{c}\text { Area } \\
{\left[\mathrm{mAU}^{*} \mathrm{~S}\right]}\end{array}$ & $\begin{array}{l}\text { Height } \\
\text { [mAU] }\end{array}$ & $\begin{array}{c}\text { Area } \\
\frac{\circ}{0}\end{array}$ \\
\hline- & & & & -------- & 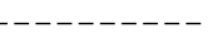 & ---1 \\
\hline 1 & 15 & & .287 & $.68425 e 4$ & 889.07800 & 386 \\
\hline 2 & 18. & & 0.3451 & $1.67493 e 4$ & 737.13922 & 8614 \\
\hline
\end{tabular}

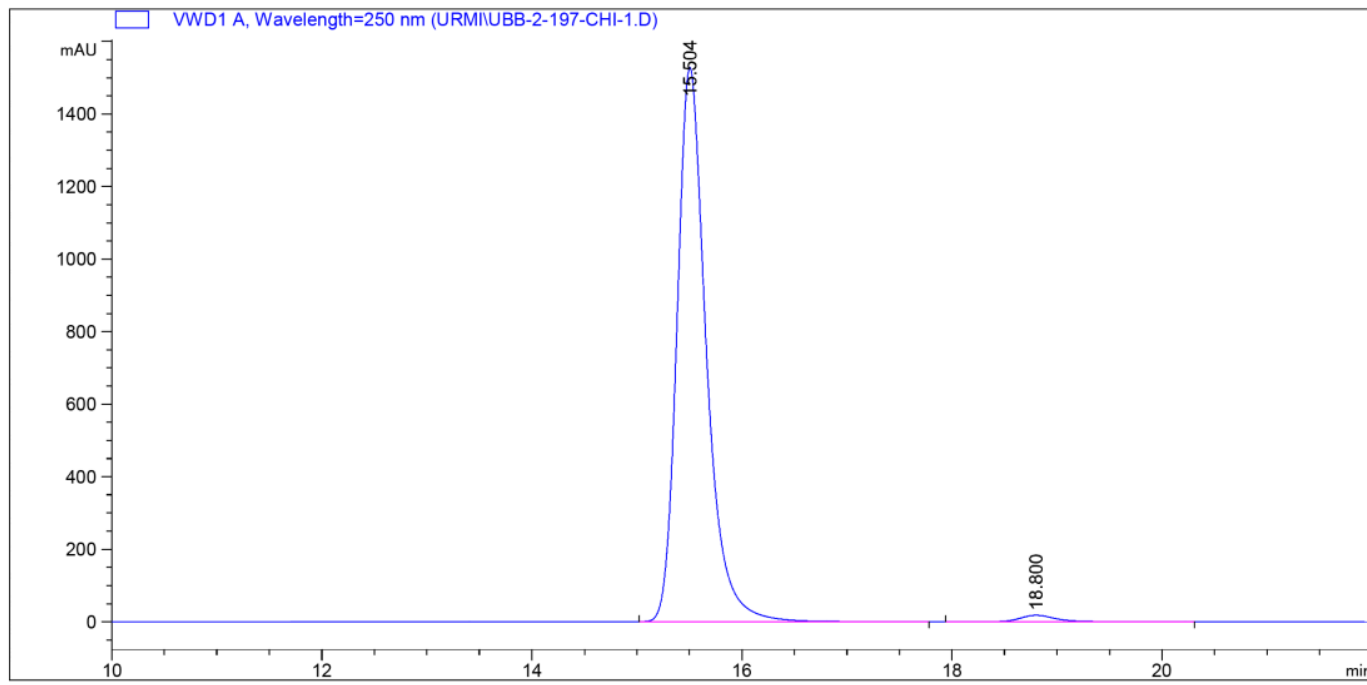

\begin{tabular}{|c|c|c|c|c|c|c|}
\hline $\begin{array}{c}\text { Peak } \\
\text { \# }\end{array}$ & $\begin{array}{c}\text { RetTime } \\
\text { [min] }\end{array}$ & Type & $\begin{array}{l}\text { Width } \\
\text { [min] }\end{array}$ & $\begin{array}{c}\text { Area } \\
{\left[\mathrm{mAU}{ }^{*} \mathrm{~S}\right]}\end{array}$ & $\begin{array}{l}\text { Height } \\
{[\mathrm{mAU}]}\end{array}$ & $\begin{array}{c}\text { Area } \\
\frac{\circ}{0}\end{array}$ \\
\hline 1 & 15.504 & BB & 0.2923 & $2.95039 e 4$ & 1527.45984 & 98.5668 \\
\hline 2 & 18.800 & $\mathrm{BB}$ & 0.3574 & 428.99579 & 17.97794 & 1.4332 \\
\hline
\end{tabular}



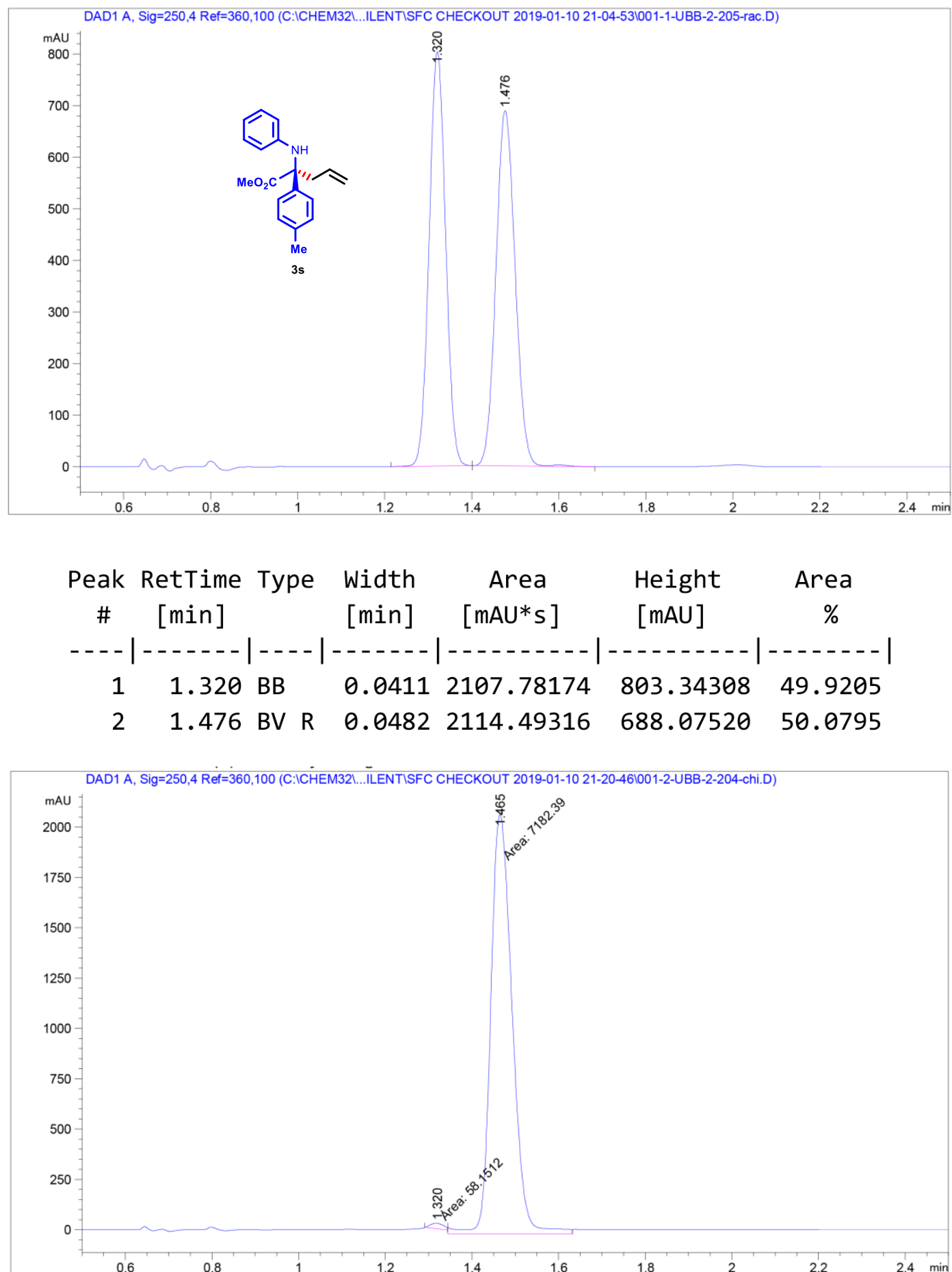

\begin{tabular}{|c|c|c|c|c|c|c|}
\hline $\begin{array}{c}\text { Peak } \\
\text { \# }\end{array}$ & $\begin{array}{c}\text { RetTime } \\
\text { [min] }\end{array}$ & Type & $\begin{array}{l}\text { Width } \\
\text { [min] }\end{array}$ & $\begin{array}{c}\text { Area } \\
{\left[\mathrm{mAU}^{*} \mathrm{~s}\right]}\end{array}$ & $\begin{array}{l}\text { Height } \\
\text { [mAU] }\end{array}$ & $\begin{array}{c}\text { Area } \\
\%\end{array}$ \\
\hline & & & & & & \\
\hline$\frac{1}{2}$ & 1.465 & & .0574 & $\begin{array}{r}30.13110 \\
7182.39355\end{array}$ & 2084.14917 & $\begin{array}{r}0.8031 \\
99.1969\end{array}$ \\
\hline
\end{tabular}



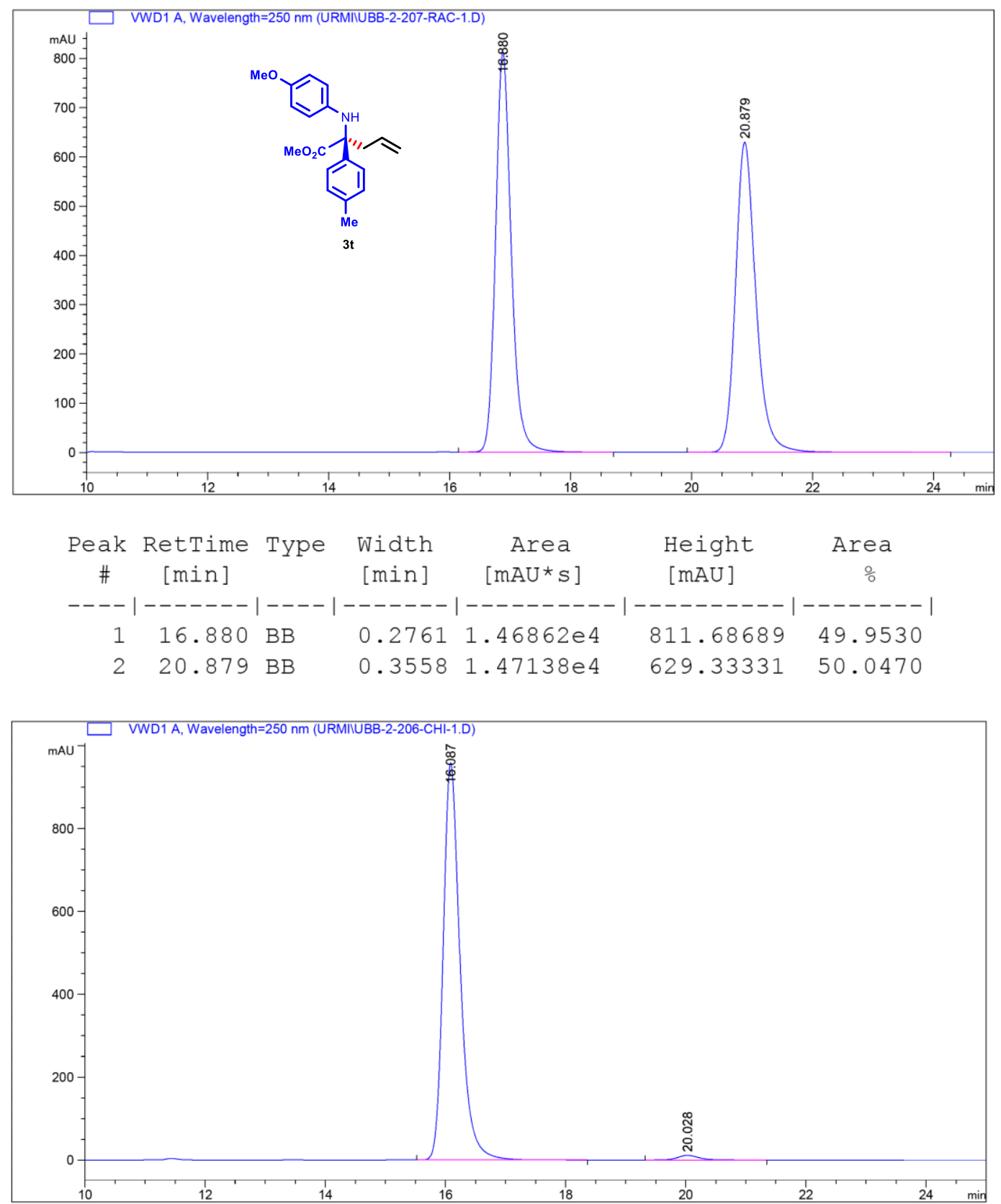

\begin{tabular}{|c|c|c|c|c|c|c|}
\hline $\begin{array}{c}\text { Peak } \\
\quad \#\end{array}$ & $\begin{array}{c}\text { RetTime } \\
\text { [min] }\end{array}$ & Type & $\begin{array}{l}\text { Width } \\
\text { [min] }\end{array}$ & $\begin{array}{c}\text { Area } \\
{\left[\mathrm{mAU}^{*} \mathrm{~S}\right]}\end{array}$ & $\begin{array}{l}\text { Height } \\
{[\mathrm{mAU}]}\end{array}$ & $\begin{array}{c}\text { Area } \\
\frac{\circ}{0}\end{array}$ \\
\hline-1 & -1 & & & --------- & -------- & ------- \\
\hline 1 & 16.087 & BB & 0.2979 & $1.88002 e 4$ & 958.01343 & 98.5517 \\
\hline 2 & 20.028 & $\mathrm{BB}$ & 0.3749 & 276.28302 & 11.11694 & 1.4483 \\
\hline
\end{tabular}



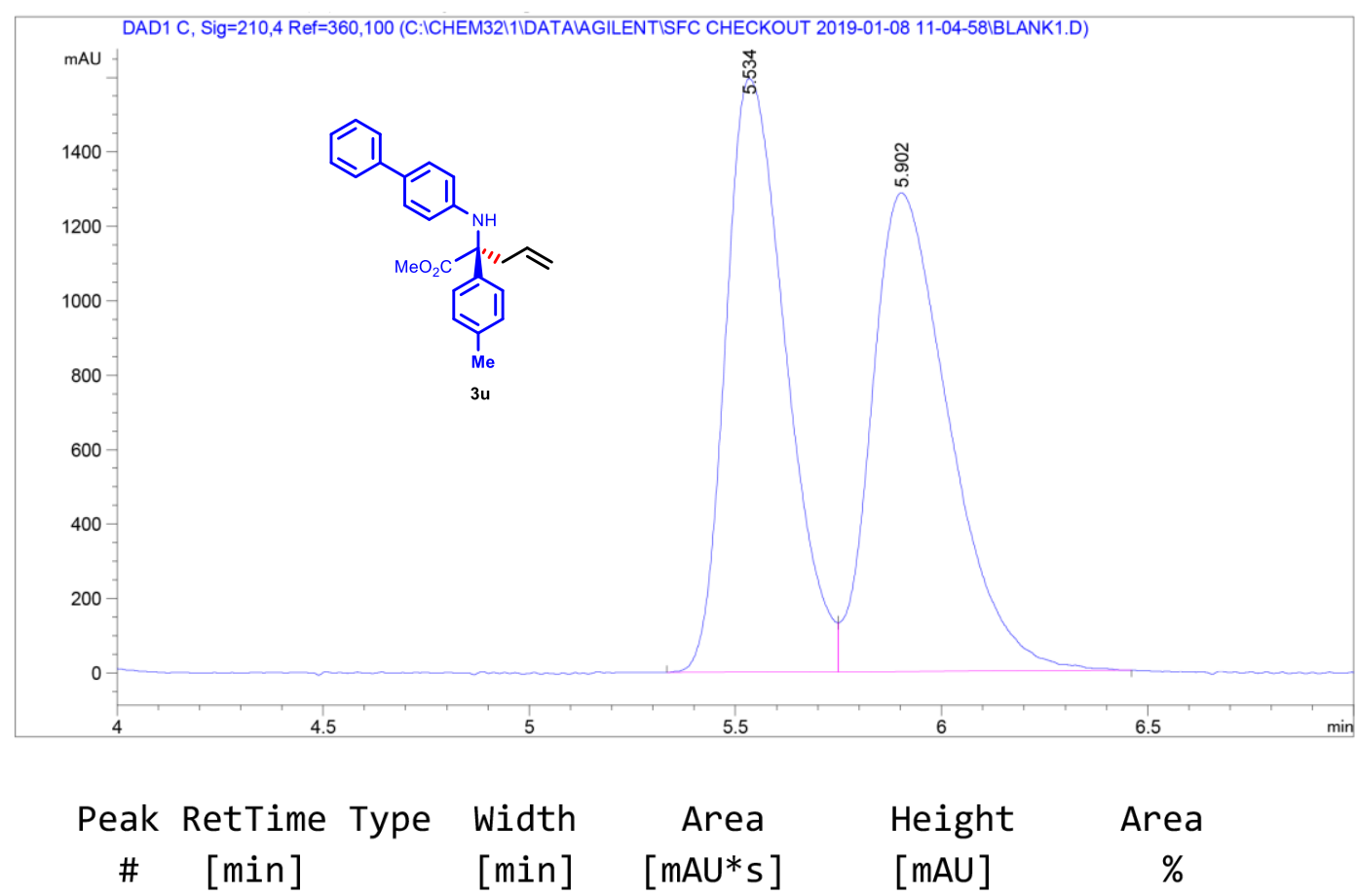

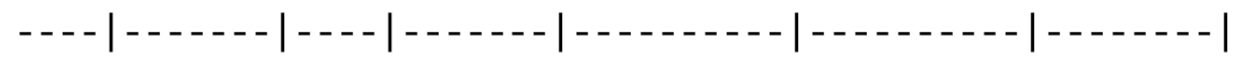

$\begin{array}{llllll}1 & 5.534 \text { BV } \quad 0.1503 & 1.61689 \mathrm{e} 4 & 1595.19592 & 49.0617\end{array}$

$\begin{array}{lllllll}2 & 5.902 \text { VV R } & 0.1898 & 1.67874 \mathrm{e} 4 & 1286.16174 & 50.9383\end{array}$

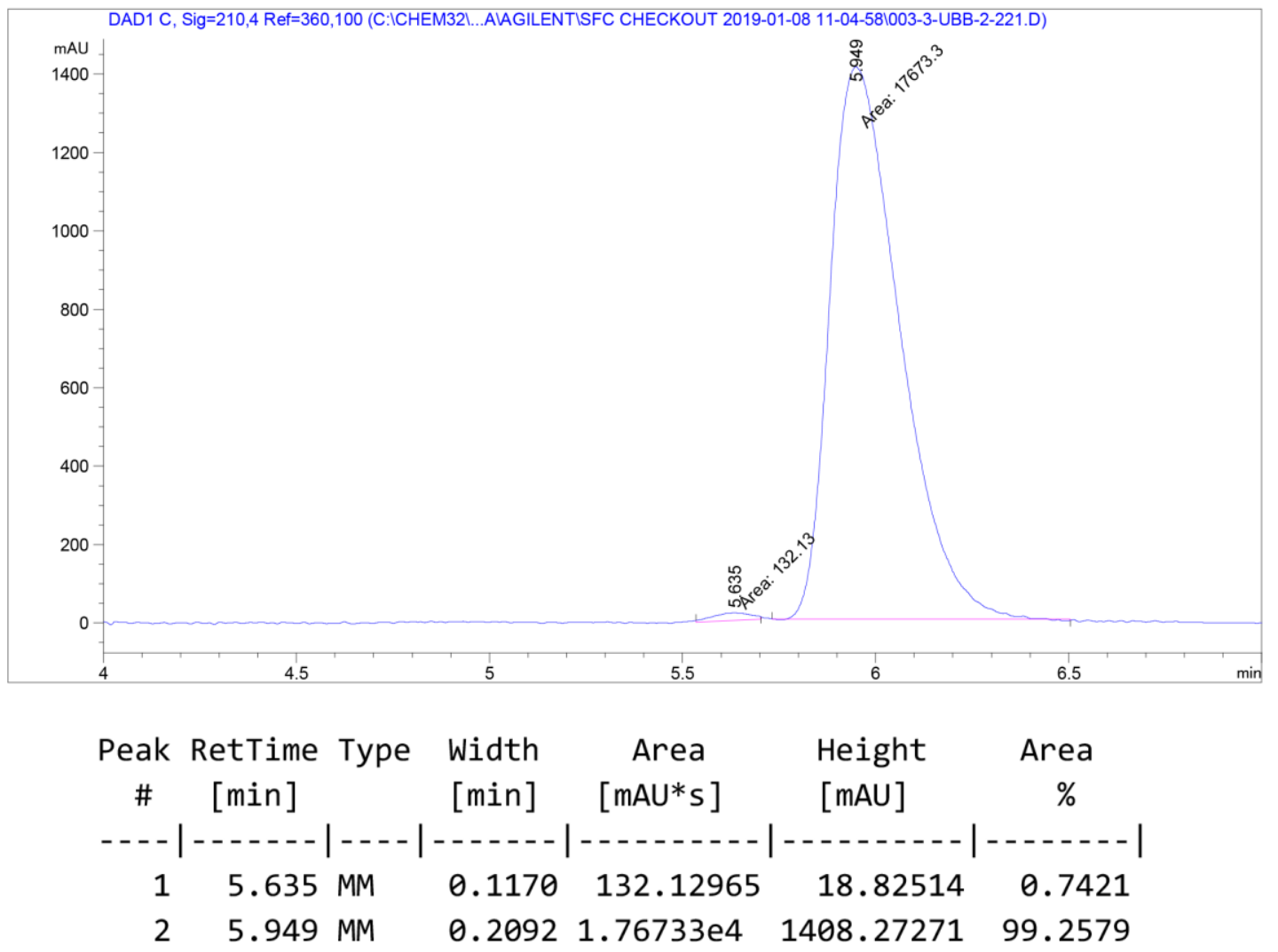



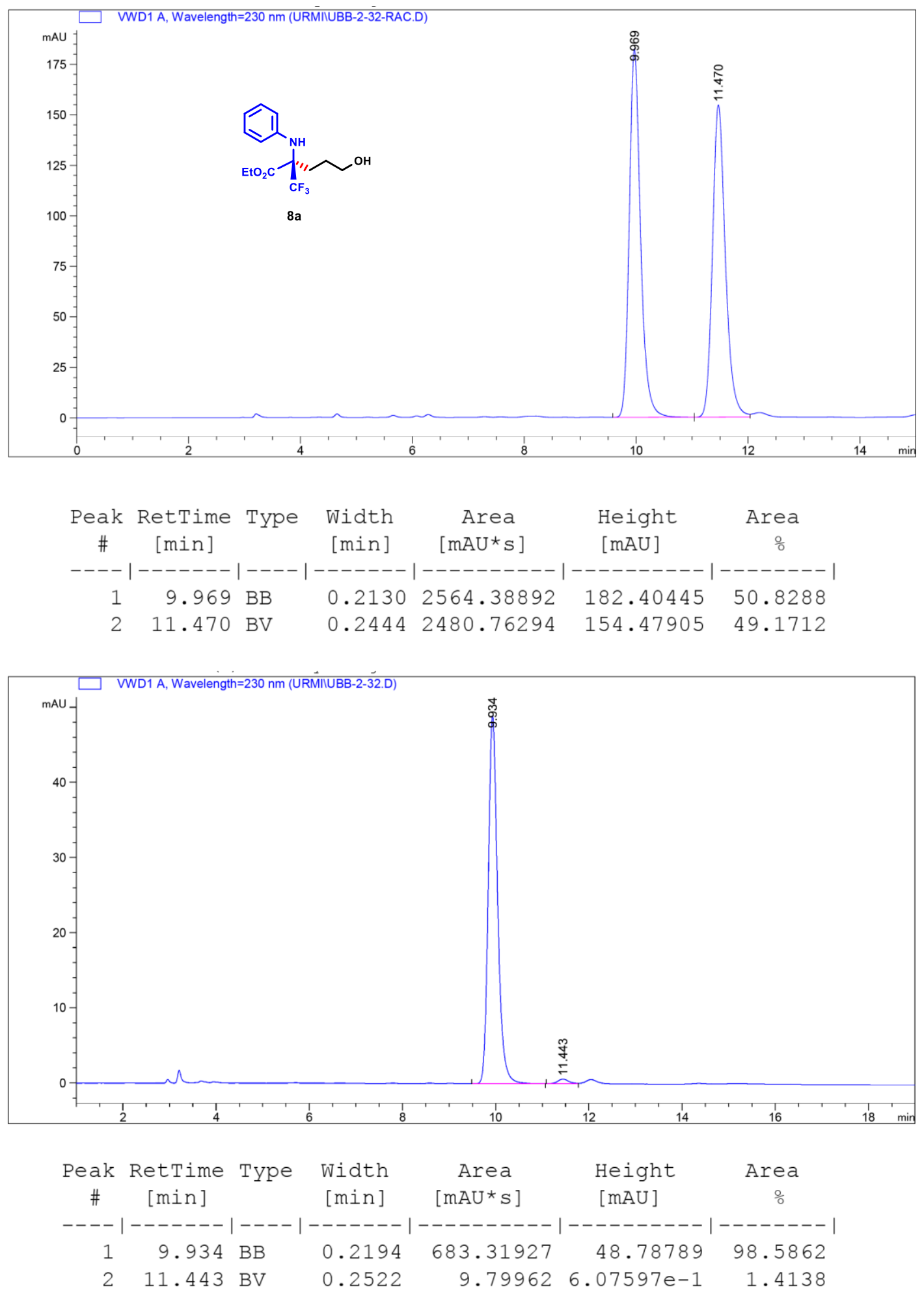


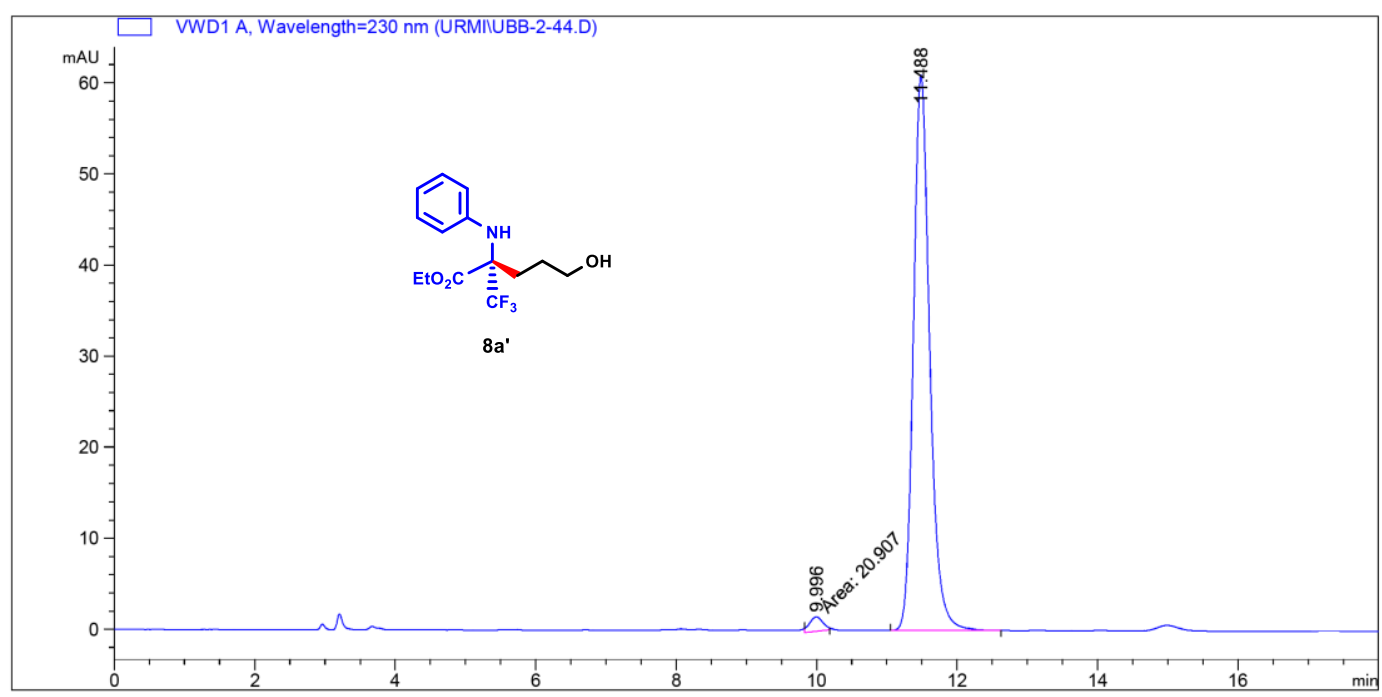

\begin{tabular}{|c|c|c|c|c|c|c|}
\hline $\begin{array}{l}\text { eak } \\
\#\end{array}$ & $\begin{array}{c}\text { RetTime } \\
\text { [min] }\end{array}$ & Type & $\begin{array}{l}\text { Width } \\
\text { [min] }\end{array}$ & $\begin{array}{c}\text { Area } \\
{\left[\mathrm{mAU}^{\star} \mathrm{S}\right]}\end{array}$ & $\begin{array}{l}\text { Height } \\
{[\mathrm{mAU}]}\end{array}$ & $\begin{array}{c}\text { Area } \\
\frac{\circ}{0}\end{array}$ \\
\hline & & & & 20.90 & & \\
\hline & & & & 982.84045 & 60.97437 & 9171 \\
\hline
\end{tabular}



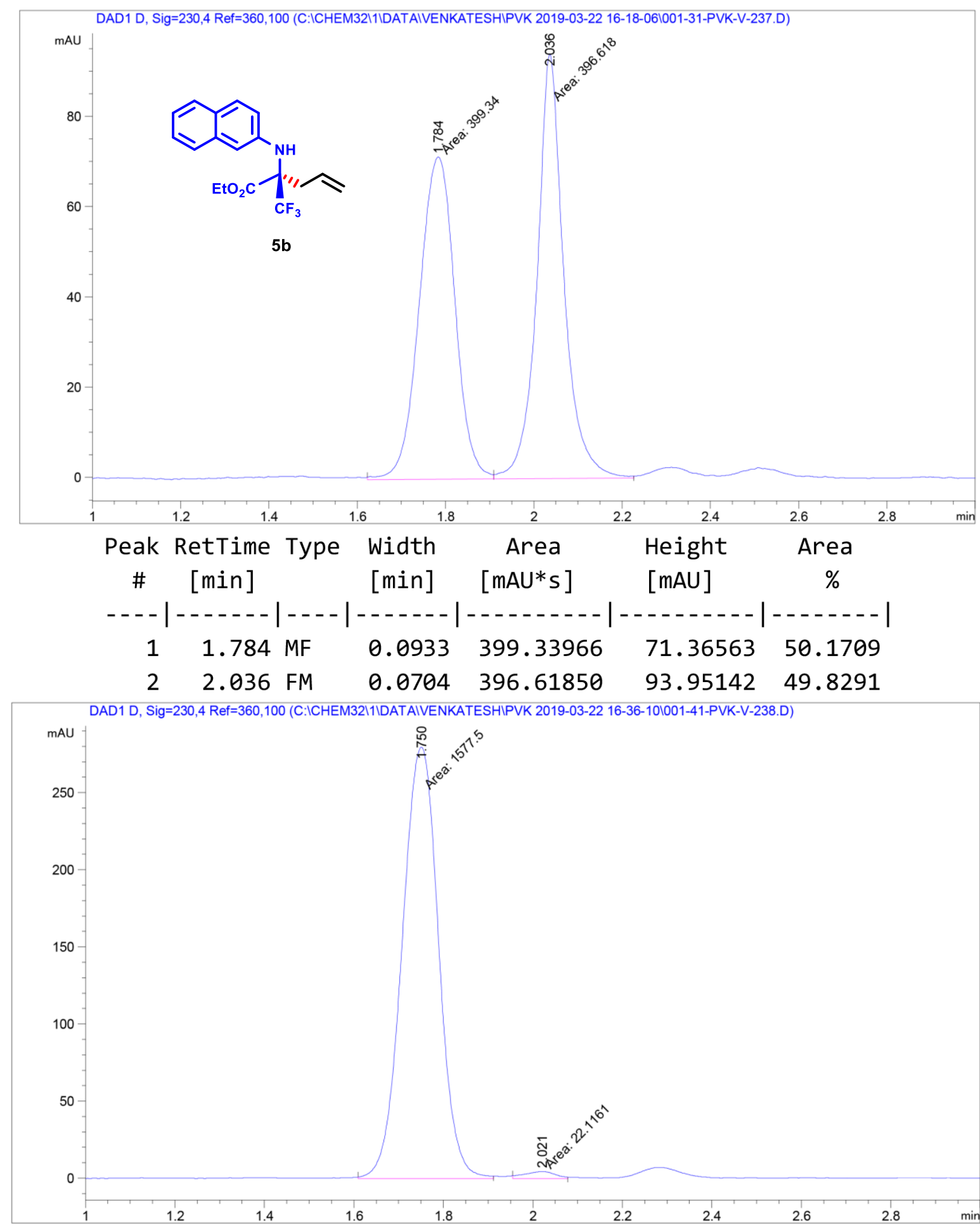

\begin{tabular}{|c|c|c|c|c|c|c|}
\hline $\begin{array}{c}\text { Peak } \\
\quad \#\end{array}$ & $\begin{array}{c}\text { RetTime } \\
\text { [min] }\end{array}$ & Type & $\begin{array}{l}\text { Width } \\
{[\mathrm{min}]}\end{array}$ & $\begin{array}{c}\text { Area } \\
{\left[\mathrm{mAU}^{*} \mathrm{~s}\right]}\end{array}$ & $\begin{array}{l}\text { Height } \\
{[\mathrm{mAU}]}\end{array}$ & $\begin{array}{c}\text { Area } \\
\%\end{array}$ \\
\hline & & & & & & \\
\hline 1 & & & & 1577.4 & 279 & 98.6 \\
\hline 2 & 2.021 & & & 22.11608 & 4.54654 & 1.3826 \\
\hline
\end{tabular}



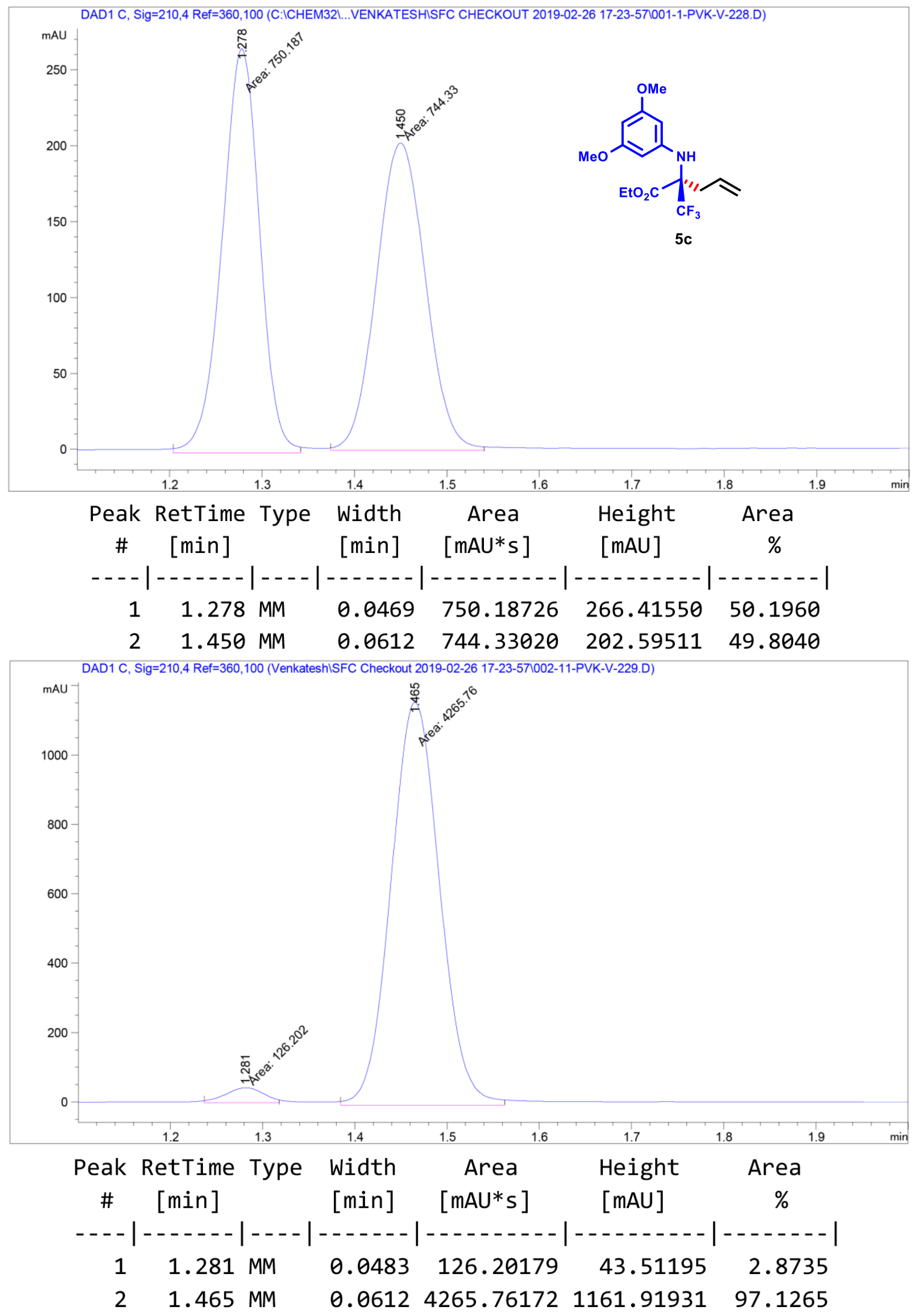

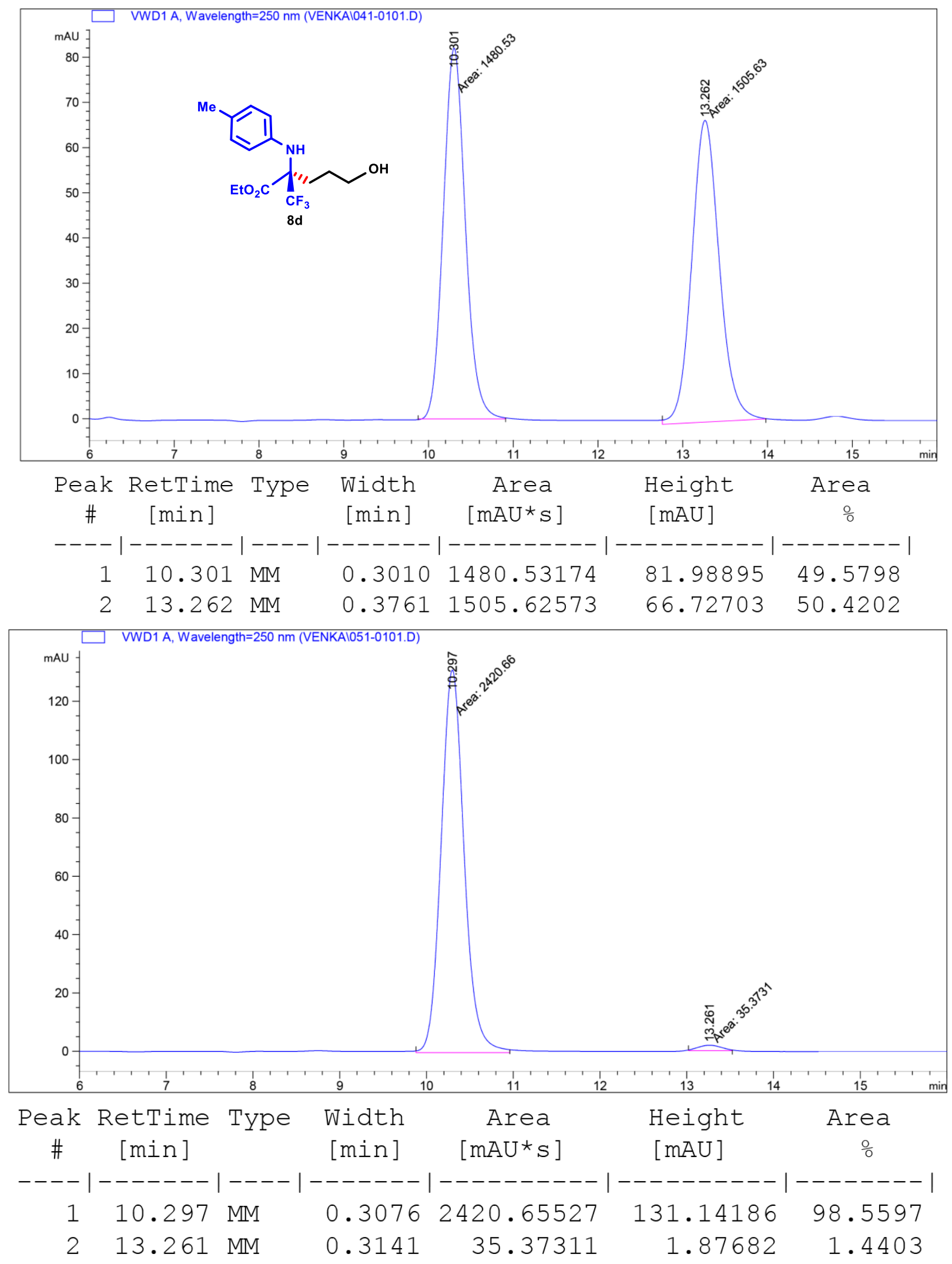

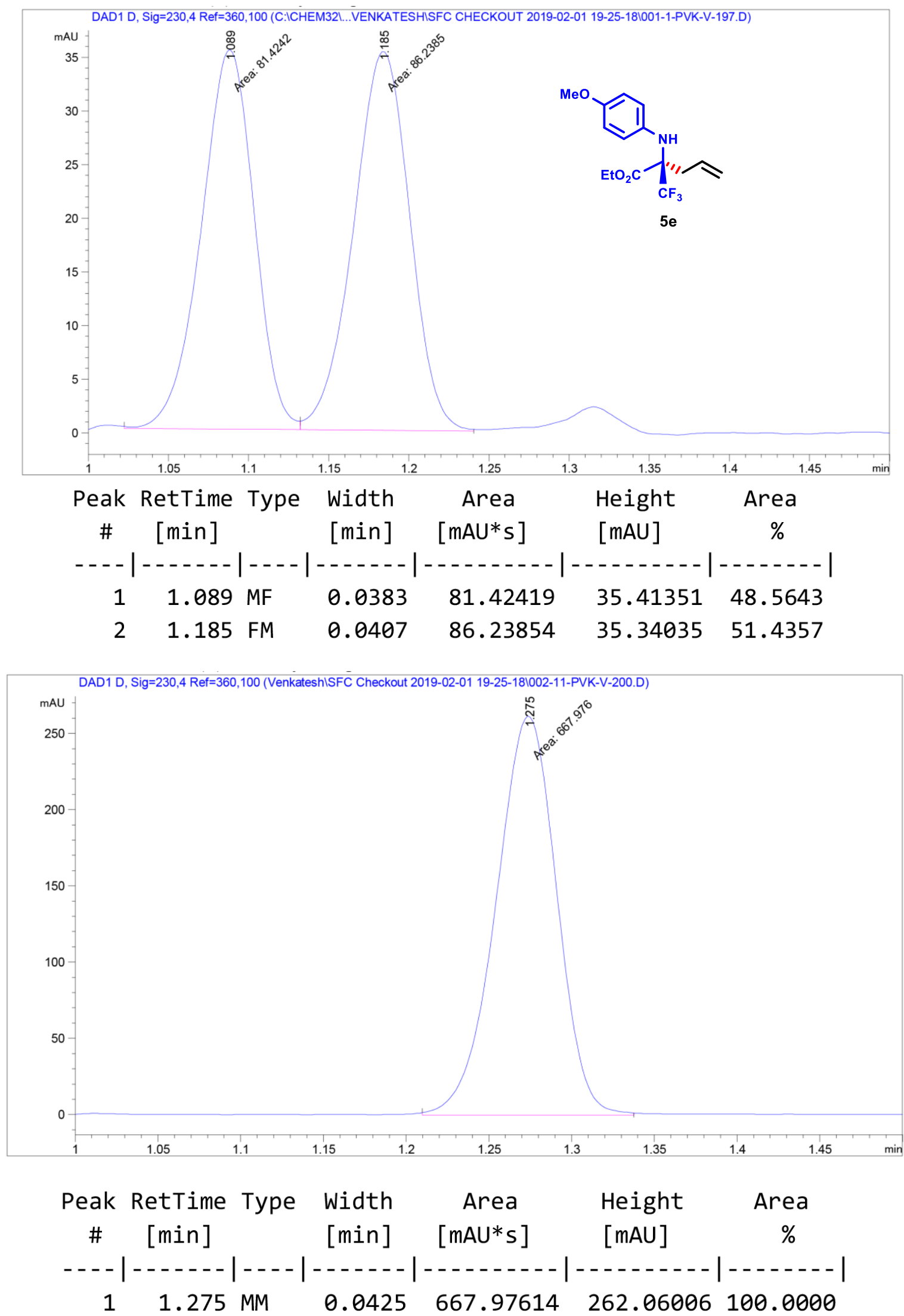

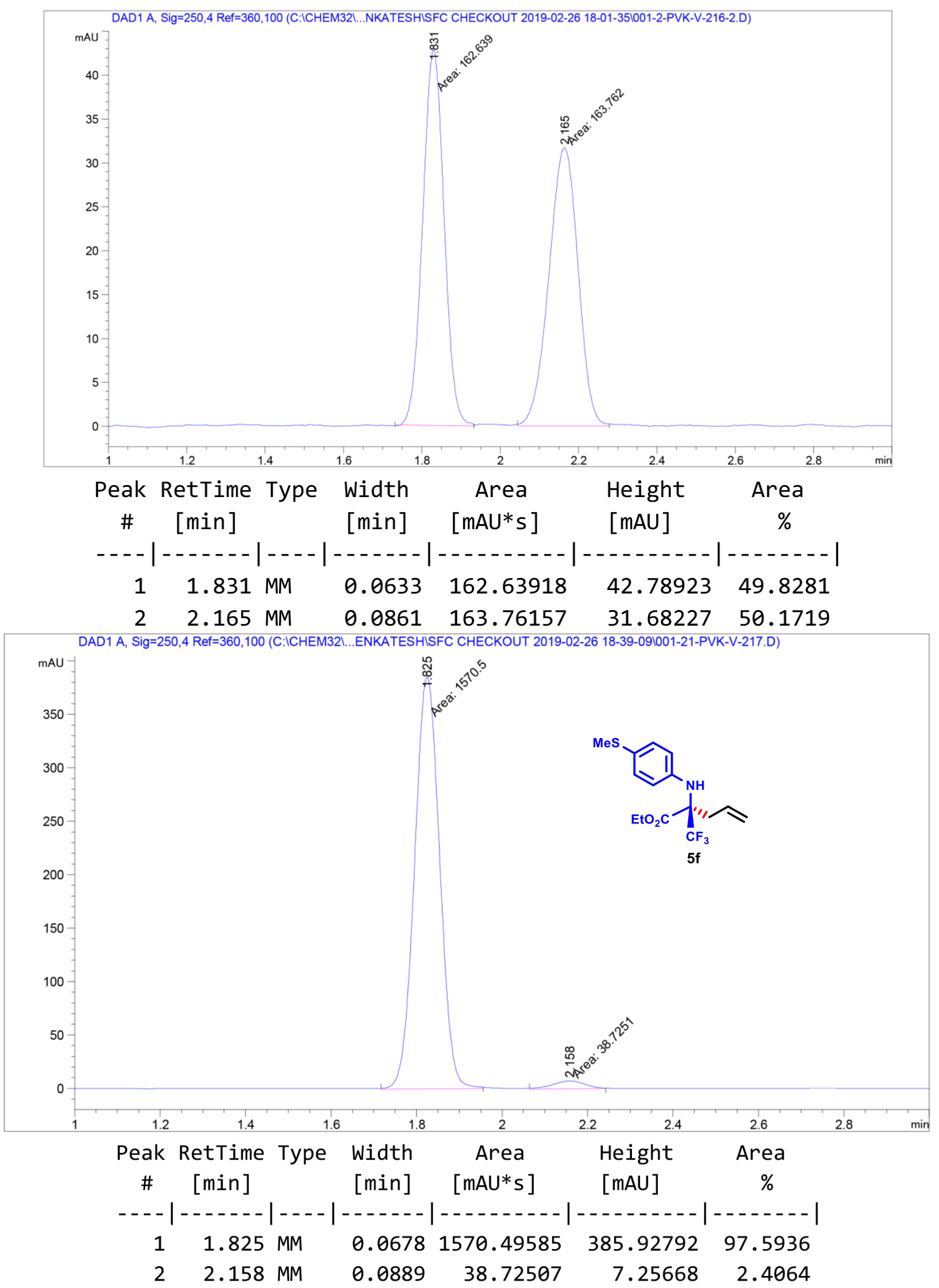


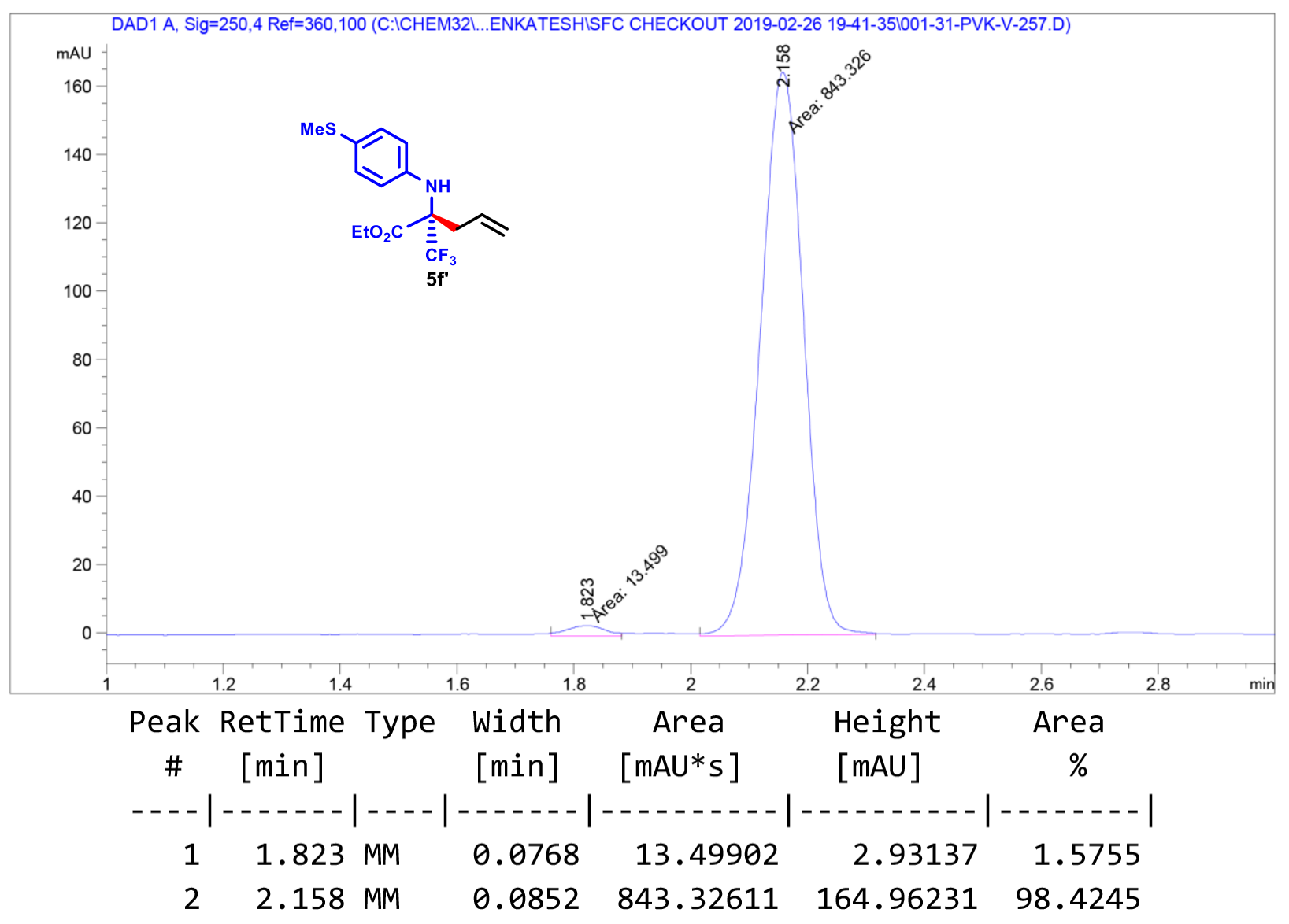



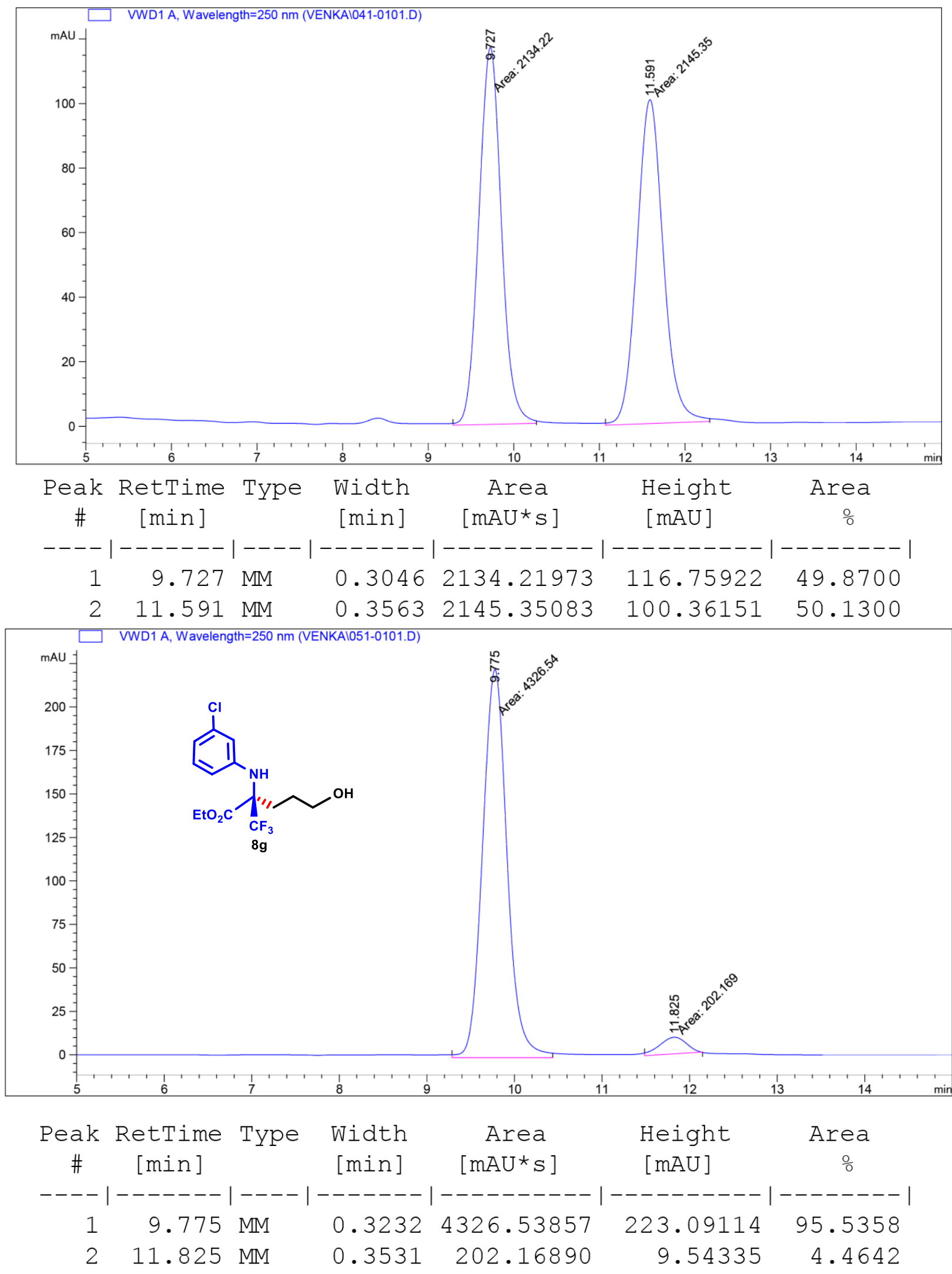


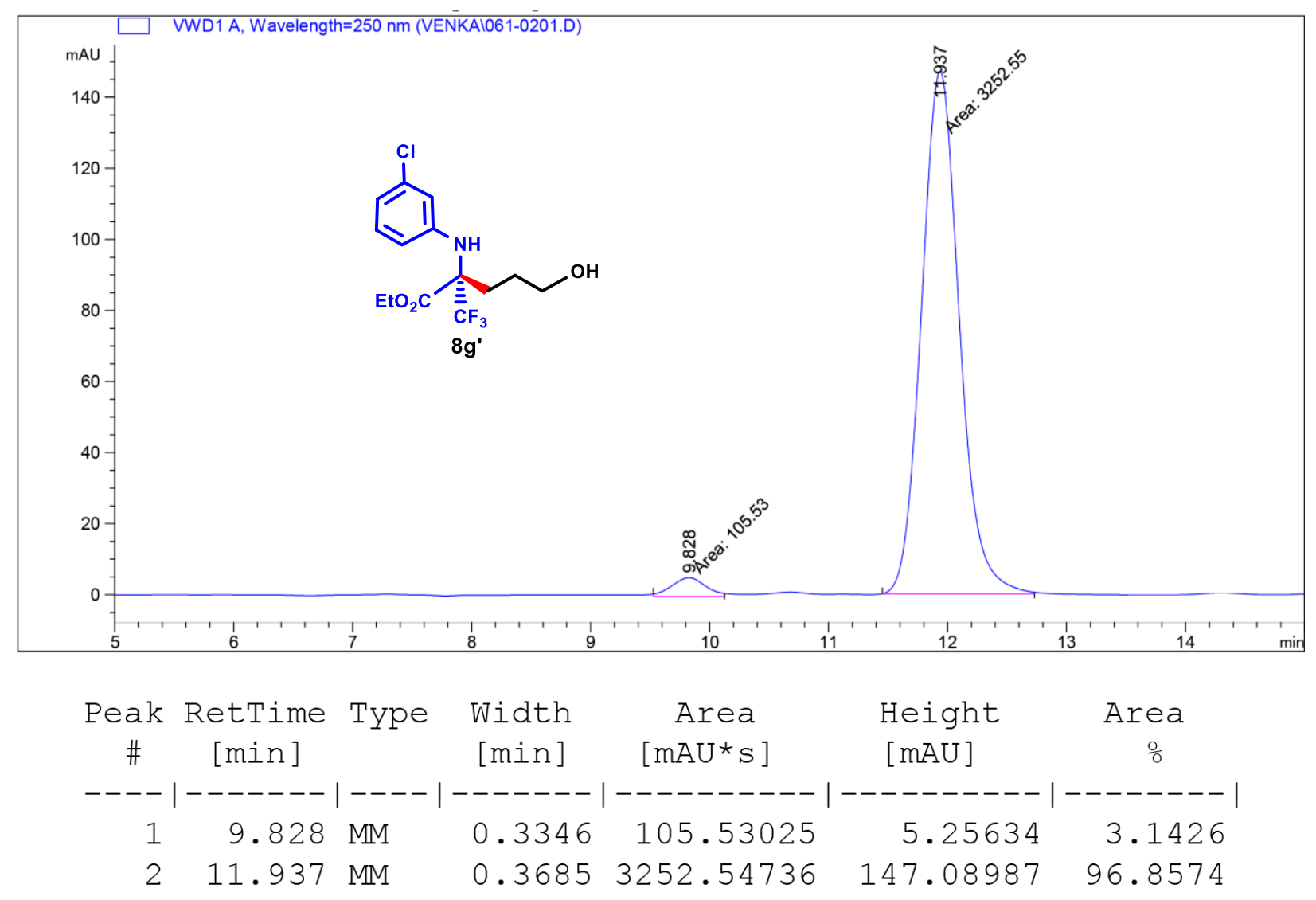



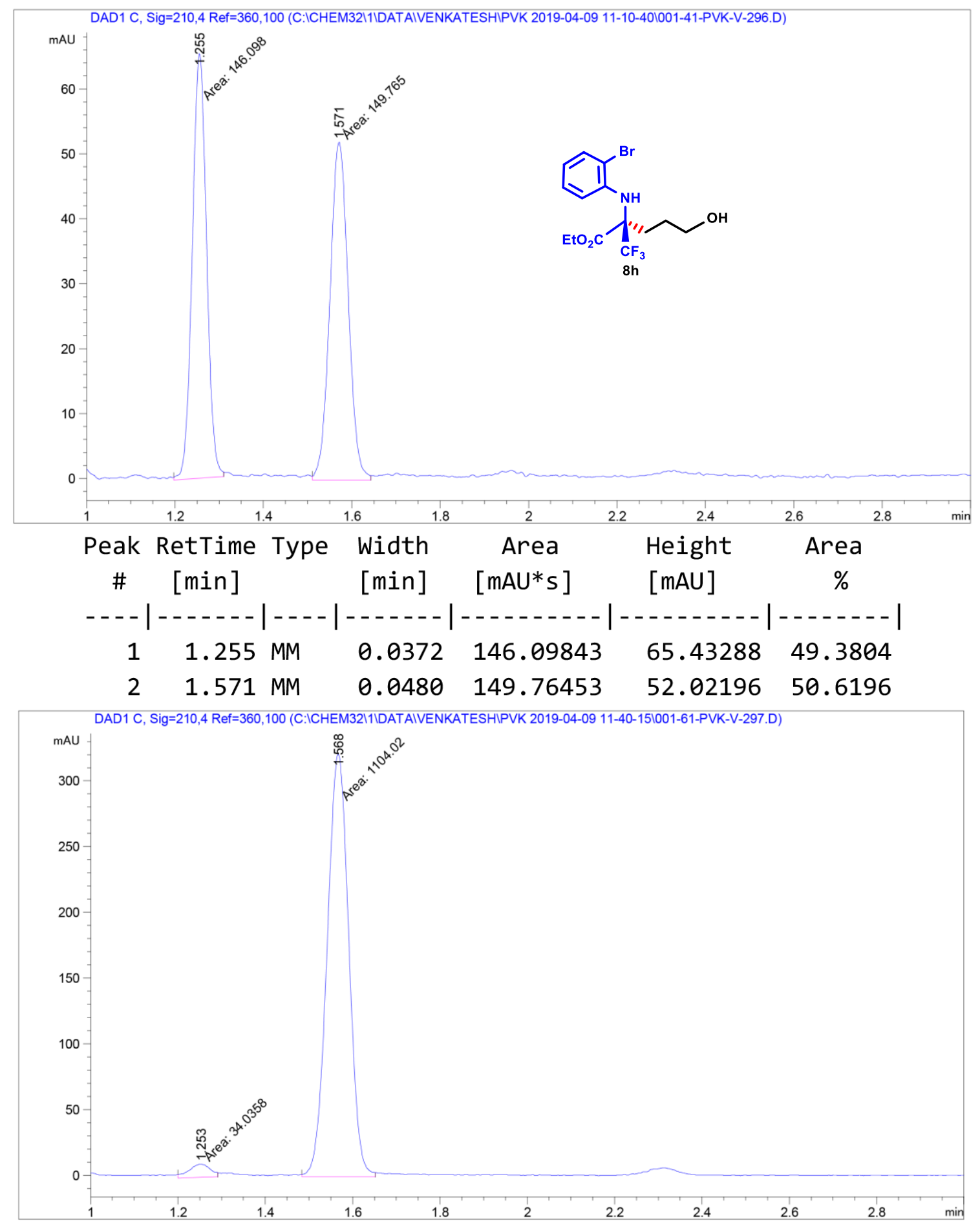

\begin{tabular}{cccccc}
$\begin{array}{c}\text { Peak RetTime Type } \\
\text { \# }\end{array} \quad \begin{array}{c}\text { Width } \\
{[\text { min }]}\end{array}$ & $\begin{array}{c}\text { Area } \\
{[\text { min }]}\end{array}$ & $\begin{array}{c}\text { Height } \\
{[\text { mAU*s }]}\end{array}$ & \multicolumn{1}{c}{$\begin{array}{c}\text { Area } \\
{[\mathrm{mAU}]}\end{array}$} & $\%$ \\
\hline 1 & 1.253 MM & 0.0573 & 34.03575 & 9.90219 & 2.9907 \\
2 & 1.568 MM & 0.0573 & 1104.02100 & 320.98459 & 97.0093
\end{tabular}



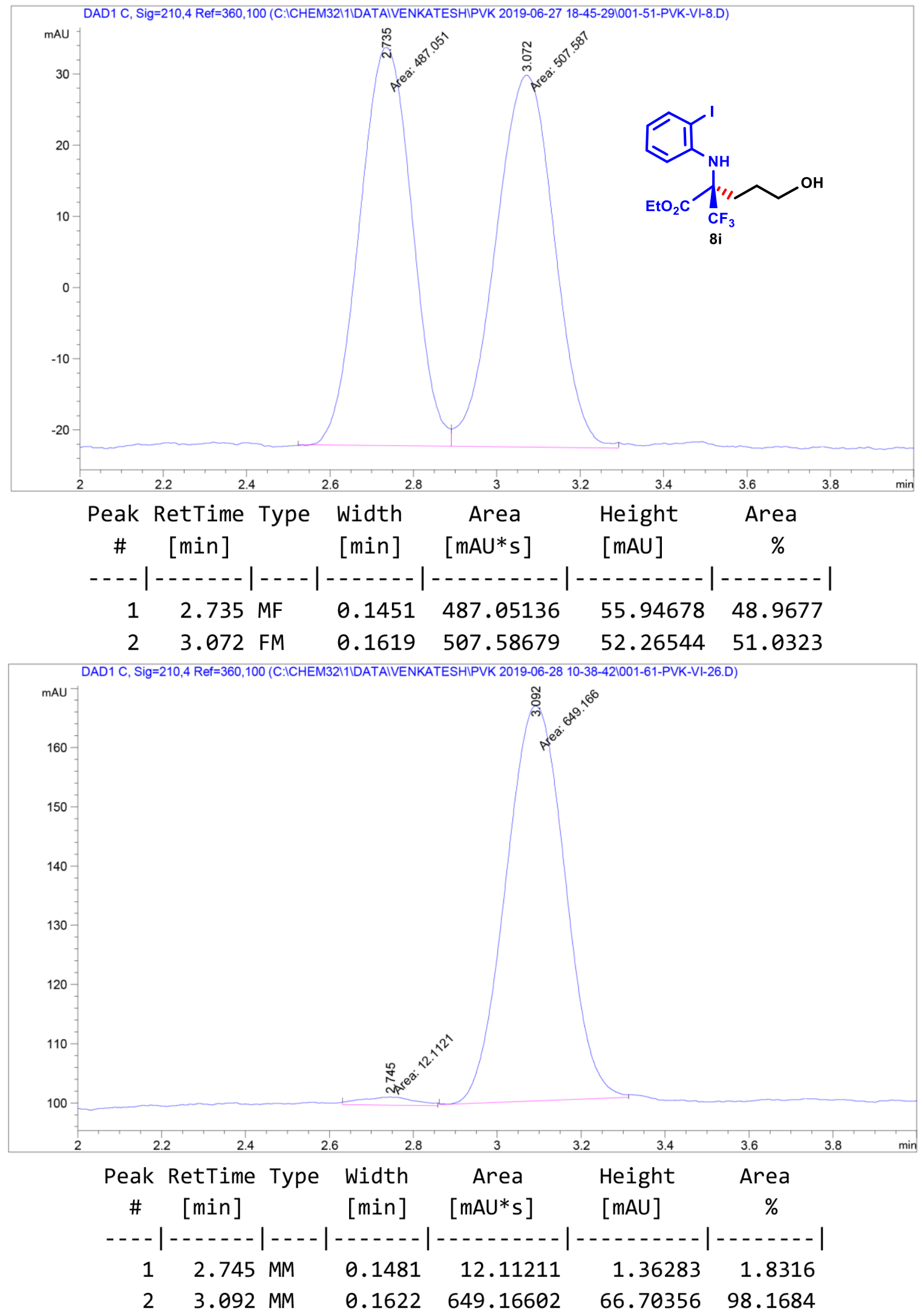


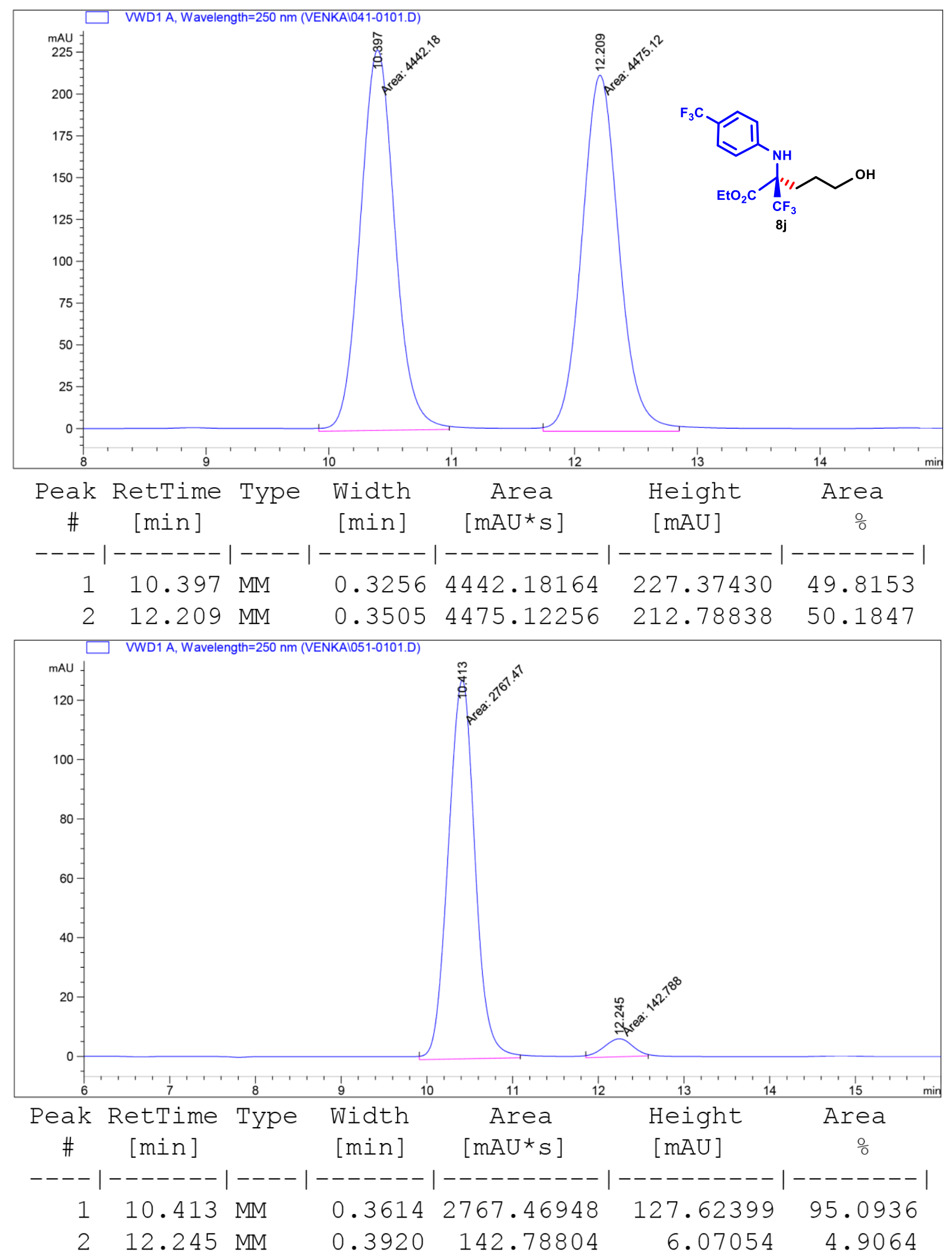



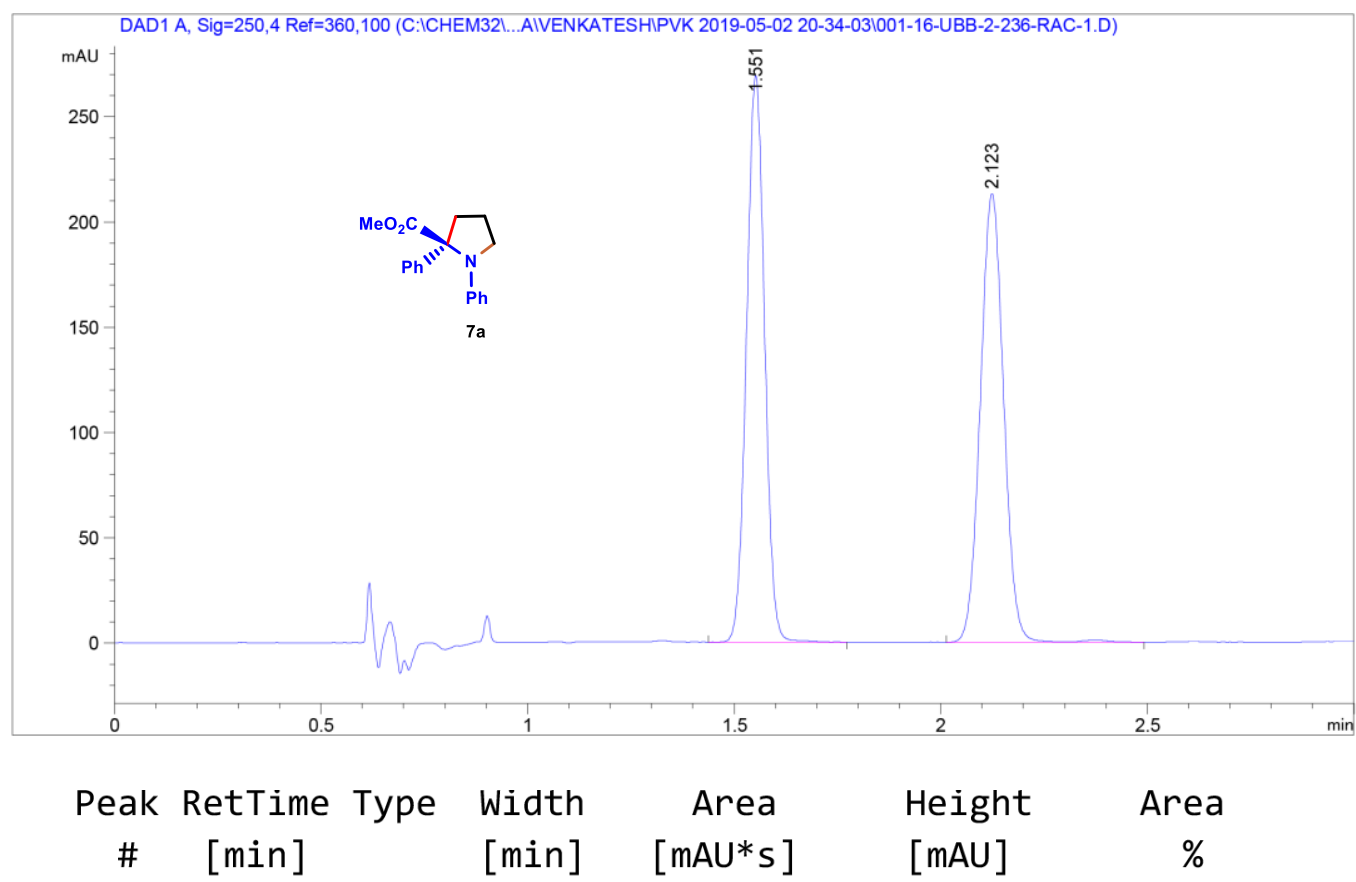

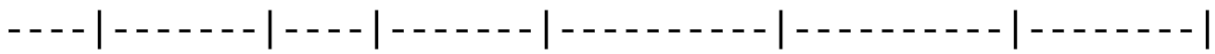

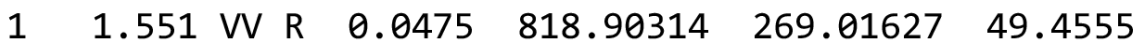

$\begin{array}{lllllll}2 & 2.123 & \text { BV R } & 0.0605 & 836.93573 & 213.03470 & 50.5445\end{array}$

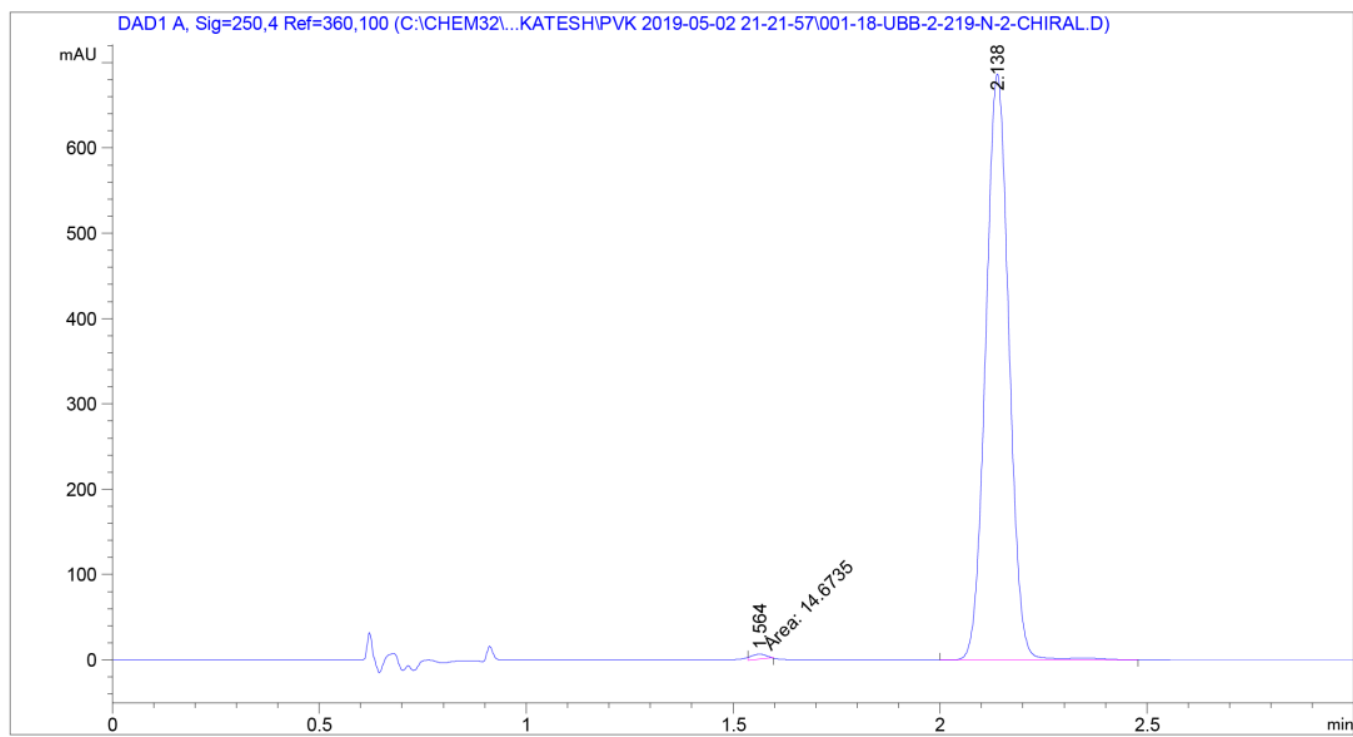

\begin{tabular}{|c|c|c|c|c|c|c|}
\hline $\begin{array}{c}\text { eak } \\
\#\end{array}$ & $\begin{array}{c}\text { RetTime } \\
\text { [min] }\end{array}$ & Type & $\begin{array}{l}\text { Width } \\
\text { [min] }\end{array}$ & $\begin{array}{c}\text { Area } \\
{\left[\mathrm{mAU}^{*} \mathrm{~s}\right]}\end{array}$ & $\begin{array}{l}\text { Height } \\
\text { [mAU] }\end{array}$ & $\begin{array}{c}\text { Area } \\
\%\end{array}$ \\
\hline & & & & & & \\
\hline 1 & & & & 14.67346 & 6.14141 & 146 \\
\hline 2 & 2.138 & $V \mathrm{R}$ & 609 & 2679.70410 & 86.42737 & 99.4554 \\
\hline
\end{tabular}



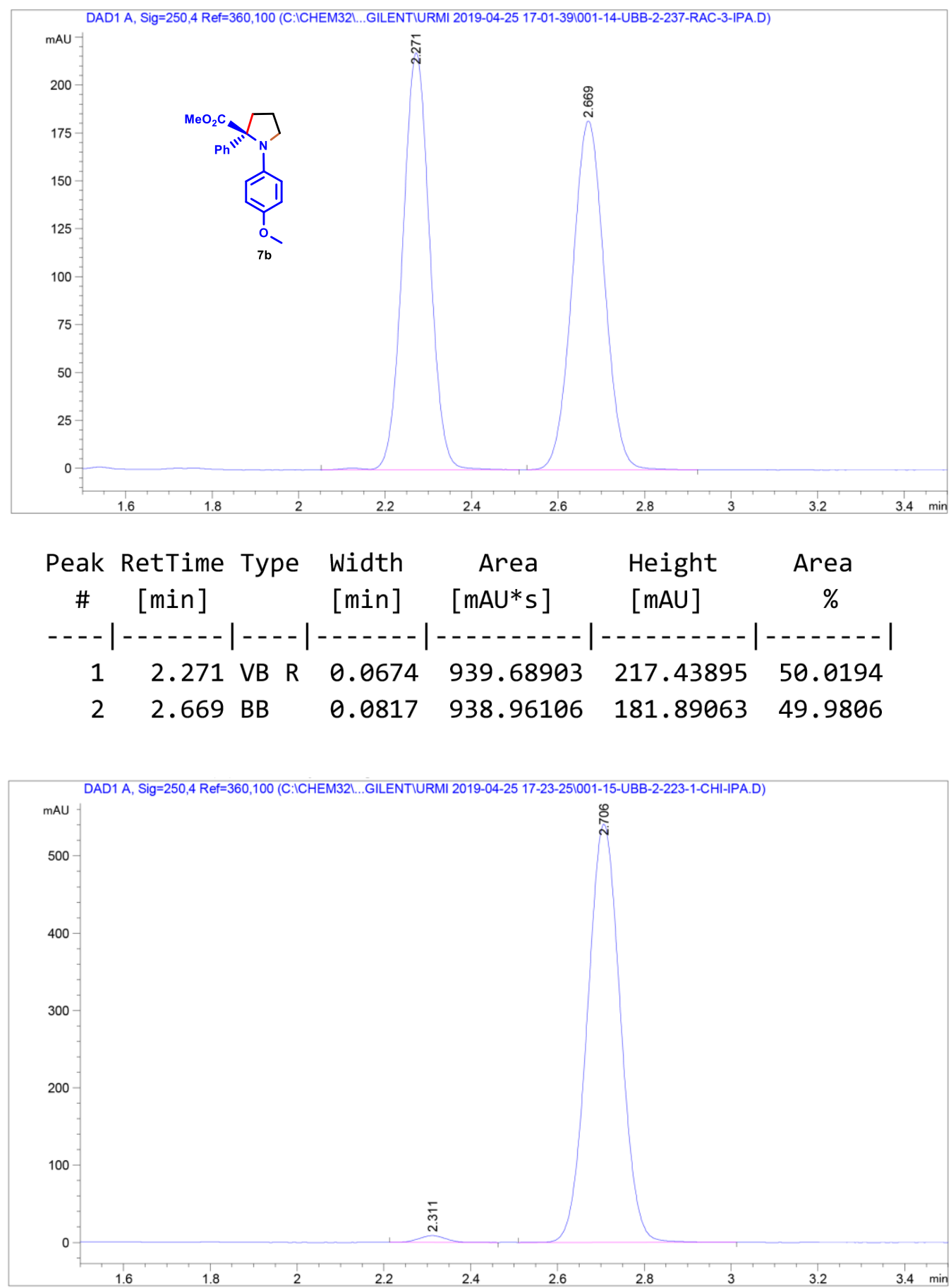

\begin{tabular}{|c|c|c|c|c|c|c|}
\hline $\begin{array}{l}\text { eak } \\
\#\end{array}$ & $\begin{array}{c}\text { RetTime } \\
\text { [min] }\end{array}$ & Type & $\begin{array}{l}\text { Width } \\
\text { [min] }\end{array}$ & $\begin{array}{c}\text { Area } \\
{\left[\mathrm{mAU}^{*} \mathrm{~s}\right]}\end{array}$ & $\begin{array}{l}\text { Height } \\
{[\mathrm{mAU}]}\end{array}$ & $\begin{array}{c}\text { Area } \\
\%\end{array}$ \\
\hline & & & & 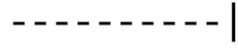 & 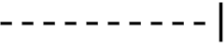 & ---- \\
\hline 1 & 2.31 & & 679 & 37.70299 & 8.72114 & 1.3048 \\
\hline 2 & 2.706 & $\mathrm{BV} \mathrm{R}$ & .0820 & 2851.96533 & 540.98425 & 98.6952 \\
\hline
\end{tabular}



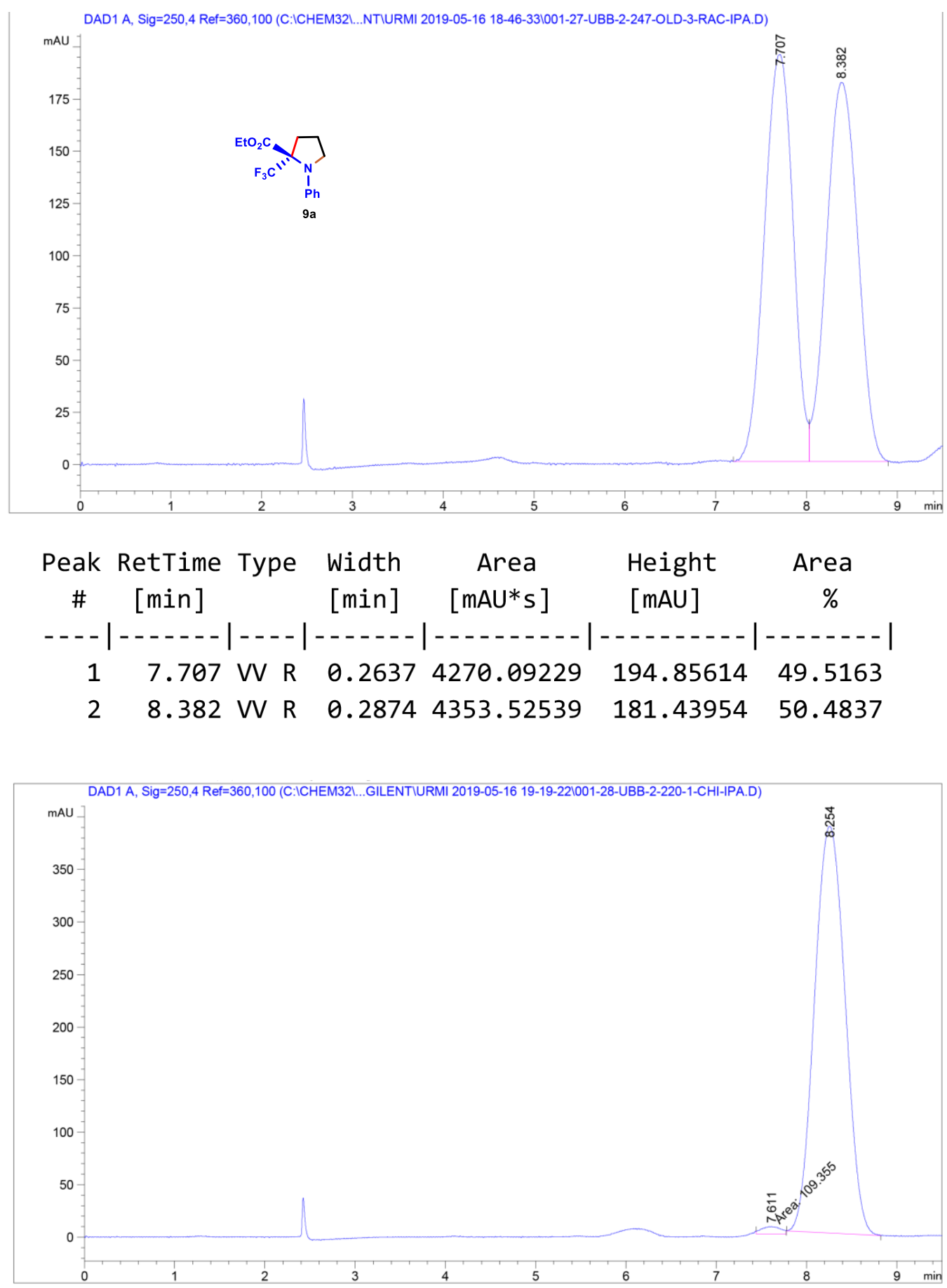

\begin{tabular}{|c|c|c|c|c|c|}
\hline $\begin{array}{c}\text { Peak } \\
\text { \# }\end{array}$ & $\begin{array}{l}\text { RetTime Type } \\
\text { [min] }\end{array}$ & $\begin{array}{l}\text { Width } \\
\text { [min] }\end{array}$ & $\begin{array}{c}\text { Area } \\
{\left[\mathrm{mAU}^{*} \mathrm{~s}\right]}\end{array}$ & $\begin{array}{l}\text { Height } \\
{[\mathrm{mAU}]}\end{array}$ & $\begin{array}{c}\text { Area } \\
\%\end{array}$ \\
\hline & & & & & \\
\hline 1 & & & 109.35461 & & 1.1870 \\
\hline 2 & $8.254 \mathrm{BV} R$ & .3449 & 9103.52832 & 387.05408 & 98.8130 \\
\hline
\end{tabular}

